NBSIR 83-2786

\title{
Technical Activities 1983 Center for Analytical Chemistry
}

U.S. DEPARTMENT OF COMMERCE National Bureau of Standards National Measurement Laboratory

Washington, D.C. 20234

October 1983

Final

Issued December 1983

Prepared for

U.S. DEPARTMENT OF COMMERCE

National Bureau of Standards

Washington, D.C. 20234 

NBSIR 83-2786

TECHNICAL ACTIVITIES 1983

CENTER FOR ANALYTICAL CHEMISTRY

H. S. Hertz, R. A. Velapoldi, and J. K. Taylor, Editors

U.S. DEPARTMENT OF COMMERCE

National Bureau of Standards

National Measurement Laboratory

Washington, D.C. 20234

October 1983

Final

Issued December 1983

Prepared for

U.S. DEPARTMENT OF COMMERCE

National Bureau of Standards

Washington, D.C. 20234

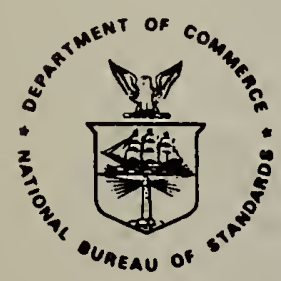

U.S. DEPARTMENT OF COMMERCE, Malcolm Baldrige, Secretary NATIONAL BUREAU OF STANDARDS, Ernest Ambler, Director 
Certain commercial equipment, instruments, or materials are identified in this report to specify adequately the experimental procedure. Such identification does not imply recommendation or endorsement by the National Bureau of Standards, nor does it imply that the materials or equipment identified are necessarily the best available for the purpose. 


\section{TABLE OF CONTENTS}

Page

\section{CENTER FOR ANALYTICAL CHEMISTRY}

A. Center Overview ................ . 1

B. Voluntary Standardization and Quality Assurance . . . . . 10

C. Laser Analytical Chemistry Group. . . . . . . . . . . . 13

1. Group Overview ................ 13

2. Selected Technical Reports ............ 14

a. Use of Laser-Enhanced Ionization in Flames for

the Analysis of Standard Reference Materials. . . . 14

b. Inclusion of Background Effects in the Computer

Simulation of LEI in Flames.......... 17

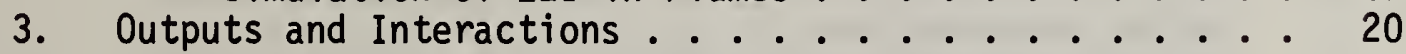

II. INORGANIC ANALYTICAL RESEARCH DIVISION

A. Division Overview ................ 23

B. Selected Technical Reports. ............ 35

1. A New Electrochemical Technique for the Estimation of Diffusion-Layer Thicknesses at Hydrodynamic

Electrodes . . . . . . . . . . 35

2. Determination of Vanadium in Botanical, Biological, and Environmental SRM's Using High-Sensitivity, Pulse-Counting Isotope Dilution Thermal Ionization Mass Spectrometry. . . . . . . . 37

3. U1 tratrace Determinations of Critical Elements in Biological Materials Using Improved Radiochemical Separations. .............. 38

4. Material Transport ............... 40

5. Analysis and Stability of Environmental Specimen Bank Samples. . . . . . . . . . . . . 41

6. The Characterization of Metastable Excited State Atomic Species Produced in Thermal Vaporization Using Resonance Ionization Mass Spectrometry (RIMS). . . . .

7. Interferences in the Determination of Germanium Using Electrothermal Atomization Atomic Absorption Spectrometry .......... . . . 47 4

8. Gases-in-Metals Analysis .............. 49

9. The Standard Potential of the $\mathrm{Ag} / \mathrm{AgCl}$ Electrode for High Accuracy $\mathrm{pH}$ Measurements. . . . . . . . . 50

10. Resonance Ionization Mass Spectrometry of Iodine and Carbon ......... 52

11. Thermal Release of Helium from Single Crystal Nickel . . 54

12. Determination of Selenium in Subcellular Fractions to Study the Glutathione Peroxidase System. . . . 57

13. The Measurement of Natural Variation in the ${ }^{32} S /{ }^{34} \mathrm{~S}$ Ratio in Atmospheric Samples ......... . 59

14. Evaluation of the Isotope Dilution Method for Ul tratrace Chromium Concentrations . . . . . . . 62 
15. Ion Optics of Single and Double Stage Mass

Spectrometers. .............. 63

16. Development of Reference Materials for Acid Rain

17. The Development of a Pulsed Thermal Atom Source For

18. Characterization of Biological SRM's Using Atomic

19. Measurement of Iodine Concentrations and Isotope

Ratios at Environmental Levels . . . . . . . . . 72

20. Certification of Uranium Isotopic Standard Reference

Materials. . . . . . . . . . . . 73

21. Neutron Depth Profiling and the Characterization of Dopant Distributions ............. . 74

22. An Automated Mass Spectrometer--Inlet System for Gas Analyses.............. 76

23. Potential Limitation in the Use of Thin Metal Films as Transmittance Standards ......... 78

24. The Effect of Manganese on the Determination of Sulfur by Optical Emission Spectrometry. ..... 80

25. Certification of NBS Buffer Materials. . . . . 82

26. Standard Reference Materials ........... 83

C. Outputs and Interactions............. 86

III. ORGANIC ANALYTICAL RESEARCH DIVISION . . . . . . . . . 101

A. Division Overview............... 101

B. Selected Technical Reports. . . . . . . . . . . 114

1. Synthesis and Characterization of Chemically Bonded

$\mathrm{C}_{18}$ Phases for Liquid Chromatography . . . . . . . . 114

2. Characterization and Comparison of Three Air

Particulate Samples for Polycyclic Aromatic

Hydrocarbon Distributions.

3. Determination of Chlorinated Pesticide Residues in Human Liver Specimens for the Pilot Environmental Specimen Bank Program. .............. 124

4. Certification of Selected Organic Compounds in SRM 1582, Petroleum Crude 0i1.......... 127

5. Determination of Relative Isomer Content in SRM 916, Bilirubin. .............. 131

6. Determination of Organic Nutrients in SRM 1549, Non-Fat Milk Powder.

7. Use of Polynuclear Aromatic Hydrocarbon Generator Columns for Calibrating Air Sampling Systems ..... . 135

8. Detection of Arson--Analys is of Accelerant Residues on Soot. ................ 137

9. Analys is of EPA Performance-Evaluation Standard Samples. ............. 138

10. Preparation and Certification of SRM Calibration Solutions. ................ 139 
11. Reference Methods for Certification of Seven Enzymes in SRM 909 ............... 142

12. Metal-Ion Affinity Chromatography Supports for Protein Separation .............. 144

13. Thin-Layer Isoelectric Focusing for Sensitive Protein Analysis ................ 145

14. Nitrogen-15 NMR Spectrum Editing by the DEPT Technique. . . . . . . . . . . 145

15. Two-Dimensional, DEPT Heteronuclear J-Resolved Carbon-13 NMR Spectroscopy ........... 149

16. Preparation and Analysis of a Quality Assurance

17. Investigation of Desorption Mechanisms in Laser Desorption and Field Desorption Mass Spectrometry. . . 155

18. Development of an Isotope Dilution/Mass Spectrometry Definitive Method for the Determination of Serum Creatinine .............. 157

19. Two-Dimensional Electrophores is System Implemented
for Protein Separation and Identification. . . . 160

20. Laser Photodissociation of Ions and Reactive Ion/Molecule Collisions: Instrumentation and First Results. .............. 161

21. Determination of Polynuclear Aromatic Hydrocarbons in Environmental Samples Using Negative Chemical Ionization Mass Spectrometry ............ 163

22. Polyethylene Methacrylate Coated Electrodes--Nonaqueous Solvent-Stable Polymer Film Electrode Modified with Methylviologen ............. 166

23. Use of a Zinc Scrubber Column for In-Line Removal of
Oxygen from HPLC Mobile Phases . . . 168

24. High-Performance Liquid Chromatographic Methods for the Determination of Nitro-Polynuclear Aromatic Hydrocarbons (N-PAH) in Complex Mixtures ....... 173

25. Spectroelectrochemical Investigations of Cyanine Dyes, Ruthenium-(bipyridine) ${ }_{2}$ dithiocarbamate Complexes, and Copper Dithiocroconates ....... 179

26. Theoretical Modeling of the Double Step

Chronocoulometry and Spectroelectrochemistry of Systems with Strong Product Deposition ......... 181

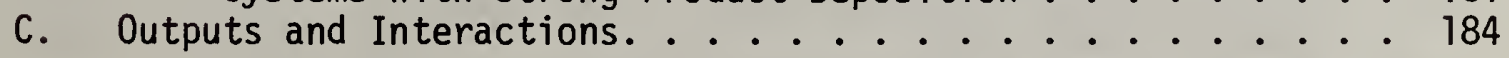

IV. GAS AND PARTICULATE SCIENCE DIVISION

A. Division Overview ................. 197

B. Selected Technical Reports. . . . . . . . 205

1. Cross-Coupled Interaction of Thermal, Electrical, Mechanical, and Chemical Parameters of Polymeric Permeation Devices. . . . . . . . . . 205

2. Band Strength Measurements of Propane by Fourier Transform Infrared Spectroscopy. . . . . . . 206 
3. Sampling and Measurement of Nitrous Oxide, Carbon

Dioxide, and Methane in the Amazon . . . . . . . . 209

4. Stability Studies of $\mathrm{NO}$ in $\mathrm{N}_{2}$ SRM's.......... . 213

5. Evaluation of Degree of Sorption in Collection of

Trace-Organic Vapors on Sorbents . . . . . . . . 216

6. Round-Robin Assessment of Gas Saturation Test Method

Proposed by EPA for Generating Vapor Pressure Data

on Organic Chemicals ............... . 218

7. Development of Techniques for Preparation and

Analys is of ppb Level Toxic Organic Standards. . . . . 221

8. Pollutant Levels in Non-protected and Protected

National Archival Storage Areas. . . . . . . . . 222

9. Toxic Gases from Lithium Battery Discharges. . . . . . 225

10. Mathematical Chemistry: Design and Evaluation of

the Analytical Measurement Process, Detection Limits, and Multidimensional Data. . . . . . . . . . . 227

11. Origins of Carbonaceous Gases and Aerosols: Survey of Recent Measurement Advances and Results....... . 231

12. Detection of $\mathrm{HCl}$ at the Sub-Parts-Per-Billion Level

Using Tunable Diode Laser Absorption . . . . . . . . 234

13. Completion of Measurements of the Temperature

Dependence of the Ozone Absorption Cross-section

in the Vacuum Ultraviolet. . . . . . . . . . . . 236

14. Reference Ultraviolet Photometer for Ozone Calibration and Assay. . . . . . . . . . . . . . . . 237

15. Completion of the Respirable Quartz SRM. . . . . . . 239

16. Use of Monte Carlo Electron Trajectory Simulation for Basic Studies in Analytical Electron Microscopy. . . . . 240

17. Visualization of the Three-Dimensional Nature of the Electron Interaction Volume in Solids. . . . . . . . 243

18. An Evaluation of X-ray Loss Due to Electron Backscatter. ................... . . 246

19. Studies of the Depth Distribution of Boron in Glass by Neutron Depth Profiling and Secondary Ion Mass Spectrometry.................... . . 248

20. Detection and Analysis of Organic Monolayers by Laser Microprobe Mass Analysis... . . . . . . . . 250

21. Standard Reference Material for Analysis of Asbestos by Electron Microscopy. . . . . . . . . . . 251

22. Visibility of Asbestos Fibers in the Scanning Electron Microscope. . . . . . . . . . . . . 253

23. An Analytical Algorithm for Calculation of Spectral Distributions of $X$-ray Tubes for Quantitative $X$-ray Fluorescence Analysis. $\cdot \dot{C}_{\alpha \text {-Coefficient Fundamental }}$

24. Comparison of Theoretical $\alpha$-Coefficient Fundamental
Parameter Method with NRLXRF for Quantitative X-ray Analysis..................... . 258

25. Final Measurements and NBS Certification of Thin-Glass Standard Reference Samples for X-ray Spectrometry and Microanalysis................... . 260

c. Outputs and Interactions. .............. 261 
I. Center for Analytical Chemistry

Harry S. Hertz, Director

Rance A. Velapoldi, Deputy Director

\section{A. Center Overview}

The Center for Analytical Chemistry develops and maintains the scientific competences and the experimental facilities necessary to provide the Nation with the basis for uniform measurements through development of measurement methodology and standards and provision of measurement services in the field of analytical chemistry. Analytical measurements provide a foundation for scientific and technological research and development concerning materials and their practical application. Moreover, analytical measurements are increasingly used as the basis for industrial, regulatory, and medical decisions. The Center serves as the National reference laboratory for assuring the reliability of analytical measurements.

The principal means by which the Center has served as a National reference laboratory is through the development and issuance of Standard Reference Materials (SRM's). The ubiquity of and demand for analytical measurements in modern society is reflected in the diversity of SRM's produced in the Center. These standards are widely used in such diverse areas as the metal and chemical industries and in clinical and environmental laboratories. Each standard is supported by research on homogeneity and stability and is certified as to chemical composition using the most reliable analytical methods available.

Analytical chemistry is increasingly being called upon to provide key data for highly complex sample types - hazardous chemical and nuclear wastes, body fluids, plant and animal tissues, foods, and high-technology materials. Often, analytical values as a function of location within a sample are needed. As the demands upon analytical chemistry become more sophisticated, the problem of standardization becomes more challenging both in terms of the number and the complexity of the proposed standard materials. As it is not reasonable to contemplate the development of standards to deal with every measurement problem, the Center's program must be multifaceted, seeking a variety of avenues to influence the quality of analytical measurements made in the United States. In addition to the issuance of standards, the Center exerts influence through close contacts with various communities by means of workshops, symposia, and participation in voluntary standards committees. Through such contacts, priorities are established for the production of standards and mechanisms are set in place for their effective use.

NOTE: Certain commercial equipment, instmuments, or materials are identified in this report to specify adequately the experimental procedure. Such identification does not imply recommendation or endorsement by the National Bureau of Standards, not does it imply that the materials or equipment identified are necessarily the best available for the purpose. 
During 1983 and 1984 we are devoting considerable effort in the Center to better define our user communities and the various mechanisms for interacting with these communities. This effort is part of the larger issue of better definition of the role and function of the Center as the National reference laboratory for analytical chemistry. The matrix on page 3 presents our current thoughts on this issue and we are now seeking the best implementation mechanisms for our efforts.

Through better definition of the role of the reference laboratory, we intend to clarify opportunities for assuring traceability of analytical measurements to national standards. In an era of increased reliance on voluntary standards it is vital, in our opinion, to assure the integrity of the measurement system through traceability to national standards. In this era, a scientifically accurate measurement base is the best support mechanism for both the industrial and regulatory sectors of the communities we serve.

The program of the Center strives to achieve a balance among the provision of currently needed standards and research efforts on new types of standards and methods of measurement. The work of the Center is performed in three divisions, each focusing upon specific sample types - inorganics, organics, and gases and particles. Within each division there are several groups representing separate analytical chemical disciplines, e.g., mass spectrometry and electrochemistry. One group, the Laser Analytical Chemistry Group, is attached to the Center office. Each division is responsible for a program in basic and applied research and maintains contacts with appropriate communities.

In the following, the major recent activities and technical directions of the divisions and groups mentioned above are given. Much work in progress is omitted but will be included in subsequent Annual Reports.

1. Outputs and Interactions

a. Publications

Schaffer, R., Sniegoski, L. T., Welch, M. J., White V, E., Cohen, A., Hertz, H. S., Mandel, J., Paule, R. C., Svensson, L., Björkhem, I., and Blomstrand, R., Comparison of Two Isotope Dilution/Mass Spectrometric Methods for the Comparison of Total Cholesterol, Clin. Chem. 28 (1), 5-8 (1982).

May, W. E., Chesler, S. N., Hertz, H. S., and Wise, S. A., Analytical Standards and Methods for the Determination of Polycyclic Aromatic Hydrocarbons in Environmental Samples, J. Environ. Anal. Chem. 12, 259-275 (1982).

White V, E., Welch, M. J., Sun, T., Sniegoski, L. T., Schaffer, R., Hertz, H. S., and Cohen, A., The Accurate Determination of Serum Glucose by Isotope Dilution/Mass Spectrometry -- Two Methods, Biomed. Mass Spectrom., 395-405 (1982). 


\begin{tabular}{|c|c|c|c|c|}
\hline 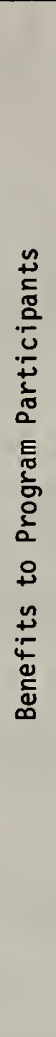 & 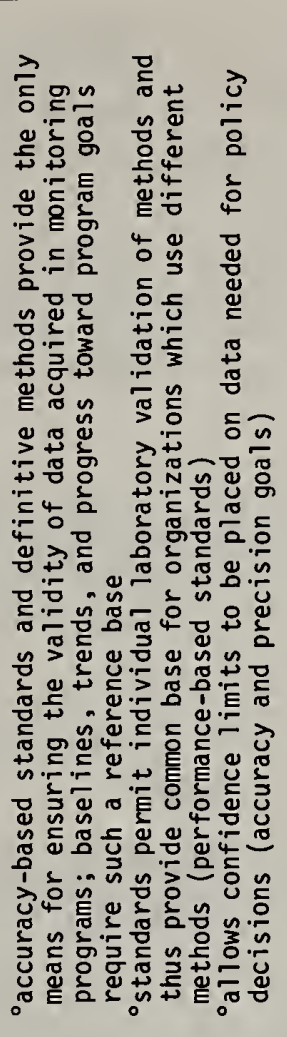 & 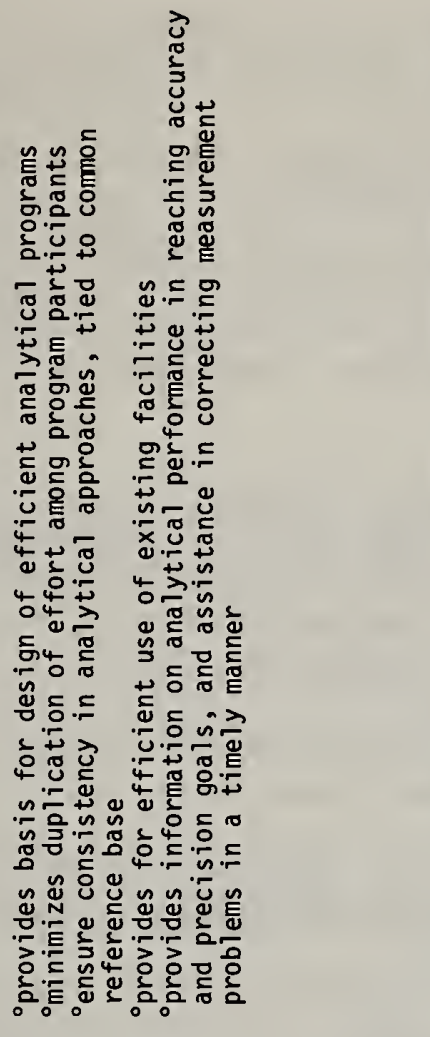 & 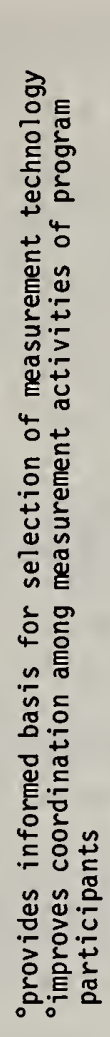 & 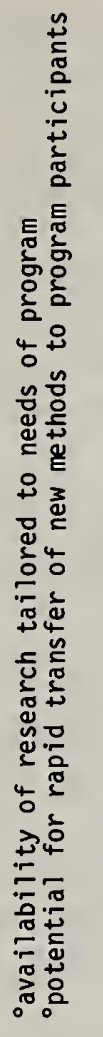 \\
\hline 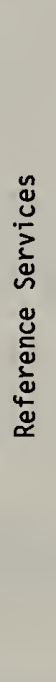 & 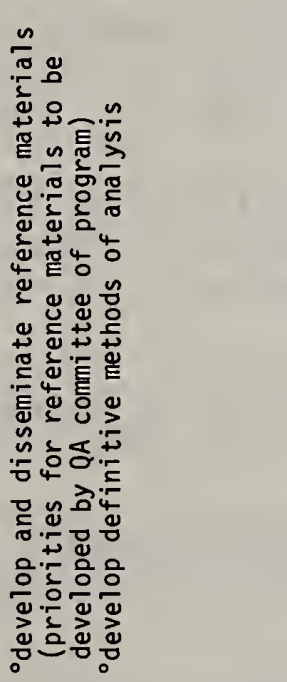 & 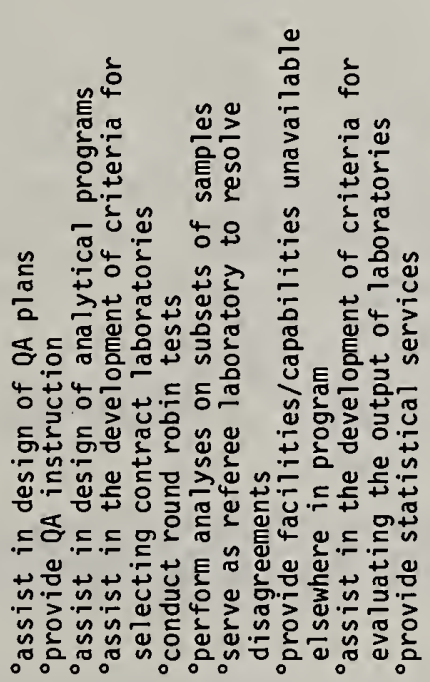 & 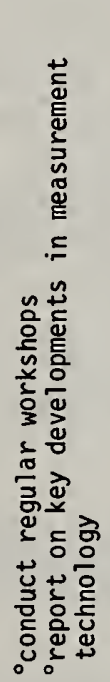 & 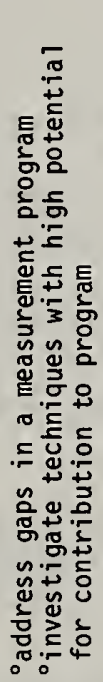 \\
\hline 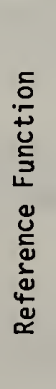 & 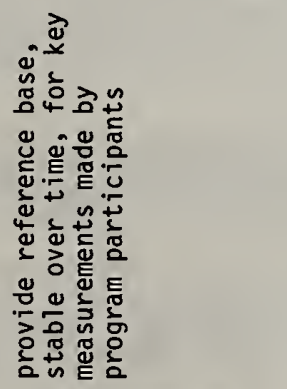 & 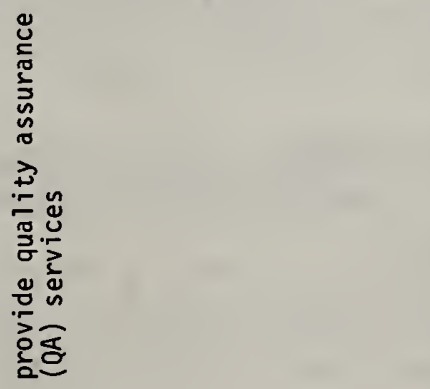 & 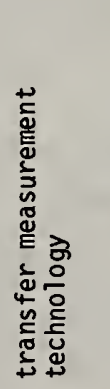 & 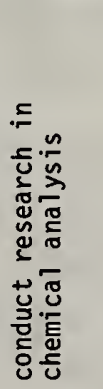 \\
\hline
\end{tabular}


May, W. E., Brown-Thomas, J. M., Chesler, S. N., Guenther, F. R., Hilpert, L. R., Parris, R. M., Richie, K. L., Sonnefeld, W. J., Wise, S. A., and Hertz, H. S., Interlaboratory Comparisons of Quantitative Analyses of Individual Compounds in Simple and Complex Mixtures, Oak Ridge Press, in press.

Yap, W. T., Schaffer, R., Hertz, H. S., White V, E., and Welch, M. J., On the Difference Between Linear and Non-Linear Models in Bracketing Procedures in Isotope Dilution/Mass Spectrometry, Biomed. Mass Spectrom., Vol. 10, 262-264 (1983).

May, W. E., Chesler, S. N., Hilpert, L. R., Hertz, H. S., Rebbert, R. E., Vogt, C. R., and Wise, S. A., "Characterization of Polycylics Aromatic Hydrocarbons in Air Particulate Extracts by Liquid and Gas Chromatographic Methods," "Identification and Analysis of Organic Pollutants in Air," L. R. Keith, ed., Butterworth Publications.

Reimann, C. W., Velapoldi, R. A., and Taylor, J. K., "Annual Report 1982, Center for Analytical Chemistry", NBSIR 82-2620.

Reedjik, J., Siedle, A. R., Van Hest, J. A. M., and Velapoldi, R. A., Coordination Compounds of Benzotriazole and Related Ligands, Inorg. Chim. Acta, 1983, in press.

Reimann, C. W., and Velapoldi, R. A., "Analytical Methods", in Encyclopedia of Chemical Technology, John Wiley and Sons, N.Y., in press.

Velapoldi, R. A., White, P. A., May, H. E., and Eberhardt, K. R., "Spectrofluorimetric Determination of Polycyclic Aromatic Hydrocarbons in Aqueous Effluents from Generator Columns", Anal. Chem. 55, 1896 (1983).

Burke, R. W. and Velapoldi, R. A., Collators. ISO Commission on Physiocochemical Measurements and Standards; Recommended Reference Materials for the Realization of Physicochemical Properties: Wavelength and Transmittance. ISO Proceedings, August 1983.

Taylor, J. K., "Validation of Analytical Methods," Anal. Chem. 55, 600A-608A (1983).

Becker, D. A., Used Lube 0i1: Hazardous Waste vs. Valuable Resource, Proceedings, Conference on Trace Substances in Environmental Health, XVI, pp. 22-27.

Becker, D. A., and Hsu, S. M., NBS Provisional Tests for Re-refined Engine $0 i 1$, Proceedings, Fourth Conference on Measurement \& Standards for Recycled $0 i 1$, September 14-16, 1982 (in press).

Becker, D. A., Analysis for PCBs in 0i1: The ASTM/NBS Round Robin, Proceedings, Fourth Conference on Measurement \& Standards for Recycled 0il, September 14-16, 1982 (in press).

Weeks, S. J., Becker, D. A., and Hsu, S. M., "ASTM/NBS Basestock Consistency Study Data", NBS Spec. Pub1. 661 (1983). 
Becker, D., Recycling 0il, Encyclopedia of Chemical Technology, Third Edition, Volume 19, 1982, pp. 979-985.

Hsu, S. M., Ku, C. S., and Becker, D. A., "Re-refined Base 0il Characterization and Consistency Monitoring," Paper in Base 0ils for Automotive Lubricants, SP-526, pp. 87-104 (Society of Automotive Engineers, 400 Commonwealth Drive, Warrendale, PA 15096, 1982).

b. $\quad \underline{\text { Talks }}$

H. S. Hertz, "The Development and Role of Definitive Methods in Clinical Chemistry" - 31st Annual Conference on Mass Spectrometry and Allied Topics, Boston, May 6, 1983.

H. S. Hertz, "SRM's and Definitive Methods: Their Roles in Clinical Chemistry", CAP/American Society for Clinical Pathologists Spring Meeting, Chicago, April 1983.

C. W. Reimann, "Trends Affecting Analytical Laboratories: Problems and Opportunities" - O'Hara Exposition Center - Rosemont, Illinois.

C. W. Reimann, "Analytical Chemistry in the Materials Sciences", - Johns Hopkins Hospital, Baltimore, Maryland, December 8, 1982.

C. W. Reimann, "An Assessment of Sciences Precision of Chemical Compositional Data Used in Support of Toxicology and Nutrition Programs," - National Academy of Sciences, February 10, 1983.

R. A. Velapoldi, "Review of the Center for Analytical Chemistry", and "Spectrophotometry and Spectrofluorimetry: Standards and Analyses", - State Bureau of Metrology, National Institute of Metrology, Beijing, China, October 18-19, 1982.

R. A. Velapoldi, Above two seminars at Shanghai Institute for Testing Technology, Shanghai Bureau of Standardization and Metrology,. Shanghai, China, October 23-24, 1982.

R. A. Velapoldi, "Accurate Measurements and Analytical Chemistry", Guest Lecturer for Course on Chromatography" by H. McNair at NBS, April 20, 1983.

R. A. Velapoldi, "Microspectrofluorimetric Measurements and Standards for Biological/Cytological Research", - Baylor College of Medicine, Houston, Texas, August 29, 1983.

R. A. Velapoldi, "Microspectrofluorimetry for Petrographic Analysis" - Conoco, Inc., Ponca City, Oklahoma, August 30, 1983.

J. K. Taylor, "Essential Features of a Laboratory Quality Assurance Program", ASTM Symposium on Statistics in the Environmental Sciences, Philadelphia, Pennsylvania, October 7, 1983. 
J. K. Taylor, "Quality Assurance of Chemical Measurements", EPA Region VII, Kansas City, M0, October 25-26, 1983.

J. K. Taylor, Quality Assurance Workshop - ADABSE Meeting, NBS, October 28, 1983.

J. K. Taylor, "Quality Assurance of Chemical Measurements", California Department of Health, Berkeley, California, February 14-15, 1983.

J. K. Taylor, "Quaiity Assurance of Chemical Measurements", California Department of Health, Los Angeles, California, February 17-13, 1983.

J. K. Taylor, "Quality Assurance of Chemical Measurements", ACS Short Course, Pittsburgh Conference, Atlantic City, New Jersey, March 4-5, 1983.

J. K. Taylor, "Quality Assurance of Chemical Measurements", Army Environmental Hygiene Agency, Edgewood, Maryland, March 28-29 and 30-31, 1983.

J. K. Taylor, "An Adequate Quality Assurance Program - The Prerequisits for Reliable Data", ACS MARM, White House, Pennsylvania, April 6, 1983.

J. K. Taylor, "Quality Assurance of Chemical Measurements", Ford Motor Co., Dearborn, Michigan, April 11-12, 1983.

J. K. Taylor, "How to Conduct a Collaboration Test of a Method", ASTM D-22 Meeting, Louisville, Kentucky, April 20, 1983.

J. K. Taylor, "Overview of Quality Assurance", FSDA Seminar, Rockville, Maryland, April 21, 1983.

J. K. Taylor, "Quality Assurance of Chemical Measurements, NBS, May 3-4, May 12-13, and May 17-18, 1983.

J. K. Taylor, "Quality Assurance of Chemical Measurements", Standard 0il Company of Ohio, Cleveland, Ohio, May 18, 1983.

J. K. Taylor, "Quality Assurance for the Laboratory", Maryland Department of Health, Baltimore, Maryland, May 24, 1983.

J. K. Taylor, "Quality Assurance of Chemical Measurements", Chemical Institute of Canada, University of Alberta, Edmonston, Alberta, Canada, July 8-9, 1983.

J. K. Taylor, "What is Quality Assurance?", ASTM Conference on Quality Assurance of Environmental Measurements, Boulder, Colorado, August 8, 1983.

J. K. Taylor, "Quality Assurance, Evaluating Analytical Methodology and Laboratory Performance", FSDA, New York Regional Laboratory, Brooklyn, New York, August 25, 1983. 
J. K. Taylor, "Quality Assurance of Chemical Measurements", ACS Short Course, ACS Meeting, Washington, D.C., August 29-30, 1983.

J. K. Taylor, "Interpretation of Collaboration Test Results", ACS Meeting, Washington, D.C., September 1, 1983.

\section{c. Committee Assignments}

Harry S. Hertz

Secretary, American Society for Mass Spectrometry

Chairman, ASMS, Cormittee on Education

Member, ASTM, Committee D19 on Water

Member, CEQ, Interagency Task. Force on Environmental Data and Monitoring Air Pollution Data and Monitoring Working Group, Task Group in Anticipatory Monitoring

Secretary, NCCLS

Technical Advisor, 0IML, SP26, Subcommittee SR5 on Reference Materials for Calibration of Instruments Meant for Checking Substances During Medico-Biological and Research Analyses

Curt W. Reimann

Chairman, Regulatory Affairs Committee, American Chemical Society

Member, Instrumentation Advisory Panel, ANALYTICAL CHEMISTRY

Co-Chairman, Symposium on Improving the Analytical Chemistry/Regulatory Interface

Member, Project Group on Quality Assurance, Interagency Task Force on Environmental Cancer and Heart and Lung Disease

Advisor, Food Chemicals Codex

Member, Analytical Laboratory Managers Association

Rance A. Velapoldi

Member, Interagency Toxic Substances Data Committee

Working Advisor, ASTM, Committee E7 on Nondestructive Testing, Subcommittee E7.03 on Liquid Penetrant and MPI Methods, Task Group E7.03.03 on E-270 Glossary Revision

Member, ASTM, Committee E13 on Molecular Spectroscopy, Subcommittee E13.06 on Molecular Luminescence

Task Group E13.06.01 on Presentation of Corrected Fluorescence Spectra

Task Group E13.06.02 on Recommended Practices on Molecular Fluorescence Spectroscopy 
John K. Taylor

Member, IS0/TC48, Laboratory Glassware and Related Apparatus

ISO/TC48/SCl, Volumetric Glassware

ISO/TC146, Air Quality

ISO/TC146/SC3, Ambient Measurements

ANSI TAG, ISO/TC172, Optical Instruments

ASTM DI9, Water

Chairman, ASTM D19.01,07, International Standards

ASTM D19.05, Inorganic Constituents

ASTM D19.07, Sediments

ASTM D22, Atmospheric Analys is

ASTM D22.04, Workplace Atmospheres

ASTM 22.01, Quality Control

Member ASTM D22.09, USTAG to TC 146

ASTM E34, Occupational Safety and Health

ASTM E36, Criteria for the Evaluation of Testing \& Inspection Laboratories

ASTM E41, Laboratory Apparatus

ASTM E41.01, Glass and Plastic Apparatus

ASTM E41.06, Weighing Devices

ASTM E41.07, Microchemical Apparatus

Collaborative Testing Subcommittee - Standard Methods

Advisory Committee - CHEMTECH (ACS)

ACS Committee on Environmental Chemistry - Subcommittee on

Environmental Analytical Chemistry

NBS Museum Committee

NBS Performance Review Board, SES

NBS Representative - Interagency Advisory Committee on Water Data

Donald A. Becker

Member, ASTM, Committee D-2, Petroleum Products and Lubricants Subcommittees $B, C, E, L, N$, and $P$

\section{d. Center Colloquium Series}

October 13, 1982

November 10, 1982

December 8, 1982
- Dr. Thomas L. Isenhour, University of North Carolina, National Science Foundation, Chapel Hill, NC, "Computer Interpretation of Analytical Data with Examples from GC/FTIR."

- Dr. Phillip D. LaFleur, Eastman Kodak Company, Rochester, New York, "Analytical Chemistry at Kodak Research Laboratories."

- Dr. Kenneth A. Rahn, Center for Atmospheric Chemistry Studies, University of Rhode Island, Narragansett, RI, "Is the Midwest Really the Source of Northeastern Acidic Aerosol? New Evidence from Elemental Tracers." 
February 23, 1983

March 16, 1983

March 23, 1983

Apri1 13, 1983

May 4, 1983

May 11, 1983

June 10,1983
- Dr. Nan Newell, Office of Technology

Assessment, U.S. Congress, "The Future of Biotechnology."

- Dr. Robert J. Charlson, Department of Civil Engineering and Institute for Environmental Studies, The University of Washington, Seattle, WA, "Factors Controlling the Acidity of Natural Rainwater."

- Dr. Shier Berman, National Research Council of Canada, Ottawa, Canada, "The Development of Reference Materials for Marine Chemistry."

- Dr. Karlheinz Ballschmiter, Department of Analytical Chemistry, University of U1m, U1m, F.R. Germany, "Studies of the Global Baseline Pollution: A Challenge in Organic Trace Analysis."

- Dr. Jaromir Ruzicka, The Technical University of Denmark, Lyngby, Denmark, "From a Test Tube to Integrated Microconduits: A New Concept of Solution Handling Based on Flow Injected Analysis."

- Dr. Robert J. Huggett, Virginia Institute of Marine Sciences, College of William and Mary, Gloucester Point, VA, "Chemical and Biological Studies of an Estuary Highly Contaminated by Polynuclear Aromatic Hydrocarbons."

- Dr. Michael E. Lipschutz, Department of Chemistry, Purdue University, West Lafayette, IN, "Thermal History of Solar Systems Objects as Revealed by Trace Elements." 


\section{B. Voluntary Standardization and Quality Assurance \\ 1. Voluntary Standardization}

The availability of carefully evaluated methods, standardized by the consensus of knowledgeable analysts is recognized as an essential component of the quality assurance of chemical measurements. Where such methods are closely related to standard reference materials and to appropriate quality assurance programs, a high quality of data is possible. Closely related to this is the requirement for evaluated and standardized analytical data.

Accordingly, the Center has played and continues to play an active role in the voluntary standardization process, both nationally and internationally. More than one-third of the staff engages in some aspect of this activity and leadership is provided in the following important standardization committees and organizations:

ASTM C -26
ASTM D-19
ASTM D-22
ASTM E-02
ASTM E-07
ISO/TC146
ISO/TC147
IUPAC
NCCLS

Nuclear Fuel Cycle Water

Sampling and Analysis of Atmospheres

Emission Spectroscopy Nondestructive Testing

Air Quality

Water Quality

Commission on Atomic Weights

National Committee for Clinical Laboratory Standards

Altogether the Center is represented on more than one hundred separate committees, and task groups of various standardization organizations. Such activity provides the opportunity for direct transfer of Center technology to a wide range of constituency. The contacts made at meetings of such organizations provide valuable input into the long-range planning of the Center.

The Center specifically interacted with the standardization community during the past year in the co-sponsoring of a Conference on the Quality Assurance of Environmental Measurements, in cooperation with ASTM and EPA. This conference, held at the University of Colorado, Boulder, $\mathrm{CO}$, during the week of August 8-12, 1983, was attended by 140 persons. The coordinator was co-chairman and presented the opening address at the conference.

\section{Quality Assurance}

To further the quality assurance program of the Center, a pilot quality circle was established in cooperation with the Organic Analytical Research Division. A quality circle consists of a group of people engaged in similar activities who meet periodically to discuss a broad scope of subjects relating to quality measurements. The group consisted of the group leaders of the Division and the Division Chief and his Deputy. The Center Quality Assurance Coordinator organized the circle and provided initial leadership. The group was joined at times by a statistician who has been responsible for analyzing much of the SRM data produced by that Division. 
The circle initially renewed basic statistical concepts and how they relate to evaluating the uncertainties of organic analytical data. The scope then changed to the consideration of measurement uncertainties in SRM's previously certified or presently being analyzed by the group. This has led to a general consideration of sources of error in organic analysis. The circle is continuing to operate largely under its own leadership with invitation of technical consultants as pertinent.

In the previous year, a generic quality assurance plan was developed in cooperation with the Office of Standard Reference Materials for the certification of SRM's. The plan formalizes the procedures that have evolved and have been followed over a long period of time. Its major features include a strong emphasis on planning the experimental work and an improved system for documenting all aspects of the certification process.

In order to test the plan, prior to its general use, three pilot projects were initiated, one in each of the Divisions. The subjects include the certification of uranium isotopic abundance standards, a mixed p-aminobenzoic acid - phenacetin standard, and calibrated permeation tubes. The considerable differences in the individual material were believed to provide a good test of the QA plan. All of the projects are now in an advanced stage and their progress has been periodically appraised on the basis of their amenability to the requirements of the QA plan. No problems have been experienced and the practicality of the plan has thus been established. In light of the favorable experience the plan is expected to be generally applied in future SRM certification.

The Center continues to assist others in their quality assurance efforts. The 2-day seminar, "Quality Assurance of Chemical Measurement", was presented three times at NBS to a total of 140 persons. In addition it was presented on two occasions to the Army Environmental Hygiene Agency, to EPA Region VII, and on separate occasions to two F\&DA field laboratories. Cooperation has been established with the American Chemical Society in presenting it as a short course which has been done twice in the past year.

The Center continues its interest in cooperating with state laboratories. Assistance has been provided to the NBS Office of Weights and Measures in the development and implementation of a Plan for Certifying the Capability of State Metrology Laboratories. A handbook on good measurement practices for such laboratories is under development. In the chemical area, the quality assurance seminar was presented to analytical chemists of the state of California in Berkeley and again in Los Angeles. A much abbreviated introductory version was presented to the environmental laboratory of the state of Maryland in Baltimore and this is expected to be extended in the future.

The Center is participating in a part-time research associate program with the Ford Motor Company. The project is concerned with investigating the merits of several approaches to quality assurance in a diversified industrial laboratory. In connection with this program, the quality assurance seminar was presented at the Ford Central Laboratory Seminar to their personnel. 
In recognition of quality assurance as the central theme of much of the Center's external program, the Center Director has made a number of contacts, especially with other government groups. As a result of this, it is expected to develop a generic quality assurance plan during the coming year for a marine environmental monitoring program involving some five other agencies.

In cooperation with the NBS Office of Standard Reference Materials, a handbook for SRM users has been planned and has advanced to the writing stage. The handbook is based on the premise that a better understanding of SRM's and their role in the chemical measurement process will improve the quality of analytical data. It will attempt to answer many of the questions raised by users of SRM's and provide guidance on the use of SRIH's in specific measurement problems.

John K. Taylor 


\section{Laser Analytical Chemistry Group}

1. Group Overview

The main long-term objective of this group is to develop new approaches to performing accurate chemical analysis. Current emphasis is on the use of lasers as spectroscopic probes. This emphasis remains strong, particularly as a result of our collaboration with the Mass Spectrometry Groups. However, a gradual shift toward the study of the material transport process is occurring as described below.

We have completed the theoretical understanding of the point charge model of laser enhanced ionization (LEI) in flames, and much has been learned in this last year's completed study. of the finite differences numerical model. We have mapped the time dependent field effects in one dimension and results show that the spatial shape of the electric field is an important parameter for assuring accurate analyses. This model shows that differences in field distribution between the anode and cathode can adversely affect the accuracy of an analytical result for the pulsed laser mode of excitation but not for the $\mathrm{CW}$ mode. Unfortunately, the necessary photon flux cannot be generated in the $\mathrm{CW}$ mode. The next best approach is to use a $\mathrm{kHz}$ pulse rate laser with adequate peak flux which simulates $\mathrm{CW}$ operation for the aspirated flame. Our plans for FY 84 include demonstrating this effect using a recently acquired copper vapor laser. We will continue our theoretical studies by using the existing mathematical model to evaluate new settings of the various experimental variables.

Our effort continues in establishing the accuracy of LEI by analyzing elements in existing Standard Reference Materials and applying LEI to the analysis of complex samples, some of which will become SRM's. In some cases it is necessary to remove elements that ionize easily in the flame; this was done by developing an ion exchange separation in collaboration with the Inorganic Mass Spectrometry Group. This year, in addition to analyzing more samples, we will be studying sources of variability in the measurement process. We will also incorporate a wavelength meter into the computer controlled system so that desired optical transitions can be accessed more easily.

Since most atomic spectroscopies best utilize atoms in the vapor phase, it would be considered useful if methods could be devised to transport atoms from the solid or liquid phase directly into the vapor without compositional bias. Spark erosion or laser ablation may do this, but studies to date are incomplete with respect to compositional bias during the transport step. Careful and detailed long term research must be devoted to this subject. The payoff is extremely high if such a method of unbiased atomic transport can be designed since the result could be matrix independent accurate analysis by many spectroscopic techniques with concomitant decrease in the need for matched matrices of standards to samples. This year we will investigate the plasma physics in the DC spark and laser ablation processes to evaluate various experimental designs. In addition, we will continue to study the relationship of chemical composition to size of the particulate matter in these processes by using microprobe and conventional analytical techniques on size-separated particles. 
We were pleased to have Professor F. E. Lytle with us on a six month sabbatical. His interaction with the Inorganic Mass Spectrometry Group on the Resonance Ionization Mass Spectrometry (RIMS) project as well as others proved to be most beneficial.

We continue to collaborate strongly with many groups in the Center: Inorganic Analytical Research Division - Mass Spectrometry Group on RIMS and on ion separation techniques; Atomic Spectroscopy Group on spark material transport; Organic Analytical Research Division - Mass Spectrometry Group on laser photofragmentation; and the Gas and Particulate Science Division Microanalysis Research Group on material transport particle analysis; Gas Metrology Group on the analysis of nitrogen dioxide with the IR diode laser; Atmospheric Chemistry Research Group on $\mathrm{HCl}$ analysis with the IR diode laser, material transport particle classification, and the designs of signal processing of carbon-14 detectors. Summaries of these collaborations can be found in the Divisions' sections of this report.

\section{Selected Technical Reports}

a. Use of Laser-Enhanced Ionization in Flames for the Analysis of Standard Reference Materials (11310)

G. C. Turk and H. M. Kingston (551)

Performance evaluation of laser-enhanced ionization (LEI) spectrometry for trace metal analysis has continued with the application of the technique to the analysis of standard reference materials. Fuel 0i1 (SRM 1634a), Incoloy and Inconel Specialty Alloys (SRM's 1244-1247), and Trace Elements in Water (SRM 1643a,b) are among the samples which have been analyzed using LEI.

The incoloy and inconels are specialty alloys with high $\mathrm{Ni}, \mathrm{Fe}$, and $\mathrm{Cr}$ contents. Because of the complex spectral background resulting from the major elements, the determination of trace elements using conventional optical methods can be difficult. The high sensitivity and double-resonance selectivity of stepwise excitation LEI can be useful for accurate analys is of trace metals in these samples. We have demonstrated this with the determination of Co in the proposed series of incoloy and inconel SRM's.

Laser excitation of Co was done in a stepwise manner, with one laser at $252.14 \mathrm{~nm}$ exciting the atom to an intermediate level, and a second laser at $591.68 \mathrm{~nm}$ further exciting the atom from the intermediate level to within one $\mathrm{eV}$ of the ionization potential of $\mathrm{Co}$, where efficient collisional ionization in the flame occurs. A 170-fold sensitivity enhancement is realized through the use of the stepwise excitation method rather than with the first step wavelength alone. Figure 1 shows a LEI wavelength scan taken while aspirating a solution of SRM 1244 inconel into the air-acetylene flame. The solution was prepared from an acid digestion of a 1 gram sample diluted to $2 \mathrm{~L}$ with distilled water. The scan was taken with the second step wavelength fixed and the first step wavelength scanned in the vicinity of the Co first step line. Note the comparable level of the Co signal, which is enhanced by stepwise excitation, to the nearby single photon lines from $\mathrm{Fe}$, which is present at approximately 


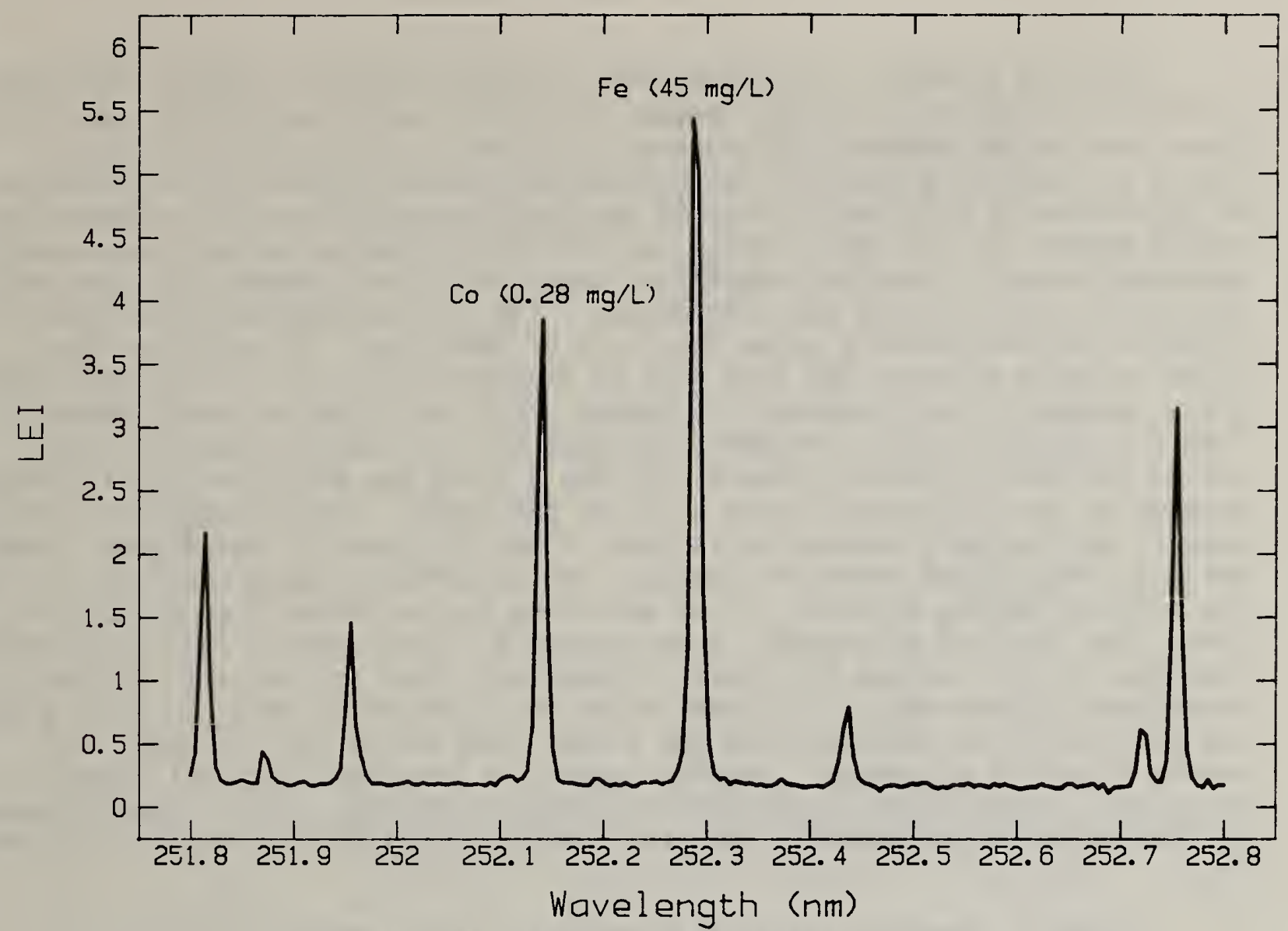

Figure 1. LEI spectral scan taken while aspirating $1 \mathrm{~g} / 2 \mathrm{~L}$ solution of inconel SRM 1244. A second step laser wavelength is fixed at $591.68 \mathrm{~nm}$, which enhances Co signal at $252.14 \mathrm{~nm}$ by a factor of 170 .

160 times the co concentration. Listed below are the average results obtained from three replicate measurements each of two independent sample preparations for each SRM in the series. Also included is the average result obtained for SRM 1289 high alloy steel, which was used as a quality control standard.

Table 1. Cobalt in Incoloy and Inconel Alloys.

\begin{tabular}{|c|c|c|}
\hline SRM & Co $(\mu \mathrm{g} / \mathrm{g})$ & $\begin{array}{c}\text { Standard Deviation } \\
\text { of Average }\end{array}$ \\
\hline 1244 & 613 & 6 \\
\hline 1245 & 738 & 7 \\
\hline 1246 & 745 & 12 \\
\hline 1247 & 915 & 8 \\
\hline 1289 & $345(350 \pm 40)^{a}$ & 2 \\
\hline
\end{tabular}


Six trace elements were determined in Trace Elements in Water, SRM 1643a (previously certified) and the renewal SRM 1643b (being certified). Work with these samples was hampered by broadband spectral background interferences caused by alkali and alkaline earth elements present in the sample. Because of the extremely high sensitivity of the low ionization potential elements to single photon LEI and their excess concentration relative to the trace metals, broadband signals from the Lorentzian "wings" of strong absorption lines are observed several $\mathrm{nm}$ from the line center. The noise associated with these signals can substantially raise the limit of detection. In addition, the alkaline earth elements can give rise to broadband molecular LEI signals. For these reasons, it was necessary to remove alkali and alkaline earth elements from the sample, using a procedure designed for such purpose based on the use of Chelex-100 chelating resin. Figure 2 shows how effectively this procedure works for LEI determination of $\mathrm{CO}_{0}$ in SRM 1643b. The interference in this case is particularly severe, as is seen in the top trace of the figure, taken while aspirating the untreated sample. The background signal observed is from the wings of the $\mathrm{Na} D$ lines, $2.7 \mathrm{~nm}$ away from the Co second step excitation line. The level of $\mathrm{Na}$ present in the sample is approximately 200 times greater than that of $\mathrm{Co}$, and the Co signal is completely lost in the noise of the $\mathrm{Na}$ background interference. The lower trace was taken after removal of the alkali and alkaline earth elements, and the signal from the $26 \mathrm{ng} / \mathrm{g}$ of $C_{0}$ in the sample is easily discerned. Varying degrees of broadband spectral interferences were observed for all of the six elements studied, with all being removed by the Chelex-100 procedure. The table below summarizes the results obtained.

Table 2. Analysis of Trace Elements in Water SRM's Using LEI Flame Spectrometry.

\begin{tabular}{|c|c|c|c|c|}
\hline \multirow[b]{2}{*}{ Element } & \multicolumn{2}{|c|}{ SRM $1643 a$} & \multicolumn{2}{|r|}{ SRM 1643b } \\
\hline & Average $^{\mathrm{a}}$ & Certified Value & Average $^{\mathrm{b}}$ & Std. Dev. of Ave \\
\hline $\mathrm{Cd}$ & 10.2 & $10 \pm 1$ & 20.0 & 0.2 \\
\hline Co & 20.5 & $19 \pm 2$ & 26.0 & 0.1 \\
\hline $\mathrm{Cu}$ & 17.8 & $18 \pm 2$ & 21.8 & 0.1 \\
\hline$M n$ & 31.0 & $31 \pm 2$ & 30.1 & 0.3 \\
\hline $\mathrm{Ni}$ & 52.3 & $55 \pm 3$ & 46.6 & 0.2 \\
\hline $\mathrm{Pb}$ & 26.9 & $27 \pm 1$ & 23.9 & 0.3 \\
\hline
\end{tabular}

af 2 samples prepared.

bof 7 samples prepared. 


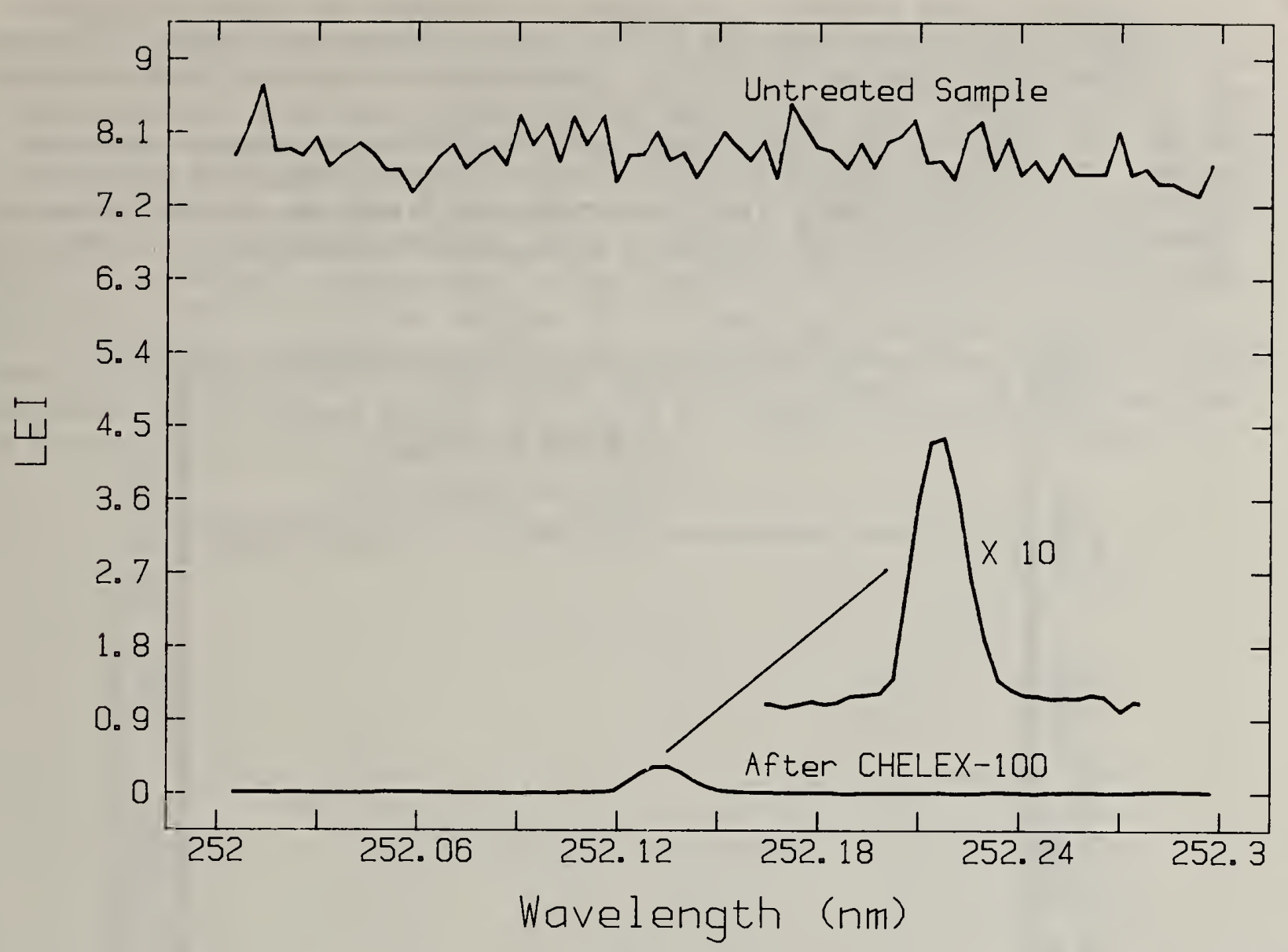

Figure 2. Stepwise excitation LEI signal for $26 \mathrm{ng} / \mathrm{g}$ of Co in water SRM 1643b before and after removal of alkali and alkaline earth elements by Chelex-100 procedure. Ten-fold scale expansion of Co signal in the treated sample is also shown. First step wavelength is scanned with second step wavelength fixed at $591.68 \mathrm{~nm}$.

b. Inclusion of Background Effects in the Computer Simulation of LEI in Flames (11310)

J. C. Travis, J. Wen, and P. K. Schenck (561)

In last year's report, we described a computer simulation of charge transport and current detection following pulsed LEI in flames (NBSIR 82-2620, section 2.a). In the model described, a fixed number of ions and electrons was deposited at time zero at a given spatial coordinate. The electrically perturbed flame in which these charges were deposited was simulated by a predicted electric field distribution and appropriate electron and ion mobilities. The sophistication of the simulation has since been considerably increased by computing the background flame conditions (electron and ion denisities and the electric field distribution) from first principles, and permitting the background to respond to the perturbation of the laser-added charges. 
Figure 3 shows a typical background calculation for a simulated airacetylene flame between infinite plane parallel electrodes spaced at $2.0 \mathrm{~cm}$ at a potential difference of $1500 \mathrm{~V}$. Because electrons move 100-1000 times faster than atomic ions in response to an electric field, a positive ion excess, or space-charge, accumulates near the cathode at steady-state, as shown. This charge results in an electric field gradient, such that the electric field may actually fall to zero in the flame, as shown, for certain combinations of background ionization rate, applied potential, and electrode spacing.

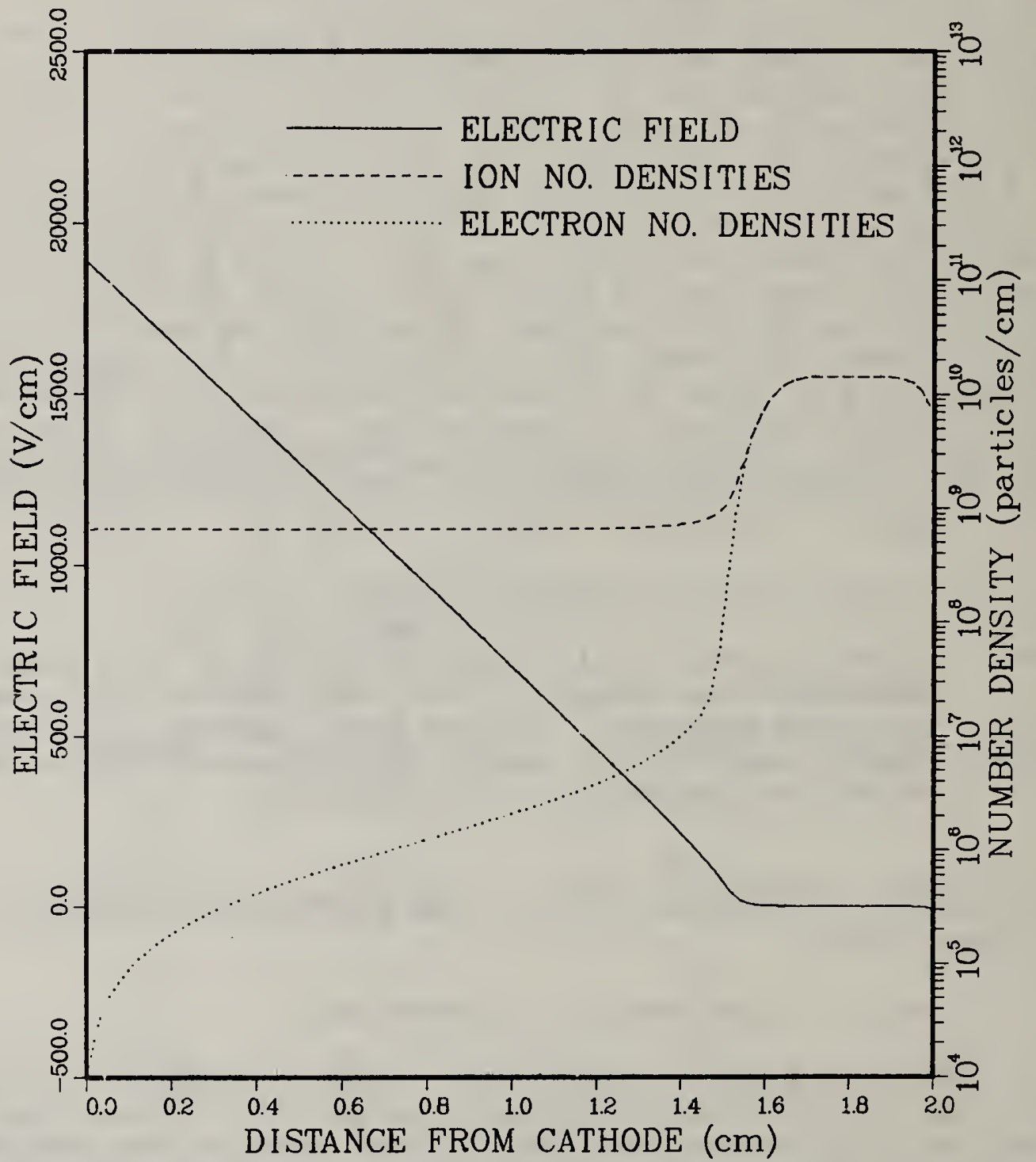

Figure 3. Calculated ion and electron densities and electric field in a simulated $\mathrm{C}_{2} \mathrm{H}_{2} /$ air flame between infinite parallel plate electrodes separated by $2 \mathrm{~cm}$ and biased at a potential difference of $1500 \mathrm{~V}$. 
Figure 4 shows the perturbations resulting to the ion and electron densities $40 \mathrm{~ns}$ after the deposition of $3 \times 10^{9}$ ions and electrons per $\mathrm{cm}^{3}$ over a width of $0.1 \mathrm{~cm}$ by an LEI event centered $0.5 \mathrm{~cm}$ from the cathode $(x=0)$. The number densities shown are differences between those calculated following laser perturbation and the steady-state background populations of figure 3 . The field is plotted in the same manner as figure 3 , but the perturbation in field due to the laser-produced charges is evident in figure 4 at this level of added charge. The negative-going peak in the electron perturbation curve constitutes a very important proof of a concept that we had advanced previously on the basis of empirical evidence: that the portion of the flame not perturbed by the electric field behaves as a conductor, making the interface between the field-perturbed "sheath" and the field-free region act as a virtual anode. The negative peak corresponds to the image charge that would be induced in a solid conductor by the free charge (positive peak) in the flame.

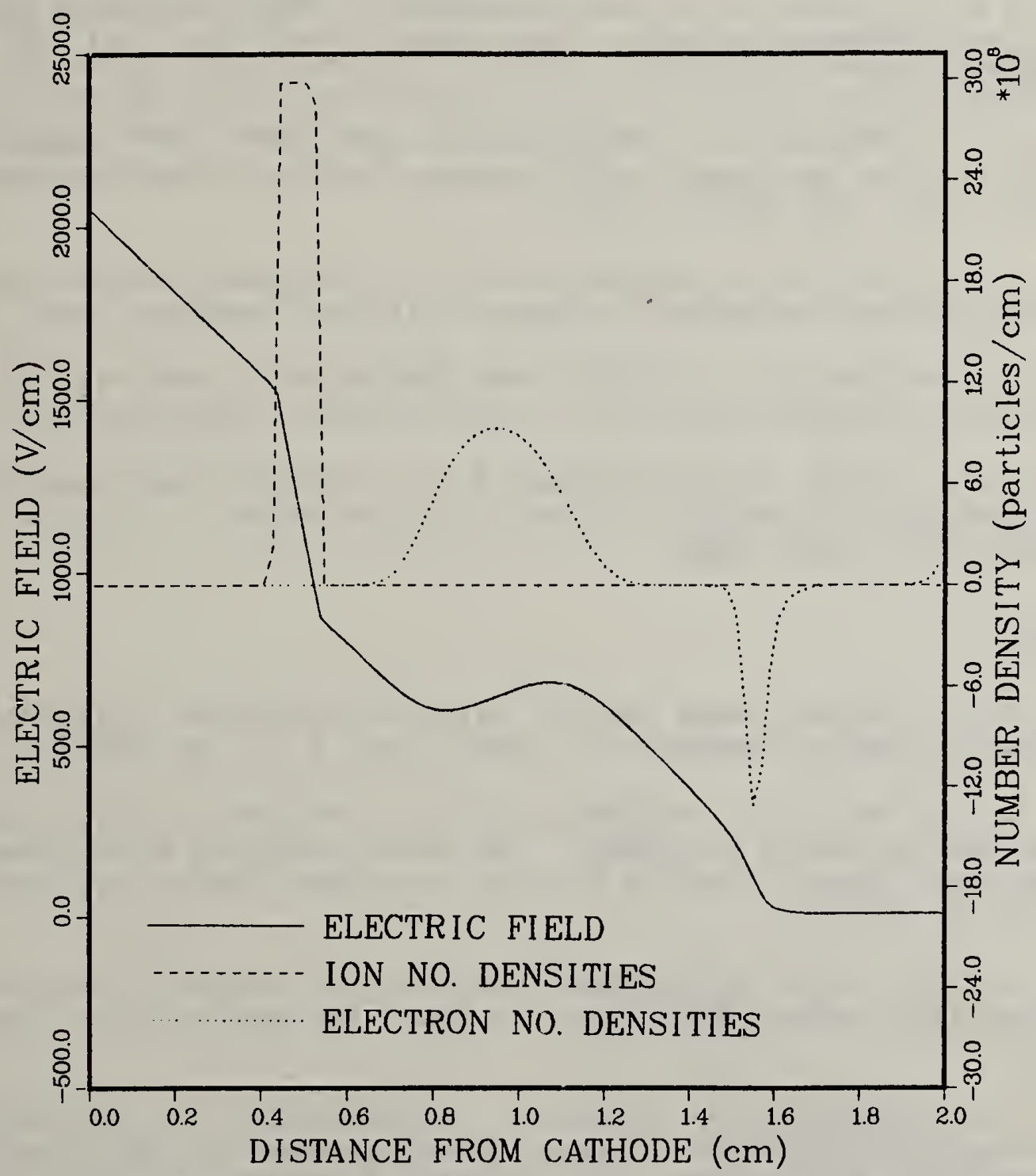

Figure 4. Perturbations to the electron and ion densities and to the electric field of figure $340-n s$ after the deposition of $3 \times 10^{9}$ ions and electrons in a $0.1 \mathrm{~mm}$ wide region centered at $0.5 \mathrm{~cm}$ from the cathode. 
Inclusion of the background conditions in the model calculations has provided an improved understanding of the role of sample matrices in perturbing the background, and with it, the LEI signal. Further additions to the model are expected to include flame convection and temperature variation with position.

\section{Outputs and Interactions}

\section{a. Publications}

Travis, J. C., "Limits to Sensitivity in Laser Enhanced Ionization," J. Chem. Ed., 59, 909-914 (1982).

Havrilla, G. J., Weeks, S. J., and Travis, J. C., "Continuous Wave Excitation in Laser-Enhanced Ionization Spectrometry," Anal. Chem., 54, 2566-2570 (1982).

Fassett, J. D., Travis, J. C., Moore, L. J., and Lytle, F. E., "Atomic Ion Formation and Measurement with Resonance Ionization Mass Spectrometry," Anal. Chem., 55, 765-770 (1983).

Travis, J. C., Turk, G. C., and DeVoe, J. R., "Trace Metal Analysis by Laser-Enhanced Ionization in Flames," Clinical Chemistry, Sept. 1983.

Maki, A. G. and Sams, R. L., "Diode Laser Spectra of cis-HONO near $850 \mathrm{~cm}^{-1}$ and trans-HONO near $1700 \mathrm{~cm}^{-1}$," J. Mol. Struct. (submitted 1983).

Kagann, R. H., Elkins, J. W., and Sams, R. L., "Absolute Band Strengths of Halocarbons F-11 and F-12 in the 8- to 16- 1 m Region," J. Geophys. Res., 88(c2), 1427-1432 (1983).

b. Talks

Travis, J. C., "Trace Element Analysis Using Laser-Enhanced Ionization in Flames," Stauffer Chemical Co., Dabbs Ferry, N.Y., Feb. 16, 1983.

Travis, J. C., Turk, G. C., and DeVoe, J. R., "Trace Metal Analysis by Laser Enhanced Ionization in Flames," 15th Annual Symposium on Advanced Analytical Concepts for the Clinical Laboratory, Gatlinburg, Tenn., April 22, 1983.

Travis, J. C., "Studies in Selective Ionization for Inorganic Analysis," Gordon Conference on Analytical Chemistry, New Hampton, N.H., August 18, 1983.

Moore, L. J., Fassett, J. D., Travis, J. C., Lucatorto, T. B. and Clark, C. W., "Isotopic Selectivity in Ultrasensitive Resonance Ionization Mass Spectrometry," 27th Annual SPIE Technical Symposium, San Diego, Calif., August 23, 1983. 
Travis, J. C. "Selective Laser Ionization for Trace Element Analysis," 30th Annual Conference of the Spectroscopy Society of Canada, Vancouver, B.C., Canada, Oct. 6-8, 1983.

G. C. Turk, C. J. Havrilla, P. K. Schenck, and J. C. Travis, "Laser-Enhanced Ionization Spectrometry for Trace Metal Analysis," IX FACSS, Philadelphia, Pa., Sept. 21, 1982. Invited

G. C. Turk, "Laser-Enhanced Ionization Spectrometry," Chemistry Department Seminar, University of Bari, Bari, Italy, June 15, 1983. Invited

G. C. Turk, J. C. Travis, and J. R. DeVoe, "Laser-Enhanced Ionization Spectrometry for Trace Metal Analysis," C.N.R.S. International Colloquium on Optogalvanic Spectroscopy and Its Applications, Aussois, France, June 23, 1983. Invited

G. C. Turk, J. C. Travis, and J. R. DeVoe, "Trace Metal Analysis by LaserEnhanced Ionization Spectrometry," 23rd Colloquium Spectroscopicum Internationale, Amsterdam, The Netherlands, July 1, 1983. 



\section{Inorganic Analytical Research Division}

Ernest L. Garner, Chief

Barry I. Diamondstone, Deputy Chief

\section{A. Division Overview}

The Inorganic Analytical Research Division conducts research, develops analytical methods, and makes qualitative and quantitative measurements of inorganic constituents, to certify standards and to provide analytical services in support of national needs. To accomplish its mission, the Division is organized into four research groups which function along competence lines. The four groups are: Activation Analysis, Atomic and Molecular Spectrometry, Electroanalytical Chemistry, and Analytical Mass Spectrometry. This structure provides the flexibility to address a wide variety of research and analytical problems requiring highly sensitive and highly accurate measurements. Working within a framework of providing maximum public benefit, research and measurement priorities are established on the basis of: (1) advancing and maintaining analytical chemical metrology; (2) providing the basis for chemical standardization; and (3) providing accurate chemical measurements for materials characterization.

As in recent years, the scientific program of the Division is diversified and supports a number of areas that have clearly identified a need for accurate baseline data. The level of activity and its cross-cutting nature are reflected by an impressive list of Standard Reference Materials that have been analyzed for certification, an extensive list of publications and talks, and a large number of interactions with industrial and academic laboratories. Guest workers (8), visiting scientists (10), postdoctorals (2), and IPA's (1) have spent all or part of the year in varying degrees of involvement in mainstream research and measurements and have made significant contributions to the success of our program.

Competence research, a cornerstone of our research program and a primary means of exploring new directions in analytical chemistry, has resuited in major breakthroughs in neutron depth profiling and ionization mechanisms research. These advances in programs initiated 2-3 years ago represent substantial gains in terms of moving research from the conceptual stage to the point of exploiting research results for their potential new measurement capabilities. Most of the recent developments in neutron depth profiling and ionization mechanisms are described in greater detail in the Group overviews and technical accomplishments of this report. Particulariy noteworthy are the research findings in neutron depth profiling during the first full year of use as a measurement tool. During this period, a radioactive nuclide $\left({ }^{22} \mathrm{Na}\right)$ was profiled for the first time and ${ }^{3} \mathrm{He},{ }^{6} \mathrm{Li}$, and ${ }^{10} \mathrm{~B}$ were quantitatively profiled in a variety of semi-conductor materials. These achievements by a team composed of research chemists from the Activation Analysis Group, a postdoctoral scientist, a research associate from industry, and a university researcher are an excellent example of collaborative investigations into research and process problems of interest to government, industry, and universities. 
Equally important in terms of impact on future measurement capability are the findings in ionization mechanisms research. From this program, resonance ionization mass spectrometry has emerged as a powerful analytical tool with increased selectivity and sensitivity for all groups of elements in the periodic table. Among numerous significant accomplishments was a demonstration of a three-photon process to ionize carbon and iodine. This achievement opens the door to a new approach for ionizing and analyzing elements on the right hand side of the periodic table which are sensitive indicators in environmental monitoring programs. Along with the successful utilization of a multi-photon ionization process, parallel developments in resonance ionization, including multielement isotope dilution, isotope ratio quantitation, and high resolution sub-doppler resonance ionization, leads towards new horizons in analytical mass spectrometry.

Continuing research in material transport and hydrodynamic modulation of electrodes has reached a point where analytical applications could begin in the near future. Results in these forward-looking areas of research, along with research to upgrade or develop new methods for existing instrumentation, will enhance the overall measurement capability of the Division. This is illustrated by the various components of methods research underway in a number of problem oriented areas for the Standard Reference Materials Program, the Recycled 0il Program, and the Nondestructive Evaluation Program. As an example, the recently equipped laboratory for gases-in-metals measurements has made substantial progress in developing methods for the analysis of oxygen, nitrogen, and hydrogen in selected metals. This technology will be utilized extensively to update existing standards as well as to certify new high technology materials as standards. Similarly, methods research in gas isotope dilution, spectrophotometry, trace elements in complex matrices, fluoride analysis, and $\mathrm{pH}$ are expected to pay dividends at a later time when specific measurements are required.

The commitment to the certification of SRM's has remained at a high level. Coverage has been extensive in terms of material types, categories, and numbers of different standards analyzed. The highlights in this area are described in Group overviews and technical reports. A Division-wide effort has been in progress this year to determine high priority trace elements in three new SRM's; Powdered Milk, Urine, and Human Serum. These materials along with future SRM's, such as clay, soil, and plastic, will provide important new standards to meet well identified measurement needs. Along with measurement services for the Standard Reference Materials Program, significant contributions were made to the quality assurance efforts of other government agencies. These include the analysis of audit samples, preparation and evaluation of materials for use as standards, isotopic tracer studies for acid rain, and the analysis of a variety of materials for trace elements.

New opportunities for research and analytical services are constantly being explored as a means of coupling base programs to the needs of other government agencies. Some progress has been made with the submission of major proposals for nutritional studies and bioavailability of trace elements, direct solid sampling using laser mass spectrometry, and isotope tracer studies for receptor modeling in acid rain. In conjunction with this outreach program to couple NBS efforts with those of other agencies, considerable attention has been given to maintaining and expanding our broad-based constituency. Among 
the most successful efforts during the year are the research collaborations developed in the competence area for neutron depth profiling and resonance ionization mass spectrometry. These efforts will continue with a high level of commitment.

For the coming year a nearly equal effort is anticipated in research, certification of standards, and analytical support for problems of national importance. Exclusive of other agency commitments, only modest changes are anticipated in current directions of base programs. For example, a minor reprogramming effort will be utilized to initiate research in high resolution chromatography. Predictably, the other agency situation is quite fluid and will be structured to provide the best match between base effort and the needs of the requesting agencies. The commitment to the certification of SRM's will remain constant and at a very high level of activity.

\section{Activation Analys is Group: Overview}

The Activation Analysis Group has as its objective basic and applied research to develop nuclear analytical techniques for increased sensitivity, better selectivity, and higher accuracy. A high level of competence has been developed for various reactor-based activation analys is techniques which include instrumental and radiochemical neutron activation analysis (INAA and RNAA), prompt gamma activation analysis (PGAA), and the nuclear track technique (NTT). The three year research program in elemental depth profiling has added the technique of thermal neutron beam depth profiling (NDP) to the scope of reactor based analytical activities. Non-reactor-based techniques include photon activation analys is (PAA), fast neutron activation analys is, and charged particle activation analysis, have been used on a more limited scale. These various techniques provide sensitive tools for fulfilling the needs of analytical chemistry in various branches of science and technology. Depending on the analytical requirements, simultaneous instrumental multielement determinations, highly selective radiochemical analysis of ultratraces, and quantitative depth profiles are available to certify standards, support other government agencies, and collaborate with academic and industrial laboratories.

Basic research and development in depth profiling have progressed to the point of yielding quantitative measurements. This facility is a unique resource in the U.S. Which is needed for investigations of the important nearsurface region of semiconductors and glasses, first wall fusion materials, and ion implantation processes. The facility is the focal point for coordinated research involving several research associates and guest workers from industry, government R\&D agencies, and universities. Quantitatively depth profiling boron in semiconductor materials has been a significant accomplishment of the past year. The data from these depth profiles have been in excellent agreement with determinations done by secondary ion mass spectrometry and are necessary to evaluate the performance of electronic devices. The recent expansion of NDP to other elements has contributed to the understanding of the behavior of helium in metals, and has resulted in depth profiles for sodium $\left({ }^{22} \mathrm{Na}\right)$ to study its diffusion in glasses and semiconductors. 
A major effort was expended in problem oriented projects and cooperative programs with other agencies. The expansion of research activities into related areas of general analytical importance such as sampling and quality assurance has focussed around specimen banking. During the past year the program to analyze trace elements in human liver and Mytizus eduzis (mussels) specimens required the efforts of both activation analysis and atomic absorption. Even though the NBS specimen bank program is a pilot effort, the experience gained provides the basis for considering specimen banking as a viable long term mechanism for evaluating environmental health and monitoring parts of our biosphere. The protocols developed for sample collection, processing, storage, and analysis, under contamination-free conditions, yield benefits beyond their application to specimens in the NBS pilot specimen bank and are applicable to other critical areas such as nutrition and health. In an effort to transfer the technology and expertise, cooperative agreements have been established with the Armed Forces Institute of Pathology and Georgetown University School of Dentistry.

The continuing efforts to enhance the capabilities of INAA and RNAA procedures have resulted in several major accomplishments. For example, mercury can now be determined at the $\mathrm{ng} / \mathrm{g}$ level in water and urine utilizing an optimized INAA procedure. This achievement resulted from a significant improvement in the performance of recently upgraded equipment. RNAA procedures such as group separations or element specific liquid extractions have been further developed to exploit the inherent sensitivities of activation analysis. These procedures are used to determine critical elements, at low concentration levels, in materials like milk and serum.

Considerable improvements in the equipment for nuclear measurements have been realized. An additional high efficiency, high resolution detector was installed, enhancing multielement and low level capabilities. A pneumatic shuttle system was installed for rapid sample transfer from the irradiation facilities to the counting rooms. It is equipped for automated operation and, in addition, can function as a sample changer. A second new sample changer is being installed in cooperation with the Smithsonian Institution. Interactions with the manufacturers of nuclear data acquisition systems have led to the general availability of a loss-free ADC and modular MCA's which are based on existing computers. A modular system has been purchased and extensive use during the next year should provide the basis for further improvements.

Research plans for the coming year include continued efforts to improve the INAA and RNAA procedures. The modifications in the irradiation facilities along with the planned upgrade of the reactor power to $20 \mathrm{MW}$ will be utilized to initiate and evaluate "absolute" activation analysis. This methodology will be based on continuous monitoring of the neutron flux spectrum at the irradiation site. It will allow definitive determinations of essentially all elements that can be activated as long as their nuclear parameters are known with sufficient precision and accuracy. In the long range, isotopic abundances, half lifes and decay schemes may have to be redetermined. Upgrading the reactor to higher power levels will allow the facility to be used for additional purposes; such as beryllium depth profiling. These improvements in the current irradiation facility will enhance both sensitivity and resolution for neutron depth profiling measurements. It is anticipated that 
investigations of near surface regions in materials may extend beyond the capabilities of NDP. Therefore, a proposal to use charged particles for depth profiling is being developed. This proposal could result in a CAC facility that is available for depth profiling work as well as for other activation analysis programs. Such a facility would allow the Group to become independent of the reactor as a neutron source as well as adding new methodologies to the scope of work. Independently, a major effort will be put towards evaluating "non-conventional" activation analysis procedures. Very short lived isotopes are of interest for new applications, and $14 \mathrm{MeV}$ and charged particle activation will be evaluated for the determination of light elements, e.g., gases in metals. With the increased sensitivities of INAA and RNAA new approaches utilizing chromatographic procedures will be tested for the determination of organometallic and biological species.

Rolf L. Zeisler, Group Leader; M. James Blackman, Kathleen A. Fitzpatrick, Ronald F. Fleming, Robert R. Greenberg, John K. Langland, Richard M. Lindstrom, George J. Lutz, Susan F. Stone, John T. Maki, Dietrich H. Vincent

\section{Analytical Mass Spectrometry Group: Overview}

The Analytical Mass Spectrometry Group develops and maintains the capabilities required to perform chemical composition measurements using the analytical sciences of chemical separation and mass spectrometry. For many years, the Group has been involved in chemical separations, plus the highly precise and accurate isotope ratio measurements, required to determine the atomic weights of the elements and to make definitive isotope dilution measurements of trace element concentrations. While maintaining the basic thrust in the traditional areas, the horizons of the Group have broadened markedly in the past year. Predicated on the competence building program and related basic research, substantial changes have been initiated to develop new capabilities for chemical separations and ion spectroscopy. By combining the foundation evolved from many years of measurement research and applications with the fundamental changes described below, the next few years will witness the evolution of a new generation of measurement capabilities for analytical ion spectroscopy and the realization of new compositional analys is capabilities that they portend.

Basic and applied research have been pursued in several significant areas that will eventually restructure, in a fundamental way, measurement capabilities in analytical spectrometry for chemical composition analysis. Research in the ion mechanisms competence building program has resulted in several significant accomplishments: resonance ionization mass spectrometry (RIMS) has been used to demonstrate resonance ionization feasibility and to study the spectroscopy for one-fourth of the elements in the periodic table; selective electronic energy state interrogation has resulted in the characterization of atomic metastable distributions and a better understanding of the thermodynamic nature of the thermal atomization process; a three-photon process has been used to ionize carbon and iodine, which potentially opens up most of the upper right-hand portion of the periodic table to analysis and provides access to an unexplored area of ultraviolet atomic spectroscopy; the incipient basis for multielement analysis with RIMS has been established and the ability to access more than twenty elements in a single tunable dye range has been 
demonstrated; a pulsed atom source has been developed and evaluated to provide a projected 30-fold improvement in RIMS sample utilization efficiency.

Ion optics calculations for the magnetic sector systems have been refined during the past year to eliminate software and computation inconsistencies. Refined stray magnetic field measurements have been completed on a magnet with adjustable gap widths and a more refined algorithm is being developed to fit the data. A copy of the recently developed Simion ion source computer modeling program has been acquired and efforts are underway to access the necessary on-site computing capability to integrate Simion into the overall approach to ion optics modeling. A new system for rapid switching of the high voltage supply is being developed for spark. source mass spectrometry. Variations in sample homogeneity and sparking conditions would be minimized for isotope dilution trace measurements, resulting in improved accuracies and precisions.

Last year the thermal formation of negative ions from complex emitting surfaces was combined with chemical separations to produce an isotope dilution method for determining iodine in botanical materials. Recently, a modified negative ion source design was acquired that can be operated at higher voltages with attendant higher efficiencies. During the next year this ion source will be used to explore negative ion formation characteristics in thermal sources. Possible applications to high ionization potential transition metals and nonmetals will be examined.

Recent developments in trace level sulfur measurements by isotope dilution have produced a method capable of precisely analyzing samples containing $\mu \mathrm{g} / \mathrm{g}$ quantities of sulfur. Exploratory research with this methodology has been pursued to establish the feasibility of identifying the sources of sulfur in particulate air samples. Encouraging results have been obtained from a collaborative effort with Professor Zoller of the University of Maryland to resolve isotope ratio variations in high altitude samples of particulate ejecta from the Mexican El Chichon volcanic eruption. These results indicate that sulfur isotope ratio variations can possibly be used as a tool for the "provenance" determination of acid rain. The established capability for provenance determination of archaeological artifacts using the lead isotope ratio "fingerprint" has continued in collaboration with the Smithsonian Institution. A new and extensive collection of Chinese bronze artifacts has been examined, and the resulting lead isotope ratios have been aggregated into five groups that suggest separate artifact fabrication sites. Efforts are currently underway to correlate these groups with existing geological isotope ratio data from specific locations in China.

A substantial portion of the effort in the atomic weights program this past year was directed toward producing an up-to-date compilation of atomic weight data from international research efforts. These data were compiled for the 1983 meeting of the IUPAC Commission on the atomic weights of the elements and represent the efforts of experts from Europe and the United States. Experimental atomic weight research continues on the gravimetric assay of lithium and on refinements to the chemical purification and isotopic analysis of gallium. 
High sensitivity applications of isotope dilution have highlighted the analytical applications portion of the Group's program, including ultra-low concentration measurements of uranium, vanadium, and cadmium in biological materials and other natural matrices (e.g., $5 \times 10^{-10} \mathrm{~g} / \mathrm{g} \mathrm{Cd}$ in normal urine, SRM 2670). These measurements, in combination with the lower levels of chromium and sulfur, determined in a variety of SRM's and natural matrices, are indicative of the considerably expanded measurement capabilities. A large number of certification measurements were completed for the Standard Reference Materials program including trace elements in human serum, metals, waters, and biological materials.

Collaborative efforts have been initiated between members of the Group and scientists from the Centers for Radiation Research, Absolute Physical Quantities, and Chemical Physics. The CRR-CAC collaboration has already produced several theoretical and experimental research papers dealing with isotopic ionization selectivity and the potential for measuring high abundance sensitivity ratios. Progress is anticipated during the next year in the selective state interrogation of sputtered neutrals from solid surfaces in collaboration with personnel from the Surface Science Division of the Center for Chemical Physics.

Several substantial proposals to other government agencies are in various stages of progress. If completely successful, these long-term proposals will impact the Group's program heavily in the areas of: basic research in resonant ionization pathways and highly sensitive and selective isotope measurements with laser spectroscopy; technology development and studies of nutrition and essential trace element bioavailability in humans using stable isotope tracers; and a new approach to the direct compositional analysis of solids using selective resonance ionization of neutrals sputtered from solids. Equipment funds from an other agency proposal are currently being used to establish a unique RIMS facility for high abundance sensitivity measurements.

Research in ion optics and measurement science will result in an upgrading of existing mass spectrometry instrumentation in several critical areas and allow the Group to maintain its leadership in accurate and precise isotope ratio measurements. Significant advances achieved in RIMS technology during the past year will continue to play an important role in defining new areas of potential impact in chemical composition measurements. The ability to sensitively and selectively interrogate atoms and molecules with energy state specificity provides a unique and broadly applicable level of measurement sophistication in ion spectroscopy. The impact of these developments will be felt in a trend toward matrix-free direct compositional analysis, ultrasensitive measurements of atoms and molecules in natural samples, and chemical speciation.

A full development of negative ion spectroscopy should greatly expand the utility of thermal ionization for isotope dilution and related applications for which the precision and accuracy may be usefully employed in the establishment of definitive measurements.

Plans are underway to combine the areas of chemical separations technology with the new instrumentation under development to provide a powerful new set of analytical tools for real-world samples. For example, the group-specific chelate separation schemes can be developed to match the evolving multielement RIMS isotope dilution capabilities that utilize specific laser-dye combinations 
and element groupings. In general, by combining the established measurement expertise in isotope ratios and mass spectrometry with the above new developments, quantum level advances can be realized in the determination of chemical compositions.

Larry J. Moore, Group Leader; I. Lynus Barnes, Ellyn S. Beary, William A. Bowman, III, Karen A. Brletic, Emile C. Deal, John D. Fassett, John W. Gramlich, Emerson F. Heald, William R. Kelly, Howard M. Kingston, George M. Lambert, Lawrence A. Machlan, John R. Moody, Thomas J. Murphy, Paul J. Paulsen, Lura J. Powe11, Ronald W. Shideler, Mary E. Stone

\section{Atomic and Molecular Spectrometry Group: Overview}

The objective of the Atomic and Molecular Spectrometry Group is to conduct research on fundamental chemical and physical phenomena related to sampling and spectrochemical analysis. Experimental control of critical processes can then be used to improve analytical measurement capability. This research includes the development of new and improved techniques to lower the detection limits for elemental analysis and to improve the precision and accuracy of these measurements. Efforts toward achieving these goals have been in the area of material transport, matrix modification, gases in metals, high accuracy spectrophotometry and expanded multielement capabilities.

Research in material transport has proceeded in three basic phases. The first phase involved the design and construction of a controlled waveform spark source to be used in the fundamental studies on spark sampling. The second phase of the research has involved the modification of a commercial spark stand to accept the standard tungsten counter electrode as well as a laminar flow jet counter electrode. The third phase was the production, collection, and analysis of aerosol particulates from SRM brass and titanium based alloys. Differences in composition between spheres and aggregates produced in the discharge varied with the materials. Future studies will involve a quantitative assessment of the sampling process.

Significant progress has been made in a continuing program to develop methods for the determination of gases in metals. Methods have been developed for hydrogen in cast iron and steels using state-of-the-art instrumentation. Sample preparation and analys is procedures are presently being developed for the determination of oxygen and nitrogen in metals and methods should be available for certifying these gases in SRM's in the near future. In a related area, methods have been developed for the determination of carbon and sulfur in cast irons, steels, and other metals.

In high accuracy spectrophotometry, two problems continue to limit the production and potential utilization of the NBS thin metal film transmittance SRM. Fortunately, the problem of significant wedge effects, observed in many of the earlier films, has been minimized by systematically modifying and empirically determining optimum coating conditions. Currently the transmittance uniformity of films supplied by a commercial contractor is about 0.1 percent. The second problem deals with a general concern about using thin metal film filters in instruments that have an interreflection error. These films 
attenuate radiation not only by absorption but also by reflection. This reflected component could, in instruments of specific design, produce artifically large interreflection effects. A modified design of a metal-on-quartz filter that should eliminate the reflected radiation from the optical path is presently being tested in a broad range of commercial spectrophotometers. A major development in using the high accuracy spectrophotometer has been the extension of the wavelength range from the current 200-800 nm to $2500 \mathrm{~nm}$ $(2.5 \mu \mathrm{m})$. This new capability is expected to have important applications in the areas of defense, agriculture, and medicine where IR measurements are important. In addition, a special multi-purpose fluorescent glass standard, for improved characterization of dye penetranst used in non-destructive crack testing procedures, was produced and evaluated.

Major thrusts during the past year included characterizing Standard Reference Materials (SRM's) and providing analytical services to characterize highly complex samples for other government agencies as well as other divisions at NBS. To meet these demands, new spectrochemical techniques were developed and expertise in old established techniques such as Kjeldahl nitrogen, spectrophotometric determination of fluoride, and gases in metals was renewed.

A great deal was accomplished in the area of spectrometric analysis during the past year. The methods employed include AAS, ICP, and DCP. The following SRM's have been characterized for major, minor, and/or trace constituents: Trace Elements in Water, Powdered Milk, Urine, Pine Needles, Tomato Leaves, Bovine Liver, Human Serum, Fly Ash, Cast Iron, Urban Particulates, Incoloys and Inconels, Wear Metals in Oil, High Purity Heptane and Isooctane, Manganese Ore, Rutile, Brasses, Bronzes, Trace Elements on Filters, XRF-films, and Aqueous Spectrometric Standard Solutions. AAS was used to determine several elements in samples of human liver for the tissue bank, four elements in a heart-tissue sample survey for the Johns Hopkins University, and germanium in samples of serum, plasma, urine, red blood cells, and bile from patients at the Georgetown University Hospital. In addition, ICP, DCP, and AAS techniques were used to determine trace elements in samples of acid rain, solid waste leachates, nuclear waste glasses, and EPA drinking water standard solutions. Optical emission spectrometry (spark) was again used extensively for homogeneity studies of SRM's (irons, steels, brasses, and bronzes). This technique was also used to produce elemental profiles for several steels.

Another significant project was the development of a training program for visiting scientists in state-of-the-art sample preparation, atomic absorption, and emission spectrometry procedures. During the past year scientists from the National Sanitation Foundation, B. F. Goodrich, Materials Research Corporation, and U.S. Army Aberdeen Proving Ground participated in this program. In addition, at the request of the U.S. State Department, a program is underway to train four scientists from the Assay and Weights Administration in Cairo, Egypt.

Research during the coming year will focus on the development of multielement procedures, primarily on solid sampling techniques to be used in conjunction with AAS and ICP. Other activities will include the development of a procedure for the analysis of silicon in biological materials and the development of new methodologies for tin analysis in sea water. In the area 
of metal analysis, the Kjeldahl method for nitrogen will be applied to trace nitrogen determinations in steels, and new or improved methods for oxygen and nitrogen in metals will be investigated. The extension of the wavelength range of the high-accuracy spectrophotometer to the near-infrared region will be completed and the evaluation of new primary sources in atomic absorption and fluorescence will be undertaken. Material transport research will focus on the examination of the dependence of particle size distributions on spark source parameters and the chemical analysis of specific size fractions by ICP and AAS. Investigations into sample introduction techniques for plasma emission spectrometry will include the development of continuous flow injection for microsampling by DCP and ICP, and a feasibility study for using hydride generation as a technique for introducing samples into the DCP and ICP.

Theodore C. Rains, Group Leader; David E. Brown, Robert W. Burke, Therese A. Butler, Barry I. Diamondstone, Michael S. Epstein, Radu Mavrodineanu, Kimberly I. Moran, John A. Norris, Douglas J. Patin, Theresa A. Rush, Robert L. Watters, Jr., Yong-Kang Zhang

\section{Electroanalytical Chemistry Group: Overview}

The Electroanalytical Chemistry Group conducts basic research and utilizes electrochemical principles to develop techniques and apply them to chemical analyses. The techniques in practice include amperometry, anodic stripping voltammetry, conductimetry, coulometry, ion chromatography, potentiometry, and voltammetry. Research activities are directed toward attaining a more thorough understanding of the complex electrochemical systems encountered in the development and implementation of electroanalytical methods. In this past year the Group has added a full-time research chemist and a NRC/NBS Postdoctoral Research Associate. In addition, two guest workers from the University of Maryland were active through the year, and a Visiting Professor from Tennessee State University joined the Group during the last quarter. During the summer, two students actively participated in the Group's work. This increase in personnel allowed the Group to expand into the following new areas of research: fundamental studies in potentiometric measurements, hydrodynamic modulation, and applications of anodic stripping voltammetry.

This year the Group hosted a very successful symposium entitled "Workshop in Direct Potentiometric Measurements in Blood," co-sponsored by CAC/NBS and the National Committee for Clinical Laboratory Standards (NCCLS). The purpose of this meeting was to provide a forum for research scientists and manufacturers and users of clinical instrumentation to discuss the current status and needs of direct potentiometric measurements in blood, with particular emphas is on the measurement of sodium. The workshop was international in scope with eight of the sixty-three attendees being from foreign countries. The proceedings of the workshop will be published.

The new $\mathrm{pH}$ facility has been well utilized for several projects. Three new lots of SRM pH buffers (sodium bicarbonate/sodium carbonate, borax, and potassium dihydrogen phosphate/disodium hydrogen phosphate) and one new lot of SRM pD buffer (sodium bicarbonate/sodium carbonate) have been certified using hydrogen $\mathrm{Ag}-\mathrm{AgCl}$ cells without liquid junction. The $\mathrm{pH}$ values of two solutions of dilute hydrochloric acid have been determined with the same 
apparatus. These solutions represent a preliminary effort in the development of a new generation of pH standards, which will be matrix specific, in this case for acid rain measurements. Concurrent with these pH determinations, fundamental studies of the standard potential of the silver/silver chloride electrode are in progress. This work is described in detail as a technical highlight.

Research in voltammetry is in progress in the area of hydrodynamic modulation. Platinum, gold, and amalgamated gold vibrating wire microelectrodes have been designed and constructed at NBS along with the related hardware and electronic circuitry. A new method for estimating diffusion-layer thickness at hydrodynamic electrodes has been conceived and tested for rotating disc electrodes and vibrating electrodes. The theory of the relaxation of the Nernstian diffusion layer has been extended to predict the frequency response of the vibrating wire electrode. The development of the vibrating wire voltammetric technique and its application to flow-injection analysis is in progress. This should substantially reduce background currents and enhance sensitivity for ultra-trace metal analyses. A spin-off of this research has been the discovery that the vibrating wire electrode may have applications for extremely low ionic strength DC conductivity measurements.

The instrumentation for ion chromatography (IC) has been heavily used for the analysis of several SRM's (milk powder, fly ash, vanadium in oil, base oil, and wear metals in oil), acid rain samples, and water quality audit solutions. The IC analysis of total cyanide has been extended to cyano-metal complexes by modifying existing reflux-distillation procedures.

Work on an acid rain project has progressed significantly toward the development of reference materials and measurement methodologies to provide quality assurance for the National Acid Precipitation Assessment Program. This work is detailed in a technical highlight.

Coulometry has been used, in the controlled current mode, for highaccuracy assays of several preparations of uranium oxide from Oak Ridge National Laboratory and in the controlled-potential mode for the assay of rutile. Anodic stripping voltammetry is being applied to the analysis of acid rain samples, glass films, and hair.

The Group is maintaining its proficiency in traditional chemical analyses through the determination of total manganese and available oxygen in manganese ore by permanganate titration. In addition, a cooperative effort is underway with the Metallurgy Division (564) to study the characteristics of plating bath solutions used in the pulsed electrodeposition of nickel-chromium alloys.

An informal agreement was initiated with the Department of Chemistry, University of Maryland, to collaborate in the investigation of air particulate and aerosol samples from remote areas and areas of volcanic activity. The major objectives of this project are to develop the methodologies necessary for trace-level sulfur and chlorine measurements and to use these methodologies to establish baseline values. 
The expansion in personnel and in scope of research, within the Group, is expected to continue through active collaboration with area universities and by making full use of the postdoctoral, research associate and visiting scientists programs. The agreement with the University of Maryland wi il continue. In conjunction with the NCCLS, it is anticipated that a research associate will develop methods and reference materials for the potentiometric measurement of calcium in biological fluids. Research in $\mathrm{pH}$ will continue as a long-term commitment to provide reliable and thermodynamically significant measurements and standards for one of the most widely used analytical parameters. In the next year, this activity will focus on resolving the problems associated with residual liquid junction potentials, specifically as they apply to acid rain measurement. Research will be initiated to develop advanced expertise in conductance measurements with the view towards issuing a Standard Reference Material for aqueous conductivity and towards understanding the fundemental relationships among conductivity, ionic mobility, diffusion, and ionic strength. Flow-injection analys is with electrochemical detection will be explored in depth. The outlook for the acquisition of an advanced ion chromatographic system is promising. This instrument will allow the Group to expand this technique into speciation studies for metals and metallo-complexes, and into cationic analyses.

William F. Koch, Group Leader; George Marinenko, Kenneth W. Pratt, Roger M. Stone, Rubye P. Torrey, Yung-Chi Wu 
B. Selected Technical Reports

(Inorganic Analytical Research Division)

1. A New Electrochemical Technique for the Estimation of Diffusion-Layer Thicknesses at Hydrodynamic Electrodes (27201)

K. W. Pratt, Jr.

In voltammetry, the maximum analytical sensitivity of convective electrodes is governed by the thickness of the diffusion layer established at the electrode. Hence, the diffusion-layer thickness, $\delta$, provides a figure of merit for the sensitivity of a given electrode. The value of $\delta$ may be used both in intercomparisons of electrodes of different geometries and in optimization of a specific electrode. Measurement of $\delta$ by standard techniques requires the determination of the "electrochemical" surface area of the electrode, which generally is greater than the "geometric" surface area.

In the past year, a new technique has been developed at NBS which allows the value of $\delta$ to be determined for any hydrodynamic electrode without measuring the surface area of the electrode. Prior knowledge of the hydrodynamic conditions prevailing at the electrode is not required. The determination of $\delta$ by this new technique is based on a comparison of the steady-state and transient currents observed at the same electrode in a single solution. The steady-state limiting current, $\mathrm{I}_{S S}$, is measured under the hydrodynamic conditions of interest at a potential $E_{1}$, corresponding to the mass-transport limited reaction of the electroactive species at the electrode. The transient faradaic response of the electrode, $I(t)$, is then recorded in quiescent solution for a potential step at time $t=0$ from a value $E_{0}$, where no reaction occurs at the electrode, to $E_{1}$, the value used in the steady-state measurements. These currents are described by equation 1 for the steady-state case and by equation 2, the cottrell equation, for the transient case:

$$
\begin{array}{r}
I_{s s}=\frac{n F A D C}{\delta} \\
I(t)=\frac{n F A D^{1 / 2}}{\pi^{1 / 2} t^{1 / 2}}
\end{array}
$$

In these equations, the parameters $n, F, A, D$, and $C$ represent the number of electrons in the electrode reaction, Faraday constant, electrode surface area, diffusion constant, and concentration of the electroactive species, respectively. Using the same electrode and solution in both experiments ensures the equality of $n, F, A, D$, and $C$.

Representative graphs of $I_{S S}$ and $I(t)$ as functions of time are presented in figure 5. For $t=t_{0}$, the point at which $I(t)=I_{s s}$, the right members of equations 1 and 2 are equal. Simplification yields the following expression:

$$
\delta=\left(\pi D t_{0}\right)^{1 / 2}
$$




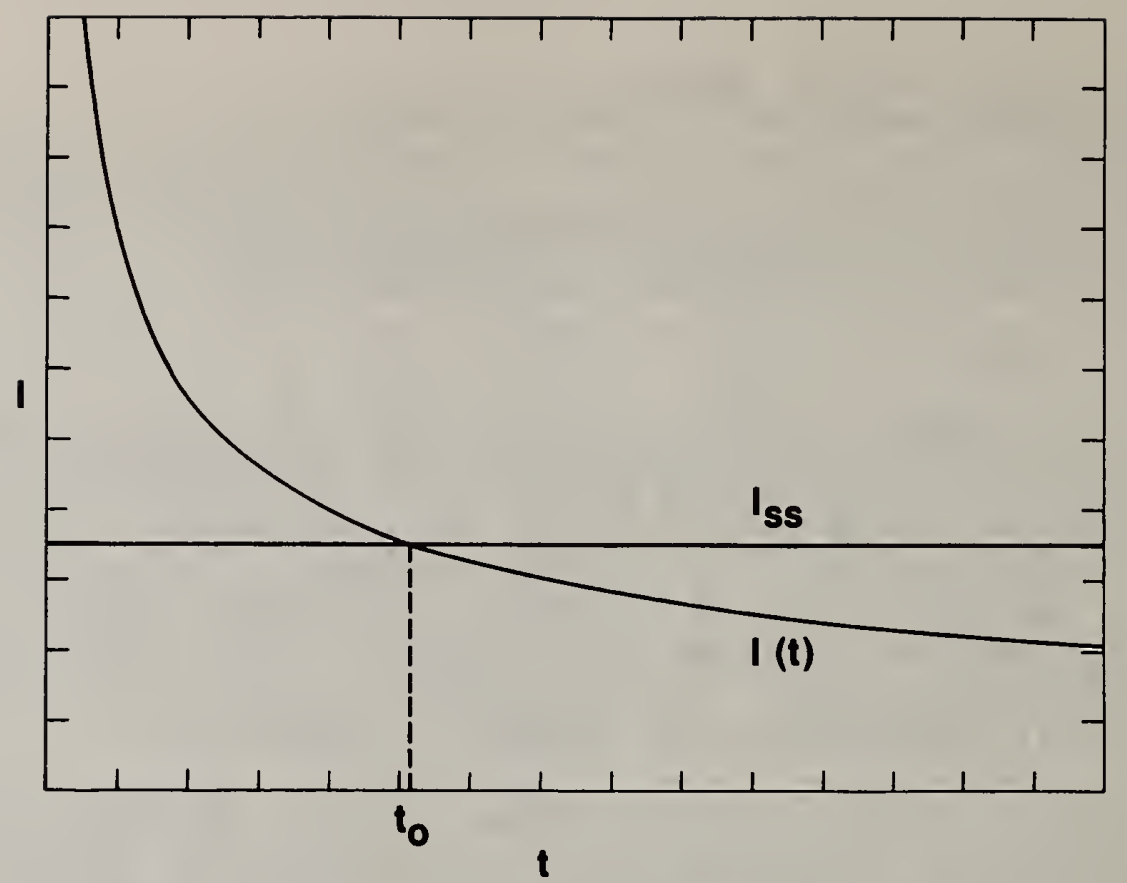

Figure 5. Steady-state and transient faradaic currents for a typical electrode. $I_{s s}=I(t)$ at $t=t_{0}$.

Equation 3 is used to calculate the value of $\delta$, using the experimental $t_{0}$. Values for $D$ may be calculated from ionic mobilities, available from the literature. Neither $A$ nor $C$ needs to be experimentally determined.

Experimental determinations of diffusion layer thickness have been conducted at NBS using rotating disc and vibrating electrodes, for which $\delta$ ranged from 2.5 to $15 \mu \mathrm{m}$. Recordings of $\mathrm{I}(\mathrm{t})$ were obtained using a triggered-sweep oscilloscope and a pulsed potentiostat with $2 \mu \mathrm{s}$ response time. Non-faradaic components were eliminated from the $I(t)-t$ curve by graphic subtraction of the non-faradaic transient recorded in pure supporting electrolyte. Values of $\delta$ obtained by the new method agreed within 10 percent of those obtained using standard techniques. Errors can be attributed to bulk depletion resulting from repetitive pulsing of the electrode. Comparative tests employing a potentiostat designed at NBS proved that the errors in $\delta$ were not related to the response time of the potentiostat.

This technique has been applied to the rotating disc electrode (RDE), for which an expression for $\delta$ based on hydrodynamic theory is known. Using the RDE, this technique has also been employed to determine diffusion coefficients of electroactive species. This same technique is also suited to the direct determination of the Schmidt number, Sc. In conjunction with a signal generator and synchronization circuity developed in this laboratory, theoretical models based on this technique have been developed which describe the experimentally observed electrochemical frequency response characteristic of vibrating wire electrodes.

Future efforts in this area will be directed toward increasing the accuracy of the method and extending the results to a comparative evaluation of the sensitivity of pulsed and hydrodynamic techniques in voltammetry. 


\section{Determination of Vanadium in Botanical, Biological, and Environmental SRM's Using High-Sensitivity, Pulse-Counting Isotope Dilution Thermal Ionization Mass Spectrometry (15310)}

J. D. Fassett, H. M. Kingston

Vanadium is an essential trace element in man and other species of animal and plant-life. The relationship of $V$ to human health is currently being investigated. Vanadium concentration is regulated within a narrow range in the blood stream of healthy individuals but has been shown to vary for both cancer and heart patients. Like many trace elements, $V$ is toxic when ingested at elevated levels. Most cases of $V$ toxicity are related to the use of fossil fuels, whose combustion products are typically $V$ enriched.

A high-sensitivity, isotope dilution thermal ionization mass spectrometric procedure, capable of determining $V$ at the ultratrace level has been developed which makes possible the certification of many biological and botanical standards for $V$ content at natural levels. New procedures in both mass spectrometry and chemistry have been developed to extend earlier work done at NBS to the ultratrace levels.

A series of successively lower $50 \mathrm{~V}$ enriched standards were prepared and calibrated. These calibrated spike solutions and standards permitted all samples, regardless of their $V$. content, to be optimally spiked. A separation procedure that uses a chelating resin was developed. Vanadium-48 and ${ }^{51} \mathrm{Cr}$ radiotracers were used to optimize the separation procedure which chelates the $V$ and then allows it to be selectively removed from a small column of resin. Each sample is oxidized prior to analysis and is contained in a single drop of $6 \mathrm{~mol} / \mathrm{L}$ hydrochloric acid.

The separated $V$ samples are loaded onto rhenium filaments containing a slurry of high purity, spectroscopic graphite. The advantages of this procedure are that a relatively large $\mathrm{V}$ ion beam is formed with low $\mathrm{Cr}, \mathrm{Ti}$, and $\mathrm{V}$ blanks; vanadium oxide formation which can cause unpredictable and undesirable behavior is suppressed; and, the high temperature ionization filament used in triple filament procedures is avoided, thus reducing $\mathrm{Cr}$ background. The loading blank is measured by directly loading $\mathrm{ng}$ amounts of the $\mathrm{V}$ spike and is less than $50 \mathrm{pg}$. Measurements were made on a thermal ionization mass spectrometer equipped with an ion-counting detector, for maximum detection sensitivity.

The determination of $\mathrm{V}$ is complicated by two isobaric interferences, ${ }^{50} \mathrm{cr}$ and ${ }^{50} \mathrm{Ti}$. To accurately measure ${ }^{50} \mathrm{~V} /{ }^{51} \mathrm{~V}$, both ${ }^{52} \mathrm{Cr}$ and ${ }^{48} \mathrm{Ti}$ must be monitored and corrections must be made for these interferences. These elements are essentially separated during the chemical processing. However, residual signals for both elements come from the purified $V$ samples and from the filament loading process. Typical corrections are less than 0.25 percent. The limiting factor in the determination of $V$ is the analytical blank which has ranged from 0.9-4.0 ng. The source of this blank must be identified and the level of the blank must be significantly reduced in order to determine the low $\checkmark$ levels in some important matrices such as human serum, which has a concentration of $0.02-0.04 \mathrm{ng} / \mathrm{g}$. 
Table 3 summarizes the values obtained and the associated measurement uncertainties for the materials analyzed to date. The biological, botanical, and environmental materials which have now been certified for $V$ should provide significant support to initial investigative efforts. However, additional materials such as serum and foodstuffs need to be analyzed to fulfill the needs of researchers evaluating the effects of $V$ on human health.

Table 3. The Concentration of Vanadium in Selected SRM's.

\begin{tabular}{lc}
\multicolumn{1}{c}{ SRM } & Concentration, $\mu \mathrm{g} / \mathrm{g}$ \\
& $2.316 \pm 0.006^{\mathrm{a}}$ \\
1572, Citrus Leaves & $0.2446 \pm 0.0049$ \\
1643b, Trace Elements in Water & $0.04520 \pm 0.00012$ \\
1633a, Fly Ash & $296.7 \quad \pm 0.9$
\end{tabular}

$\underline{\mathrm{RM}}$

$8505, V$ in Crude $011 \quad 390.4 \pm 1.1$

$a_{ \pm}$value represents measurement precision, $s$, for at least six independent determinations.

3. U1tratrace Determinations of Critical Elements in Biological Materials Using Improved Radiochemical Separations (15310)

R. R. Greenberg

In view of the continuing concern over the levels of anthropogenic inputs to the environment, accurate and reliable analytical methods for the determination of toxic elements are needed. The need to determine these elements at lower and lower concentrations necessitates improvements in the analytical methodology. When coupled to an appropriate radiochemical separation, neutron activation analysis (NAA) has the capability of determining many biologically important elements at the required trace and ultratrace levels.

A radiochemical procedure was previously developed to determine $\mathrm{Ag}$, As, $\mathrm{Cr}$, Mo, $\mathrm{Sb}$, and Se via NAA with the use of hydrated manganese dioxide (HMD) inorganic ion exchanger [1]. Copper and cadmium were subsequently extracted from the eluent using $\mathrm{Bi}$ and $\mathrm{Zn}$ diethyldithiocarbamates (DDC) in chloroform, and their concentrations were determined. Unfortunately, most or all of the $P$ contained in a sample is also retained by HMD when the above procedure is used, and $32 \mathrm{p}$ can interfere with the determination of the elements of interest by elevating the background level of radiation. This can be particularly serious when analyzing biological materials such as animal tissues and body fluids, milk, leafy vegetables, etc. where the $P$ concentration can be as high as one 
percent on a dry weight basis. In addition, the determination of $\mathrm{Cd}$, after extraction with $\mathrm{Zn}(D D C)_{2}$, can, at the $\mathrm{ng} / \mathrm{g}$ level and below, suffer from the background radiation produced by the small fraction of radioactive $\mathrm{Zn}$ which accompanies Cd through the separation procedure.

Research was initiated to reduce the level of $P$ retained on the HMD resin and also to reduce the fraction of radioactive $\mathrm{Zn}$ accompanying $\mathrm{Cd}$. A procedure has been developed which, with carefully controlled experimental parameters, reduces the $32 \mathrm{p}$ level on the HMD column by 80-90 percent and has only minimal effects on the elements of interest. Basically, the procedure involves preconditioning the HMD with $1 \mathrm{M} \mathrm{HNO}_{3}-1 \mathrm{M} \mathrm{H}_{3} \mathrm{PO}_{4}$ followed by eluting the ${ }^{32} \mathrm{P}$ with dilute $(0.0025 \mathrm{M}) \mathrm{H}_{3} \mathrm{PO}_{4}$ in $1 \mathrm{M} \mathrm{HNO}_{3}$. With these modifications $\mathrm{Ag}$, As, Mo, and $\mathrm{Sb}$ are quantitatively retained (>99.5 percent), $\mathrm{Cr}$ and Se are nearly quantitatively retained (98.5-99.5 percent) and $\mathrm{Cu}$ and $\mathrm{Cd}$ are quantitatively eluted.

This procedure has been used to analyze Bovine Liver (SRM 1577) and the new Milk Powder (proposed SRM 1549), which is currentiy being certified. The observed concentrations in the Bovine Liver are compared with the certified values in Table 4 . The amount of $32 \mathrm{P}$ retained by the HMD was reduced by 80-90 percent resulting in a reduction of the background levels for most elements by a factor of 5-10. This lower level of background radiation not only improved the counting statistics by a factor of $2-3$, but also allowed the samples to be counted closer to the detector for an additional improvement in sensitivity.

Table 4. Concentrations Observed in Bovine Liver, SRM 1577.

\begin{tabular}{lrrr} 
Element & \multicolumn{1}{c}{ This Work } & \multicolumn{1}{c}{ Certified } \\
Ag $(n g / g)$ & 58 & $\pm 2^{\mathrm{a}}$ & $(60)^{\mathrm{b}}$ \\
As $(\mathrm{ng} / \mathrm{g})$ & 50 & \pm 2 & $55 \quad \pm 5$ \\
Cd $(n g / g)$ & 292 & \pm 8 & $270 \pm 40$ \\
Cr $(n g / g)$ & 85 & \pm 5 & $88 \pm 12$ \\
Cu $(\mu \mathrm{g} / \mathrm{g})$ & $192 \pm 4$ & $193 \pm 10$ \\
Mo $(\mu \mathrm{g} / \mathrm{g})$ & $3.18 \pm 0.03$ & $(3.4)^{\mathrm{b}}$ \\
Sb $(n g / g)$ & $5.6 \pm 0.7$ & $(5)^{\mathrm{b}}$ \\
Se $(\mu \mathrm{g} / \mathrm{g})$ & $1.08 \pm 0.03$ & $1.1 \pm 0.1$
\end{tabular}

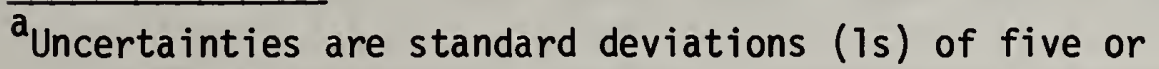
more samples.

${ }^{b}$ Non-certified, information only value.
}

Although $\mathrm{Cd}$ can be determined in most biological materials as part of the multielement HMD-DDC procedure, its concentration in the Milk Powder was too low to be determined with the interference produced by $\mathrm{Zn}(D D C)_{2}$ carry through. Efforts were directed at reducing the levels of radioactive $\mathrm{Zn}$ which accompanied the $\mathrm{Cd}$ through the separation procedure. Several modifications to the 
extraction procedure, including washing the $\mathrm{Zn}(D D C)_{2} / \mathrm{chloroform}$ solution (containing $\mathrm{Cd}$ ) twice with aqueous solutions of $100 \mathrm{mg}$ of unirradiated $\mathrm{Zn}^{+2}$, back-extracting the $\mathrm{Cd}$ into $2 \mathrm{M} \mathrm{HCl}$, and eliminating the $\mathrm{HMD}$ step (to minimize decay of the ${ }^{115} \mathrm{Cd}$ isotope) produced a significantly improved signal-to-noise ratio for $\mathrm{Cd}$. The observed recovery for this separation procedure, while not quantitative was highly reproducible, $95.2 \pm 0.4$ (1s)\%. Five samples of Milk Powder were analyzed and the observed Cd concentration was $0.47 \pm 0.07$ (1s) $\mathrm{ng} / \mathrm{g}$.

Neutron activation analysis has the capability of determining many elements at biologically important levels when coupled to an appropriate radiochemical separation. Improved radiochemical separations have been developed to determine eight biologically active elements at the trace and ultratrace levels frequently required in current biomedical research. Two of these elements, Cd and Sb have been determined at the sub-ppb level in the Milk Powder. Individual separation procedures have previously been developed for $\mathrm{Hg}, \mathrm{Pt}$, Se, and $\mathrm{Sn}$, and continuing research will be directed at improving the methodologies for other biologically important elements such as $\mathrm{Cr}$ and $\mathrm{Ni}$.

\section{Reference}

[1] Gallorini, M., Greenberg, R. R., and Gills, T. E., Anal. Chem., 50, 1479 (1978).

\section{Material Transport (15310)}

R. L. Watters, Jr., J. A. Norris, M. S. Epstein

Many analytical spectrometric techniques consist of a step which converts the sample into vapor followed by an excitation step. Various forms of atomic and ionic spectroscopy are then used to derive the analytical signal. The overall accuracy of such techniques can be limited by bias in the sampling step. When the bulk sample is transported into a chemical and physical form amenable to analysis, the vapor composition may not match that of the original material. By focussing on the processes of sampling for analytical spectrometry we define a broad area of research on material transport devoted to the study of a variety of sampling techniques. The short term goal is to investigate the spark discharge as a sampling device.

The spark discharge produces atoms, ions, and particles by erosion of the cathodic sample. The relative populations and compositions of these species provide the elements necessary to define the material balance of this sampling process. This year we have restricted our attention to the study of the aerosol produced by the spark. The particles exiting an enclosed spark standard were collected using an electrostatic precipitator and examined under an analytical electron microscope (AEM). The spark source is similar to many industrial units of the controlled waveform type which are used for emission spectrometry. A second spark source was constructed to study the affects of specific electronic parameters on aerosol production. Electronic triggering of this source enables a wide range of spark repetition rates to be used. 
A Standard Reference Material Brass Sample (SRM C1101) was chosen to compare the $\mathrm{Zn} / \mathrm{Cu}$ ratio in the collected aerosol with the bulk composition. The certified values are 69.50 percent $\mathrm{Cu}$ and 30.30 percent $\mathrm{Zn}$ for a $\mathrm{Zn} / \mathrm{Cu}$ ratio of 0.436 . The electronically triggered source was used to generate aerosol for excitation with the inductively coupled plasma (ICP). The intensity ratio of $\mathrm{Zn} / \mathrm{Cu}$ emission from the ICP stabilized after a few seconds and remained constant for 60 seconds. Furthermore, peak spark current, duration, and repetition rate seemed to have little effect on the $\mathrm{Zn} / \mathrm{Cu}$ intensity ratio. However, observation of the aerosol using the AEM revealed two particle size domains. A sparsely populated fraction consisted of 500 nanometer diameter copper spheres in which $\mathrm{Zn}$ could not be detected. The predominant fraction consisted of aggregates of 10 nanometer particles. Although these particles contained both $\mathrm{Cu}$ and $\mathrm{Zn}$, the exact concentration ratio has yet to be measured. From these observations, it appears that the major mass fraction of sampled brass is contained in the aggregates and that the composition of the sampled material remains constant regardless of spark source parameters mentioned.

Particles from a titanium based alloy (SRM 654) and a stainless steel material (SRM 1154) have also been examined using the AEM. Differences in composition between spheres and aggregates are apparent in these materials as well, and the relative population of the spheres in the stainless steel aerosol is greater than the other aerosols.

It is thought that the aggregates result from vapor-phase condensation of the sampled material and that spheres result from ejection of molten material from the sample surface. It is, therefore, likely that the relative populations of these size fractions will vary with sample matrix. In order to quantitatively measure the size fraction populations, an aerosol charge mobility analyzer will be coupled to the electronically triggered source. Each fraction will then be isolated for independent chemical analysis using ICP, direct current plasma, and atomic absorption techniques. Observed variations in particle size and composition, and the degree to which these variations can be controlled, will then be related to matrix type. The optical equipment for investigating condensation processes using laser fluorescence is presently being assembled for the next major phase of the study.

\section{Analysis and Stability of Environmental Specimen Bank Samples (15310)}

K. A. Fitzpatrick, S. F. Stone, R. Zeisler

Several tasks of the Pilot Environmental Specimen Bank program (see previous reports $[1,2]$ ) have been accomplished in the past year. The first sample type, human livers, is being collected and analyzed on a continuing basis. From the previously banked and analyzed human liver specimens, a set of 12 was selected and reanalyzed to evaluate their stability during long term storage. A second sample type, Mytilus edulis, representing an aquatic accumulator, was added to the bank.

Human Livers. The collection of liver samples is still carried out by a contractor in Seattle. By the end of this fiscal year, a total of 4501 iver samples will have been banked. Homogenization and analysis of the 30 baseline samples collected during the second year are completed. This brings the total 
number of analyzed livers to 66 . The trace element concentrations found during the second year of the study are comparable to those found during the first year.

The reanalysis of the first year samples was carried out to evaluate the effect of the different storage conditions on elemental concentrations. In comparing data from the samples stored at liquid nitrogen vapor temperature $\left(-120{ }^{\circ} \mathrm{C}\right.$ to $\left.-190{ }^{\circ} \mathrm{C}\right)$, at $-80^{\circ} \mathrm{C}$, at $-25{ }^{\circ} \mathrm{C}$, and freeze dried at room temperature, no statisticaliy significant changes (see [1]) have been found for trace element concentrations.

Mussels. The marine mussel, Mytilus edulis, was added to the bank as the second environmental sample. A set of 6000 mussels was collected from Narragansett Bay, R.I., as part of the first year study to develop the sampling and analytical protocols. The mussels were separated into batches of 70 animals each and banked under the four storage conditions: $\operatorname{LN}_{2}\left(-120{ }^{\circ} \mathrm{C}\right.$ to $\left.-190{ }^{\circ} \mathrm{C}\right),-80^{\circ} \mathrm{C},-25^{\circ} \mathrm{C}$, and freeze dried at room temperature. Two batches have been homogenized. Individual aliquots of the homogenate are stored at $\mathrm{LN}_{2}$ vapor temperature and, to date, ten subsamples from each batch have been analyzed.

The implementation of a completely instrumental analytical protocol was initiated. Concentration for 28 trace elements were determined using neutron activation analysis (INAA). This technique is complemented by an $x$-ray fluorescence procedure to obtain data for additional elements and also to provide a check on quality assurance for selected values obtained from INAA. An intercomparison of the two techniques has been initiated. Table 5 shows the mean values and their standard deviations for each of the elements obtained from INAA in the ten subsamples. Additional data are given for a freshwater mussel homogenate from the German Pilot Environmental Specimen Bank. This analysis was done as part of the ongoing exchange of specimens between the two programs and subsequent intercomparisons. The German homogenate shows elevated europium and chromium levels and an unusually high hafnium concentration. The difference in the bromine concentrations is explained by bromine being a component of sea salt, which would be present in the marine mussel tissues.

The future activities in this program will focus on the dissemination of the results to the potential user communities and on the technology transfer to other related programs. A cooperative program is being developed with the Army Institute of Pathology. The goal is to investigate possible relationships between trace constituent concentrations in different human organs and diseases. In continuation of the initial pilot program, the sampling and analysis procedures for the measurements of trace constituents in human dietary intake will be reviewed. Collaboration in this area has been initiated with USDA, FDA and IAEA.

References

[1] Annual Report 1981, CAC, NBSIR 81-2425, p. 9-16.

[2] Annual Report 1982, CAC, NBSIR 82-2620, p. 10-21. 
Table 5. Trace Elements in Marine and Freshwater Bivalves.

(Concentration in $\mu \mathrm{g} / \mathrm{g}$ )

Mytilus Edulis

M2N0054

$\mathrm{Na}$

$\mathrm{Mg}$

AT

C1

$\mathrm{K}$

$\mathrm{SC}$

Mn

$\mathrm{Fe}$

Co

$\mathrm{Cu}$

As

Se

$\mathrm{Br}$

$\mathrm{Rb}$

Mo

$\mathrm{Ag}$

Cd

Cs

La

Ce

Sm

$\mathrm{Eu}$

$\mathrm{Hg}$

Th

Hf

$\begin{array}{clc}54200 & \pm 1000 \\ 6430 & \pm 540 \\ 760 & \pm 70 \\ 100100 & \pm 4900 \\ 11700 & \pm 1500 \\ 0.138 & \pm & 0.007 \\ 2.05 & \pm & 0.20 \\ 21.9 & \pm & 1.2 \\ 571 & \pm & 27 \\ 0.538 & \pm & 0.014\end{array}$

$\leq 70$

$122 \pm$

$2.47 \pm$

$443 \pm$

$7.24 \pm$ $\leq 1.4$

$0.104 \pm$ $\leq 4$

$0.255 \pm$

$0.683 \pm$

$0.59 \pm$

$0.98 \pm$

$0.101 \pm$

$0.0193 \pm$

$0.48 \pm$

$0.139 \pm$

$0.070 \pm$
5

1.0

0.11

40

0.56

0.013

0.007

0.003

0.09

0.10

0.014

0.0004

0.24

0.006

0.018
Dreissena Polymorphy

Mussel Homog.

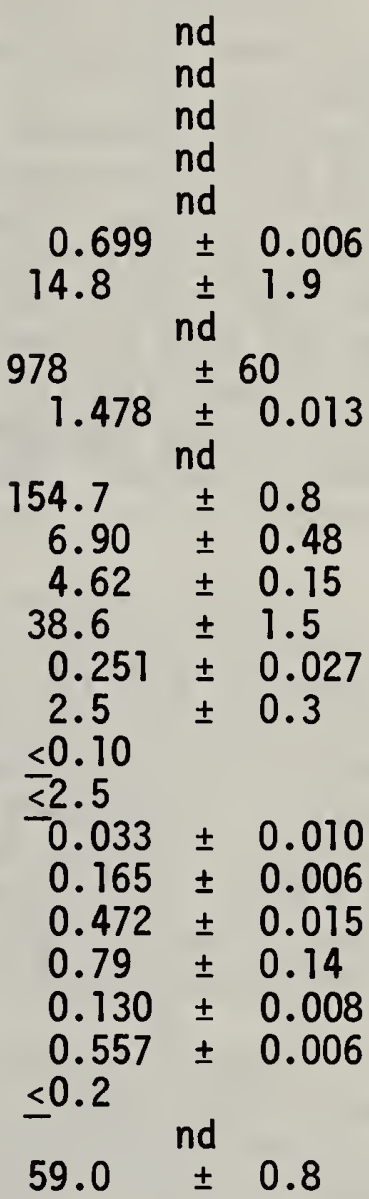

nd $=$ not determined. Errors represent sample standard deviation and measurement uncertainty. 
6. The Characterization of Metastable Excited State Atomic Species Produced in Thermal Vaporization Using Resonance Ionization Mass Spectrometry (RIMS) (26108)

\author{
J. D. Fassett, L. J. Moore, J. C. Travis (550), F. E. Lytle
}

Resonance ionization mass spectrometry (RIMS) combines mass spectrometry with laser photo-ionization to provide a selective and sensitive method of producing ions from a gas phase reservoir of atoms. As part of a multiyear competence research program in ionization mechanisms and ion structures, a RIMS system has been established at NBS. This system is one of the first in the U.S., where there are presently four or five laboratories actively investigating applications of RIMS in analytical chemistry. Only recently have the results of these investigations begun to appear in the literature. The NBS RIMS system was described in last year's report [1] and at that time preliminary results were discussed. In the past year the system has been considerably upgraded, allowing it to be better utilized in a variety of research modes. This report summarizes the broad elemental utility of the system.

The NBS system is conceptually different from the RIMS systems established elsewhere $[2,3]$. By designing the system around a tunable dye laser with frequency-doubled output, it was hoped that an experimentally versatile instrument would be established that could ionize a majority of elements in the periodic table. Experiments have shown this original design concept to be correct. Resonance ionization has been demonstrated for 25 elements in this laboratory during the past year. Fully 75 percent of all elements ionized by RIMS to date were first studied here at NBS. The majority of these elements have extremely high ionization potentials and thus are difficult to ionize by conventional means.

Several important changes have been made in the RIMS system. The initial results indicated that the atomic spectroscopy was a much more complex phenomenon than previously anticipated [1]. Routine atomic spectroscopy was made possible by completely automating the wavelength scanning capabilities of the laser system. A computer-controlled stepper motor was coupled to the grating of the dye laser with a minimum step size of $0.5 \mathrm{pm}$ in the UV. An autotracker was added to the frequency doubler which maintains maximum conversion of the visible light to the UV as the dye laser frequency is scanned. Software was implemented to collect the data and plot ion-intensity versus wavelength information in real time and to manipulate these data in a variety of useful ways. The interaction of the laser beam with the atom plume was also changed by the removal of the $200 \mathrm{~mm}$ lens which was previously used to focus the UV light through the mass spectrometer. The saturation effects, which occurred with focussing, severely distorted the laser optical spectra. These changes have helped to make the spectroscopy more tractable.

The production of neutral atomic beams by thermal vaporization and resonance ionization has been demonstrated for 25 elements. Table 6 summarizes the results for those elements investigated with the wavelength range of the dye Rhodamine $610,292-304 \mathrm{~nm}$, and in figure 6 the breadth of types of atomic metastable distributions is illustrated, ranging from $\sim 100$ percent ground state 
Table 6. Resonance Ionization Mass Spectrometry Spectroscopic Survey Between 292-304 nm.

\begin{tabular}{|c|c|c|c|c|c|c|}
\hline ELEMENT & $\begin{array}{l}\text { FIL./ } \\
\text { TECH. }\end{array}$ & $\begin{array}{c}\text { TEMP } \\
\mathbf{K} \\
\end{array}$ & $\begin{array}{r}\text { SPE } \\
\text { GSO } \\
\end{array}$ & $\begin{array}{l}\text { TRAL } \\
\text { ESO } \\
\end{array}$ & $\begin{array}{l}\text { LINE } \text { S }^{a} \\
\text { UNTAB } \\
\end{array}$ & $\begin{array}{l}\text { MAX. } \\
\text { INT. }\end{array}$ \\
\hline Co & $\operatorname{Re} / \mathrm{H}$ & 1195 & 4 & 2 & 2 & 30. \\
\hline Dy & $\mathrm{Re} / \mathrm{H}$ & 1470 & 1 & 0 & 10 & .72 \\
\hline Er & $\mathrm{Re} / \mathrm{H}$ & 1561 & 1 & 0 & 4 & 6.4 \\
\hline $\mathrm{Fe}$ & $\mathrm{Re} / \mathrm{H}$ & 1228 & 4 & 17 & 0 & 138. \\
\hline Hf & $\mathrm{Hf}$ & $(3.75 \mathrm{~A})$ & 5 & 7 & 1 & 15.6 \\
\hline In & $\operatorname{Re} / C$ & 1070 & 1 & 0 & 0 & .84 \\
\hline $\mathrm{Lu}$ & $\mathrm{Re} / \mathrm{H}$ & 1760 & 1 & 0 & 0 & .44 \\
\hline Mo & $\mathrm{Re} / \mathrm{H}$ & 1184 & 2 & 0 & 1 & 47. \\
\hline N1 & $\mathrm{Re} / \mathrm{H}$ & 1228 & 3 & 8 & 18 & 11.1 \\
\hline Re & $\mathrm{Re}$ & 2291 & 2 & 34 & 23 & 4700 \\
\hline S r & $\mathrm{Re} / \mathrm{H}$ & 1294 & 1 & 0 & 0 & 1.5 \\
\hline $\mathrm{T} \mathbf{a}$ & $\mathbf{T} \mathbf{a}$ & $(2.75 \mathrm{~A})$ & 2 & 15 & 4 & 137. \\
\hline $\begin{array}{l}\text { Th } \\
\text { T1 }\end{array}$ & $\begin{array}{l}\mathrm{Re} / \mathrm{C} \\
\mathrm{Re} / \mathrm{C}\end{array}$ & $\begin{array}{l}1967 \\
1794\end{array}$ & $\begin{array}{l}1 \\
4\end{array}$ & $\begin{array}{r}0 \\
11\end{array}$ & $\begin{array}{r}36 \\
4\end{array}$ & $\begin{array}{l}85 . \\
27 .\end{array}$ \\
\hline $\mathbf{U}$ & $\mathrm{Re} / \mathrm{C}$ & 1933 & 2 & 2 & 180 & 3.5 \\
\hline V & $\mathrm{Re} / \mathrm{C}$ & 1828 & 2 & 11 & 1 & 3.7 \\
\hline $\mathbf{Y}$ & $\mathrm{Re} / \mathrm{C}$ & 1736 & 5 & 6 & 2 & 40 \\
\hline $\mathrm{Zr}$ & $\mathrm{Re} / \mathrm{C}$ & 2033 & 2 & 2 & 10 & 3.8 \\
\hline
\end{tabular}

${ }_{\text {a }} S O=G r o u n d-S t a t e-0 r 1 g 1$ at 1 ng. ESO=Exc1ted-State-0riginat $1 \mathrm{ng}$. Tabulated from NBS Monograph 145, Part 1.

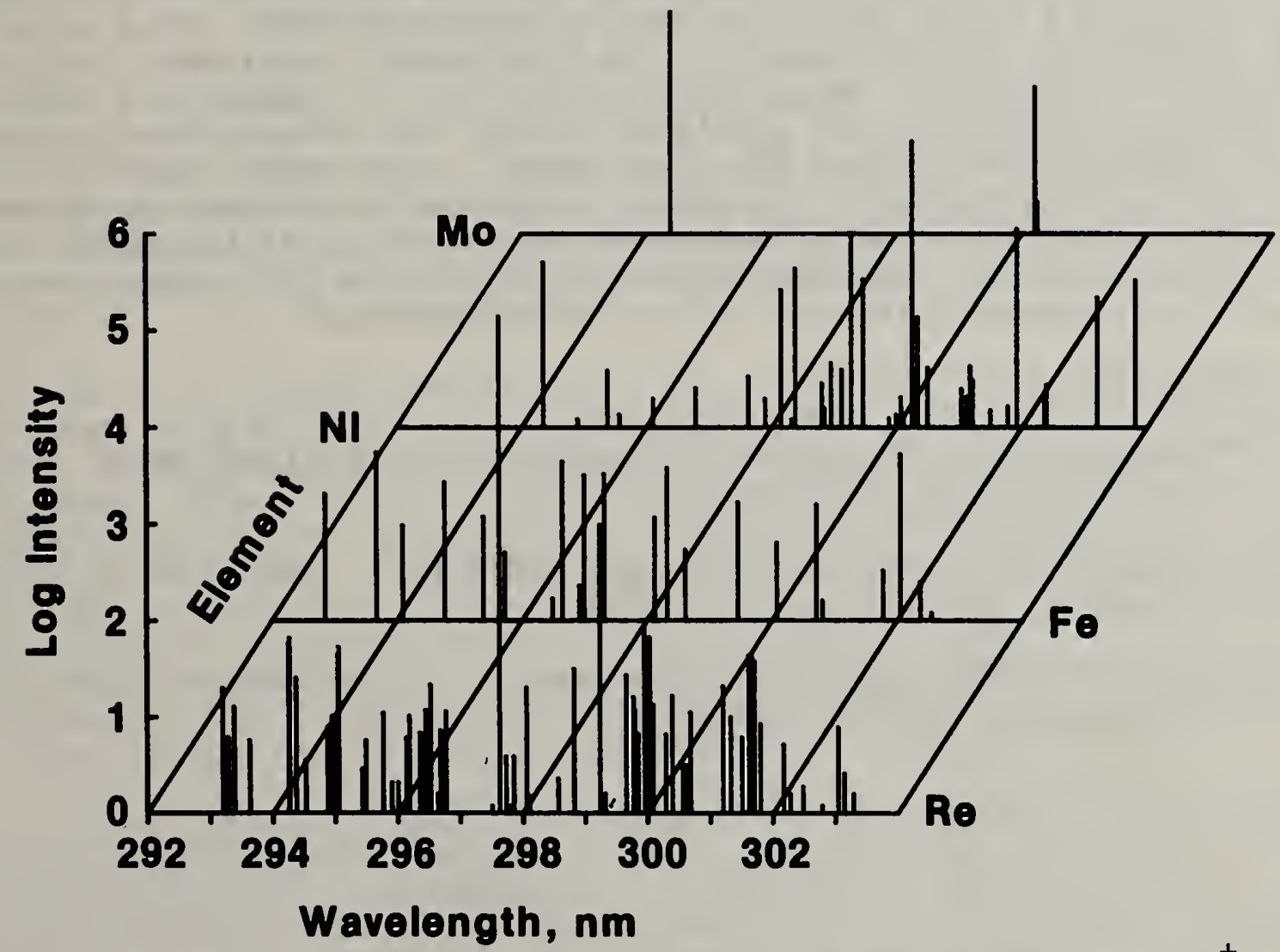

Figure 6. RIMS spectra, ion intensity versus wavelength, of ${ }^{187} \mathrm{Re}^{+}$, ${ }^{60} \mathrm{Ni}^{+},{ }^{56} \mathrm{Fe}^{+}$, and ${ }^{98} \mathrm{Mo}^{+}$. 
populations for Mo, to broadly populated lower energy level states for Fe and $\mathrm{Ni}$. In addition to the elements in the table, $\mathrm{Li}, \mathrm{Sn}, \mathrm{Mg}, \mathrm{Os}$, and $\mathrm{Pb}$, as well as I and C have also been photoionized. The samples were prepared for these experiments by two methods designed to generate reduced elemental species on the thermal filament substrate: a hydrogen reduction process and a carbonization process (designated $H$ and $C$, respectively in the table). Sample sizes were typically one microgram.

Peaks corresponding to the predicted ground-state-originating resonant transitions were observed. Also observed were peaks corresponding to transitions from atomic excited states and untabulated, unexplained peaks. An anlysis of the excited-state-originating lines produced the following observations. Although the relative intensities of lines are dependent upon photon absorption cross sections, the wavelength-dependent photon flux, the geometry of the laser/atom plume interaction volume, as well as the populations of the transition-originating state, it was shown that the line intensities semiquantitatively follow a Boltzmann distribution of excited state populations as defined by the temperature of the filament.. This result implies that equilibrium conditions are established in the thermal vaporization source and that excited-state atomic species which are formed in the vaporization process are long lived.

The extent to which metastable excited-states affect analytical measurements using RIMS will be strongly dependent upon the element and its electronic structure and to a lesser degree the vaporization temperature. For a large number of elements, excited states will be significantly populated. The implications are manifold. Absolute sensitivity will be reduced by a factor corresponding to the fractional population of the level from which excitation occurs, ionization efficiencies being equivalent. Furthermore, spectral selectivity will be reduced as the number of observed transitions per element increases. A positive aspect of metastable atom formation is the possibility of using these spectral lines analytically in RIMS. This fact widens the potential multielement analytical utility of the technique.

\section{References}

[1] Fassett, J. D., Travis, J. C., Moore, L. J., and Lytle, F. E., Anal. Chem., 55, 765 (1983).

[2] Donahue, D. L., Young, J. P., and Smith, D. H., Int. J. Mass Spectrom. Ion Phys., 43,293 (1982).

[3] Miller, C. M., Nogar, N. S., Gancarz, A. J., and Shields, W. R., Anal. Chem., 54, 2377 (1982). 
7. Interferences in the Determination of Germanium Using Electrothermal Atomization Atomic Absorption Spectrometry (15310)

Y. K. Zhang, T. C. Rains

Spirogermanium- $\mathrm{HCl}\left(\mathrm{C}_{2} \mathrm{H}_{5}\right)_{2} \mathrm{Ge} \longrightarrow{ }^{\mathrm{N}-\left(\mathrm{CH}_{2}\right)_{3} \mathrm{~N}\left(\mathrm{CH}_{3}\right)_{2}} \cdot 2 \mathrm{HCl}$, an antineoplastic agent, is being clinically evaluated on carcinoma cells and as an anti-malaria drug at the Georgetown University Hospital, Washington, DC. This material is a white powder which is soluble in water and 95 percent ethanol and has a molecular formula of $\mathrm{C}_{17} \mathrm{H}_{36} \mathrm{GeN}_{2} \cdot 2 \mathrm{HCl}$. Presently, there is no established clinical method for the determination of germanium in biological materials. The determination of germanium is difficult by any method because it forms volatile halide compounds. In atomic absorption spectrometry, germanium forms a highly stable oxide species at relatively high temperatures which precludes the efficient production of germanium atoms. The high temperature nitrous oxide-acetylene flame has a detection limit of approximately $1.5 \mu \mathrm{g} \mathrm{Ge} \mathrm{mL}{ }^{-1}$. However, the small quantity of sample available for analys is in some biological samples precludes the use of any flame technique.

Electrothermal atomic absorption spectrometry (ETAAS) is frequently used for trace analysis. However, the improved sensitivity for germanium by ETAAS was not attained when the biological material was sampled off the walls of the graphite tubes. With the addition of the L'vov platform to the graphite tube, the absorption signal for germanium improved by an order of magnitude. This supports the theory that the germanium is being volatilized as the oxide. When the sample is volatilized off the wall of the graphite tube, the Ge0 passes into the inner gas cavity, which is cooler than the wall temperature. Under these conditions, the $\mathrm{GeO}$ is not completely dissociated into the atomic form. When the L'vov platform is used, the Ge0 remains on the platform longer permitting the inner gas cavity to reach a higher temperature; therefore, the $\mathrm{GeO}$ is more likely to be dissociated.

The L'vov platform used in this study is similar to the one described by Henderberger et al. [1]. A 5 by $8 \mathrm{~mm}$ curved section is cut from the grooved end of a pyrotytically coated tube. No difference has been shown between used and unused tubes. The sides of the chip or platform are filed so that the platform will fit into the furnace, and the platform is inserted into another graphite tube using a pair of tweezers. The platform is centered directly under the sample port by using a metal probe. The window is reinstalled and the injection tip adjusted to ensure that it does not come in contact with the platform. There should be a $1 \mathrm{~mm}$ gap between the platform and the bottom of the graphite cell. If the platform rests on the bottom of the graphite tube, a longer cooling down period will be required after atomization.

Initial studies without the L'vov platform verified the reports of interferences found by other workers. Chloride and phosphate ions severely interfere with the determination of germanium. To alleviate these difficulties, a series of matrix modifiers was evaluated. Nickel nitrate is widely used in ETAAS as a matrix modifer to suppress the volatilization of As, Se, and Sn. Figure 7 shows a comparison of the absorption of germanium with and without nickel. Not only did the nickel increase the sensitivity of germanium but it also permitted 


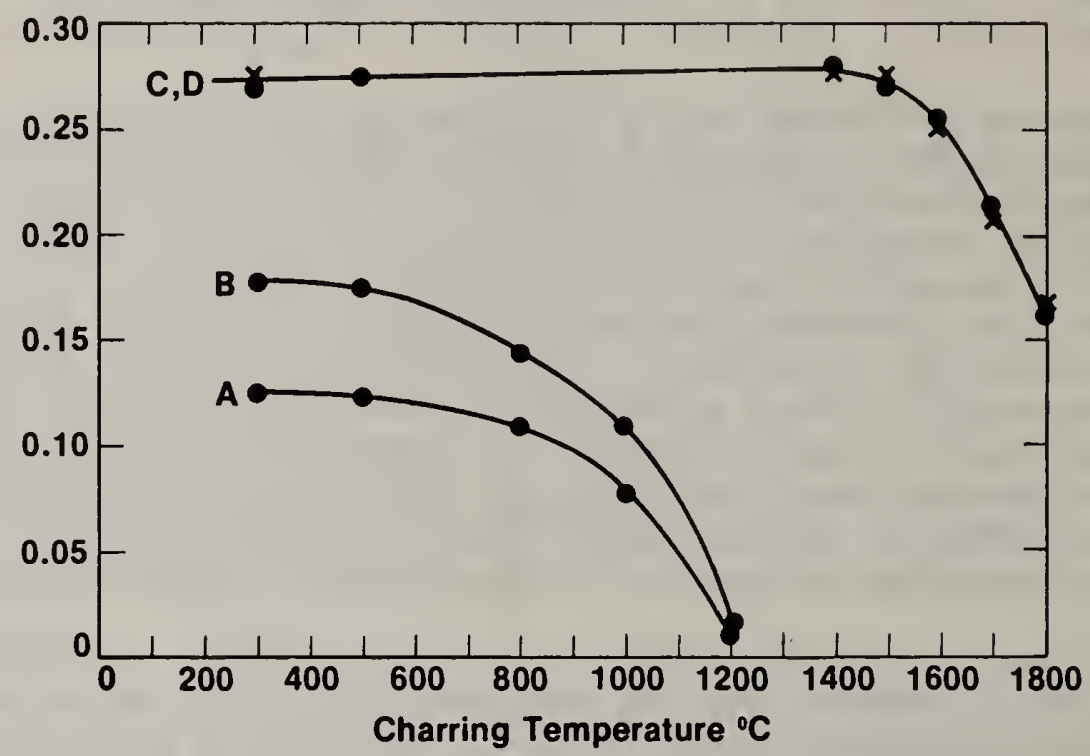

Figure 7. Effect of charring temperature on absorption of $4 \mathrm{ng}$ of germanium with and without matrix modifiers.
A. $1 \% \mathrm{HNO}_{3}$
B. $1 \% \mathrm{HNO}_{3}$ plus $1 \%$ ammonium tartrate
C. $1 \% \mathrm{HNO}_{3}$ plus $1000 \mu \mathrm{g} \mathrm{Ni} / \mathrm{ml}(.-$.
D. $1 \% \mathrm{HNO}_{3}, 1 \%$ ammonium tartrate plus $1000 \mu \mathrm{g} \mathrm{Ni} / \mathrm{mL}(\mathrm{x}-\mathrm{x})$

the use of a higher charring temperature. Nickel concentrations of $1000 \mu \mathrm{g} / \mathrm{mL}$ did not alleviate the interferences resulting from concentrations of chloride greater than 0.5 percent. Ammonium tartrate was investigated but showed little improvement over a 1 percent $\mathrm{HNO}_{3}$ solution (Figure 7). However, with the addition of nickel nitrate plus ammonium tartrate the interference of chloride was completely removed and a charring temperature of $1200{ }^{\circ} \mathrm{C}$ could be used.

With no matrix modifier, 1 to $10 \mu \mathrm{g} / \mathrm{mL}$ of $\mathrm{Na}, \mathrm{K}, \mathrm{Ca}, \mathrm{Mg}$, and $\mathrm{PO}_{4}$ completely suppressed the absorption signal of $0.2 \mu \mathrm{g}$ of germanium. With the addition of $1000 \mu \mathrm{g}$ of nickel and 1 percent ammonium tartrate, concentrations of $5000 \mu \mathrm{g} / \mathrm{mL}$ of $\mathrm{Na}, \mathrm{K}$, and $\mathrm{Ca}$ produced no adverse effect. Under the same conditions, a slight suppression of $0.2 \mu \mathrm{g} / \mathrm{mL}$ of Ge was observed at the $5000 \mu \mathrm{g} / \mathrm{mL}$ level of magnesium. The presence of $1000 \mu \mathrm{g}$ of $\mathrm{PO}_{4}{ }^{-3}$ produced a severe suppression of the signal from $0.2 \mu \mathrm{g} / \mathrm{mL}$ of $\mathrm{Ge}$. By increasing the concentration of nickel to $4000 \mu \mathrm{g} / \mathrm{mL}$ the effect of the signal from $2000 \mu \mathrm{g} / \mathrm{mL}$ of $\mathrm{PO}_{4}^{-3}$ on the signal from $0.2 \mu \mathrm{g} / \mathrm{mL}$ of $\mathrm{Ge}$ was eliminated. 
In summary, a method has been developed to determine germanium by ETAAS at the submicrogram level using nickel and ammonium tartrate as matrix modifers in conjunction with the L'vov platform. The procedure has been used to determine germanium in samples of serum, plasma, red blood cells, urine, and bile from patients treated with the drug at Georgetown University Hospital.

\section{Reference}

[1] Hinderberger, E. J., Kaiser, M. L., and Koirtyohann, S. R., Atomic Spectrosc., $2,1-7$ (1981).

\section{Gases-in-Metals Analys is (15310)}

B. I. Diamondstone, D. E. Brown, G. J. Lutz

The capability to make quantitative measurements of gases-in-metals has not been available at NBS for the last 15 years. As a result of outside requests, the Division has begun to reestablish this capability with the support of the Office of Standard Reference Materials. Earlier determinations of gases-in-metals involved the use of hot-extraction and vacuum-fusion techniques for hydrogen while analyses for oxygen and nitrogen were done by vacuum-fusion, inert gas fusion and neutron activation techniques. Of primary importance has been the evaluation of newly received instrumentation to determine its capabilities. In addition, the operators of the equipment must gain the experience necessary for the analyses of various gases in a wide variety of materials.

The gases-in-metals instrumentation has been combined, in a single laboratory, with the carbon and sulfur analyzers. The new equipment consists of a furnace and detector combination for the simultaneous determination of oxygen and nitrogen as well as a similar unit for the determination of hydrogen.

Initial studies involved the analyses of a number of commercially available standards as well as NBS Standard Reference Materials (SRM's) to evaluate the precision and accuracy of the instrumentation. Participation in a number of ASTM round robins has also provided valuable information during the evaluation period. Both of the above exercises required considerable investigation into "proper" methods of sample preparation. This work is ongoing and is recognized as a critical part of any analysis for gases in metals.

An important goal of the gases-in-metals program is to establish two independent techniques for the certification of $\mathrm{H}_{2}, \mathrm{~N}_{2}$, and $\mathrm{O}_{2}$ in metal SRM's. Work is underway to utilize activation analysis as a supporting technique. A recent literature survey confirmed that $14 \mathrm{MeV}$ neutron activation of oxygen is specific for most metals and the lower limit is a few tenths of a ppm. Lower levels can be determined by ${ }^{3} \mathrm{He}$ activation using an accelerator. Preliminary samples have been delivered to the 14 MEV facility at Texas A\&M. If the information obtained from Texas A\&M is promising, then further studies involving NBS personnel will be planned. An extensive library search is underway to locate potential backup techniques for $\mathrm{H}_{2}$ and $\mathrm{N}_{2}$ analyses. 
The most significant accomplishment of the program, to date, has been the completion of the analysis of SRM 352b, an unalloyed titanium which will be certified for $\mathrm{H}_{2}$ and will replace SRM 352a. Although the data have not been finalized, preliminary values indicate that this material will be certified with an uncertainty considerably less than its predecessor.

The increased need for SRM's certified for gases will result in more pressure to produce accurate and precise values for gases-in-metals at continually decreasing concentrations. Considerable effort will be made to update "information only" values on existing SRM's as well as to produce new ones. Emphasis during the next few years will be on developing backup techniques for certification and carefully evaluating the effects of sample preparation on observed concentrations.

\section{The Standard Potential of the $\mathrm{Ag} / \mathrm{AgCl}$ Electrode for High Accuracy pH Measurements (15310)}

Y. C. Wu, G. Marinenko, K. W. Pratt, Jr., W. F. Koch

The $\mathrm{Ag} / \mathrm{AgCl}$ electrode is a thermodynamically reversible electrode of the second kind. Its usefulness has been demonstrated for many years and as a result it has been adopted as a reference electrode. The use of the $\mathrm{Ag} / \mathrm{AgCl}$ electrode for $\mathrm{pH}$ determinations was originated at NBS in the late thirties [1]. Today this electrode is used routinely at NBS to certify pH measurements in a number of SRM's.

The measurement of $\mathrm{pH}$ is directly related to $\mathrm{E}^{\circ}$ of $\mathrm{Ag} / \mathrm{AgCl}$ through the following relationship:

$$
\mathrm{pH}=-\log \mathrm{a}_{\mathrm{H}^{+}}=\log \mathrm{m}_{\mathrm{Cl}}{ }^{-}+\frac{E-E^{\circ}}{\mathrm{RT} \ln 10} F+\log \left(\gamma_{\mathrm{Cl}}{ }^{-}\right)_{I}
$$

where $a_{H^{+}}$is the activity of hydrogen ion, $\mathrm{m}_{\mathrm{Cl}}{ }^{-}$is the concentration of chloride ion, $E$ is the emf of the cell, $E^{\circ}$ is the emf of $\mathrm{Ag} / \mathrm{AgCl}$ electrodes at their standard states, $F$ is the Faraday constant, $\gamma_{\mathrm{C}}{ }^{-}$is the activity coefficient of chloride ion, and $\log \left(\mathrm{r}_{\mathrm{Cl}}{ }^{-}\right)_{\mathrm{I}}$ is evalauted at $\mathrm{m}_{\mathrm{Cl}}{ }^{-} \rightarrow 0$ and at constant ionic strength of the test solution. The value of $E^{\circ}$ is the measure of accuracy for determining $\mathrm{pH}$. Recently, Bates [2] has reported that values for $\mathrm{E}^{\circ}$ of $\mathrm{Ag} / \mathrm{AgCl}$ determined in cells without-transference from over 30 independent measurements vary from 0.2222 to $0.2228 \mathrm{~V}$ at $25^{\circ} \mathrm{C}$. The extreme difference is about $0.0006 \mathrm{~V}$, which amounts to differences of approximately $0.01 \mathrm{pH}$ units in subsequent $\mathrm{pH}$ measurements.

Variations in $\mathrm{E}^{\circ}$ of such magnitude $(0.6 \mathrm{mV})$ must be caused by the $\mathrm{Ag} / \mathrm{AgCl}$ electrode itself, because the other factors in the system for determining $E^{\circ}$ can be systematically eliminated. In fact, experts met in 1956 and decided to assign an uncertainty of $0.2 \mathrm{mV}$ to the $E^{\circ}$ measurements for this electrode. The sources of problems associated with this electrode have been investigated by numerous workers in electrochemistry. The areas studied have included methodology, electrode preparation, and operational precautions. The 
characteristics of this electrode needed to be better defined in order to reduce the uncertainties associated with $\mathrm{pH}$ measurements done on SRM's at NBS.

When classical experiments were repeated, it was observed that oxygen entered the electrode and was probably the major cause of the observed variations in $E^{\circ}$. Although this observation was not new, the effect had never been fully discussed and investigated. To verify the effect of oxygen on the behavior of the electrode, the following experiments were conducted.

A. Electrode Preparation: A thermal electrolytic type of preparation was selected because the method is simple and avoids contamination. Classical procedures were followed, except when electrodes were necessarily exposed to either ordinary or argon atmosphere.

B. Cell EMF Measurement: The cell setup, temperature control, and measuring device were similar to those described by Durst [3] with a few minor modifications. Special care was taken to have the electrode and $\mathrm{HCl}$ solution under either ordinary or argon atmosphere.

Four sets of experimental conditions were compared:
(1) $E_{e l}(0)^{-S(0)}$
(2) $\mathrm{E}_{\mathrm{el}}(0)^{-S(A r)}$
(3) $E_{e l(A r)}-S(0)$
(4) $\mathrm{E}_{\mathrm{el}}(\mathrm{Ar})^{-S(A r)}$

where el $(0)=$ electrode exposed to air, el $(A r)=$ electrode exposed to argon, $\mathrm{S}(0)=\mathrm{HCl}$ solution saturated with air, $\mathrm{S}(\mathrm{Ar})=\mathrm{HCl}$ solution saturated with argon. The range of results for approximately one hundred electrode measurements under the four sets of experimental conditions respectively are:

(1) $E^{\circ}=0.22260 \mathrm{~V}$ and up

(2) $E^{\circ}=0.22250-0.22260 \mathrm{~V}$

(3) $E^{\circ}=0.22240-0.22250 \mathrm{~V}$

(4) $E^{\circ}=0.22230-0.22240 \mathrm{~V}$

The results clearly demonstrate the oxygen effect on $\mathrm{E}^{\circ}$ for the $\mathrm{Ag} / \mathrm{AgCl}$ electrode. However, the overall reproducibility for each set was only $\pm 0.05 \mathrm{mV}$ as compared with 0.01 to $0.02 \mathrm{mV}$ as reported in the literature. Work is continuing in the areas of oxygen adsorption and desorption measurements to quantitatively determine the effects of oxygen on $E^{\circ}$. This work is necessary to lower the uncertainties in existing $\mathrm{pH}$ measurements.

References

[1] Hamer, W. J. and Acree, S. F., "Potentiometric Method for the Accurate Measurement of Hydrogen-ion Activity," J. Res. NBS, 23, 647 (1939).

[2] Bates, R. G. and Macaski11, J. B., "Standard Potential of the Ag/AgCl Electrode," Pure App1. Chem., 50, 1701 (1978).

[3] Durst, R. A., "Standardization of pH Measurements," NBS Special Publication 260-53 (1975). 
J. D. Fassett, L. J. Moore, J. C. Travis (550), T. B. Lucatorto (533),

C. W. Clark (531)

The elements iodine and carbon possess long-lived radionuclides $\left({ }^{129} \mathrm{I}, \mathrm{t}_{1 / 2}=1.7 \times 10^{9} \mathrm{y} ;{ }^{14} \mathrm{C}, \mathrm{t}_{1 / 2}=5.7 \times 10^{3} \mathrm{y}\right)$ that are important as environmental tracers of natural and anthropogenic activity. The measurement of the radionuclides by mass spectrometry has provided major challenges; the high ionization potentials $(C=11.25 \mathrm{eV} ; \mathrm{I}=10.45 \mathrm{eV})$ make these elements extremely difficult to ionize by conventional means. Mass spectrometric techniques based upon negative ionization have been the most successful, however, these techniques have not been capable of measuring the high isotopic abundance ratios of these elements with the requisite sensitivity to address the most difficult tracer and dating problems.

The feasibility of ionizing both iodine and carbon by resonance ionization mass spectrometry (RIMS) has been demonstrated. In contrast to the two-photon RIMS that has been done for other elements, a three photon process was used in this study to ionize the elements. This process consisted of a two-photon transition via a virtual state followed by the absorption of a third, ionizing photon. Theoretical calculations for carbon indicate that saturation of the absorption cross-section for a two-photon transition is within the capabilities of existing instrumentation. Thus, sensitivity of the ionization step will not be sacrificed in the resonance ionization process.

The use of a two-photon transition for the ionization of both iodine and carbon provides an extra dimension to the RIMS process by introducing the potential of isotopic selectivity. If the photons that these elements absorb are polarization labelled, Doppler-free spectroscopy of these elements is theoretically possible. The isotopic shifts of both elements are believed to be large enough that Doppler-free resonance ionization will allow the selective ionization of one isotope. The wavelength-dependent selective ionization of one isotope in the presence of a second would help to overcome the abundance sensitivity limitations imposed by the mass spectrometer and allow large isotopic ratios to be measured by conventional instrumentation. Research is actively being pursued in this area.

The samples were prepared by drying AgI (for iodine) and graphite (for carbon) onto the rhenium filament substrate. Sample sizes were typically 10-100 $\mathrm{gg}$. The resonance ionization signals for the two-photon transitions of iodine $(279.7 \mathrm{~nm})$ and carbon $(287.0)$ were observed at nominal temperatures of $650{ }^{\circ} \mathrm{C}$ and $1750{ }^{\circ} \mathrm{C}$, respectively. The laser beam from the frequency doubler was focused with a $30 \mathrm{~cm}$ lens, before it entered the mass spectrometer ionization region; which produced a peak power of $10^{2} \mathrm{gW} / \mathrm{cm}^{2}$. Wavelength scanning and RIMS spectra were collected for both elements. A relatively large off-resonance background was present in both cases, probably resulting from the dissociation/ionization of molecular species with little wavelength dependence. The molecular ion, $\mathrm{C}_{2}{ }^{+}$, was observed for carbon. For iodine, there existed a relatively large $\mathrm{Ag}^{+}$signal. Neither of these ions showed any wavelength dependence. 
Carbon has two low-lying energy levels within $50 \mathrm{~cm}^{-1}$ of the ground state, as well as three nearly equivalent energy levels at the excited state. The experimental spectrum is seen in figure 8 . The predicted spectrum consists of eight lines and thus, one of the predicted lines is unresolved. If the distribution of electronic state populations follows the Boltzmann relationship as previously shown for other elements studied by RIMS, then 55 percent of the atoms leaving the filament will be in the excited state, $43.5 \mathrm{~cm}^{-1}$ above the ground state.

The spectroscopy of iodine showed several transitions from extremely high excited state levels that could not have been populated by equilibrium thermal processes. Possibly, iodine is selectively populating certain metastable excited state levels significantly above the ground state in the molecular dissociation process induced by the laser. Evidence for this phenomenon has been reported elsewhere, but this is the first example observed in this laboratory.

The ability to form and measure ions with the sensitivity and selectivity reported here, coupled with an understanding of the basic processes involved, extend the potential utility of RIMS to detection of many of the elements on the right hand side of the periodic table. Furthermore, the use of RIMS to characterize vapor compositions and to identify photodecomposition products can be applied to the study of vaporization phenomena and to tailor the formation of atom reservoirs for specific measurement applications.

\section{Thermal Release of Helium from Single Crystal Nickel (26109)}

D. H. Vincent, J. T. Maki, K. S. Grabowski

The diffusion and trapping of helium, as well as other inert gases in metals, became of great practical interest during recent years because of material problems related to nuclear energy production, e.g., the void formation in structural materials under neutron bombardment in fission reactors and the ablation of first wall materials in future fusion reactors. A new approach to the problem of identifying trapping sites and determining trap binding energies for helium in metals at low helium concentrations has been developed. The approach is based on using ion implantations of ${ }^{3} \mathrm{He}$ and subsequent depth profiling of the implanted helium using a thermal neutron beam. The thermal release of the helium is studied by measuring the depth distribution of the helium remaining in the sample after each anneal. While this method has been used before to observe helium release from molybdenum, niobium, and copper, this work will represent the first unambiguous determinations of detrapping energies. Since the solubility of helium in metals is quite low, studies often require the introduction of helium atoms into a metal by implantation as helium ions in an accelerator. If such implantations are performed at ion energies above a few keV, with the sample held at room temperature, the implanted helium is quantitatively trapped. Each helium atom is trapped very close to the end of its range in the metal, so that concentration profiles of the implanted helium can be used to determine range distributions of the implanted ions. An example of such a range distribution is shown in figure 9 . If a metal sample containing implanted helium is brought to a sufficiently high temperature, some or al1 of the helium atoms will be released from their trapping sites. 


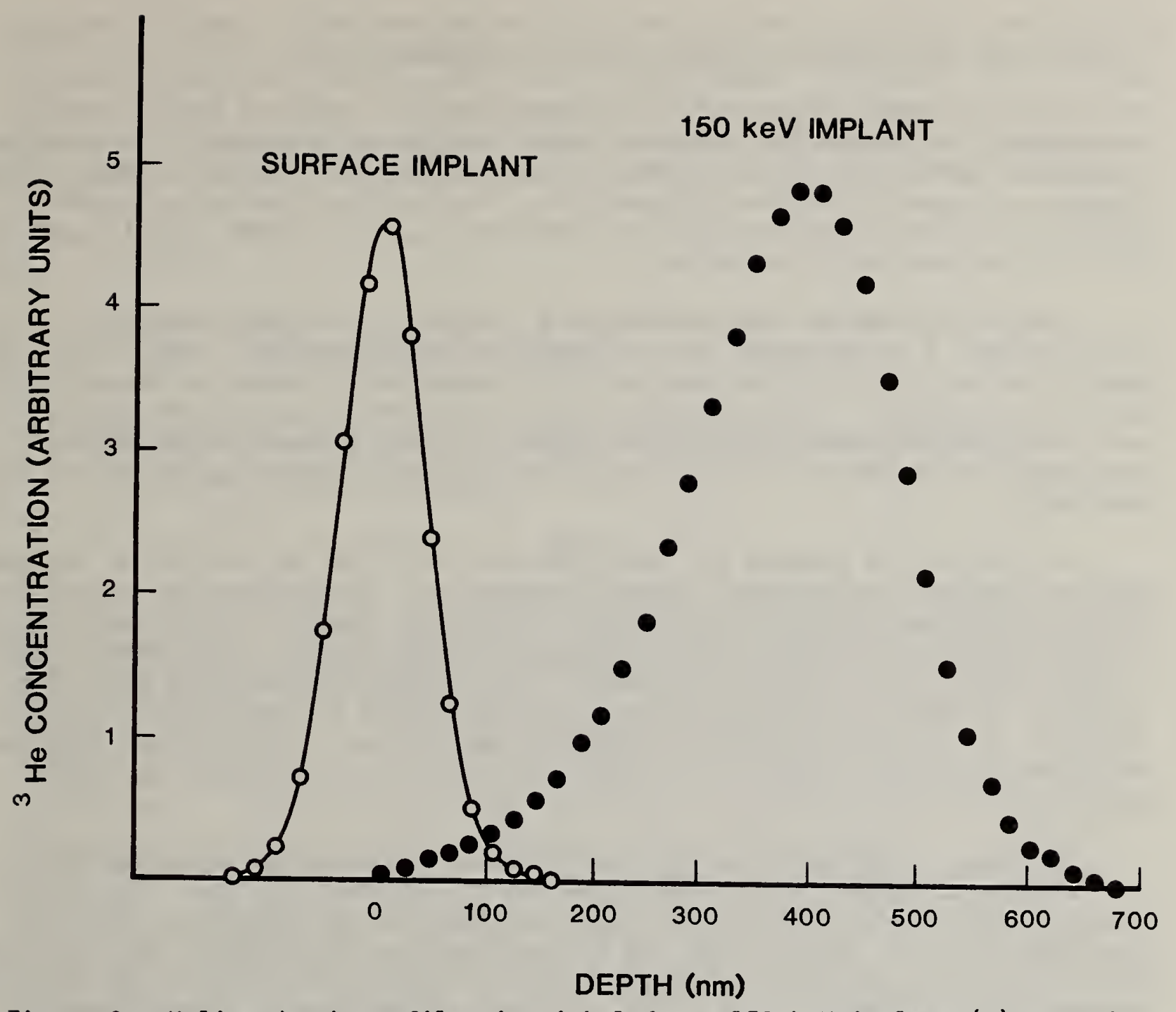

Figure 9. Helium depth profiles in nickel for a $150 \mathrm{keV}$ implant (•), and for a $250 \mathrm{eV}$ surface implant (o) which represents the system resolution function.

Once the helium is detrapped, it is released from the metal surface almost instantaneously, indicating a very large diffusivity for helium in metals.

Most of the measurements dealing with helium in nickel have been done as desorption experiments in which helium-implanted samples were subjected to a temperature ramp, and the helium release was observed by mass spectrometry. Implantation energies were usually low (of the order of 1 to $10 \mathrm{keV}$ ), and implantation surface doses were comparatively high, so that the formation of helium atom aggregates was likely before release. The present work differs from previous investigations in two respects. First, a relatively high implantation energy is used $(150 \mathrm{keV})$, and the implantation dose is kept low enough to avoid, as much as possible, the formation of larger helium aggregates (bubbles). Secondly, helium release is observed not by measuring the amount of gas released through the surface of the sample, but by observing changes in the helium concentration inside the sample as a function of depth. Using this method, it will be possible not only to measure activation energies for helium 
detrapping, but also to relate these activation energies to specific trapping sites along the range distribution of the implanted ions.

The activation energy for helium detrapping is likely to depend on the particular kind of lattice defect at which the helium is trapped. In order to reduce the variety of trapping sites as much as possible, single crystal nickel samples are used for helium implantation. This confines the helium traps mainly to isolated vacancies and excludes trapping at extended defects such as dislocations and grain boundaries.

Samples for helium implantation were prepared by cutting slices of approximately $1 \mathrm{~mm}$ thickness from a single crystal nickel rod. One surface of each slice was carefully polished and its crystallinity tested by obtaining a Laue $x$-ray pattern. These patterns indicate that the normal of the samples is well away from any ion channeling direction, so that helium ion channeling should not occur during implantation if the sample is perpendicular to the ion beam axis.

A first set of samples has been implanted with ${ }^{3} \mathrm{He}$ ions at an ion implantation accelerator at the Naval Research Laboratory. Precautions were taken to prevent ion beam heating of the samples during implantation. Ion beam currents of approximately $300 \mathrm{nA}$ were used, and implantation surface doses were $2 \times 10^{16}$, $1 \times 10^{16}, 6 \times 10^{15}$, and $1 \times 10^{15}{ }^{3} \mathrm{He}-i o n s / \mathrm{cm}^{2}$. The largest surface dose $\left(2 \times 16^{3} \mathrm{He}-\right.$ ions $/ \mathrm{cm}^{2}$ ) produced a maximum concentration of about 0.9 atom percent ${ }^{3} \mathrm{He}$ at the peak of the range distribution for $150 \mathrm{keV}{ }^{3} \mathrm{He}$ ions. This can be compared with the solubility of helium in nickel at room temperature which is $1 \times 10^{7}$ atom percent.

The depth distribution of the helium after implantation was measured at the neutron depth profiling facility at the NBS research reactor. The distance from the surface of specific implanted ${ }^{3} \mathrm{He}$ atoms was determined by measuring the energy loss of protons from the nuclear reaction ${ }^{3} \mathrm{He}(n, p)^{3} \mathrm{H}$. This reaction has a large cross section at thermal neutron energies (5237 barns) and produces protons with an energy of $572.5 \mathrm{keV}$. The energy spectrum of protons emerging from the surface of the sample as it is irradiated with thermal neutrons was measured with a silicon surface barrier detector. This energy spectrum was converted to a depth distribution for the implanted ${ }^{3} \mathrm{He}$ atoms with the aid of the known proton stopping power $(d E / d x)$ in nickel. Figure 9 shows the depth distribution of helium atoms in a sample implanted with $150 \mathrm{keV}{ }^{3} \mathrm{He}-i$ ions to a surface dose of $2 \times 10^{16}{ }^{3} \mathrm{He}$-ions $/ \mathrm{cm}^{2}$. This depth distribution is broadened by the energy resolution of the detector and the associated electronic equipment. To indicate the width of the resolution function, a spectrum from a surface implantation of helium ions ( $250 \mathrm{eV}$ implantation energy) is also shown in Figure 9. The mean projected range of the $150 \mathrm{keV}$ implant is $372 \mathrm{~nm}$. This compares well with the value of $367 \mathrm{~nm}$ obtained by interpolation from a set of theoretical mean ranges at various energies given by Biersack et al. [1].

Annealing experiments on the implanted samples are in progress. Activation energies for detrapping of the implanted helium will be determined by a combination of isochronal and isothermal anneals. It is planned to extend the present work to include ${ }^{3} \mathrm{He}$ profiling and thermal release studies of nickelbased metallic glasses. Both the trapping efficiencies and the detrapping behavior will be investigated. A further application of the technique of ${ }^{3} \mathrm{He}$ 
depth profiling will be the decoration of radiation-induced defects in pure metals, in metallic glasses, and in silicate glasses. Of these, the silicate glasses may be the most promising materials for an application of this method, since helium is known to permeate these materials easily at room temperature. Preliminary experiments on this new application of ${ }^{3} \mathrm{He}$ depth profiling are planned.

Reference

[1] Biersack, J. P., Fink, D., Henkelmann, R., and Muller, K., J. Nuc1. Mater. 85/86, 1165 (1979).

12. Determination of Selenium in Subcellular Fractions to Study the Glutathione Peroxidase System (15310)

R. Zeisler, A. G. Katki, C. E. Myers

Cardiac mitochondria generate significant quantities of hydrogen peroxide as a by-product of normal metabolism. This toxic product must be eliminated before it causes severe damage to mitochondria and surrounding organelles. For this reason, the existing defenses of mitochondria to hydrogen peroxide attack are of interest. There are two major enzyme classes able to destroy hydrogen peroxide: catalases and glutathione dependent peroxidases. The latter are all known to be soluble enzymes which contain selenium in their active sites. Indeed, tissue activity of this enzyme appears to be strictly dependent on dietary selenium. Selenium deficiences may result in diseases which have been associated with a resultant lack of glutathione peroxidase activity. This enzyme class may also be involved in protection against chemical carcinogens and radiation damage. However, there are considerable variations in dietary selenium levels in man and animals. Also, the existence of healthy, selenium deficient subjects suggest that other mechanisms of peroxidase removal must exist. Katki and Myers [1] showed the presence of membrane bound glutathione peroxidase-like activity in mitochondria which may be selenium independent.

The interrelationships among selenium, peroxide detoxification mechanisms, and enzyme separations have been studied. In addition, the influence of drugs on the peroxidase system has been investigated using selenium determinations in enzyme and cell fractions. Instrumental as well as ultrasensitive radiochemical neutron activation analysis procedures have been implemented for the determination of selenium and other essential trace elements in a series of samples provided by the National Cancer Institutes (NCI). In an attempt to purify a membrane-bound, selenium-independent, glutathione peroxidase, beaf heart mitochondria were investigated for the presence of this new enzyme. After preparing mitochondria and intermembrane space by digitonin treatment, the preliminary separation scheme resulted in purified enzyme fractions in which selenium and other trace elements were determined (figure 10). The determination of selenium in the final preparation is of utmost importance since this is the only true measure of the dependence of the peroxidase system on selenium concentration. The newly developed radiochemical activation 


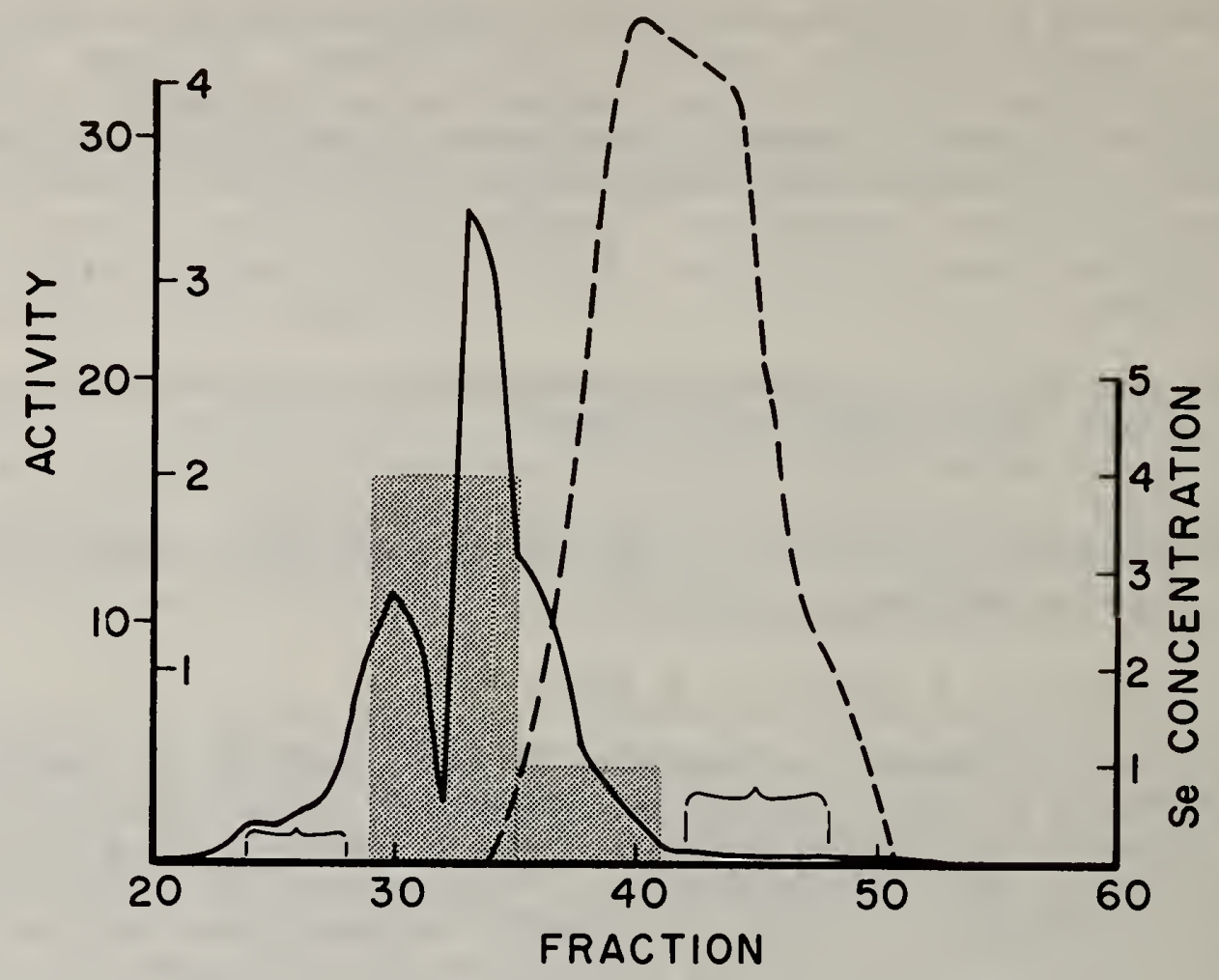

Figure 10. Specific Activity and Selenium Concentrations in Enzyme Fractions.

Solid Line: Specific peroxidase activity, 0 to $30 \mathrm{nmoL} / \mathrm{min}$. $\mathrm{mg}$ (protein)--Reduction of Peroxide

Broken Line: Specific transferase activity, 0 to $4 \mathrm{nmoL} / \mathrm{min}$. mg (protein)--Reduction of Peroxide

Shaded Areas: Selenium concentration, 0 to $5 \mu \mathrm{g} / \mathrm{g}$ (dry matrix) in freeze-dried pooled fractions. In fractions 24 to 28 and 42 to 48 , selenium is below the INAA detection limit of $0.7 \mu \mathrm{g} / \mathrm{g}$.

analysis procedures provide a sensitivity of $\sim 10^{-12} \mathrm{~g}$ for selenium, i.e., the selenium concentration in nanogram amounts of enzyme can be used to quantify the enzyme, as highly purified enzymes become available.

Future work will utilize the ability to determine nanogram quantities of enzymes to identify the proteins directly on chromatography columns or on two-dimensional protein maps.

Reference

[1] Katki, A. G. and Myers, C. E., "Membrane-bound Glutathione Peroxidase-Like Activity in Mitochondria," Biochem. Biophys. Res. Com., 96, 85 (1980). 
13. The Measurement of Natural Variation in the ${ }^{32} S /{ }^{34} \mathrm{~S}$ Ratio in Atmospheric Samples (15310)

\section{W. R. Kelly, P. J. Paulsen}

A new procedure for the measurement of sulfur isotopic ratios by thermal ionization mass spectrometry using the AsS molecular ion has been developed during the last two years. Previous reports [1] provided examples of precise and accurate sulfur determinations by isotope dilution in a variety of Standard Reference Materials. The chemical blank $(\sim 0.3 \mu \mathrm{g} \mathrm{S})$ is the limiting source of uncertainty for quantities of sulfur below $100 \mu \mathrm{g}$ and the calibration of the tracer is the limiting source of error $(20.2 \%$ rel., $1 \mathrm{~s})$ above this amount $[1,2]$. Another important application which has been explored during the past year is measurement of natural variations in the ${ }^{32} \mathrm{~S} /{ }^{34} \mathrm{~S}$ ratio.

Sulfur has four stable isotopes with the following average relative abundances: ${ }^{32} \mathrm{~S} \sim 95.02$ percent, ${ }^{33} \mathrm{~S} \sim 0.75$ percent, ${ }^{34} \mathrm{~S} \sim 4.21$ percent, and ${ }^{36} \mathrm{~S} \sim 0.02$ percent. The abundances of the sulfur isotopes vary in nature as a result of mass dependent fractionation principally during S-0 bond breaking in sulfur reducing bacteria. Over geological time this has resulted in large variations in the ${ }^{32} \mathrm{~S} /{ }^{34} \mathrm{~S}$ ratio. These differences are expressed as enrichments or depletions in the ${ }^{34} \mathrm{~S}$ abundance relative to Canyon Diablo meteoritic troilite (Fes) defined by the following equation:

$$
\delta^{34} \mathrm{~S} / 00=\left[\frac{\left({ }^{34} S /{ }^{32} S\right)_{\text {sample }}}{\left({ }^{34} S /{ }^{32} S\right)_{S T D}}-1\right] \times 1000
$$

The vast majority of samples found in nature fall between +40 and $-40 \delta^{34} \mathrm{~S}$ units, but samples with $\delta^{34} S$ values as large as -65 and +95 have been observed. This variation has been used to trace the origin of ore and oil deposits, natural waters, and sulfur in the atmosphere [3]. Of particular interest is the problem of determining the source of atmospheric sulfur compounds at a receptor site. The main thrust of our work has been the development and refinement of the thermal ionization technique for the determination of sulfur isotopic ratios on small samples. As a demonstration of the capabilities of the technique developed, $\delta^{34} \mathrm{~S}$ values have been determined in a number of samples collected by Project Airstream after the eruption of the El Chichon volcano in southern Mexico. Project Airstream has been sampling the stratosphere for more than a, decade in a transect from $60^{\circ} \mathrm{N}$ to the equator [4]. The eruption of El Chichon in March-April 1982 had the largest impact on the atmosphere of any eruption since the 1883 eruption of Krakatoa. The main impact has been the large amount of $S$ injected into the stratosphere, estimated to be more than $10^{6} \mathrm{~T}$ or about 10 times more than that from Mount St. Helens. The source of this enormous amount of sulfur is not completely known. Aqueous extracts of filter samples were obtained through the cooperative efforts of E. J. Mroz of Los Alamos Scientific Laboratory and W. H. Zoller of the University of Maryland. Values of $\delta^{34} S$ have been determined in samples from three different flights of Project Airstream. The chemical and mass spectrometric procedures used are similar to those described in previous reports. Measurement precisions were about 0.1 percent. The results given in Table 7 are the first 
Table 7. $\delta^{34} \mathrm{~S} 0 / 00$ Values in Project Airstream and El Chichon Samples.

Sample

Flight $4(4 / 20 / 82)$

Filter 8

9

10

11 $\underline{\mu g S}$

31

172

201

49

Flight $5(7 / 21 / 82)$

Filter 5

7
8
10

11

Flight $6(10 / 27 / 82)$

Filter 7

8

9

10

11

El Chichon

Elemental S

Lake Water

41

63

67

114

68

118

131

159

115

123

300

$\sim 200$
Sample S

$\delta^{34} 50 / 00^{a}$

26

$+13.2+2.2$

$-3.3$

191

$+32.3+0.7$

$-1.0$

183

$+29.2+1.5$

$-1.9$

288

$+28.9+0.7$

$-0.9$

40

$+3.4+2.7$

$-2.9$

45

$+3.7+1.7$

$-1.9$

66

$+3.3 \pm 7.6$

81

$+5.0+1.2$

$-1.3$

57

$+4.3+1.6$

$-1.8$

74

$+11.0+1.5$

$-1.8$

87

$+11.6+1.4$

$-1.7$

99

$+13.2+0.5$

$-0.7$

100

$+11.8+1.1$

$-1.3$

103

$+14.5+1.7$

$-2.0$

6000

$+0.78 \pm 0.4$

4000

\footnotetext{
Uncertainties are computed as the linear sum of the measurement precision (2s) and the bias due to chemical blank.
} 
sulfur isotopic measurements on Project Airstream samples. The sample sizes shown in column 2 range from about 30 to $200 \mu \mathrm{g} \mathrm{S}$. Column 3 lists the (sample $\mathrm{S} / \mathrm{blank} S$ ) weight ratios. It was assumed that the blank could have a composition of $\delta^{34} \mathrm{~S} 0 / 00= \pm 40$ which accounts for the asymmetry in the uncertainties given in the last column. The variations in $\delta^{34} \mathrm{~S} 0 / 00$ range from +0.8 to +32 . A graphical representation of a portion of the data is given in Figure 11 (a) and (b) where the $\delta^{34} S$ values have been plotted on contour plots from Mroz et al. [5]. Figure 11 (a) constructed with data from before the eruption of ET Chichon shows a homogeneous distribution of sulfate in the atmosphere.

Figure 11 (b) constructed from data after the eruption shows a very heterogeneous distribution of sulfur with extremely high values at $19 \mathrm{~km}$ at latitude $10^{\circ} \mathrm{N}$. The very large $\delta^{34} \mathrm{~S}$ values of +32 , in this region indicate that this is not juvenile sulfur from the El Chichon magma but may be due to the injection of overlying evaporites deposits which can have high $\delta^{34} S$ values. Further interpretations must wait for additional data.
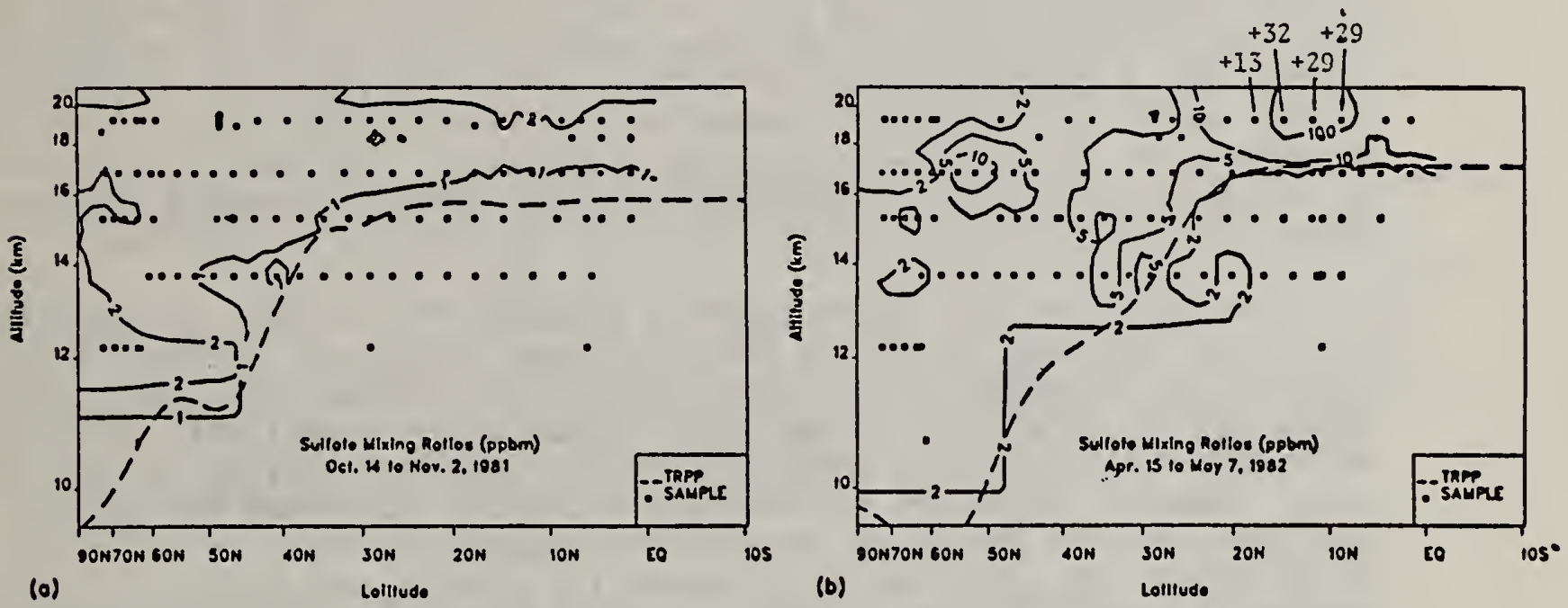

Figure 11. Contour plots of sulfate, concentrations before (a) and after (b) the eruption of El Chichon volcano. $\delta^{34} \mathrm{~S} 0 / 00$ values are plotted for four stratospheric samples (b).

References

[1] Paulsen, P. J. and Kelly, W. R., "Determination of Sulfur by Isotope Dilution Thermal Ionization Mass Spectrometry as $\mathrm{AsS}^{+}$ Ions," submitted to Anal. Chem.

[2] Kelly, W. R. and Paulsen, P. J., "Precise Determination of Sulfur at High Concentrations by Isotope Dilution Thermal Ionization Mass Spectrometry," submitted to Talanta.

[3] Krouse, H. R., "Sulur Isotopes in Our Environment," in Handbook of Environmental Isotope Geochemistry, Vol. 1, The Terrestrial Environment, 1980. 
[4] Sedlacek, W. A., Mroz, E. J., Lazrus, A. L., and Gandrud, B. W., "A Decade of Stratospheric Sulfate Measurements Compared with Observations of Volcanic Eruptions," J. Geophys. Res. 88, 3741-3776 (1983).

[5] Mroz, E. J., Mason, A. S., and Sedlacek, W. A., "The Stratospheric Sulfate from El Chichón and the Mystery Volcano," submitted to Geophyd. Res. Letts.

\section{Evaluation of the Isotope Dilution Method for Ultratrace Chromium} Concentrations (15601)

K. A. Brletic, L. J. Powell

The accurate determination of chromium concentrations in samples containing less than $100 \mathrm{ng} / \mathrm{g}$ of chromium is a problem of major concern to nutritionists. Many of the results published over the past 10-15 years are unreliable and, as a result, estimates of chromium concentration in biological fluids have varied by more than a factor of 1000 during this period. A long-term goal of this project has been to develop a method for the accurate measurement of chromium concentrations at the sub-nanogram per gram level. This year's efforts resulted in the certification of four environmental and clinical standard Reference Materials, several of which contained less than $20 \mathrm{ng} / \mathrm{g}$ of chromium.

Last year's annual report outlined a method for the mass spectrometric analys is of chromium capable of increased sensitivity over previous methods. At that time the method had received only minimal evaluation; and, in fact no determinations were made at the sub- 100 nanogram per gram level. Briefly described, the chromium is separated from the matrix by solvent extraction into methyl isobutyl ketone and is then back extracted into water and reduced. The mass spectrometric procedure requires the sequential drying of silica-gel, approximately 200 nanograms of chromium(III) nitrate, and boric acid onto a rhenium filament followed by high temperature drying. This loading technique yields ion currents of $1-4 \times 10^{-11} \mathrm{~A}$.

To work at this level, a thorough assessment of the analytical blank had to be made. Efforts to reduce the blank included further purification of reagents and more extensive cleaning of apparatus. In addition, an attempt was made to reduce the quantities of reagents used in the chemical separation procedure of the analysis. This, however, resulted in lower recovery of the chromium as well as a greater concentration of impurities in the final sample, which inhibited the ionization of the chromium in the mass spectrometer. Overall, these efforts led to a four-fold reduction in the magnitude and variability of the analytical blank.

The concentration of chromium was determined in SRM 1643b, Trace Elements in Water; SRM 909, Human Serum; SRM 2670, Toxic Metals in Urine (Elevated and Normal Levels); and SRM 1549, Powdered Milk. The average values and associated uncertainties reported at the 95 percent confidence level are summarized in Table 8. 


\section{Table 8}

SRM 1643b, Water

SRM 909, Human Serum

SRM 2670, Urine - Elevated Normal

SRM 1549, Powdered Milk

\section{Concentration (ng/g)}

$18.6 \pm 0.3^{\mathrm{a}}$

$89.5 \pm 3.0^{\mathrm{a}}$

$84.5 \pm 6.9^{\mathrm{a}}$

$12.4 \pm 5.8^{\mathrm{a}}$

$<10.0$

$\mathrm{a}_{95}$ percent confidence level.

The analytical blanks ranged from 1-15 nanograms depending upon the complexity of the analytical procedure. Large (multigram) samples were used to offset the effects of the blank. For SRM 1549, Powdered Milk, the level of chromium in the analytical blank was greater than the level in the sample and, therefore, the determined concentration must be viewed as an upper limit.

In summary, the method outlined above makes it possible to measure chromium concentrations at levels of 20 nanograms per gram with an accuracy of a few percent. The analytical blank, however, is clearly a limiting factor in terms of increased sensitivity, and thus remains a major barrier, particularly when the total amount of chromium in the sample is less than 10 nanograms. Additional research is needed to reduce the analytical blank to the picogram level to adequately satisfy many of the analytical requirements of clinical and nutritional studies. However, this technique is sufficiently useful and accurate to meet many of the needs for chromium analysis.

15. Ion Optics of Single and Double Stage Mass Spectrometers (15310)

I. L. Barnes, P. Morales

Historically high precision or "analytical" mass spectrometers have been constructed so that the ion beam enters the magnetic field normal to the magnetic pole faces. While this permits instruments to be constructed with well defined and predictable geometries, there are severe constraints, the two most important of which are the lack of focussing in the vertical or " $z$ " direction and the fact that the dispersion (or resolution) is directly proportional to the magnet radius (i.e., to increase the dispersion by a factor of 10, the magnet must be 10 times as large). Greater dispersion would permit increased accuracy in the measurement of isotopic ratios, especially very large ratios. This would also allow the use of multiple collectors, again increasing the accuracy of the measurement.

A program is underway to develop a theoretical background for the ion optics of mass spectrometers using non-normal entry of ion beams. This work is being carried out in conjunction with researchers at the Institute of Physics, University of Mexico. Such instruments would produce increased dispersion (for 

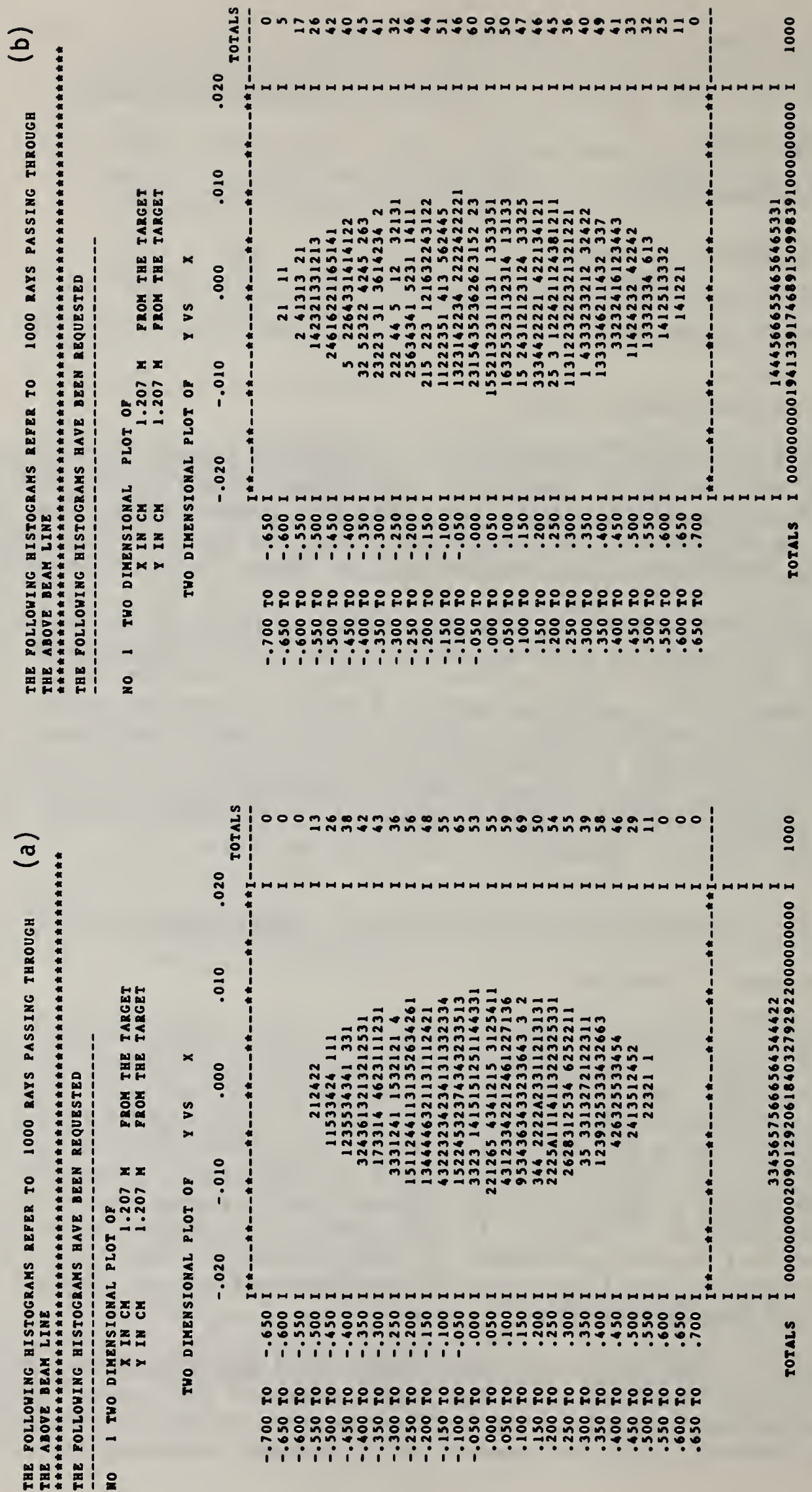

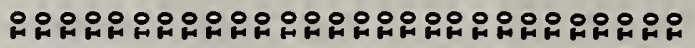

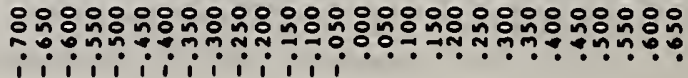

물 నึ์ E岳 品 $=$ व


농ำ ปี으 ำ 터ำ 4 जि = 동으넝 $=$ 동동

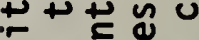
उ थ के 틍 就这岂 幽更 응 ๖ั

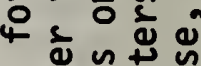

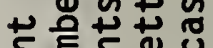
동드 응 르요

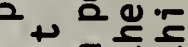

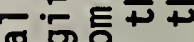

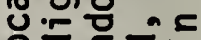
40

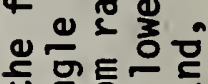

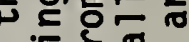

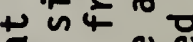
大

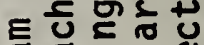
\%

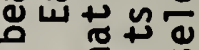
ᄃ. . ธ 흠ำำ 4 ํํ워ำ - 幺

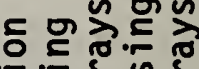
品的的 ư

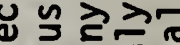
约 ज织 윤 $U=\omega$ 的 出苛出. 䒘塞言会岂

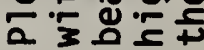
ำ 
a given radius magnet) and would also give simultaneous focussing in the horizontal and vertical directions. Since precise knowledge of the value of the stray magnetic field is an important part of the theoretical background, previous work involved careful measurements of the stray field of an NBS designed magnet and the development of a mathematical expression describing the field. During the past year these measurements have been repeated on a magnet which could be adjusted to give different width gaps. These data are currently being processed to determine the constants which give the best fit. The computer programs TRANSPORT and TURTLE have been modified to include the expression for a specific magnet and the output of these programs gives much more realistic results. An example of the ion beam cross-section at the focal points of two systems is shown in figure 12 .

A new program "MASMEX", which uses only fundamental physical expressions for the position of the ion beams coming from as many as 27 points on the representation of a real filament, was written here last year. The program has the advantage that the coordinates of the beams may be obtained at any point in the complete system so that the effects of small changes in the magnetic field may be examined. During this past year a fundamental "bug" was discovered which has now been corrected. A plotting subroutine is being added which will reduce the large amount of data and make the program easier to use.

During the next year, we plan to add a segment to each program to model the focussing properties of the ion source so that realistic ion beams focussed, partially focussed, and defocussed may be examined at any point in the complete mass spectrometer.

\section{Development of Reference Materials for Acid Rain Studies (15310)}

W. F. Koch, G. Marinenko, Y. C. Wu

Acid rain is a topic of national and international concern. The ramifications of this phenomenon involve social, political, economic, environmental and energy-related issues. To assess and propose meaningful remedial strategies, chemical analyses must be done on rainfall samples. Since the acid rain problem is not restricted to specific times or locales, these analyses must be performed over long periods of time at numerous locations around the world. The analyses are carried out both in the field and in laboratories. Since 1975, the National Bureau of Standards has had an agreement with the US EPA to provide a means of intercalibrating the various measurement stations and of establishing a foundation for long term trend analysis. Initially, the project entailed the production and characterization of audit materials, dubbed, Simulated Precipitation Reference Materials (SPRM). In 1980, the scope of the project was significantly broadened to include research leading to the development of a Standard Reference Material (SRM) for acid rain. In addition, measures aimed at improving the accuracy and reliability of the analytical methods used in acid rain studies were to be evaluated. In the past year, significant progress has been made toward achieving the research goals.

A major change has been made in the composition and storage of the fifth series of SPRM. This series has been stored in polyethylene bottles rather 
than glass ampoules and consists of a set of five solutions, all concentrates requiring a 50-fold dilution before they can be used. Three of the solutions have been characterized for the major anions and cations found in rainwater, as well as pH, acidity, and conductivity. The other two solutions in the set contain trace metal ions stabilized with $0.5 \mathrm{~mol} / \mathrm{L}$ nitric acid.

The move to polyethylene bottles was necessitated by the fact that the moderately acidic solutions are not stable in glass ampoules. This has been established through several long term stability studies. The results of one such study is shown in Table 9. A solution prepared in May 1981 was split between glass ampoules and polyethylene bottles. A divergence between the values for $\mathrm{pH}$ and conductance was apparent after the first month and continues to date. In another experiment the $\mathrm{pH}$ and conductivitiy of a dilute solution of sulfuric acid ( $\sim \mathrm{pH} 4$ ) in polyethylene has remained stable for over three years.

Table 9

\begin{tabular}{lcccc} 
& \multicolumn{2}{c}{ Glass } & & \multicolumn{2}{c}{$\begin{array}{l}\text { Polyethylene } \\
\text { Date }\end{array}$} & pH & Conductance & & pH & Conductance \\
$6 / 81$ & 4.12 & $36.0 \mu \mathrm{S} / \mathrm{cm}$ & 3.87 & $52.4 \mu \mathrm{S} / \mathrm{cm}$ \\
$3 / 82$ & 4.30 & 29.3 & 3.90 & 53.0 \\
$1 / 83$ & 4.50 & 23.9 & 3.85 & 54.0 \\
$4 / 83$ & 4.34 & 27.1 & 3.84 & 54.8 \\
$7 / 83$ & 4.44 & 26.6 & 3.91 & 54.7
\end{tabular}

Three new studies have been initiated this year to enhance our knowledge of the stability of anions and hydrogen ion in dilute solutions. One study involved the monitoring of SPRM (V) samples that were stored in polyethylene following a 50-fold dilution. Preliminary results are very encouraging. Another set of solutions has been prepared containing phosphate as well as the other major cations and anions. This was done to simulate the composition of rainwater in the Midwest and plains states. Early indications are that such solutions, particularly when dilute, are not stable. Additional studies on these solutions using a bactericide have yet to be carried out. The third study entailed the collection, characterization, storage, and monitoring of a sample of natural rainwater. It is still too early to draw any conclusions about the stability of these solutions.

Research in methods development has focussed primarily on the measurement of $\mathrm{pH}$ in low ionic strength solutions. The residual liquid junction potential resulting from the use of buffer solutions to calibrate the electrode system can seriously bias measurements of the $\mathrm{pH}$ of rainwater. Work is underway to account for this phenomenon and to determine if one type of junction performs better than others. Also in progress is the preparation of two strong acid solutions (nominally $\mathrm{pH} 3.8$ and 4.3) which will be analyzed for pH using hydrogen, $\mathrm{Ag} / \mathrm{AgCl}$ cells without liquid junctions. This is a preliminary study to test the feasibility of using such solutions for the calibration of $\mathrm{pH}$ measurements in rainwater samples. An interlaboratory comparison using the solutions is planned for this fall. 
The overall goal of this project is to provide quality assurance for the analytical measurements of rainwater samples as part of the National Acid Precipitation Assessment Program. It is anticipated that the research involving measurements in wetfall will continue for two more years. At the same time, it is planned to expand the project to include dry deposition studies. Other related projects include fundamental studies in the measurement of $\mathrm{pH}$ and conductivity and will continue on a long-term basis.

\section{The Development of a Pulsed Thermal Atom Source For Use in RIMS (26108)}

J. D. Fassett, R. W. Shideler, L. J. Moore

The development and application of a resonance ionization mass spectrometer (RIMS) system is described in accompanying reports. This system consists of a pulsed laser, operating at $10 \mathrm{~Hz}$, and a continuous thermal atomization source. It has been demonstrated that thermal sources can be used to produce neutral atom beams for a wide range of elements. However, the duty cycle mismatch between the continuous atom source and the laser results in a substantial loss in sample utilization efficiency, i.e., sensitivity of the process. To exploit the inherently high photoionization efficiency of the laser and ultimate sensitivity of RIMS, a better match is required between the ionization and atomization processes.

The atomization and ionization processes can be better matched by either pulsing the atomization source or by using a higher repetition rate laser or continuous (CW) laser for photoionization. Work is underway in other laboratories to develop CW RIMS systems [1]. With the present state of laser technology, CW systems have many disadvantages relative to pulsed laser systems. They lack both the elemental coverage and the ionization efficiency of pulsed systems, and thus are applicable only to specialized problems. The atomization process can be pulsed by either ablative techniques, such as ion and atom sputtering or laser heating, or by pulsed vaporization. Ablation has definite promise and is being investigated by several laboratories. However, the energetics of these ablative processes and their inability to selectively produce atoms strongly suggests that their general application needs to be firmly established.

A pulsed thermal vaporization source, based upon the known ability to produce stable neutral atom beams whose temperature-intensity behavior is classically defined, was constructed. The nature of the thermal atom pulse was evaluated by temporal scanning of the atomization pulse relative to the laser pulse. Changes in the atomization filament were required to achieve the most pronounced pulsed atom beam. When iron samples were used to test this system, a 30-fold improvement in sample utilization over the continuous atom source was demonstrated for the pulsed thermal vaporization source.

The pulsed thermal source was made by modifying the solid-state, constantcurrent filament supply that has been developed and used in this laboratory for thermal ionization. This supply provides up to 10 amps of current. In the pulsed mode of operation, a 10 amp current pulse is applied to the filament for a selected period of time. The 10 amp pulse can be applied on top of an 
established base current level. The thermal pulse can be triggered in conjunction with the laser or a delay can be incorporated such that the laser pulse is triggered at the time of maximum atom density.

The temporal nature of the thermal pulse was determined by delaying the laser relative to the pulser. A photodiode detector of the laser pulse was used to trigger both an oscilloscope and a pulse generator. The pulse generator which provided a pulse delay ( $\mathrm{ns}$ to $\mathrm{ms}$ ) was used to trigger the thermal pulser. The oscilloscope was used to monitor the position of the thermal pulse relative to the laser pulse. The ion signal versus the time delay was determined by manually scanning the relative position of the two pulses in the $100 \mathrm{~ms}$ region between pulses. The ion signal was integrated at each time position in the scan for $20 \mathrm{~s}$ or 200 laser pulses.

The first experiments with the standard Re filament assembly indicated that the mass of the filament limited its temperature response and thus, sample utilization. As a result, a second filament was prepared whose length and width were reduced. For this filament, a 4.5 ms current pulse produced the experimental signal versus time curve that is illustrated in figure $13(\mathrm{a})$. The signal decayed to 50 percent of maximum in $3.5 \mathrm{~ms}$ and to one percent of maximum in $30 \mathrm{~ms}$. In contrast to the standard filament, the baseline was reached between thermal pulses. A sample utilization improvement factor of 12 is calculated from this curve.

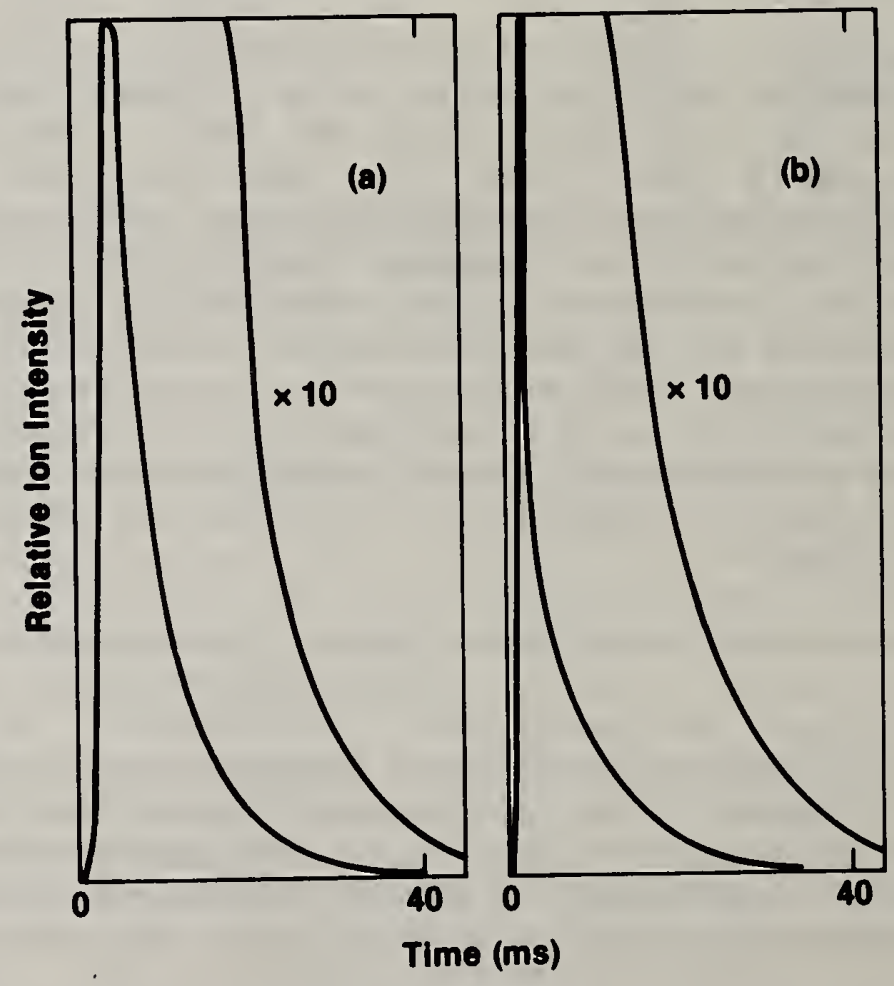

Figure 13. Profile of thermal atom pulse with miniaturized filament assembly. RIMS signal versus time after thermal pulse initiation: (a) no current offset and $4.5 \mathrm{~ms}$ current pulse width; (b) 0.9 amp offset and $3.0 \mathrm{~ms}$ current pulse width. 
Since this second miniaturized filament reached baseline between thermal pulses, an attempt was made to apply the current pulse on top of a constant current base level. A base level of 0.9 amps was established and midlevel ionization signal achieved with a $3 \mathrm{~ms}$ pulse. The experimental signal-versustime curve also is illustrated in figure 13 (b). In this experiment, it took less than $1 \mathrm{~ms}$ to decay to 50 percent of the maximum. However, it still required $30 \mathrm{~ms}$ to decay to one percent of the maximum signal. The best observed enhancement, a factor of 31 , was achieved in this case.

Further attempts to reduce the size of the filament were unsuccessful. It is obvious that reducing the size of the filament to achieve the fastest heating response must be balanced against the ability to load a sample upon the filament such that the sample sees an isothermal heating pattern. Improvements in sample loading will be required to address this problem. The 30 -fold increase in sample utilization improves the efficiency of the pulsed atomization-pulsed ionization RIMS system to about $10^{-3}$. The elemental coverage and analytical sensitivity make the potential applications extremely exciting.

\section{Reference}

[1] Miller, C. M. and Nogar, N. S., Anal. Chem., 55, 1608 (1983).

18. Characterization of Biological SRM's Using Atomic Spectrometry Techniques (15601)

T. C. Rains, T. A. Rush, T. A. Butler, M. S. Epstein, R. L. Watters, Jr.

There is increasing concern about both the essentiallity and toxicity of substances in man's diet. An important aspect of characterizing the intake of such substances is the trace element analysis of foods. The accumulation of certain elements in the body can be monitored by analyzing trace elements in biological fliuds. The accuracy of these analyses is of great concern to nutritionists, and is, in part, assured by the use of well characterized reference materials. Two biological SRM's are being prepared and analyzed for this purpose. Numerous major, minor, and trace elements are being determined in a large supply of powdered milk to be issued as an NBS SRM. In addition, a similar program is underway in which samples of freeze-dried urine, having normal and elevated levels of trace elements of interest, are prepared and analyzed.

The elemental concentrations in milk and urine vary over a wide range. Biological matrices which have a relatively high concentration of $\mathrm{Na}, \mathrm{Ca}$, or Mg produce interelement effects during the analysis of most analytes by electrothermal atomic absorption spectrometry (ETAAS). This difficulty has been largely alleviated by using matrix modification techniques and the L'vov platform. In the direct current plasma emission spectrometry technique (DCP), a solution of $\mathrm{Li}(1000 \mu \mathrm{g} / \mathrm{mL})$ was added as a spectroscopic buffer. With the inductively coupled plasma (ICP) technique, high concentrations of cations did not create any difficulty. However, it was essential to match the acid concentration to achieve optimal precision and accuracy. 
The major difficulty encountered in the determination of trace elements such as $\mathrm{Cd}, \mathrm{Fe}$, and $\mathrm{Pb}$ is contamination. The sources of contamination include the apparatus, the reagents, the environment, and the analysts. Glass of any kind is attacked by strong acids and must not be used. Teflon-containers have been evaluated by Murphy [1] and are acceptable for these analyses. Another source of contamination is the plastic cups used routinely with the automatic pipetting system of the ETAAS instrument. Reused plastic cups which had been soaked overnight in 20 percent $\mathrm{HNO}_{3}$ and then rinsed with copious amounts of distilled water produced significant blanks. To minimize the difficulty encountered in $\mathrm{Fe}, \mathrm{Cd}$, and $\mathrm{Pb}$ analyses, only new cups were used. To ensure low and reproducible blanks, each new cup had to be soaked according to the previously described procedure.

Tables 10 and 11 show the results obtained for SRM 1549 (Powdered Milk) and SRM 2672a (Urine). The powdered milk was digested with a mixture of $\mathrm{HNO}_{3}$ and $\mathrm{HClO}_{4}$ and then the solution was analyzed for all the major and trace elements except $\mathrm{Si}$ and $\mathrm{Hg}$. To prevent losses of mercury during the digestion, the sample was digested under reflux conditions. Silicon was solubilized with a sodium carbonate fusion in separate solutions. For the analysis of freeze dried urine, the urine was reconstituted with water. The DCP analysis was performed on the reconstituted urine after appropriate dilutions were made and Tithium was added as a spectroscopic buffer. For the FAAS and ETAAS analyses, the urine was digested in $\mathrm{HNO}_{3}$ and the appropriate matrix modifier was added.

In conclusion, the use of matrix modifiers and spectroscopic buffers greatly improved the precision and accuracy of FAAS, ETAAS, and DCP analyses. In addition, the care taken to minimize the sources of contamination significantly reduced blank levels. Further work is needed to improve the detection limits of silicon in biological materials.

\section{Reference}

[1] Murphy, T. J., "The Role of the Analytical Blank in Accurate Trace Analysis," NBS Spec. Pub1. 422, 7th IMR Symposium, U.S. Gov. Printing Office, August 1976. 
Table 10. SRM 1549, Powdered Milk.

\begin{tabular}{|c|c|c|c|}
\hline Element & Method & Concentra & tion, wt. \\
\hline $\begin{array}{l}\mathrm{Ca} \\
\mathrm{K} \\
\mathrm{Na} \\
\mathrm{P}\end{array}$ & $\begin{array}{l}\text { ICP } \\
\text { ICP } \\
\text { FES } \\
\text { ICP } \\
\text { DCP }\end{array}$ & $\begin{array}{l}1.27 \\
1.79 \\
1.70 \\
0.501 \\
1.052\end{array}$ & $\begin{array}{l}(0.01) \\
(0.01) \\
(0.01) \\
(0.002) \\
(0.009)\end{array}$ \\
\hline $\begin{array}{l}\mathrm{Cd} \\
\mathrm{Co} \\
\mathrm{Cu} \\
\mathrm{Fe} \\
\mathrm{Hg} \\
\mathrm{Mn} \\
\mathrm{Pb} \\
\mathrm{Si} \\
\mathrm{Sn} \\
\mathrm{Zn}\end{array}$ & $\begin{array}{l}\text { ETAAS } \\
\text { ETAAS } \\
\text { DCP } \\
\text { ETAAS } \\
\text { CVAAS } \\
\text { DCP } \\
\text { ETAAS } \\
\text { DCP } \\
\text { DCP } \\
\text { FAAS } \\
\text { ICP }\end{array}$ & $\begin{array}{l}\quad 0.031 \\
<0.1 \\
0.68 \\
2.5 \\
0.0004 \\
0.29 \\
<0.1 \\
<50 \\
<0.5 \\
46.3 \\
45.2\end{array}$ & $\begin{array}{l}\frac{\mu \mathrm{g} / \mathrm{g}}{(0.009)} \\
(0.05) \\
(0.3) \\
(0.0001) \\
(0.03)\end{array}$ \\
\hline
\end{tabular}

\footnotetext{
average of replicate determinations, one standard deviation of a single determination in parentheses.
}

Table 11. SRM 2672a, Freeze Dried Urine.

\begin{tabular}{clcccc} 
Element & Method & \multicolumn{2}{c}{$\frac{\text { Normal }^{\mathrm{a}}}{\text { Elevated }^{\mathrm{a}}}$} \\
\cline { 3 - 4 } & FAAS & 104 & $(1.8)$ & 105 & $(1.2)$ \\
$\mathrm{Ca}$ & DCP & 105 & $(0.9)$ & 106 & $(0.7)$ \\
$\mathrm{Cd}$ & ETAAS & $0.0023(0.0001)$ & $0.97(0.006)$ \\
$\mathrm{Cu}$ & DCP & $0.126(0.008)$ & $0.370(0.016)$ \\
& ETAAS & $0.128(0.013)$ & $0.362(0.012)$ \\
$\mathrm{Cr}$ & ETAAS & $0.0098(0.0017)$ & $0.183(0.010)$ \\
$\mathrm{Pb}$ & ETAAS & $<0.005$ & $0.099(0.011)$ \\
$\mathrm{Hg}$ & CVAAS & $<0.002$ & $0.105(0.003)$
\end{tabular}

\footnotetext{
average of replicate determinations, one standard deviation of a single determination in parentheses.

FAAS - Flame atomic absorption spectrometry

DCP - Direct current plasma spectrometry

ETAAS - Electrothermal atomic absorption spectrometry

CVAAS - Cold Vapor atomic absorption spectrometry

ICP - Inductively coupled plasma

FES - Flame emission spectrometry
} 
19. Measurement of Iodine Concentrations and Isotope Ratios at Environmental Levels (15310)

R. M. Lindstrom, G. J. Lutz

Iodine-129, a long-lived $\left(t_{1 / 2}=15.7 \mathrm{My}\right)$ isotope produced in high yield in uranium fission, is an ideal tracer for assessing the environmental impact of nuclear testing and nuclear energy production. Because of its long half-life and low energy of its radioactive emissions, 129 I cannot be measured directly by radiation counting at the present-day environmental level of $<10^{-6} \mathrm{~Bq} / \mathrm{g}$, which corresponds to $<10^{9}$ atoms $/ \mathrm{g}$ or $<2 \times 10^{-13} \mathrm{~g} / \mathrm{g}$. Neutron activation analysis is a sensitive method for measuring concentrations of 129 I and stable 127 I found in environmental samples, the $129 \mathrm{I}$ being converted to the more readily measured $130 \mathrm{I}$ isotope having $10^{5}$ times as much activity. The procedure used at NBS involves the separation of iodine from the sample by combustion, intense neutron irradiation, removal of interfering radioactivities by gas-phase separation using a selective ion-retention medium, and low-level gamma-ray assay.

In the early phases of this work, repeated measurements of samples expected to be low in ${ }^{129}$ I yielded occasional high concentrations, many times the level expected. Contamination of laboratories by this commerciallyavailable isotope in the course of tracer work is insidious and difficult to detect because of its low-energy emissions and long half-life. Stringent precautions must be taken to prevent contamination of samples containing background levels of $129 \mathrm{I}$. Pre-irradiation sample manipulations at NBS are confined to laboratories and apparatus where ${ }^{129}$ I has never been used. A11 sample handling is done in a clean area within one laboratory, in which the air supply to the work bench is filtered through activated charcoal to remove elemental iodine and through a HEPA filter to remove particulates. Oxygen flowing over the sample during combustion is filtered through silvered quartz wool and activated charcoal. The combustion tube and boat are precleaned by ignition in flowing oxygen, and the activated charcoal for the combustion trap is crushed and sieved in a class 100 clean room and baked in vacuum before use. Cross-contamination is avoided by using a new combustion tube and boat for each new material; agreement among replicate samples of the same material burned in the same combustion tube is a stringent test of the blank. These measures have been sufficient to eliminate entirely the occasional high values seen earlier, so that the blank is now comparable to the detection limit. During the past year, reproducible measurements of ${ }^{129} \mathrm{I}$ at the sub-pg/g level were performed routinely.

Stable $127 \mathrm{I}$ is measured in the same samples by a short irradiation and gamma assay before the long irradiation. In order to avoid the uncertainty associated with yield determination, an epithermal irradiation capability in the NBS Reactor has been established, allowing non-destructive measurements of stable iodine to be made in botanical materials down to a few tenths of a microgram per gram.

NBS measurements help to provide quality control to other laboratories involved in monitoring environmental levels of ${ }^{129} \mathrm{I}$. Two Standard Reference 
Materials have been characterized to assist in assuring comparability. SRM 1572, Citrus Leaves, was found to have a ${ }^{129}$ I concentration of $(2 \pm 1) \times 10^{8}$ atoms $/ \mathrm{g}(0.04 \pm 0.02 \mathrm{pg} / \mathrm{g})$, and in SRM 1646, Urban Particulate, $(17 \pm 5) \times 10^{-8}$ atoms/g. The ${ }^{129} \mathrm{I} / 127 \mathrm{I}$ isotopic ratio in both materials is $2 \times 10^{-8}$, which appears to be characteristic of the present-day environmental average in the northern hemisphere. For comparison, the ${ }^{129} \mathrm{I} / 127 \mathrm{I}$ ratio in the pre-1945 biosphere has been estimated to be $2 \times 10^{-12}$, near unity in uranium fission and $10^{-6}$ near reprocessing plants.

Emphasis in the coming year will be on improving the sensitivity of 129 I measurements by reducing the continuum background due to fission products in the gamma spectrum, and by irradiating at as high a flux as the triple-capture interference will allow. In the longer term, specificity and probably sensitivity can be improved by the use of coincidence counting techniques tailored to the decay scheme.

\section{Certification of Uranium Isotopic Standard Reference Materials (15310)}

J. W. Gramlich, L. A. Machlan, J. R. Moody

Highly accurate uranium isotopic standards are necessary to calibrate mass spectrometers used in the nuclear industry for the precise isotopic analysis of nuclear materials. The mass dependent isotopic fractionation, inherent in the mass spectrometric ionization process, must be calibrated against standards of known isotopic composition if accurate results are to be obtained. This information is necessary for the accountability and safeguarding of nuclear materials. The purpose of this project was to reissue several uranium isotopic SRM's which are used to calibrate mass spectrometric measurements. In addition to the reissuance of current uranium isotopic SRM's, this project will yield accurate measurement of the isotopic composition of SRM 960, a uranium metal certified only for uranium assay. Since there is no National standard of uranium with natural isotopic composition that is certified for isotopic composition, the certification of this SRM will be a significant contribution to the nuclear community.

The new SRM' $s$, at nominal $235 \mathrm{U}$ abundances of 3,2 , and 0.5 percent and in the form of $\mathrm{U}_{3} \mathrm{O}_{8}$, were obtained from Oak Ridge National Laboratory. The newly developed semi-micro Davies-Gray titration was used in both the preparation of synthetic mixtures for the determination of the major isotopes, $235 \mathrm{U}$ and $238 \mathrm{U}$; and the determination of the minor isotopes, ${ }^{234} \mathrm{U}$ and ${ }^{236} \mathrm{U}$. Spark source mass spectrometry was used to verify that no impurities were present which could interfere with the titration or with the mass spectrometry measurements.

The synthetic mixtures for the $235 \mathrm{U}$ and $238 \mathrm{U}$ determination were prepared by combining weighed aliquots of two separated isotope solutions, $235 \mathrm{U}$ and ${ }^{238} \mathrm{U}$, whose concentrations had been accurately and precisely determined by the semimicro Davies-Gray titration method. These synthetic mixtures, with known isotopic ratios, were used to calibrate the mass spectrometers used for the isotopic assay of the SRM materials. The minor isotopes, ${ }^{234} \mathrm{U}$ and ${ }^{236} \mathrm{U}$, were measured using isotope dilution. Known amounts of each SRM were spiked with a known amount of $233 \mathrm{U}$, SRM 995. The minor isotopes in these three new materials are considerably lower in concentration than in the old SRM's, thus increasing 
the difficulty of accurate measurements. Low mass scatter from $238 \mathrm{U}$ interferes with the baseline measurements for ${ }^{236} \mathrm{U}$ : at a ratio of $10^{6}$ for $238 \mathrm{U} / 236 \mathrm{U}$ this can become a significant contribution to the errors in the $236 \mathrm{U}$ measurements. A similar relationship exists between $235 \mathrm{U}$ and ${ }^{234} \mathrm{U}$. By optimizating the analytical and instrumental parameters, the minor isotopes $234 \mathrm{U}$ and $236 \mathrm{U}$ were measured with an average uncertainty (1s) of 0.5 percent. Measurements were made both under computer control and by manual scanning followed by hand calculation of the data: the results of the two methods of data collection are in good agreement. The isotopic measurements required for certification of these SRM's are essentially complete and the statistical evaluation of the data is in progress.

Based on the precision obtained using the Davies-Gray titrations $(<0.01$ percent), as well as the quality of the mass spectrometric measurements, it is expected that the new uranium isotopic standards will be substantially more accurate than the presently available standards. The issuance of these new SRM's will conclude this research effort. These SRM's are expected to substantially improve accountability measurements in the nuclear industry.

21. Neutron Depth Profiling and the Characterization of Dopant Distributions (26109)

R. F. Fleming, R. G. Downing, J. T. Maki, D. H. Vincent

Increasingly, problems in science and industry require measurement of the concentration distribution of constituents in the near-surface region of materials. This is most significant in the semiconductor industry where nondestructive profiling reference measurements are needed to cross-calibrate other methods as well as to perform simple analyses. In the case of boron, its distribution after an implantation, its diffusion during material processing, and its homogeneity throughout glass overcoats must be known to design state-of-the-art microcircuitry of reproducible integrity. In this first year of operation, the synergism between neutron depth profiling (NDP) and other techniques such as secondary ion mass spectroscopy (SIMS) [1], spreading resistance probe (SRP), Fourier transform IR (FTIR) and prompt gamma-ray emission (PGRE) has been demonstrated for the analysis of boron in semiconductor materials.

The NDP facility utilizes a high quality, intense thermal neutron beam at the NBS 10 MW reactor [2]. The depth profiling of boron or a number of other elements (e.g., He, $\mathrm{Li}, \mathrm{Na}$ ) depends on the fact that when the target isotope absorbs a neutron, monoenergetic charged particles are isotropically emitted. The amount of energy these particles lose before reaching a detector outside the sample surface is directly related to the depth at which the reaction takes place. Therefore, the energy spectrum of the charged particles can be unfolded to give an absolute measurement of the depth distribution of the reacting element. The particles stopping power in a particular matrix must therefore be known.

Figure 14 is an overlay of two NDP measurements made on a borophosphosilicate glass (BPSG) film. One measurement was made after the sample was 


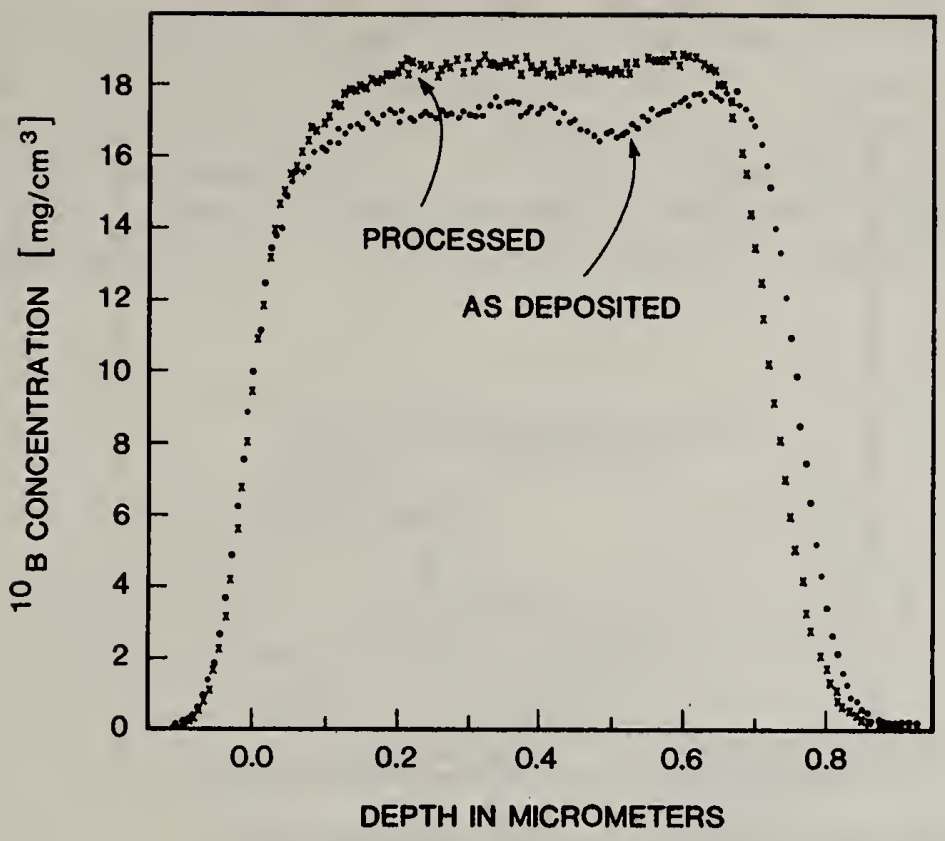

Figure 14. Boron concentration profile of a borophosphosilicate glass film on a silicon substrate. The comparison is between the deposited glass $(\cdot)$ and the same film after annealing $(x)$. Data are not corrected for detector resolution.

deposited on a silicon wafer and the other measurement was made on the same wafer after physical processing of the sample. BPSG is used as an insulating layer and for forming tapered contours over steep steps of oxide on silicon for high density integrated circuits. NDP was able to determine the sample thickness, the homogeneity of the boron distribution, and the absolute boron concentration at discrete depths using a single non-destructive measurement. Together the plots show the leveling of the boron distribution after processing. The integrals of the two peaks are equivalent indicating that no boron loss occured during processing. In addition, these measurements were used to establish a calibration curve for FTIR, PGRE, and NDP in collaboration with researchers from industry and university labs.

With respect to other elements of interest, perhaps the most significant accomplishment this year has been the profiling of ${ }^{22} \mathrm{Na}$ for the first time. In figure 15, the sodium profile of ${ }^{22} \mathrm{Na}$ is shown, diffused in a single layer thick BPSG film on a silicon substrate. In addition, collaborative work with other National labs is being carried out to study diffusion properties of ${ }^{6} L i$ in copper and lattice damage during implantation of $1{ }^{0} \mathrm{~B}$ in copper. In the coming year NDP will continue to be applied to the profiling of $\mathrm{He}, \mathrm{Li}, \mathrm{B}, \mathrm{Na}$, and $\mathrm{Bi}$. The successful profiling of boron and ${ }^{22} \mathrm{Na}$ points toward the extension of NDP to other elements such as beryllium profiling using ${ }^{7} \mathrm{Be}$. Further improvements in resolution and accuracy should result as this unique facility continues to develop. 


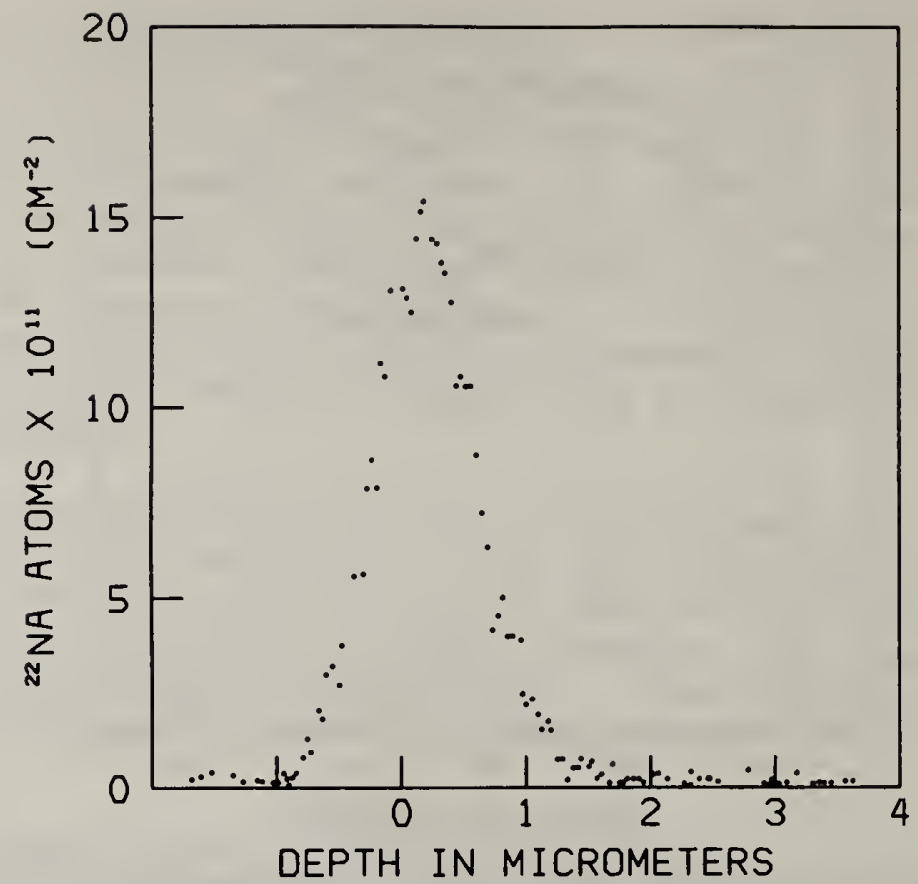

Figure 15. Neutron depth profile of ${ }^{22} \mathrm{Na}$ in BPSG film, not corrected for detector resolution.

References

[1] Downing, R. G., Fleming, R. F., Simons, D. S., and Newbury, D. E., Microbeam Analysis-1982, K. F. J. Heinrich, ed., (San Francisco Press, 1982), pp. 219.

[2] Downing, R. G., Fleming, R. F., Langland, J. K., Vincent, D. H., Proceeding of the Sixth Intern. Conf. on Ion Beam Analysis, 1982, Nucl. Instr. Method, in press.

\section{An Automated Mass Spectrometer--Inlet System for Gas Analyses (15601)}

I. L. Barnes, W. A. Bowman, R. L. Shideler, P. Morales

Isotope dilution mass spectrometery is an analytical technique which is widely applied to the determination of trace constituents in solid materials. In many cases this method is highly precise and very accurate and has been accepted as a definitive method.

IDMS has not yet been applied to the determination of the normally gaseous light stable elements, i.e., carbon, hydrogen, oxygen, nitrogen and sulfur. However, the same principles as for solid samples should apply, with the added advantage that gas phase measurements are more precise than those made with thermal ionization since the measurements are usually compared to a reference gas which is analyzed alternately with the sample. 
A program was initiated to construct an instrument and develop the methodology for the IDMS analyses of gases with the initial effort to be concentrated on the analys is of carbon dioxide in nitrogen and air. To accomplish this, a $30 \mathrm{~cm}$ radius, $90^{\circ}$ deflection mass spectrometer has been constructed. The instrument has an electron bombardment ion source and a specially designed (using the new ion optics programs) triple collector to collect the three masses for $\mathrm{CO}_{2}(44,45$, and 46), simultaneously (figure 16).

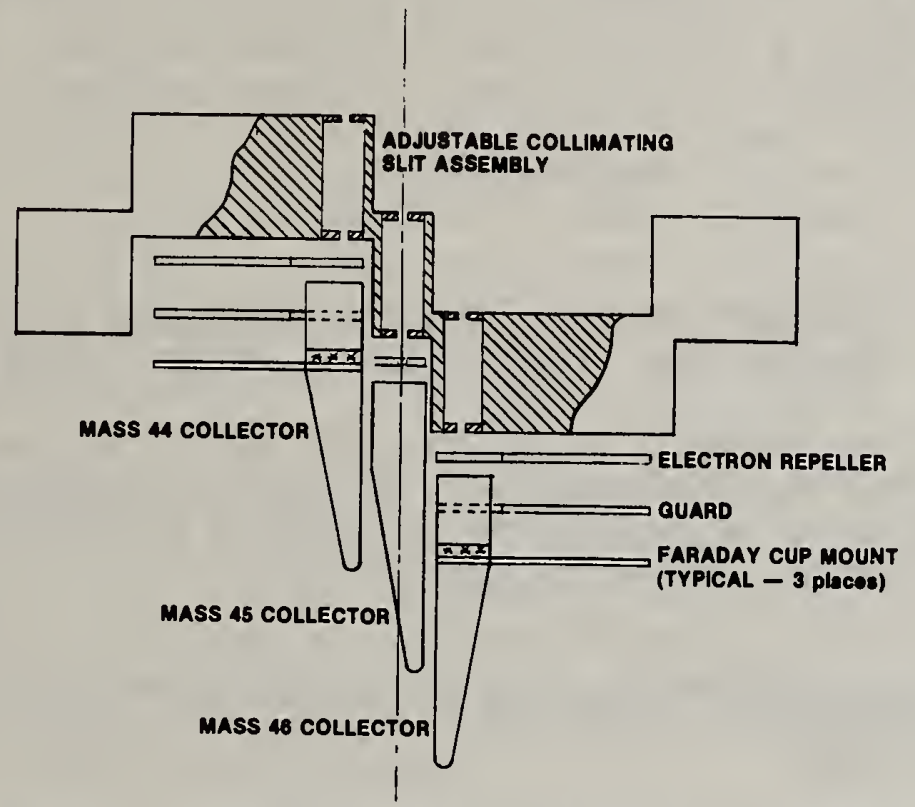

Figure 16. Triple filament collector assembly. Designed specifically for carbon dioxide, each collector contains an adjustable slit assembly, a deep Faraday cup, and guard and repeller plates.

The critical part of this instrument, however, is the specially designed inlet system. Such a system must actually consist of two identical systems as closely matched as possible. A schematic diagram of the new inlet system is shown in figure 17. The pressure in the sample and standard sides must be matched as perfectly as the best technology permits. This is accomplished in the new system by the use of a mechanism consisting of two matched stainless steel bellows mounted on slide assemblies with precision lead screws driven by computer controlled stepper motors. Each side of the system is connected to one side of a high precision capacitance manometer operated in the null mode. A binary coded decimal (BCD) output from the manometer interfaced to a computer is used to control the stepper motors to produce equal pressure. In addition, the output signal from the mass 44 signal from the mass spectrometer is monitored with a high precision digital voltmeter (DVM). A mismatched signal indicates mismatched pressures and possibly an impure sample. Each of the air operated valves indicated in Figure 17 is controlled by a small solenoid which is controlled by a NBS designed IEEE-488 interface connected to the computer. Any, or all, valves may be opened or closed independently or simultaneously. An additional feature of the new instrument is the use of high precision ratio DVM's so that the ratios $44 / 45$ and $44 / 46$ can be measured directly and at the same time. 


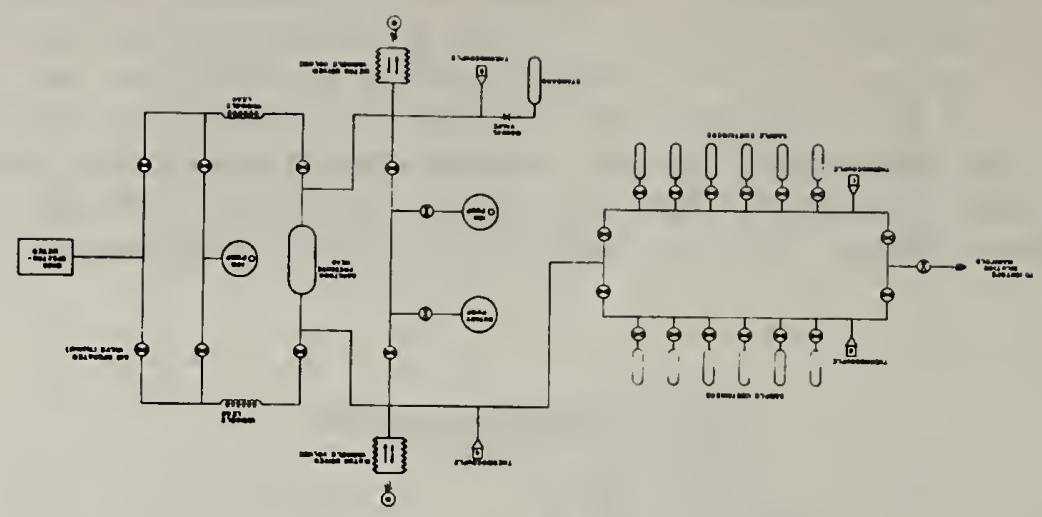

Figure 17. Schematic diagram of automated sample preparation, storage, and inlet system for light stable elements.

The initial measurements of the isotopic ratios of several $\mathrm{CO}_{2}$ reference materials made with the instrument were an order of magnitude more precise than the best reported to date and it is expected that this will be improved further as the computer program is refined.

\section{Potential Limitation in the Use of Thin Metal Films as Transmittance Standards (15601)}

R. W. Burke, R. Mavrodineanu, M. V. Smith

A continuing research activity in high accuracy spectrophotometry is focussed on the development of transmittance standards whose spectral properties permit their use throughout the ultraviolet, visible, and near infrared regions of the spectrum. Semi-transparent metal films have long been considered the best of the extremely limited number of materials that can be used for this purpose. Filters prepared by evaporating thin films of chromium, inconel, or nichrome onto quartz substrates exhibit excellent optical neutrality from $200 \mathrm{~nm}$ to $2.5 \mu \mathrm{m}$. Because of this neutrality, their transmittance is, except for the most stringent applications, independent of wavelength, spectral bandpass, and heterochromatic stray radiation.

During the production of SRM 2031 (chromium on quartz), there were, in addition to long term stability, two other potential problems of major concern. The first dealt with finding a suitable means for protecting the chromium film from environmental exposure and physical damage. This problem was solved when it was found that the exposed metal layer could be adequately protected by covering it with a second clear quartz plate. This second plate was ground and polished in such a manner that a rigid assembly of the two plates could be achieved by optical contact. The second problem dealt with the possible consequences of the intrinsic property of semi-transparent metal films to attenuate incident radiation not only by absorption but also by significant reflection. It was our concern that, for instruments of less than optimal design, this reflected radiation could produce erroneous indications of the accuracy of the photometric scale because of the substantially larger 
contribution to the measured transmittance of interreflections between the film and the irradiating or detecting optics.

Although concern about the reflection problem still existed at the time, SRM 2031 was issued in March 1980 on the basis of a Tengthy in-house study and collaborative measurements performed by the National Physical Laboratory in Great Britain, the Michelson Laboratory in China Lake, CA, and nine other laboratories in the United States, six of which were manufacturers of spectrophotometers. At the time, none of these laboratories encountered any difficulties in using this type of filter and no problems were reported in the subsequent use of SRM 2031 until Apri1. 1983. At that time, a representative of a Swedish manufacturer of spectrophotometers informed NBS that a check of the transmittance accuracy of one line of their instruments with NBS SRM 930d, Glass Filters and SRM 2031, produced discrepant results. One of these instruments was tested by NBS and the discrepancies were confirmed. An examination of this instrument indicated that reflections from the chromium-on-quartz filters could be responsible for the observed differences. To confirm this iypothesis, the chromium-on-quartz filters were placed in special holders in wich they were tilted at an angle of 5 to 6 . degrees to the incident beam. Inder these conditions the indicated accuracy of the instrument was comparable :0 that obtained with the glass filters (SRM 930d). These findings confirmed in initial opinion that, for this particular instrument, isochromatic reflections generated by the chromium films are primarily responsible for degrading the accuracy of the measured transmittance. Subsequent discussions with the manufacturer have led to a change in optical design that substantially reduces the instrument's interreflection error.

A modification of SRM 2031 is presently being evaluated that should prevent reflective metal film transmittance standards from magnifying the interreflection effects in spectrophotometers whose optical design inherently produces interreflection errors. This modification is illustrated in figure 18. Cross-sectional views of the existing SRM 2031 and the proposed modification are shown in 18 (A) and 18 (B) respectively. In the case of the modified filter, the chromium layer is evaporated onto a quartz plate that has been ground and polished at a $7^{\circ}$ angle to the front face. The quartz cover plate has an identical but opposite wedge. In this modification the chromium layer receives the incident radiation at an angle and whatever portion is reflected is eliminated from the optical path since it is absorbed by the blackened walls of the sample compartment. A second important advantage of this particular design of tilted filter is that it does not shift the beam significantly. Because the front and the rear surfaces of the assembly are perpendicular to the incident radiation and only the film is tilted, the shift of the transmitted beam at the photocathode is kept at a minimum.

Six sets of these modified filters have been prepared and are presently undergoing extensive in-house testing. Pending the results of these tests, the filters will be tested further in the broadest range of commercial spectrophotometers available to establish their general effectiveness for calibrating instruments with inherent interreflection errors. 


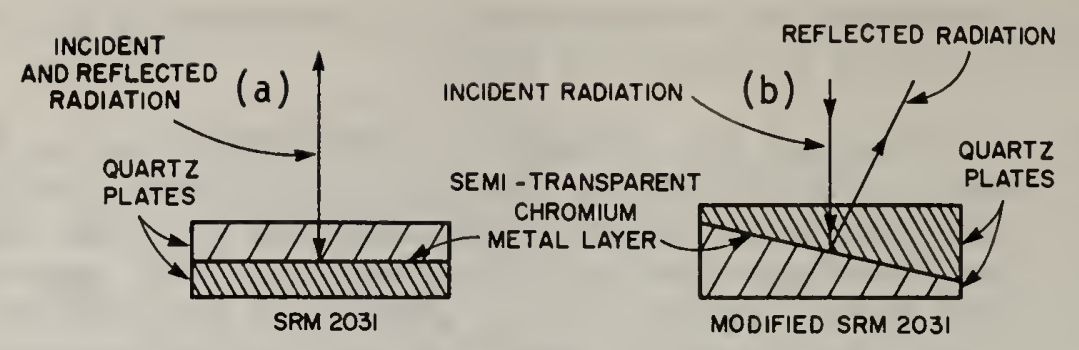

Figure 18. Cross-sectional views of SRM 2031 (a) and its proposed modification (b) for minimizing interreflection effects.

24. The Effect of Manganese on the Determination of Sulfur by Optical Emission Spectrometry (15310)

J. A. Norris, D. E. Brown

An apparent bias in the emission spectrochemical determination of sulfur in steels was reported previously [1]. This discrepancy became apparent during the redetermination of sulfur, in a number of SRM's, by isotope dilution mass spectrometry (ID/MS). At the time the report was written, there was not sufficient information available to determine a definite cause for the discrepancy.

A major cause of bias in emission spectroscopy is the presence of a spectral line of another element at or very near the analytical line of the element being determined. Most SRM's are complex mixtures of many elements at varying concentrations. Consequently, quantifying the interference due to a particular element is a difficult procedure. Sometimes these assignments can be made successfully for wavelengths above $2000 \AA$ where abundant photographic information is available. For the vacuum region, below $200 \AA$, little information is available; thus determining interferences requires special materials. A listing of glow discharge emission lines cites only $\mathrm{Ca}, 1807.74 \AA$; $\mathrm{Ti}$, $1806.98 \AA$, and $\mathrm{Ta}, 1806.82 \AA$ as possible interferences with $\mathrm{S} 1807.31 \AA$. Another compendium of vacuum ultraviolet emission lines indicates that $\mathrm{Ni}$, 1807.56 $\AA$ and 1807.245 $\AA$; $A 1,1807.40 \AA$; and Mo, $1807.63 \AA$ might interfere. However, these data were obtained under excitation conditions considerably different from those normally used for spectrochemical analysis.

Special ferrous base binary alloys, prepared by SKF STAL of Sweden, were used to identify elements that interfere with the analysis of sulfur. These alloys contained $\mathrm{B}, \mathrm{Al}, \mathrm{Si}, \mathrm{P}, \mathrm{V}, \mathrm{Cr}, \mathrm{Mn}, \mathrm{Co}, \mathrm{Ni}, \mathrm{Cu}, \mathrm{As}, \mathrm{Nb}, \mathrm{Mo}, \mathrm{Sn}$, and $\mathrm{W}$ in concentrations ranging from ppm levels for some elements to 24 percent for $\mathrm{Cr}$ and 22 percent for $\mathrm{Ni}$. All materials were analyzed on the vacuum spectrometer using calibration curves based on existing SRM's.

of all the elements tested, only $\mathrm{Mn}$ and $\mathrm{Ni}$ had an appreciable influence or the sulfur determination. The influence of $\mathrm{Ni}$ was an order of magnitude less than that of manganese. The results obtained are shown in Table 12. 
Table 12. Influence of Manganese on the Determination of Sulfur.

\begin{tabular}{|c|c|c|c|c|}
\hline Binary \# & $\begin{array}{l}\text { Assignec } \\
\% \mathrm{Mn}\end{array}$ & $\begin{array}{l}\text { Values } \\
\% \mathrm{~S} \\
\end{array}$ & $\begin{array}{c}\text { Determined Value } \\
\% \mathrm{~S}\end{array}$ & $\begin{array}{l}\text { \% Sulfur Bias } \\
\text { Per } \% \mathrm{Mn} \\
\end{array}$ \\
\hline $\begin{array}{l}6012 \mathrm{H} \\
6013 \mathrm{G} \\
6014 \mathrm{G}\end{array}$ & $\begin{array}{l}0.553 \\
1.08 \\
2.04\end{array}$ & $\begin{array}{l}0.0112 \\
0.0120 \\
0.0080\end{array}$ & $\begin{array}{l}0.0123 \\
0.0142 \\
0.0113\end{array}$ & $\begin{array}{l}0.00199 \\
0.00203 \\
0.00162\end{array}$ \\
\hline
\end{tabular}

The only available information on a possible bias between the SKF sulfur determination and our sulfur calibration comes from the values for sulfur in two base steel materials that are a part of the binary series. The results obtained for these samples are shown in Table 13.

Table 13. Sulfur Determined in SKF Base Steels.

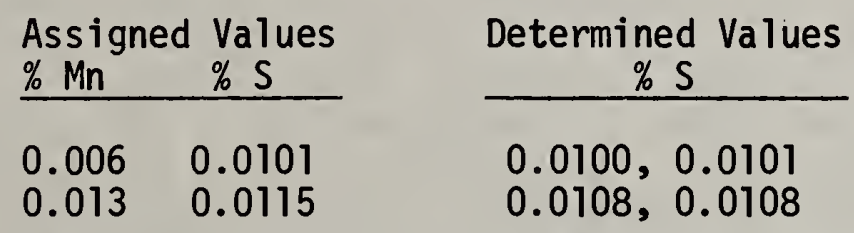

These data show that a correction of 0.002 percent $S$ per percent $\mathrm{Mn}$ is the present best estimate of the manganese influence on sulfur. Several recent homogeneity studies have been reevaluated and the sulfur values have been corrected to reflect the influence of manganese. This correction significantly improves the accuracy for samples having a major amount of manganese. The corrected values are shown in Table 14.

Table 14. Corrected Sulfur Values (\%).

\begin{tabular}{|c|c|c|c|}
\hline SRM & Certificate $S$ & $\begin{array}{l}\text { Uncorrected } \\
\text { Sulfur } \\
\end{array}$ & $\begin{array}{c}\text { Corrected } \\
\text { Sulfur } \\
\end{array}$ \\
\hline 22 & $0.022 \pm 0.002$ & 0.022 & $0.0204 \pm 0.0011$ \\
\hline 12 & $0.039 \pm 0.005$ & 0.045 & $0.044 \pm 0.0024$ \\
\hline 1225 & $0.014 \pm 0.001$ & 0.0161 & $0.0152 \pm 0.0008$ \\
\hline 1226 & $0.0044 \pm 0.0006$ & 0.0051 & $0.0045 \pm 0.0003$ \\
\hline 1227 & $0.026 \pm 0.002$ & 0.0305 & $0.0297 \pm 0.0024$ \\
\hline 1228 & $0.018 \pm 0.001$ & 0.0194 & $0.0187 \pm 0.0008$ \\
\hline 1269 & $0.0061 \pm 0.0004$ & 0.0085 & $0.0058 \pm 0.0004$ \\
\hline 1270 & $0.0065 \pm 0.0010$ & 0.0064 & $0.0051 \pm 0.0004$ \\
\hline & $0.020 \pm 0.001$ & 0.0218 & $0.0211 \pm 0.0036$ \\
\hline & $0.017 \pm 0.001$ & 0.0171 & $0.0168 \pm 0.000$ \\
\hline
\end{tabular}

Reference

[1] 1981 Annual Report, Center for Analytical Chemistry. 


\section{Certification of NBS Buffer Materials (15310)}

Y. C. Wu, G. Marinenko

The pioneering work on the development of the National $\mathrm{pH}$ Scale was conducted at NBS under the leadership of S. F. Acree, W. J. Hamer, and R. G. Bates. The National pH Scale, which evolved from their work, has gained worldwide recognition and acceptance. The NBS pH scale is defined over a range of $\mathrm{pH} 3.5$ to $\mathrm{pH} 10$ in terms of seven $\mathrm{pH}(\mathrm{S})$ values assigned to seven primary reference standards. Traceability of this scale is maintained by Standard Reference Materials (SRM's) of these seven buffers which are evaluated at NBS and the measured $\mathrm{pH}(S)$ values certified. Depletion of the reserve quantities of these SRM buffers requires certification of the $\mathrm{pH}$ values for new lots of these materials.

For the purpose of primary certification (absolute measurement), accurate emf measurements must be performed in a galvanic cell without a liquid junction. Since the measured $\mathrm{pH}$ value is computed directly from $E^{\circ}$ and the measured emf of the cell, accurate values for the potentials of the hydrogen and $\mathrm{Ag} / \mathrm{AgCl}$ electrodes must be assured. Performance of the hydrogen electrodes was checked periodically by making emf measurements in $0.01 \mathrm{~m} \mathrm{HCl}$ against a common $\mathrm{Ag} / \mathrm{AgCl}$ reference electrode. The emf of all hydrogen electrodes was within $0.02 \mathrm{mV}$, which contributes less than $0.001 \mathrm{pH}$ uncertainty to the certified $\mathrm{pH}$ of the standard buffers.

The $\mathrm{Ag} / \mathrm{AgCl}$ electrodes used in this work are of somewhat different design than those used previously. They are formed on high purity silver wire using the thermal electrolytic method. Particular attention is given to the exclusion of oxygen. Electrodes produced by this procedure are more stable and more reproducible than those produced by the previous method. The lifespan of the $\mathrm{Ag} / \mathrm{AgCl}$ electrodes is also significantly extended, as indicated by the reproducibility of $E^{\circ}$ of these electrodes measured periodically. Maintenance of the instrumentation required for $\mathrm{pH}$ measurements is a continuous process, involving periodic calibration of the digital barometer, quartz thermometer, and digital voltmeter against the National standards.

Using the above approach and the improved facilities, three new buffer materials were evaluated this year and their $\mathrm{pH}(\mathrm{S})$ values certified, at $5^{\circ}$ increments, between 0 and $50{ }^{\circ} \mathrm{C}$ :

\section{$\mathrm{pH}$ at $25^{\circ} \mathrm{C}$}

(1)
SRM 191a/192a $\quad \mathrm{NaHCO}_{3} / \mathrm{Na}_{2} \mathrm{CO}_{3}$

SRM 187c Borax

SRM 186Id/186IId $\quad \mathrm{KH}_{2} \mathrm{PO}_{4} / \mathrm{Na}_{2} \mathrm{HPO}_{4}$
10.010

9.180

6.863

Further work is in progress on the development of reference solutions for the calibration of glass electrodes for $\mathrm{pH}$ measurements of low ionic strength solutions such as acid precipitation. 
26. Standard Reference Materials (15310)

B. I. Diamondstone

The certification of Standard Reference Materials (SRM's) represents one of our major outputs and accounts for approximately one-third of the Division's total staff effort. This work is diverse in nature, requires a high degree of analytical skills and covers the full range of acceptance testing, homogeneity evaluation, and accurate measurements leading to the certification of inorganic constituents. As in the past, this support falls into two categories; STRS-Scientific and Technical Research Services and WCF--Working Capital Funds. Input in these areas is provided by the entire scientific staff with a considerable portion representing cooperative efforts between groups within the Division.

During the past year, six different areas of rsearch were funded by the Office of Standard Reference Materials under the STRS program (Table 15). The STRS portion of the funding is intended for the preliminary research necessary to develop the methodologies required for the actual production of SRM's. On the other hand, the WCF projects result in the actual certification for chemical composition of a marketable standard. Once again, analyses were carried out on more than 75 different SRM's covering a variety of matrices including metals, fossil fuels, body fluids, foods, nuclear materials, and biologicals (Table 16). In many cases, each certification involves several or all of the groups in the Division and can require 20-30 individual analyses on each SRM.

A major effort has been put forth to apply existing and newly acquired methodologies to the determination of trace and ultratrace level elements in urine, serum, and milk. Work is nearly complete on these materials and they should be issued as SRM's in the near future. Nearly a dozen separate techniques have been used to certify these three materials. In the area of metals, work continues towards the certification of a number of new SRM's such as inconels, incoloys, and manganese ores. The process of updating existing SRM's is carried out on a continual basis. For the first time in approximately 15 years, NBS has produced a certifiable value for hydrogen in a metal SRM. This year the first set $(\mathrm{Cd}, \mathrm{Pb}, \mathrm{Ag}, \mathrm{Zn})$ of a new series of aqueous spectrometric standard solutions has been prepared. These standards are intended for use with any of a number of spectrometric techniques including AAS, ICP, DCP, and spectrophotometry.

During the coming year work will begin on several new materials leading to the certification of a number of new SRM's. These materials include soil, clay, tissues, ores, and metals. Additional sets of aqueous spectrometric standards are also planned. Each year a significant amount of work goes into updating existing SRM's, i.e., changing "information only" numbers to certified values and performing analyses on previously undetermined elements. 
Table 15. STRS Projects Supported by OSRM During FY 83.

Task

Gases in Metals

Measurement of $\mathrm{pH}$

Analytical

Methodologies

Gas Isotope Dilution Mass Spectrometry

High-Accuracy

Spectrophotometry

Neutron Depth

Profiling
Description

Effort, SY

Research leading to the develop-

0.7 ment of an in-house capability to certify oxygen, nitrogen, and hydrogen in a broad range of metals. Develop $14 \mathrm{MeV}$ neutron technology used in the measurement of oxygen in steels.

Research to develop the capability of making high-accuracy measurements of $\mathrm{pH}$ suitable for certification purposes.

Continued research to develop analytical methodologies related to the certification of Standard Reference Materials.

Continued research leading to the development of gas ID/MS methods for determining elements such as sulfur, oxygen, and nitrogen in many metals and $\mathrm{CO}$ and $\mathrm{CO}_{2}$ in nitrogen and air.

Research to extend the measurement capabilities of the high-accuracy spectrophotometer into the wavelength region from $800 \mathrm{~nm}$ to $3.5 \mathrm{~nm}$. Also, investigate the wedge effect of chromium coatings on optical filters.

Research to develop accurate thermal neutron beam method for measuring the compositional profile of boron- 10 in silicon.
0.5

0.5

0.2

3.3 
Table 16. WCF Work Carried Out on Standard Reference Materials During FY 83.

SRM Number

$1233,346 \mathrm{a}$

$1219,1151-1154 a, 1155,343 a$

$864-867,1244-1247$

2670

2671

2672

953

$1633 a$

$191 a, 192 a$

2031

930d

1084, 1085

1083

1573,1575

1549

$1643 \mathrm{~b}$

1577 , b

C1252, 400, 399, 398,

$457,496,395,394$

C2403-C2410

C1290-C1292, C2400-C2402

2423-2427

$25 d$

670

909

1618

2121

120c, 694

1832

33e, 1761-1767

$1264 a$

1227
Title

Valve Steels

Stainless Steels

Inconels and Incoloys

Trace Elements in Urine

Fluorine in Urine

Mercury in Urine

Neutron Density Monitor Wires

Trace Elements in Fly Ash

pH Sodium Carbonate--Sodium Bicarbonate

Metal on Quartz Filters

0.6

Glass Filters for Spectrometry

0.2

Wear Metals in 0 il

0.3

Base Stock 0 il

0.3

Tomato Leaves, Pine Needles

0.2

Powdered Milk

1.2

Trace Elements in $\mathrm{H}_{2} \mathrm{O}$

0.8

Trace Elements in Bovine Liver

0.1

Copper Benchmarks

0.1

Brasses

0.4

White Irons

0.4

Ductile Iron

0.4

Rutile

0.2

Manganese Ore

0.2

Trace Elements in Serum

0.6

Fuel 0 il

0.2

Atomic Absorption Standard Solutions

Phosphate Rocks

0.2

XRF Films

0.4

Low Alloy Steels

0.3

High Carbon Steel

0.3

Plain Steel 
C. Outputs and Interactions

(Inorganic Analytical Research Division)

\section{Publications}

Barnes, I. L., Murphy, T. J., and Michiels, E. A. I., "Certification of Lead Concentration in Standard Reference Materials by Isotope Dilution Mass Spectrometry," J. Assoc. Off. Ana 1. Chem., 65, 953 (1982).

Bechtler, G., Epstein, M. S., Geary, T. D., Havemann, W., and Attoe, P., "Provisional Guidelines for Listing Specifications of Flame Emission Spectrometers," Clin. Chim. Acta, 122, 111F (1982).

Burke, R. W., Paulsen, P. J., Maienthal, E. J., and Lambert, G. M., "Validation of the Sulfur Concentration of Selected Iron-Base NBS Standard Reference Materials by Isotope Dilution Spark Source Mass Spectrometry," Talanta, 29, 809 (1982).

Coplen, T., Kendal1, C., and Barnes, I. L., "Two New Carbonate Stable Isotope Standards," Geostandards Newsletter, VI, 257 (1982).

Cronin, D. J. and Kingston, H. M., "An Examination of the MCC-1 Leach Test Method: Comparison of Glass Compositions," Materials Characterization Center DOE, Summary Report PNL-4382, Battelle, Workshop on the Leaching Mechanisms of Nuclear Waste Forms, 144 (1982).

Downing, R. G., Fleming, R. F., Simons, D. S., and Newbury, D. E., "NeutronInduced Reactions and Secondary Ion Mass Spectrometry: Complementary Tools for Depth Profiling," Microbeam Analysis-1982, K. F. J. Heinrich, ed., San Francisco, pp. 219 (1982).

Epstein, M. S., Geary, T. D., Gower, G., Tavsch, W., Mills, K. J., and Polt, D., "Provisional Guidelines for Listing Specifications of Atomic Absorption Spectrometers," Clin. Chim. Acta, 122, 117F (1982).

Fleming, R. F., "Neutron Self-Shielding Factors for Simple Geometries," Int. J. of Applied Radiation and Isotopes, 33, 1263 (1982).

Greenberg, R. R., "Radiochemical Procedures Used at NBS for High-Sensitivity Certification Analysis of Standard Reference Materials," Trans. Am. Nucl. Soc., 43, 269 (1982).

Kingston, H. M., Cronin, D. J., and Epstein, M. S., "An Examination of the MCC-1 Leach Test Methods: Experimental Technique," Materials Characterization Center DOE, Summary Report PNL-4382, Battelle, Workshop on the Leaching Mechanisms of Nuclear Waste Forms, 133 (1982).

Kingston, H. M., Greenberg, R. R., Beary, E. S., Hardas, B. R., Moody, J. R., Rains, T. C., and Liggett, W. S., "The Characterization of the Chesapeake Bay: A Systematic Analysis of Toxic Trace Elements," National Technical Information Service, Order No. PB82-265-265, (1982). 
Kingston, H. M. and Lutz, G. J., "Adsorption and Stability Prior to Analysis of Simulated Nuclear Waste Leachate," Nuclear and Chemical Waste

Management, 3 , 245 (1982).

Koch, W. F., Marinenko, G., and Stolz, J. W., "Simulated Precipitation Reference Materials, IV," National Bureau of Standards (U.S.), NBSIR 82-2581, (1982).

Mielenz, K. D., Weidner, V. R., and Burke, R. W., "Heterochromatic Stray Light in UV Absorption Spectrometry: A New Test Method," Applied Optics, 2l, 3354 (1982).

Moody, J. R., "NBS Clean Laboratories for Trace Element Analysis," Anal. Chem., 54, 1358A (1982).

Moody, J. R. and Beary, E. S., "Purified Reagents for Trace Metal Analysis," Talanta, 29, 1003 (1982).

Burke, R. W. and Mavrodineanu, R., "Accuracy in Analytical Spectrophotometry," NBS Special Publication 260-81, (1983).

Burke, R. W. and Velapoldi, R. W., "Recommended Reference Materials for Realization of Physicochemical Properties: Transmittance and Wavelength," Draft Documents for IUPAC Commission I.4, (1983).

Epstein, M. S., "Determination of U1tratrace Levels of Lead in Reference Fuels by Graphite Furnace Atomic Absorption," Atomic Spectrosc., 4, 62 (1983).

Fassett, J. D., Moore, L. J., Travis, J. C., and Lytle, F. E., "Atomic Ion Formation and Measurement with Resonance Ionization Mass Spectrometry," Anal. Chem. , 55, 765 (1983).

Greenberg, R. R., "Neutron Activation Analysis Techniques Used For U1tra-trace Level Elemental Characterization of New NBS Milk Powder Standard Reference Material," Trans. Am. Nucl. Soc., 44, 28 (1983).

Greenberg, R. R. and Kingston, H. M., "Trace Element Analysis of Natural Water Samples by Neutron Activation Analysis with Chelating Resin," Ana1. Chem., 55, 1160 (1983).

Kelly, W. R. and Fassett, J. D., "Determination of Picogram Quantities of Uranium in Biological Tissues by Isotope Dilution Thermal Ionization Mass Spectrometry with Ion Counting Detection," Anal. Chem., 55, 1040 (1983).

Koch, W. F., "The Determination of Trace Levels of Cyanide by Ion Chromatography with Electrochemical Detection," J. Res. Nat. Bur. Stand. (U.S.), 88, 157 (1983).

Koch, W. F., Stolz, J. W., Kelly, W. R., Paulsen, P. J., Lindstrom, R. M., Fleming, R. F., and Greenberg, R. R., "Methods and Procedures Used at the National Bureau of Standards to Certify NBS Sulfur in Coal SRM's for Sulfur Content, Calorific Value, Ash Content," T. E. Gills, ed., Nat. Bur. Standard. (U.S.) Spec. Publ. 260 (1983). 
Marinenko, G., Koch, W. F., and Etz, E. S., "High-Precision Coulometric Titration of Uranium," J. Res. Nat. Bur. Stand. (U.S.), 88, 117 (1983).

Messman, J. D., Epstein, M. S., Rains, T. C., and O'Haver, T. C., "Performance Characteristics of a Continuum-Source Wavelength-Modulated Atomic Absorption Spectrometer," Anal. Chem., 55, 1055 (1983).

Moody, J. R., "Sampling and Storage of Materials for Trace Elemental Analysis," Trends in Anal. Chem., 2, 116 (1983).

Pella, P. A., Kingston, H. M., Sieber, J. R., and Feng, L. Y., "Effect of Sample Dissolution Procedures on X-ray Spectrometric Analysis of Biological Materials," Anal. Chem., 55, 1193 (1983).

Pratt, K. W. and Johnson, D. C., "The Vibrating Wire Electrode as an Amperometric Detector for Flow-Injection Systems," Anal. Chim. Acta, 148, 87 (1983).

Watters, R. L., Jr., "Practical Limits of Precision in Inductively Coupled Plasma Spectrometry," American Laboratory, p. 16 (1983).

Wise, S. A., Fitzpatrick, K. A., Harrison, S. H., Zeisler, R., Langland, J. K., Kratochvil, B., Rains, T. C., Rush, T. A., Butler, T. A., Gramlich, J. W., Powel1, L. J., Brletic, K. A., Murphy, T. J., Bailey, J., Maienthal, E. J., Koch, W. F., Kline, W. F., Allen, C. F., Chesler, S. N., Hilpert, L. R., Greenberg, R. R., MacCrehan, W. A., and Anderson, D. F., "The Pilot National Environmental Specimen Bank--Analysis of Human Liver Specimens," Zeisler, R., Harrison, S. H., and Wise, S. A., eds., Nat. Bur. Stand. (U.S.) Spec. Publ. 656, p. 127 (1983).

Barnes, I. L., Gills, T. E., and Reed, W. P., "Copper Standard Reference Materials," ASTM Special Publication, "Copper Cathodes," in press.

Beary, E. S., Paulsen, P. J., and Lambert, G. M., "The Determination of Selenium and Tellurium in Copper Reference Materials Using Isotope Dilution Spark Source Mass Spectrometry," in press.

DeBievre, P., Gallet, M., Holden, N., and Barnes, I. L., "Isotope Abundances and Atomic Weights of the Elements," J. of Phys. and Chem. Ref. Data, in press.

Domalski, E. S., MacCrehan, W. A. Moody, J. R., Tewari, Y. B., and Walker, J. A., "Characterization of Mill Scale Wastes," Annual Report of Office of Recycled Materials, National Bureau of Standards, in press.

Downing, R. G., Fleming, R. F., Langland, J. K., and Vincent, D. H., "Neutron Depth Profiling at the National Bureau of Standards," Proceedings of the Sixth International Conference on Ion Beam Analysis, Tempe, AZ, May 23-27, 1983, Nucl. Instr. and Method., in press.

Epstein, M. S. and Blunde11, C. G., "Temperature Regulation System for a D.C. Plasma/Echelle Spectrometer," Anal. Chem., in press. 
Epstein, M. S. and Winefordner, J. D., "Summary of the Usefulness of Signalto-Noise Treatment in Analytical Spectrometry," Prog. Anal. Atom.

Spectrosc., in press.

Fassett, J. D. and Kelly, W. R., "Interlaboratory Analysis Program of Anion Exchange Resin Beams Loaded with Nanogram Amounts of Uranium and Plutonium Using Thermal Ionization Mass Spectrometry," in press.

Fassett, J. D., Moore, L. J., Shideler, R. W., and Travis, J. C., "Pulsed Thermal Ion Source for Use in Resonance Ionization Mass Spectrometry," in press.

Fassett, J. D., Moore, L. J., and Travis, J. C., "The Characterization of Thermally Produced Metastable Excited State Atomic Species Using Resonance Ionization Mass Spectrometry," Int. J. Mass Spectrom. Ion Phys., in press.

Fleming, R. F., Pei, P. and Hsu, S. M., "Test Methods for Total Chlorine in Lubricating Base 0ils," in press.

Gramlich, J. W. and Machlan, L. A., "Isotopic Variations in Commercial High Purity Gallium," in press.

Kelly, W. R. and Paulsen, P. J., "Precise Determination of Sulfur ai High Concentrations by Isotope Dilution Thermal Ionization Mass Spectrometry," in press.

Kingston, H. M., Cronin, D. J., and Epstein, M. S., "Investigation of a Precise Static Leach Test for the Testing of Simulated Nuclear Waste Materials," in press.

Kingston, H. M., Paulsen, P. J., and Lambert, G. M., "The Determination of Selenium and Tellurium in Stainless Steel, White Cast Iron, and Nickel Base Alloy Standard Reference Materials by Isotope Dilution Spark Source Mass Spectrometry," in press.

Ku, C. S., Wu, Y. C., and Hsu, S. M., "Oxidative Degradation Mechanism of Lubricants," in press.

Lucatorto, T. B., Clark, C. W., and Moore, L. J., "Ultrasensitive Mass Spectrometry Based on Two-Photon, Sub-Doppler Resonance Ionization," in press.

Moody, J. R., "Recent Trends in Laboratory Design in the USA," Proceedings of Lab Expo 82 (London), in press.

Moore, L. J., Kingston, H. M., Murphy, T. J., "The Use of Isotope Dilution Mass Spectrometry for the Certification of Standard Reference Materials," in press.

Paulsen, P. J. and Kelly, W. R., "Determination of Sulfur by Isotope Dilution Thermal Ionization Mass Spectrometry as AsS ${ }^{+}$Ions," in press. 
Powe11, L. J. and Murphy, T. J., "The Determination of Atomic Weights at NBS--The Case of Silver and Its Impact on the Faraday," in press.

Powe11, L. J. and Paulsen, P. J., "The Determination of Microgram Per Gram Concentrations of Hafnium in Zirconium Metal and Zircaloy 4 Metal Standard Reference Materials by Isotope Dilution Spark Source Mass Spectrometry," in press.

Winefordner, J. D. and Epstein, M. S., "Atomic Spectrometric Methods," Chapter in physical methods of Chemistry, B. W. Rossiter and J. F. Hamilton, eds., John Wiley \& Sons, New York, in press.

Wu, Y. C. and Hsu, S. M. "Lubricant Degradation Mechanism for Engine 0i1," NBS Special Publication for the Conference on Measurements and Standards for Recycle 0il-IV, in press.

Yaffe, Y., Flessel, C. P., Wesolowski, J. J., del Rosario, A., Guirguis, G. N., Martias, V., Gramlich, J. W., Kelly, W. R., DeGarmo, T. E., and Coleman, G. C., "Identification of Lead Sources in California Children Using the Stable Isotope Ratio Techniques," in press.

Zeisler, R., Harrison, S. H., and Wise, S. A., "Trace Elements in Human Livers Using Quality Control in the Complete Analytical Process," Biol. Trace Element Res., in press.

Zeisler, R., Langland, J. K., and Harrison, S. H., "Cryogenic Homogenization Procedure for Biological Tissues," Anal. Chem., in press.

Greenberg, R. R., Fleming, R. F., and Zeisler, R., "High Sensitivity Neutron Activation Analysis of Environmental and Biological Standard Reference Materials," submitted for publication.

Kingston, H. M. and Greenberg, R. R., "An Elemental Ratioing Technique for Assessing Concentration Data From a Complex Water System," submitted for publication.

Lutz, G. J., Rook, H. L., and Lindstrom, R. M., "Determination of I-129 at Natural Levels by Thermal Neutron Activation Analysis," submitted for publication.

Rains, T. C., "Atomic Absorption Spectrometry," Chapter in Treatise on Water Analysis, R. A. Minear, ed., Academic Press, submitted for publication.

Rains, T. C., Watters, R. L., Jr., and Epstein, M. S., "Application of Atomic Absorption and Plasma Emission Spectrometry for Environmental Analysis," submitted for publication.

Zhang, Y. K., "Determination of Germanium in Biological Fluids by Electrothermal Atomization Atomic Absorption Spectrometry," submitted for publication. 
2. Talks

Moody, J. R., "Sampling for Trace Analysis," Society for Applied Spectroscopy Continuing Education Course, Beltsville, MD, October 4, 1982. Invited

Epstein, M. S., "Atomic Absorption Spectrometry," 2nd NBS-ADABSE Analytical Chemistry Workshop, National Bureau of Standards, Gaithersburg, MD, October 24, 1982. Invited

Watters, R. L., Jr., "Atomic Emission Spectrometry Using the Inductively Coupled Plasma (ICP) Technique," NBS-ADABSE Analytical Chemistry Workshop, National Bureau of Standards, Gaithersburg, MD, October 24, 1982. Invited

Rains, T. C., "State of the Art of Atomic Absorption Spectrometry," NBS-ADABSE Analytical Chemistry Workshop, National Bureau of Standards, Gaithersburg, MD, October 26, 1982 . Invited

Zeisler, R., "Trace Elements in Human Livers Using Quality Control in the Complete Analytical Process," lst Annual Scientific Meeting of the Society for Environmental Geochemistry and Health, Greenville, NC, November 2, 1982.

Dowing, R. G., "Thermal Neutron Depth Profiling in Boron and Silicon," 7 th International Conference on the Application of Accelerators in Research and Industry, Denton, TX, November 8, 1982.

Greenberg, R. R., "Radiochemical Procedures Used at NBS for High-Sensitivity Certification Analysis of Standard Reference Materials," American Nuclear Society Meeting, Washington, DC, November 16, 1982. Invited

Moody, J. R., "Special Facilities for Trace Metal Analysis at NBS," University of Illinois (Champaign), Chemistry Colloquium, November 16, 1982. Invited

Rains, T. C., "State of the Art of Sample Preparation for Inductively Coupled Plasma," 21st Eastern Analytical Symposium, New York, NY, November 18, 1982. Invited

Watters, R. L., Jr., "Random and Non-Random Variability in ICP Calibration Functions," 21 st Eastern Analytical Symposium, New York, NY, November 18, 1982 . Invited

Moody, J. R., "Techniques for Trace Metal Analysis at NBS," U.S.G.S. National Water Quality Control Laboratory Colloquium, Arvada, C0, November 19, 1982. Invited

Rains, T. C., "Using Matrix Modification to Alleviate Interferences in Graphite Furnace Atomic Absorption Spectroscopy, "21st Eastern Analytical

Symposium, New York, NY, November 19, 1982.

Fleming, R. F., "Neutron Depth Profiling of Elemental Concentrations," Be11 Laboratories, Murray Hi11, NJ, December 1, 1982. 
Fassett, J. D., "Resonance Ionization Mass Spectrometry," Division Seminar, National Bureau of Standards, Gaithersburg, MD, December 1, 1982.

Rains, T. C., "New Innovations in Electrothermal Atomization/Atomic Absorption Spectrometry," Milwaukee Section of Society for Applied Spectroscopy, Milwaukee, WI, February 15, 1983. Invited

Barnes, I. L., "Focussing Properties of Stray Magnetic Fields," Institute of Physics, University of Mexico, March 2, 1983. Invited

Rains, T. C., "Atomic Absorption and Emission Spectrometry," ACS Short Course, Pittsburgh Conference, Atlantic City, NJ, March 5, 1983.

Barnes, I. L., "Isotope Dilution Mass Spectrometry and Its Use in Chemical Analysis," Graduate School of Chemistry, University of Mexico, March 9, 1983. Invited

Rains, T. C., "The Art of Sample Preparation for Atomic Absorption Spectrometry," Assay and Weights Administration, Gamalia, Cairo, March 20, 1983. Invited

Rains, T. C., "Instrumental Method of Analysis," Assay and Weights Administration, Gamalia, Cairo, March 21, 1983. Invited

Rains, T. C., "Determination of Trace Elements in Water and Air Particulate by AAS," Assay and Weights Administration, Gamalia, Cairo, March 23, 1983. Invited

Rains, T. C., "Interferences in Atomic Absorption Spectrometry," Assay and Weights Administration, Gamalia, Cairo, March 27, 1983. Invited

Gramlich, J. W., "The Application of Thermal Ionization Isotope Dilution Mass Spectrometry to the Certification of NBS Standard Reference Materials," Department of Chemistry, Arizona State University, Tempe, AZ, March 29, 1983. Invited

Rains, T. C., "State of the Art of AAS," Egyptian Organization for Standardization," Gamalia, Cairo, March 29, 1983. Invited

Rains, T. C., "Characterization of Standard Reference Materials by AAS," National Research Center, Dokki, Cairo, March 30, 1983. Invited

Rains, T. C., "Determination of Major, Minor, and Trace Elements in Ferrous and Non-ferrous Metals, Ores, and Ceramics by AAS," Assay and Weights Administration, Gamalia, Cairo, March 31, 1983. Invited

Vincent, D. H., "Neutron Depth Profiling at the National Bureau of Standards," New York Area Regional Meeting on Ion Beam Analysis, Brooklyn, NY, Apri1 6, 1983.

$\mathrm{Ku}, \mathrm{C} . \mathrm{S}$. , Wu, Y. C., and Hsu, S. M., "Oxidative Degradation Mechanism of Lubricants," Am. Soc. Lubricant Engineers Annual Meeting, Houston, TX, April 25, 1983. 
Fleming, R. F., "Advantages of Neutron Activation Analysis with Special Emphasis on Semiconductor Technology," Ford Motor Company, Dearborn, MI, April 27, 1983.

Fleming, R. F., "Analytical Chemistry Applications of Neutrons," Great Lakes Chapter of the Health Physics Society, Dearborn, MI, April 28, 1983. Invited

Rains, T. C., "Trace Elemental Analysis of Environmental Materials," Florida Society of Environmental Analysis, Tampa, FL, April 29, 1983. Invited

Kelly, W. R., "The Use of Stable Traces in Source Apportionment. Discussions on the Deep Creek Lake Source--Receptor Study," National Bureau of Standards, Gaithersburg, MD, May 3, 1983. Invited

Fleming, R. F., "Elemental Depth Profiling Using Thermal Neutrons," Eastman Kodak Company, Rochester, NY, May 9, 1983.

Brletic, K. A., Powell, L. J., and Garner, E. L., "A High Sensitivity Procedure for Chromium Using Silica-Gel Boric Acid Ionization Enhancement," 31st Annual Conference on Mass Spectrometry and Allied Topics, Boston, MA, May 12, 1983.

Paulsen, P. J., Beary, E. S., and Lambert, G. M., "The Determination of Selenium and Tellurium in NBS Copper Base SRM's by SSMS Isotope Dilution," 31 st Annual Conference of Mass Spectrometry and Allied Topics, Boston, MA, May 12, 1983.

Fassett, J. D., Moore, L. J., and Travis, J. C., "RIMS--Resonance Ionization Mass Spectrometry of 18 Elements From Thermal Vapor Atom Reservoirs," 31 st Annual Conference of Mass Spectrometry and A1lied Topics, Boston, MA, May 13, 1983.

Fassett, J. D., Moore, L. J., and Travis, J. C., "A Laser Mass Spectrometry Laboratory for Stable Isotope Measurements and Vaporization Diagnostics," 31 st Annual Conference of Mass Spectrometry and Allied Topics, Boston, MA, May 13, 1983.

Downing, R. G., "Neutron Depth Profiling and Semiconductor Analysis," Motorola Surface Research and Development Laboratory, Pheonix, AZ, May 20, 1983.

Kelly, W. R., "The Measurement of Sulfur Isotopic Ratios by Thermal Ionization Mass Spectrometry," Carnegie Institution of Washington, Washington, DC, May 25, $1983 . \quad$ Invited

Barnes, I. L., "Ultimate Mass Dispersion and Resolution in Magnetic Sector Machines," Symposium on New Techniques and Potential Applications of Ultrasensitive Mass Spectrometry, National Bureau of Standards, Gaithersburg, MD, May 31, 1983. Invited 
Fassett, J. D., "Recent Developments in RIMS," Symposium on New Techniques and Potential Applications of Ultrasensitive Mass Spectrometry, National Bureau of Standards, Gaithersburg, MD, May 31, 1983.

Kelly, W. R., "Cosmogenic and Geophysical Measurements," Symposium on New Techniques and Potential Applications of Ultrasensitive Mass Spectrometry, National Bureau of Standards, Gaithersburg, MD, May 31, 1983. Invited

Moore, L. J., "Resonance Ionization Mass Spectrometry--Potential Applications," Symposium on New Techniques and Potential Applications of Ultrasensitive Mass Spectrometry, National Bureau of Standards, Gaithersburg, MD, May 31, 1983. Invited

Zeisler, R., "The Table Top Accelerator as an Analytical Tool," Symposium on New Techniques and Potential Applications of Ultrasensitive Mass Spectrometry, National Bureau of Standards, Gaithersburg, MD, May 31 , 1983.

Fleming, R. F., Vincent, D. H., and Downing, R. G., "Neutron Depth Profiling for Materials Characterization," 5 th Symposium on Applied Surface Analysis, Dayton, $\mathrm{OH}$, June 10, 1983.

Greenberg, R. R., "Neutron Activation Analysis Techniques Used for U1tra-trace Level Elemental Characterization of New NBS Milk Powder Standard Reference Material," American Nuclear Society Meeting, Detroit, MI, June 15, 1983. Invited

Lutz, G. J., "The Determination of Iodine in Biological Standard Reference Materials by Instrumental Photon and Epithermal Neutron Activation Analysis," American Nuclear Society Meeting, Detroit, MI, June 15, 1983. Invited

Fleming, R. F., "Applications of Neutron Depth Profiling," National Bureau of Standards, Gaithersburg, MD, June 15, 1983.

Koch, W. F. and Marinenko, G., "Atmospheric Deposition Reference Materials: Measurement of $\mathrm{pH}$ and Acidity," 76th Annual Meeting of the Air Pollution Control Association, Atlanta, GA, June 22, 1983.

Rains, T. C., Rush, T. A., and Butler, T. A., "Trace Element Characterization of Urine for a Standard Reference Material by Atomic Absorption and Emission Spectrometry," 23rd Colloquium Spectroscopicum International Amsterdam, The Netherlands, June 27, 1983.

Rains, T. C., "Trace Elemental Analys is of Water by AAS," EPA, Annapolis, MD, July 21, 1983. Invited

Moody, J. R., Machlan, L. A., and Gramlich, J. W., "Characterization of Uranium Absolute Isotopic Standard Reference Materials," 53rd Society of Analytical Chemistry Meeting, Edinburgh, Scotland, July 22, 1983. 
Norris, J. A., "Qualitative and Semi-quantitative Analysis, Data Handling, and Statistics, Direct Reading Spectrochemical Analysis and Laboratory Instruction in Photographic Emission Spectroscopy," 28th Annual Course on Modern Industrial Spectroscopy, Arizona State University, Tempe, AZ, August 1, 1983.

Moore, L. J., Fassett, J. D., Travis, J. C., Lucatorto, T. B., and Clark, C. W., "Isotopic Selectivity in Ultrasensitive RIMS," Symposium on Laser-Based U1trasensitive Spectroscopy, SPIE Conference, San Diego, CA, August 22, 1983. Invited

Moore, L. J., Fassett, J. D., Travis, J. C., and Lucatorto, T. B., "Resonance Ionization Mass Spectrometry," Lawrence Livermore National Laboratory, Nuclear Chemistry Division, Livermore, CA, August 25, 1983. Invited

Rains, T. C., "Atomic Absorption and Plasma Emission Spectrometry," American Chemical Society National Meeting, Washington, DC, August 27-28, 1983.

Beary, E. S. and Moody, J. R., "Laboratory Design for the Safe Distillation of Acids," American Chemical Society, National Meeting, Washington, DC, August 29, 1983. Invited

Koch, W. F., "Acid Precipitation Standards: Measurement of pH," American Chemical Society National Meeting, Washington, DC, August 29, 1983.

Moore, L. J., "Stable Isotope Measurements with Thermal and Resonance Ionization Mass Spectrometry," Symposium on Stable Isotopes in Nutrition, American Chemical Society National Meeting, Washington, DC, August 29, 1983. Invited

Moody, J. R., "Installation and Proper Use of Fume Hoods for Perchloric Acid in Analytical Laboratories," American Chemical Society National Meeting, Washington, DC, August 29, 1983. Invited

Stone, S. F., "A Protocol for the Multielement Analysis of Banked Marine Bivalues," American Chemical Society National Meeting, Washington, DC, August $30,1983$.

Fleming, R. F., Downing, R. G., Lindstrom, R. M., Riley, J. E., Jr., and Vincent, D. H., "Materials Analysis Using Thermal Neutron Reactions: Methods," American Chemical Society National Meeting, Washington, DC, August $31,1983$. Invited

Riley, J. E., Jr., Downing, R. G., Fleming, R. F., Lindstrom, R. M., and Vincent, D. H., "Materials Analys is Using Neutron Reactions: Applications," American Chemical Society National Meeting, Washington, DC, September 1, 1983. Invited

Zeisler, R., "Quality Assurance and Standardization in Sampling and Sample Preparation of Biological Materials," 10th Annual Meeting of the Federation for Analytical Chemistry and Spectroscopy Societies, Philadelphia, PA, September 26, 1983. Invited 
Epstein, M. S., "Laser-excited Atomic Fluorescence Spectroscopy in Florida," 10th Annual Meeting of the Federation for Analytical Chemistry and Spectroscopy Societies, Philadelphia, PA, September 27, 1983. Invited

Rains, T. C., "Sample Preparation Workshop," 10th Annual Meeting of the Federation for Analytical Chemistry and Spectroscopy Societies, Philadelphia, PA, September 27, 1983.

\section{Committee Assignments}

I. Lynus Barnes

Member, Department of Health, Education, and Welfare, The Chemistry Task Force of the National Shellfish Sanitation Program Washington Editorial Review Board International Union of Pure and Applied Chemistry, Commission of Atomic Weights and Isotopic Abundances

Secretary, IUPAC, Commission on Atomic Weights and Isotopic Abundances, Subcommittee for Assessment of Isotopic Abundances

Robert W. Burke

Member, ASTM D-34, Waste Disposal

ASTM E-03, Chemical Analysis of Metals

Collator, Transmittance and Wavelength Section, IUPAC Commission

I.4, Physicochemical Measurements and Standards

Barry I. Diamonds tone

Member, ASTM D-34, Waste Disposal

ASTM D-34.02.01, Section on Batch Extractions

ASTM E-03, Chemical Analysis of Metals

ASTM E-03.01, Gases in Metals

ASTM E-03.93, Long Range Planning

Michael S. Epstein

Member, ASTM D-19, Water

Editorial Advisory Board, Progress in Analytical Atomic Spectroscopy

Chairman, Baltimore-Washington Section of the Society for Applied Spectroscopy

Conference Committee, Society for Applied Spectroscopy

Delegate, FACSS Governing Board for the Division of Analytical Chemistry of the American Chemical Society

Ronald F. Fleming

Member, Committee on Accelerator-Based Neutron Research at NBS ASTM E-10.05, Nuclear Radiation Metrology

John W. Gramlich

Member, Institute of Nuclear Materials Management Committee N15.9.3, Non-Destructive Assay (Physical Methods) 
Robert R. Greenberg

Member, NBS Chemistry Storeroom Committee

Trace Analysis Committee, Biomedical Division, American Nuclear Society

Howard M. Kingston

Member, ASTM C-26.05, Methods of Test

ASTM C-26.07, Waste Materials

Task Group Leader, C-26.05, Leachate Analysis Methods

William F. Koch

Member, ASTM D-19, Water

ASTM D-19,02, General Specifications and Technical Resources

ASTM D-19.05, Inorganic Constituents in Water

ASTM D-22, Air

Chairman, Teller's Committee, Chemical Society of Washington

John K. Langland

Member, NBS Electronics Storeroom Committee

Richard M. Lindstrom

Alternate Member, NBSR Hazard Evaluation Committee

Lawrence A. Machlan

Member, ASTM C-26, Nuclear Fuel Cycles ASTMC-26.05, Test Methods

Secretary, ASTM C-26.01, Editorial and Terminology

George Marinenko

Member, ASTM D-19, Water

ASTM D-19.09, Saline and Brackish Waters

ASTM D-19.05, Inorganic Constituents in Water

ASTM D-19.05.03.06, Ozone

Registration Committee of National Meeting of the Electrochemical Society

Chairman, ASTM D-19.05.04.03, Voltammetry

ASTM D-19.09.01.14, Chlorine in Saline Water

ASTM D-19.09.06, Redox Potentials

ASTM D-19.09.07, pH in Saline Water

National Capital Section of the Electrochemical Society

Blum Award Arrangements, Committee of the Electrochemical

Society

Councilor, National Capital Section of the Electrochemical Society

John R. Moody

Member, ASTM D-19, Water

ASTM D-19.05, Inorganic Constituents in Water

Audit Committee, Chemical Society of Washington

Contamination Control Standards and Practices for Federal

State 209c Institute for Environmental Sciences 
Thomas J. Murphy

Member, American Chemical Society Committee on Analytical Reagents American Chemical Society Committee on Membership Affairs International Union of Pure and Applied Chemistry (IUPAC), Commission on Atomic Weights and Isotopic Abundances International Union of Pure and Applied Chemistry (IUPAC), Subcommittee for the Assessment of Isotopic Abundances, Commission on Atomic Weights and Isotopic Abundances

Chairman, American Chemical Society Admissions Committee Audit Committee, Chemical Society of Washington

Councilor, Chemical Society of Washington

John A. Norris

Member, ASTM E-02.01, Fundamental Practices

ASTM E-02.02, Statistics

ASTM E-02.04, Samping and Standards

ASTM E-02.06, Lead, Tin, Zinc

ASTM E-02.07, Aluminum

ASTM E-02.08, Refractory Metals

ASTM E-02.09, Ferrous

ASTM E-02.10, Miscellaneous Non-Metallic Materials

Representative, SAS to Joint Committee on Atomic and Molecular Physical Data

Secretary, ASTM E-02, Emission Spectroscopy

Lecturer, Annual Short Course, "Modern Industrial Spectroscopy," Arizona State University, Tempe, AZ

Lura J. Powe 11

Member, Audit Committee, Chemical Society of Washington

Board of Directors, NBS Child Care Association

Chairman, Committee on Isotope Ratio Measurements, American Society for Mass Spectrometry

Treasurer, Chemical Society of Washington

President, Standards Committee for Women

Theodore C. Rains

Member, Proposal Evaluation Committee for National Science Foundation AOAC Committee on Automated Methods

Editorial Board of Annual Reports on Analytical Atomic Spectroscopy (The Chemical Society of London) and the Canadian Journal of Spectroscopy

Subcommittee No. 6 on Methods of Air Sampling and Analysis

ASTM E-02.10, Non-Metallic Materials

ASTM E-02.13, Nomenclature

ASTM D-01, Paint and Related Coatings

ASTM D-19, Water

Chairman, FACSS Governing Board

Past-President, Society for Applied Spectroscopy

Professor-in-charge, ACS Short Course on "Atomic Absorption Spectroscopy"

Task Force Leader, Scientific Instrumentation Information Network and Curricula (SIING) for project on "Atomic Absorption Spectroscopy" Column Editor, Journal of Applied Spectroscopy 
Ronald W. Shideler

Member, NBS Electronics Storeroom Committee

Yung-Chi Wu

Member, ANSI 2228 Incineration

Council Member, ASME Codes and Standards

Rolf L. Zeisler

Member, IAEA Advisory Group on Nuclear Techniques in Environmental Health Studies of Mineral Pollutants

Technical Program Committee International Symposium on the Use and Development of Low and Medium Flux Research Reactors NBSR Hazard Evaluation Committee

Chairman, Trace Element Analysis Committee, Biomedical Division, American Nuclear Society

4. Other

a. Seminars

October 14, 1982 - Professor William H. Zoller, Analytical Nuclear and Environmental Chemistry Division, Department of Chemistry, University of Maryland, "The Chemistry of Remote Area Aerosols."

December 16, 1982 - Dr. John A. Miller, Air Resources Laboratory, NOAA, "Acid Precipitation."

January 27, 1983 - Dr. R. Jack Pickering, United States Geological Survey, "Atmospheric Deposition Monitoring Under the National Acid Precipitation Assessment Plan (NAPAP)."

February 2, 1983 - Mr. Walter Slavin, Applied Research Department, Perkin-Elmer Corporation, "Practical Experiences with the Modern Graphite Furnace."

April 6, $1983 \quad$ - Mr. William P. Reed, Production and Certification Programs, Office of Standard Reference Materials, National Bureau of Standards, Washington, DC, "Research and Measurement Needs for the Certification of Standard Reference Materials, Plans, and Perspectives for FY 84."

May 25, 1983 - Dr. Walter Mertz, Beltsville Human Nutrition Research Center, U.S. Department of Agriculture, Beltsville, Maryland, "Health Implications of Modern Nutrition Research--Relation to Progress in Analysis." 
b. Conferences Sponsored

September 19-20, $1983 \quad-\quad$ R. Zeisler, Eighth U.S./German Seminar of State and Planning on Environmental Specimen Banking, National Bureau of Standards, Gaithersburg, MD.

September 21, $1983 \quad-\quad$ R. Zeisler, International Review of Environmental Specimen Banking, National Bureau of Standards, Gaithersburg, MD.

c. Seminars Organized

W. F. Koch, Workshop on Direct Potentiometric Measurements in Blood, National Bureau of Standards, Gaithersburg, MD, May 18-20, 1983. 


\title{
III. Organic Analytical Research Division
}

\author{
Willie E. May, Acting Chief \\ Dennis J. Reeder, Deputy Chief
}

\section{A. Division Overview}

The Organic Analytical Research Division conducts research to develop and improve techniques for the preparation, purification, characterization, and analysis of organic compounds and uses these techniques in the solution of analytical problems related to national needs. The Division's research is carried out by five groups organized along functional lines: Bioanalytical Techniques, Electrochemistry, Gas Chromatography, Liquid Chromatography, and Mass Spectrometry. During the past year, the Division has been actively engaged in the application of organic analytical chemistry to measurement problems related to energy, the environment, health and clinical chemistry, and food and nutrition. Results of Division research are utilized by industry, other Federal agencies, public and private institutions, and research programs at the National Bureau of Standards. Research efforts during the past year have resulted in over 50 articles being published or in press in archival journals.

As in past years, our research efforts have been in support of basic organic analytical chemistry, in support of Other Agency requests, and in short-term projects performed on a service analysis basis. This year, substantial progress has been made in research on basic techniques in organic analytical chemistry and in furthering our understanding of measurement principles in this area. The Division is now actively involved in three competence building programs selected for funding by the NBS Director: Organic Electrochemistry, Analys is of Ionization Mechanisms and Ion Structures in Organic Mass Spectrometry, and Bioanalytical Techniques--The Use of Proteins in Analytical Chemistry. In addition, we have continued our research activities in liquid chromatographic retention mechanisms, funded out of our Division's STRS base. Details concerning these activities are provided in the group overviews and technical reports that follow.

During the past year, we have performed service analyses in support of several NBS and other government agency programs. Some examples follow. We completed our first set of organic analyses, organochlorine pesticides in $\mathbf{3 0}$ human liver samples, in support of the Environmental Specimen Bank program. In addition, we analyzed over 28 oil samples for PCB content in support of the NBS Recycled Materials program. We also assisted the Quality Assurance Branch of EPA/Cincinnati by analyzing their performance evaluation and quality control standards, prepared by an outside contractor, to establish traceability to NBS. Details concerning this activity are given in technical report 9.

Collaborative research efforts have begun with the two other divisions in the Center for Analytical Chemistry. Research in conjunction with the Gas and Particulate Science Division is providing a foundation for the development of an isotope dilution gas chromatography/mass spectrometry procedure for analys is of low ppb levels of toxic organics in air. Joint studies have been initiated with the Inorganic Analytical Research Division for neutron activation analyses of electrophoretic gels to provide information on the distribution of silver in gels and give insight into the mechanism of silver staining. 
The major portion of our efforts in support of the NBS Standard Reference Materials (SRM) program was focused on the development of synthetic calibration mixtures to complement existing matrixed SRM's. We also supplied information values for a number of SRM's, certified in previous years, in an attempt to increase their utility. Details concerning these and other SRM activities are given in technical reports $2,4,5,6,10$, and 11 and in the group overviews. Our projected SRM research activities for the coming year include the beginning of a staged and comprehensive program for the development of SRM's for dioxins and dibenzofurans.

The toxic chemicals handling laboratory, completed during FY 1982, has become fully operational--a protocol for its use has been written and put into use. A new gas chromatography laboratory was completed in February. We are currently completing the design of a bioanalytical facility and expect to begin construction early in FY 1984.

Our programs in liquid chromatographic retention mechanisms and mass spectrometric ionization mechanisms were bolstered by NRC postdoctoral fellows in the respective areas. Each will remain with us for an additional year. We also plan to actively pursue postdoctoral fellows for the gas chromatography, organic electrochemistry, and bioanalytical areas during the coming year. We are continuing our collaborative efforts with the College of American Pathologists by the addition of a new research associate, Dr. Kwokei $\mathrm{Ng}$, to replace Dr. Michael Welch, who has joined our full-time staff. Our full-time hiring priorities include a senior level liquid chromatographer and a biochemist with experience in enzymes and enzyme immobilization.

In keeping with research planning which we have been actively doing for the past several years, we anticipate pursuing some very exciting research areas during the coming year. We will continue to work on high-priority analytes, such as creatinine, for clinical chemistry; to extend the usage and capabilities of the NMR facility; and to further improve our protein separation capabilities while developing standard marker proteins for two-dimensional electrophoresis. We also plan to expand our efforts in interfacing liquid chromatography and/or metal-ion affinity chromatography with electrophoretic methods. We will continue to apply the use of electrochemical detection combined with liquid chromatographic separations to the analysis of trace organic and organometallic species. The spectroelectrochemical study of metal croconates will be completed in FY 1984 and the analytical applications of chemically modified electrodes will be pursued. Our efforts in liquid chromatography/mass spectrometry will continue, as will our studies of the photodissociation of ions and structures of ions and molecules. Our studies in liquid chromatographic retention mechanisms will continue and a program in supercritical fluid chromatography will be initiated.

Finally, as mentioned in last year's report, we have begun to focus a portion of our research efforts in the areas of nutrient and hazardous waste analyses. Our research in both areas is beginning to bear fruit and these new thrusts will continue. Our research in the nutritional area will be expanded to include serum as well as food analysis. National concern regarding the presence of dioxins and dibenzofurans in the environment is demanding a stronger effort in that area. 
1. Bioanalytical Techniques Group: Overview

The Bioanalytical Techniques Group has continued its programs in the competence area by further research on protein separations using electrophoresis. The Spectroscopy Group was merged into the Bioanalytical Techniques Group in November, with one of the members devoting time to investigation of protein staining mechanisms. Additionally, the continued work in the clinical chemistry area has resulted in the development of isotope dilution/mass spectrometric (ID/MS) methods for creatinine and urea.

Electrophoretic methods for separation and detection of proteins were implemented and improved. The "rocket" technique using antiserum in an agarose matrix was shown to be useful for quantifying human albumin. This method was used to determine the albumin content of SRM 909. Further work using this technique is planned.

Thin-layer isoelectric focusing (TL-IEF) shows promise as a rapid method for screening proteins. This technique makes use of high-field gradients $(>400 \mathrm{volts} / \mathrm{cm})$ to produce rapid focusing in thin $(125 \mu \mathrm{m})$ gels. To dissipate the Joule heat thus formed with up to $20 \mathrm{~W}$ of power, an efficient thermoelectric device cools the plates to constant temperature. Silver staining of the focused proteins gives results within one hour after the electrophoretic run of 40 minutes. Up to 60 bands of focused proteins have been observed in serum samples. The extraordinary sensitivity of this method is evidenced by the small amount of protein required. Typically, $1 \mu \mathrm{L}$ of a $1: 20$ dilution of serum is used for separations. This means that we are detecting approximately 5-50 $\mathrm{ng}$ of protein per band.

The two-dimensional electrophoresis (2-DE) work moved forward with the acquisition of equipment capable of running ten large gels at one time. The set-up and implementation of the larger capacity was greatly helped by the talents of Professor Frederic Giere, a summer faculty hire from Lake Forest College, Lake Forest, IL. Professor Giere has had many years experience in teaching workshops on 2-DE. The system was used to examine fish serum, bacterial membrane proteins, and enzymes as marker proteins. Preliminary studies showed great heterogeneity among commercially available molecular weight marker proteins.

The studies that began last year to assess the effect of composition of sodium dodecyl sulfate (SDS) preparations on 2-DE separations were extended and completed (see Technical Report 12). The results of that work were presented at an invited session of the annual meeting of the Electrophoresis Society.

Several publications are being drafted to report the results of the protein separations stemming from our collaborative work with the Sandy Hook Marine Laboratory.

We initiated joint studies with Rolf Ziesler of the Inorganic Analytical Research Division for neutron activation analysis of electrophoretic gels to provide information on the distribution of silver in the gels and give insight into the mechanism of silver staining. A major effort leading to this work 
was the compilation and comparison of silver staining methods from available literature.

Work in the spectroscopy area resulted in five publications and several major developments. A new pulse sequence has been developed for doing twodimensional, heteronuclear J-resolved ${ }^{13} \mathrm{C}$ NMR, with polarization transfer by the DEPT technique. The sequence has been applied to analysis of the anomeric and ring form equilibria of digitoxose. Spectrum editing of the ${ }^{15} \mathrm{~N}$ NMR spectra of aminoglycoside antibiotics has been implemented by the DEPT technique. In addition, two-dimensional, proton-proton shift correlated NMR (COSY) has been applied to the almost complete assignment of the proton NMR spectra of the angiotensin SRM and also the different forms of digitoxicose. Further, NMR methods have been developed for the analysis of water in SRM urea and of lactose in SRM 1549, Non-Fat Dry Milk. Certification of SRM 1595, Tripalmitin, has been completed. Recertification of SRM dextrose is underway.

Continuing activities in the clinical area have focused on improvements to the wet-chemical steps, including spiking, analyte isolation, and derivative formation, in order to minimize errors in the ID/MS definitive methods. A manuscript describing our serum urea ID/MS definitive method is being reviewed within NBS prior to submission to Analytical Chemistry for publication. Creatinine doubly labeled with carbon-13 was synthesized for the ID/MS method we are developing for creatinine in serum. We had been using creatinine- $d_{3}$ with the deuterium atoms in nonreactive positions in the molecule, but the precision was much less than satisfactory and there was evidence of bias between independently prepared sets of standards. With the creatinine- ${ }^{13} \mathrm{C}_{2}$, the precision was very much better, and no bias was observed between sets of standards.

We began planning, design, and initial work on a triple-module modernized laboratory for biotechnology. This laboratory will have a ten-foot hood for handling many of the volatile solutions used in electrophoresis. In addition, an adjoining section will house many of the instruments that are now scattered in various laboratories. The laboratory will provide working space for approximately six chemists and is expected to improve efficiency in operations.

During FY 1984, we will continue to improve our separation capabilities, while developing protein marker standards. The hire of a biochemist with experience in enzymes and enzyme immobilization is expected to add considerably to the expertise of the group. We are actively recruiting during this time. We plan to expand our efforts in interfacing techniques, such as liquid chromatography, metal-ion affinity chromatography, and other separations methods with the analytical tools of electrophoresis.

A workshop on 2-D electrophores is standardization is planned for FY 1984 with preliminary planning underway. Contacts for plenary lectures have been made.

Dennis J. Reeder, Group Leader; Lyle A. Alexander, Alex Cohen, Bruce Coxon, Andrew E. Davidson, Alexander J. Fatiadi, Frederic A. Giere, Diane K. Hancock, Cindy L. Hsu, Maria L. Luzarraga, Millard Maienthal, Robert Schaffer 


\section{Electrochemistry Group: Overview}

The competence building program in organic electrochemistry has continued to make good progress toward its research goals this year, and we have been able to apply the results of some of our research to NBS programmatic areas. During the fifth year of this effort, our staffing has remained stable and most of the major pieces of equipment are now operational. Some needs still exist for instrumentation, especially in the area of automation.

Our research continued to be focused on the study of organic and organometallic redox reactions using state-of-the-art electrochemical techniques: cyclic voltammetry, chronoamperometry, chronocoulometry, and rotating ringdisk electrochemistry; hybrid techniques, such as spectroelectrochemistry and liquid chromatography with electrochemical detection; the preparation and evaluation of chemically modified electrodes; and mathematical modeling of electrochemical processes in support of the group research activities.

In one study, the electrochemical behavior of the laser dye, 4-[2-(5phenyloxazoly1)]-1-methylpyridinium tosylate was examined. In aqueous solution, the one-electron reduction of this cation is characterized by very strong product deposition as evidenced by a pronounced stripping wave on the oxidative scan. An extensive spectroelectrochemical study using gold-minigrid optically transparent thin-layer electrodes is nearly complete, with results showing characteristic and diagnostic behavior associated with a product-deposition process. In double potential step chronocoulometry, unusual charge-time features are also observed related to the product deposition. This behavior has been mathematically modeled on the assumption of phase equilibrium between the deposited product and the solution adjacent to the electrode. Analytical equations were developed for the charge-time plot in chronocoulometry and the Nernst plot in spectroelectrochemistry. These equations not only describe the experimental behavior but also provide the means to obtain kinetic and thermodynamic information, such as the diffusion coefficient, formal potential, electrochemical stoichiometry, and solubility of the reduced form of the dye, from the experimental data.

Cyclic voltammetry, chronocoulometry, and spectroelectrochemistry are also being used to study the electrode reactions of copper(II) complexes of dithiosquarate, dithiocroconate, and dicyanomethylenedithiocroconate in nonaqueous media. The formal redox potentials and the number of electrons involved in the redox processes are being evaluated spectroelectrochemically. From the electrochemical data, we are attempting to determine the effects of the ligands on the copper(II) reduction process.

The development of selective analytical sensors and catalytic surfaces for electrosynthesis may be achieved by the chemical modification of electrodes. Furthermore, modification by means of polymer films offers the advantages of inherent chemical and physical stability, incorporation of large numbers of catalytic sites, and relatively facile electron transport across the film. As reported last year, we have followed two approaches in the fabrication of polymer-film electrodes: electrochemically initiated polymerization and spindip coating. In both cases, two types of polymers have been studied, those which covalently bind the electroactive sites and ion-exchange polymers which electrostatically sequester the electroactive sites. In addition, we have investigated the electrochemical behavior of doped and undoped polymer films 
of polyacetylene and polypyrrole which are electronic conductors, in contrast to previously studied polymer films which were ionic conductors with site-tosite charge transfer.

Our investigation of the behavior of charged and uncharged film penetrants has been continued. The overall catalytic efficiency of these films will depend on the mobility of the substrate molecules across the film. With the completion of our computer-controlled system for the acquisition of currenttime or charge-time transient responses, this area of investigation is now proceeding at a much faster and more efficient rate. In-depth studies have been performed on $\left[R u(I I)(b p y)_{2}(4-V P)_{2}\right] \cdot 2 P F_{6}$ and $\left[R u(I I)(b p y)_{2}(4-V P)_{2}\right] \cdot 2($ styrene sulfonate) polymer films, where the two films differ as to the degree of cross-linking. Polymeric ion-exchanger films of polyethylene methacrylate and Nafion have been derivatized with cationic molecules such as methyl viologen, organic laser dyes, and transition metal complexes. These derivatized films have been evaluated using cyclic voltammetry and charge-time transient studies. Our investigation of the mass transport processes within these polymeric films is critical for the fundamental understanding of the electronic and structural changes occurring during the electrochemical conversion.

In conjunction with the Liquid Chromatography Group, an analytical method has been developed for the determination of phenol and ortho-cresol in crude oil based on the extraction of the diluted oil with aqueous base followed by neutralization of the phenolate anions. The final determination was made using reversed-phase liquid chromatography coupled to oxidative detection at a glassy carbon electrode.

Work is continuing on several aspects of the chemistry, electrochemistry, and analytical determination of nitro-polynuclear aromatic hydrocarbons ( $\mathrm{N}-\mathrm{PAH}$ ). We are collaborating with Alexander Fatiadi of the Bioanalytical Techniques Group by evaluating the products of nitration reaction mixtures and various purification schemes. The goal of this project is to provide suitable standards for the analytical and electrochemical studies of $\mathrm{N}-\mathrm{PAH}$. We are also studying the photodegradation products of $\mathrm{N}-\mathrm{PAH}$ in solution. The electrochemical reduction reactions of these compounds are being investigated in aqueous and nonaqueous media using normal, reverse, and differential pulse polarography and cyclic voltammetry. Coulometry is also being used in conjunction with liquid chromatography to study the complex, multistep reduction of N-PAH. Collaboration with the Liquid Chromatography Group has resulted in the development of two approaches to the determination of N-PAH in diesel exhaust particulate extracts. These liquid chromatographic methods will be used along with gas chromatography/mass spectrometry for the certification of several $\mathrm{N}-\mathrm{PAH}$ in SRM 1650, Diesel Exhaust Particulates.

Our plans for the coming year call for continued research on several of the above projects with special emphasis in the area of chemical modification of electrodes as selective organic sensors. We expect to continue to adapt our research efforts to address the analytical needs of the Center.

Richard A. Durst, Group Leader; Elmo A. Blubaugh, Richard T. Burke, Belle Liang, William A. MacCrehan, Gwendolyn S. Marbury, Scott R. Mund, William T. Yap 


\section{Gas Chromatography Group: Overview}

Three areas of analytical research have been explored by the Gas Chromatography Group during the past year. Our program in gas chromatography combined Fourier transform infrared spectroscopy (GC/FTIR) was continued. A new laboratory was designed and constructed to house the GC/FTIR and the instrument was installed and optimized therein. A number of inlet configurations and columns were utilized in an attempt to increase the apparent sensitivity and resolution of the system; however, GC/FTIR performance is still below that which is needed to obtain useful spectroscopic identifications of components in complex environmental samples. We hope to be able to attract a postdoctoral fellow or similar person to work in this area, as a complete redesign of the GC interface will probably be required if high quality data is to be realized.

We have modified the gas saturation system developed and used previously by the Liquid Chromatography Group to measure vapor pressures of polynuclear aromatic hydrocarbons (PAH) at temperatures between $60-300{ }^{\circ} \mathrm{C}$. The work done previously in the Liquid Chromatography Group covered the $10-50{ }^{\circ} \mathrm{C}$ range. The $\log P$ versus $1 / T$ plots obtained using this system are linear and agree with literature values. A variant of this system is being employed in this laboratory for the introduction of well-defined amounts of gaseous PAH internal standards onto sampling tubes containing porous polymers (XAD-2). Using these spiked tubes and the sampling system developed last year, we have determined a set of potassium correlated PAH profiles from wood burning stove emissions.

We have also pursued a number of basic chromatographic investigations. Split, splitless, and on-column injection techniques have been modified to gain an increase in chromatographic precision. The performance of two sulfurspecific detectors, electrolytic conductivity detector, and a flame photometric detector were compared using sulfur PAH standards. Although the electrolytic conductivity detector was considerably more sensitive, it suffered from high noise and baseline drift. A post-column trapping technique was developed to remove polar solvents, such as methanol, while allowing nonpolar analytes eluting with the solvent to pass only slightly broadened to the detector. In collaboration with the Liquid Chromatography and Mass Spectrometry Groups, quantification and identification of minor PAH constituents in Washington, St. Louis, and Philadelphia dust samples have been accomplished using high resolution techniques.

As in past years, the group has been actively involved in the Standard Reference Materials program. We have participated in the certification analyses for the following materials:

1. SRM 1581, Organics in Drinking Water - Completed analysis of halocarbons and developed delivery system.

2. SRM 1582, Petroleum Crude 0i1 - Information values for phenol and 0 -cresol provided.

3. SRM 1583, Pesticides in Isooctane - Completed analysis of all analytes. 
4. SRM 1585, Polychlorinated Biphenyl Congeners in Isooctane Completed analysis of all analytes.

5. SRM 1586, Unlabeled and Deuterium Labeled Priority Pollutants Chemical purities completed; ampouling completed and analyses proceeding.

6. SRM 1584, Phenols in Methanol - Analysis of 11 analytes in progress.

In collaboration with the Mass Spectrometry Group, a synthetic leachate sample was prepared, ampouled, and analyzed. This surrogate standard will be used by EPA/Las Vegas for proficiency testing. In addition, two sets of solutions used by EPA/Research Triangle Park as marker compounds for its "Master Analytical Scheme" have been analyzed for nitrobenzene and t-butanol.

Five sets of check samples were analyzed for EPA/Cincinnati. Each sample set contained related halogenated compounds ranging from chlorinated solvents to pesticides at concentrations of between 1 to $1000 \mu \mathrm{g} / \mathrm{g}$. A total of 114 ampoules were analyzed and over 650 separate analytical determinations were made. The samples (except for one set) were analyzed on our computercontrolled auto-injection GC system.

In collaboration with the Bioanalytical Techniques Group, the purity of 17 samples of sodium dodecyl sulfate (SDS) was determined. This analys is required the use of a double extraction technique on the hydrolyzed SDS samples. The results of the analyses were correlated with protein separations performed by two-dimensional electrophoresis (2-DE).

A new project, funded by the Law Enforcement Standards Laboratory, was initiated in the group. The objective of the program was to determine arson accelerants from soot collected remotely from the point of fire origin. It was found that soot originating from common accelerants (except for alcohol) contained traces of PAH, while soot arising from the normal contents of a house (wood, paper, plastics, synthetics, etc.) do not contain the PAH markers.

In support of the U.S. Pilot Environmental Specimen Bank program, the pesticide contents of 30 human liver tissue samples were analyzed by electron capture gas chromatography. Also, 28 samples of oil submitted by the recycled oil repository were analyzed for polychlorinated biphenyls (PCB). The samples were analyzed using our bidimensional chromatographic technique.

A new gas chromatography laboratory was completed and the group has occupied the facility since February. The group acquired a second automated gas chromatographic system and will receive a new integration/control system shortly. 
In FY 1984, the group will continue in its research program as previously reported. Special emphasis will be placed on high resolution techniques and new methods of analysis for compounds such as dioxins and dibenzofurans. The group also plans to collaborate with the Liquid Chromatography Group in developing a program in supercritical fluid chromatography.

Stephen N. Chesler, Group Leader; Delmo Enagonio, Franklin R. Guenther, Reenie M. Parris, Richard E. Rebbert

\section{Liquid Chromatography Group: Overview}

The research activities in the Liquid Chromatography Group involve both basic and applied components. A major effort continues to be focused on gaining a clearer understanding of the chemical and physical processes that contribute to retention in reversed- and normal-phase liquid chromatography (LC). We have also extended our efforts in the development and use of multidimensional LC systems (chromatographic selectivity) in combination with selective detectors or detection systems for the determination of individual compounds in complex mixtures. These research efforts have resulted in 25 publications being co-authored by members of the group (and frequently with members of other groups) during the past year.

Specific research accomplishments for the past year are as follows: (1) Evaluated the feasibility for modifying LC column selectivity by mixing $\mathrm{C}_{18}$ materials with different selectivities both intimately and by short columns in a series. We found that columns with specific selectivities could be "customized." (2) Developed synthesis conditions and procedures for characterization of monomeric, oligomeric, and polymeric $C_{18}$ phases. Evaluated selectivity differences among these different phase types using selected probe molecules. (3) In conjunction with the Gas Chromatography (GC) and Mass Spectrometry (MS) Groups, provided a detailed characterization of air particulate samples collected in Washington, DC (SRM 1649), St. Louis, MO (SRM 1648), and Philadelphia, PA. Several polynuclear aromatic hydrocarbons (PAH) were identified (based on GC and LC retention times, mass spectra, and fluorescence spectra) for the first time on air particulate samples. Quantitative data were provided for over 100 components per sample. (4) In conjunction with the GC Group, analyzed over 30 liver specimens for determination of organochlorine residues. An interlaboratory comparison exercise for measurement of these compounds between NBS and two German laboratories was completed. (5) In conjunction with the Electrochemistry Group, completed the development of two methods for determination of nitro-PAH in complex mixtures (technical report 23). (6) Developed methods for analys is of ascorbic acid, nicotinic acid, and nicotinamide in powdered milk formulations in preparation for supplying concentration values for those analytes in SRM 1549, Non-Fat Dry Milk. In addition, we made contributions to several Other Agency projects.

As in past years, the group has been actively involved in the NBS Standard Reference Materials (SRM) program. We have participated in analyses leading to the certification or characterization of the following materials: 
1. SRM 1582, Petroleum Crude 0i1 - Completed analysis for six PAH and, in conjunction with the Electrochemistry Group, two phenols (technical report 23 ).

2. SRM 1514, p-Aminobenzoic Acid in Phenacetin - Completed analysis of nominal $0.7,2.0$, and 5.0 mole percent sets (technical report 10).

3. SRM 1584, Priority Pollutant Phenols in Methanol - Ampouling complete; analysis for five phenols completed (technical report 10).

4. SRM 1549, Non-Fat Dry Milk - LC values provided for vitamin C, vitamin $D_{2}$, nicotinic acid, nicotinamide, and lactose. This SRM will be certified for trace metals content and is intended primarily for evaluating procedures and equipment used for trace metals analysis.

5. SRM 1648, Urban Particulate Matter - Provided information values for $13 \mathrm{PAH}$. This SRM was issued in 1978, certified for inorganic constituents only.

6. SRM 911 , Bilirubin - Determined the relative proportions of the three structural isomers of bilirubin (technical report 5 ). This SRM was certified in 1971 for bilirubin content, but no information was provided on the individual isomer content.

7. SRM 1650, Diesel Soot - Ampouling has been completed and analyses for selected $\mathrm{PAH}$ and nitro-PAH are in progress.

8. No number assigned yet, Nitrated-PAH in Solvent - Stability of five nitrated PAH in several solvents and conditions of storage being investigated; ampouling of the SRM solution is scheduled for January 1984 (technical report 10).

Our program in LC retention mechanisms has been bolstered by the addition of Dr. Lane Sander as a NAS/NRC Postdoctoral Fellow from the University of Washington. Dr. Sander's work has focused mainly on stationary phase contributions to LC retention. Dr. Sander has been primarily responsible for the synthesis and characterization of the monomeric, oligomeric, and polymeric $C_{18}$ phases mentioned earlier. This year, with the addition of Dr. Joe Foley, a NAS/NRC Postdoctoral Fellow from the University of Florida, we will broaden our investigations of LC retention mechanisms to include the role of the mobile phase.

During the coming year, we will continue our. efforts in LC/MS (in conjunction with the Mass Spectrometry Group), the development and use of multidimensional approaches for the analysis of complex mixtures, and work in the area of post- and pre-column derivatization. We plan to begin work in the area of both packed and capillary supercritical fluid chromatography. Our efforts in the environmental area will continue to decrease, while we expand our clinical and food/nutritional programs.

Willie E. May, Group Leader; Bruce Benner, Jr., Iona N. Black, Jeanice M. Brown-Thomas, Richard G. Christensen, Walter. F. Kline, Linda D. Fong, Sam A. Margolis, Lane C. Sander, Stephen A. Wise 


\section{Mass Spectrometry Group: Overview}

The Mass Spectrometry Group has pursued research in a number of areas involving both basic and applied research. Efforts are continuing to develop mass spectrometric methods for the accurate quantitation of individual organic compounds in complex matrices, to refine the liquid chromatography/mass spectrometry (LC/MS) interface, and to use it for quantitation in conjunction with the triple quadrupole mass spectrometer system. Research has also continued on the laser induced photodissociation of ions and the collision induced dissociation of ions as part of the competence building program in mass spectrometry, "Analysis of Ionization Mechanisms and Ion Structures." Investigations have begun on two projects in conjunction with the Gas and Particulate Science Division; one on the mechanistic relationship between field desorption mass spectrometry and laser desorption mass spectrometry, and the other on development of gas chromatography/mass spectrometry (GC/MS) methods and standards for trace organic chemicals in air.

Quantitative measurements made in the Mass Spectrometry Group over the past year have been used for the certification of several SRM's including SRM 1582 (Wilmington Crude 0i1), SRM 1583 (Pesticides in Isooctane), and SRM 909 (Human Serum).

Development of isotope dilution definitive methods for the quantitative determination of human serum constituents has continued in collaboration with the Bioanalytical Techniques Group. Human Serum SRM 909 has now been certified for four organic analytes and measurements have begun on creatinine. The concentration of glucose in SRM 909 has been remeasured because the glucose level has been found to change with time. As mentioned previously, a manuscript describing the ID/MS method for serum urea has been completed and will be submitted to Analytical Chemistry. A manuscript describing our ID/MS method for uric acid is in preparation. Work has begun on preparation of a labeled tripalmitin to be used in an ID/MS method for triglycerides.

Research in the environmental area has focused on the development of accurate quantitative methods for the determination of trace level toxic organic compounds in a variety of sample types. Research supported by the Department of Energy (DOE) has led to the development of GC/MS methods for the quantitation of organic compounds in aqueous leachates of hazardous wastes. The method, which involves solvent extraction of an aqueous leachate followed by capillary column GC/MS with selected ion monitoring, provides the sensitivity and selectivity necessary for quantitation at the part-per-billion level. The method has been used to measure organic compounds in aqueous leachates of two DOE-supplied materials: (1) solvent refined coal vacuum still bottoms and (2) an H-coal filter cake material. Capillary column GC/MS using positive chemical ionization has been used to determine the concentrations of seven compounds in a petroleum crude oil sample, which is to be issued as SRM 1582. The analytes include five polynuclear aromatic hydrocarbons (PAH) and a sulfur- and a nitrogen-heterocyclic compound. Preliminary investigations on the use of methane negative chemical ionization (NCI) mass spectrometry indicate that this may be a sensitive and highly selective technique for the determination of certain types of compounds including several PAH and pesticides. A gas chromatography/negative chemical ionization mass spectrometry (GC/NCIMS) method was used to measure the concentrations of three PAH 
in SRM 1582. We have also used GC/NCIMS to measure the concentrations of heptachlor epoxide, aldrin, and $\gamma$-BHC in SRM 1583, Pesticides in Isooctane.

In conjunction with the Gas Chromatography and Liquid Chromatography Groups, the development of isotopically labeled marker compound standards for use in EPA's "Master Analytical Scheme for the Analys is of Organic Compounds in Water" and "Comprehensive Scheme for Solids, Sediments, and Sludge" and stability studies of the marker compound solutions were completed during the past year.

Capillary column GC/MS with selected ion monitoring detection was used for the determination of 12 toxic organic compounds in a gravimetrically prepared liquid hazardous waste which was delivered to the EPA for use in its quality assurance monitoring programs. Measurements made on this sample have enabled us to assess the accuracy and precision achievable in the analysis of a hazardous waste sample. Work is continuing on the development of a solid hazardous waste quality assurance material for the EPA. This material is to be prepared so that known amounts of selected organic compounds will be obtained in aqueous leachates.

As part of a joint project with the Gas and Particulate Science Division for the EPA on research leading to standards for hazardous chemicals, the Mass Spectrometry and Gas Chromatography Groups are developing a method for measuring toxic organic compounds in gases at the ppb level. A cold trapping technique followed by GC/MS using isotope dilution methodology adapted from the definitive methods has been applied to bromobenzene at $30 \mathrm{ppb}$ in nitrogen with promising results. We are working to extend the method to $1 \mathrm{ppb}$ and to establish the accuracy and precision of the method. Such a method will provide EPA with a direct way to determine the sensitivity of a GC/MS system and NBS with an independent method for certifying organic compounds present at low levels in gases.

Studies on the collision of ions with reactive neutral species, a part of the competence building program in mass spectrometry entitled "Analysis of Ionization Mechanisms and Ion Structures," have begun using a newly acquired triple quadrupole mass spectrometer. The MS/MS instrument is also being used in conjunction with our LC/MS interface in an attempt to perform direct LC/MS/MS determination of dibenzothiophene in SRM 1582, Wilmington Crude 0il. Research in laser photodissociation of organic ions has continued this year. Ions generated by electron impact ionization have been subjected to laser induced dissociation in both the ion source and the first field free region of a double focusing mass spectrometer. Design has been completed for an apparatus to allow study of the photodissociation in the first field free region of ions generated by field ionization and field desorption and construction is underway.

A study of the mechanisms involved in the laser desorption (LD) and field desorption (FD) mass spectrometry of organic compounds has been initiated. The LD work was done on a laser microprobe mass analyzer (LAMMA) in collaboration with the Gas and Particulate Science Division. Several organic acids and salts have been examined in this work that looks at some of the existing theories of ion formation proposed for desorption techniques. 
During the past year, Dr. Corazon Vogt, University of Missouri, Columbia, MO, conducted sabbatical research with the Mass Spectrometry Group. Dr. Vogt assisted in the development of methods for the quantitative determination of trace level organics in aqueous leachates of hazardous wastes. We were also fortunate to have Dr. Zhao Min from the People's Republic of China working in our group for six months. She was involved in both the definitive methods development and ionization mechanisms programs.

We plan to continue the ongoing projects with which the group is involved including the environmental projects with special attention to further investigation of the utility of negative chemical ionization, the development of isotope dilution definitive methods, and the study of the photodissociation of ions and the collision of ions with reactive neutral molecules as sources of information on the structures of ions and molecules.

Edward White V, Group Leader; Gary D. Byrd, Laurence R. Hilpert, Zhao Min, Stanley Meiselman, Kristy L. Richie, Lorna T. Sniegoski, Corazon R. Vogt, Michael J. Welch 
B. Selected Technical Reports

(Organic Analytical Research Division)

1. Synthesis and Characterization of Chemically Bonded $C_{18}$ Phases for

Liquid Chromatography (15320)

L. C. Sander, S. A. Wise

To a large extent, the chromatographic properties of a reversed-phase liquid chromatographic (LC) sorbent are dependent on the conditions of the bonded phase synthesis. Pronounced differences in selectivity have been observed between monomeric and polymeric alkyl bonded phases, especially in the analysis of polycyclic aromatic hydrocarbons. An understanding of the origin of these differences is important both theoretically and practically. Such knowledge would be valuable in methods development and would permit the retention behavior of certain compounds to be more accurately predicted. In the past, we have examined selectivity differences for various commercially available $C_{18}$ materials. However, these studies were somewhat limited since the exact details of the synthesis conditions were unavailable. In this study, a number of monomeric, polymeric, and oligomeric (intermediate) $C_{18}$ bonded phases were prepared (i.e., synthesized) on a variety of silica substrates and the selectivity differences were evaluated. The goals of this study were threefold: to examine changes in selectivity with phase type (monomeric, polymeric, oligomeric), to observe the effects of surface coverage on selectivity for polymeric phases, and finally to study any effects that the silica substrate might impart to solute retention.

Five different base silicas were used in the preparation of the bonded phase sorbents. The physical properties of these substrates vary considerably, as shown in Table 17; however, all five silicas have $300 \AA$ pore diameters. The various LC phases prepared are listed in Table 18. Surface concentration values $\left(\mu \mathrm{mol} / \mathrm{m}^{2}\right.$ ) for the oligomeric series of phases were larger than the monomeric phases, and the polymeric phases had the largest $C_{18}$ surface concentrations of all phase types. Furthermore, within the polymeric phases, chain density increases with the amount of water added during phase synthesis. Selectivity differences among the phases were examined using a 16 polycyclic aromatic hydrocarbon mixture (SRM 1647). Separations of this mixture on three representative phases are illustrated in figure 19. The best separations were obtained with moderately loaded polymeric phases. The oligomeric phases gave only partial resolution of benzo[k]fluoranthene and benzo[a]pyrene (peak numbers 12 and 13), while on the monomeric phases these and several other components were unresolved. A simple empirical LC test was devised to gauge the relative monomeric or polymeric nature of a phase. The elution order of a three component mixture, phenanthro[3,4-c]phenanthrene (PhPh), 1,2:3,4:5,6:7,8tetrabenzonaphthalene (TBN), and benzo[a]pyrene ( $B[a] P)$ was observed to be strongly dependent on the type of phase and surface coverage. Using a mobile phase of 85 percent acetonitrile/water, the mixture was analyzed on each of the columns (figure 19.). On monomeric phases, the elution order was $B[a] P$, $\mathrm{PhPh}, \mathrm{TBN}$; on oligomeric phases, $\mathrm{PhPh}, \mathrm{B}[\mathrm{a}] \mathrm{P}, \mathrm{TBN}$; and on polymeric phases, $\mathrm{PhPh}, \mathrm{TBN}, \mathrm{B}[\mathrm{a}] \mathrm{P}$. Depending on the elution order of a mixture, new phases 
could be rapidly screened and column selectivity toward more complex PAH mixtures predicted.

The effect of pore size of the silica substrate was also investigated for the polymeric phases. Polymeric phases were produced on four silica substrates with $60,100,150$, and $300 \AA$ pore diameters. The 100 and $150 \AA$ substrates were experimental silicas of the same composition as silica A, Table 17 . The $300 \AA$ silica is listed as silica $C$, and the $60 \AA$ silica, an irregular substrate material not listed in Table 17. Differences in selectivity were clearly evident as being a function of pore size (figure 20). Using the threecomponent test mixture, the polymeric nature of the phases was judged to increase with increasing pore size. Polymeric phases produced on small pore substrates had chromatographic properties similar to those of monomeric phases, and separation of all 16 components was not possible. Baseline separation of a11 components was possible using the larger (150 and $300 \AA$ ) pore substrates. Future studies on bonded phases will be directed toward the production of new phases using ultralong alkyl chains with both monomeric and polymeric surface modification.

Table 17. Physical Properties of Silica Substrates.

\begin{tabular}{|c|c|c|c|c|}
\hline Silica & $\begin{array}{l}\text { Specific Surface } \\
\text { Area }\left(\mathrm{m}^{2} / \mathrm{g}\right)^{\mathrm{a}}\end{array}$ & Shape & $\begin{array}{l}\text { Pore Volume } \\
\quad(\mathrm{mL} / \mathrm{g})\end{array}$ & $\begin{array}{c}\text { Percent Carbon } \\
\text { (Silica Background) }\end{array}$ \\
\hline Silica $A$ & 45 & spherical & 0.42 & 0.06 \\
\hline Silica B & 60 & spherical & 0.6 & 0.10 \\
\hline Silica C & 90 & spherical & 0.66 & 0.41 \\
\hline Silica D & $200^{b}$ & spherical & 2.0 & 0.18 \\
\hline Silica $E$ & 250 & irregular & 1.52 & 0.11 \\
\hline
\end{tabular}

\footnotetext{
${ }^{a}$ Nominal values reported by the manufacturer.

banufacturer's surface area value is $250 \mathrm{~m}^{2} / \mathrm{g}$; the value listed was determined by BET measurement.
} 


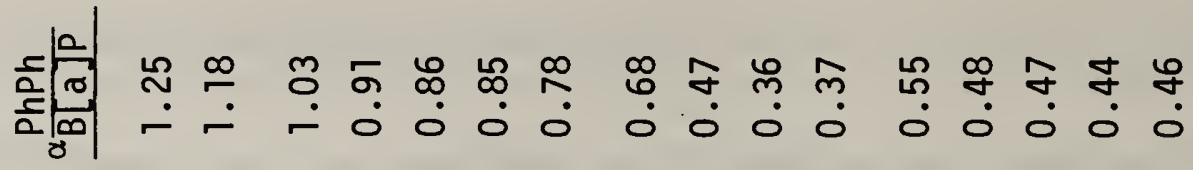

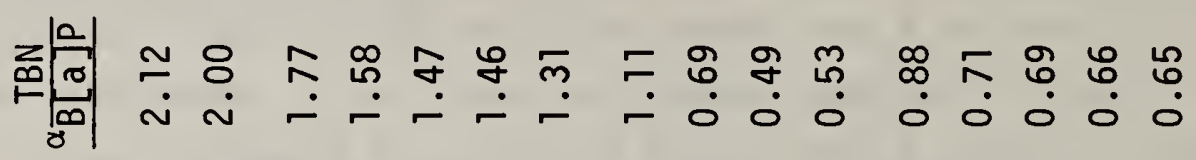

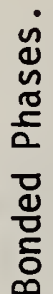

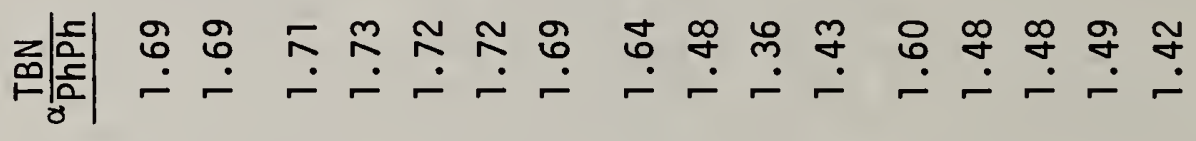

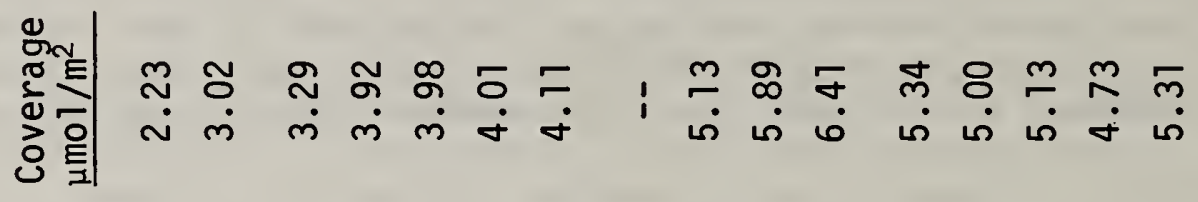

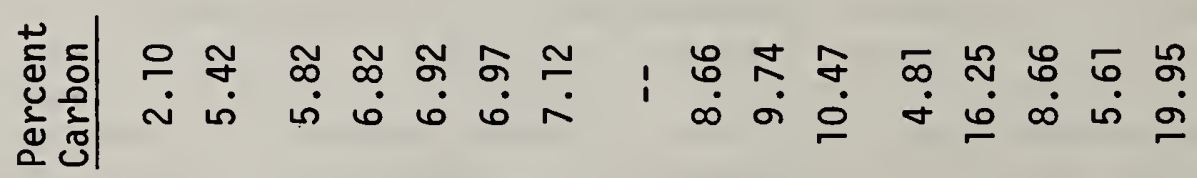

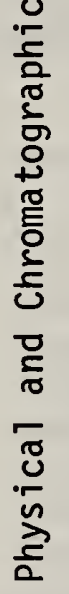

$\infty$

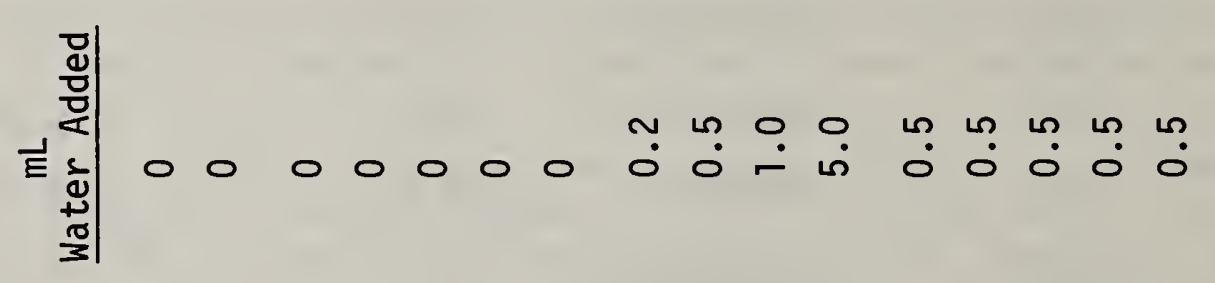

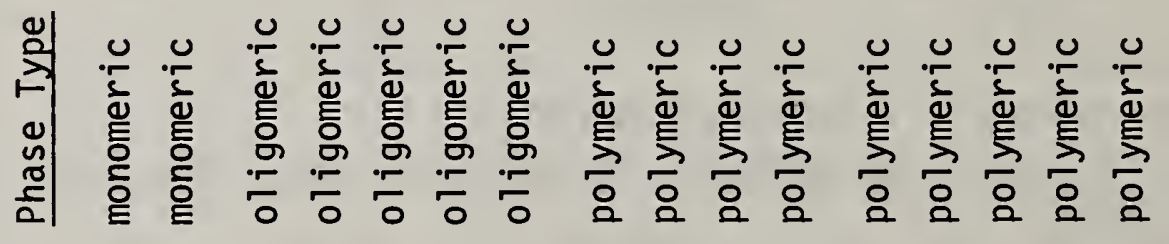

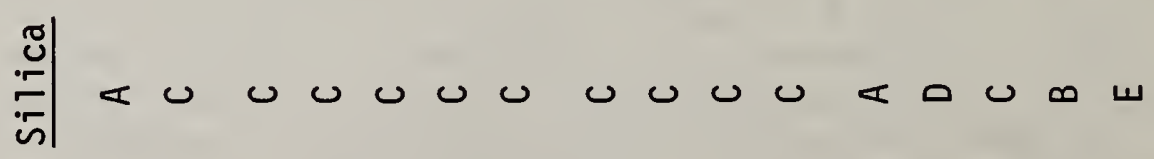

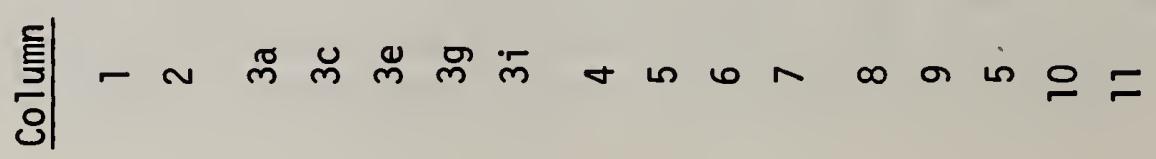



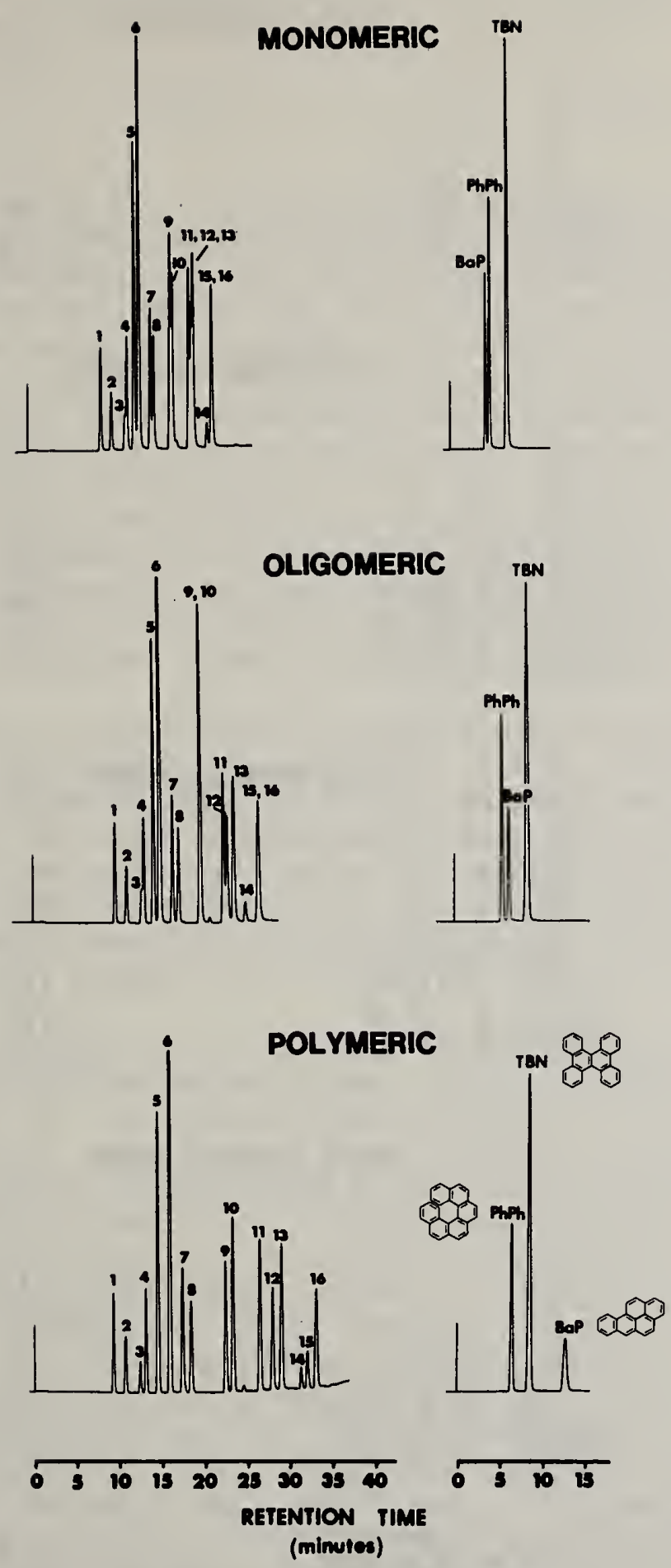

Figure 19. Separation of polycyclic aromatic hydrocarbons (SRM 1647) on representative monomeric, oligomeric, and polymeric phases. Separation of the sixteen-component mixture was performed using gradient elution, 40-100 percent acetonitrile in water over 30 minutes at $2 \mathrm{~mL} / \mathrm{min}$. The three-component mixture was run isocratically at 85 percent acetonitrile in water, and the order of elution is indicative of phase type. 

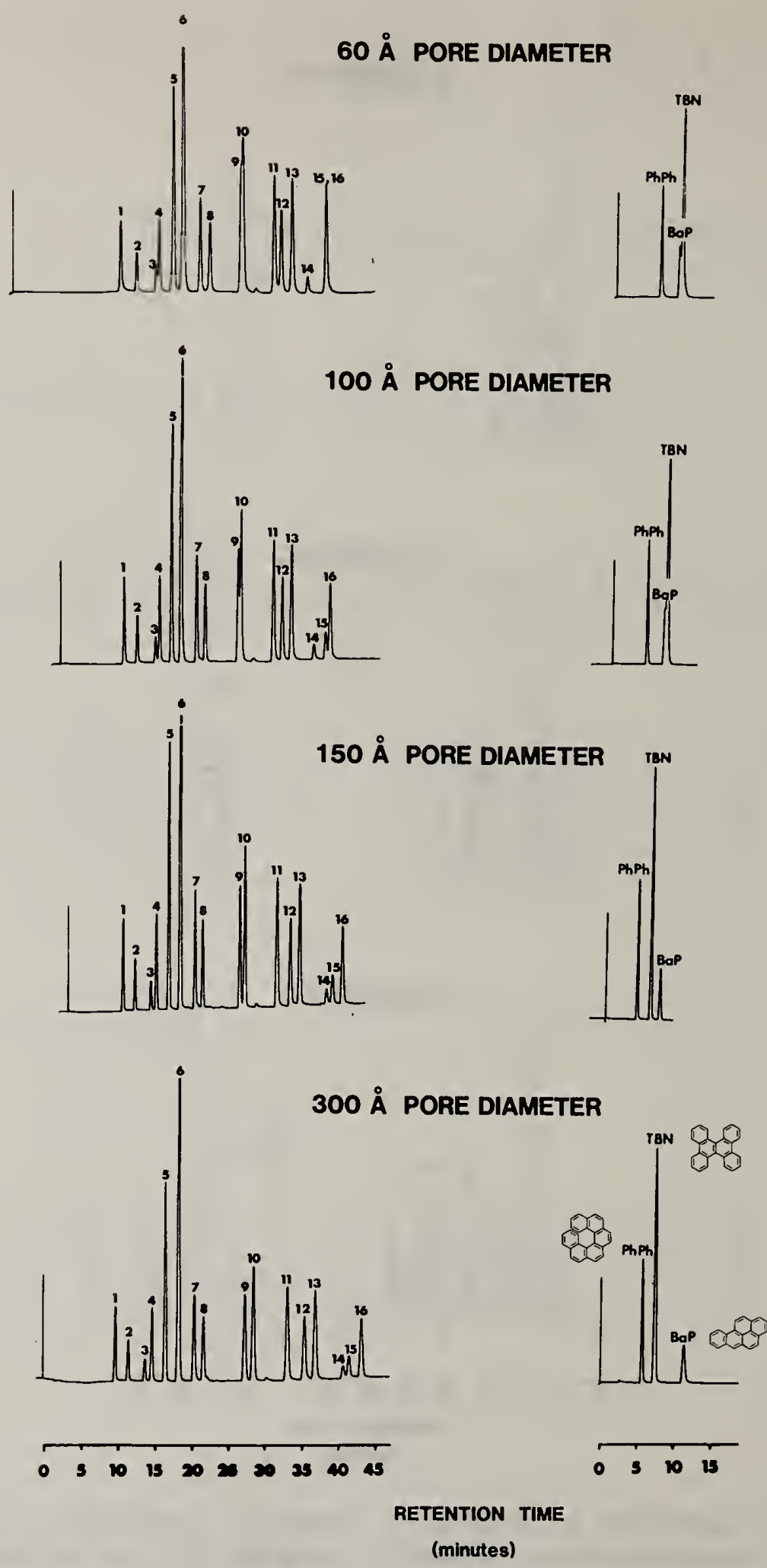

Figure 20. Selectivity comparison for polymeric phases prepared on narrow and wide pore silica substrates. Note especially components 9 and 10 (benz[a]anthracene and chrysene). 
2. Characterization and Comparison of Three Air Particulate Samples for Polycyclic Aromatic Hydrocarbon Distributions (15320)

S. N. Chesler, L. R. Hilpert, W. E. May, R. E. Rebbert, C. R. Vogt,

S. A. Wise

In a previous report [1], the methodology and results for the determination of selected polycyclic aromatic hydrocarbons (PAH) in SRM 1649 (Urban Dust/Organics) were reported. Since this air particulate material and a second air particulate SRM sample (SRM 1648, Urban Particulate Matter, which is certified for inorganic species only) are available to other laboratories to serve as benchmark materials for interlaboratory comparisons and methods development, additional characterization of the PAH content of these two materials was undertaken. A third air particulate sample, collected in Philadelphia, PA, by the Environmental Protection Agency (EPA) for both chemical and biological characterization, was also analyzed. Normally, only 10 to 15 major PAH components are measured in such samples. However, since these three materials are available in large quantities and have been widely distributed, these samples were characterized not only for the major PAH constituents but also for the numerous minor PAH components.

After extraction of the samples with methylene chloride, the PAH constituents were isolated by clean-up on a short silica column, followed by norma 7-phase liquid chromatography (LC) on an aminosilane column to isolate a total PAH fraction. The major PAH components were then determined in the total PAH fraction from each sample using high resolution capillary gas chromatography (GC) and reversed-phase LC with fluorescence detection. The results of the GC and LC analyses of the PAH mixtures from the three air particulate samples are summarized in Tables 19-21.

Quantification of the minor components in complex PAH mixtures is often difficult because of the co-elution of many components even when using high resolution capillary GC. To overcome this problem, the total PAH mixture from each sample was further separated into eight fractions using normal-phase LC on an aminosilane column. Normal-phase LC provides separation of PAH based on the number of aromatic carbons, i.e., alkyl-substituted PAH elute with the parent PAH. These fractions were then analyzed by reversed-phase LC, GC, and gas chromatography/mass spectrometry (GC/MS) to identify and quantify the individual PAH. To quantify the minor components in each fraction, one of the major components in each fraction, which had been quantified previously in the total PAH fraction, was used as an internal standard, e.g., phenanthrene in fraction 2, fluoranthene in fraction 3, benz[a]anthracene in fraction 4, benzo[a]pyrene in fraction 5 , etc. Using this approach, over 100 components in each air particulate sample were quantified. Of these compounds, approximately 40 unsubstituted PAH and 15 methyl-substituted PAH were identified based on GC and LC retention data, fluorescence spectra, and mass spectrometric data. The remaining compounds were tentatively identified as alkylsubstituted PAH based on mass spectrometric and fluorimetric data. Several compounds were positively identified for the first time in air particulate samples, e.g., pentaphene and dibenzo[b,k]fluoranthene. 
The last two PAH fractions from the normal-phase LC fractionation were of particular interest. Fraction 7 contained six isomers of molecular weight 278. Using LC retention data in conjunction with fluorescence spectra, these isomers were identified as dibenz $[a, c]$ anthracene, dibenz $[a, j]$ anthracene,

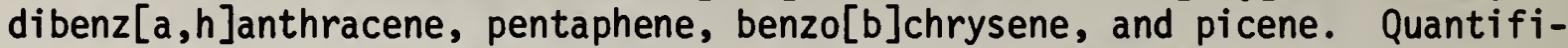
cation of these six isomers without isolation into a separate fraction would be extremely difficult, since they are only minor components which elute in the same region as several major constituents. The last fraction contained six isomers of molecular weight 302 (dibenzopyrene or dibenzofluoranthene isomers). Two of these isomers were identified as naphtho[1,2,3,4-def]chrysene and dibenzo[ $b, k]$ fluoranthene.

of particular interest in the comparison of the results from these three samples is the possibility of source identification based on relative ratios of selected PAH. For example, the ratio of benzo[e]pyrene to benzo[a]pyrene is 1.1 for the Washington, DC, sample versus 1.7 for the St. Louis and Philadelphia samples. Future research will investigate the potential use of PAH ratios for source identification and apportionment, particularly using ratios of minor PAH components which are not generally quantified.

Reference

[1] Center for Analytical Chemistry Annual Report, NBSIR 82-2620 (1982). 
Table 19. Concentration $(\mu \mathrm{g} / \mathrm{g})^{\mathrm{a}}$ of Selected PAH in Washington, DC, Sample (SRM 1649) as Determined by GC and LC Methods.

Compound Measured

Phenanthrene

Anthracene

Pyrene

Fluoranthene

Benz[a]anthracene

Chrysene

Triphenylene

Benzo[e]pyrene

Benzo[a]pyrene

Perylene

Indeno $[1,2,3-c d]$ pyrene

Benzo[ghi]perylene

Dibenz $[a, h] a n t h r a c e n e$

Benzo[b]fluoranthene Benzo[k]fluoranthene
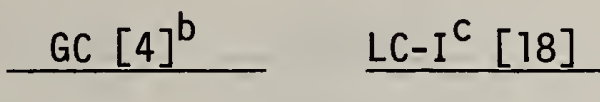

$\underline{L C-I I}{ }^{C}[9]$

$4.5 \pm 0.3$

$7.2 \pm 0.2$

$6.3 \pm 0.4$

$6.2 \pm 0.2$

$7.3 \pm 0.2$

$7.0 \pm 0.5$

$6.8 \pm 0.4$

$2.4 \pm 0.1$

$2.8 \pm 0.3$

$2.4 \pm 0.1$

$\left\{4.6 \pm 0.2^{d}\right.$

$3.5 \pm 0.1$

$3.3 \pm 0.2$

$3.0 \pm 0.3$

$2.6 \pm 0.4$

$2.6 \pm 0.1$

$0.84 \pm 0.09$

$0.80 \pm 0.04$

$0.65 \pm 0.02$

$3.3 \pm 0.3$

$3.4 \pm 0.4$

$3.6 \pm 0.2$

$4.7 \pm 0.2$

$3.9 \pm 0.8$

$5.2 \pm 0.6$

$\overline{a_{\text {Values }} \text { represent }}$ the mean \pm standard deviation for the given number of determinations.

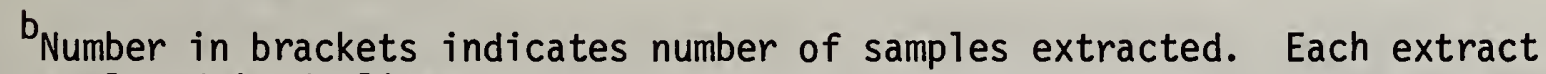
analyzed in duplicate.

${ }^{C}$ LC-I, no LC clean-up, 7-methylfluoranthene used as internal standard; LC-II, no LC clean-up, perylene- $d_{12}$ used as internal standard; LC-III, LC clean-up, perylene- $d_{12}$ used as internal standard.

$d_{G C}$ value includes both chrysene and triphenylene.

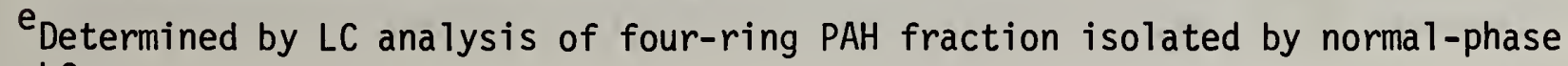
LC. 
Table 20. Concentration $(\mu \mathrm{g} / \mathrm{g})^{\mathrm{a}}$ of Selected PAH in St. Louis, M0, Sample (SRM 1648) as Determined by GC and LC Methods.

\begin{tabular}{|c|c|c|c|c|c|}
\hline Compound Measured & & {$[4]^{b}$} & $\underline{L C-I^{C}}[3]$ & 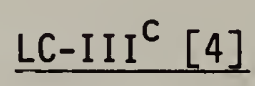 & \\
\hline Phenanthrene & 3.8 & $\pm 0.2^{c}$ & -- & $4.6 \pm 0.3$ & \\
\hline Anthracene & & -- & - & $0.36 \pm 0.01$ & \\
\hline Pyrene & 7.3 & \pm 0.4 & $7.4 \pm 0.2$ & $6.8 \pm 0.2$ & \\
\hline Fluoranthene & 9.2 & \pm 0.5 & $7.9 \pm 0.6$ & $8.7 \pm 0.4$ & \\
\hline Benz [a]anthracene & 3.2 & \pm 0.2 & $2.8 \pm 0.1$ & $3.2 \pm 0.1$ & \\
\hline $\begin{array}{l}\text { Chrysene } \\
\text { Triphenylene }\end{array}$ & $\{7.8$ & $\pm 0.6^{d}$ & $\begin{array}{c}6.6 \pm 0.1 \\
--\end{array}$ & $\begin{array}{c}6.6 \pm 0.2 \\
--\end{array}$ & $\begin{array}{l}6.9^{e} \\
2.6 \pm 0\end{array}$ \\
\hline Benzo[e]pyrene & 5.2 & \pm 0.3 & - & -- & \\
\hline Benzo[a]pyrene & 3.1 & \pm 0.2 & $2.6 \pm 0.2$ & $3.4 \pm 0.2$ & \\
\hline Perylene & 0.76 & \pm 0.07 & $0.65 \pm 0.02$ & $0.69 \pm 0.02$ & \\
\hline Indeno $[1,2,3$-cd $]$ pyrene & 4.4 & \pm 0.15 & $4.8 \pm 0.2$ & $4.7 \pm 0.2$ & \\
\hline Benzo[ghi]perylene & 4.7 & \pm 0.1 & $5.5 \pm 0.8$ & -- & \\
\hline $\begin{array}{l}\text { Benzo[b]fluoranthene } \\
\text { Benzo[k] fluoranthene } \\
\text { Benzo[j]fluoranthene } \\
\text { Benzofluoranthenes (Total) }\end{array}$ & $\begin{array}{r}3.7 \\
3.5 \\
9.8 \\
16.9\end{array}$ & $\begin{array}{l} \pm 0.5 \\
\pm 0.2 \\
\pm 0.5 \\
\pm 0.8\end{array}$ & $\begin{array}{c}3.3 \stackrel{--}{ \pm} 0.1 \\
-- \\
--\end{array}$ & $\begin{array}{c}3.4 \stackrel{-}{ \pm} 0.05 \\
-- \\
--\end{array}$ & \\
\hline
\end{tabular}

aalues represent the mean \pm standard deviation for the given number of determinations.

$b_{\text {Number }}$ in brackets indicates number of samples extracted. Each extract analyzed in duplicate.

${ }^{C}$ LC-I, no LC clean-up, 7-methylfluoranthene used as internal standard; LC-II, no LC clean-up, perylene- $d_{12}$ used as internal standard; LC-III, LC clean-up, perylene- $d_{12}$ used as internal standard.

$d_{G C}$ value includes both chrysene and triphenylene.

${ }^{e}$ Determined by $L C$ analysis of four-ring PAH fraction isolated by normal-phase LC. 
Table 21. Concentration $(\mu \mathrm{g} / \mathrm{g})$ of Selected PAH in Philadelphia, PA, Sample (EPA) as Determined by GC and LC Methods.

Compound Measured

Phenanthrene

Anthracene

Pyrene

Fluoranthene

Benz[a]anthracene

Chrysene

Triphenylene

Benzo[e]pyrene

Benzo[a]pyrene

Perylene

Indeno $[1,2,3-c d]$ pyrene

Benzo[ghi]perylene

Benzo[b]fluoranthene

Benzo[k]fluoranthene

Benzo[j]fluoranthene

Benzofluoranthenes (Total)

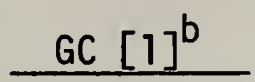

$3.5 \pm 0.1$

$7.3 \pm 0.1$

$13.6 \pm 0.2$

$11.4 \pm 0.2$

$\left\{29.7 \pm 2.5^{d}\right.$

$16.4 \pm 0.7$

$9.5 \pm 0.1$

$2.2 \pm 0.2$

$12.5 \pm 0.7$

$12.6 \pm 0.7$

$9.9 \pm 1.0$

$11.1 \pm 1.4$

$26.7 \pm 1.1$

$48.5 \pm 2.0$
2.9

0.4

$\underline{\text { LC-III }[1]^{\mathrm{C}}}$

6.6

$11.8 \pm 0.2$

$11.7 \pm 0.2$

$\begin{aligned} 23.1 & \pm 0.1 \\ 9.8 & \pm 0.1\end{aligned}$

$7.5 \pm 0.1$

$2.1 \pm 0.1$

$13.1 \pm 1.0$

$11.0 \pm 0.1$

$--$

$\overline{\text { Values represent }}$ the mean \pm standard deviation for triplicate measurements of a single extract.

$b_{\text {Number }}$ in brackets indicates number of samples extracted and analyzed.

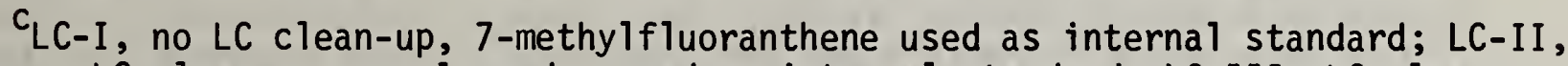
no LC clean-up, perylene- $d_{12}$ used as internal standard; LC-III, LC clean-up, perylene- $d_{12}$ used as internal standard.

$d_{G C}$ value includes both chrysene and triphenylene.

e Determined by LC analysis of four-ring PAH fraction isolated by normal-phase LC. 
3. Determination of Chlorinated Pesticide Residues in Human Liver Specimens for the Pilot Environmental Specimen Bank Program (15320)

S. N. Chesler, W. F. Kline, S. A. Wise

As part of the NBS/EPA Pilot Environmental Specimen Bank program, analytical methodology for the determination of organochlorine pesticide residues in human liver samples was implemented during the past year. Thirty individual human liver samples, which were obtained in accordance with the NBS/EPA National Environmental Specimen Bank protocol for sample collection [1], were cryogenically homogenized as described by Zeisler, et al. [2] and aliquots were analyzed for organochlorine pesticide residues. Aliquots from these same 30 liver specimens were placed in storage under various conditions as part of the storage evaluation study to determine the feasibility of long-term storage of biological samples for trace analysis. The results from the analyses of these 30 liver specimens will provide the baseline values for comparison to results obtained in the future from analyses of stored aliquots.

The sample preparation consisted of mixing the liver homogenate with sodium sulfate, chromatographic extraction of the sample/sodium sulfate mixture with hexane:acetone (2:1), and chromatographic clean-up of the extract on Florisil. Internal standards were added to the sample prior to extraction to facilitate quantitation. The percent extractable fat was determined for each sample by taking an aliquot of the extract to dryness in a small tared flask. The mass of residue was assumed to be fatty lipid material. The Florisil column chromatography separated the chlorinated pesticides from the polar or lipid material. The eluant from the Florisil column was concentrated to an appropriate volume and analyzed by capillary gas chromatography (GC) with selective electron capture detection.

The data obtained from the analyses of the 30 individual samples are summarized in Table 22. Eight different chlorinated pesticides were observed in nearly all of the samples. The range, mean, and median values based on both wet weight and fat weight are given. In a strict analytical sense, the wet weight values are the most appropriate in that they reflect the concentrations of the pesticides in the actual liver sample. The values, based on percent extractable fat, assume that all of the pesticides observed were originally associated with the extractable fat. This assumption, while lacking absolute analytical validity, has become a convention among researchers in this area since it facilitates the comparison of data obtained from the analys is of different organs in addition to compensating for different extraction techniques [3]. The average percent standard deviation associated with the gas chromatographic analys is of each sample is shown in the last column in Table 22. Approximately 30 samples will be analyzed in the next year to provide additional baseline data. Selected samples, which have been stored for over one year, will be reanalyzed to evaluate the stability of the samples under various conditions of storage. 


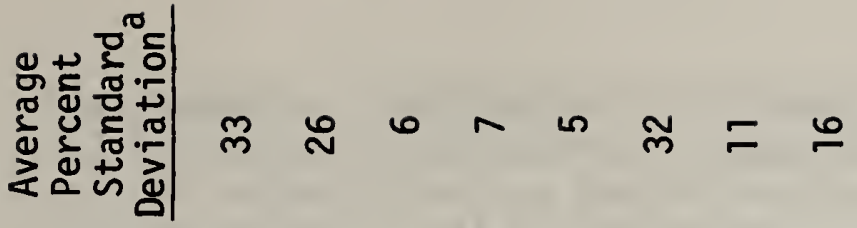

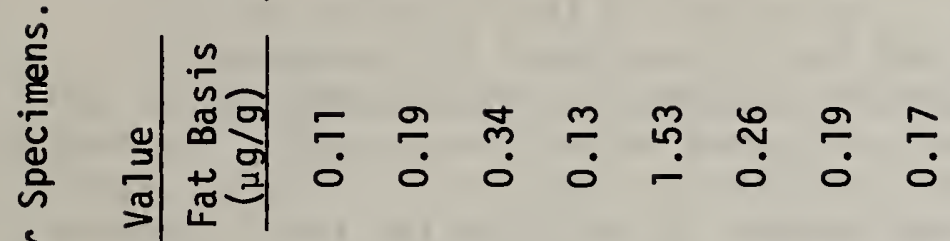

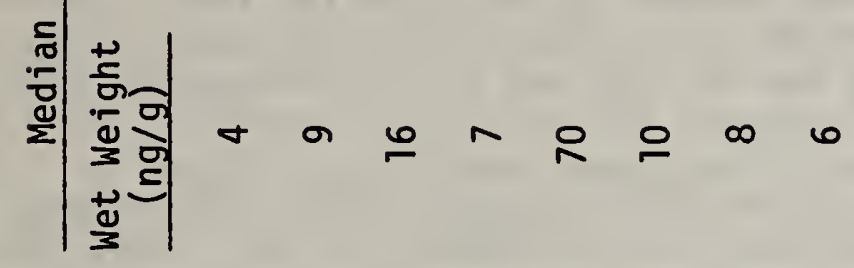

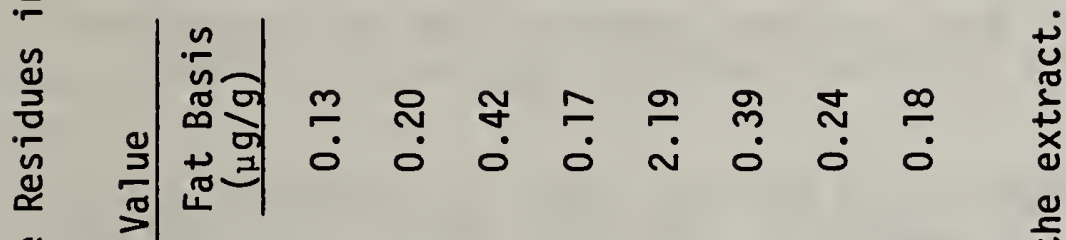

䞤苛

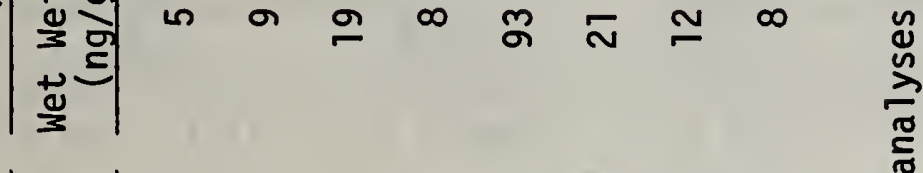

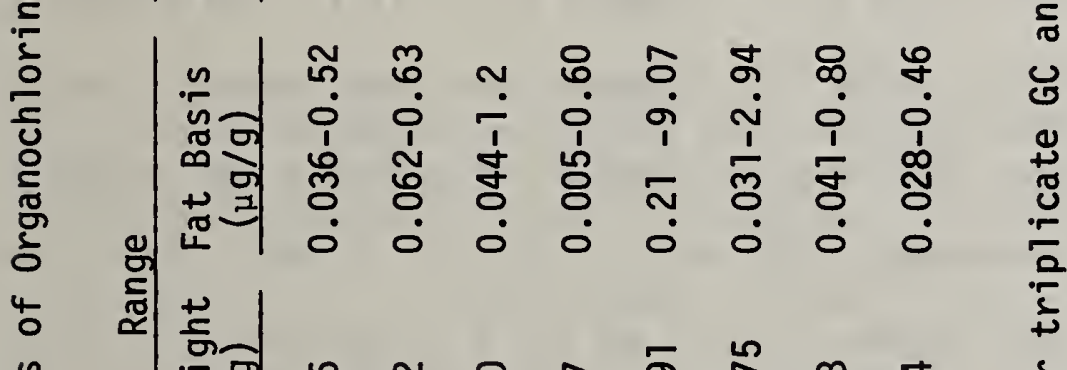

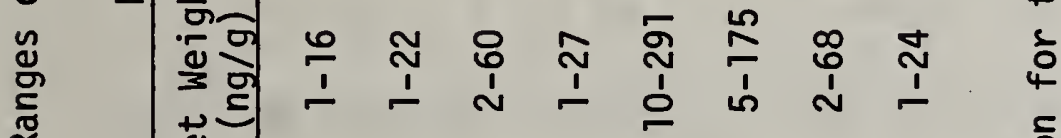

20.

ำ

$\frac{0}{\frac{1}{r}}$

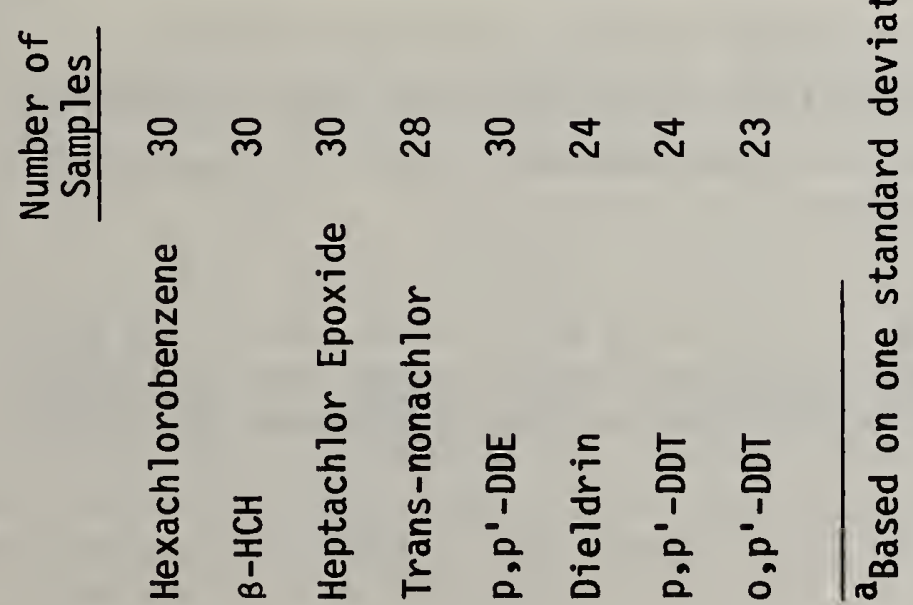


As part of a cooperative program with a similar Pilot Environmental Specimen Bank Program in the Federal Republic of Germany, aliquots of liver specimens from each pilot program were exchanged and analyzed for the determination of chlorinated pesticides. The results of this interlaboratory comparison of analyses between NBS and the University of UIm in Germany are summarized in Table 23. The agreement of the results is good considering that somewhat different analytical methods were used by each laboratory. The results obtained by the University of UIm for dieldrin are low due to suspected losses in the LC clean-up procedure. No explanation is available for the difference in the results obtained for $\mathrm{B}-\mathrm{HCH}$ in the U.S. sample. The NBS results for $p, p^{\prime}-D D T$ in the German liver sample may be high due to the presence of high concentrations of polychlorinated biphenyls (PCB's) in this sample. The PCB's are isolated in a separate fraction in the German analytical procedure, whereas they are in the same fraction as the chlorinated pesticide residues in the NBS procedure and several isomers may co-elute with p,p'-DDT. Additional liver samples and extracts have been exchanged between these two laboratories in order to further investigate the interlaboratory comparability of these analyses.

Table 23. Interlaboratory Comparison of Determinations of Organochlorine Pesticide Residues in Human Liver Specimens ( $\mathrm{ng} / \mathrm{g}$ extractable fat).

\begin{tabular}{|c|c|c|c|c|c|}
\hline \multirow[b]{3}{*}{ Hexachlorobenzene } & \multicolumn{2}{|c|}{ U.S. Sample (L1S0347) } & \multicolumn{3}{|c|}{ German Sample (006) } \\
\hline & $\mathrm{NBS}^{\mathrm{a}}$ & U1m & $\mathrm{NBS}^{\mathrm{a}}$ & $\mathrm{U} 1 \mathrm{~m}^{\mathrm{b}}$ & $U 1 m^{\mathrm{C}}$ \\
\hline & $72 \pm 15^{d}$ & $73 \pm 2$ & $2580 \pm 230$ & $1711 \pm 169$ & $1923 \pm 32$ \\
\hline $\mathrm{B}-\mathrm{HCH}$ & $383 \pm 37$ & $12 \pm 1$ & $888 \pm 73$ & $892 \pm 73$ & $982 \pm 18$ \\
\hline$p, p^{\prime}-D D E$ & $2340 \pm 140$ & $2138 \pm 60$ & $1840 \pm 40$ & $1794 \pm 65$ & $1858 \pm 14$ \\
\hline Dieldrin & $1020 \pm 190$ & $585 \pm 22$ & -- & --- & -- \\
\hline$p, p^{\prime}-D D T$ & $334 \pm 80$ & $330 \pm 10$ & $40 \pm$ & $20 \pm$ & $20 \pm 1$ \\
\hline
\end{tabular}

Three sample aliquots extracted from one jar; three GC analyses per sample extract.

${ }^{b}$ One sample (jar) extracted; three GC analyses per sample extract.

CThree samples (jars) extracted; three GC analyses per sample extract.

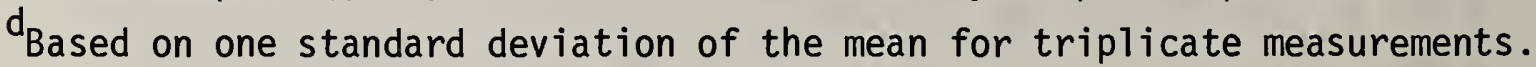

References

[1] Zeisler, R., Harrison, S. H., and Wise, S. A., eds., The Pilot National Environmental Specimen Bank: Analysis of Human Liver Specimens, NBS Special Publication 656, Washington, DC (1983). 
[2] Zeisler, R., Langland, J. K., and Harrison, S. H., Cryogenic Homogenization Procedures for Biological Tissues, Analytical Chemistry (in press).

[3] Morgan, D. P. and Roan, C. C., Chlorinated Hydrocarbon Pesticide Residues in Human Tissues, Archival Environmental Health 20, 452-457 (1970).

4. Certification of Selected Organic Compounds in SRM 1582, Petroleum Crude 0i1 (15320)

J. M. Brown-Thomas, L. R. Hilpert, W. F. Kline, W. A. MacCrehan, W. E. May,

R. M. Parris, R. E. Rebbert, C. R. Vogt

The objective of this program was to develop a Standard Reference Material (SRM) with certified concentrations for selected organic compounds in a complex matrix. This SRM will be useful in chemical characterization and methods development research programs and quality assurance efforts of laboratories involved in trace organic analysis in general, and the characterization of fuels in particular. A petroleum crude oil (Wilmington crude) was selected as the matrix to be used in this effort. This material will serve as a companion to SRM 1580, Organics in Shale 0il, that was certified in 1980 for many of the same compounds. The use of both SRM's will provide analysts with two points of calibration for the measurement of compounds that are certified in both materials.

In general, the certification of SRM's is accomplished through utilization of a single definitive method or at least two independent analytical methods. In order for a parameter to be certified, the results of the analyses by the independent methods must be in agreement. The limits of uncertainty given on the Certificate of Analysis reflect the agreement between the methods. The concentrations of dibenzothiophene, phenol, o-cresol, and six polycyclic aromatic hydrocarbons (PAH) were determined by two methods each. Carbazole, benzo[e]pyrene $(B[e] P)$, benzo[ghi]perylene $(B[$ ghi $] P)$, and indeno[ $1,2,3-c d]-$ pyrene (IP) were measured using only one analytical procedure. A summary of the results obtained and the methods used for analysis are presented in Table 24.

The sequential, high-performance liquid chromatographic (HPLC/HPLC) procedure that was used for determination of six PAH has been reported previously $[1,2,3]$ and was used in the certification of SRM's 1580 and 1649 . The high-performance liquid chromatographic/electrochemical detection (HPLC/EC) procedure for determination of phenol and o-cresol was developed specifically for this SRM and is similar to that reported by Shoup et al. [4].

The phenolic compounds were extracted from the oil into an aqueous phase by basic conversion to the phenolate anions with base. They were subsequentiy neutralized and then analyzed using a C-8 column with a mobile phase of 30 percent acetonitrile $/ 5$ percent tetrahydrofuran in water with a $0.05 \mathrm{~mol} / \mathrm{L}$ ammonium acetate electrolyte. The electrochemical detector consisted of a $1.2 \mathrm{~mm}$ glassy carbon electrode, and an applied potential (versus $\mathrm{Ag} / \mathrm{AgCl}$ 


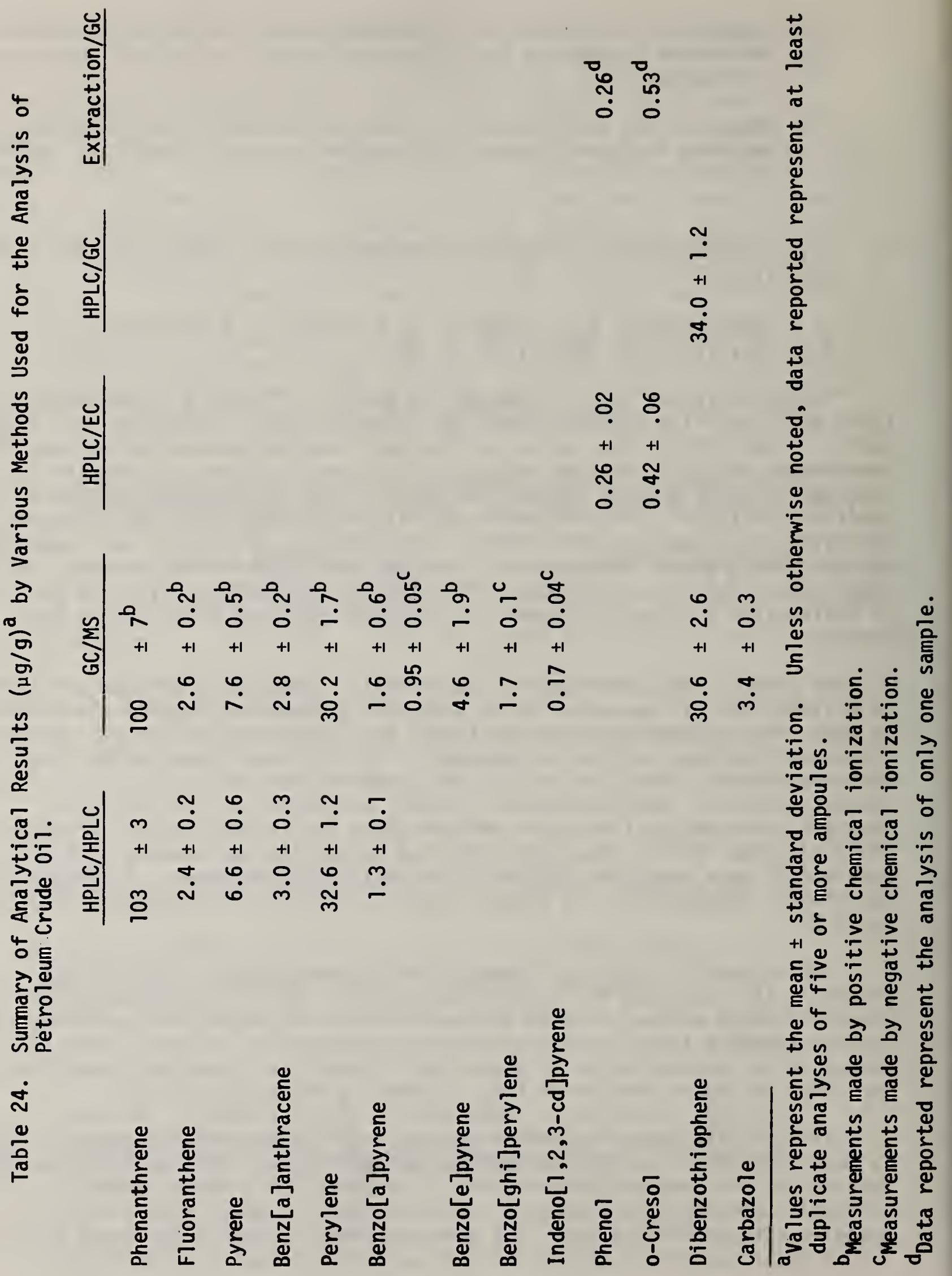


$3 \mathrm{~mol} / \mathrm{L} \mathrm{KCl}$ ) of $-1.00 \mathrm{~V}$ was used. Quantification was accomplished by use of standard additions.

A sample of SRM 1580 was analyzed to assess the accuracy of the procedure. The certified concentrations of phenol and o-cresol are $407 \pm 50$ and $385 \pm 50$, respectively. The concentrations, as determined using this procedure, were $375 \pm 15$ and $391 \pm 5$, respectively.

Measurements for the PAH, dibenzothiophene, and carbazole were made by gas chromatography/mass spectrometry (GC/MS) using both positive (CI) and negative chemical ionization (NCI). The NCI GC/MS procedure used for the determination of $B[a] P, B[$ ghi] $P$, and $I P$ is described in detail in technical report 21 of this section. For the positive CI GC/MS procedure, measurements were made on samples of the diluted oil without any sample pre-treatment or clean-up. Chromatographic separations were carried out on high resolution fused silica capillary columns with a liquid phase of either SE-52 or DB-5. The columns were connected directly to the ion source of the mass spectrometer through a specially designed interface. The interface allows the methane reagent gas to be brought in coaxially with the capillary column so that it sweeps the effluent from the column into the ion source. Selected ion monitoring defection was used to obtain additional selectivity; the quasi-molecular ion $(M+H)$ was monitored for each analyte. Quantitative measurements were made using perylene- $d_{12}$ as the internal standard. Relative response factors for the analytes were determined from a solution of the analytes plus perylene- $d_{12}$ gravimetrically prepared to mimic the concentrations of the analytes in the oil.

The sequential liquid chromatographic/gas chromatographic (HPLC/GC) procedure used for determination of dibenzothiophene involved the isolation of the analyte from the oil matrix by HPLC using a semi-preparative aminosilane column using a 7 percent methylene chloride in hexane mobile phase. The fraction collected from the HPLC column was then analyzed by capillary GC with flame photometric detection.

Detector calibration experiments were performed to determine the linearization exponent to be used for samples to be analyzed that day. The three calibration mixtures were composed of dibenzothiophene in hexane with 2-methyldibenzothiophene (2-M-DBTP) present for volume correction. The concentration of 2-M-DBTP was identical in each calibration mixture, while the relative concentration of DBTP was $1.0,2.0$, and 3.0 units, respectively. A linearization exponent was obtained from the slope of the regression line calculated from a plot of $l o g$ concentration DBTP versus log DBTP/2-M-DBTP response. This exponent was then used in the standard additions calculations to determine the concentration of DBTP.

The gas chromatograms of the LC fractionated samples gave well-resolved peaks for DBTP and for three other peaks which eluted after it. The first and largest peak in the chromatogram has a retention time identical to 4-methyldibenzothiophene; the second, either 2- or 3-M-DBTP; and the third was not identified but may be 7-M-DBTP. The first peak was used for volume correction since it gave the highest measured precision. The other two peaks were used similarly as a confirmatory check. For all samples analyzed, the concentration 
of DBTP determined by use of any of the three possible volume correction peaks agreed to within experimental error.

The extraction/GC procedure used for the determination of phenol and 0 -cresol has been reported previously $[1,5]$ and was used in the certification of SRM 1580. However, at the low concentrations of phenols present in the petroleum crude oil, we had problems distinguishing the analyte signals from background signals. We were able to salvage useful data from only one of the eight samples analyzed.

This material will be issued as SRM 1582 upon completion of statistical analysis of the data generated by the various methods described in this report. Certified values will be given for phenanthrene, fluoranthene, pyrene, benz[a]anthracene, perylene, benzo[a]pyrene, and dibenzothiophene. Information values will be provided for the other analytes measured.

\section{References}

[1] Hertz, H. H., Brown, J. M., Chesler, S. N., Guenther, F. R., Hilpert, L. R., May, W. E., Parris, R. M., and Wise, S. A., Determination of Individual Organic Compounds in Shale 0i1, Anal. Chem. 52, 1650-1657 (1980).

[2] May, W. E., Brown-Thomas, J. M., Hilpert, L. R., and Wise, S. A., Certification of Selected Polynuclear Aromatic Hydrocarbons in SRM 1580, Organics in Shale 0il, Anal. Methods and Biol. Fate of Polynuclear Aromatic Hydrocarbons, M. Cooke and A. J. Dennis, eds., Battelle Press, Columbus, Ohio, 1-16 (1981).

[3] Brown, J. M., Wise, S. A., and May, W. E., Determination of Benzo[a]pyrene and Recycled 0ils by a Sequential HPLC Method, J. Environ. Sci. Health A15 (6), 613-623 (1980).

[4] Shoup, R. E. and Meyer, G. S., Determination of Environmental Phenols by Liquid Chromatography/Electrochemistry, Anal. Chem. 54, 1164-1169 (1982).

[5] Guenther, F. R., Parris, R. M., Chesler, S. N., and Hilpert, L. R., Determination of Phenolic Compounds in Alternate Fuel Matrices, J. Chromatog. 207, 256-261 (1981). 
J. M. Brown-Thomas, R. G. Christensen, A. Cohen, W. E. May, R. Schaffer

SRM 976 is known to consist of three structural isomers of bilirubin, differentiated as III $\alpha$, IX $\alpha$, and $X_{I I I}$. Bilirubin IX $X_{\alpha}$ is the natural product. The III $\alpha$ and XIII $\alpha$ forms arise from the IX $\alpha$ as a result of a fragmentation and recombination reaction. It is believed that the reaction that gave rise to the III $\alpha$ and XIII $\alpha$ forms in the bilirubin used for SRM 916 took place early in the process of its isolation. McDonagh [1] discovered these isomers, isolated each by thin-layer chromatography, and measured their molar absorptivities. He estimated their proportions in SRM 916 to be $10: 75: 15$, respectively. We had confirmed the ratio by using his molar absorptivities and TLC. Now we undertook to measure the isomer proportions by liquid chromatographic analysis and to confirm their molar absorptivities.

Conditions were: Silica column, methylene chloride solvent containing 0.7 percent $(\mathrm{v} / \mathrm{v})$ acetic acid, detection at $450 \mathrm{~nm}$. The relative peak areas found (mean of five measurements \pm standard error of the mean) were $102.6 \pm 0.9$, $794.6 \pm 2.6$, and $104.0 \pm 1.6$ for the III $\alpha$, IX $\alpha$, and XIII $\alpha$ isomers, respectively. These are not representative of the relative amounts of the isomers, however, because the individual molar absorptivities are known to be different.

The determination of molar absorptivities was accomplished by separating quantities of the bilirubin isomers on a preparative scale liquid chromatographic column, using a chromatographic scheme similar to that described above and in reference [2]. After the isomer-containing fractions were collected, the solvent was removed by using a rotary evaporator; then each of the fractions was subjected to another similar fractionation. This resulted in three isomer fractions that were obtained in smaller volumes of solvent, thus minimizing possible contamination from solvent impurities and/or column bleed. Solutions of each isomer fraction were made in methylene chloride containing 0.7 percent acetic acid and the absorbances at the peak maxima and at $450 \mathrm{~nm}$ were noted. Each solution was then subjected to an analytical liquid chromatography separation to determine its proportional content of the three isomers.

The "contamination" isomers present in each solution ranged from less than 0.5 percent to about 5 percent. By an iterative process, the extinction coefficients were corrected to eliminate errors from that source. The millimolar absorptivities at $450 \mathrm{~nm}$ were found to be: $62.35,61.67$, and 49.00 for the III $\alpha, I X \alpha$, and XIII $\alpha$ isomers, respectively. The precision of these measurements is estimated to be about one percent relative standard deviation. The presence of any systematic bias, such as that from possible inclusion of solvent in the bilirubin crystals, is unknown.

Using these molar absorptivities at $450 \mathrm{~nm}$ and the relative peak areas noted above, the relative amounts of the isomers in SRM 916 were calculated to be: $0.099 \pm 0.002,0.774 \pm 0.008$, and $0.127 \pm 0.002$ for the III $\alpha, I X_{\alpha}$, and XIIIa isomers, respectively. The uncertainties are dominated by the contributions from the absorptivity determinations. Now that the necessary techniques have been perfected, we plan to make a more definitive measurement. 


\section{References}

[1] A. F. McDonagh and F. Assisi, Commercial Bilirubin: A Trinity of Isomers, FEBS Letters 18, 315-317 (1971).

[2] T. A. Woodbridge and D. A. Lightnor, Separation of the III $\alpha$, IX $\alpha$, and XIII $\alpha$ Isomers of Bilirubin and Bilirubin Dimethylester by HighPerformance Liquid Chromatography, J. Liquid Chromatog. 1, 657-658 (1978).

6. Determination of Organic Nutrients in SRM 1549, Non-Fat Milk Powder (15320)

I. N. Black, L. D. Fong, S. A. Margolis

Liquid chromatographic (LC) methods have been developed for the analysis of ascorbic acid, nicotinic acid (NA), and nicotinamide (NAM). The method for ascorbic acid uses a semi-preparative amine column, and the analyte is eluted isocratically with a solvent composed of 30 percent $0.005 \mathrm{~mol} / \mathrm{L}$ monobasic sodium phosphate in acetonitrile with 0.1 percent mercaptoethanol. Detection is UV $(254 \mathrm{~nm})$. At a flow rate of $3.5 \mathrm{~mL} / \mathrm{min}$, the ascorbic acid elutes at 213.5 minutes and is resolved from isoascorbic acid and dehydroascorbic acid (figure 21). The limit of detection for ascorbic acid was below $1.0 \mu \mathrm{g}$.

Figure 21. Separation of ascorbic acid, dehydroascorbic acid, and isoascorbic acid by HPLC using an amine column with isocratic elution.

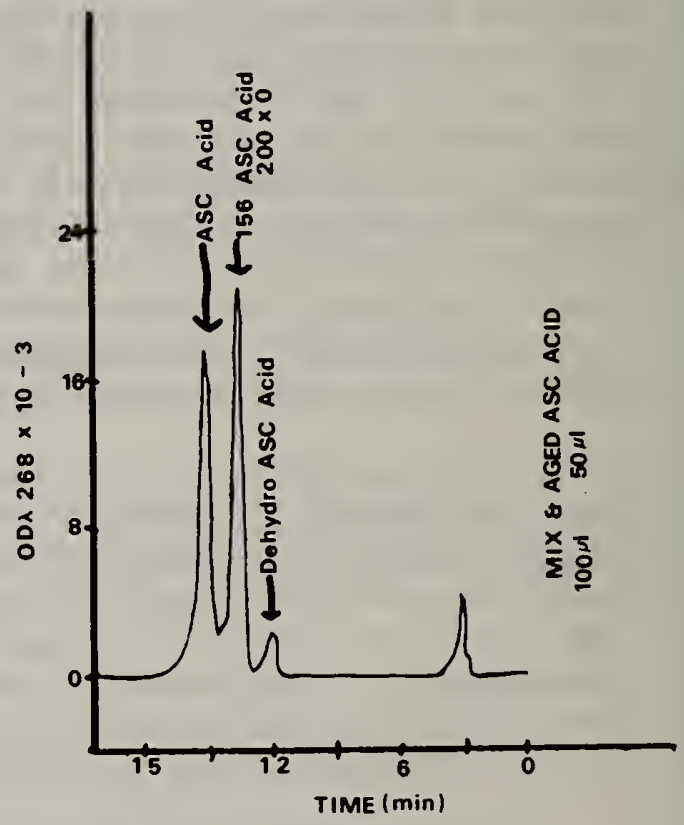

Several methods have been evaluated for the analysis of the NA and NAM. Reversed-phase LC methods using ion-pairing and an octadecylsilane column separated either one compound or the other, but no method was capable of resolving both compounds with $k$ 's of 5 to 10 . A method using an analytical amine column and a solvent similar to that used for ascorbic acid analys is retained NA with a $k^{\prime}$ of about 10 , but did not retain NAM. A strong cation 
exchange resin equilibrated with $0.01 \mathrm{~mol} / \mathrm{L}$ formic acid: acetonitrile 95:5 ( $v: v$ ) was used to retain both $N A$ and NAM. The analytes were eluted from the column with a 13-40 percent linear gradient (15 minutes), $0.1 \mathrm{~mol} / \mathrm{L}$ ammonium acetate $\mathrm{pH} 6.0$, acetonitrile $75: 25(\mathrm{v}: \mathrm{v})$. The NA and NAM were well separated (figure 22) and the limits of detection were less than $0.1 \mu \mathrm{g}$ for each analyte.

Figure 22. Separation of nicotinic acid and nicotinamide by HPLC using a cation exchange column with gradient elution.

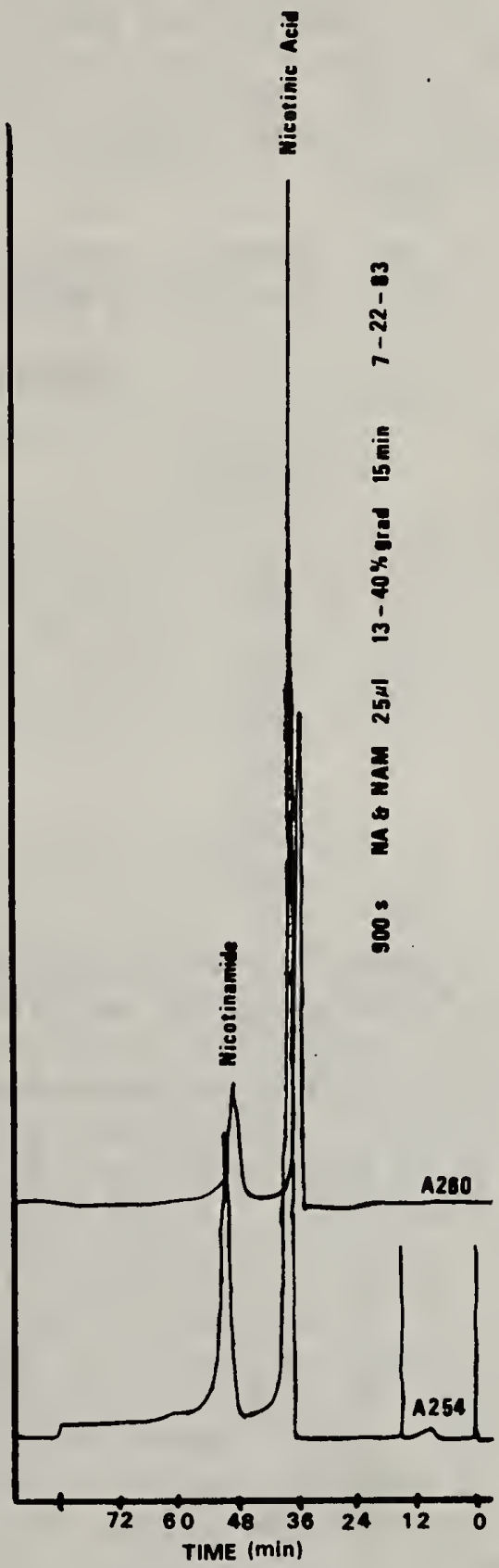

The method for extracting and stabilizing the ascorbic acid from non-fat dry milk consists of dissolving 0.1-1.0 g of sample in $2 \mathrm{~mL}$ of a 0.1 percent aqueous solution of dithiothreotol, precipitating the proteins with $2 \mathrm{~mL}$ of meta phosphoric acid $\left(\mathrm{HPO}_{3}\right)$, and then adding $1 \mathrm{~mL}$ of acetonitrile. The suspension is centrifuged and an aliquot of the clear supernatant fluid is 
analyzed by the method described above. We are using dithiothreotol to reduce dehydroascorbic acid to ascorbic acid and to prevent oxidation of ascorbic acid. The optimum conditions for the extraction of ascorbic acid are being evaluated. Table 25 summarizes our effort to date. The optimum concentration for the meta phosphoric acid lies above 8 percent, and the time intervals between the various additions appear to be unimportant if completed within one hour. The identity of the ascorbic acid peak from the chromatogram was confirmed by collecting the peak in five consecutive fractions and demonstrating that the material in each fraction exhibited a UV spectrum characteristic of ascorbic acid. The ascorbic acid content in SRM 1549 (non-fat dry milk), when analyzed by the AOAC method at the FDA, is $72 \mu \mathrm{g} / \mathrm{g}$. Our preliminary results are $55-60 \mu \mathrm{g} / \mathrm{g}$.

Table 25. Effect of $\mathrm{HPO}_{3}$ and Time on Ascorbic Acid Extraction from Non-Fat Dry Milk.

- - - - Ascorbic Acid Content ( $\mu \mathrm{g} / \mathrm{g}$ Sample) - - - -

\begin{tabular}{|c|c|}
\hline $\begin{array}{l}\text { Percent } \\
\mathrm{HPO}_{3} \\
\end{array}$ & No Delay \\
\hline 0 & 3 \\
\hline 2 & 42 \\
\hline 4 & 57 \\
\hline 6 & 52 \\
\hline 8 & 65 \\
\hline 12 & 54 \\
\hline 16 & 57 \\
\hline
\end{tabular}

One Hour Delay in

$\mathrm{HPO}_{3}$ Addition

55
One Hour Delay in

$\mathrm{CH}_{3} \mathrm{CN}$ Addition

The method for extracting NA and NAM from non-fat dry milk and rice flour consists of hydrolyzing the nicotinic acid containing organic molecules to yield NA. The hydrolysis is performed with a 10 percent suspension of calcium hydroxide at elevated temperatures. Experiments with NAD and NADH indicate that they will be completely hydrolyzed to NA and NAM in three hours at $75^{\circ} \mathrm{C}$. Under these same conditions, NAM is partially hydrolyzed to NA in the absence of food materials. In the presence of rice flour, NA is released from its organic sources, but NAM does not appear to be hydrolyzed to NA. Furthermore, only 30 percent of the added NA is recovered as NA after the hydrolysis reaction. The remainder appears to be converted to a compound with a retention time that is similar to that for NAM. We are now searching for optimum conditions for stabilizing and extracting NA and NAM from non-fat dry milk and rice flour. The vitamin content of these materials is being determined by the standard additions method of analysis. Preliminary results indicate that the rice flour contains $30 \mu \mathrm{g} / \mathrm{g}$ NA. Determination of NA in SRM 1549 is in progress. 
7. Use of Polynuclear Aromatic Hydrocarbon Generator Columns for Calibrating Air Sampling Systems $(19105,15320)$

S. N. Chesler, F. R. Guenther, W. H. Zoller (University of Mafyland)

A method for measuring vapor pressures of high molecular weight polycyclic aromatic hydrocarbon (PAH) at temperatures between 60 and $300{ }^{\circ} \mathrm{C}$ has been developed. This study is a continuation of work done in the Liquid Chromatography Group [3] and utilizes a method similar in principle. The work done previously covered the $10-50{ }^{\circ} \mathrm{C}$ range and benz[a] anthracene (molecular weight 228) was the largest compound studied. In this method, an all-glass sampling system, along with a gas chromatographic (GC) oven, allows an extensive temperature range to be studied $\left(60-300{ }^{\circ} \mathrm{C}\right)$. Vapor pressure data is important in the prediction of transport mechanisms from industrial processes or waste sites, the adsorption tendencies of PAH on airborne particulate matter, and in the calculation of Henry's law constants to predict water/air distributions. This study has also resulted in the construction of a constant vapor density generator to use in the calibration of air sampling equipment.

The vapor generator columns were constructed with $6 \mathrm{~mm}$ 0.D. glass packed with 100 mesh glass beads. Three zones of the pure crystalline compound under study are packed within the last $3 \mathrm{~cm}$ of glass beads. A fused silica capillary column provides the physical linkage between the generator column and a cold trap. The generator column is housed within a GC oven, and a thermocouple (situated within the generator column at the entrance to the fused silica line) is used to measure temperature.

To measure the vapor pressure at a particular temperature, the oven is equilibrated at that temperature for a period of one-half hour. The vapor is vented during this time. To begin trapping, the trap line is opened at a valve and the vent line closed. The vapor is then directed through the fused silica line, through the trap and valve, and into a water filled inverted volumetric flask. After collecting a known volume of saturated vapor, the trap is washed out with methylene chloride. A suitable internal standard is added and the resulting solution analyzed by GC. With the collected volume known (volumetric flask) and the amount collected known (GC analysis), the vapor density can be calculated. Then by calculating back to the generator column conditions (temperature/pressure corrections), the saturated vapor density can be found. Vapor densities can be converted to vapor pressures through use of the ideal gas law. The vapor pressure values are in reasonable agreement with some literature values obtained for the same temperature range (Table 26). However, a bias exists between these values and the data obtained previously [3] when extrapolated to the same temperature region. Reasons for these differences are being investigated.

Vapor density measurements for anthracene, fluoranthene, pyrene, 1-methylpyrene, chrysene, benzo[a]anthracene, benzo[e]pyrene, and perylene have been made. Table 26 shows some vapor pressure data from this work and, for comparison, some literature values. Measurements of benzo[a]pyrene, benzo[ghi]perylene, indeno[ghi]pyrene, and some nitro-PAH are planned. 
Table 26. Vapor Pressure in Pascals at $100^{\circ} \mathrm{C}$.

Present Work

Anthracene

Pyrene

1-Methylpyrene

Benz[a]anthracene

Chrysene

Benzo[e]pyrene
2.92

1.81

1.20

0.137

0.0154

0.00994
Literature

$3.144[1], 0.0632[2], 1.37[3]$

$1.793[1], 1.150[2], 0.976[3]$

$0.114[2], 0.0201[3]$

$0.0160[2]$

A semi-portable 1-methylpyrene generator column has also been developed. This column is packed in a similar method, as stated above. 1-Methylpyrene was chosen because it is not generally found (relative to other PAH) in environmental samples and can therefore be used as an internal standard for the analysis of PAH in air. The generator column is made of $6 \mathrm{~mm} \mathrm{0.D.} \mathrm{pyrex}$ glass wrapped with 28 gauge Nichrome wire. A tapered glass tip leads from the column to a fine tip from which the vapor is emitted. The tip is heated from a separate circuit of wrapped Nichrome wire. The entire generator column is insulated with ceramic fiber cloth. A proportional temperature controller monitors and heats the main body of the column. A Variac is used to heat the tip to a temperature well above the column temperature.

In use, the column is equilibrated at the desired temperature for one hour. Then, several calibration runs are made by collecting the vapor for 30 minutes with an inverted miniature glass funnel in which $100 \mu \mathrm{L} / \mathrm{min}$ of air is drawn. The collected condensate is then washed out with methylene chloride and an internal standard added. The resulting solution is then measured by GC. After calibration, the generator column can be used to spike 1-methylpyrene into air sampling traps. In this way, 1-methylpyrene is introduced into the system as a vapor and is allowed to interact with adsorbents in a similar way as components in sampled air. This method for introduction of internal standards should allow more accurate accounting for losses that occur during sample work-up.

References

[1] Bradley, R. S. and Cleasby, T. G., J. Chem. Soc. 1690-1692 (1953).

[2] Hoyer, H. and Peperle, W., Z. Elektrochem. 62, 61-66 (1958).

[3] Sonnefeld, W. J., Zoller, W. H., and May, W. E., Ana1. Chem. 55, $275-280$ (1983). 
8. Detection of Arson--Analysis of Accelerant Residues on Soot (16630)

S. N. Chesler, F. R. Guenther

The purpose of this project was to determine if residues or products from accelerant combustion could be detected on soot. The ultimate goal was to be able to determine, from collected soot, if accelerants had been involved in a fire when other physical evidence has been destroyed. Our immediate goal was to determine if any unconsumed vapors would be adsorbed onto soot as it leaves flaming accelerants. This hypothesis proved to be incorrect, as we could find no trace of an accelerant pattern on soot generated in the laboratory. Our attention thus turned to investigation of higher molecular weight species that may be produced in the sooting process.

To produce soot in the laboratory, we burned a number of accelerants in pyrex beakers and collected the soot on watch glasses positioned over the top of the beakers. The soot was then scraped and collected. Approximately $200 \mathrm{mg}$ was then extracted with hexane using sonic vibration. The resulting solution was centrifuged and the supernate transferred to a clean GC sample vial. These samples were then concentrated under a flow of purified $\mathrm{N}_{2}$ to approximately $200 \mu \mathrm{L}$ and a $1 \mu \mathrm{L}$ aliquot injected onto a $30 \mathrm{~m} \times 0.25 \mathrm{~mm} \mathrm{I} . \mathrm{D}$. capillary column coated with an immobilized SE-52 phase to $0.25 \mu \mathrm{m} f i l m$ thickness. Accelerants studied included unleaded and leaded gasoline, kerosene, paint thinner, charcoal lighter fluid, methanol, toluene, and hexane.

Gas chromatographic (GC) analysis showed a clearly recognizable pattern among all accelerant soots, except from methanol, of polycyclic aromatic hydrocarbons (PAH). Although small amounts of PAH are produced in any combustion process, the magnitude of the concentration of PAH on the soot samples was much greater than expected from wood or material burns. Subsequent testing of soot collected from burning wood, rug, and small amounts of plastics showed no pattern of PAH at this concentration level. It seems very probable that soot PAH profiles might serve as useful indicators of arson.

This method will be subjected to stringent field testing. We are in the process of obtaining samples from actual fires when arson is known to have occurred, and the presence of accelerants are suspected, as well as samples from accidental fires. So far, we have examined samples from a single fire and have observed a PAH profile from soot collected at the fire scene. While arson is not suspected in this nonresidential fire, certain aerosol cans and other materials of unknown composition were consumed. These results may point to a possible interference in the method. We will conduct further investigations in an attempt to eliminate this source of interference and therefore false diagnosis. One possible explanation for this data is that large amounts of plastics produce soot mimicking accelerant soot in a flaming fire of high temperatures. 


\section{Analysis of EPA Performance-Evaluation Standard Samples (15320)}

S. N. Chesler, F. R. Guenther, R. E. Rebbert

This work was undertaken as part of the Center for Analytical Chemistry effort to assist the Quality Assurance Branch of EPA in Cincinnati with their performance evaluation and quality control studies and to establish traceability to NBS. Our participation in this program was begun in late FY 1982 and will continue into FY 1984. In particular, the following types of samples were analyzed: quality control samples (QCS) for water quality analyses for volatile organics--series 17; QCS for drinking water analyses for (1) chlorinated hydrocarbon pesticides--series 4 and (2) trihalomethanes--series 3 ; and QCS for priority pollutants/hazardous wastes/toxic chemicals for chlorinated hydrocarbon pesticides--series 18. In addition, at EPA's request, we also analyzed QCS for purgeables (sample with 30 volatile compounds in methanol).

In the volatile organic series, four solutions with different concentrations of nine volatile halocarbons in methanol were analyzed by gas chromatography with a flame ionization detector (FID). These compounds were: chloroform, 1,1,1-trichloroethane, 1,2-dichloroethane, carbon tetrachloride, trichloroethylene, bromodichloromethane, dibromochloromethane, tetrachloroethylene, and bromoform. The concentrations of the various compounds varied from 25 to $600 \mu \mathrm{g} / \mathrm{g}$. Triplicate measurements were made on six ampoules for each solution. In this case, the less sensitive FID was used instead of the more sensitive electron capture detector (ECD), which is usually employed. However, the ECD is 500-1000 times less responsive to 1,2-dichloroethane than trichloroethane, which eluted from the column only 0.3 minutes before it. Thus, the dichloroethane is lost in the tail of the trichloroethane when an ECD is used; both of these compounds are easily detected when a FID is used.

For the chlorinated hydrocarbon pesticides--series 4, an ECD was utilized to measure the concentration of lindane, endrin, and methoxychlor in six ampoules selected from the lot. At least five injections were made from each ampoule to determine the average concentrations, which varied from less than 1 to almost $100 \mu \mathrm{g} / \mathrm{g}$. In this study, our results showed an ampoule-to-ampoule variation in the lindane concentration and the appearance of an unknown compound whose concentration changed inversely with the concentration of lindane. This suggests that in some ampoules the lindane had decomposed and produced the unknown compound.

The four solutions of the trihalomethane series were also analyzed with an ECD. Again, at least five measurements were made for each of the six ampoules from each solution. The average concentrations of the compounds (chloroform, bromodichloromethane, dibromochloromethane, and bromoform) determined in methanol varied from 40 to $660 \mu \mathrm{g} / \mathrm{g}$.

The chlorinated hydrocarbon pesticide--series 18 was analyzed with an ECD. Six injections from six ampoules from each of four solutions were used to determine concentrations of the six compounds (heptachlor, aldrin, $p, p^{\prime}-D D E$, dieldrin, $p, p^{\prime}-D D D$, and $\left.p, p^{\prime}-D D T\right)$. The average concentrations varied from 0.1 to $1.1 \mu \mathrm{g} / \mathrm{g}$. 
Although the purgeable samples contained 30 compounds, EPA requested that we analyze for chloromethane, chloroethane, and bromomethane only. These components were analyzed by an internal standard (vinyl chloride) method with a $30 \mathrm{~m} \times 0.25 \mathrm{~mm}$ I.D. capillary column coated with $1 \mu \mathrm{m}$ film of an immobilized SE-52 phase. A post-column trap packed with activated alumina was installed between the column exit and the FID. This trap selectively removes the methanol solvent, but allows the analytes of interest to pass through to the detector unhindered and be measured without interference from the solvent. Our analytical results and those of EPA agreed closely for all analytes except for bromomethane. Differences in results for this analyte are being resolved.

Tables of these results are not given since these samples will be used by EPA in future performance evaluation studies and confidentiality is required. This cooperative effort with EPA/Cincinnati will continue into FY 1984 . In particular, a formal means for showing traceability to NBS will be developed for these and future quality control samples.

\section{Preparation and Certification of SRM Calibration Solutions (15320)}

J. M. Brown-Thomas, S. N. Chesler, F. R. Guenther, D. K. Hancock,

W. F. Kline, W. E. May, R. M. Parris, R. E. Rebbert, D. J. Reeder

The Standard Reference Materials (SRM's) that have been certified for trace organic analysis fall into two general categories--those to be used for calibration of analytical instrumentation and those to be used for quality assurance of analytical procedures. Our major thrusts during the past few years have been directed toward the development of SRM's for trace organic analytes in complex natural matrices, i.e., SRM 1580 (Organics in Shale 0i1) and SRM 1649 (Urban Dust/Organics) for the latter purpose.

During FY 1983, in addition to certifying another complex matrix SRM (1582, Organics in Petroleum Crude), we have produced and certified a number of calibration SRM's (solutions or mixture). Each of these SRM's, with the exception of SRM 1514 (Differential Thermal Analysis Purity Set), contain a number of selected analytes dissolved in a pure solvent. SRM 1514 is composed of three sets of p-aminobenzoic acid--phenacetin mixtures. These SRM's can be used for calibrating analytical instrumentation. Many calibration SRM's are also useful as standard addition analytes in the analysis of complex mixtures.

Halocarbons in Methanol for Drinking Water Analysis, SRM 1539, has been completed and is now available. This SRM contains seven halocarbons ranging from chloroform to bromoform and, when diluted $1: 10^{5}$ in halocarbon-free water, produces a water-containing halocarbon at commonly encountered concentration levels $(0.5-50 \mathrm{mg} / \mathrm{L})$.

Chlorinated Pesticides in Isooctane, SRM 1583, has been certified and will be available shortly. This SRM contains six common pesticides-- $\delta-$ and $\gamma-B H C$, aldrin, heptachlor epoxide, $p, p^{\prime}-D D E$, and $p, p^{\prime}-D D T--a t$ certified levels near $1 \mu \mathrm{g} / \mathrm{g}$. This SRM will serve the residue chemist and provide for the first time an NBS traceable standard for environmental measurements of pesticides in water, food, tissues, and airborne particulates. A related material, SRM 1585, 
Polychlorinated Biphenyls ( $P C B$ ) Congeners in Isooctane, contains eight individual PCB congeners. Each of these congeners, listed in the table below, were chosen for their specific chemical toxicological or environmental effects.

PCB

Number

Congener

Rationale

3 4-Monochlorobiphenyl

15 4,4'-Dichlorobiphenyl

$282,4,4^{\prime}$-Trichlorobiphenyl

$522,2^{\prime}, 5,5^{\prime},-$-Tetrachlorobipheny 1

$773,3^{\prime}, 4,4^{\prime}$-Tetrachlorobiphenyl

$1012,2^{\prime}, 4,5,5^{\prime}$-Pentachlorobiphenyl

A prevalent, incidentally generated $P C B$

A prevalent, incidentally generated $P C B$

A predominant peak of Aroclors 1016, 1242

$1382,2^{\prime}, 3,4,4^{\prime}, 5^{\prime}$-Hexachlorobiphenyl

A predominant peak of Aroclors 1016, 1242

A very toxic, less metabolized PCB

A predominant peak of Aroclors 1254, 1260

$1532,2^{\prime}, 4,4^{\prime}, 5,5^{\prime}$-Hexachlorobipheny 1

A predominant peak of Aroclors 1254, 1260

A predominant peak of Aroclors 1254, 1260

The congeners are certified at the $\mu \mathrm{g} / \mathrm{g}$ level and also can be used as qualitative markers for determination of the presence of incidentally generated PCB and the various commercially produced PCB mixtures (Aroclors).

SRM 1586, Isotopically Labeled and Unlabeled Priority Pollutants in Methanol, has been prepared specifically to be of use to those environmental analysts who use the U.S. Environmental Protection Agency (EPA) Standard Methods $1624,1625,624,625,524$ or 525 . SRM 1586 consists of two separate solutions of ten priority pollutants. The compounds in one solution carry either a deuterium or carbon-13 label, while the second solution contains the same compounds with no isotopic labels. The compounds, EPA compound class, type of label, and nominal concentrations are listed in the table below.

Compound Type

Base/Neutrals

Purgeables/

Volatiles
Selected Compound (Isotopic Label)

Phenol $\left(d_{6}\right)$

2,4-Dichlorophenol

$\left(3,5,6-d_{3}\right)$

2-Nitrophenol $\left(3,4,5,6-d_{4}\right)$

Bis-2-Ethylhexyl Phthalate

$$
\left(3,4,5,6-d_{4}\right)
$$

Nitrobenzene $\left(d_{5}\right)$

Naphthalene $\left(d_{8}\right)$

Benzo[a]pyrene $\left(d_{12}\right)$

Carbon Tetrachloride $\left({ }^{13} \mathrm{C}\right)$

Chlorobenzene $\left(d_{5}\right)$

Benzene $\left(d_{6}\right)$
Nominal Concentration $\mu \mathrm{g} / \mathrm{mL}$

100

100

100

60

100

100

100

100 
These SRM solutions will be used either together or separately to calibrate and/or test the user laboratory's employment of the various EPA standard methods. The SRM was certified by the use of gravimetry, gas chromatography (GC), and liquid chromatography (LC); isotopic purity was verified by mass spectrometry.

SRM 1584 is a methanol solution of the eleven phenols included in the EPA's list of priority pollutants. The concentrations range from 15 to $65 \mu \mathrm{g} / \mathrm{mL}$. This SRM will be useful for calibrating gas and liquid chromatographic instrumentation, for phenol analysis, and can also be used for fortifying water samples. This SRM was certified by use of gravimetry, GC, and $L C$.

SRM 1514 is composed of a set of four vials containing "pure" phenacetin and phenacetin doped with nominately $0.7,2$, and 5 mole percent p-aminobenzoic acid. These materials are intended for use in evaluating the method of determining purity by differential scanning calorimetry. The actual amounts of p-aminobenzoic acid present were determined by liquid chromatography and confirmed by spectrofluorimetry.

The preparation and certification of (1) Nitrated-Polycyclic Aromatic Hydrocarbonss (N-PAH) and (2) Dioxin Calibration Solution SRM's are planned for early FY 1984 . The N-PAH SRM project represents a joint effort between NBS and the Coordinating Research Council, Inc. (CRC), Atlanta, GA. The CRC is funding a commercial laboratory to synthesize 20 pure ( $>99$ percent) N-PAH. We will dissolve them in an appropriate solvent and certify their concentrations. This SRM will be intended for use in calibrating instrumentation to be used in the analysis of $\mathrm{N}-\mathrm{PAH}$ in complex mixtures.

We are currently investigating the stability of $\mathrm{N}-\mathrm{PAH}$ solutions using 2-nitronaphthalene, 2-nitrofluorene, 9-nitroanthracene, 1-nitropyrene, and 6 -nitrobenzo[a]pyrene as representative compounds from the class. Methylene chloride, methanol, and nitromethanol solutions have been prepared. The solutions are being stored at room temperature or at $-10^{\circ} \mathrm{C}$ in either clear or amber ampoules. We have found nitromethane and methanol solutions of these $\mathrm{N}$-PAH stored at $-10{ }^{\circ} \mathrm{C}$ to be stable for at least six months.

The Dioxin Calibration Solution SRM is being prepared using NBS funding and will have certified concentrations $(2100 \mathrm{ng} / \mathrm{mL})$ of ${ }^{13} \mathrm{C}$ labeled and unlabeled 2,3,7,8-tetrachlorodibenzo-p-dioxin (the most hazardous of the family of 75 different chlorinated dioxins) in separate isooctane solutions. 
11. Reference Methods for Certification of Seven Enzymes in SRM 909 (15606)

\section{R. Schaffer}

The catalytic (activity) concentrations of the seven enzymes acid phosphatase (ACP), alkaline phosphatase (ALP), aspartate aminotransferase (AspAT), alanineaminotransferase (AlaAT), creatine kinase (CK), lactate dehydrogenase (LDH), and $\gamma$-glutamyltransferase $(\gamma-G T)$ were determined in NBS Human Serum Standard Reference Material (SRM 909). Separate teams of clinical chemistry experts selected specific analytical methods for each of the enzymes and then performed them. The results obtained by each team in the third (and last) round of team testing provided the activity concentrations assigned to the SRM. All of the methods are spectrophotometric and are primarily those recommended as candidate reference methods by the Standards Committee of the American Association for Clinical Chemistry and/or the Experts Panel on Enzymes of the International Federation of Clinical Chemistry. However, the reaction temperature for all of these analyses was $29.77{ }^{\circ} \mathrm{C}$, the melting point of gallium for which NBS issues SRM 1968. Interlaboratory standard deviations of less than 10 percent were achieved (see Table 27 and figure 23). Measurements made one year after the conclusion of the multilaboratory studies showed unchanged enzyme activities, i.e., activities within the uncertainties of the assigned values. This work is described in detail in NBS Special Publication 260-83 on "The Measurement of the Catalytic (Activity) Concentration of Seven Enzymes in NBS Human Serum SRM 909," published in June 1983.

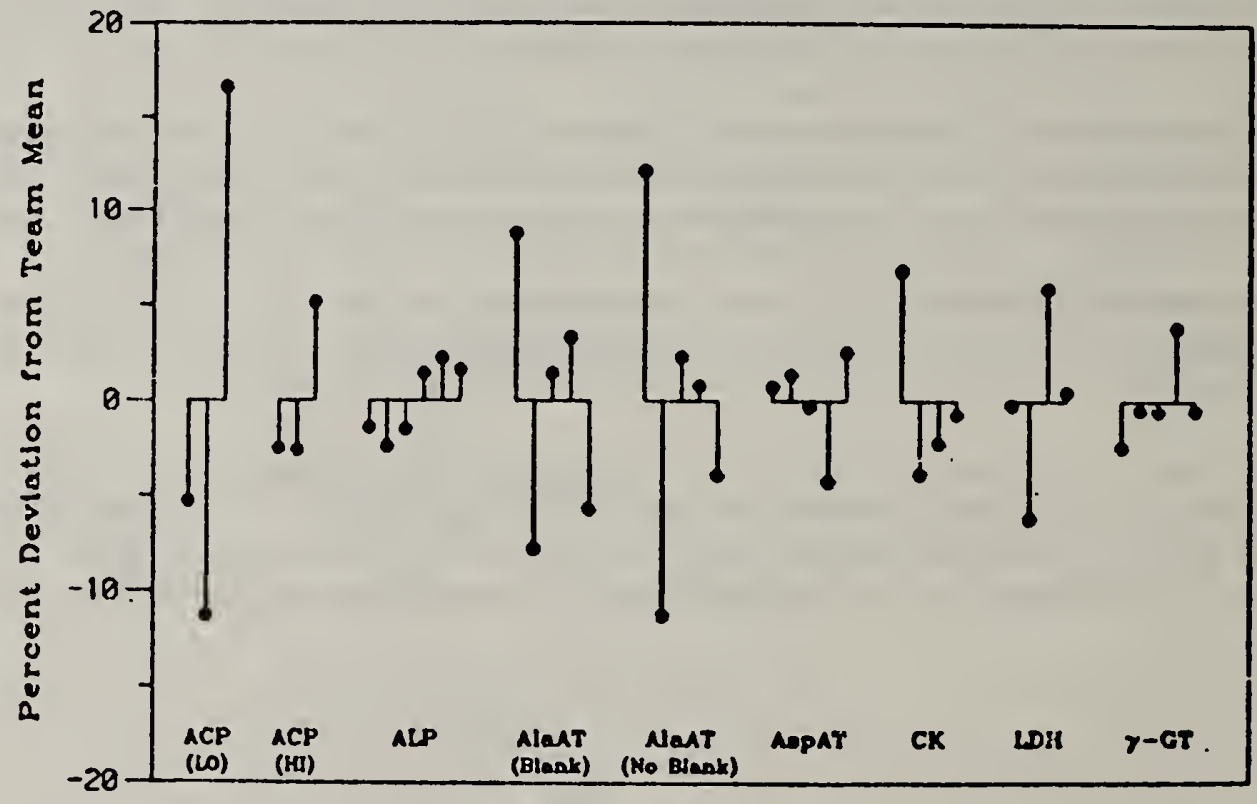

Figure 23. Summary of all results in terms of percent deviations from enzyme team means. Each laboratory is represented by its mean value (closed circle, $\bullet$ ). 


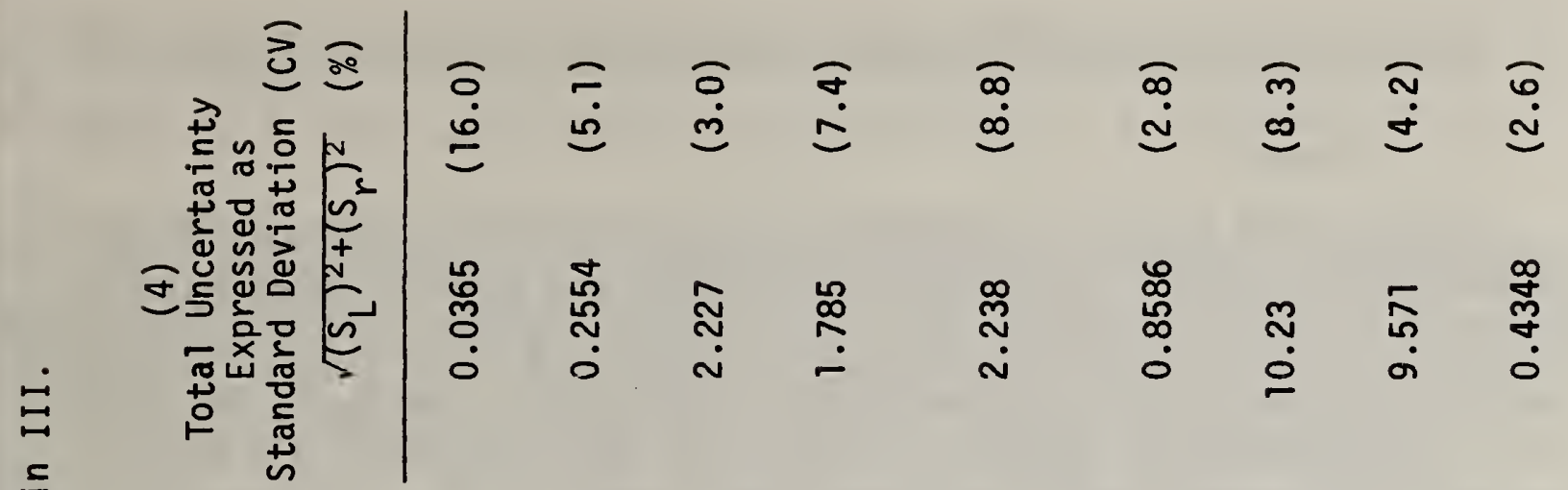

$$
\begin{aligned}
& \text { 产 }
\end{aligned}
$$

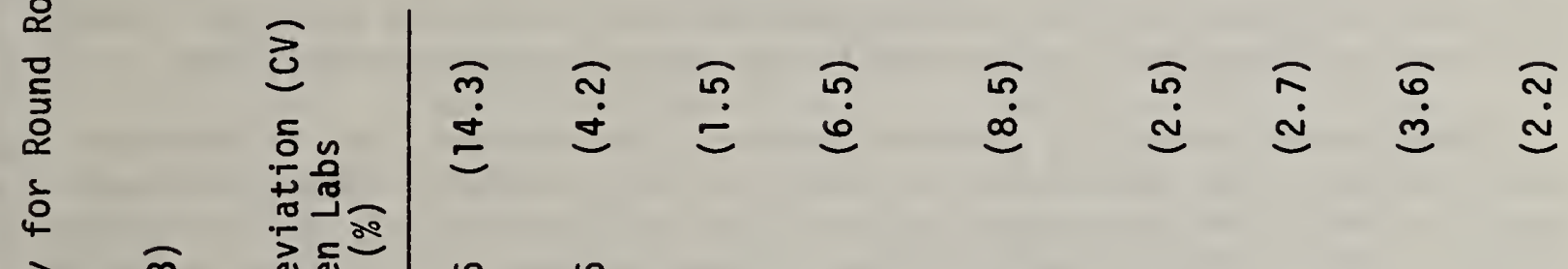

$$
\begin{aligned}
& \text { 跤 } \\
& \frac{5}{\frac{5}{2}}
\end{aligned}
$$

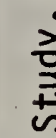

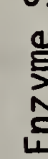



$$
\begin{aligned}
& \frac{1}{2}
\end{aligned}
$$

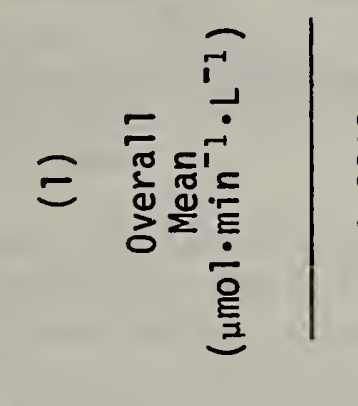

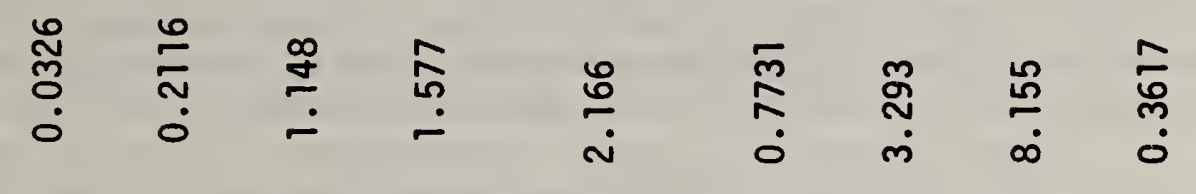

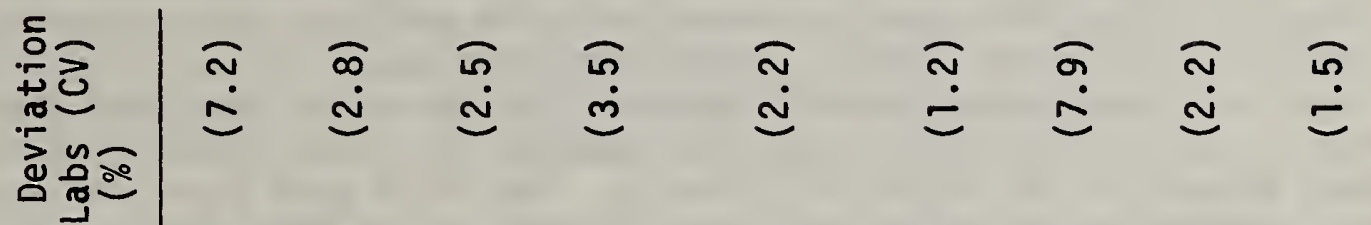

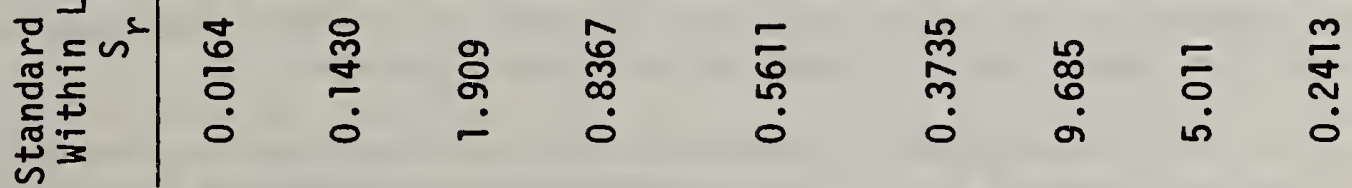

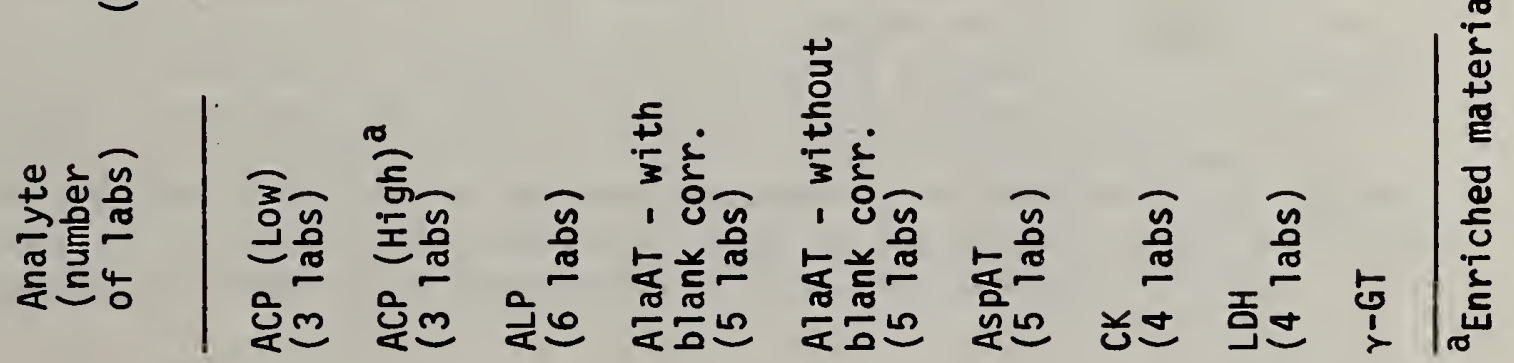




\section{Metal-Ion Affinity Chromatography Supports for Protein Separation (26111)}

L. A. Alexander, A. E. Davidson, A. J. Fatiadi, C. L. Hsu, S. A. Margolis,

D. J. Reeder

Descriptions of the development and use of immobilized metal ions on chromatography supports for separating proteins and other biomaterials are beginning to appear in the scientific literature. Exploiting the interactions between solutes and immobilized metal ions appears to be a viable direction for exploring new ways to achieve protein separations. We began synthesis of several chromatographic supports to which the following metal ions were covalently attached: $\mathrm{Mn}^{2}, \mathrm{Fe}^{2+}, \mathrm{Fe}^{3}, \mathrm{Co}^{2}, \mathrm{Ni}^{2}{ }^{+}, \mathrm{Cu}^{2}, \mathrm{Zn}^{2}$, and $\mathrm{Cd}^{2}{ }^{+}$. The primary support material was polystyrene-divinylbenzene copolymer beads with covalent attachment of the metal ions by an iminodiacetate ligand.

These column materials were tested in a low-pressure liquid chromatographic system using flow rates of approximately $1 \mathrm{~mL} / \mathrm{min}$. Human serum was placed on the column and sequentially eluted with increasing ionic strength buffers. Peak fractions were obtained, concentrated, and analyzed by sodium dodecylsulfate (SDS) polyacrylamide gel electrophoresis, thin-layer isoelectric focusing, and two-dimensional electrophoresis.

of the materials tested, the column support coupled with $\mathrm{Cu}^{+}{ }^{+}$appears to be the most interesting. Three major protein peaks are consistently eluted, using a three-step sodium acetate, $\mathrm{pH} 5.5$ gradient with increasing ionic strength. The first peak contained albumin and many other proteins. The protein distribution in the second and third peaks is now being investigated. There is a large proportion of protein in the second peak that has a basic charge and migrates to an apparent isoelectric point (pI) of about 9 . This peak appears to be devoid of albumin. The third peak has a more neutral distribution of proteins, with the majority of protein having a pI of about 7 . Albumin is absent in this peak as well.

We are systematically exploring the interactions of proteins with a wide variety of supports to further understand the mechanisms of interactions of the metal-binding ligands with individual protein molecules. By studying these specific interactions, we expect to gain a greater knowledge of protein structure and the mechanisms of separation. Use of these and similar supports may play an important analytical, as well as industrial, role in biomolecule separations. 


\title{
13. Thin-Layer Isoelectric Focusing for Sensitive Protein Analysis (26111)
}

\author{
C. L. Hsu, D. J. Reeder, K. L. Richie
}

Separation of protein mixtures into component parts is a goal of many techniques in biotechnology. Separation by isoelectric focusing is accomplished in a $\mathrm{pH}$ gradient stabilized by carrier ampholytes between two electrodes. In this technique, proteins migrate until they align themselves at their isoelectric point (pI) at which point a protein possesses no net overall charge and will therefore concentrate as migration ceases. Resolution in isoelectric focusing is a function of the square root of the voltage gradient applied. Typically, either long $(>10 \mathrm{~cm})$ polyacrylamide gels in glass tubes or flat, thick $(1-2 \mathrm{~mm})$ gels are used in these separations. High voltages that would increase resolution in the traditional-type gels result in distortion of the protein bands due to the heating caused by direct current flowing through a high resistance. We have developed capabilities for preparing thin acrylamide gels containing ampholytes on glass or thin plastic plates. This technique makes use of high field gradients $(>400$ volts $/ \mathrm{cm}$ ) to produce rapid focusing in thin $(125 \mu \mathrm{m})$ gels. The improved cooling characteristics of thin-layer gels also allow ampholyte concentrations to be increased to improve $\mathrm{pH}$ gradient production. To dissipate the heat $(>20 \mathrm{w})$ formed, an efficient thermoelectric device cools the plates to constant temperature even with 20 watts input. Silver staining of the focused proteins gives results within one hour after the electrophoretic run of 40 minutes. Up to 60 bands of focused proteins have been observed in serum samples. The extraordinary sensitivity of this method is evidenced by the small amount of protein required. Typically, $1 \mu \mathrm{L}$ of a 1:20 dilution of serum is used for separations. Thus, individual protein components in the 10-50 ng range may be detected. The faintest bands are estimated to contain about 1-5 ng of protein.

Thin-layer isoelectric focusing (TL-IEF) thus shows promise as a rapid method for separating and screening proteins. We have used the technique to differentiate serum proteins obtained from fish, to assess the purity of human insulin produced by bio-engineered bacteria, to evaluate enzyme purity, and to assess different types of bacterial vaccines.

\section{Nitrogen-15 NMR Spectrum Editing by the DEPT Technique (15320)}

\section{B. Coxon}

NMR spectrum editing by the DEPT (Distortionless Enhancement by Polarization Transfer) method [1] was originaliy developed for the purpose of confirmation of spectral assignments in $13 \mathrm{C}$ NMR spectra. The end result of this technique is a set of separated $\mathrm{CH}, \mathrm{CH}_{2}$, and $\mathrm{CH}_{3}$ subspectra with signal:noise ratios enhanced by transfer of magnetization from the protons to the ${ }^{13} \mathrm{C}$ nuclei. Since each subspectrum contains either $\mathrm{CH}, \mathrm{CH}_{2}$, or $\mathrm{CH}_{3}$ signals, this allows the ready identification of these structural types. Sensitivity enhancement by magnetization transfer is achieved in this instance by methods in which several pulses are applied (in some cases simultaneously) to the protons and ${ }^{13} \mathrm{C}$ nuclei. The implementation of such pulse sequences has been made possible by the 
installation (during 1982) of an advanced pulse programmer in our WM-400 NMR spectrometer. This accessory also permits control of the RF phases $( \pm x, \pm y)$ of the observation and decoupling channels.

We have recently extended spectrum editing by the DEPT technique to the structural analysis of aminoglycoside antibiotics by ${ }^{15} \mathrm{~N}$ NMR. The major chemical difference between ${ }^{13} \mathrm{C}$ and ${ }^{15} \mathrm{~N}$ sites is that most $\mathrm{CH}$ protons do not exchange or exchange only very slowly, whereas most $\mathrm{NH}$ protons undergo rapid chemical exchange. This is particularly true for the aminoglycoside antibiotics, for which we have observed that $\mathrm{NH}$ proton exchange is enhanced by the polyhydroxylic nature of the compounds. For this reason, these antibiotics were examined as their solutions in 9:1 v/v $\mathrm{CF}_{3} \mathrm{CO}_{2} \mathrm{H}: \mathrm{CD}_{3} \mathrm{CO}_{2} \mathrm{H}$, a solvent mixture that protonates all nitrogen atoms except those of amide groups, and thereby suppresses the rapid $\mathrm{NH}$ proton exchange which occurs at higher $\mathrm{pH}$. For the purpose of a deuterium lock signal, the less expensive $\mathrm{CD}_{3} \mathrm{CO}_{2} \mathrm{D}$ solvent was not used, in order to avoid the complication of partially deuterated amino groups.

The pulse sequence for proton decoupled, ${ }^{15} \mathrm{~N}$ DEPT NMR is indicated in figure 24, in which $\theta$ represents a proton pulse (at $400 \mathrm{MHz}$ ) of variable width, and $1 / 2 \mathrm{~J}$ is a fixed delay time defined by an average value of the one-bond coupling constants ${ }^{1} \underline{\mathrm{J}}_{15} \mathrm{NH}$ of the antibiotics. For the purpose of ${ }^{15} \mathrm{~N}$ spectrum editing (for example, the proton decoupled ${ }^{15} \mathrm{~N}$ NMR spectrum of isofortimicin), a set of three raw spectra $\left(\theta_{1}, \theta_{2}\right.$, and $\left.\theta_{3}\right)$ was acquired by use of the proton pulses $\theta=\pi / 4, \pi / 2$, and $3 \pi / 4$, respectively (see figure $25(d)$, for $\theta=\pi / 4$, for example). Linear combinations of these raw spectra, according to the equations,

$$
\begin{aligned}
& \mathrm{NH}=\theta_{2}-0.40 \theta_{1}-0.28 \theta_{3} \\
& \mathrm{NH}_{2}=0.58 \theta_{1}+0.29 \theta_{2}-0.98 \theta_{3} \\
& \mathrm{NH}_{3}=\theta_{1}-0.68 \theta_{2}+0.69 \theta_{3} .
\end{aligned}
$$

then gave a set of proton decoupled $\mathrm{NH}, \mathrm{NH}_{2}$, and $\mathrm{NH}_{3}$ subspectra (see figures 25 (a), 25 (b), and 25 (c), respectively).

The ${ }^{15} \mathrm{~N}$ assignments indicated by these subspectra neatly confirm those which we had made earlier by analysis of the multiplicities of the proton coupled, ${ }^{15} \mathrm{~N}$ spin multiplets of isofortimicin (see figure 25 (e)) combined with ${ }^{15} \mathrm{~N}$ chemical shift correlations with structural analogs. The nonideal values of the coefficients in the linear combinations indicate pulse imperfections, due possibly to nonoptimum design of the decoupler coils in our $15 \mathrm{~mm}{ }^{15} \mathrm{~N}$ probe or to the use of overly long proton pulse widths $(\pi / 2=75 \mu \mathrm{s})$ because of insufficient pulse power at $400 \mathrm{MHz}$. Future work will be directed toward improvement of the experimental conditions.

References

[1] Doddre11, D. M., Pegg, D. T., and Benda11, M. R., J. Magn. Reson. 48, 323-327 (1982); Bruker literature, with Hul1, W. E., (1982). 


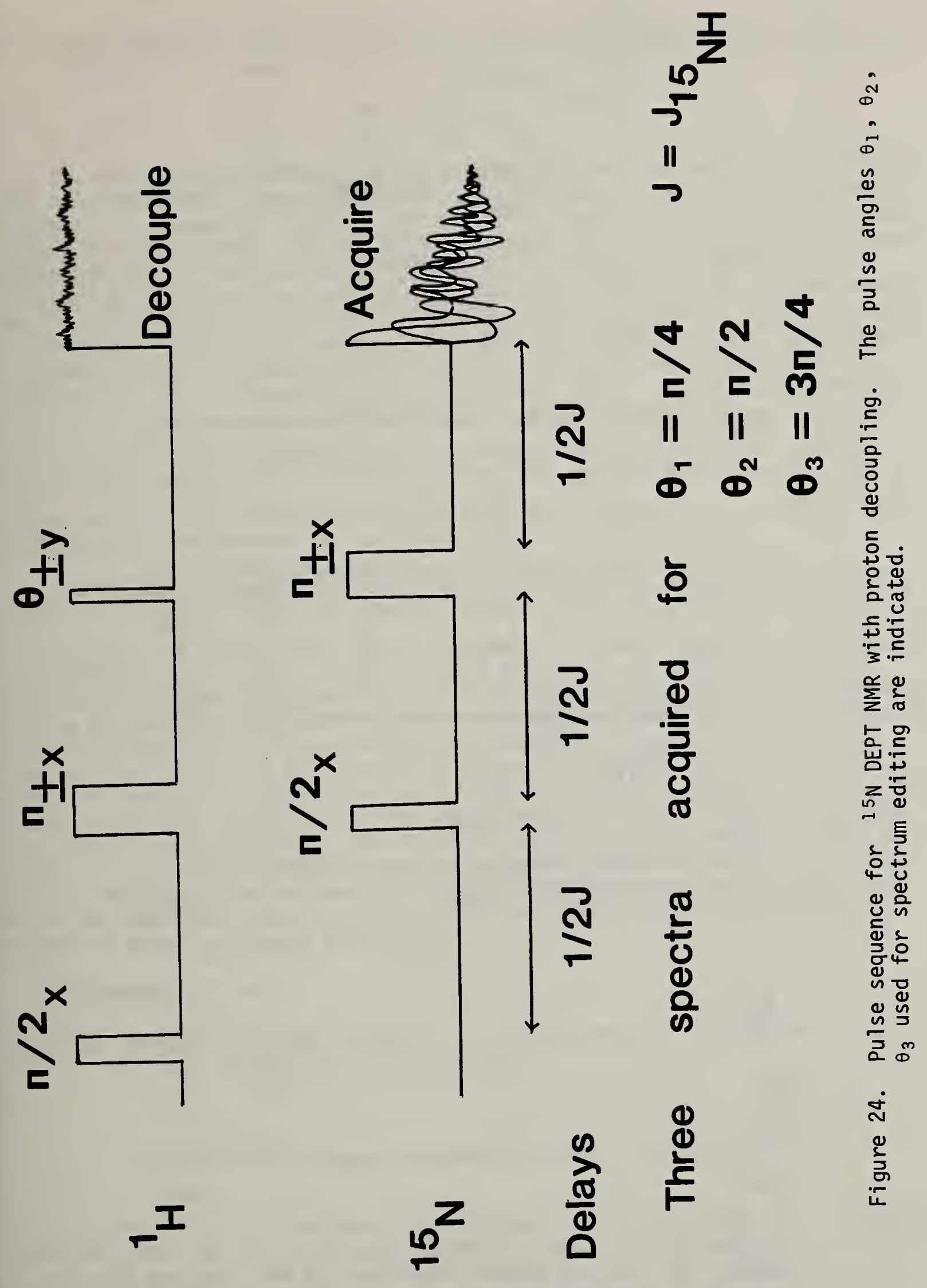



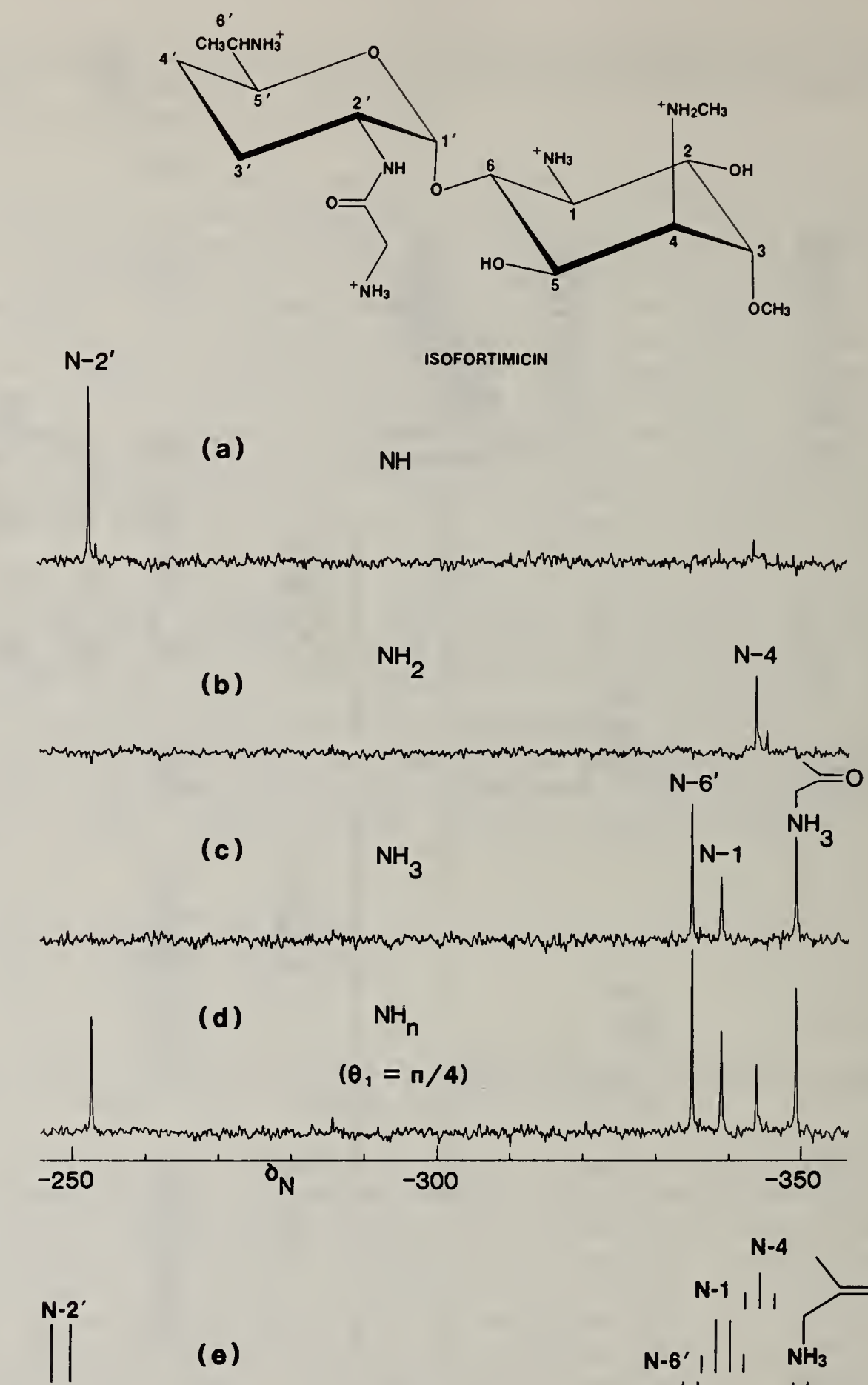

(e)

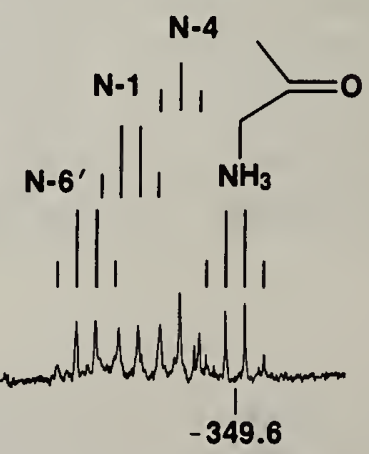

Figure 25. ${ }^{15} \mathrm{~N}$ NMR spectra of a solution of isofortimicin in $9: 1 \mathrm{~V} / \mathrm{V}$ $\mathrm{CF}_{3} \mathrm{CO}_{2} \mathrm{H}: \mathrm{CD}_{3} \mathrm{CO}_{2} \mathrm{H}$. (a) $-(\mathrm{c})$ edited sub-spectra of $\mathrm{NH}, \mathrm{NH}_{2}$, and $\mathrm{NH}_{3}$ groups; (d) complete proton decoupled ${ }^{15} \mathrm{~N}$ NMR spectrum obtained by use of the pulse angle $\theta_{1}=\pi / 4$ in the DEPT sequence; (e) proton coupled (normal acquisition without polarization transfer). 
15. Two-Dimensional, DEPT Heteronuclear J-Resolved Carbon-13 NMR Spectroscopy (15320)

B. Coxon

The DEPT technique discussed in the previous section has been combined with two-dimensional, heteronuclear J-resolved NMR by the spin-flip method [1] to give a new pulse sequence for two-dimensional, DEPT Heteronuclear (2D DEPTH), J-resolved NMR. The 2D DEPTH sequence (shown in figure 26) has been implemented for ${ }^{13} \mathrm{C}$ NMR at $100.6 \mathrm{MHz}$, using $5 \mathrm{~mm}$ samples. The first four pulses of the sequence are used to transfer magnetization from the protons to the ${ }^{13} \mathrm{C}$ nuclei, and, as in the spin-flip method, the last ${ }^{1} \mathrm{H} \pi$ pulse is used to invert the proton spin states so that $\mathrm{J}$-modulation of the ${ }^{13} \mathrm{C}$ magnetization continues during the entire $t_{1}$ period and is not refocused at the time of acquisition. In this sequence, the fixed delay $D 2$ is defined by the reciprocal of an average value of the one-bond coupling constant ${ }^{1}{ }_{-} \mathrm{CH}$. The $2 \mathrm{D}$ DEPTH data matrix is generated by incrementing the total variable delays $\left(t_{1} / 2\right)$ from a minimum value of $1 / 2 \mathrm{~J}_{\mathrm{CH}}$ plus the width of the $\theta$ pulse. The latter pulse may be assigned the various values $\pi / 6, \pi / 4, \pi / 2,3 \pi / 4$, or $5 \pi / 6$, depending upon the purpose of the experiment.

The 2D DEPTH sequence has been used to analyze the anomeric and ring form equilibria of $\underline{\underline{D}}$-digitoxose in $\mathrm{DMSO}-\underline{d}_{6}$ solution, the ${ }^{1} \mathrm{~J}_{\mathrm{CH}}$ values being useful for categorizing the various forms. A contour plot and associated projection of a $2 D$ DEPTH J-resolved ${ }^{13} \mathrm{C}$ NMR spectrum of a mixture of four anomers of digitoxose is shown in figure 27. Like the INEPT (Insensitive Nuclei Enhancement by Polarization Transfer) version of $2 D$ heteronuclear J-resolved NMR, the 2D DEPTH sequence offers sensitivity enhancement by means of polarization transfer and proton $T_{1} s$ that are perhaps shorter than ${ }^{13} \mathrm{C} T_{1} s$, but has the added advantage of "correct" multiplicities in the $2 \mathrm{D} \mathrm{J}$-spectra. For example, figure 28 shows a comparison of the J-spectra obtained by $2 D$ DEPTH, 2D spinflip, and 2D INEPT methods. The 2D DEPTH and 2D spin-flip methods yield the correct multiplicity (triplet) for the methylene carbon signal, whereas the 2D INEPT method yields an incorrect multiplicity (doublet). Some spectral artifacts have been noted in the 2D DEPTH method and studies of methods for removal of these artifacts are planned.

\section{References}

[1] Bodenhausen, G., Freeman, R., Niedermeyer, R., and Turner, D. L., J. Magn. Reson. 24, 291-294 (1976). 


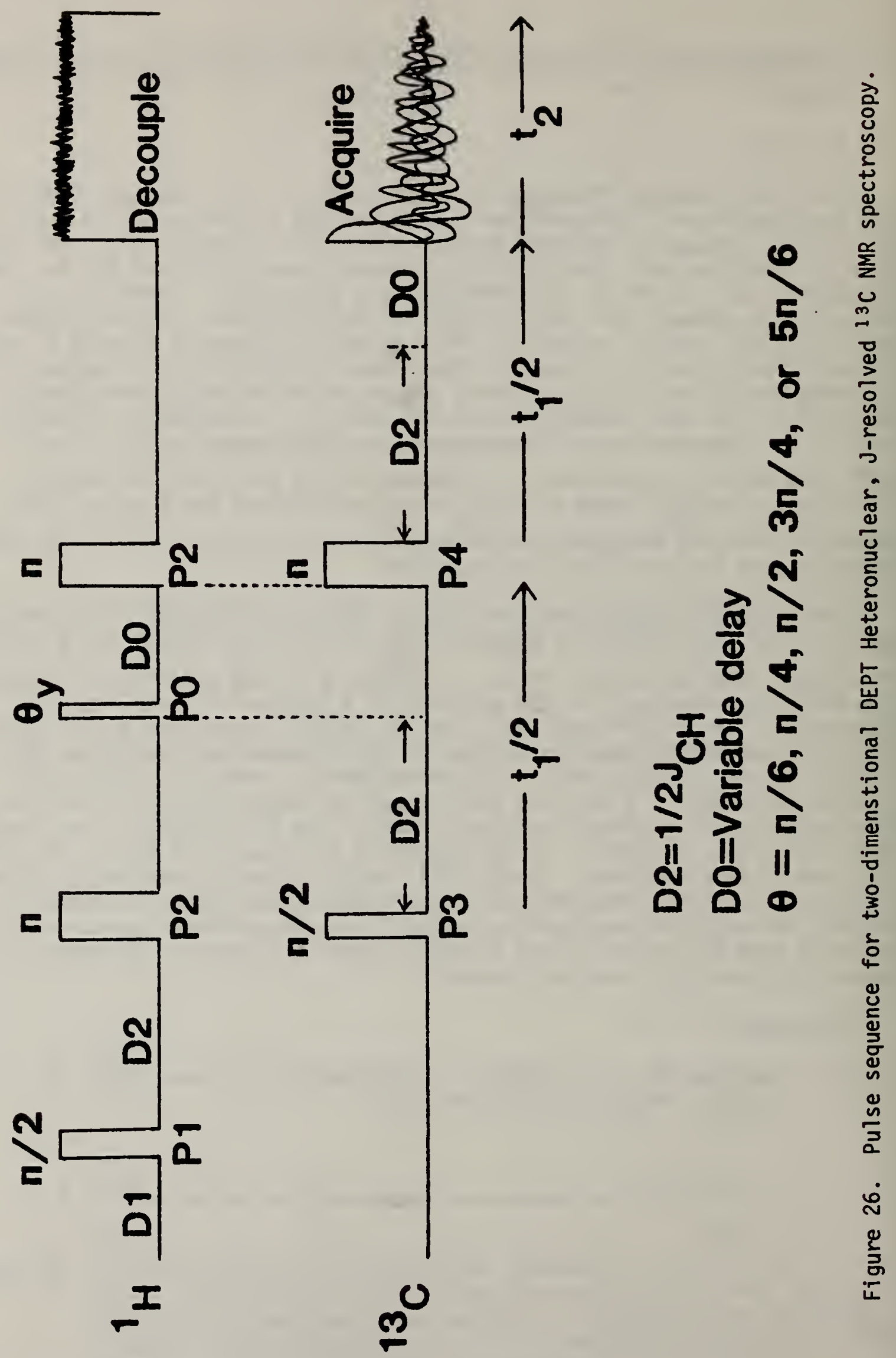




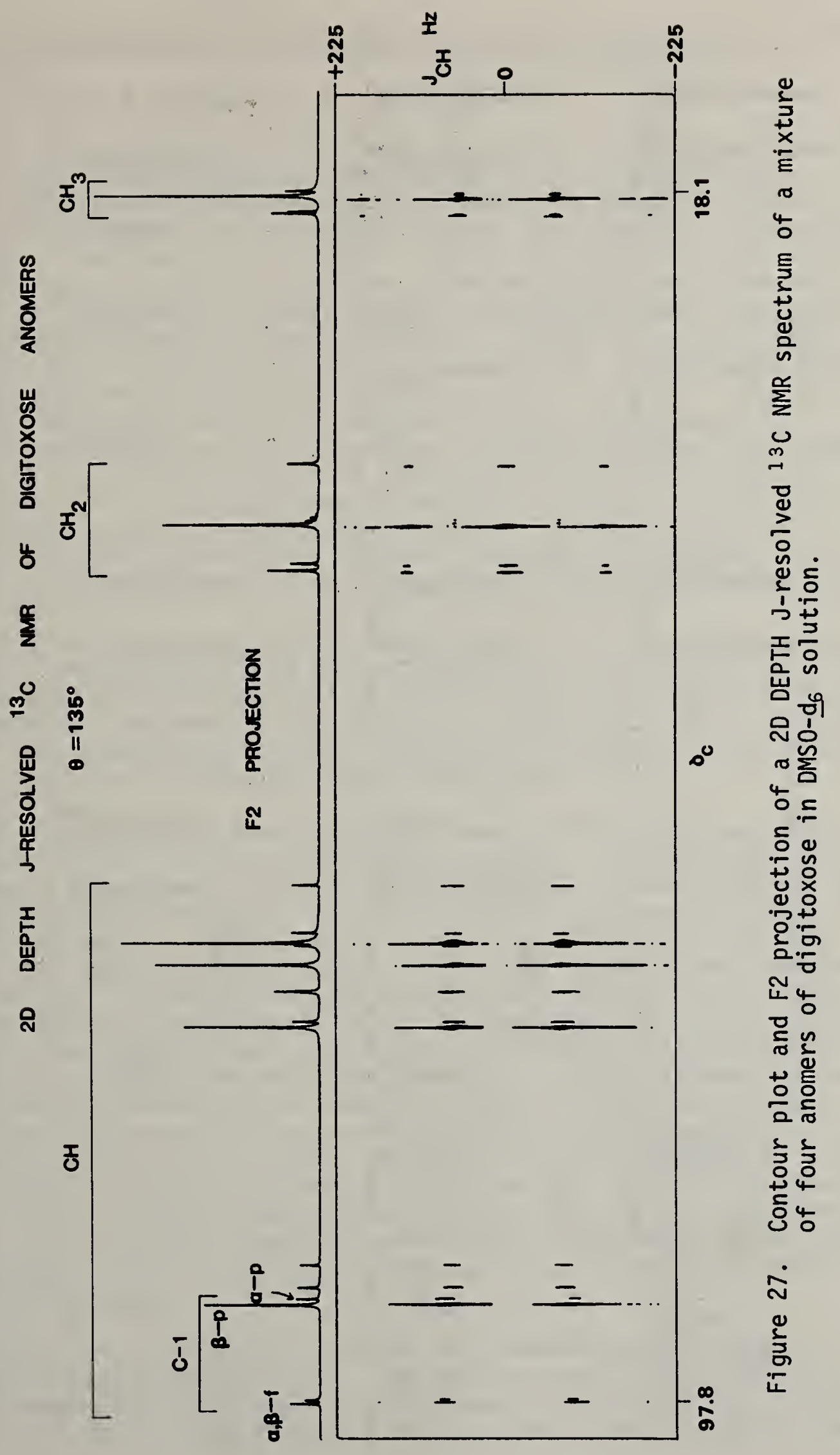



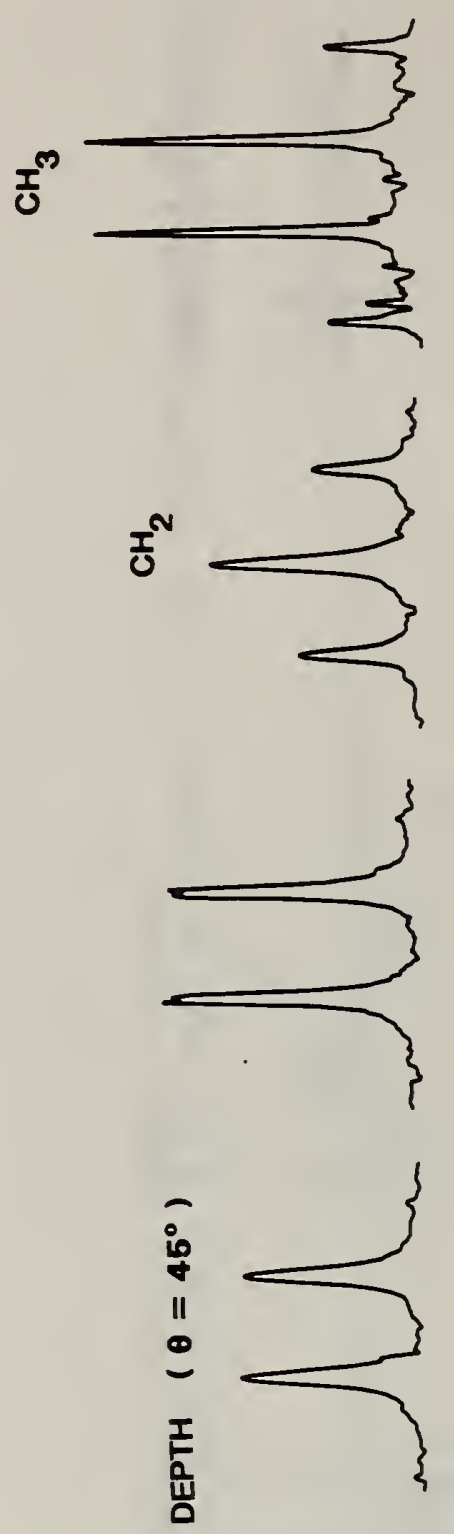

ส
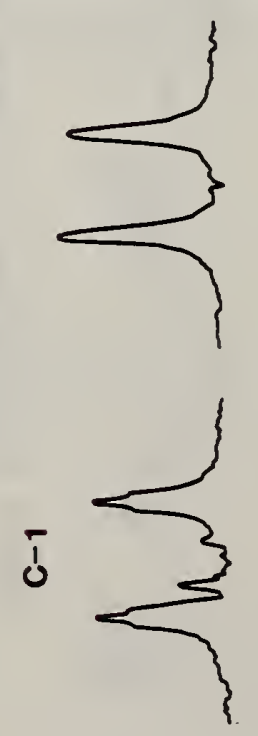
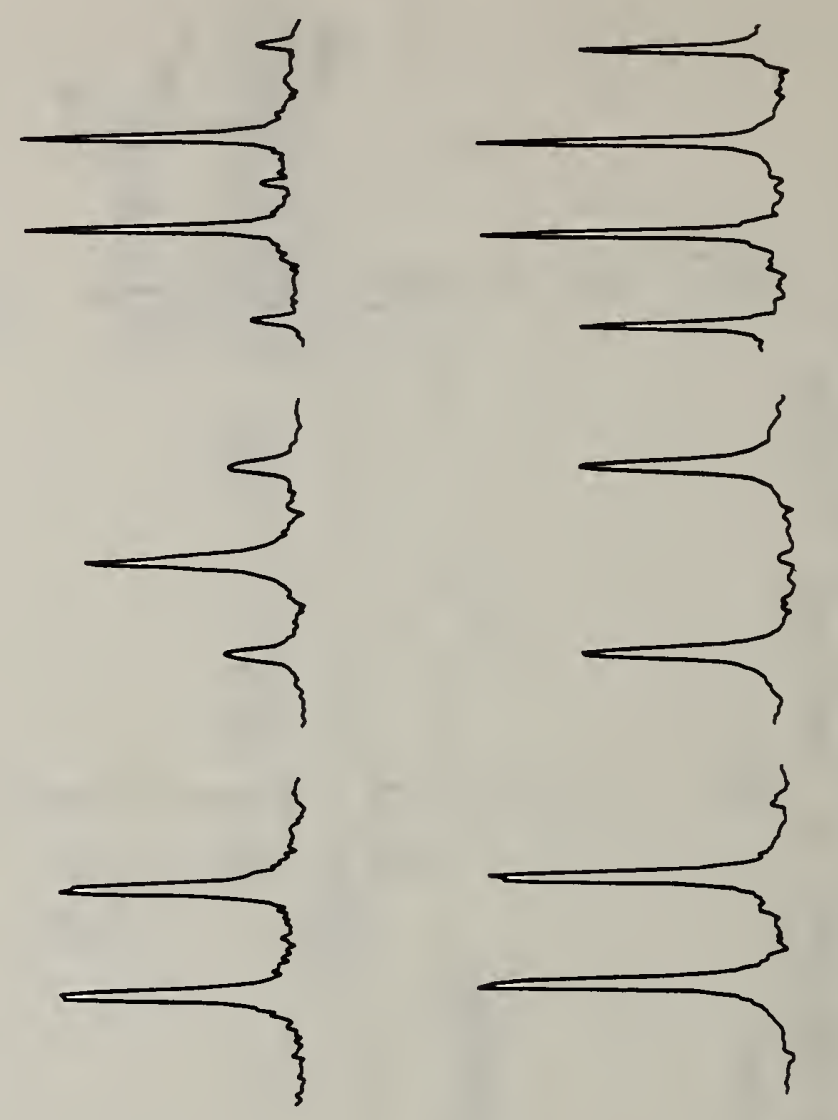

ลิ

Im

논여

듬

을

व 4 i

둥

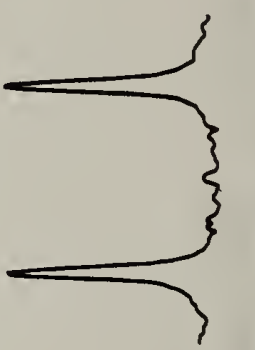

ज ํㅟำ

วิธ่

들

U u

.

웡

옹

๑ U

ก

过

ช0.

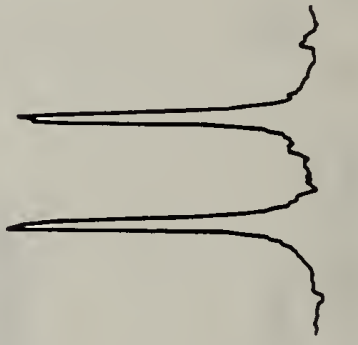

증

웡

O

삔

ธٓ㇒

둥

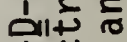

1 .

$\infty \mathrm{d}$

4 放。

014

$20^{2}$

옹 U⿺辶

के

in $x$

的哭齐

을

$\sum$ 宸4

u

占

0 岂

는

ํㅡㅇ

무울

论 跤

드워

的政

둥

워

过䗆
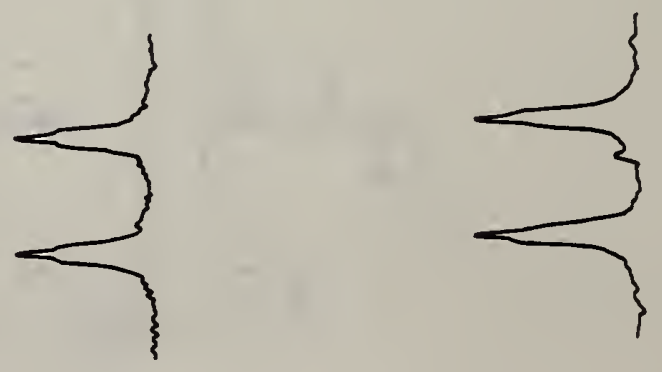

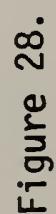


16. Preparation and Analysis of a Quality Assurance Sludge Material (15320)

D. Enagonio, L. R. Hilpert, R. C. Paule (500), C. R. Vogt

The Resource Conservation and Recovery Act of 1976 (RCRA) established a Federal program to provide comprehensive regulation of hazardous wastes.

Materials are designated as hazardous wastes based on a number of criteria including chemical composition and the ability of toxic organic compounds to migrate from, or leach out of, the waste. Accurate quantitative data on the concentrations of toxic organic compounds in the waste or aqueous leachates of the waste are vital to any program for the proper management of hazardous wastes. The use of Standard Reference Materials (SRM's), where available, or the use of well-characterized materials as standards and methods whose accuracy and precision are known can help ensure the accuracy of analyses. During the past year, we have characterized and analyzed a liquid sludge material by combined high resolution capillary column gas chromatography/mass spectrometry to determine the accuracy and precision achievable in the analysis of a hazardous waste. This work, supported by the EPA, is part of a continuing project on the development of methods and standards for the analysis of hazardous wastes. The liquid sludge material, prepared and analyzed at NBS, is currently being used by the EPA in its quality assurance monitoring programs.

A sample of an industrial organic sludge from a solvent recovery/hazardous waste storage facility was diluted with a high-boiling ligroin (bp $90-110^{\circ} \mathrm{C}$ ) and fortified with known amounts of 11 priority organic pollutants so that the resulting concentrations were in the $10-50 \mu \mathrm{g} / \mathrm{g}(\mathrm{ppm})$ range. Fifty liters of the material were prepared, and $100 \mathrm{~mL}$ aliquots were packaged in 500 clean, amber sample vials and sealed with teflon-lined caps.

A stratified random sampling scheme was used to select 15 samples from the total batch of material for quantitative analysis. Fifteen separate strata were used, and a random sample within each stratum was chosen. This ensured full examination of the sample set and at the same time ensured a degree of random choice of samples. Duplicate analyses of the 15 samples were made, and the order of the individual analyses was randomized. Quantitative measurements for phenol, m-dichlorobenzene, hexachlorobenzene, pentachlorophenol, phenanthrene, fluoranthene, pyrene, benzidine, di-n-propyl phthalate, di-2-ethylhexyl phthalate, and benz[a]anthracene were made by gas chromatography/mass spectrometry (GC/MS) with selected ion monitoring detection. Table 28 shows the results of the quantitative determinations. Because samples of this material are currently being used in quality assurance monitoring programs by the EPA, actual analyte concentrations are not given. In most cases, the experimental concentration agreed well with the gravimetric concentration. Benzidine showed the poorest agreement. The best precision was obtained for the di-2-ethylhexyl phthalate and the polycyclic aromatic hydrocarbons fluoranthene, pyrene, and benz[a]anthracene; poorer precision was observed for the benzidine, phenol, and m-dichlorobenzene.

For each of the 11 analytes, a within- and between-bottle analysis of variance (ANOVA) was performed $[1,2]$. The results showed no statistically significant between-bottle variability. Furthermore, approximately one-half of the calculated between-bottle components of variance were slightly negative, 
Table 28. Analytes in Quality Assurance Sludge at the $10-50 \mu \mathrm{g} / \mathrm{g}(\mathrm{ppm})$ Level Determined by GC/MS.

Percent Difference Between

Analyte Gravimetric Concentration and

\begin{tabular}{|c|c|c|}
\hline Analyte & Experimental Concentration & $C v^{b}$ \\
\hline Phenol & -5.9 & 5.7 \\
\hline m-Dich lorobenzene & -3.6 & 4.7 \\
\hline Hexachlorobenzene & 0.9 & 2.1 \\
\hline Pentach lorophenol & 1.8 & 2.5 \\
\hline Phenanthrene & -1.3 & 3.4 \\
\hline Fluoranthene & 4.1 & 1.5 \\
\hline Pyrene & 5.4 & 1.6 \\
\hline Benzidine & 31.4 & 3.3 \\
\hline Di-n-propyl Phthalate & 0.0 & 2.4 \\
\hline Di-2-ethylhexyl Phthalate & 5.4 & 1.1 \\
\hline Benz $[a] a n t h r a c e n e$ & 8.7 & 1.4 \\
\hline
\end{tabular}

and the other half were small and slightly positive, a behavior characteristic of a homogeneous material.

The GC/MS data was also used to characterize the GC/MS measurement variability. A within- and between-day ANOVA was made for the 11 analytes to characterize the overall variability of the GC/MS measurements. This yielded statistically significant between-day components of variance for almost all analytes. It is therefore necessary to take this component of variability into account in the experimental design.

The standard error (standard deviation of the weighted average) for each analyte was calculated from the within- and between-day components of variance. The coefficient of variation (Table 28), which is an expression of uncertainty, was about 3 percent for all analytes. These data indicate that accurate quantitative results for the determination of toxic organic compounds in a complex matrix, such as an industrial sludge, can be obtained using high resolution capillary column GC/MS with selected ion monitoring detection. 
References

[1] Paule, R. C. and Mandel, J., Consensus Values and Weighting Factors, J. Res. NBS 87, 377-385 (1982).

[2] Mandel, J. and Paule, R. C., Interlaboratory Evaluation of a Material with Unequal Numbers of Replicates, Ana 1. Chem. 42, 1194-1197 (1970); and Correction, Anal. Chem. 43, 1287 (1971).

17. Investigation of Desorption Mechanisms in Laser Desorption and Field Desorption Mass Spectrometry (26108)

G. D. Byrd, E. White V, D. S. Simons (553)

Large organic molecules that are not sufficiently volatile nor thermally labile for analysis by electron impact ionization can often be successfully characterized using various types of desorption mass spectrometry. Some of the more common desorption methods used in mass spectrometry include field desorption (FDMS), laser desorption (LDMS), and fast atom bombardment (FAB). The future development of these ionization methods as analytical tools will depend on knowledge of the basic phenomena involved in each case. Overall similarities in the mass spectra produced by various desorption sources suggest that these methods may share one or more common ionization mechanisms. In this work, some of the proposed mechanisms suggested for FDMS and LDMS are examined. The FDMS work is being done on a double focusing instrument and the LDMS work is being carried out on a laser microprobe mass analyzer in conjunction with the Gas and Particulate Science Division (553).

The two methods were investigated by looking at a series of simple homologous dicarboxylic acids $\left[\mathrm{HOOC}-\left(\mathrm{CH}_{2}\right)_{n}-\mathrm{COOH}, \mathrm{n}=0\right.$ to 5$]$. Both methods produced $(\mathrm{M}+\mathrm{H})^{+}$and $\left(\mathrm{M}+\mathrm{H}-\mathrm{H}_{2} \mathrm{O}\right)^{+}$in most cases, but LDMS gave more fragmentation, some of which may be due to partial pyrolysis of the sample. Also, an increase in fragmentation/pyrolysis with increasing chain length of the acids was observed in LDMS but not in FDMS. One explanation of these results is that the minimum energy necessary for desorption and ionization is applied in the case of FDMS by the control of the emitter wire current, while the nearly instantaneous heating of a localized area in LDMS supplies excess energy to the desorbed species. The amount of heating that takes place in LDMS depends somewhat on the ability of the sample to absorb the photons and disperse the heat generated. In FDMS, the transfer of heat to the sample is more uniform and the absorption properties of the sample are not important.

An important mechanism believed to be operational during the desorption process is that of gas phase ion-molecule reactions which are thought to be responsible for the production of protonated, cationized, and anionized species which are often the major peaks in the spectra. Such reactions may occur just above the surface in LDMS where easily ionized species such as alkali cations attach themselves to neutral organics that are also thermally desorbed. In order to verify this mechanism for the dicarboxylic acids, a metal cation was co-desorbed along with the organic species by defocusing the laser beam such that the copper metal substrate was included in the target 


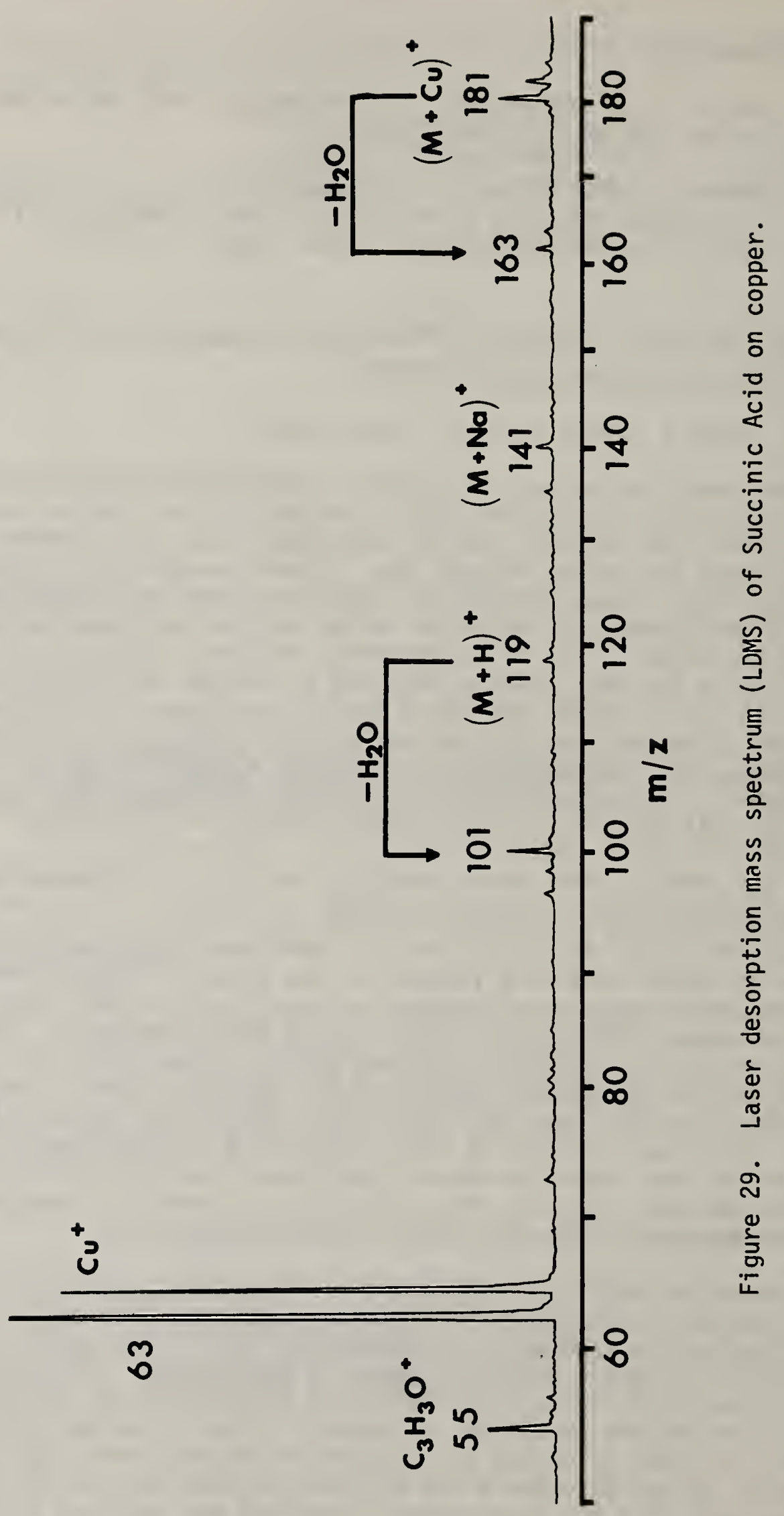


area. Recent experiments in gas phase reactions between metal ions and organics have shown that $\mathrm{Cu}^{+}$reacts with carboxylic acids to release $\mathrm{H}_{2} \mathrm{O}$ from the collision complex. As shown in figure 29, this product is formed during the LD experiment, and thus the experiment adds to the mounting evidence for the importance of gas phase reactions in LDMS.

Some variations in sample mounting were tried to examine the role played by the substrate material in the desorption process. An attempt to desorb sample ions from a liquid substrate by LD proved successful. In this experiment, a volatile organometallic solid, ferrocene, was suspended in glycerol and mounted in the LDMS source. A remarkably clean spectrum consisting almost completely of $\mathrm{M}^{+\cdot}$ and no glycerol-related peaks was observed. This demonstrates the difference in the desorption characteristics of the sample and the substrate material chosen in this case and suggests possible applications of desorption techniques to volatile species suspended in liquids. Future experiments will look further at the role of the substrate material in the desorption process.

18. Development of an Isotope Dilution/Mass Spectrometry Definitive Method for the Determination of Serum Creatinine $(15320,26108)$

A. Cohen, H. S. Hertz, R. Schaffer, M. J. Welch, E. White V

Definitive methods are carefully designed methods which, after rigorous testing, have been shown to provide highly accurate and precise results [1]. Such methods are used at NBS for the certification of analyte levels in reference serum pools, which then serve as accuracy transfer standards for the evaluation of reference and field methods. We have developed definitive methods for cholesterol, glucose, uric acid, and urea and used these methods to certify concentrations in a freeze-dried human serum pool, Standard Reference Material (SRM) 909, as well as in other reference sera. Creatinine, another clinically important analyte in serum, is used as a measure of renal function. Development of a definitive method for serum creatinine has now been completed and certification of the creatinine level in SRM 909 is in progress.

The definitive method for creatinine utilizes isotope dilution/mass spectrometry, as have all other definitive methods for organic serum analytes. Initial development work used a creatinine- $d_{3}$ as the labeled internal standard. Results were very imprecise, possibly due to isotope effects. We synthesized a creatinine $-13 \mathrm{C}_{2}$ from glycine $-{ }^{13} \mathrm{C}$ and methyl iodide $-{ }^{13} \mathrm{C}$ according to the following scheme:

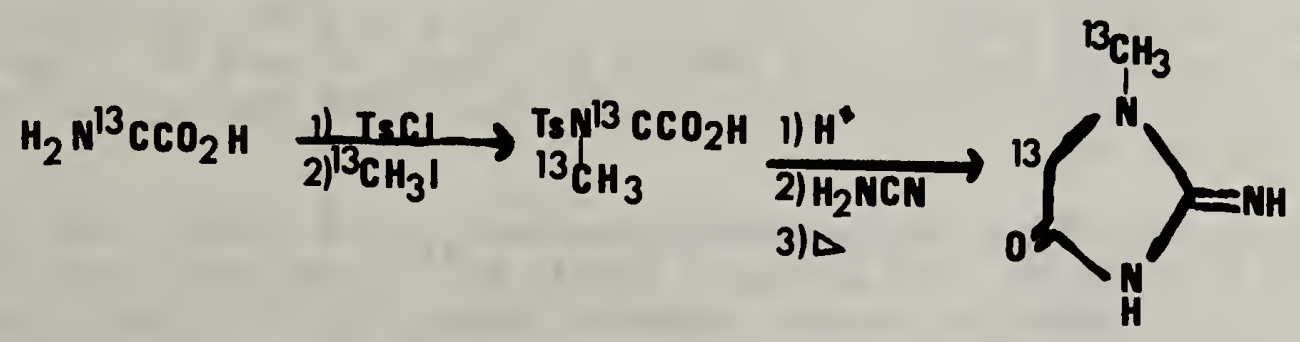


Using this new labeled material, the precision of the method was substantially improved (CV's of $<0.2$ percent).

A brief description of the method is as follows: A weighed quantity of creatinine- ${ }^{13} \mathrm{C}_{2}$ is added to a known weight of serum, such that the labeled/ unlabeled creatinine ratio is approximately $4: 1$. After an overnight equilibration, the serum is put on a weak acid resin column and washed through with water. Any creatine present is eluted while creatinine is retained. The creatinine is then eluted with an aqueous ammonia solution and this fraction is freeze-dried. For derivatization, creatinine is heated in the presence of 2,4-pentanedione, acetic acid, and ethanol for three days at $85-86^{\circ} \mathrm{C}$ to form the ethyl ester of $\mathrm{N}$-(4,6-dimethy1-2-pyrimidiny 1)- $\mathrm{N}$-methylglycine. Excess reagents are evaporated and the derivative is dissolved in distilled heptane for analysis.

Isotope ratio measurements are made on a low resolution, magnetic sector mass spectrometer modified with an NBS-built microcomputer system, which controls switching of the magnetic field and handles data acquisition, output, and reduction. Samples are introduced via a gas chromatograph directly coupled to the mass spectrometer and equipped with a nonpolar capillary column.

The electron impact mass spectrum of the creatinine derivative has a very intense $\left(\mathrm{M}-\mathrm{COOC}_{2} \mathrm{H}_{5}\right)^{+}$ion at $\mathrm{m} / \mathrm{z} 150$ and 152 for the unlabeled and labeled forms, respectively. These two ions are monitored sequentially; the measured intensities of each are corrected for background and then summed. The summed intensities are then ratioed for each sample or standard. The standards are mixtures of known amounts of creatinine- ${ }^{3}{ }^{3} C_{2}$ and SRM 914 (creatinine) derivatized as described above and are used for calibration of the measured intensity ratios of serum samples. The technique of bracketing is used for calibration and the following scheme is employed: For each sample, the two standards whose unlabeled/labeled weight ratios most closely bracket that of the sample are chosen. One standard is injected into the GC, followed five minutes later by an injection of the sample, and ten minutes later by the other standard. After a retention time of approximately 26 minutes, each is measured. The intensity ratio for the sample is compared with the intensity ratios for the standards to precisely find the weight ratio for the sample. The samples are remeasured on a second day with the order of standards reversed. The results from the two days are averaged for the reported result. To test for the presence of measurement bias, some samples are remeasured, using $(M+1)^{+}$ions generated by isobutane chemical ionization.

Prior to the measurement of serum samples, two independently prepared sets of standards are tested for consistency with use of the bracketing technique described above. The results of such a test for the standards used in bracketing serum samples from SRM 909 are shown in Table 29. The agreement between the two sets is satisfactory, with an apparent bias of 0.12 percent between the two sets.

The results of two independently prepared sets of samples from SRM 909 are shown in Table 30 . The agreement within and between sets meets our criteria for a definitive method. However, before certification is complete, another set will be run and confirmatory measurements, using isobutane chemical 
Table 29. Consistency Tests for Two Independently Prepared Sets of Standards, $A-D$ and $E-H$, by Use of Bracketing.

\begin{tabular}{|c|c|c|c|c|}
\hline \multirow[b]{2}{*}{ Standard } & \multirow[b]{2}{*}{$\begin{array}{c}\text { Bracketed } \\
\text { By } \\
\end{array}$} & \multicolumn{2}{|c|}{$\frac{\text { Untabeled }}{\text { Labeled Ratios }}$} & \multirow[b]{2}{*}{$\begin{array}{l}\text { Difference } \\
\text { Percent }\end{array}$} \\
\hline & & $\begin{array}{l}\text { Mean ID/MS } \\
\text { Determined } \\
\text { Weight Ratio } \\
\end{array}$ & $\begin{array}{l}\text { Weighed-In } \\
\text { Ratio } \\
\end{array}$ & \\
\hline A & $-a$ & & 0.2679 & \\
\hline B & $E, F$ & 0.24965 & 0.2493 & +0.14 \\
\hline C & $E, F$ & 0.23705 & 0.2370 & +0.02 \\
\hline D & $F, G$ & 0.2196 & 0.2193 & +0.14 \\
\hline$E$ & $A, B$ & 0.25765 & 0.2582 & -0.21 \\
\hline$F$ & $C, D$ & 0.22825 & 0.2284 & -0.06 \\
\hline G & $D, H$ & 0.21625 & 0.2166 & -0.16 \\
\hline H & $-a$ & & 0.2083 & \\
\hline
\end{tabular}

Table 30. Creatinine in Human Serum SRM 909 by Isotope Dilution/Mass Spectrometry--Two Independently Prepared Sets.

\section{Mean mg Creatinine/} g Dry Serum

\begin{tabular}{ccc} 
Sample & Set 1 & Set 2 \\
\hline 1 & 0.2155 & 0.2155 \\
2 & 0.2155 & 0.2154 \\
3 & 0.2154 & 0.2151 \\
4 & 0.2157 & 0.2156 \\
5 & 0.2157 & 0.2154 \\
6 & & 0.2153 \\
Mean & 0.2156 & 0.2154 \\
CV (\%) & 0.06 & 0.08 \\
Difference Between Set Means $=0.09$ percent
\end{tabular}


ionization, will be made on a subset of samples selected from these three sets to provide additional evidence for the absence of significant bias.

Future work in this program will include measurement of creatinine in the proficiency testing sera of the College of American Pathologists and possibly other reference sera, remeasurement of glucose in SRM 909, and development of a definitive method for triglycerides.

References

[1] "Guidelines for the Development of Definitive Methods for Use in Clinical Chemistry for the National Reference System in Clinical Chemistry," NRSCC 1, National Committee for Clinical Laboratory Standards, Villanova, PA 19085 (1979).

19. Two-Dimensional Electrophores is System Implemented for Protein Separation and Identification $(26111,15310)$

F. A. Giere, D. K. Hancock, D. J. Reeder

As part of our efforts in the separation and characterization of proteins, with the eventual goal of using them as analytical reagents, work has continued on refining two-dimensional electrophoresis (2-DE). Our initial experience with the technique indicated that the resolution and sensitivity were high, but that reproducibility and staff-hour requirements were unsatisfactory. If multiple gels could be cast and run simultaneously, gel-to-gel reproducibility and efficiency could be improved. Various options were explored and a commercially available system was chosen to satisfy our requirements.

A 2-DE laboratory has been set up using this system. This allows the simultaneous casting and running of 20 first dimension iso-gel rods. Ten $18 \times 16 \mathrm{~cm}$ gradient gels can also be simultaneously cast and then run in the second dimension. This gives us a throughput of $30 \mathrm{gels} /$ week compared to the four previously possible.

A photographic system for documentation of the 2-DE gels has been established. Panatomic-X film, a $35 \mathrm{~mm}$ camera with a Wratten \#55 gel filter, and a light box with daylight bulbs have been found to give excellent results.

Various methods of protein staining have been tested on the second dimension gels, and these various techniques are now being compared using sets of identical 2-DE gels. Of particular interest are the silver stains, which give the greatest sensitivity but also unfortunately the greatest experimental difficulties. In collaboration with R. Zeisler of the Inorganic Analytical Research Division, neutron activation analysis of silver-stained gels is being used to gain information on silver distributions in the gels and on conditions that may lead to enhancement of the stain and sensitivity of the technique. Initial results show that low background gels can be prepared and that a uniform distribution of silver occurs in ammoniacal silver stains while silver is concentrated in the protein bands in the non-ammoniacal stains. Extension 
of this technique should allow detection of metal-binding enzymes and phosphoproteins.

Interlaboratory reproducibility continues to be a major problem with 2-DE. This is due, in part, to variations in source materials for casting and running gels. The composition of the acrylamide, which serves as the separation matrix, has been examined by high-performance liquid chromatography (HPLC). Differences between freshly recrystallized and commercially available acrylamide have been determined and correlation of these differences with electrophoretic separation is under further study. A definitive relationship has been found between the purity of sodium dodecylsulfate (SDS) and electrophoretic resolution. Impure SDS was shown to cause band spreading, loss of staining, and streaking artifacts in the 2-D gels.

Interlaboratory comparison would be further aided by use of marker compounds that would identify molecular weight and isoelectric point parameters. Potential proteins have been identified as candidate marker compounds. Future work will involve modification of these proteins with chromophores for easy visualization.

Several collaborative projects using 2-DE have been pursued with two other agencies. Work continues with Sandy Hook Marine Laboratories on differentiation of several species of flounder and on coordinating their serum agglutination properties with 2-DE patterns. A 2-DE comparison study of bacterial cell wall lipopolysaccharides is being carried out to clarify results obtained at Walter Reed on bacterial phenotypes used in hybrid vaccines.

20. Laser Photodissociation of Ions and Reactive Ion/Molecule Collisions: Instrumentation and First Results (26108)

R. G. Christensen, H. S. Hertz, D. J. Pereles, R. L. Sams (550),

M. J. Welch, E. White V

As part of the continuing mass spectrometry competence building program, "Analysis of Ionization Mechanisms and Ion Structures," we have been investigating the potential of the laser induced photodissociation and the collision induced dissociation of ions for organic structure determination and analytical specificity.

A schematic of the three different experimental set-ups for investigating the photodissociation of ions is shown in figure 30 . In each case, the beam from an argon ion laser with an output power of at least one watt at each of ten wavelengths between 357 and $529 \mathrm{~nm}(3.47-2.34 \mathrm{eV})$ is directed into a double focusing mass spectrometer of Mattauch-Herzog geometry. The signal from ions created by photodissociation is extracted by chopping the laser beam and measuring the intensity of the in-phase ion signal with a lock-in amplifier.

A first set of experiments, begun two years ago, was conducted by directing the laser beam through the source (entry point 1) into the first field-free region of the mass spectrometer. Ions created by electron impact and then photodissociated in the first field-free region (metastable ions) were detected 


\section{CHOPPER}

\section{\begin{tabular}{|l|l|l|l|l|l|}
\hline ARGON ION LASER & ARGS \\
\hline LENSES & &
\end{tabular} \\ (1)}

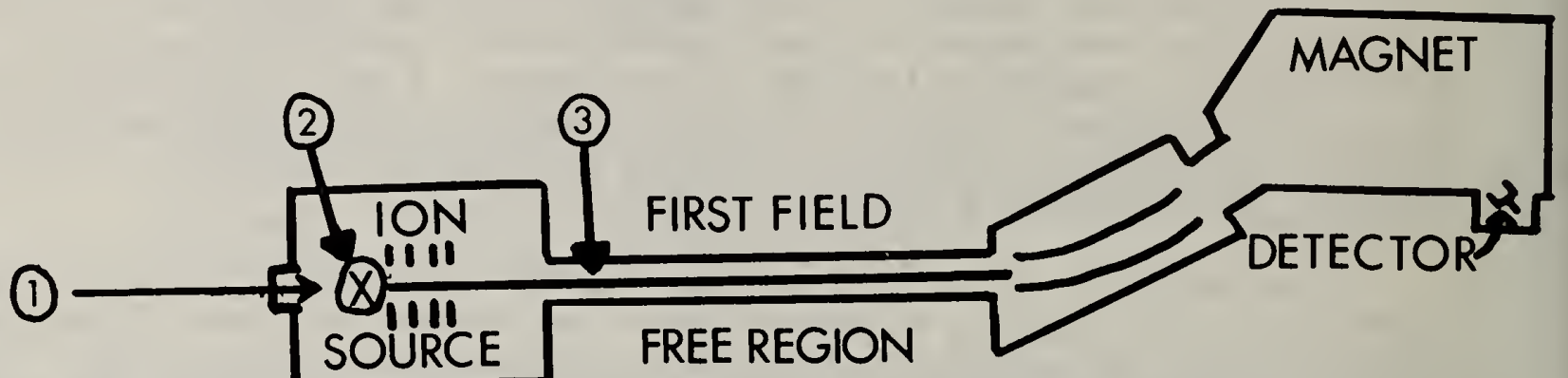

ELECTROSTATIC ANALYZER

Figure 30. Schematic of the experimental arrangements for the laser induced photodissociation of ions. Mirrors have been omitted.

by either accelerating voltage scans or by linked scans of the electric and magnetic sectors. These experiments showed that the experimental set-up is capable of detecting ions created by photodissociation. We have confirmed that photodissociation of organic ions occurs for at least some ions from most of the compounds examined, that the extent of dissociation is wavelength dependent even in the visible region and different for different ions, and that the ion source temperature affects the internal energy of the ions to the extent of about $0.2 \mathrm{eV} / 100^{\circ} \mathrm{C}$.

In a second type of experiment conducted recently, we have looked at photodissociation in the ion source by bringing the laser beam through the electron impact ionization region perpendicular to the ion beam (entry point 2). After consideration of the residence times of the ions in the laser beam and the focusing of the laser beam, the arrangement was expected to produce moderately stronger signals at the cost of the loss of parent-daughter specificity provided by the metastable ion experiments. Instead it was found that one watt at $514.5 \mathrm{~nm}$ dissociated about a quarter of the molecular ions of butylbenzene. The extent of dissociation was also found to be pressure dependent with no detectable dissociation observed at an indicated gauge pressure of about $4 \times 10^{-6}$ torr and maximum dissociation at lower pressures. Additional experiments have led us to the tentative conclusion that the pressure in the ionization region is very much higher than expected and that charge exchange ionization is occurring. We intend to investigate further since these effects can produce gross changes in the relative intensities of peaks in photodissociation mass spectra, thus limiting their usefulness.

One promising use of the photodissociation of ions for structure determination is its application to ions of low internal energy, such as those formed by field ionization, field desorption, chemical ionization, and fast atom bombardment. The photon-induced fragmentation should provide structural information for molecules not amenable to electron impact ionization similar 
to that obtainable from low energy collision induced dissociation, but with the advantage of precise energy control by selecting the wavelength. Modification of the mass spectrometer to bring the laser beam into the first field-free region (entry point 3 ), while allowing the use of field ionization and desorption, and the construction of a device to permit multiple passes of the laser beam through the ion beam are underway.

Experiments on collision induced dissociation of ions began early in 1983 after modifying an existing instrument to a triple quadrupole mass spectrometer. Initial efforts were devoted to understanding the complex focusing properties of the instrument, comparison of results on several test compounds with results reported in the literature, and experiments which taught us what to expect from variations in the ion energy of collisions. We completed our part of a round robin experiment designed to discover what factors affect comparability of collision induced dissociation spectra from different mass spectrometers. Reactive collisions have been observed to occur and the reaction to depend on the ion energy. Plans for the near future include addition of a data acquisition system, exploration of reactive collision gases, and application of collision induced dissociation to quantitation by combined liquid chromatography/mass spectrometry/mass spectrometry with both reactive and unreactive collision gases.

21. Determination of Polynuclear Aromatic Hydrocarbons in Environmental Samples Using Negative Chemical Ionization Mass Spectrometry $(15320,26108)$

G. D. Byrd, L. R. Hilpert, C. R. Vogt

Negative chemical ionization mass spectrometry (NCIMS) is a relatively new ionization technique which provides a highly selective and sensitive means of determining certain classes of organic compounds in complex mixtures. NCI mass spectra can be obtained from organic compounds by resonance capture of thermal electrons if the molecules have positive electron affinities, and if the electrons emitted from a hot filament are reduced to energies of a few electron volts through collisions with inert gases such as $\mathrm{CH}_{4}, \mathrm{Ar}$, or $\mathrm{N}_{2}$. The major species formed is usually the molecular anion, $M^{-}$, which often yields relatively large ion currents and little fragmentation. The specificity of NCIMS, coupled with a high resolution separation technique such as fused silica capillary column gas chromatography, provides a highly selective means for the determination of NCI-sensitive compounds in complex environmental samples, often with a minimum amount of sample clean-up.

During the past year, we have investigated the NCIMS of polynuclear aromatic hydrocarbons. We have observed a remarkable selectivity of detection of benzo[a]pyrene $(B[a] P)$ over the benzo[e]pyrene (B[e]P) isomer. Figure 31 shows the methane NCI spectra for $B[a] P$ and $B[e] P$, respectively. The NCI spectrum for the $B[a] P$ shows a strong molecular anion at $\mathrm{m} / \mathrm{z} 252$ and a small ( $<1$ percent) adduct ion at $\mathrm{m} / \mathrm{z} 266$. In comparison, the NCI spectrum of the $\mathrm{B}[\mathrm{e}] \mathrm{P}$ shows a base peak at $\mathrm{m} / \mathrm{z} 266$ and a much less intense molecular anion at $\mathrm{m} / \mathrm{z} 252$. Stockl and Budzikiewicz [1] have shown that alkyl radical species present in the $\mathrm{CH}_{4}$ plasma can react with substrate molecules to give 

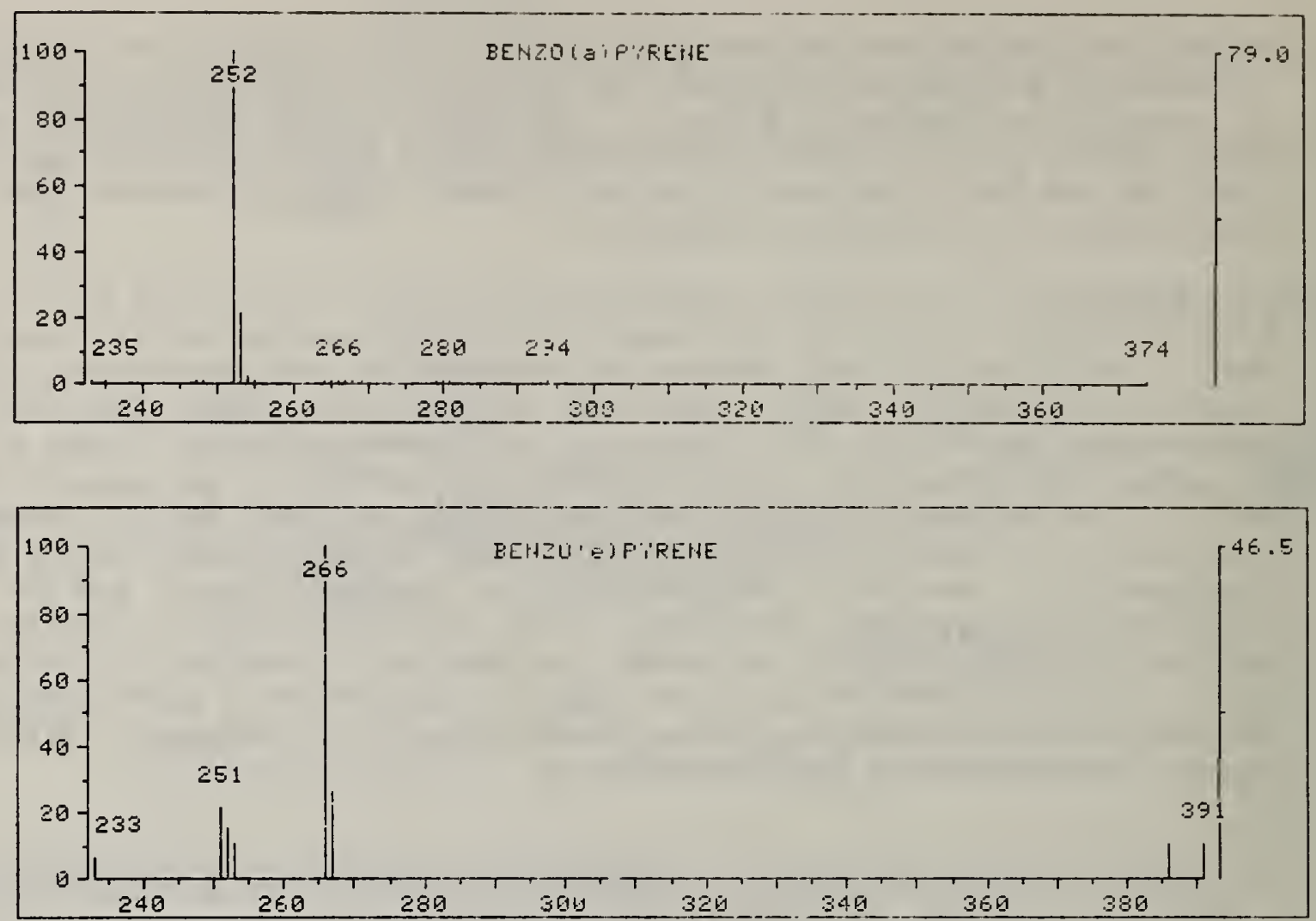

Figure 31. Methane negative chemical ionization mass spectra of benzo[a]pyrene and benzo[e]pyrene.

$\left(M+\mathrm{C}_{n} \mathrm{H}_{2}\right)^{-\cdot}$ ions, and we believe this process yields the $\left(M+\mathrm{CH}_{2}\right)^{-}$species at $\mathrm{m} / \mathrm{z} 266$. The NCI spectra of indeno[1,2,3-cd]pyrene and benzo[ghi]perylene also show strong molecular anions at m/z 276 and small adduct ions at $\mathrm{m} / \mathrm{z} 290$.

Quantitative determinations for benzo[a]pyrene, indeno[1,2,3-cd]pyrene, and benzo[ghi]perylene in SRM 1582 (Petroleum Crude 0i1) were made by gas chromatography (GC) NCIMS using $B[a] P-d_{12}$ as an internal standard and detection by selected ion monitoring. Earlier attempts to determine $B[a] P$ in a Wilmington crude oil by GC/MS, using both electron impact and positive chemical ionization (CI), proved unsuccessful due to the low concentration of the analyte in the $0 i 1$ and the many interferences encountered, even in the selected ion monitoring analysis of the diluted oil. Values for the $B[a] P$ concentration determined by electron impact and positive CI GC/MS fell in a range of approximately 1.0-2.3 ppm with coefficients of variation of 15-30 percent.

Figure 32 shows a selected ion record chromatogram for the negative CIMS analysis of a Wilmington crude oil sample. The $B[a] P$ peak is free of interferences and is easily integrated. Five ampoules of SRM 1582 were measured in triplicate. The results of these determinations are shown in Table 31 . The GC/NCIMS value for the B[a]P concentration in SRM 1582 is in reasonable agreement with the value of $1.2 \mathrm{ppm}$ determined using a multidimensional HPLCspectrofluorimetric procedure. 


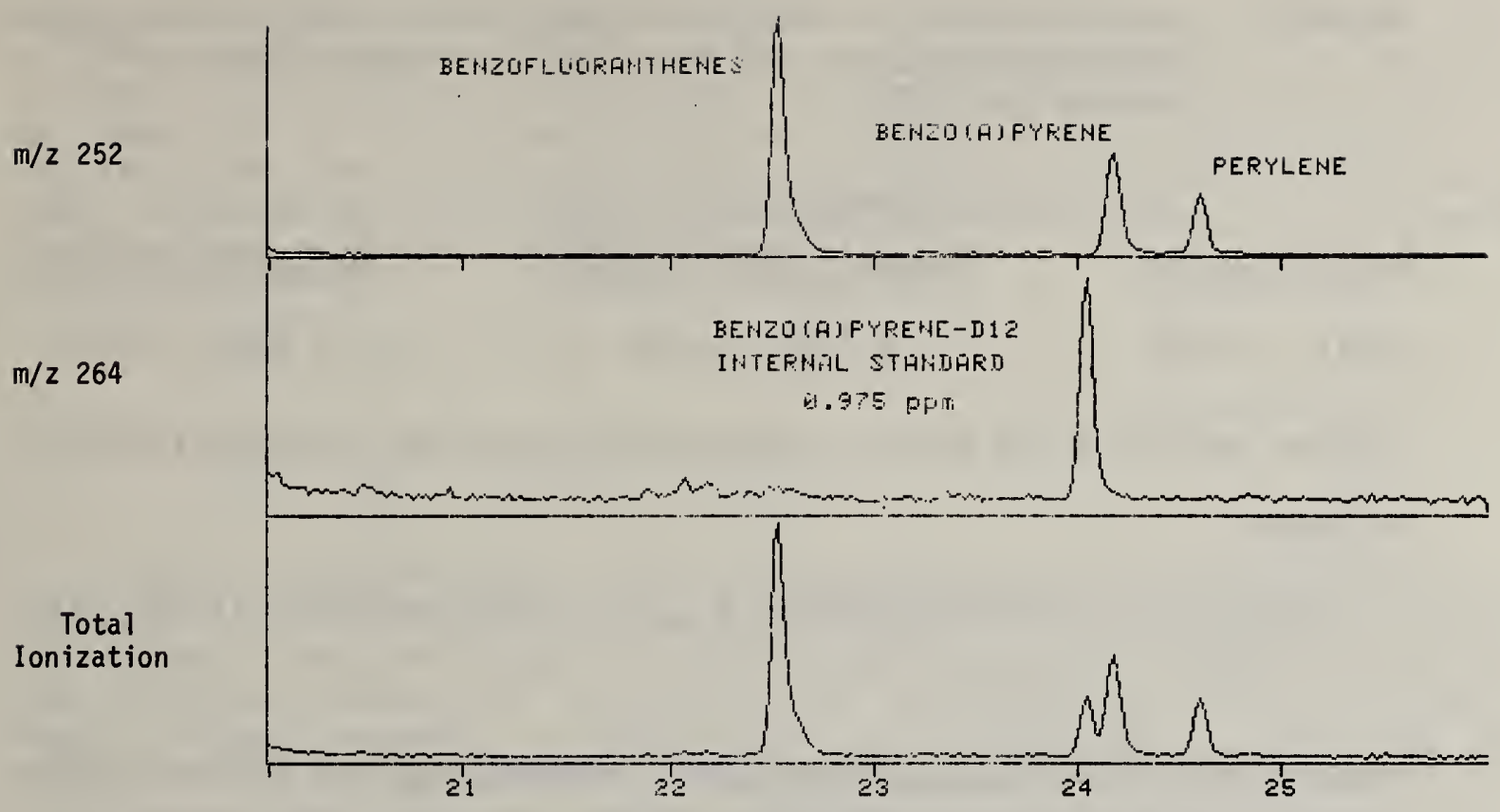

$\mathrm{m} / \mathrm{z} 276$

Total

Ionization

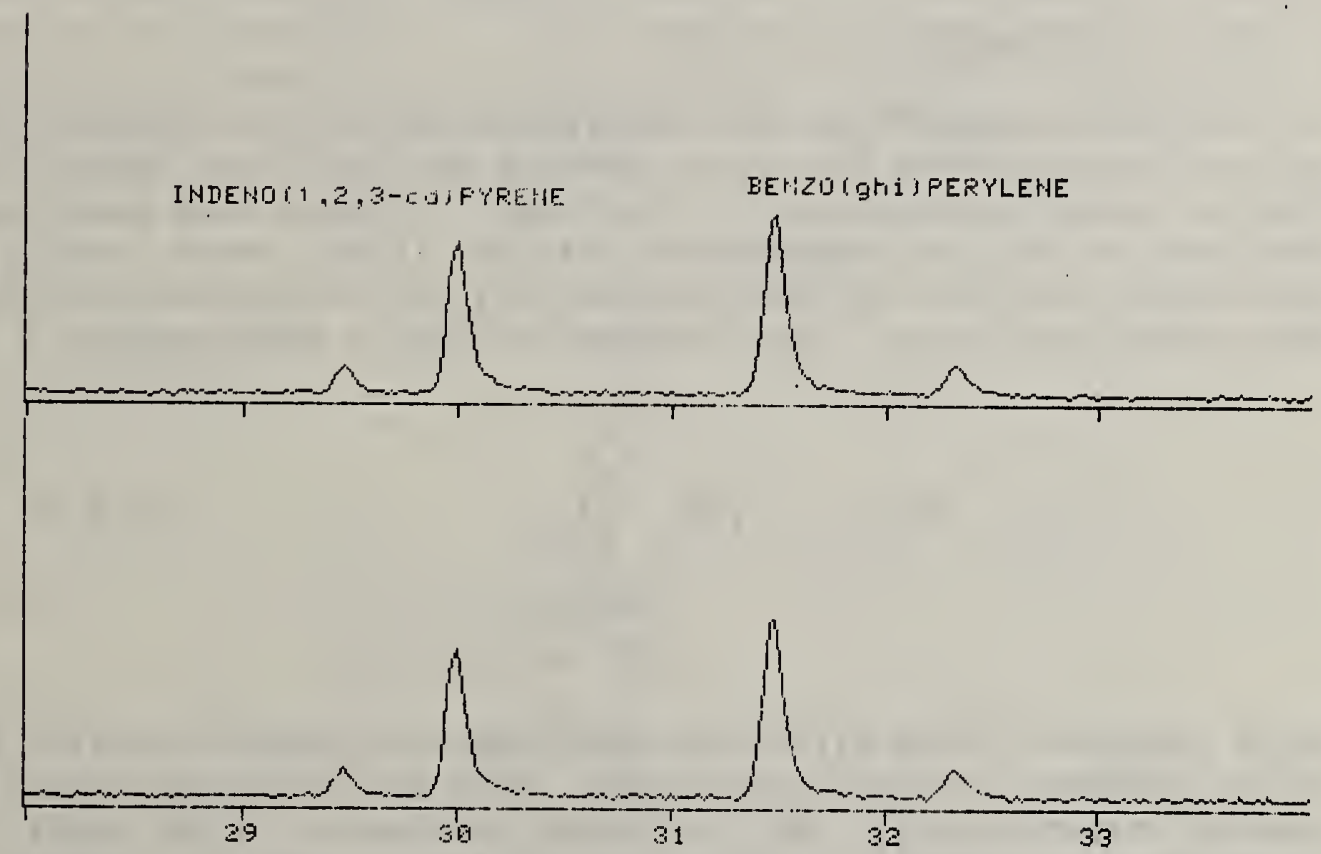

Figure 32. Negative chemical ionization selected ion record chromatograms $\mathrm{m} / \mathrm{z} 252,264$, and 276 for analysis of Wilmington crude oil. 
Table 31. Concentrations of Selected Polynuclear Aromatic Hydrocarbons in Wi lmington Crude Oil Measured by Negative Chemical Ionization GC/MS.

$\begin{array}{lcc}\text { Benzo[a]pyrene } & \begin{array}{c}\text { Concentration }(\mu \mathrm{g} / \mathrm{g})^{\mathrm{a}} \\ \text { Indeno[1,2,3-cd]pyrene }\end{array} & \text { Benzo[ghi]perylene } \\ 0.951 \pm 0.048 & 0.169 \pm 0.044 & 1.697 \pm 0.135\end{array}$

$\mathrm{a}_{\text {Values represent }}$ the mean \pm standard deviation for 15 determinations. Reference

[1] Stock1, D. and Budzikiewicz, H., Org. Mass Spectrom. 17 (8), 376 (1982).

22. Polyethylene Methacrylate Coated Electrodes--Nonaqueous Solvent-Stable Polymer Film Electrode Modified with Methylviologen (26101)

E. A. Blubaugh, R. A. Durst

We wish to report on the preparation and electrochemical evaluation of polymer film modified electrodes for use as analytical sensors when derivatized with an appropriate catalyst. Platinum electrodes have been coated with the copolymer of ethylene methacrylic acid/Na (PEM), which forms a uniform and continuous film, and cationic species may be incorporated via ion exchange at the carboxylate sites. The structure of PEM is shown below:

$$
-\left(\mathrm{CH}_{2}-\mathrm{CH}_{2}\right)_{\bar{n}}-\cdots-\left(\begin{array}{c}
\left.\mathrm{CH}_{2}-\frac{1}{\mathrm{C}}\right)_{\mathrm{m}} \\
\frac{\mathrm{COOH}}{\mathrm{COO}^{-} \mathrm{Na}^{+}}
\end{array} \quad \cdot \mathrm{n} / \mathrm{m} \cong 25\right.
$$

These copolymer films offer the advantages of enhanced tensile strength, ease of preparation, and most importantiy, they are stable to a wide range of organic solvents (e.g., DMF, propylene carbonate, $\mathrm{CH}_{3} \mathrm{CN}, \mathrm{CH}_{2} \mathrm{Cl}_{2}$ ), which are important with respect to their utilization as chemical sensors.

These ion-exchange polymers may fulfill the above goal for the following reasons. Ion-exchange based electrode coatings have the potential technological advantages of being regenerable and easy to prepare. The electroactive catalyst is incorporated by immersing the polymer film/electrode into a solution of the catalyst. A disadvantage of these ion-exchange polymers is that the electrostatic trapping in the ion-exchange process is a chemically reversible step. That is, the catalyst may be leached from the polymer film. However, relative solution/polymer film solubilities may be exploited to minimize the leaching problem. 
We have incorporated a variety of redox catalysts in polymer films of PEM. Some examples of these cationic electroactive species are methylviologen, [ruthenium-trisbipyridine] ${ }^{2+}$ and 5-pheny]-2-(4-pyridy])oxazole. ${ }^{1}$ :0TS (a laser dye). We have concentrated our efforts on the study of methylviologen and the laser dyes. We are primarily interested in the methylviologen as a redox catalyst for the catalytic reduction of organo-halides. The following reactions are important to investigate:

$$
\mathrm{MVII}_{\mathrm{MV}^{+}+\mathrm{R}-\mathrm{X} \rightarrow \mathrm{MV}^{-} \rightarrow \mathrm{MV}^{+}}^{+}+\mathrm{R}^{+}+\mathrm{Cl}^{-}
$$

Figure 33 presents the cyclic voltammogram for the redox reactions in equation 1 for the reduction of methylviologen. This reaction takes place in the polymer $f i l m$, and analys is of the current-potential curves indicate that the reaction is chemically reversible. As the reduction of the entrapped methylviologen proceeds, the film turns a bright blue color, due to the cation radical $\mathrm{MV}^{+}$. We have added solutions of organo-halide compounds (e.g., 1,2-dibromoethane) to the solution containing the derivatized polymer film electrode and observed enhanced reduction current for the catalytic reduction of the organo-halide (reaction 2). This behavior unfortunately persists for only a few cyclic voltammograms. Future work will involve investigation of the cause of instability of these methylviologen derivatized polymer films of PEM and their use as analytical sensors.
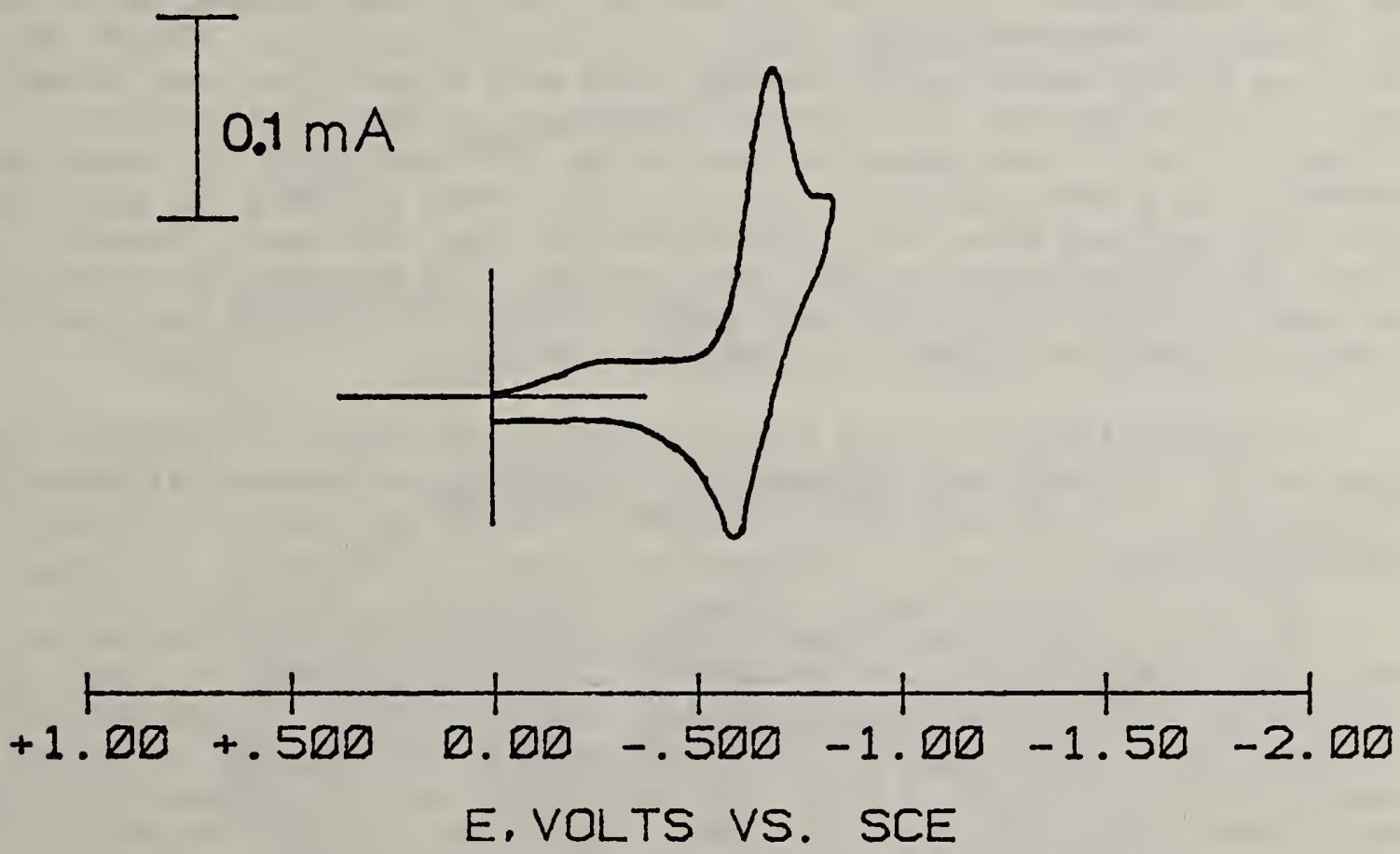

Figure 33. Cyclic voltammogram for incorporatted methyl viologen in polyethylene methacrylic acid/Na copolymer film electrode in $0.200 \mathrm{M} \mathrm{Na}_{2} \mathrm{SO}_{4}$ solution. Scan rate $=50 \mathrm{mV} /$ second. 
23. Use of a Zinc Scrubber Column for In-Line Removal of Oxygen from HPLC Mobile Phases $(15320,26101)$

W. A. MacCrehan, W. E. May

There are several reasons why the removal of atmospheric oxygen from liquid chromatographic mobile phases may be desirable or necessary. In the case of molecular fluorescence detection, the signal may be "quenched" by intersystem energy transfer from the excited state of the fluorophore to singlet oxygen. For reductive electrochemical detection, oxygen must be reduced to very low levels, since the oxygen reduction current contributes deleteriously to the residual current and detector noise.

We have discovered a simple, but effective, way to quantitatively reduce oxygen to water in high-performance liquid chromatographic mobile phases, using a zinc scrubber column (consisting of a short HPLC column, dry-packed with zinc particles). When a neutral supporting electrolyte is added to the LC eluent, oxygen is reduced by the following reactions:

$$
\begin{aligned}
& \mathrm{Zn}+\mathrm{O}_{2}+4 \mathrm{H}^{+} \rightarrow \mathrm{Zn}^{+}+\mathrm{H}_{2} \mathrm{O}_{2} \\
& \mathrm{Zn}+\mathrm{H}_{2} \mathrm{O}_{2}+2 \mathrm{H}^{+} \rightarrow \mathrm{Zn}^{+}+2 \mathrm{H}_{2} \mathrm{O}
\end{aligned}
$$

We felt that it was important to investigate whether both reactions 1 and 2 went to completion, since the incompleteness of reaction 2 would allow the undesirable production of hydrogen peroxide in the HPLC mobile phase. To test for the production of $\mathrm{H}_{2} \mathrm{O}_{2}$, an aliquot of zinc scrubbed eluent was collected and subjected to differential pulse polarographic analysis. Figure $34-A$ shows the scan of the unadulterated eluent, with only a reduction wave evident for $\mathrm{Zn}_{2}$. To confirm that $\mathrm{H}_{2} \mathrm{O}_{2}$ did not contribute to the signal observed, an aliquot of $\mathrm{Na}_{2}$ EDTA was added to complex the $\mathrm{Zn}^{+}{ }_{2}$, shifting its reduction potential to a more negative value. $\mathrm{Had}_{2} \mathrm{O}_{2}$ been present, its wave should have been apparent after the "subtraction" of the zinc wave. However, as figure 34-B illustrates, no $\mathrm{H}_{2} \mathrm{O}_{2}$ was detected. To demonstrate the expected response for $\mathrm{H}_{2} \mathrm{O}_{2}$, an aliquot was added to the level of $\mathrm{H}_{2} \mathrm{O}_{2}$ that would be expected if only reaction 1 occurred (figure 34-C).

The effectiveness of the oxygen reduction efficiency is apparent when we examine the residual current curve for reductive electrochemical detection (figure 35). The current level obtained is quite low and is suitable for high sensitivity work.

The zinc scrubber may also be used in conjunction with fluorescence detection to eliminate oxygen-quenching. The data presented in Table 32 show the relative signal enhancement obtained when the oxygen is removed with the scrubber, when ratioed to the signal obtained in non-degassed saturated mobile phase (70 percent $\mathrm{CH}_{3} \mathrm{CN}$ in $\mathrm{H}_{2} \mathrm{O}$ ). In some cases, such as pyrene, the signal enhancement factor (9) could provide a significant increase in sensitivity as well as improve the reproducibility of fluorescence measurement. 


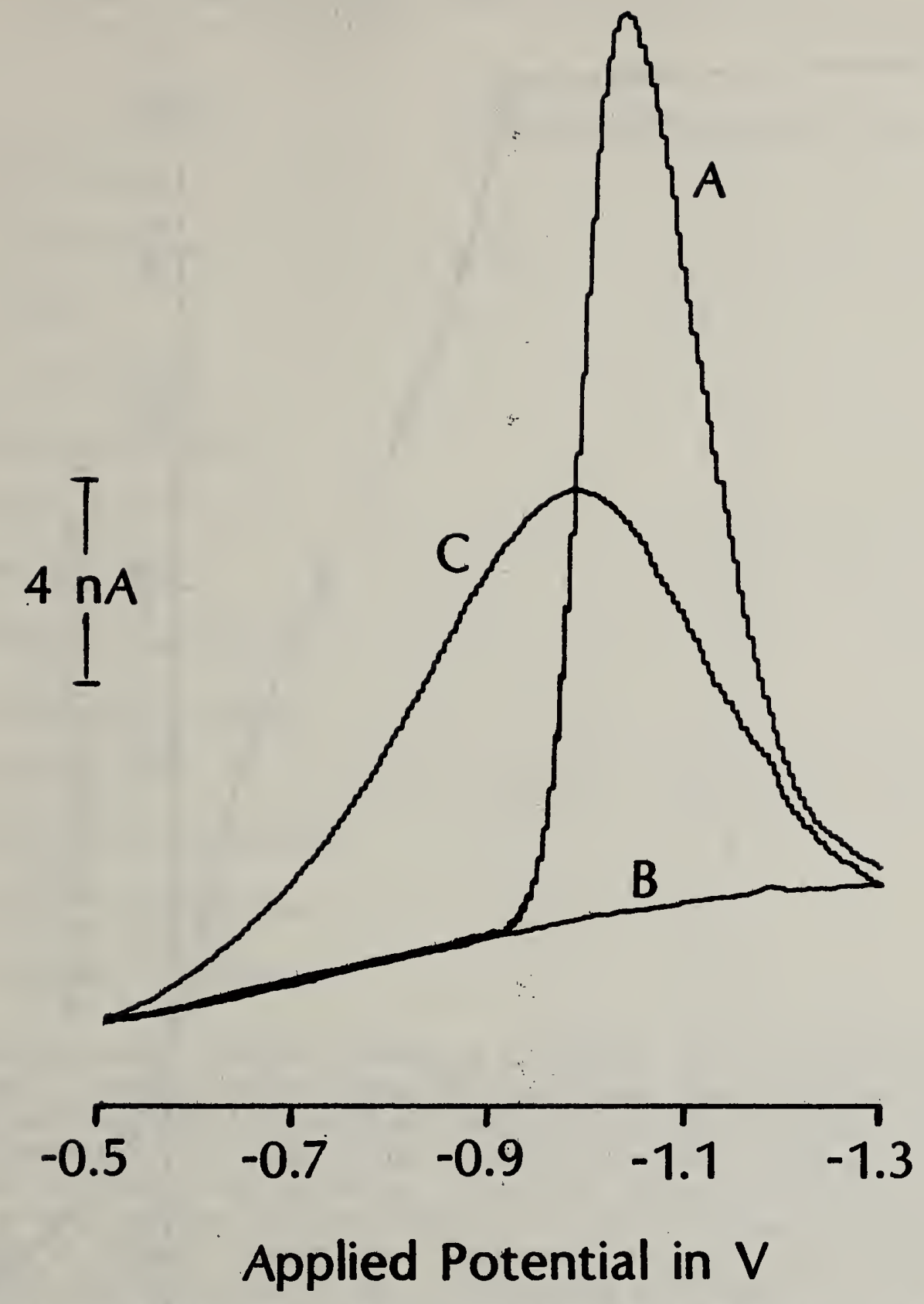

Figure 34. Differential pulse polarographic analysis of zinc scrubbed eluent. Conditions: sample--eluent diluted $1: 10$ with $0.1 \mathrm{~mol} / \mathrm{L}$ sodium phosphate buffer $\mathrm{pH} 7.0$; electrode--SMDE medium drop; reference electrode--Ag/AgCl $3 \mathrm{~mol} / \mathrm{L} \mathrm{KCl}$; scan rate--5 $\mathrm{mV} / \mathrm{s}$. A--scrubbed eluent alone; $\mathrm{B}$--addition. of $4 \times 10^{-3} \mathrm{~mol} / \mathrm{L} \mathrm{Na}{ }_{2}$ EDTA; C--addition of $1.3 \times 10^{-4} \mathrm{~mol} / \mathrm{L}$ $\mathrm{H}_{2} \mathrm{O}_{2}$. 


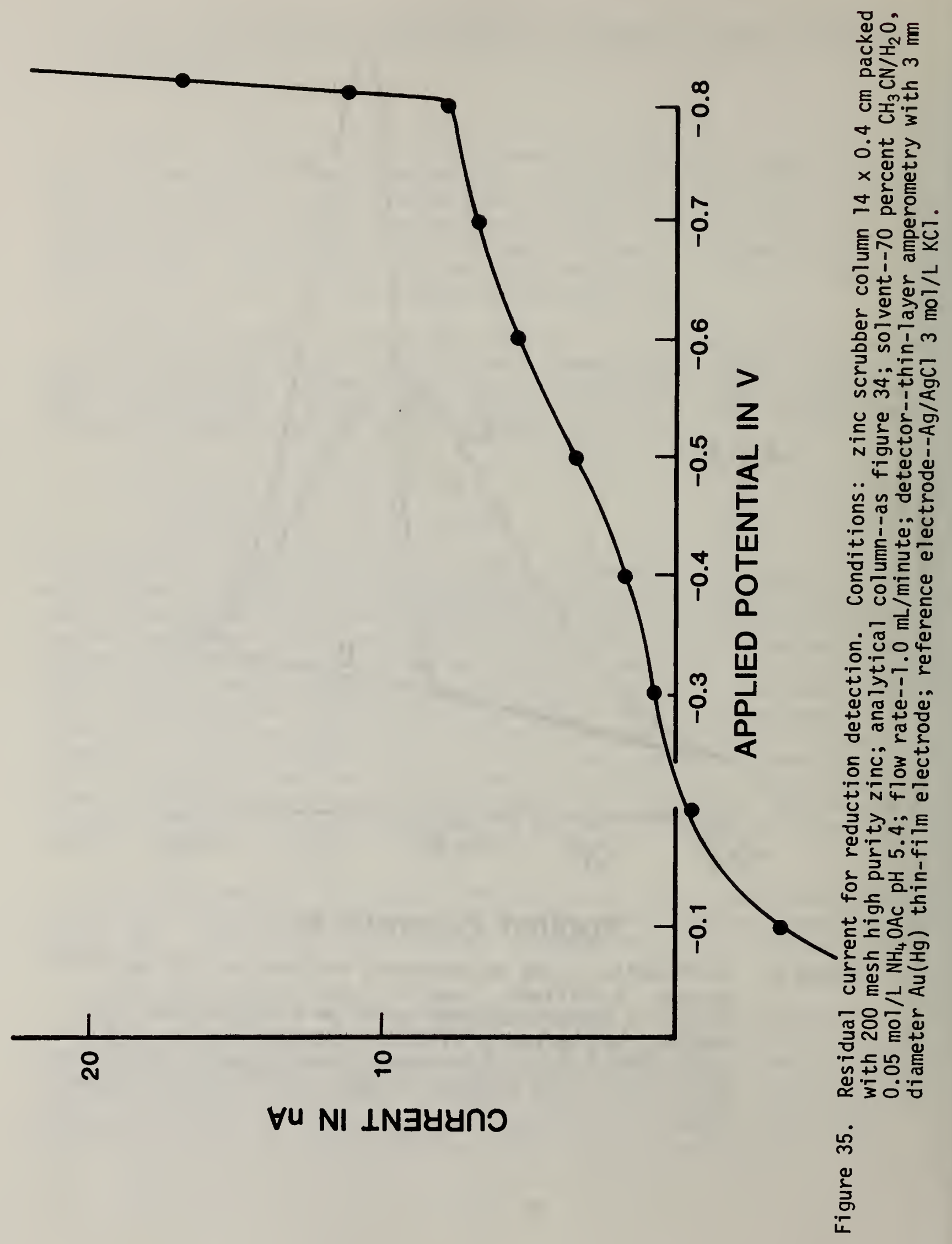


Table 32. Fluorescence Enhancement Using Zinc Scrubber Column, Relative to Air Saturated Solution.

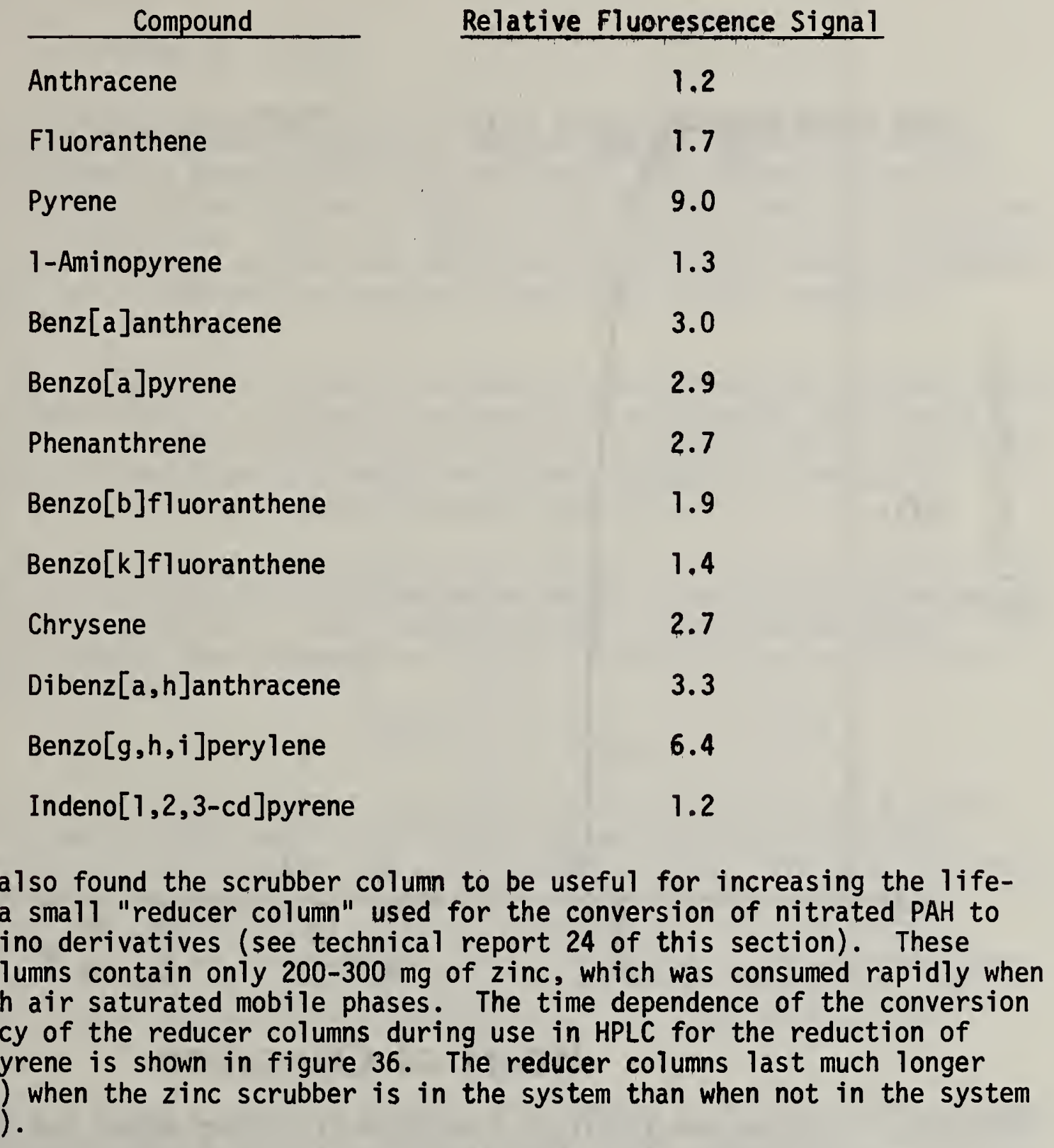




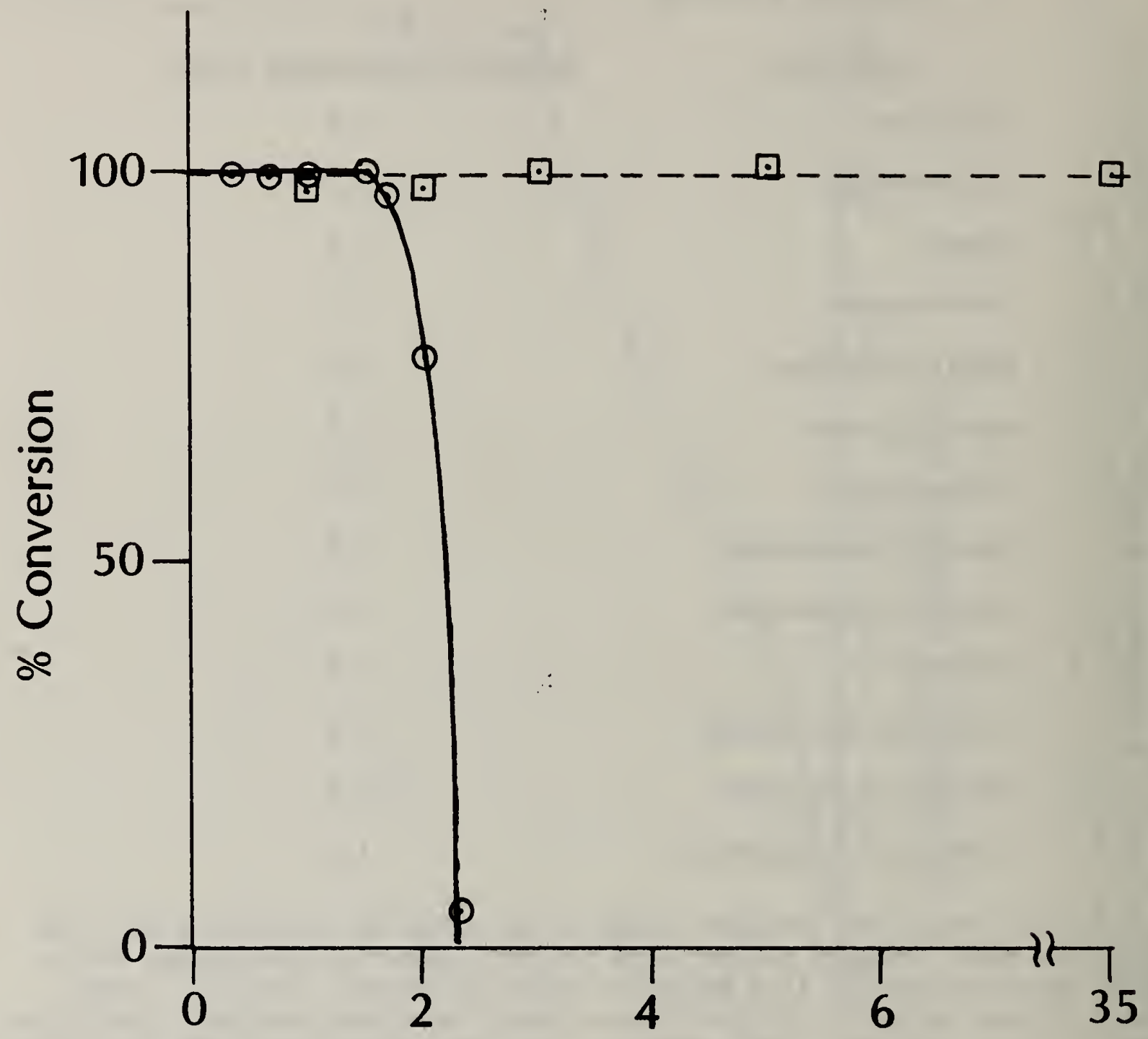

Hours of Operation

Figure 36. Fluorescence signal as a function of reducer column age when used at constant flow. Conditions: as figure 35 except, analyte 1-nitropyrene; flow rate-- $1.5 \mathrm{~mL} / \mathrm{min}$; open circles/solid line-wi thout zinc scrubber; squares/dashed line--with zinc scrubber. 
24. High-Performance Liquid Chromatographic Methods for the Determination of Nitro-Polynuclear Aromatic Hydrocarbons (N-PAH) in Complex Mixtures $(15320,26101)$

\section{W. A. MacCrehan, W. E. May}

Many studies have demonstrated the high mutagenicity of diesel and gasoline engine particulate emissions. Recent work has shown that unburned polynuclear aromatic hydrocarbons (PAH) may react with nitrogen oxides to produce nitrated PAH derivatives (N-PAH). The N-PAH compounds that are formed are said to represent as much as 50 percent of the "direct-acting" mutagenicity of diesel particulate extracts when determined by the Ames Salmonella bioassay [1]. Thus, it is important to develop accurate and reliable methods for measuring these compounds.

During the past year, we have continued to develop the two high-performance liquid chromatographic (HPLC) detection methods, mentioned in last year's report [2], for the HPLC determination of N-PAH in complex mixtures--molecular fluorescence following on-line reduction to the corresponding amine and reductive differential pulse electrochemical detection.

The fluorescence detection approach relies on a simple, quantitative conversion of the N-PAH to the corresponding amine using a short column packed with zinc metal particles. This "reducer column" follows the analytical column but precedes the fluorescence detector. The following reaction has been shown to occur:

$$
\mathrm{Ar}-\mathrm{NO}_{2}+\mathrm{Zn} \stackrel{\mathrm{pH} 5.2}{\longrightarrow} \mathrm{Ar}-\mathrm{NH}_{2}+\mathrm{Zn}^{+2}
$$

The measured conversion efficiency was found to be greater than 99.5 percent for the following N-PAH tested: 2-nitronaphthalene, 9-nitroanthracene, 2-nitrofluorene, 1-nitropyrene, and 6-nitrobenzo(a)pyrene. The peak dispersion effect of using the reducer column was minimized by its small dimensions $(0.4 \times 4 \mathrm{~cm})$ and by mixing small particle zinc metal $(7 \mu \mathrm{m})$ with silica particles $(20 \mu \mathrm{m})$ in an optimized ratio of $1: 2$ by weight. The peak-width-athalf-height is increased by only about one second for a fifteen-second peak. Thus, very little chromatographic resolution is lost using this on-line reduction.

Figure 37 shows a gradient elution chromatogram of several N-PAH standards using the reduction/fluorescence detection approach. The limit of detection is quite good--about $15 \mathrm{pg}$ for 1-nitropyrene. This is quite adequate for the measurement of N-PAH in organic solvent extracts of diesel soot.

Selectivity in the fluorescence detection comes from the ability to control both the excitation wavelength and resulting emission wavelength. In fact, for detection of selected N-PAH compounds, the excitation and emission wavelengths may be programmed during the chromatographic run, optimizing these parameters for each compound. However, despite the selectivity of this fluorescence detection approach, the diesel particulate extracts have too many fluorescent matrix constituents (such as PAH and nitrogen-containing aromatic 


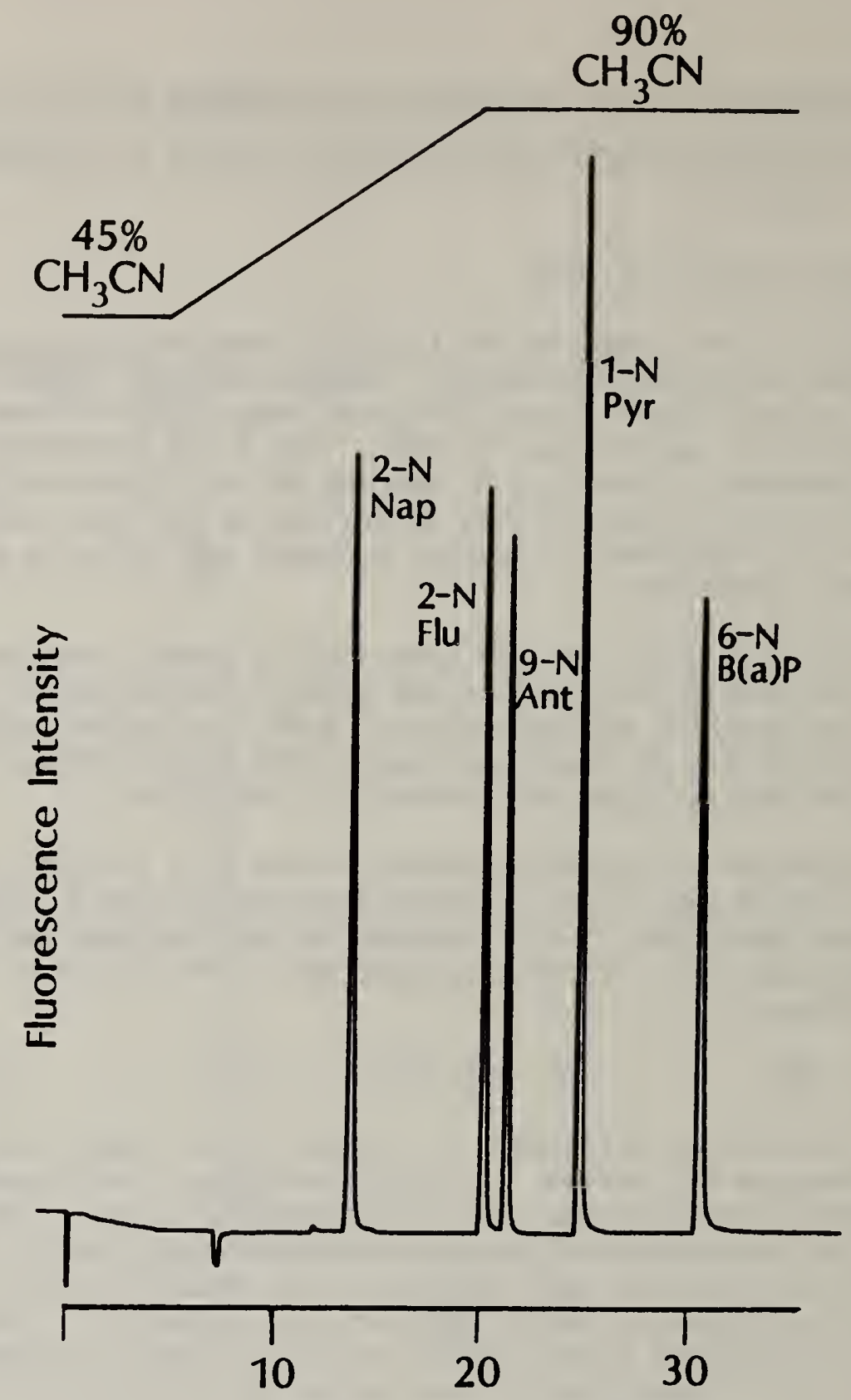

Minutes

Figure 37. HPLC/fluorescence detection of some N-PAH standards after on-line reduction of the amines. Conditions: column--octadecylsilane bonded-phase silica, $5 \mu \mathrm{m}$ particles, $25 \times 0.46 \mathrm{~cm}$; solvent-gradient of $\mathrm{CH}_{3} \mathrm{CN} / \mathrm{H}_{2} \mathrm{O}$ as marked, $0.025 \mathrm{~mol} / \mathrm{L} \mathrm{NH}_{4} \mathrm{OAC} \mathrm{pH} 5.4$; flow rate--1.5 mL/minute; zinc/oxygen scrubber $14 \times 0.4 \mathrm{~cm}, 100$ mesh particles; zinc--reducer column $4 \times 0.3 \mathrm{~cm}$, packed with $\mathrm{Zn} /$ silica; detector--fluorescence with excitation and emission wavelengths optimized for each component using $2.5 \mathrm{~nm}$ slit widths; sample--a $2 \mu \mathrm{L}$ injection of a mixture of $10.0 \mu \mathrm{g} / \mathrm{g} 2$ 2-nitronaphthalene $(2-\mathrm{N}$ Nap), $10.7 \mu \mathrm{g} / \mathrm{g} 2$ 2-nitrofluorene $(2-\mathrm{N}$ Flu $), 11.7 \mu \mathrm{g} / \mathrm{g} 9-n i t r o-$ anthracene $(9-N$ Ant $), 11.0 \mu \mathrm{g} / \mathrm{g} 1-$ nitropyrene $(1-N \mathrm{Pyr})$, and $8.0 \mu \mathrm{g} / \mathrm{g}$ 6-benzo[a]pyrene $(6-N \mathrm{~B}[\mathrm{a}] \mathrm{P})$. 
compounds) for the signals from $\mathrm{N}-\mathrm{PAH}$ to be measured without interference by a direct injection HPLC analysis. A semi-preparative, normal-phase fractionation of the diesel particulate extracts has been developed to overcome this selectivity difficulty. Using a hexane/methylene chloride mobile phase and a propylaminocyano bonded-phase column, a fraction may be collected that includes $\mathrm{N}-\mathrm{PAH}$ from between two to seven aromatic rings, yet excludes all interfering $\mathrm{PAH}$ and other aromatic nitrogen-containing compounds. The detection of several $\mathrm{N}$-PAH in a methylene chloride extract of diesel particulates, using the fractionation and reduction/fluorescence approach, is shown in figure 38 . Future work will center on developing an optimum excitation/emission wavelength program and the extension of the technique to include dinitro-PAH.

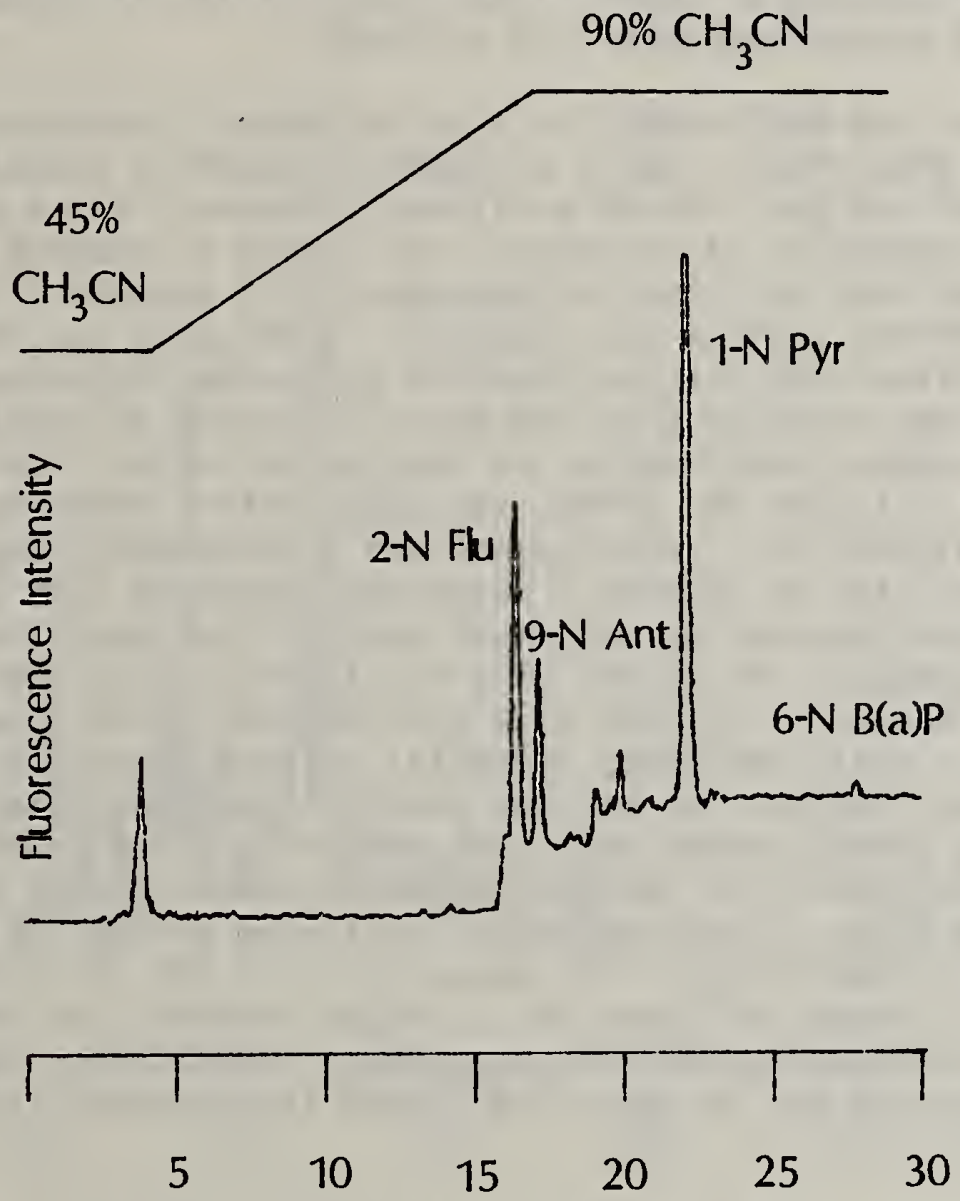

Minutes

Figure 38. Fractionated diesel particulate sample using wavelength programmed fluorescence detection. Conditions: column--octadecylsilane bonded-phase silica, $5 \mu \mathrm{m}$ particles, $25 \times 0.46 \mathrm{~cm}$; solvent-methylene chloride extract of diesel particulates fractionated on $\mathrm{PAC}$ column; flow rate-- $1.5 \mathrm{~mL} /$ minute; zinc/oxygen scrubber $14 \times 0.4$ $\mathrm{cm}, 100$ mesh particles; zinc--reducer column $4 \times 0.3 \mathrm{~cm}$, packed with $\mathrm{Zn/silica;}$ detector--fluorescence with the following optimized wavelengths $\left(2.5 \mathrm{~nm}\right.$ slit widths) -- 2-nitrofluorene $\lambda_{\mathrm{ex}}=285 \mathrm{~nm}$, $\lambda_{\mathrm{em}}=370 \mathrm{~nm}, 9-$ nitroanthracene $\lambda_{\mathrm{ex}}=260 \mathrm{~nm}, \lambda_{\mathrm{em}}=505 \mathrm{~nm}, 1$-nitropyrene $\lambda_{\mathrm{ex}}=360 \mathrm{~nm}, \lambda_{\mathrm{em}}=430 \mathrm{~nm}, 6-\mathrm{nitrobenzo}[\mathrm{a}]$ pyrene $\lambda_{\mathrm{ex}}=300$ $\mathrm{nm}, \lambda_{\mathrm{em}}=475 \mathrm{~nm}$. 
The second approach under development for the HPLC detection of N-PAH compounds is reductive electrochemical detection. This approach utilizes the relatively low electrochemical reduction potential of nitro-aromatic compounds at a mercury-gold thin-film electrode. When this detector cell is used in conjunction with the zinc-oxygen scrubber (to remove atmospheric oxygen to low levels), very low residual currents are observed at the potential for the detection of $\mathrm{N}$-PAH.

In order to optimize the sensitivity and selectivity of the electrochemical detection approach, we are making a thorough investigation of the electrochemical behavior of N-PAH. This information will then be used to aid in the design of an optimum detection strategy.

The simplest and most sensitive electrochemical detection mode we have investigated is amperometry, where a constant potential (usually $-0.75 \mathrm{~V}$ for $\mathrm{N}-\mathrm{PAH}$ ) is applied and the current monitored. However, since all species with their reduction potential at or below $-0.75 \mathrm{~V}$ give a detector response, the amperometric mode does not have the selectivity to measure $\mathrm{N}-\mathrm{PAH}$ directiy in unfractionated diesel particulate extracts. Other work has shown that the diesel soot contains many electroreducible oxygenated hydrocarbons, such as quinones, which may co-elute with the $\mathrm{N}$-PAH and cause an additive interference. When the normal-phase fractionation is used prior to the reversed-phase chromatography to isolate the N-PAH, the amperometric detection approach produces clear signals for 1-nitropyrene and 6-nitrobenzo(a)pyrene (figure 39). However, we would like to develop a detection technique that would not rely on the same sample preparation procedure used in the reductive/fluorescence approach. Fortunately, the selectivity of electrochemical detection may be substantially enhanced by the use of a differential pulse waveform. In this detection mode, a small amplitude potential pulse--centered at the potential where the analyte displays the maximum change in current/potential behavior-is combined with gated current detection measuring the difference current resulting from the pulse. A selectivity enhancement results since only compounds having their current/potential half-wave within the pulse interval will provide a current output. The detection of $\mathrm{N}-\mathrm{PAH}$ in an unfractionated diesel extract is shown in figure 40 . A pulse interval was chosen that was optimized for the detection of 1-nitropyrene. Signals for 1-nitropyrene and 6-nitrobenzo(a)pyrene may be seen with little interference from unresolved matrix components.

These HPLC procedures will be used in conjunction with gas chromatography/ mass spectrometry (GC/MS) to certify the concentrations of selected $\mathrm{N}-\mathrm{PAH}$ in a diesel soot material (SRM 1650).

References

[1] Schuetzle, D., Env. Health Persp. 47, 65-80 (1983).

[2] Center for Analytical Chemistry Annual Report, NBSIR 82-2620 (1982). 


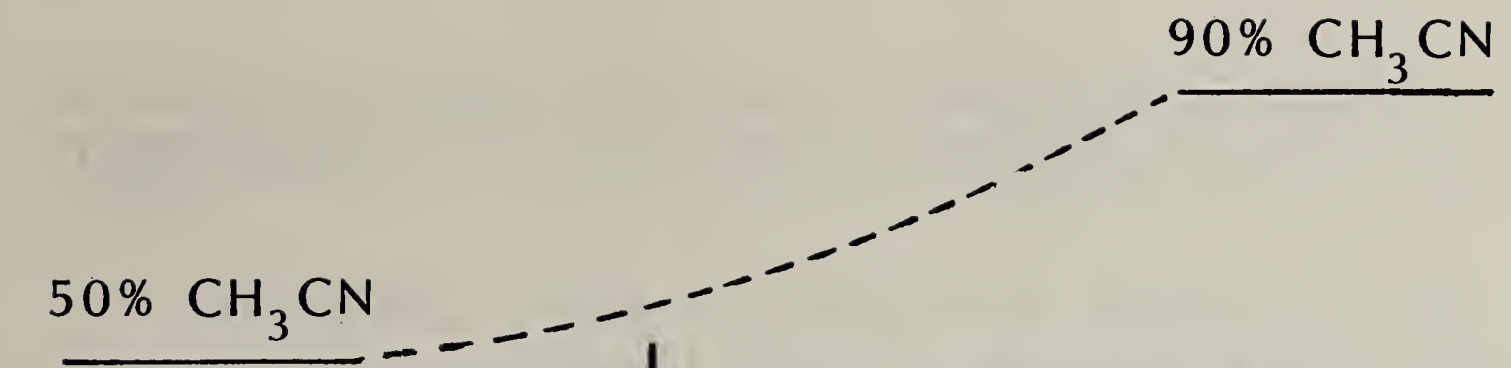

Amperometric Detection at $-0.7 \mathrm{~V}$

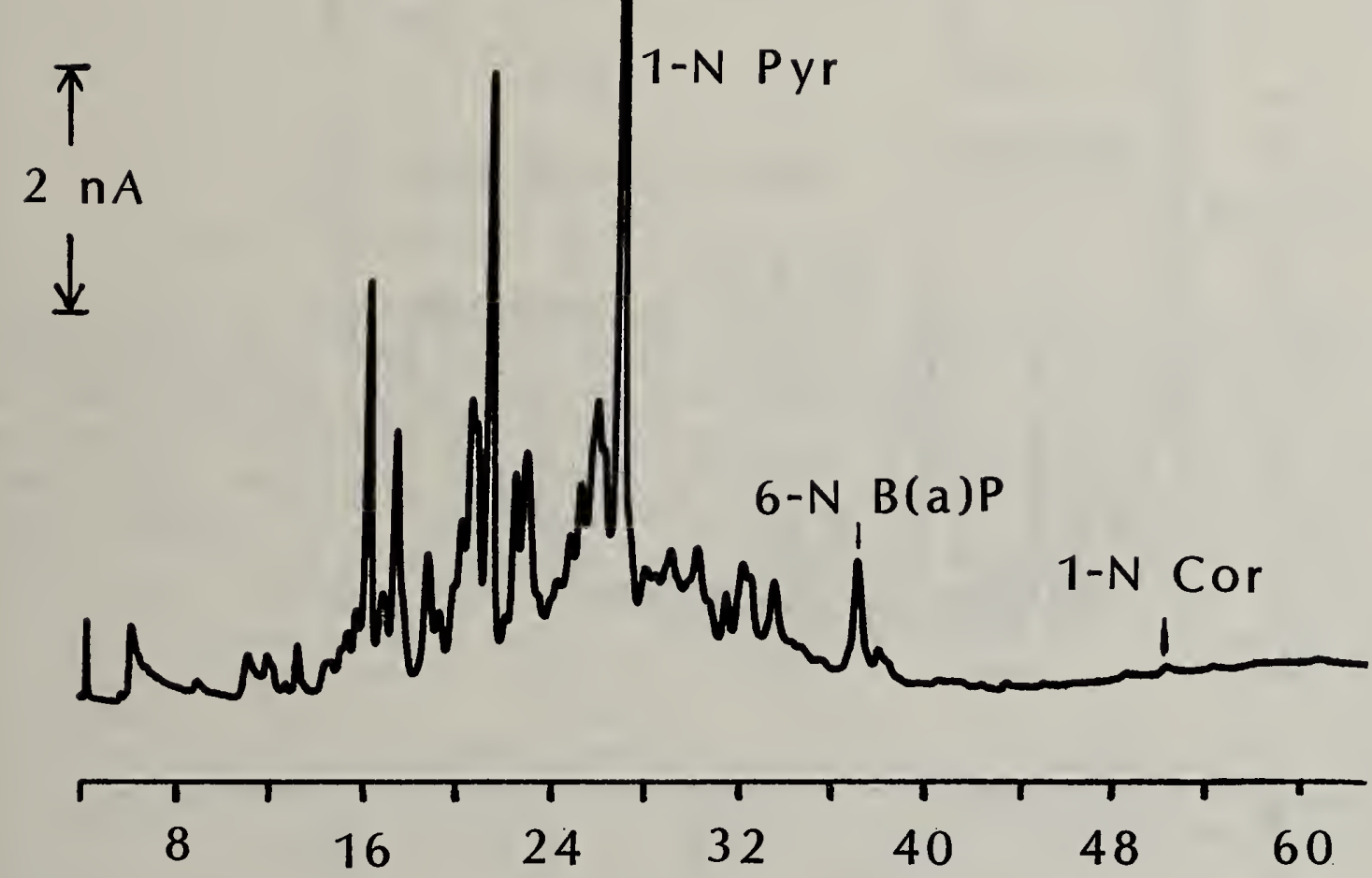

\section{Minutes}

Figure 39. Fractionated diesel particulate sample using amperometric detection. Conditions: column--octadecylsilane bonded-phase silica, $5 \mu \mathrm{m}$ particles, $25 \times 0.46 \mathrm{~cm}$; solvent--methylene chloride extract of diesel particulates fractionated on PAC column; flow rate--1.5 mL/ min; zinc/oxygen scrubber $14 \times 0.4 \mathrm{~cm}, 100$ mesh particles; zinc-reducer column $4 \times 0.3 \mathrm{~cm}$, packed with $\mathrm{Zn} /$ silica; detector-amperometry at $-0.7 \mathrm{~V}$ (versus $\mathrm{Ag} / \mathrm{AgCl} 3 \mathrm{~mol} / \mathrm{L} \mathrm{KCl}$ reference), electrode gold-mercury thin film $3.0 \mathrm{~mm}$ diameter. 


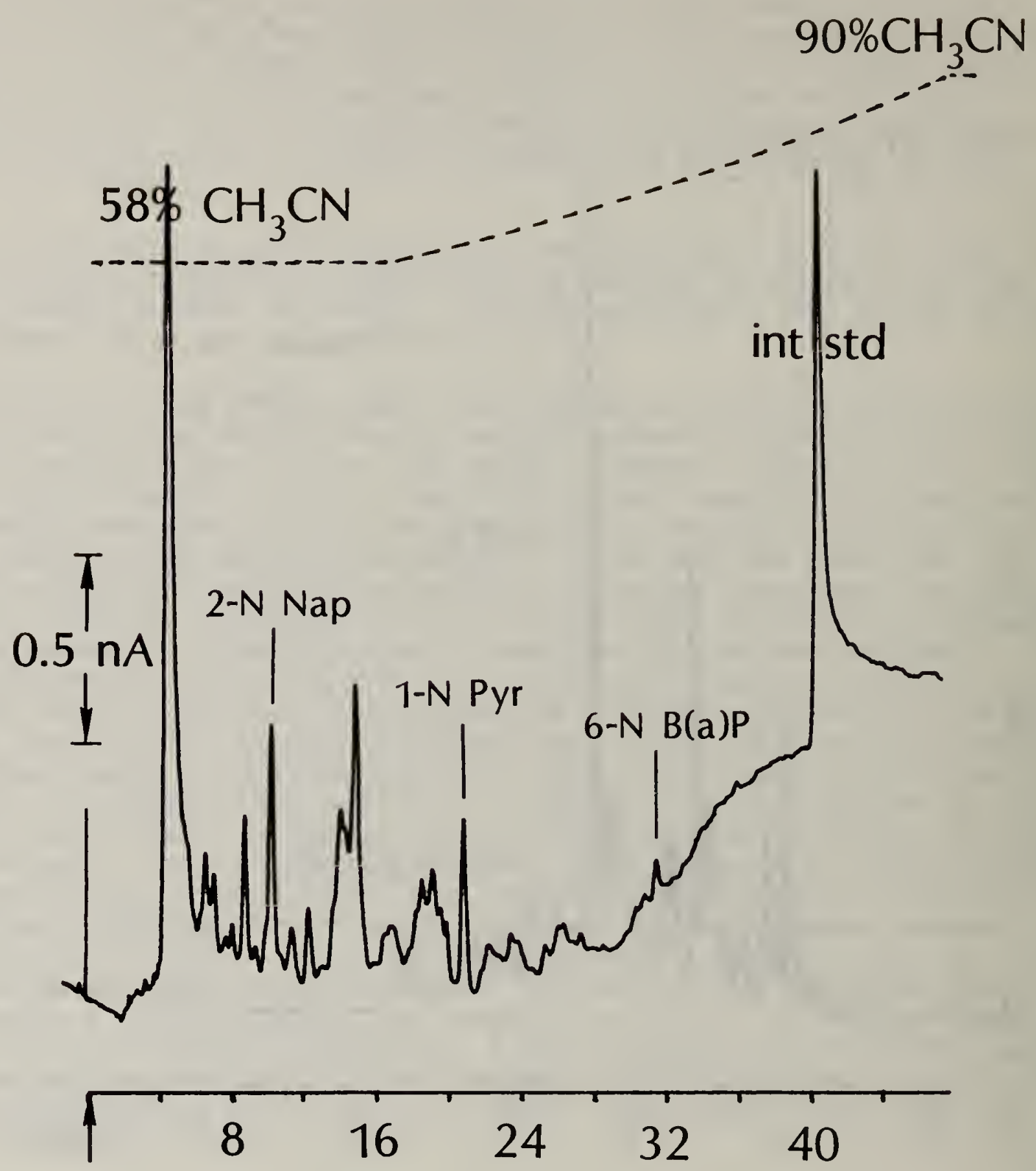

Minutes

Figure 40. Unfractionated diesel particulate sample using differential pulse electrochemical detection. Conditions: sample--methylene chloride extract of diesel particulates; detector electrode-gold-mercury thin film $1.2 \mathrm{~mm}$ diameter; base potential -0.57 , pulse height $5 \mathrm{mV}$. 
25. Spectroelectrochemical Investigations of Cyanine Dyes, Ruthenium-

(bipyridine) ${ }_{2}$ dithiocarbamate Complexes, and Copper Dithiocroconates (26101)

E. A. Blubaugh, R. T. Burke, R. A. Durst, G. S. Marbury, W. T. Yap

Several spectroelectrochemical studies are currently in progress to help elucidate the electrochemical behavior of a variety of interesting research materials. One of these studies concerns the vinylogous family of polyine monocationic dyes, illustrated by the typical group structure:

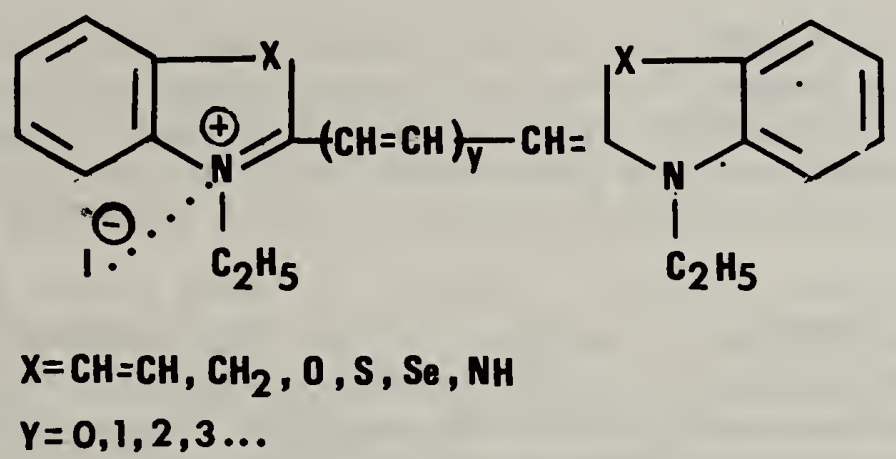

These compounds are very strong absorbers of radiation in the visible spectral region with their wavelengths of maximum absorbance determined by the length of the conjugated chain, the end group heteroatom, and the structure of the end group. These dyes are widely used as sensitizers in photographic emulsions, laser dyes, and energy conversion catalysts in photovoltaic cells. Using the model compound 1,1'-diethyl-4,4'-dicarbocyanine iodide (kryptocyanine), a series of spectra were recorded spectropotentiostatically over a period of 90 minutes. The very sharp isosbestic point observed indicates that the product, assumed to be a radical, is exceptionally stable. A Nernstian plot of the applied potential versus $\log [R] /[0]$ was linear and, from the slope and intercept, values of $n=1.10$ and $E^{01}=0.882 \mathrm{~V}$ versus SSCE were obtained. In addition to providing thermodynamic data on these compounds, these spectroelectrochemical studies point to a potential new application. Because of the exceptionally large absorptivities of both the reactant and product, and the apparent reversibility of the electron transfer process, their use in electrochromic display devices is suggested.

A great deal of interest and work by a number of research groups has been directed toward the characterization of bipyridine complexes of ruthenium. This interest is generated, in part, because of the usefulness of these ruthenium complexes for heterogeneous catalysis, for solar energy conversion, and in electrochromic devices. This work focuses on the electrochemical characterization of a series of complexes with the general formula: 
$\operatorname{Ru}\left(\right.$ II) $(\text { bpy })_{2} S-\stackrel{\text { II }}{C}-R$

where bpy $=2,2$ - bipyridine, and

$\mathrm{R}=-\mathrm{O}-\mathrm{CH}_{3},-\mathrm{N}\left(\mathrm{C}_{2} \mathrm{H}_{5}\right)_{2},-\mathrm{N}\left(\mathrm{CH}_{3}\right)_{2},-\mathrm{N}$

Cyclic voltammograms of these sulfur-containing complexes show two redox couples. The couple located in the positive potential region is the Ru(III)/ $\mathrm{Ru}(\mathrm{II})$ process, while the redox couple observed at negative potentials is ascribed to the reduction of the bipyridine ligand to the anion radical. Scan rate studies indicate that the $\mathrm{Ru}$ redox couple is a diffusion controlled process.

The spectroelectrochemical behavior of the four complexes was also investigated. A Nernst plot of spectropotentiostatic data for the xanthate complex gave values for $n$ and $E^{\circ 1}$ of 1.0 and $0.706 \mathrm{~V}$ versus SSCE, respectively. Results for the dithiocarbamate complexes were similar with values of $n=1$ and $E^{\circ 1}$ about $0.5 \mathrm{~V}$ versus SSCE. The observation that the $E^{\circ}$. values for the dithiocarbamate complexes were less positive than that for the xanthate complex presumably reflects the relative stabilization of the ruthenium oxidation states by the sulfur-containing ligands. It is proposed that the greater stabilizing power of the dithiocarbamate ligands is due to delocalization of some positive charge into the ligand system by the following resonance forms:

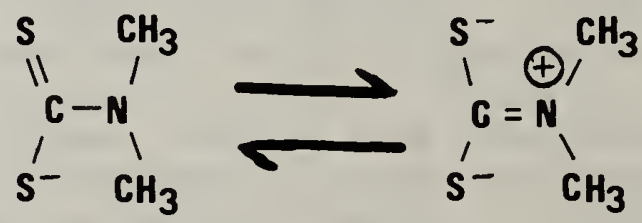

The xanthate ligand, however, does not posses capability for positive charge delocalization to the same extent.

A third study concerns the electrochemical behavior of dithiocroconate complexes of $\mathrm{Cu}$ (II). The ligands are oxocarbon analogs in which two of the oxygen atoms have been replaced by sulfur. Certain of these compounds have been shown to possess the properties of photoconductivity and rectification. Cyclic voltammetric studies of the $\mathrm{Cu}(\mathrm{II}) / \mathrm{Cu}(\mathrm{I})$ redox couple for the dithiocroconate and dicyanomethylene dithiocroconate complexes in dimethylformamide showed reversible behavior with $E^{\circ 1}$ values of $-0.34 \mathrm{~V}$ and $-0.05 \mathrm{~V}$ versus SSCE, respectively. Spectroelectrochemical Nernst plots gave values of $-0.306 \mathrm{~V}$ and $-0.0536 \mathrm{~V}$, respectively, again showing the expected trend toward less negative reduction potentials with increased ligand conjugation which is 
better able to delocalize the electron density by $\pi$ back bonding with the metal $d_{x z}$ and $d_{y z}$ orbitals. The Nernst plots provided $n$ values of 1.34 and 1.49 for the two complexes and further work is in progress to try to account for these noninteger values.

26. Theoretical Modeling of the Double Step Chronocoulometry and Spectro-
electrochemistry of Systems with Strong Product Deposition (26101)

E. A. Blubaugh, R. T. Burke, R. A. Durst, W. T. Yap

A theoretical model has been developed to explain and to analyze the double-potential step chronocoulometric data for systems with products which strongly deposit onto the electrodes, such as in the case of the cyanine laser dyes. Two of the most striking characteristics of the charge, $Q$, as functions of time, $t$, of such a system are: (1) the net charge passed in the reverse step, $Q_{r}$, plotted against $\theta=\sqrt{t-\tau}+\sqrt{\tau}-\sqrt{t}$, where $\tau$ is the time duration of the step, yields a set of parallel straight lines with constant slope for different concentrations of the dye $\left(C^{*}\right)$; and (2) intercept $\left(Q_{0}\right)$ of these lines with the $Q$-axis increasing with $\tau$. We make the assumption that the concentration of the product adjacent to the electrode surface is fiyed at the product solubility, $s_{R}$, for the condition of phase equilibrium between the deposited phase and the solution phase. Then solving the diffusion equations with appropriate initial and boundary conditions for the oxidized and reduced species, we obtained the following charge-time relationships:

$$
Q(t<\tau)=K C * \sqrt{t}+Q_{0}
$$

and

$$
Q_{r}=Q(\tau)-Q(t>\tau)=K \gamma s_{R} \theta+K\left(C^{*}-s_{R} \gamma\right) \sqrt{\tau}+Q_{0}
$$

where $K=2 n F A \sqrt{D_{0}} / \sqrt{\pi}$ and $\gamma=\sqrt{D_{R} / D_{0}}$.

Figure 41 shows plots of $Q$ as a function of $\sqrt{t}$ (unlabeled 1 ine) and $Q_{r}$ as a function of $\theta$ for various $\tau$ (curves 1 to 5 ). The points are data from experiments performed in aqueous solution containing $1.0 \mathrm{mmol} / \mathrm{L}$ of the laser dye and $0.1 \mathrm{~mol} / \mathrm{L} \mathrm{KCl}$. The values of $\tau$ for curves 1 to 5 are $6.35 \mathrm{~s}, 12.75 \mathrm{~s}$, $19.05 \mathrm{~s}, 25.4 \mathrm{~s}$, and $31.75 \mathrm{~s}$, respectively.

In a system with strong product deposition, the spectropotentiostatic Nernst plot (figure 42) is biphasic instead of a single straight line. By applying the condition of phase equilibrium between the deposited phase and the solution in the thin-layer cell, we obtain the following relation between the two Nernst plot variables, the applied potential $E$ and $r(E)=\left[A(E)-A_{R}\right]$ / $\left[A_{0}-A(E)\right]$,

$$
\ln r(E)= \begin{cases}\ln [\theta(E)(1-x f)+x(1-f)]-\ln (1-x) & \text { for } E>E_{S} \\ \ln \theta(E)+\ln [f /(1-x f-f \theta[E])] & \text { for } E<E_{S}\end{cases}
$$




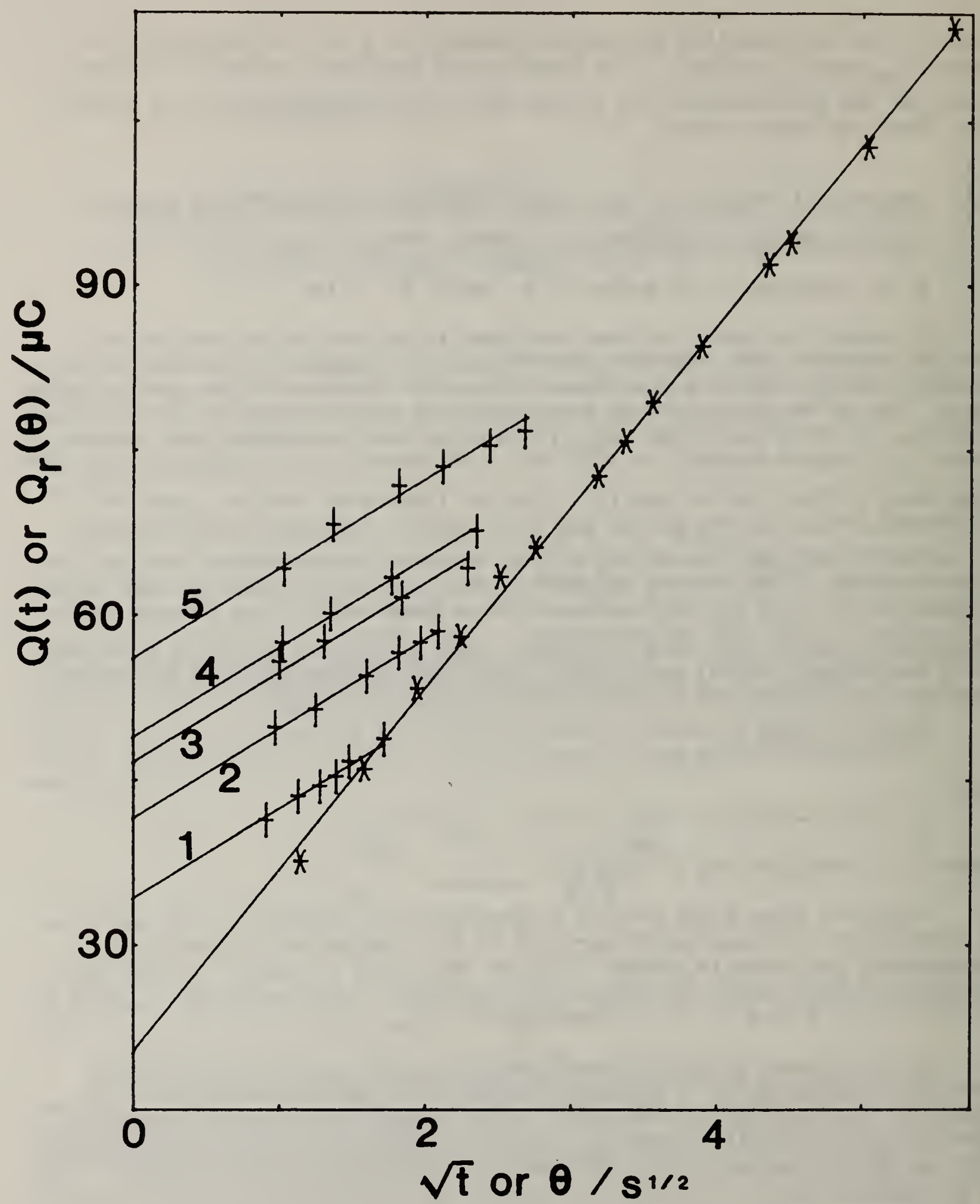

Figure 41. Plots of $Q\left(t_{<\tau}\right)$ versus $\sqrt{t}$ and $Q_{r}(t>\tau)$ versus $\theta$. For $t<\tau$, experimental points are denoted by * and the line is eq. (1). For $t>\tau$, experimental points are denoted by + and the lines are eq. (2) with different values of $\tau$. 


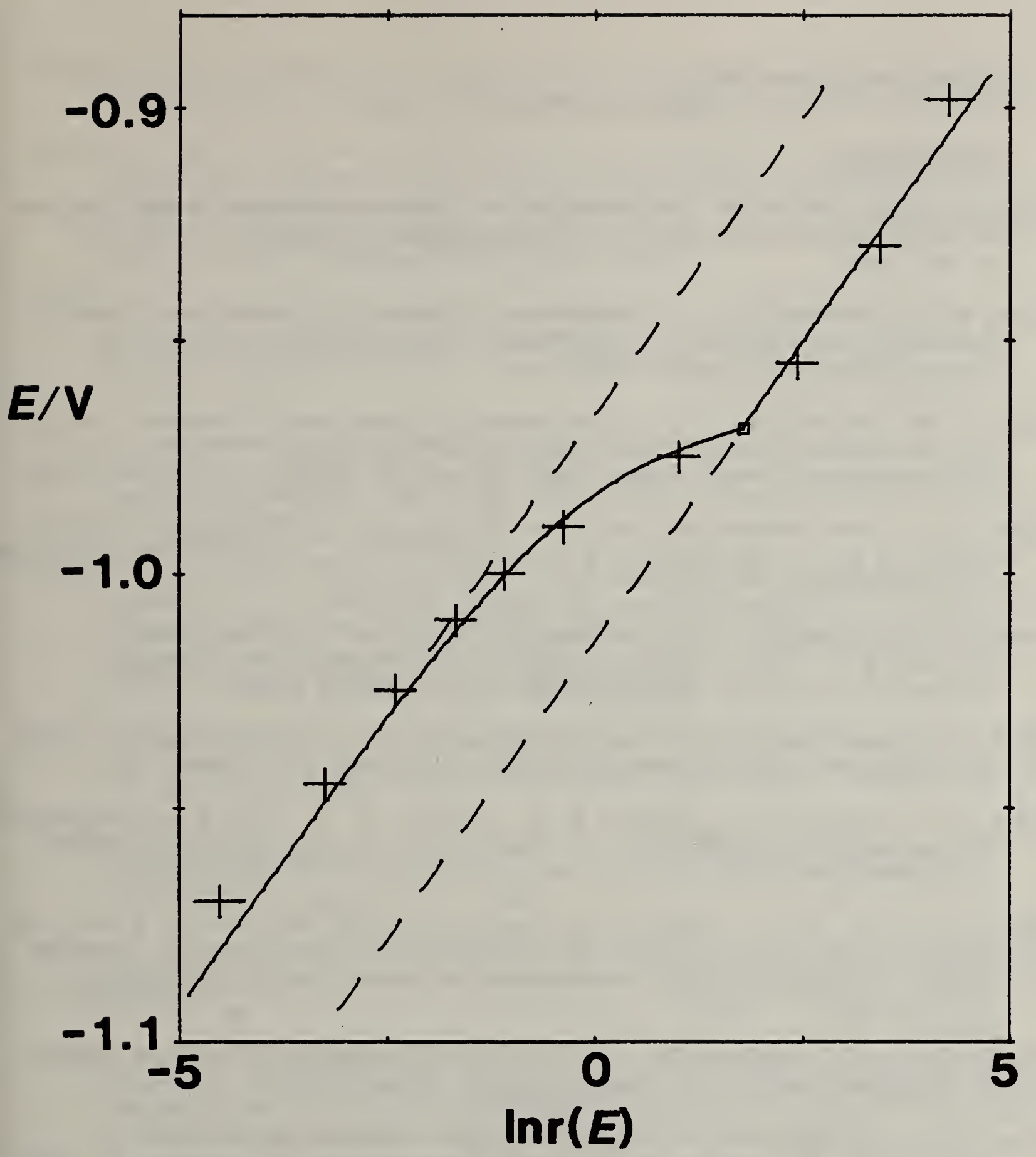

Figure 42. Plot of $E$ versus in $r(E)$. Experimental points are measured at $368 \mathrm{~nm}$. The solid curve is eq. (3).

where $f=s_{R} / C^{*}, \theta(E)=\exp \left[n\left(E-E^{\circ}\right) / R T\right]$, and $x$ denotes the ratio of the molar absorptivity of the reduced form to that of the oxidized form, $A(E)$ denotes the absorbance at $E$, and $E_{S}$ is the potential at which deposition first occurs.

Figure 42 shows a Nernst plot of an experiment using $2.4 \mathrm{mmol} / \mathrm{L}$ of laser dye in $0.1 \mathrm{mmol} / \mathrm{L} \mathrm{KCl}$. With the application of this model, the diffusion coefficient, the formal potential, the number of electrons involved in the electrochemical reactions, and the solubility of the product can be estimated from the experimental data obtained from chronocoulometry and spectroelectrochemistry. 
C. Outputs and Interactions

(Organic Analytical Research Division)

1. Publications

Bunding, K. A., Be11, M. I., and Durst, R. A., Surfaced-Enhanced Raman Spectrum of $\mathrm{N}$-Methylpyridinium Ion on a Silver Electrode, Chem. Phys. Lett. 89 , 54-58 (1982).

Coxon, B. and Reynolds, R. C., Synthes is of Nitrogen-15 Labeled Amino Sugar Derivatives by Addition of Phthalimide- ${ }^{15} \underline{\mathrm{N}}$ to a Carbohydrate Epoxide, Carbohyd. Res. 110, 53-54 (1982).

Doane, L. M. and Fatiadi, A. J., Electrochemical Oxidation of Croconate Salts and Evidence of Chemical Equivalency of Carbonyl Oxygen Atom and Dicyanomethylene Group, Angew. Chem. Intern. Ed. Engl. 21, 635-636 (1982).

Durst, R. A., The International pH Scale, Methodology and Physiology of Blood Gases and pH, B. Oeseburg and W. G. Zijlstra, eds., Private Press, Groningen, The Netherlands, 186-189 (1982).

Durst, R. A., Blubaugh, E. A., Bunding, K. A., Fultz, M. L., MacCrehan, W. A., and Yap, W. T., Organic Electrochemical Techniques Having Potential

Clinical Application, Clin. Chem. 28 (9), 1922-1930 (1982).

Fultz, M. L. and Durst, R. A., Mediator Compounds for the Electrochemical Study of Biological Redox Systems, Anal. Chim. Acta 140, 1-18 (1982).

Kline, W. F., Enagonio, D., Reeder, D. J., and May, W. E., Liquid Chromatographic Determination of Valproic Acid in Human Serum, J. Liq. Chromatog. $\underline{5}$ (9), 1697-1709 (1982).

Kong, R. C., Lee, M. L., Tominaga, Y., Pratap, R., Iwao, M., Castle, R. M., and Wise, S. A., Capillary Column Gas Chromatographic Resolution of Isomeric Polycyclic Aromatic Sulfur Heterocycles in a Coal Liquid, J. Chromatog. Sci. 20, 502-510 (1982).

MacCrehan, W. A., A Centrifugal Filtration System Modified to Minimize Losses of Organomercury Cations, Anal. Chem. 54, 838-839 (1982).

Margolis, S. A., Reference Materials for Organic Nutrient Measurement: Proceedings of a Workshop Held at NBS, Gaithersburg, MD, October 23, 1980, NBS SP 635,43 pages (1982).

May, W. E., Chesler, S. N., Hertz, H. S., and Wise, S. A., Analytical Standards and Methods for the Determination of Polycyclic Aromatic Hydrocarbons in Environmental Samples, Intern. J. Environ. Anal. Chem. 12, 259-275 (1982).

Schaffer, R., Mandel, J., Sun, T., Cohen, A., and Hertz, H. S., Standard Reference Materials: Evaluation by an ID/MS Method of the AACC Reference Method for Serum Giucose, NBS SP 260-80, 43 pages (1982). 
Sniegoski, L. T., White V, E., and Konash, P. L., Synthesis of 2-Naphthalene-d Sulfonic Acid, J. Labeled Compounds and Radiopharm. 19 (9), 1081-1087 (1982).

Sonnefeld, W. J., Zoller, W. H., May, W. E., and Wise, S. A., On-Line Multidimensional High-Performance Liquid Chromatographic Determination of Polycyclic Organic Material in Energy Related Fuel 0ils, Polynuclear Aromatic Hydrocarbons: Phys. and Biol. Chem., M. Cooke and A. J. Dennis, eds., Battel1e Press, Columbus, $\mathrm{OH}, 755-764$ (1982).

White V, E., Welch, M. J., Sun, T., Sniegoski, L. T., Schaffer, R., Hertz, H. S., and Cohen, A., The Accurate Determination of Serum Glucose by Isotope Di Tution/Mass Spectrometry -- Two Methods, Biomed. Mass Spectrom. 9 , 395-405 (1982).

Wise, S. A., Allen, C. F., Chesler, S. N., Hertz, H. S., Hilpert, L. R., May, W. E., Rebbert, R. E., and Vogt, C. R., Characterization of Air Particulate Material for Polycyclic Aromatic Compounds, NBSIR 82-2595, 57 pages (1982).

Wise, S. A., Bowie, S. L., Chesler, S. N., Cuthrell, W. F., May, W. E., and Rebbert, R. E., Analytical Methods for the Determination of Polycyclic Aromatic Hydrocarbons in Air Particulate Matter, Polynuclear Aromatic Hydrocarbons: Phys. and Biol. Chem., M. Cooke and A. J. Dennis, eds., Battelle Press, Columbus, OH, 919-929 (1982).

Yap, W. T. and Doane, L. M., Determination of Diffusion Coefficients by Chronoamperometry with Unshielded Planar Stationary Electrodes, Anal. Chem. 54 (8), 1437-1439 (1982).

Ausloos, P., Rebbert, R. E., Schwarz, F. P., and Lias, S. G., Pulse- and Gamma-Ray Radiolysis of Cyclohexane: Ion Recombination Mechanisms, Rad. Phys. and Chem. 21, 27 (1983).

Botto, R. E. and Coxon, B., Nitrogen-15 Nuclear Magnetic Resonance Spectroscopy of Neomycin B and Related Aminoglycosides, J. Am. Chem. Soc., 1027-1028 (1983).

Bowers, G. N., Jr., Alvarez, R., Cali, J. P., (NBS retired), Eberhardt, K. R., Reeder, D. J., Schaffer, R., and Uriano, G. A., Standard Reference Materials: The Measurement of the Catalytic (Activity) Concentration of Seven Enzymes in NBS Human Serum SRM 909, NBS SP 280-83, 95 pages (1983).

Christensen, R. G., White V, E., Meiselman, S., and Hertz, H. S., Quantitative Trace Analysis by Reversed-Phase Liquid Chromatography-Mass Spectrometry, J. Chromatogr. 271, 67-70 (1983).

Covington, A. K., Bates, R. G., and Durst, R. A., Definition of pH Scales, Standard Reference Values, Measurement of $\mathrm{pH}$ and Related Terminology, Pure and Appl. Chem. 55, 1467-1476 (1983). 
Davidson, R. M., Margolis, S. A., White V, E., Coxon, B., and Oppenheimer, N. J., A New, Facile Synthesis of 2-amino(pento- and Hexo-furano)oxazoline Derivatives, Carbohyd. Res. 111, C16-C19 (1983).

Davidson, R. M., White V, E., Margolis, S. A., and Coxon, B., Synthesis of Nitrogen-15 Labeled 2-Amino(glycofurano) Oxazolines Via Glycosylamine Intermediates, Carbohyd. Res. 116, 239-254 (1983).

Durst, R. A., pH Values, Scales, and Standards, Geigy Scientific Tables, 8th ed. (3), 54-57 (1983).

Fatiadi, A. J., Preparation and Synthetic Applications of Cyano Compounds, The Chem. of Functional Groups, Suppl. C, S. Patai and Z. Rappoport, eds., John Wiley and Sons, London, 1057-1303 (1983).

Fatiadi, A. J., Priority Toxic Pollutants in Human Urine: Their Occurrence and Analys is (Monograph), NBS/EPA, NBSIR 83-2690 (1983).

Hertz, H. S., Chesler, S. N., Gump, B. H., and May, W. E., Intercalibration: A Means for Interlaboratory Validation of Environmental Data, A Summary of Activities Related to offshore Drilling of Petroleum at the Natl. Bureau of Stds. 1975-1981, 68-70 (1983).

Kline, W. F., Allen, C. F., Chesler, S. N., Hilpert, L. R., and Wise, S. A., Development of Analytical Methodology for the Determination of Organochlorine Compounds in Human Liver Samples, in The Pilot Natl. Environ. Specimen Bank -- Anal. Of Human Liver Samples, R. Zeisler, S. H. Harrison, and S. A. Wise, eds., NBS SP 656, 91-97 (1983).

Maas, A. H. J., Weisberg, H. F., Zijlstra, W. G., Durst, R. A., and SiggaardAndersen, 0., Reference Method for PH Measurement in Blood, J. Clin. Chem. Clin. Biochem. 21, 313-321 (1983).

MacCrehan, W. A., Determination of Organomercury Cations in Tissue Samples Using Liquid Chromatography with Amperometric Detection, in The Pilot Natl. Environ. Specimen Bank -- Anal. of Human Liver Samples, R. Zeis Ter, S. H. Harrison, and S. A. Wise, eds., NBS SP 656, 115-119 (1983).

Margolis, S. A. and Coxon, B., Comparison of Amino Acid Analysis of Angiotensin by Proton NMR and Classical Methods, Abstracts of Am. Soc. of Biol. Chem. 74th Mtg., San Francisco, CA, (1983).

May, W. E., Gump, B. H., Chesler, S. N., and Hertz, H. S., Analysis of Polynuclear Aromatic Hydrocarbons in Marine Samples by Coupled-Column Liquid Chromatography, A Summary of Activities Related to Offshore Drilling of Petroleum at the Nat7: Bureau of Stds. 1975-1981, $150-155$ (1983).

May, W. E., Miller, M. M., Tewari, Y., Brown-Thomas, J. M., and Goldberg, R., The Solution Thermodynamics of Some Slightly Soluble Hydrocarbons in Water, J. Chem. Eng. Data 28, 197 (1983). 
Perry, B. W., Doumas, B. T. (Chairman), Bayse, D. D., Butler, T., Cohen, A., Fellows, W., Garber, C. C., Howe11, B., Koch, T., Krishnamurthy, S., Louderbach, A., McComb, R. B., Miller, D., Miller, R. R., Rand, R. N., and Schaffer, R., A Candidate Reference Method for Determination of Bilirubin in Serum. Test for Transferability, Clin. Chem. 29 (2), 297-301 (1983).

Sniegoski, L. T. and White V, E., Synthesis of 1-Dodecyl-d $d_{25}$ Phosphate, J. Labeled Compounds and Radiopharm. 20 (2), 303-309 (1983).

Wise, S. A., High-Performance Liquid Chromatography for the Determination of Polycyclic Aromatic Hydrocarbons, Chapter 5, Handbook for Polycyclic Aromatic Hydrocarbons, A. Bjørseth, ed., Marcel Dekker, NeW York, NY, 183-256 (1983).

Wise, S. A., The National Environmental Specimen Bank: Background and History, in The Pilot National Environ. Specimen Bank -- Analysis of Human Liver Samples, R. Zeisler, S. H. Harrison, and S. A. Wise, eds., NBS SP 656, $1-4(1983)$.

Wise, S. A., Fitzpatrick, K. A., Harrison, S. H., and Zeisler, R., Operation of the U.S. Pilot National Environmental Specimen Bank Program, in Environmental Specimen Banking and Monitoring as Related to Banking, R. A. Lewis, N. Stein, and C. W. Lewis, eds., Univ. of Saarland, Germany, 108-129 (1983).

Wise, S. A. and May, W. E., The Effect of $C_{18}$ Surface Coverage on Selectivity in Reversed-Phase Liquid Chromatography of Polycyclic Aromatic Hydrocarbons, Anal. Chem. 55, 1479-1485 (1983).

Yap, W. T., Durst, R. A., Blubaugh, E. A., and Blubaugh, D. D., Chronoamperometry of Polymer-Modified Electrodes: Charge Transport by Diffusion and Migration, J. Electroanal. Chem. 144, 69-75 (1983).

Yap, W. T., Schaffer, R., Hertz, H. S., White V, E., and Welch, M. J., On the Difference Between Linear and Non-Linear Models in Bracketing Procedures in Isotope Dilution/Mass Spectrometry, Biomed. Mass Spectrom. 10 (4), 262-264 (1983).

Zeisler, R., Harrison, S. H., and Wise, S. A., Analysis of Human Liver Specimens in the U.S. Pilot Environmental Specimen Bank Program, in Environ. Specimen Banking and Monitoring as Related to Banking, R. A. Lewis, N. Stein, C. W. Lewis, eds., Univ. of Saarland, Germany, 331-351 (1983).

Bunding, K. A., Durst, R. A., and Be11, M. I., Surface-Enhanced Raman Spectroscopy of $\mathrm{N}$-Methylpyridinium Cation and Pyridine: Identification of Surface Species, J. Electroanal. Chem., in press.

Durst, R. A., Clinical Electrode Potentiometry: Sources of Error, Reference Methods and Materials, Proceedings of the Workshop on Direct Potentiometric Measurements in Blood, in press.

Fatiadi, A. J., The Oxidation of Organic Compounds by Active Manganese Dioxide, Oxidative Procedures in Org. Chem. Involving Metal Compounds, W. J. Mijs, ed., Plenum Press, London, in press. 
Fultz, M. L. and Durst, R. A., Investigation of Two Multichannel Image Detectors for Use in Spectroelectrochemistry, Talanta, in press.

Kamat, P. V., Fox, M. A., and Fatiadi, A. J., Dye Loaded Polymer Electrodes, Photoelectrochemical Sensitization by Croconate Violet in Polymer Solutions and Films, J. Amer. Chem. Soc., in press.

Margolis, S. A. and Konash, P. L., The HPLC Anal.ysis of Diastereomers and Structural Analogs of Angiotensins I and II, Anal. Biochem., in press.

Mattammal, M. B., White V, E., Zenser, T. V., and Davis, B. B., Mass Spectrometry of 2-Substitutes-Y-Arylthiazoles III. Identification of Microsomal NitroReduction Products by Mass Spectrometry, Biomed. Mass Spectrom., in press.

May, W. E., Brown-Thomas, J. M., Chesler, S. N., Guenther, F. R., Hilpert, L. R., Parris, R. M., Richie, K. L., Sonnefeld, W. J., Wise, S. A., and Hertz, H. S., Interlaboratory Comparisons of Quantitative Analyses of Individual Compounds in Simple and Complex Mixtures, Oak Ridge Press, in press.

May, W. E., Chesler, S. N., Hilpert, L. R., Hertz, H. S., Rebbert, R. E., Vogt, C. R., and Wise, S. A., Characterization of Polycyclic Aromatic Hydrocarbons in Air Particulate Extracts by Liquid and Gas Chromatographic Methods: Identification and Analysis of Organic Pollutants in Air, L. R. Keith, ed., Butterworth Publications, in press.

Wise, S. A., Analytical Techniques for the Determination of Petroleum Hydrocarbons and Combustion Products, Pollution and the Protection of Water Quality, Hemisphere Publishing, New York, NY, in press.

Wise, S. A., Interlaboratory Comparisons and the Use of Reference Materials for Quality Control of Analytical Methodologies, Pollution and the Protection of Water Quality, Hemisphere Publishing, New York, NY, in press.

Wise, S. A., Campbell, R. M., May, W. E., Lee, M. L., and Castle, R. N., Normaland Reversed-Phase Liquid Chromatographic Separations of Polycyclic Aromatic Sulphur Heterocycles, Polynuclear Aromatic Hydrocarbons: Combustion Processes, Environ. Effects, and Biochem., M. Cooke, A. J. Dennis, and G. L. Fisher, eds., Battelle Press, Columbus, $\mathrm{OH}$, in press.

Wise, S. A., Sander, L. C., and May, W. E., Modification of Selectivity in Reversed-Phase Liquid Chromatography of Polycyclic Aromatic Hydrocarbons Using Mixed Stationary Phases, J. Chromatog., in press.

Velapoldi, R. A., White, P. A., May, W. E., and Eberhardt, K., Spectrofluorometric Determination of Polycyclic Aromatic Hydrocarbons in Aqueous Effluents from Generator Columns, Anal. Chem., in press.

Yap, W. T., Blubaugh, E. A., Durst, R. A., and Burke, R. T., Spectroelectrochemistry of a System Containing a Species which Deposit onto the Electrode, J. Electroanal. Chem., in press. 
Yap, W. T., Burke, R. T., Blubaugh, E. A., and Durst, R. A., Chronocoulometry of a System with Strong Product Adsorption, J. Electroanal. Chem., in press.

Fatiadi, A. J., Permanganate Ion: An 01d, But Still Novel Oxidant in Organic Chemistry, Oxidate Procedures Involving Metal Compounds in Org. Chem., submitted for pubTication.

MacCrehan, W. A. and May, W. E., Oxygen Removal in Liquid Chromatography Using Zinc/0xygen Scrubber Column, Anal. Chem., submitted for publication.

May, W. E. and Wise, S. A., Liquid Chromatographic Determination of Polycyclic Aromatic Hydrocarbons in Air Particulate Extracts, Anal. Chem., submitted for publication.

Parris, R. M., Guenther, F. R., May, W. E., Chesler, S. N., Analys is of PCB's in 0i 1: Technique on SRM Development, Proc. Conf. on Measurements and Stds. for Recycled $0 i 1$-- IV, National Bureau of Standards, Gaithersburg, MD, (september 14-16, 1982), submitted for publication.

Roberts, G. D. and White V, E., Silver Sulfonates as Mass Standards in Field Desorption Mass Spectrometry, Biomed. Mass Spectrom., submitted for publication.

Welch, M. J., Cohen, A., Hertz, H. S., Ruegg, F. C., Schaffer, R., Sniegoski, L. T., and White V, E., An Isotope Dilution Mass Spectrometric Method for the Accurate Determination of Serum Urea, Anal. Chem., submitted for publication.

2. Talks

D. J. Reeder, "Materials Variations in Polyacrylamide Gel Electrophoresis," Two-Dimensional Gel Electrophoresis Journal Club Seminar, October 5, 1982. Invited

W. E. May, "On-Line Multidimensional Liquid Chromatographic Determination of PAH in Complex Samples," ASTM E-19 Committee on Chromatography, New Orleans, LA, October 12, 1982. Invited

R. G. Christensen, "Quantitative Trace Analysis by Reversed-Phase LC/MS Employing Continuous Preconcentration," Second Workshop on LC/MS and MS/MS of the International Association of Environmental Analytical Chemistry, Montreaux, Switzerland, October 21, 1983. Invited

S. A. Wise, "Normal- and Reversed-Phase Liquid Chromatographic Separations of Polycyclic Aromatic Sulfur Heterocycles," Seventh International Symposium on Polynuclear Aromatic Hydrocarbons, Columbus, OH, October 26, 1982.

E. A. BTubaugh, "Electrochemical Techniques: Cyclic Voltammetry and Hybrid Electrochemical Techniques," Second NBS/ADABSE Analytical Chemistry Workshop, NBS, Gaithersburg, MD, October 27, 1982. 
R. A. Durst, "Electrochemistry," Second NBS/ADABSE Analytical Chemistry Workshop, NBS, Gaithersburg, MD, October 27, 1982. Invited

R. Schaffer, "Certified and Uncertified Values Assigned to SRM's," Capital Section of the AACC, Columbia, MD, November 17, 1982. Invited

S. A. Wise, "Determination of Polycyclic Aromatic Compounds in Environmental Samples," Eastern Analytical Symposium, New York, NY, November 17-19, 1982. Invited

A. J. Fatiadi, "The Chemistry and Electrochemistry of 0xo- and Pseudo-0xocarbons," Georgetown University, Washington, DC, November 18, 1982. Invited

W. E. May, "On-Line Multidimensional Liquid Chromatography," Eastern Analytical Symposium, New York, NY, November 18, 1982. Invited

B. Coxon, "Nitrogen-15 NMR Spectroscopy of Aminoglycosides," American University, Washington, DC, November 30,1982 . Invited

R. A. Durst, "Polymer Modified Electrodes for Organic Electrochemistry," Special Symposium on NBS Research, NBS, Gaithersburg, MD, January 10, 1983. Invited

R. A. Durst, "Electrochemical Approaches to Organic Analysis," California Department of Health Services, Los Angeles, CA, January 20, 1983. Invited

R. A. Durst, "Novel Electrochemical Techniques for Organic Analysis," IBM Research Laboratory, San Jose, CA, January 21, 1983. Invited

W. A. MacCrehan, "Liquid Chromatographic, Organometal Speciation Using Electrochemical Detection," Gordon Research Conference on Chemical Oceanography, Ventura, CA, January 31, 1983. Invited

R. A. Durst, "Overview of Strategies for the Characterization and Analysis of Organic Compounds," Lawrence Berkeley Laboratory, Berkeley, CA, February 1, 1983. Invited

R. A. Durst, "Organic Electrochemical Research at the National Bureau of Standards, "Lawrence Livermore Laboratory, Livermore, CA, February 2, 1983. Invited

R. A. Durst, "Electrochemical Approaches to Organic Analys is," Department of Health Services, Berkeley, CA, February 4, 1983. Invited

W. E. May, "Multidimensional HPLC Techniques Applied to the Determination of Polycyclic Aromatic Compounds in Complex Mixtures," Emory University, Atlanta, GA, February 22, 1983. Invited

J. M. Brown-Thomas, "Introduction to Basic Principles in High-Performance Liquid Chromatography," Alcorn State University, Lorman, MS, February 24, 1983. Invited 
W. E. May, "Multidimensional HPLC Techniques Applied to the Determination of Polycyclic Aromatic Compounds in Complex Mixtures," Alcorn State University Lorman, MS, February 24, 1983. Invited

F. R. Guenther, "Characterization of Wood Combustion Emissions," 34th Pittsburgh Conference on Analytical Chemistry and Applied Spectroscopy, Atlantic City, NJ, March 7, 1983.

W. E. May, "HPLC Determination of Nitrated-PAH in Complex Mixtures," 34th Pittsburgh Conference on Analytical Chemistry and Applied Spectroscopy, Atlantic City, NJ, March 7, 1983.

W. A. MacCrehan, "Conversion of Nitro-Polycyclic Aromatic Hydrocarbons to the Amines Using an On-Line Liquid Chromatographic Reducer Column," 34th Pittsburgh Conference on Analytical Chemistry and Applied Spectroscopy, Atlantic City, NJ, March 8, 1983.

J. M. Brown-Thomas, "Determination of Organic Nutrients in Milk by Multidimensional High-Performance Liquid Chromatography," 34th Pittsburgh Conference on Analytical Chemistry and Applied Spectroscopy, Atlantic City, NJ, March 10, 1983.

W. F. Kline, "The Determination of Organochlorine Pesticide Residues in Human Liver Specimens from the NBS/EPA Pilot National Environmental Specimen Bank Program," 34th Pittsburgh Conference on Analytical Chemistry and Applied Spectroscopy, Atlantic City, NJ, March 10, 1983.

W. E. May, "Trace Organic Analytical Applications of HPLC," Bishop College, Dallas, TX, March 21, 1983. Invited

W. E. May, "Liquid Chromatographic Methods for Determination of Physico-chemical Properties of Organic Compounds," Bishop College, Dallas, TX, March 21, 1983. Invited

W. E. May, "Trace Organic Analytical Applications of HPLC," Prairie View University, Prairie View, TX, March 22, 1983. Invited

W. E. May, "Liquid Chromatographic Methods for Determination of Physico-chemical Properties of Organic Compounds," Prairie View University, Prairie View, TX, March 22, 1983. Invited

S. A. Wise, "Liquid Chromatography for the Determination of Polycyclic Aromatic Compounds in Environmental Samples," Department of Health Services, State of California Air and Industrial Hygiene Laboratory, Berkeley, CA, March 23, 1983. Invited

W. E. May, "Trace Organic Analytical Applications of HPLC," Huston-Tillotson College, Austin, TX, March 24, 1983. Invited

W. E. May, "Liquid Chromatographic Methods for Determination of Physico-chemical Properties of Organic Compounds, Huston-Tillotson College, Austin, TX, March 24, 1983. Invited 
W. E. May, "Trace Organic Analytical Applications of HPLC," Alabama A\&M University, Huntsville, AL, March 25, 1983. Invited

W. E. May, "Liquid Chromatographic Methods for Determination of Physico-chemical Properties of Organic Compounds," Alabama A\&M University, Huntsville, AL, March 25, 1983. Invited

B. Coxon, "Two-Dimensional DEPT Heteronuclear J-Resolved ${ }^{13} \mathrm{C}$ NMR and ${ }^{15} \mathrm{~N}$ NMR Spectrum Editing," 24th Experimental NMR Conference, Asilomar, CA, Apri1 13, 1983.

W. E. May, "Liquid Chromatographic Methods for the Determination of Polynuclear Aromatic Hydrocarbons (PAH) and Nitro-PAH in Environmental Samples," Florida A\&M University, Tallahassee, FL, April 14, 1983. Invited

L. T. Sniegoski, "The Use of Isotope Dilution/Mass Spectrometry for the Accurate Determination of Organic Constituents in Serum," Women's Forum, NBS, Gaithersburg, MD, April 20, 1983. Invited

W. E. May, "Environmental Applications of High Performance Liquid Chromatography," ACS, Norfolk, VA, April 22, 1983. Invited

J. M. Brown-Thomas, "Determination of Organic Nutrients in Milk by Multidimensional High-Performance Liquid Chromatography, Hampton Institute, Hampton, VA, Apri1 29, 1983. Invited

S. A. Wise, "Modification of Selectivity in Reversed-Phase Liquid Chromatography of Polycyclic Aromatic Hydrocarbons Using Mixed Stationary Phases," Seventh International Symposium on Column Liquid Chromatography, Baden-Baden, Germany, May 2-6, 1983.

M. J. Welch, "The Accurate Determination of Serum Urea and Creatinine by Isotope Dilution/Mass Spectrometry," ASMS, Boston, MA, May 10, 1983.

L. R. Hilpert, "Accuracy and Precision in the GC/MS Determination of Toxic Organic Compounds in Hazardous Wastes," ASMS, Boston, MA, May 11, 1983.

L. R. Hilpert, "Hazardous Waste Analysis -- Sticking to Measurements and Avoiding Controversy," ASMS Workshop on Sampling and Analysis of Hazardous Wastes, Boston, MA, May 11, 1983. Invited

W. A. MacCrehan, "Reductive LCEC of Nitro-Polynuclear Aromatic Hydrocarbons (N-PAH) Diesel Exhaust Particulates Using a Zinc/0xygen Scrubber Column," 1983 International Symposium on LCEC and Voltametry, Indianapolis, IN, May 16, 1983. Invited

R. A. Durst, "Clinical Electrode Potentiometry: Sources of Error, Reference Methods and Materials," Workshop on Direct Potentiometric Measurements in Blood, NBS, Gaithersburg, MD, May 20, 1983. Invited 
W. E. May, "Liquid Chromatographic Determination of Nitrated Polynuclear Aromatic Hydrocarbons in Complex Mixtures," 10th Annual Meeting of the National Organization for the Professional Advancement of Black Chemists and Chemical Engineers, Knoxvi11e, TN, May 27, 1983.

W. E. May, "Liquid Chromatographic Determination of Physico-chemical Properties of Hydrophobic Substances," 10th Annual Meeting of the National Organization for the Professional Advancement of Black Chemists and Chemical Engineers, Knoxville, TN, May 27, 1983.

R. A. Durst, "Ionic Activity and Single Ion Activity Coefficients for Calcium Ion at $25{ }^{\circ} \mathrm{C}$," 1st Meeting of the European Working Group on Ionized Calcium, Os10, Norway, June 17, 1983. Invited

R. G. Christensen, "Quantitative Trace Organic Analysis by Combined LC/MS," Symposium on LC/MS 186th ACS National Meeting, Washington, DC, August 29, 1983.

B. Coxon, "Spectrum Editing of the Nitrogen-15 NMR Spectra of Aminoglycosides by the DEPT Technique," 1983 American Chemical Society Meeting, Washington, DC, August 29, 1983.

B. Coxon, "Two-Dimensional DEPT Heteronuclear J-Resolved ${ }^{13} \mathrm{C}$ NMR of Digitoxose," 1983 American Chemical Society Meeting, Washington, DC August 29, 1983.

B. Coxon, "Two-Dimensional Proton-Proton Chemical Shift Correlated NMR Spectroscopy of Digitoxose and Its Anomers," 1983 American Chemical Society Meeting, Washington, DC, August 29, 1983.

B. Coxon, "Structure of the Reaction Product of 4-pheny1-2,3-dioxobutyro-1,4lactone, a Dehydroascorbic Acid/Analog, with 0-phenylene Diamine," 1983 American Chemical Society Meeting, Washington, DC, August 31, 1983.

W. F. K1 ine, "The Determination of Organochlorine Pesticide Residues in Human Liver Specimens from NBS/EPA Pilot Environmental Specimen Bank Program," The Eighth U.S.-German Seminar of State and Planning on Environmental Specimen Banking, NBS, Gaithersburg, MD, September 19, 1983. Invited

S. A. Wise, "Status of the U.S. Pilot National Environimental Specimen Bank," International Review of Environmental Specimen Banking, NBS, Gaithersburg, MD, September 21, 1983. Invited

\section{Committee Assignments}

Willie E. May

Chairmen, ADABSE, Analytical Workshop Committee

Member, ASTM, Committee D19 on Water,

Committee E19 on Chromatography

Member, ACS

Member, Washington Chromatography Discussion Group 


\section{Bruce Coxon}

Secretary, ASTM, Committee E13.07 on Nuclear Magnetic Resonance, Committee E13 on Molecular Spectroscopy

Associate Referee, International Commission for Uniform Methods of Sugar Analysis, Subcommittee 5 on $100{ }^{\circ} \mathrm{S}$ Point of Sugar Scale

Referee, U.S. National Committee on Sugar Analysis, Subcommittee 5 on $100{ }^{\circ} \mathrm{S}$ Point of Sugar Scale

Richard A. Durst

Member, Advisory Board, Analytical Chemistry

Member, ASTM, Committee D19 on Water

Member, Board of Advisory Editors, Ion-Selective Electrode Reviews

Member, Department of the Interior, Office of Water Data Coordination, Task Group 5 on Chemical and Physical Quality of Water and Sediments

Secretary, IFCC, Scientific Committee, Analytical Section, Expert Panel on $\mathrm{pH}$ and Blood Gases

Member, Instrument Society of America, Subcommittee SP76.02 on Electrochemical Analyzers

Member, IUPAC, Committee $V$ on Analytical Chemistry, Subcommittee 5 on Electroanalytical Chemistry

Member, NCCLS, Subcommittee on Electroanalytical Methods

Member, NCCLS, Subcommittee on pH and Blood Gases

Laurence R. Hilpert

Member, ASTM, Committee D34 on Waste Disposal

\section{Dennis J. Reeder}

Member, AACC, Committee on Standards

Study Group on Cortisol

Study Group on Total Serum Protein

Associate Member, IFCC, Expert Panel on Drug Effects in Clinical Chemistry

Member, NCCLS, Subcommittee on Total Protein

Robert Schaffer

Member, AACC, Committee on Standards

Subcommittee on Cholesterol

Subcommittee on Creatinine

Subcommittee on Electrolytes

Subcommittee on Glucose

Subcommittee on Iron

Sbucommittee on Theophylline

Subcommittee on Urea

Subcommittee on Uric Acid

Member, NCCLS, Area Committee for Clinical Chemistry

Subcommittee on Calibration Reference and Control Materials

Subcommittee on Quantities and Units

Subcommittee on Reference Methods and Materials

Member, Council for National Reference System in Clinical Chemistry 
4. other

a. Seminars

October 6, $1982 \quad$ - Branimir Simic-Slavaski, Case Western Reserve University "Surface-Enhanced Raman Scattering from Adsorbed Phthalacyanines and Their Electrochemical Behavior".

October 19, 1982 - Anders Colmsj6, University of Stockholm, "The Shpol'skij Effect and Its Applications in Analytical Chemistry".

November 16, 1982 - James B. Callas, University of Washington, "ReversedPhase Chromatography -- Adsorption or Partitioning Chromatography?".

November 19, 1982 - Robert C. Allen, Medical University of South Carolina, "Two-Dimensional Electrophoresis: Denaturing and NonDenaturing Conditions".

December 1, $1982 \quad$ - George N. Bowers, Jr., Hartford Hospital, "Standardization of Ionic Calcium Measurements".

January 19, $1983 \quad$ - Alan Lowe, DuPont Company, "HPLC Columns for High Speed and High Resolution Analyses".

January 31, 1983 - Elwood 0. Titus, Food and Drug Administration, "The Role of Calcium in Trans Membrane Systems".

February 17, 1983 - Marko Branica, Ruder Baskovic Institut, Yugoslavia, "Electrochemical Determination of Metal-Ligand Interactions in Natural Waters".

February 24, 1983 - 0tto S. Wolfbeis, Institut fur Organische Chemie, Austria, "Synthesis and Application of Fluorescent Reagents to be Used in Analytical Chemistry and Biochemistry".

March 14, 1983

- James Alexander Apffel, Free University, The Netherlands, "Quantitation in On-Line Multidimensional HPLC".

April 11, 1983 - Thomas Doyle, Food and Drug Administration, "HighPerformance Liquid Chromatography of Enantiomers Using Chiral Stationary Phases".

April 12, 1983

- William P. Reed, NBS Office of Standard Reference Materials, "Research and Measurement Needs for the Certification of Standard Reference Materials, Plans, and Perspectives for FY 84".

April 21, 1983 - Dennis Gere, Hewlett-Packard Company, "Bioanalytical Applications of Supercritical Fluid Chromatography". 
May 4, 1983

July 6, 1983 - David Firestone, Richard Niemann, and James Sphon, Food

- Jaromir Ruzicka, The Technical University of Denmark, "From a Test Tube to Integrated Microcondiut by Flow Injection Analysis -- A New Approach to Sample Handling in the Research Laboratory". and Drug Administration, "Analytical Methods for the Determination of Dibenzodioxins in Environmental Samples".

\section{b. Conferences Sponsored}

October 24-29, 1982 - W. E. May, Workshop on "A Survey of State-of-the-Art Instrumental Methods for Chemical Analysis," jointly sponsored by the National Bureau of Standards and the Association for the Development and Advancement of Black Scientists and Engineers (ADABSE), National Bureau of Standards, Washington, DC.

September 21, 1983 - S. A. Wise, "The International Review of Environmental Specimen Banking, National Bureau of Standards, Washington, $D C$.

\section{c. Seminars Organized}

S. A. Wise, "The 8th U.S.-German Seminar of State and Planning on Environmental Specimen Banking," National Bureau of Standards, Washington, DC, September 19-20, 1983.

d. SRM Certification/Information Values

1) SRM 911 , Bilirubin

2) SRM 1581, Organics in Drinking Water

3) SRM 1582, Petroleum Crude 0il

4) SRM 1583, Pesticides in 0so-octane

5) SRM 1584, Phenyls in Methanol

6) SRM 1585, Polychlorinated Biphenyl Congeners in Iso-octane

7) SRM 1586, Unlabeled and Deuterium Labeled Priority Pollutants

8) SRM 1514, Purity Established for Differential Thermal Analysis

9) SRM 1648, St. Louis Urban Dust

10) SRM 1649, Urban Dust, Organics 
IV. Gas and Particulate Science Division

Harry L. Rook, Chief

Raymond L. McKenzie, Deputy Chief

A. Division Overview

The Gas and Particulate Science Division conducts research to develop new or improved chemical measurement procedures and uses those procedures to certify the chemical composition of NBS standards and to solve analytical problems related to national needs. The research is directed toward the measurement of chemical composition on a microscopic scale and the measurement of gaseous molecules in complex mixtures or in atmospheric samples. The Division's programs are carried out by four groups with the research effort divided approximately equally between gas analytical research and microanalytical research.

During 1983, the Division has completed a long range plan for research in trace gas analysis and standards and has initiated research in two major areas. In toxic organic gas analysis, our efforts have been devoted to the development of standards and methods of analysis for gases at concentrations ranging from a few parts-per-billion (ppb) to 10 parts-per-million (ppm). Although the microgravimetric gas mixture preparation procedure described last year is capable of making predictable multicomponent gas mixtures with concentrations of individual components below one $\mathrm{ppb}$, analytical methods for most components were limited by detection sensitivity and imprecision. This year, considerable effort has been devoted to developing methods for the gas chromatographic separation and analysis of multicomponent gaseous organic mixtures where component concentrations were as low as one ppb. The precision of the GC analysis procedure has been improved by over a factor of four such that the individual components of benzene, carbon tetrachloride, vinyl chloride and tetrachloroethylene now can be analyzed with an uncertainty of less than $\pm 15 \%$ at a few ppb concentrations. Collaborative research with the Organic Analytical Research Division to develop isotope dilution gas chromatographic-mass spectrometric procedure for similar gas mixtures has been promising. Recent analysis on gas mixtures analyzed by both methods has given data which agree within $\pm 20 \%$. Work is continuing on these methods to improve agreement. The results of these research programs will allow us to obtain analytical data by two independent methods of analysis for the certification of Standard Reference Materials containing trace levels of volatile toxic organic materials.

The second area of major research activity has been in trace atmospheric gas analysis. Initial research to develop analytical methods for $\mathrm{HCl}$ and $\mathrm{N}_{2} \mathrm{O}$ at atmospheric concentrations has been described in past reports. During the current year, planning meetings have been held with program managers at NASA and EPA to define needs for trace gas standards and calibration mixtures for trace atmospheric species. The importance of accurate analytical methods and standards for these gases is underscored by the fact that NASA alone is spending more than $\$ 50$ million this year in tropospheric and stratospheric trace gas research and measurements. Currently, no accurate calibration gases or standards exist to put these measurements on a common scale. 
As a result of our planning, the Division has initiated a research program to develop analytical methods with the sensitivity and selectivity to allow accurate analysis of specific atmospheric species below $1 \mathrm{ppb}$. Concurrent with this effort, studies on methods of preparing stable gas mixtures at these levels has been initiated. Initial analytical research has focused on diode-laser absorption photometry and electron capture gas chromatography. This year, a technique has been developed for the measurement of gaseous $\mathrm{HCl}$ below one $\mathrm{ppb}$ using direct diode laser absorption. This technique has proven to be more than ten times more sensitive than the technique developed using photothermal detection discussed in last year's report. Also, a new method has been completed for the analysis of $\mathrm{N}_{2} \mathrm{O}$ at atmospheric levels using gas chromatography with electron capture detection. This method is the result of two years research and now allows for the analysis of $\mathrm{N}_{2} \mathrm{O}$ with a precision of \pm 0.1 percent in binary gas mixture standards and \pm 0 .3 percent precision in atmospheric air. The method is a major improvement over currently used procedures and is being applied to the certification analyses of a new series of atmospheric gas SRM's.

Other significant accomplishments of the past year include: The completion of the ozone ultraviolet absorption cross-section values at four atmospheric temperatures; the development of a model which elucidates a mechanism for the loss of nitrous oxide in compressed gas cylinders; the ultratrace detection of $\mathrm{NO}_{2}$ in air by photothermal spectroscopy; and the completion of absolute band strength measurements on seven vibrational band systems in Freon-11 and 12.

In the Microanalysis Research Group and the X-Ray Fluorescence Group, this year's programs have focused on improved first-principal quantitation methods and on elemental, isotopic, and molecular compositional mapping. During 1983, research on combined analyses using the Analytical Electron Microscope (AEM) and the Laser Microprobe Mass Analyzer (LAMMA) has led to the first complete compositional mapping of particles smaller than one micrometer in diameter. Compositional maps of heterogeneous single particles and aggregates of submicrometer particles contained information on morphology and major, trace, and isotopic composition of elements of interest. Such complete analys is of particles allows information to be derived on particle origin and, to some degree, atmospheric transformation. A second area where substantial progress has been made is in automated data transfer and analysis of chemical or isotopic images. A procedure had been developed and tested to transfer digital image information to a dedicated computer for identification and quantitative data analysis. A prime concern of this research has been the retention of analytical accuracy and precision during the image processing. Other important accomplishments include: development of new thin film glass standards for AEM and LAMMA calibration; research into improved accuracy and precision of isotopic ratio analys is by LAMMA and Secondary Ion Mass Spectrometry (SIMS); the first automated digital chemical image transfer; and completion of a new XRF quantitation algorithm for the calculation of the spectral output distribution of $x$-ray tubes.

Future research in gas analytical chemistry has been reviewed this year. A new long-range research plan has been developed combining research objectives in gas analytical chemistry and atmospheric chemistry. Research in the accurate measurement of trace and uitra trace atmospheric constituents is the 
lead component of the plan. Initial efforts will concentrate on laser absorption photometry, improved multicomponent methods using gas chromotography and collaborative research in both inorganic and organic isotope dilution gas mass spectrometry coupled with the research into methods of preparing stable gas mixtures containing trace constituents.

Future research in microanalysis is primarily defined by the third year portion of the Compositional Mapping research plan. Automated two-dimensional chemical images will be processed using new dedicated digital imaging systems on both the SIMS and AEM systems. Methods to insure quantitative information retention will be developed and tested. Research on quantitative sub-micrometer analysis of both thin samples and particles will be continued. Finally, a new base-funded program in chemometrics and chemical information optimization will be initiated.

\section{Microanalysis Research Group: Overview}

The Microanalysis Research Group carries out research to advance the development and application of microbeam analysis techniques for the chemical, crystallographic, and morphological characterization of matter on the micrometer and sub-micrometer spatial scale. The analytical techniques utilized are based on primary (excitation) particle beams of electrons, ions, or photons, and secondary (analytical) signals of x-rays, inelastically scattered electrons, inelastically scattered photons, and ions. The techniques include electron microprobe analysis, scanning electron microscopy, electron energy loss spectrometry, secondary ion mass spectrometry, laser micro-Raman spectrometry, and laser microprobe mass analysis. Research is conducted on several aspects of each technique, including (1) advancing understanding of the basic physics of sample excitation and attenuation of the secondary radiation; (2) developing methods of quantitation; (3) producing standards and techniques as a means of disseminating developments; and (4) applying results of the research to developing improved compositional mapping techniques and to the study of specific analytical problems in aid of NBS and other government agency activities.

In the area of microanalysis standards, a major standard was issued in FY83 representing the culmination of intensive research into the characterization of asbestos by microscopy. SRM 1876, Chrysotile Asbestos Fibers, represents the first available asbestos material for which the fiber loading has been adequately characterized to serve as a test for counter accuracy. Research has also continued in our program of developing glass standards for microanalysis. A new series of bulk glass standards is nearing completion. Thin film glass standards for analytical electron microscopy and laser microprobe mass analysis have been tested for homogeneity and resistance to degradation under electron bombardment. It is our objective to establish these films as SRM's in FY84 or FY85 depending on OSRM funding.

Several members of the group have continued to work intensively in the development of sequential analytical electron microscopy/laser microprobe mass analysis for the elemental and isotopic analysis of single particles. Particular emphasis has been placed on the development of thin films to serve as support structures for particles while contributing minimal spectral interferences. 
Combined secondary ion mass spectrometry (SIMS)/neutron depth profiling (NDP) studies have been carried out on a series of samples, including candidate materials for a new SRM boron-in-silicon depth profiling standard. This work was a joint effort with Division 551 and is advantageous to the development and quantitation of both techniques, since SIMS offers lateral spatial resolution and sensitivity while NDP provides a depth profile with minimal sample disruption.

Studies of the accuracy and precision of isotopic ratio analysis by laser microprobe mass analysis have been carried out. The detector response has been examined in detail, and the limitations imposed by the non-linear response of the detector/transient recorder have been more fully characterized.

Research plans for 1984 include activities in each of the major techniques. In secondary ion mass spectrometry, quantitative compositional imaging will be explored, and limits to accurate isotopic ratio measurements will be established. In laser Raman microanalysis, the performance of multichannel detection systems is under study. In analytical electron microscopy, the measurement of the composition of entrained particles will be considered by a combination of experiment and Monte Carlo simulation. In laser microprobe mass analysis, interelement effects and sensitivity factor variations will be studied by means of sputtered glass films. Combinations of several of these techniques will enable us to create compositional maps with improved accuracy and utility.

Plans to upgrade selected microbeam instruments are progressing. The ion microscope will be augmented with a primary mass filter which will allow selection of the primary ion species. A dedicated digital imaging system will be attached to the ion microscope in FY84. A digital imaging system is also being developed for the analytical electron microscope which will make use of the VAX computer and the DeAnza image processor. The transient recorder on the LAMMA will be upgraded to provide better dynamic range and mass storage, which should improve the precision of the isotopic measurements as well as providing full spectral coverage on each laser shot.

Dale E. Newbury, Group Leader; Adville A. Bell, David S. Bright, R. G. Downing, Edgar S. Etz, Ryna B. Marinenko, Robert L. Myklebust, Patrick J. Sheridan, David S. Simons, John A. Small, Brian R. Stallard, Eric B. Steel, Barbara B. Thorne.

\section{X-ray Fluorescence Group: Overview}

The X-ray Fluorescence Group performs research on improved techniques for sample preparation to increase the analytical sensitivity; and fundamental parameter data reduction methods to increase the accuracy of quantitative analysis of the elemental composition of thin and bulk samples. A major portion of our work also consists of the quantitative analysis and homogeneity testing of new, and renewal SRM's using a high performance automated wavelength-dispersive spectrometer.

This year several important accomplishments have been achieved. First, the development of an NBS algorithm for the calculation of the spectral output 
distribution of $x$-ray tubes has been completed. A research paper on this work has been presented at the 23rd International Spectroscopy Congress in Amsterdam, the Netherlands and has been submitted for publication in "X-ray Spectrometry". We are now working to incorporate this algorithm in an NBS fundamental parameters computer program for quantitative $\mathrm{x}$-ray fluorescence analysis. Related to the development of fundamental parameter methods, we have assessed the relative accuracy of a new theoretical $\alpha$-coefficient model proposed by LaChance [1] using a number of samples of different types such as alloys, pressed powders, and fused oxide minerals.

We have completed our fabrication of two sets of thin glass films to serve as SRM $x$-ray spectrometer standards. Of major importance in this work was the design and manufacture at NBS of a planetary substrate stage especially adapted for the ion sputtering instrument. This modification allowed a uniform deposition of glass in which the elemental composition was homogeneous across the film diameter. The new planetary stage is especially important for fabricating high quality thin films of different elemental composition for future SRM's.

Finally, several kinds of samples such as steel alloys, phosphate ores, borosilicate glass, brass alloys, and over four hundred austenite samples were measured for either elemental homogeneity and/or quantitative analysis.

Plans for activities of the X-ray Fluorescence group for FY84 will include: (1) continued development of our fundamental parameter approach to $x$-ray analysis; (2) completion of a data reduction computer program which will incorporate the NBS algorithm together with the calculation of theoretical $\alpha$-coefficients for performing quantitative $x$-ray analysis; (3) final certification of thin film glass standards for x-ray calibration; (4) performance testing of the new PW1400 automated wavelength-dispersive spectrometer; and (5) research in preconcentration technology with various cation and anion specific substrates.

Reference

[1] LaChance, G. R., Cloisse, F., Adv. X-ray Anal., 23, 87 (1980).

Peter A. Pella, Group Leader; Anthony A. Marlow, John R. Sieber, Gerald Sleater.

3. Atmospheric Chemistry Research Group: Overview

The Atmospheric Chemistry Research Group conducts research on measurement techniques for ambient concentrations of atmospheric species in both the gas and particle (liquid and solid) phases. This research includes the development of sampling/collection techniques, in situ analytical methods, and micro-sample preparation and analysis techniques. A primary objective of this research is the extension of existing techniques and the development of new analytical techniques for the detection and characterization of trace and ultratrace level atmospheric constituents. 
Research has continued this year in the study and development of spectroscopic techniques for the analysis of trace and reactive atmospheric gases. The determination of the ozone ultraviolet absorption cross-section values as a function of temperature has been completed. The values are being used internationally by the ozone monitoring community although the values have been distributed as provisional pending final error analysis of the complete data set. The construction, calibration, and evaluation of performance parameters of a new standard reference ozone photometer has been completed. The new instrument now serves as the standard reference photometer at NBS, as the reference photometer for the EPA ozone monitoring network, and most recently as the standard for a NASA intercomparison study of various ozone measurement systems used for stratospheric and tropospheric ozone concentration measurements. Research to develop sensitive reactive gas analytical techniques has resulted in a technique for the detection of $\mathrm{HCl}$ at the sub-parts-per-billion level using tunable diode laser absorption. This is the most sensitive in situ detection method available for this important reactive atmospheric trace gas. Also, significant progress has been achieved this year in the application of photothermal spectroscopy for the detection of trace levels of $\mathrm{NO}_{2}$.

Significant achievements have been obtained in the development and application of mathematical analysis of analytical data sets in evaluation of detection limit assessment, design and improvement of the analytical measurement process, and in the interpretation of multidimensional data sets. A primary application of these techniques has been the study of the origins of carbonaceous aerosols (source apportionment and receptor modeling). Other applications include: Consultation with DOE in the design and feasibility of source apportionment in the study of the generation and transport of sulfur emissions suspected to be involved in the formation of acid rain; and research to define the lower limits of detection of radiological effluent from nuclear power plant operation.

Accomplishments have been achieved in several areas of particle research and characterization. The Respirable Quartz SRM has been completed and the data turned over to the Office of Standard Reference Materials for issuance as a Standard Reference Material. Studies in collaboration with the Microanalytical Group on the characterization of organic materials adsorbed on surfaces and particles by Laser Microprobe Mass Analysis (LAMMA) have provided a demonstration of the feasibility of the technique for qualitative analysis of micrometer and sub-micrometer sized samples. We have also completed studies on the development and characterization of personal particulate exposure monitors (PEM's). The NBS sampler has been evaluated in a field study intercomparison which confirmed the performance characteristics obtained by wind tunnel testing in the laboratory. We also completed a feasibility study on the development of an in-vehicle PEM for use to measure commuter exposure to lead and specified organic pollutants characteristic of automobile emissions.

The focus of research activities for the next year will include: a) characterization of sampling and sample transport of material from a bulk sample by spark discharge; b) continued development and application of photoacoustic spectroscopic detection techniques for trace and ultratrace atmospheric gases, including reactive species which must be measured in situ; 
c) continued development and application of mathematical analysis and modeling of data sets, including receptor modeling, source apportionment and detection limit issues; d) application of carbonaceous aerosol and gas measurement techniques to the study of polar ice core samples to help determine the composition of ancient atmospheres; and e) applications of the LAMMA for analysis of organic and inorganic species on surfaces and particles.

Raymond L. McKenzie, Group Leader; Arnold M. Bass, Robert E. Continetti, Lloyd A. Currie, Robert A. Fletcher, Alan Fried, Rudy V. Kelly, George A. Klouda, David A. Krask, James E. Norris, Jeannie Sullivan, Gerald R. Tompkins.

\section{Gas Metrology Research Group: Overview}

The Gas Metrology Research Group conducts fundamental and applied research leading to the improvement of measurement science for ambient and trace gas analysis, and the development of accurate and reliable standards for a variety of gaseous species. The latter are developed to provide data quality assurance in gas measurements made world-wide, and include both non-organic and organic gaseous species. Basic measurements and research are carried out to ensure that the gas standards generated are both accurate and stable. Research is also conducted to improve the scientific understanding of chemical instability of certain gaseous species. The Group directly responds to and interacts with gas measurement and standards needs of other agencies, industrial associations, and standards setting bodies, and provides advice and guidance to the private sector as appropriate. The Group, utilizing its unique capabilities, also performs a limited number of gas analyses for external organizations.

Activities and accomplishments during the past year have included: a continuation of trace gas research using Fourier transform infrared spectroscopy (FTIR) and diode laser systems; completion of the first set of SRM's to provide data quality assurance in world-wide measurements of long-term changes of atmospheric $\mathrm{CO}_{2}$ levels; assessment of the causes for concentration instability in NO gas mixtures; the development of low parts-per-billion (ppb) level organic gas mixtures; and the application of gas analysis to a variety of other agency programs. A brief description of some of these activities are given below and in the more detailed write-ups of the technical accomplishments that follow.

The first three ambient concentration level $\mathrm{CO}_{2}$ in air SRM's (nominally 330,340 , and $350 \mathrm{ppm}$ ) were completed in FY83. These SRM's have estimated uncertainties less than $0.1 \%$ relative, and are now available for calibrations of world-wide measurements for quantifying long-term trends in increasing atmospheric levels of $\mathrm{CO}_{2}$. A second set of three SRM's covering the $300-400$ $\mathrm{ppm}$ range, are now undergoing certification analysis. This second set will also be certified for $\mathrm{ppb}$ levels of $\mathrm{N}_{2} \mathrm{O}$, and will provide information values for Freons $\mathrm{F}-11$ and $\mathrm{F}-12$ in the parts-per-trillion range. Research on the characterization of fundamental spectral properties of the Freons using FTIR has led to publication of absolute band strengths of F-11 and F-12 covering three vibrational band systems of the former and four of the latter at 
$296 \pm 1 \mathrm{~K}$, with a resolution of $0.06 \mathrm{~cm}^{-1}$ in the "atmospheric window" between 8-16 $\mu \mathrm{m}$. These results infer a $17 \%$ and $5 \%$ greater absorbance strength for $F-11$ and F-12 than band strengths used in recent greenhouse warming calculations.

Research using FTIR and conventional gas analysis techniques was applied to the examination of the causes of instability in NO gas mixtures. A terminal reaction product, nitrous acid (HONO), predicted from homogeneous gas phase kinetics, was found at concentrations much lower than predicted, and its trace presence in both stable and unstable mixtures did not allow for a meaningful correlation of HONO with NO instability. The difficulty in using HONO to quantify $\mathrm{NO}$ instability may be complicated by surface sorption in cylinders and on the glass gas cell used in FTIR analysis. Elevated levels of $\mathrm{NO}_{2}$ in $\mathrm{NO}$ mixtures may correlate with NO instability, and further work is continuing to explore this possibility. Additional studies are planned using mixtures of NO that have been quantitatively doped with known trace levels of $\mathrm{NO}_{2}$ and/or water vapor using a new flow-through FTIR gas cell to minimize wall effects during analysis.

In the area of trace organic gas standards, notable progress has been made in the preparation of multi-component organic mixtures in the low ppb (5-15 ppb) range. The techniques developed to reliably produce such mixtures were published during this year. Work is also in progress for the certification of four-component aromatic mixtures of benzene, toluene, chlorobenzene, and bromobenzene at $0.25 \mathrm{ppm}$ and $9.5 \mathrm{ppm}$.

Several special studies were undertaken during the course of the past year involving: a time-course examination of pollutant gas levels in the National Archives; analyses of toxic gases evolved from lithium batteries, for the Naval Surface Weapons Center (NSWC); and the analysis of air samples taken from the Amazon River in Brazil. The Amazon study was a joint effort with Harvard University, NASA, and the Calypso Society, to assess the concentrations of selected gas species $\left(\mathrm{CH}_{4}, \mathrm{CO}_{2}, \mathrm{~N}_{2} \mathrm{O}, \mathrm{CO}\right.$, and freons $\mathrm{F}-11$ and $\left.\mathrm{F}-12\right)$ in a remote area. Preliminary analyses of the samples taken indicate enrichments of about 4 percent for methane and about 1 percent for $\mathrm{N}_{2} \mathrm{O}$ in the air above the tropical rain forest surrounding the Amazon River, relative to air above the Atlantic Ocean.

Future research plans of the Gas Metrology Research Group include: (1) continued research using FTIR, diode-laser, and GC-electron capture for detection, analysis, and characterization of trace gas species; (2) continued analytical research into the causes of instability in reactive gas mixtures using synthetically-prepared $\mathrm{NO}-\mathrm{NO}_{2}$-water vapor mixtures; (3) development of heats of sorption data associated with the sorption of volatile toxic organics by sorbents; and (4) the application of electrochemical approaches to the analys is of reactive gas mixtures containing $\mathrm{NO}_{x}$ and $\mathrm{SO}_{2}$.

Walter L. Zielinski, Jr., Group Leader; Barry C. Cadoff, William F. Cuthrell, William D. Dorko, James W. Elkins, Ernest E. Hughes, Patricia A. Johnson, Gerald D. Mitchell, Richard D. Myers, George C. Rhoderick, William P. Schmidt, Gerald A. Sleater, James E. Suddueth. 
B. Selected Technical Reports

(Gas and Particulate Science Division)

1. Cross-Coupled Interaction of Thermal, Electrical, Mechanical, and Chemical Parameters of Polymeric Permeation Devices (15330)

G. D. Mitchell

Although gas permeation tubes have been used empirically to generate known concentrations of gases in various matrices [1-4], no definitive investigation to establish the underlying theoretical basis or to determine the effect of the various parameters on the permeation process has been undertaken. The interest in gas permeation tubes and biological control release devices led to a preliminary theoretical evaluation to establish the relation between selected (external/internal) parameters and the diffusitivity, solubility and permeability of specific species through polymeric materials. The parameters of concern are not only the gradient of the chemical potential of the species, but also such parameters as stress, strain, electric field, polarization, charge, voltage, temperature, and entropy. Cross-coupled interactions of these parameters, which in the past have not been studied in relation to polymer permeation, represent the basis for this evaluation. A comprehensive approach to the complete characterization of polymeric permeation devices is afforded by a thermodynamic-based theory that explores the cross-coupled relationship between the permeating species and substrate under the influence of any or all of the parameters mentioned above.

A dynamic model of cross-coupled interactions of thermal, mechanical, electrical, and chemical parameters and their effect upon permeation is being explored. Since permeation and control release devices are binary systems, consisting of both the polymer substrate and a permeator, and since the permeation process depends on both kinetic and thermodynamic properties of the system it is not unexpected to find that gas permeation through polymeric materials is not entirely related to any one specific property of either the permeator or the substrate. The Table presented below depicts the parameters and coefficients that are to be considered, in addition to the diffusion and solubility characteristics of the permeation device. Research aimed at improving the understanding of the limitations and role of these parameters in relationship to permeation devices is continuing.

References

[1] A. E. O'Keefe and G. C. Ortman, Anal, Chem. 38, 760 (1966).

[2] A. E. O'Keefe and G. C. Ortman, Anal, Chem. 39, 1047 (1967).

[3] M. D. Thomas and K. E. Amtower, J. Air Pollut. Contr. Assoc. 16, 623 (1966).

[4] L. A. Elfey and C. E. Decker, Anal. Chem. 40, 1959 (1968). 
Table 33. Definitions of the Coefficients Representing the Principal and Coupled Parameters.

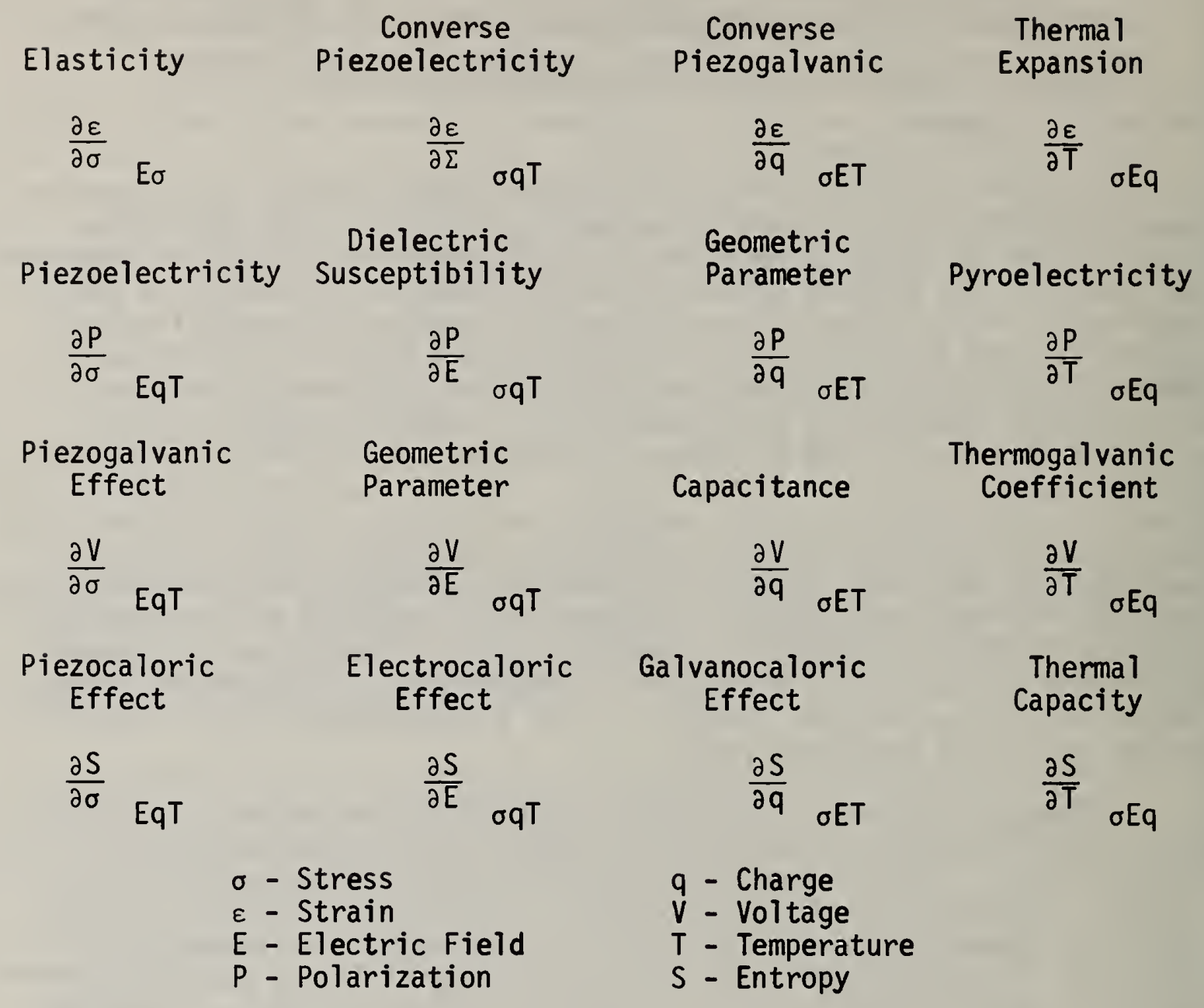

2. Band Strength Measurements of Propane by Fourier Transform Infrared Spectroscopy (15330)

G. C. Rhoderick, J. W. Elkins

The infrared properties of propane have received considerable attention in atmospheric science because of the role of propane in the tropospheric chemistry of polluted urban environments. Interest has also extended into planetary astrophysics, since the positive identification of propane in the atmospheres of Saturn and its largest satellite, Titan, by the Voyager 1 spaceprobe's FTIR spectrometer [1]. Accurate measurement of the band strengths $\left(S_{v}\right)$ permit determination of concentration values from recorded spectra. We have re-examined these values at a higher resolution $\left(0.06 \mathrm{~cm}^{-1}\right)$ with a FTIR spectrometer to improve the accuracy of spectroscopic analysis techniques for propane. 
Figure 43 shows a representative Beer's law plot, the integrated absorbance versus pressure-pathlength product, for the $v_{1}\left(v_{2}\right)$ and $v_{3}$ bands of propane. Standards prepared by gravimetry and by pressure measurements were used to fill a 5- and $10-\mathrm{cm}$ cell to one atmosphere. The integrated absorbance was measured also from a $5-\mathrm{cm}$ cell containing 0.25 percent propane in nitrogen from SRM 2647. The data from the SRM, pressure and gravimetric standards fall on the same line. Therefore, the pressure and gravimetric methods produce equivalent standards under the conditions of the experiment. The plot also demonstrates that the FTIR is a valuable tool in the verification of concentration values for SRM's.



PRESSURE - PATHLENGTH PRODUCT $(p \cdot 1)$

Figure 43. Beer's law plots with band strengths (slope of line) for the $v_{1}\left(v_{2}\right)$ and $v_{3}$ bands. The standard deviation of the least squares determination of the slope is the value in parenthesis.

The slope of the line in figure 43 is equal to the band strength. Table 34 shows previous measurements together with our measurements of the major band systems of propane between 700 and $4400 \mathrm{~cm}^{-1}$, corrected to $296 \mathrm{~K}$. The previous results of Stalmakhova et al. [2] and Kondo and Saeki [3] were obtained using conventional spectrometers at 2 and $1 \mathrm{~cm}^{-1}$ resolution, respectively. Band assignments in parentheses indicate that the band system has coincidental overlapping of bands from different vibrational states. Many of the infrared bands of propane are merged together and require deconvolution to separate individual band strengths from interfering bands. The 
Table 34. Band Strengths for Propane $\left(\mathrm{C}_{3} \mathrm{H}_{8}\right)$.

\begin{tabular}{|c|c|c|c|c|}
\hline $\mathrm{C}_{3} \mathrm{H}_{8}$ & & & $\begin{array}{l}\text { trengths at } \\
\text { (296) } \mathrm{cm}^{-2} \mathrm{~atm}\end{array}$ & \\
\hline $\begin{array}{l}\text { Band } \\
\text { System }\end{array}$ & $\begin{array}{l}\text { Observed } \\
\text { Band } \\
\text { Center } \\
\left(\mathrm{cm}^{-1}\right)\end{array}$ & $\begin{array}{l}\text { Stalmakhova } \\
\text { et al. } \\
\text { (1965) } \\
\text { Ref. } 2^{b}\end{array}$ & $\begin{array}{l}\text { Kondo } \\
\text { and Saeki } \\
(1972) \\
\text { Ref. } 3^{b}\end{array}$ & $\begin{array}{l}\text { Present } \\
\text { Work }\end{array}$ \\
\hline$v_{21}$ & 748 & 9.92 & $9.05(1.03)$ & $9.97(0.32)$ \\
\hline$v_{8}$ & 869 & 2.32 & $2.26(0.70)$ & $2.04(0.13)^{c}$ \\
\hline$v_{16}$ & 922 & 6.12 & $4.69(0.66)$ & $4.57(0.17)^{c}$ \\
\hline$v_{15}$ & 1054 & 4.38 & $4.07(0.45)$ & $3.26(0.15)$ \\
\hline$v_{7}\left(v_{20}\right)$ & 1170 & 5.21 & $5.10(0.62)$ & $3.89(0.13)$ \\
\hline$v_{14}$ & 1338 & 9.92 & $10.3(2.9)$ & $7.56(0.30)^{c}$ \\
\hline$v_{6}\left(v_{13}\right)$ & 1378 & 26.5 & $24.3(3.7)$ & $23.8(0.8)^{c}$ \\
\hline$v_{4}\left(v_{5}\right)$ & 1476 & 70.3 & $70.8(7.4)$ & $75.1(2.4)^{C}$ \\
\hline$v_{3}$ & 2887 & 372 & $218(43)$ & $188(7)^{C}$ \\
\hline$v_{1}\left(v_{2}\right)$ & 2977 & 554 & $736(59)$ & $748(24)^{C}$ \\
\hline$v_{1}+v_{22}$ & 3175 & --- & --- & $9.97(0.36)$ \\
\hline$v_{7}+v_{9}$ & $\begin{array}{l}3335 \\
4150 \\
4300\end{array}$ & $\begin{array}{l}--- \\
--- \\
---\end{array}$ & $\begin{array}{l}--- \\
--- \\
---\end{array}$ & $\begin{array}{l}1.49(0.05) \\
13.0(0.5) \\
17.9(0.6)\end{array}$ \\
\hline$v_{8}\left(v_{16}\right)^{d}$ & & 8.44 & 6.95 & $6.61(0.22)$ \\
\hline $\begin{array}{l}v_{4}\left(v_{5}\right)\left(v_{6}\right) \\
\left(v_{13}\right)\left(v_{14}\right) d\end{array}$ & & 106.7 & 105.4 & $106.6(3.4)$ \\
\hline$v_{1}\left(v_{2}\right)\left(v_{3}\right)^{d}$ & & 926 & 954 & $936(30)$ \\
\hline
\end{tabular}

ancertainties for band strengths in the last digits are given in parentheses. The uncertainties for previous work are reported values, whereas the uncertainties in the present work include both reproducibility errors and possible systematic errors (see text).

buthors in ref. 1 reported values in $10^{-8} \mathrm{~cm}^{2} \mathrm{~s}^{-1} \mathrm{~mol}^{-1}$ with uncertainties of $5-10 \%$, and in ref. 2 reported in $\mathrm{L} \mathrm{mol}^{-1} \mathrm{~cm}^{-2}$.

Measurements of these bands obtained by deconvolution.

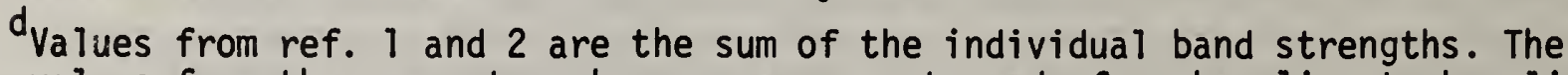
values from the present work are measurements made from baseline to baseline. 
largest disagreement between our results and previous measurements exist for these interfering bands. This discrepancy can be explained as the inability to define precise upper and lower bounds of an individual band in a merged continuum of numerous bands (errors in deconvolution), since the total band system strengths of the interfering bands agree to within a few percent between laboratories (see last three lines in Table 34 ).

Estimates of our uncertainties (reported as one standard deviation) given in Table 34 were obtained by adding in quadrature three major possible sources of error: pressure-pathlength product $(\mathrm{pl})$ errors $( \pm 0.7 \mathrm{RSD}$, relative standard deviation), reproducibility in the least squares determination of the slope of the Beer's law plot (i.e. standard deviation shown in figure 43), and a possible systematic error. A systematic error of \pm 3 percent (RSD) was estimated by comparing our FTIR results of other infrared absorbing molecules against accurate high resolution infrared measurement by other researchers. We believe our band strengths of propane are reliable, because of the higher resolution of our measurements compared to other studies and the care we took to avoid possible errors.

\section{References}

[1] Maguire, W. A., R. A. Hanel, D. E. Jennings, V. G. Kunde, and R. E. Samuelson, Nature, 292, 683-686, (1981).

[2] Stalmakhova, A. G. Finkel and L. M. Sverdlov, Optika I Spektroskopia, $18,1083-1086$, (1965).

[3] Kondo and Saeki, Spectrochim. Acta, 29A, 735-751, (1972).

3. Sampling and Measurement of Nitrous Oxide, Carbon Dioxide, and Methane in the Amazon (15330)

J. Elkins

Increased levels of certain trace gases in the atmosphere, (such as carbon dioxide $\left(\mathrm{CO}_{2}\right)$, methane $\left(\mathrm{CH}_{4}\right)$, and nitrous oxide $\left(\mathrm{N}_{2} \mathrm{O}\right)$ may increase the surface temperature of the earth [1] and may also influence the equilibrium concentration of stratospheric ozone [2]. The sources and sinks of these gases are primarily biological [3], together with significant anthropogenic sources. Global climatic models predict enhanced concentration levels of these gases in the atmosphere over biologically active areas, such as the Amazon river basin. Few reliable measurements of trace gases are reported in the literature for such a remote global region.

A unique research opportunity was offered by the Cousteau Society when scientists from NBS and Harvard University were invited to sail with the research vessel, R/V Calypso, during the Amazon Expedition in December 1982. The Amazon estuary is important because the waterway provides about 20 percent of the total fresh water source to the world's oceans. Our research was co-sponsored by the National Aeronautics and Space Administration (NASA) and the Cousteau Society in order to sample both the air and water ecosystems for 
analysis of trace gaseous species from this large river system. Water and air samples were collected at Manaus and aboard the R/V Calypso. Duplicate air samples were returned to NBS and Harvard University for analysis.

Figure 44 shows the cruise track of the R/V Calypso in December 1982 during the course of the expedition from Belem, Brazil at the mouth of the Amazon to the Caribbean island of Martinique. Station locations are marked on the figure. The interior stations (Sta. 1 \& 2) at Manaus, Brazil were sampled before boarding the Calypso. December is typically the "dry season" in the Amazon river basin and a period of low river flow. The source of the air mass over the Amazon basin are the "Trade winds", generally blowing in from either the northeast or the southeast.

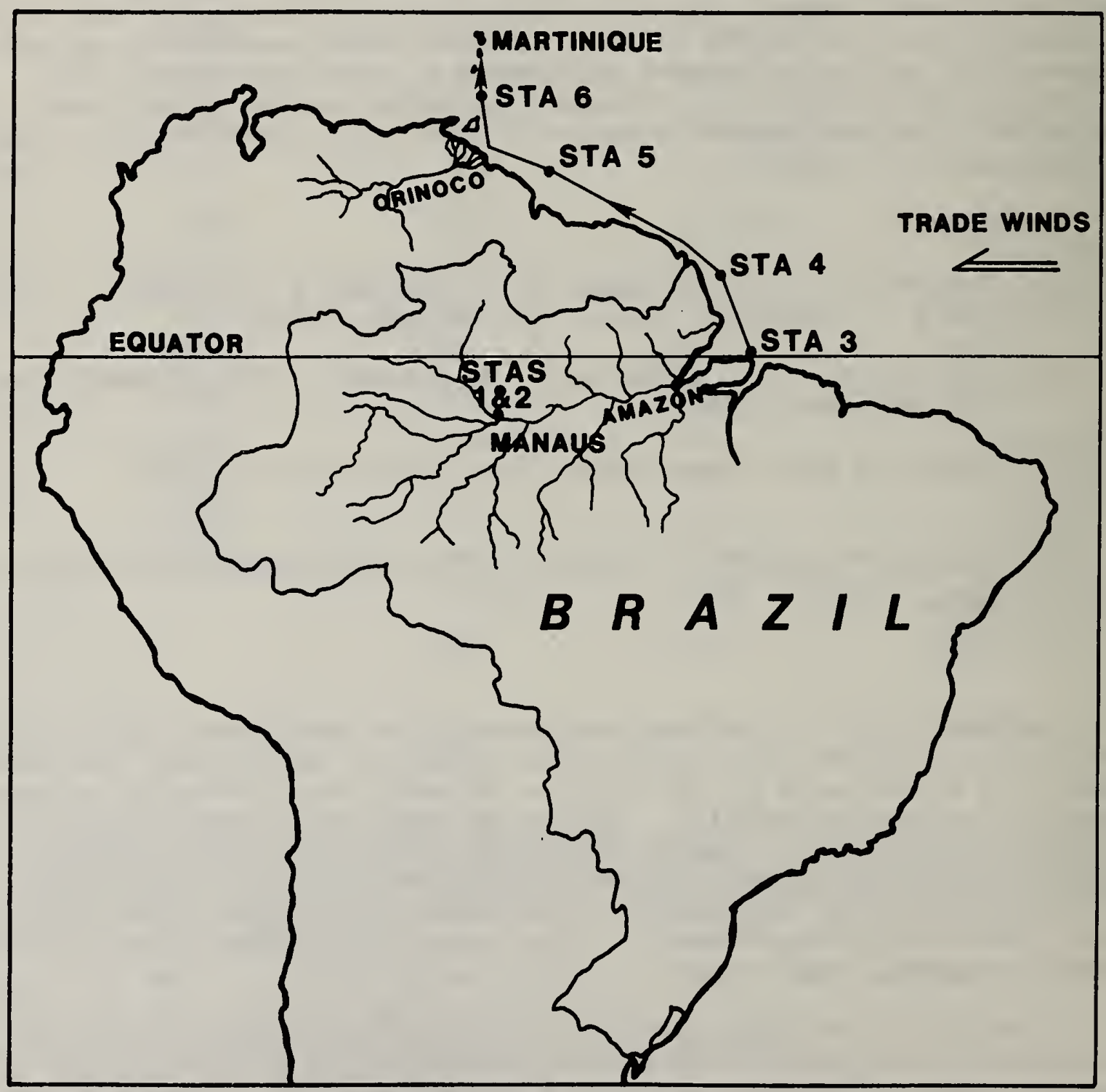

Figure 44. 
The most interesting results from the expedition were the differences in the atmospheric concentrations of these trace gases for the interior and coastal stations of the Amazon basin. Table 35 shows the average, standard deviation, and number of measurements for the concentrations of each trace gas at the stations indicated in figure 44 . The observed differences in concentration between the interior and coastal Amazon stations for each gas were calculated along with the uncertainties of the differences at the 95 percent confidence level. Identical samples from the same location were measured independently by different analytical methods at both NBS and Harvard University (Professors McElroy and Wofsy). The agreement in $\Delta C$ values between laburatories reported in the last two rows of Table 35 was excellent. The results show a statistically significant enrichment of about 3 percent and 1.5 percent for $\mathrm{CH}_{4}$ and $\mathrm{N}_{2} \mathrm{O}$, respectively, in the air above the interior station at Manaus over the air above the stations off the coast of Brazil. This enrichment of $\mathrm{CH}_{4}$ and $\mathrm{N}_{2} \mathrm{O}$ confirmed calculations from global climatic models developed at Harvard University and Goddard Institute for Space Studies. The concentration for carbon dioxide at the interior stations ranged from 338 to $419 \mathrm{ppm}$ (partsper-million), indicating large and variable sources of $\mathrm{CO}_{2}$ near the ground of the forest.

The measurements reported here are limited in scope and reflect only results from the "dry season". We have recently collected additional samples from more locations around Brazil during the "wet season" and those samples are currently being analyzed.

\section{References}

[1] Chamberlain, J. W., H. M. Foley, G. J. MacDonald, and M. A. Ruderman, in Carbon Dioxide Review: 1982, ed. by W. C. Clark, Oxford University Press: New York, 255-277, (1982).

[2] McElroy, M. B., J. W. Elkins, S. C. Wofsy, and Y. L. Yung, Rev. Geophys. Space Sci., 14, 143-150, (1976).

[3] Elkins, J. W., S. C. Wofsy, M. B. McElroy, C. E. Kolb, and W. A. Kaplan, Nature, 275, 602-606, (1978). 
Table 35. Results ${ }^{\mathrm{C}}$ for Methane, Carbon Dioxide, and Nitrous Oxide During Amazon Expedition, December 1982.

\begin{tabular}{|c|c|c|c|}
\hline SITE & $\mathrm{CH}_{4}$ CONC. (ppm) & $\mathrm{CO}_{2}$ CONC. (ppm) & $\mathrm{N}_{2} \mathrm{O}$ CONC. (ppb) \\
\hline $\begin{array}{l}\text { STA \#1, } 2 \\
\text { MANAUS } \\
\text { DEC } 8,9^{a}\end{array}$ & $1.684 \pm 0.015(11)$ & $371.0 \pm 23(11)$ & $307.2 \pm 3.3(10)$ \\
\hline $\begin{array}{l}\text { STA \#3,4 } \\
\text { COAST } \\
\text { DEC } 10,13^{\mathrm{a}}\end{array}$ & $1.637 \pm 0.013$ & $344.6 \pm 5.1$ & $302.4 \pm 1.4$ \\
\hline $\begin{array}{l}\text { STA \#5 } \\
\text { OCEAN } \\
8^{\circ} \mathrm{N} \\
\text { DEC } 15^{\mathrm{a}}\end{array}$ & $1.658 \pm 0.008$ & $345.5 \pm 4.2(5)$ & $302.5 \pm 1.5$ \\
\hline $\begin{array}{l}\text { STA \#6 } \\
\text { OCEAN } \\
12^{\circ} \mathrm{N} \\
\text { DEC } 17^{\mathrm{a}}\end{array}$ & $1.652 \pm 0.015(6)$ & $343.0 \pm 0.9(5)$ & $302.2 \pm 0.7$ \\
\hline \multicolumn{4}{|c|}{$\begin{array}{l}\text { MANAUS-COAST }(\Delta C) \\
\text { NBS }\end{array}$} \\
\hline $\begin{array}{l}\Delta \mathrm{c} \\
95 \% \mathrm{CI}^{\mathrm{b}}\end{array}$ & $0.047 \pm 0.011$ & $26.4 \pm 12.6$ & $4.8 \pm 2.1$ \\
\hline $\begin{array}{l}\text { HARVARD } \\
\Delta c \\
95 \% \mathrm{CI}^{\mathrm{b}}\end{array}$ & $0.042 \pm 0.019$ & $19.2 \pm 21.1$ & $4.0 \pm 2.8$ \\
\hline
\end{tabular}

$\overline{a_{\text {Values reported }}}$ as average \pm standard deviation. Value in parentheses is number of measurements ( $\mathrm{ppm}=$ parts-per-million, and $\mathrm{ppb}=$ parts-per-bilition). buncertainties are based on the $t$-test and reflect the $95 \%$ confidence interval (CI).

$\mathrm{C}_{\mathrm{A}} 1 \mathrm{I} \pm$ values represent one standard deviation except those for Manaus-Coast ( $95 \%$ CI (two standard deviations)). 


\section{Stability Studies of NO in $\mathrm{N}_{2}$ SRM's (15330)}

B. C. Cadoff

While SRM gas mixtures are widely recognized standards due to their concentration accuracy and stability, occasionally cylinders are not certified because of an observed instability. Generally, it is the more reactive gas species (such as $\mathrm{NO}$ and $\mathrm{SO}_{2}$ ) that are more prone to show instability, which, although over a period of time may be relatively small (e.g., 1-2 percent concentration degradation), is still unacceptable. The current research program has the long range objective of identifying the causes of instability and proposing ways of improving stability. The program has initially been focused on examining the instability of $\mathrm{NO}_{\mathrm{O}}$ in $\mathrm{N}_{2}$ gas mixtures.

The first approach was, to assess the effects of homogeneous gas phase reactions. For this study, a total of 15 cylinders were examined which had been prepared as SRM 1687a, at a nominal concentration of $1000 \mathrm{ppm} N O$ in $\mathrm{N}_{2}$. Most of the cylinders had shown small losses of NO between the initial analysis and a second analysis some 18 months later. The approach to this study involved both collection of analytical data on species present and consideration of the gas phase chemical reactions that can occur, along with the associated rate and equilibrium constants.

The presence of impurity gases in compressed gas mixtures has always been a concern; however, any cause and effect pattern on composition instability due to these impurities has been, at best, ambiguous. For example, it has been postulated that water vapor may play a role in the loss of some species such as NO. However, it has been observed that some stable cylinders contain higher concentrations of $\mathrm{H}_{2} \mathrm{O}$ than other unstable cylinders.

The reactions that have been reported to occur in the gas phase involving NO, $\mathrm{NO}_{2}, \mathrm{H}_{2} \mathrm{O}$ and $\mathrm{O}_{2}$ are few in number and are as follows:

$$
\begin{aligned}
2 \mathrm{NO}+\mathrm{O}_{2} & \longrightarrow 2 \mathrm{NO}_{2} \\
2 \mathrm{NO}_{2} & \rightleftarrows \mathrm{N}_{2} \mathrm{O}_{4} \\
\mathrm{NO}+\mathrm{NO}_{2} & \rightleftarrows \mathrm{N}_{2} \mathrm{O}_{3} \\
\mathrm{~N}_{2} \mathrm{O}_{3}+\mathrm{H}_{2} \mathrm{O} & \rightleftarrows 2 \mathrm{HONO} \\
3 \mathrm{NO}_{2}+\mathrm{H}_{2} \mathrm{O} & \rightleftarrows 2 \mathrm{HNO}_{3}+\mathrm{NO}
\end{aligned}
$$

Reaction (1) is a rather fast reaction which has been well studied and for which the rate constant is reliable. The reaction goes to completion with no reverse reaction at room temperature. When representative values for NO and $\mathrm{O}_{2}\left[955 \mathrm{ppm} \mathrm{N0}, 7 \mathrm{ppm} 0_{2}, 13.78 \mathrm{MPa}(2000 \mathrm{psi})\right]$ are substituted in the rate equation, calculation indicates that the reaction is more than 99.9 percent completed in 10 minutes. In fact, even at concentrations of $50 \mathrm{ppm}$ NO, trace $\mathrm{O}_{2}$ is converted to $\mathrm{NO}_{2}$ in approximately one hour. Hence, any trace oxygen that may be present during the preparation of a cylinder is rapidly converted to $\mathrm{NO}_{2}$. 
Reaction (5) can be excluded from consideration since recent studies have shown that, in the presence of a high concentration of $\mathrm{NO}$ relative to $\mathrm{NO}_{2}$, no detectable reaction between $\mathrm{NO}_{2}$ and $\mathrm{H}_{2} \mathrm{O}$ takes place. Therefore, the gas phase reactions that can lead to a slow degradation of an $\mathrm{NO}-\mathrm{in}-\mathrm{N}_{2}$ mixture are reactions (2), (3) and (4). [In a totally dry system equations (2) and (3) would govern.]. These reactions are relatively slow, and produce (at equilibrium) HONO as the predominant product. Earlier in this project, a computer-based kinetics program was used to model the case of $100 \mathrm{ppm} \mathrm{NO}$, with $\mathrm{NO}_{2}$ and $\mathrm{H}_{2} \mathrm{O}$ present. It was found that NO would degrade slowly, over a period of months or years. The problem with this calculation was that there is no reliable rate constant for equation (4)-literature values varied by a factor of 100 . However, the situation with respect to equilibrium constants is much better (with errors in the range of about $5 \%$ ) and an equilibrium model was finally used to compare and assess the chemical analysis of these cylinders.

Analytical data were obtained as follows: NO - chemiluminescent detector; $\mathrm{H}_{2} \mathrm{O}$ - electrolytic analyzer; all other species - FTIR using a 100 meter cell. Table 36 lists the analytical results for these cylinders. Percentages at the top of the columns are estimates of uncertainties. These cylinders are now three years old and have been analyzed for NO on three occasions, approximately 18 months apart. Two cylinders have exhibited no change (41-2-C and 41-3-C); the other 13 cylinders have undergone changes as large as 1.9 percent. However, with the exception of 41-51-C, every cylinder has been stable from the second to the third analysis. Referring to equations (2), (3) and (4) and calculating the concentrations at equilibrium, we find that, for the case of $\mathrm{NO}=955 \mathrm{ppm}, \mathrm{NO}_{2}=14 \mathrm{ppm}$ and $\mathrm{H}_{2} \mathrm{O}=20 \mathrm{ppm}$, all at $2000 \mathrm{psi}$, the products of reaction would be $6 \mathrm{ppm} \mathrm{HONO}$ and $0.8 \mathrm{ppm} \mathrm{N}_{2} \mathrm{O}_{3}$, with trace amounts of $\mathrm{N}_{2} \mathrm{O}_{4}$. As seen from Table 36 , HONO is present in only trace amounts (less than $1 \mathrm{ppm}$ ), and $\mathrm{N}_{2} \mathrm{O}_{3}$ is not detectable. If we make the assumption that HONO is lost to the walls of the cylinder, this reaction would result in a 1.5 percent loss of NO, a representative loss as seen from Table 36 . Thus, we can explain the loss of NO as resulting from gas phase reactions and subsequent loss to the walls, or in other words, a gas phase titration reaction. Of course, this is not a proof but rather a plausible explanation. In this connection it is interesting to note that the cylinders that did not undergo change between the second and third analysis all had only trace levels of $\mathrm{NO}_{2}$. The one cylinder that did change had a very high level of $\mathrm{NO}_{2}$ (25 ppm).

The mechanism of degradation which has been postulated requires that $\mathrm{NO}_{2}$ be present. The experimental results are in accord with this mechanism since stable cylinders, and cylinders which have previously undergone changes but which are now stable, contain only trace levels of $\mathrm{NO}_{2}$, far below the amount necessary to cause a detectable change in the NO concentrations. The one cylinder which has continued to degrade contains a large concentration of $\mathrm{NO}_{2}$. The expected products of reaction, $\mathrm{N}_{2} \mathrm{O}_{3}$ and $\mathrm{HONO}$, are either absent (in the case of $\mathrm{N}_{2} \mathrm{O}_{3}$ ) or present in extremely low concentrations (in the case of HONO) indicating that one.or both products are lost to the walls.

The now-stable cylinders will be reexamined after several months to redetermine NO content. It is expected that no further change will take place since only trace levels of $\mathrm{NO}_{2}$ are present. A key set of experiments are planned in which we will fill cylinders with suitable levels of $\mathrm{NO}, \mathrm{NO}_{2}$ and 





$\mathrm{H}_{2} \mathrm{O}$ and determine the resulting component concentration changes. A more detailed kinetic study can also be conducted in which varying levels of the reactants are studied.

The $\mathrm{NO}, \mathrm{NO}_{2}, \mathrm{H}_{2} \mathrm{O}$ system might also be used to assess the inertness of a particular cylinder (or a particular treatment for a cylinder) by determining whether the concentrations of species agree with those predicted by considering the appropriate equilibrium constants.

\section{Evaluation of Degree of Sorption in Collection of Trace-Organic Vapors} on Sorbents (15330)

G. Sleater, W. L. Zielinski, Jr., G. A. Ulma, Jr.

Sorbents have been widely used in the past decade for the evaluation of air pollution and of personal exposures in industrial environments due to volatile organic compounds of known or suspected human health hazards. The sorbed organics are typically measured by desorption from the sorbent using either solvent extraction or thermal means, followed by chromatographic analysis. Several significant activities relative to the importance of developing standardized, reliable, data concerning air pollutant levels and worker exposures, include: NBS' development of a set of SRM's containing quantified levels of specific organics sorbed on charcoal; EPA's proposed gas saturation procedure, based on sorbent collection of organic vapors for organic vapor pressure measurements (q.v.); and NIOSH's development of an analytical method (No. P\&CAM 127), based on sorption on charcoal followed by desorption with carbon disulfide.

It was determined by NBS and others that attempts of quantitative recovery of sorbed organics from the substrate introduced the greatest uncertainty in estimations of the levels of trace organics in air. To address this, a series of experiments were initiated during the past year, aimed at improving the understanding of the behavior of the sorption-desorption process. Three different sorbents were selected for study: coconut charcoal, Tenax, and a carbopack-like material.

Three types of experiments were designed with the objective of: (1) comparing sorbent capacities; (2) developing heat of sorption data for various volatile organics; and (3) testing the efficacy of employing heat of sorption values for selecting optimum desorbing solvents.

Weighed samples of the sorbents were incubated at room temperature in desiccators containing vapors of specific organics to compare their relative sorptive capacities. Preliminary experiments using toluene as the test vapor indicated that charcoal sorbed about 4-fold the amount of toluene sorbed by Tenax (weight increase of $38.3 \%$ versus $9.97 \%$ ). In a more defined desiccator study, sorbent capacities for acetone and benzene were examined for all three sorbents studied, with the following results: 
$\%$ Weight Increase

Acetone

Charcoal

Tenax

Carbopack
28.44

0.34

0.26
Benzene

28.47

5.02

0.24

Hence, sorbent capacities vary dramatically. Charcoal may have too great a sorptive capacity, engendering incomplete desorption prospects, while carbopack appears to have low sorbent qualities of little utility.

In a separate set of experiments, heats of sorption of various organic vapors were measured. The experimental design involved sampling the head-space vapor of an organic compound and measurement of retention data on a gas chromatographic column packed with the sorbent at 4-6 temperatures. The use of a charcoal GC column afforded very limited retention data due to the high capacity (sorption affinity) of charcoal. However, analyses on Tenax were successful. The heats $\left(\Delta \mathrm{H}_{\mathrm{a}}\right)$ were calculated from the relationship

$$
\ln v_{r}^{\prime}=-\Delta H_{a} / R T+C
$$

where $V_{r}^{\prime}$ is the corrected retention volume at column temperature $T$ in degrees Kelvin, and $R$ is the gas constant, $1.987 \mathrm{cal} / \mathrm{mol}$-degree. Linear regression analysis of $1 n V_{r}^{\prime}$ vs $1 / T$ gave the following sorption heats on Tenax:

$$
\Delta \mathrm{H}_{\mathrm{a}} \text { (kcal/mole) }
$$

2-Nitrophenol

Benzene

Chloroform

Ether

Acetone

Carbon Disulfide
19.01

14.01

13.01

12.68

11.56

10.17
Correlation

Coefficient

0.9993

0.9998

0.9998

0.9983

0.9997

These results are consistent with the capacity measurements of benzene and acetone on Tenax, given above. It is interesting that carbon disulfide, commonly used as a desorbing solvent, does not appear to be an optimum choice because of $i$ ts lower heat of sorption. Solvent desorption experiments from desiccator experiments are currently in progress to test the consistency of desorption recovery data with heats of sorption data. 
6. Round-Robin Assessment of Gas Saturation Test Method Proposed by EPA for Generating Vapor Pressure Data on Organic Chemicals (15330)

W. L. Zielinski, Jr, K. R. Eberhardt (714)

A round-robin study was conducted to evaluate a test standard proposed by EPA [1] for the determination of near-ambient vapor pressures of pure organic compounds. The study was coordinated by NBS, supported by the EPA Office of Pesticides and Toxic Substances, and involved an analytical evaluation by eight private testing laboratories, a university laboratory, and the Gas \& Particulate Science and Organic Analytical Research Divisions of CAC. Statistical evaluations of the data obtained were performed in the Statistical Engineering Division of the NBS Center for Applied Mathematics.

The test method is based upon the gas saturation technique in which the carrier gas flows through the test compound compartment and then through a two-stage adsorbent trap. In this technique the flow system is housed in a constant temperature box and the effluent flow rate is monitored over the time course of the experiment. Following the experiment, the adsorbent trap is demounted, and the level of sorbed test compound is determined by solvent extraction of the sorbent and quantitative analysis (using gas chromatography or another appropriate technique). Desorption efficiency is determined separately from sorbent spiked with the test compound, to correct for that fraction of compound not desorbed by the extracting solvent.

The private laboratories examined 10 test compounds, comprised of either solids or liquids, covering a nominal vapor pressure range of $10^{2}-10^{-5} \mathrm{~Pa}$ (about $10^{\circ}-10^{-7}$ torr). The university laboratory examined two of the test compounds to test the adaptability of the proposed test standard in the hands of capable, but inexperienced operators. NBS performed an evaluation of the experimental assembly and the proposed test standard per se to develop guidance information for the round-robin, and evaluated six of the test compounds in an independently developed alternate procedure [2] for vapor pressure measurements using either cryogenic trapping or sorption on C-18 reversed-phase particles, followed by HPLC analysis. The data obtained by the latter procedure were highly precise, consistent with reliable literature data, and were used as reference values in the statistical evaluation of the round-robin data.

Vapor pressure values ( $p$, in Pascals) were calculated from the experimental results using the expression:

$$
p=W R T / M v
$$

where $w$ is the total sorbed mass of a test compound of molecular weight $M$; $v$ is the total volume $\left(\mathrm{m}^{3}\right)$ passing through the system during the duration of the experiment, $T$ is the experimental temperature in Kelvin, and $R$ is the gas constant $\left(8.31 \mathrm{~Pa}-\mathrm{m}^{3} / \mathrm{mol}-\mathrm{deg}\right)$. The round-robin data received from the eight private testing laboratories for the 10 test compounds were subjected to statistical analysis. A summary of this analysis is given in Table 37 . This numerical summary follows the format given in ANSI/ASTM E 691-79, "Standard Practice for Conducting an Interlaboratory Test Program to Determine the Precision of Test Methods." In Table 37, column (2) lists the mean of the 


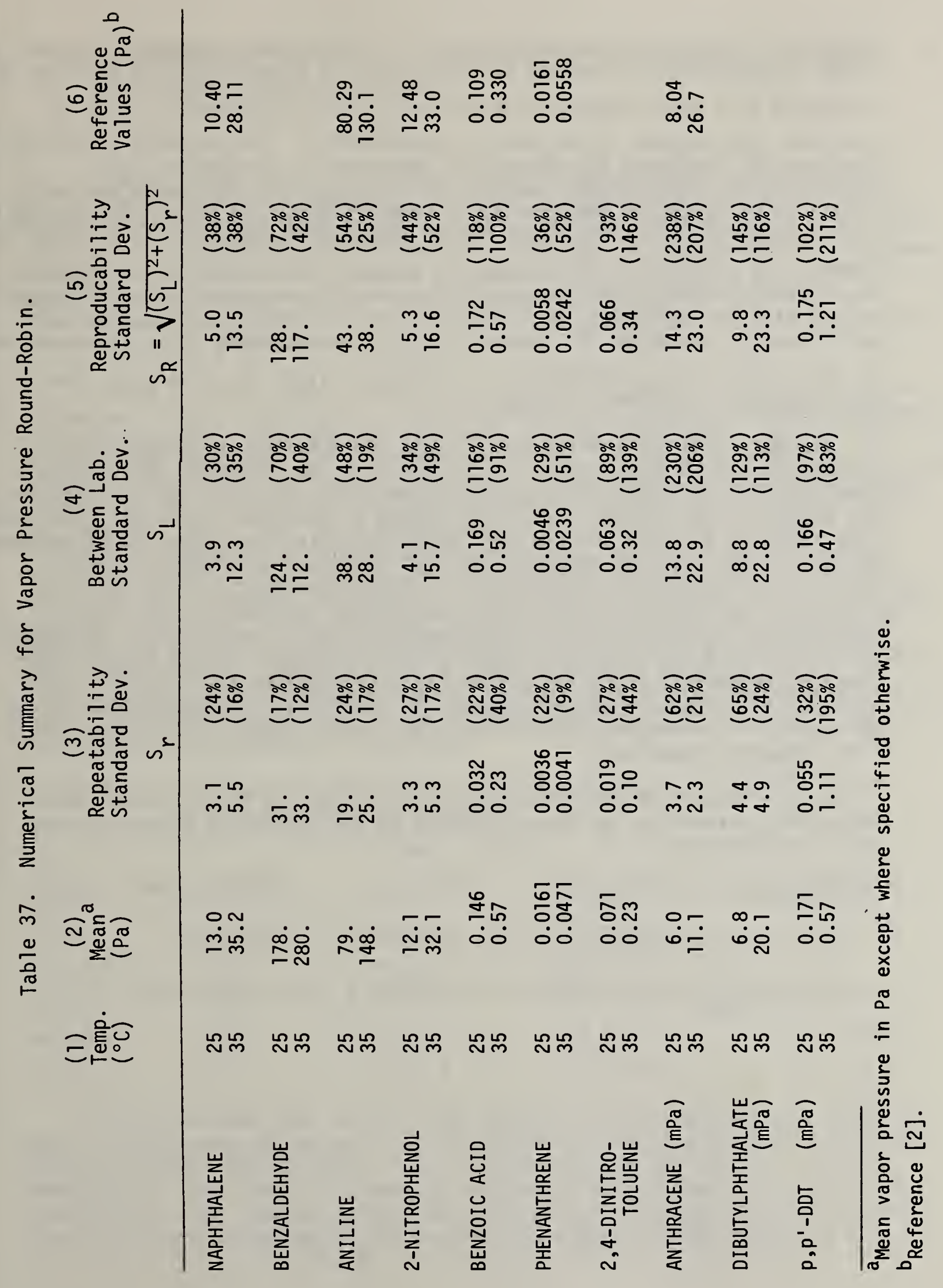


laboratory means at a given temperature for a given test compound. Column (3) is the "repeatability" standard deviation $\left(S_{r}\right)$ of three replicate analyses for a compound by a test laboratory, pooled across all laboratories (with $S_{r}$ expressed as a percent of the mean in parenthesis). The standard deviation $\left(S_{L}\right)$ in column (4) estimates the between-lab component of variation from a random-effects model one-way analysis of variance, and represents the variability due strictly to between-lab differences after the within-lab variability is accounted for. The "reproducibility" standard deviation $\left(S_{R}\right)$ in column (5) combines the within-lab and between-lab standard deviations, and represents the typical variability between individual results obtained by two different laboratories analyzing one sample each using the proposed test method (the coefficient of variation for reproducibility, representing $S_{R}$ as a percent of the mean, is given in parenthesis). Reference values for several of the test compounds are given in column (6).

The most notable observations of this round-robin evaluation of the proposed test method were that: (1) the within-lab precision was typically significantly less than the between-lab precision, pointing out the need for a more explicit description of the test method to minimize individual interpretations in using the test method; (2) the laboratories most experienced in using the test method gave the most precise, but not always the most accurate results; (3) the greatest uncertainty in obtaining results using the test method was contributed by the imprecision in the desorption efficiency determination and the use of this determination for correcting for the amount of test compound not desorbed by the extraction solvent in the actual vapor pressure experiments (especially when using charcoal as the sorbent); and, finally, (4) the precision and accuracy in vapor pressure measurement by the gas saturation technique are much improved when a sorbent is excluded (e.g., using cryogenic trapping).

A final report of this round-robin, including all test data and their statistical evaluation has been submitted to the EPA Office of Pesticides and Toxic Substances.

References

[1] Federal Register, Vol. 45, No. 227, pp. 77345-77350.

[2] Sonnefeld, W., Zoller, W., and May, W., Anal. Chem., 55, 275 (1983). 


\section{Development of Techniques for Preparation and Analys is of ppb Level}

Toxic Organic Standards (15330)

W. P. Schmidt, W. F. Cuthrell

Previous work in this long-range research program for the development of reliable, stable gas standards of toxic, volatile organic species has led to the development of benzene and tetrachloroethylene gas cylinder SRM's, each at nominal concentrations of 10 and 0.25 parts-per-million (ppm) and a benzene permeation tube SRM with a nominal permeation rate of $0.3-0.5$ micrograms/minute at $25^{\circ} \mathrm{C}$. The successful development of gas-cylinder SRM's was based on a precise method for gravimetric preparation and gas chromatographic measurement reported previously [1].

During the past year, research has been specifically directed toward the extension of this technology to the preparation and analys is of multi-component organic gas mixtures at the low. parts-per-billion (ppb) level. One of the applications evaluated has been the preparation and analys is of a five-component organic gas-cylinder mixture in which each of the organics were at concentrations below $15 \mathrm{ppb}$, by mole. This mixture was prepared at the request of EPA to conduct a field test to intercompare the efficiencies of different air sampling approaches for monitoring toxic organic species in the atmosphere. The mixture was prepared gravimetrically, and analyzed prior to and following the field test, as well as several months later to check the stability of the organic components. Analyses were conducted by intercomparing a number of primary gas mixtures prepared by gravimetry with the test mixture by repeated gas chromatographic analysis using a flame ionization detector (FID). The resultant data were subsequently evaluated by a statistical curvilinear fit to obtain concentration levels for the organic components in the test cylinder. The results are summarized in Table 38 .

Table 38. Comparison of Gravimetrically Calculated and Analyzed Levels for a Five Component Mixture.

$$
\text { (ppb, by mole) }
$$

\begin{tabular}{|c|c|c|c|c|}
\hline Species & Gravimetric & lst Analysis & 2nd Analysis & 3rd Analysis \\
\hline $\mathrm{CHCl}_{3}$ & 3.7 & $4.8 \pm 2.6$ & $3.5 \pm 0.4$ & $3.8 \pm 0.12$ \\
\hline $\mathrm{CCl}_{4}$ & 8.2 & $7.9 \pm 6.1$ & $7.3 \pm 1.0$ & \\
\hline $\mathrm{C}_{2} \mathrm{Cl}_{4}$ & 12.4 & $13.7 \pm 3.4$ & $11.4 \pm 0.5$ & $12.5 \pm 0.16$ \\
\hline Benzene & 9.0 & $6.8 \pm 0.3$ & $8.3 \pm 0.2$ & $8.4 \pm 0.28$ \\
\hline Toluene & 6.5 & $5.9 \pm 0.6$ & $6.1 \pm 0.3$ & $6.1 \pm 0.23$ \\
\hline
\end{tabular}

It may be noted that in the first analysis the measurement precision of the three halogenated compounds in the mixture is about a factor of 10 poorer than for benzene and toluene. This occurred because the FID has a lower response for this class of compounds and had to be operated at its limit of sensitivity. An electron capture detector would provide greater sensitivity for this class of compounds, but because its response characteristics are 
non-linear, it was not used. The notable improvement between the 1st and subsequent analyses (up to a factor of 20) was principally due to (a) increasing the GC sampling loop volume from 5 to $10 \mathrm{~mL}$, and (b) refinement of chromatographic conditions. The field test was conducted between the 1st and 2 nd analyses, during which time the cylinder pressure was reduced about 1000 psi (from 1950 to $900 \mathrm{psi}$ ); performance of the 2nd and 3 rd analyses resulted in a pressure loss of less than 100 psi. These data demonstrate, for the first time, the feasibility of preparing reliable, stable, multi-component organic gas mixtures in the very low ppb range.

The technology developed from the research being conducted in this program is currently being applied to the preparation and analysis of four cylinders each containing five organic species (chloroform, carbon tetrachloride, tetrachloroethylene, benzene, and vinyl chloride) in the low $(\leq 50)$ and intermediate (100-250) ppb range. These gas mixtures will be used as a primary standard for EPA in a test audit for monitoring emissions from hazardous waste incineration. Work will also be conducted in the coming year on the certification of the first NBS multi-component organic gas mixture SRM's containing benzene, toluene, chlorobenzene, and bromobenzene in a nitrogen matrix at nominal concentrations of 0.25 and $9.5 \mathrm{ppm}$, by mole.

\section{Reference}

[1] Schmidt, W. P. and Rook, H. L., "Preparation of Gas Cylinder Standards for the Measurement of Trace Levels of Benzene and Tetrachloroethylene," Analytical Chemistry (1983) 55, 290-294.

8. Pollutant Levels in Non-protected and Protected National Archival Storage Areas (16114)

E. E. Hughes, R. Myers

The Gas Metrology Group participated in an extensive survey of the quality of air within three federal buildings in which materials of great national and artistic value are stored and displayed. The study, coordinated by the Center for Building Technology, NBS, included the National Archives, the Madison Building of the Library of Congress, and the East Wing of the National Gallery of Art. The survey was conducted to determine the efficiency of the ventilating systems for removing deleterious air pollutants drawn into and circulated through the buildings.

In this study, measurements were made at eleven different locations inside the National Archives. Each location was supplied by a different ventilating system. Nitrogen oxides as $\mathrm{NO}_{x}\left(\mathrm{NO}_{2}+\mathrm{NO}\right)$ and sulfur dioxide levels were measured continuously for a minimum of 24 hours, but in most cases for at least 48 hours. Ozone measurements were measured for several short periods of time during each day.

The oxides-of-nitrogen analyzer and the sulfur dioxide analyzer were calibrated at the beginning and end of each sampling period and once every 
24 hours in between. Permeation tubes were used as calibrants for sulfur dioxide and nitrogen dioxide, and a gas mixture of nitric oxide in nitrogen was used to calibrate for nitric oxide. The ozone analyzer was calibrated at NBS. It was necessary to measure instrument temperature and barometric pressure during sampling times in order to calculate ozone levels. Although the requirements for measurement accuracy in this report were not stringent, the calibration scheme used was adequate to determine differences in the ventilating systems. The maximum uncertainty of the measurement of oxides of nitrogen did not exceed $\pm 5 \%$ relative, since the primary calibrant of the oxides of nitrogen used was a stable gas mixture. The observed variation of the sensitivity (signal/concentration) for both sulfur dioxide and nitrogen oxides analyzers calibrated with permeation tubes was about $\pm 10 \%$ relative over the more than two months course of the measurements. Because little or no ozone was measured at any of the sampling sites, it was only necessary to be sure that the ozone analyzer was functioning properly when sampling.

The results of the measurements of oxides of nitrogen showed, in general, a diurnal pattern following the morning and evening rush-hour traffic. For all but one sampling site at the National Archives, concentrations were less than the District of Columbia's ambient measurements. The measurements made at the Madison Building of the Library of Congress and at the East Wing of the National Gallery of Art were similar to the results obtained at the National Archives.

The significance of the sulfur dioxide measurements at the National Archives became obvious when compared to the results obtained at the Madison Building and the East Wing of the National Gallery. The sulfur dioxide concentration at these two sites did not exceed the lower detectable limits. At the time of the analyses, this limit was $0.0005 \mathrm{ppm}$ at the Madison Building and $0.001 \mathrm{ppm}$ at the East Wing of the National Gallery. The ambient concentrations measured by the District of Columbia were consistently above the lower detectable limits. No significant concentration of ozone was found at any of the locations sampled either in the National Archives or at the Madison Building and the East Wing of the National Gallery. Data supplied by the District of Columbia Air Monitoring Division indicated a maximum ambient concentration of $21 \mathrm{ppb}$ ozone during any sampling period. Because ozone in the ambient atmosphere is usually at low levels at the time of this study, no real conclusions could be drawn.

Figure 45 shows a typical plot of the concentration of oxides of nitrogen measured in one of the stack areas of the National Archives. Figure 46 shows a similar plot of measurements made at the Madison Building. 


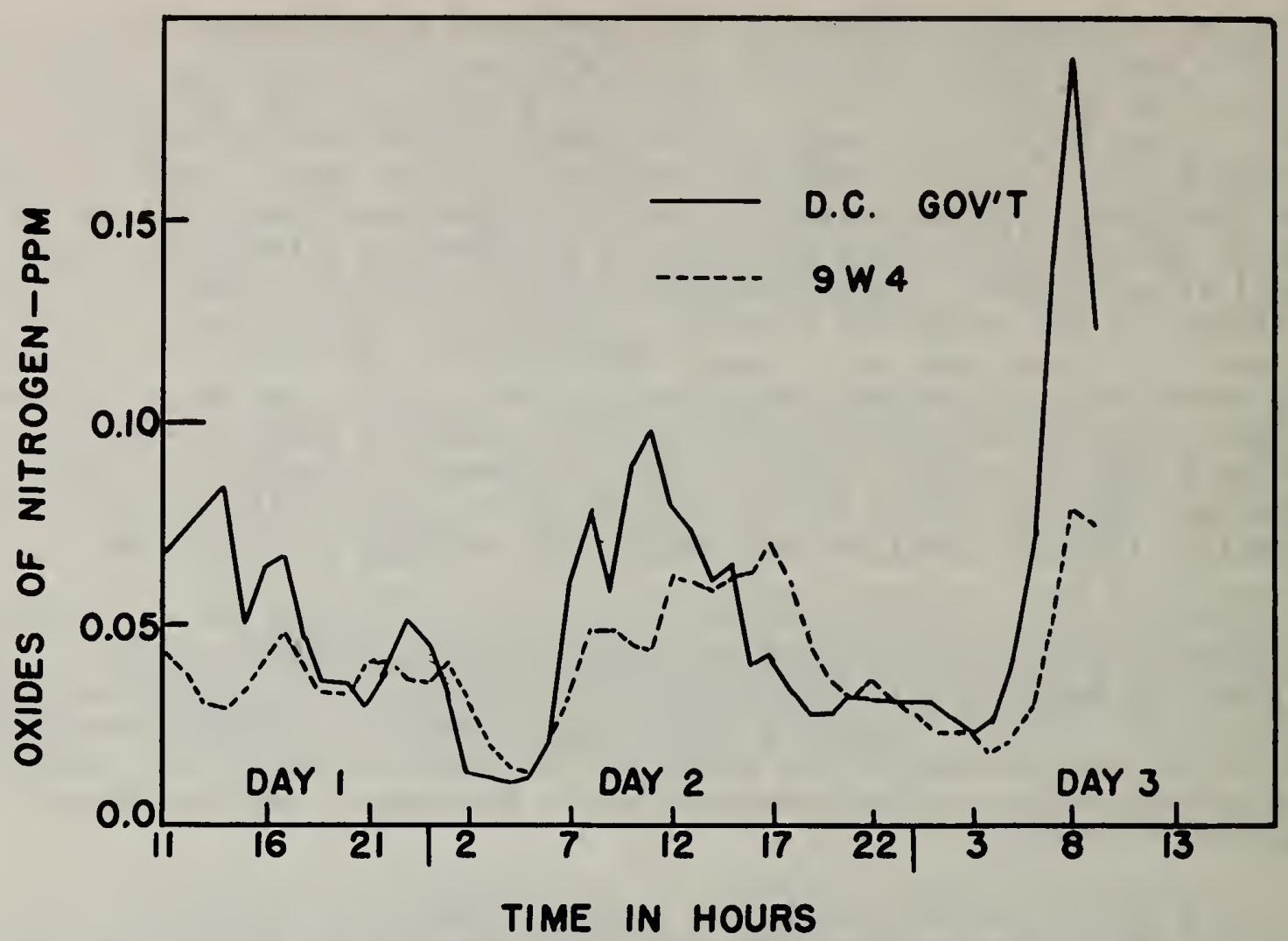

Figure 45. Concentration of oxides of nitrogen measured in stack area 9W4 of the National Archives compared to ambient outdoor measurements made by the DC Government.

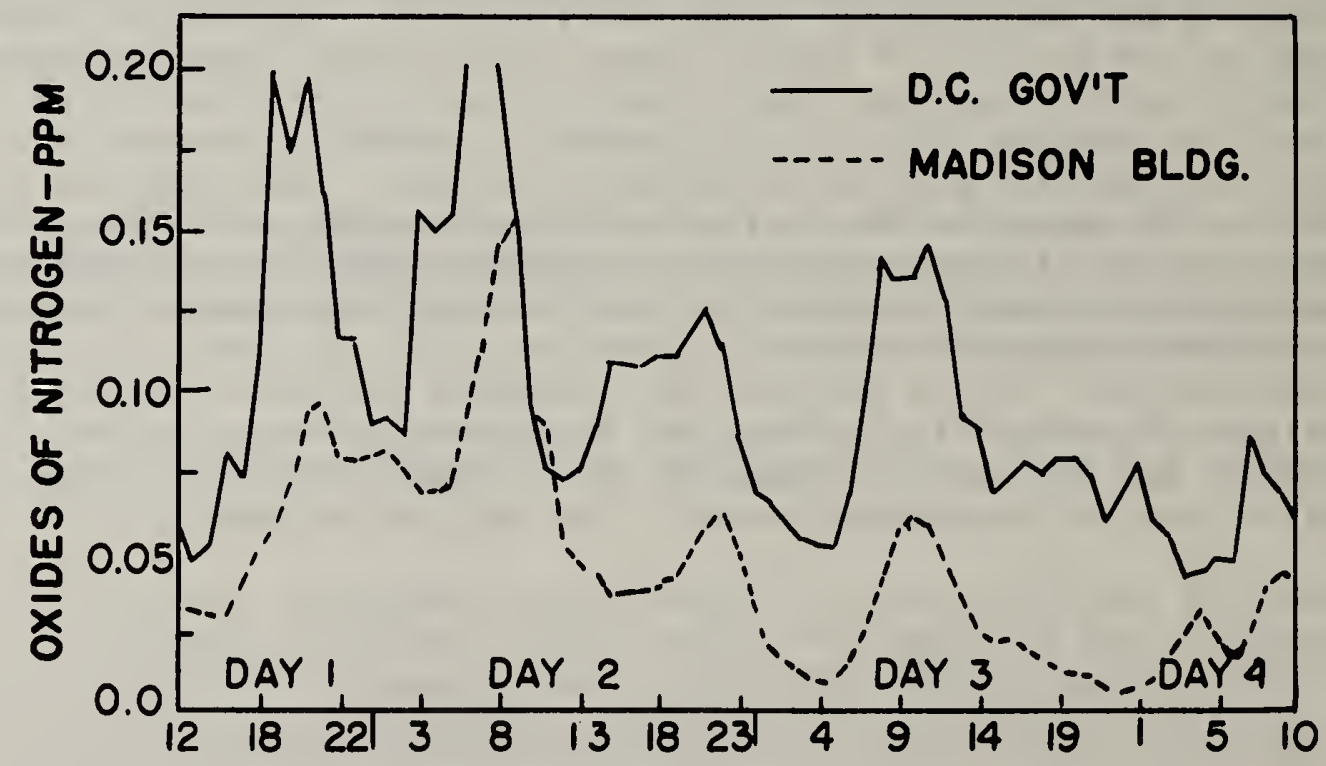

TIME IN HOURS

Figure 46. Concentration of oxides of nitrogen measured in the Madison Building of the Library of Congress compared to ambient outdoor measurements made by the DC Government. 
W. Dorko

The Naval Surface Weapons Center (NSWC) is evaluating lithium batteries as a source of power for certain military systems. The term "lithium battery" refers to a family of battery types, all containing lithium, but which may differ in size, type of electrolyte, internal design, and other construction materials. These batteries can be used aboard submarines, surface ships, aircraft, and in a navigational system called the Global Positioning System. A principal advantage of lithium batteries is their high and constant power output. Two areas of concern in the use of lithium batteries are the potential for evolution of toxic gases during discharge and the potential for battery explosion under certain adverse conditions. NBS was requested to examine the evolved gases generated by the batteries under various NSWC test conditions.

A number of lithium batteries were individually discharged in special chambers at NSWC under controlled but varying conditions. The gases evolved from each battery discharge were collected in stainless steel sample cylinders and transferred to NBS for analysis. The gaseous components of primary interest were those that exceeded one percent of the total gas mixture evolved during each discharge.

Due to the complexity of the samples, both gas chromatography and mass spectrometry were required for the analyses. Two different gas chromatographic columns were necessary to achieve the required separation. A separate mass spectrometric analysis was used to verify the chromatographic results, and to identify and quantify several additional constituents $\left(\mathrm{HCl}, \mathrm{Cl}_{2}\right.$, and $\mathrm{SOCl}_{2}$ ) that did not elute from the GC columns. A number of binary gas mixtures were prepared and used for calibration purposes, along with a multicomponent gas mixture containing $\mathrm{CO}_{2}, \mathrm{COS}, \mathrm{CS}_{2}, \mathrm{H}_{2} \mathrm{~S}$, and $\mathrm{SO}_{2}$ in nitrogen.

The results of a series of analyses are summarized in Table 39. A number of the gases that were identified and quantified $\left(\mathrm{CO}, \mathrm{H}_{2} \mathrm{~S}, \mathrm{HCl}, \mathrm{COS}, \mathrm{SO}_{2}, \mathrm{CS}_{2}\right.$, $\mathrm{SOCl}_{2}$ ) possess toxic and/or corrosive characteristics. The results will be used by NWSC to assess appropriate precautions for personnel protection during battery use. These and further studies will be undertaken to improve the understanding of the chemistry associated with the production of the observed gases. 
ํํํ

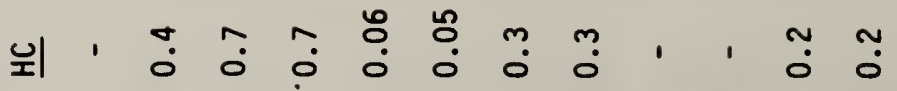

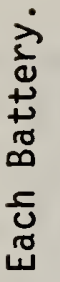

흔

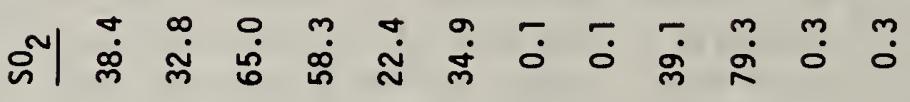

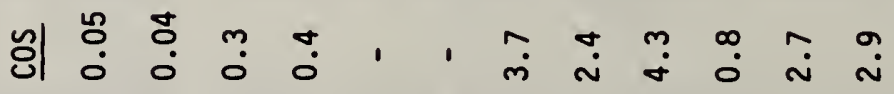

4

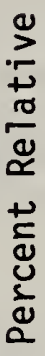

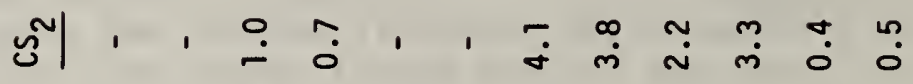

心

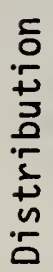

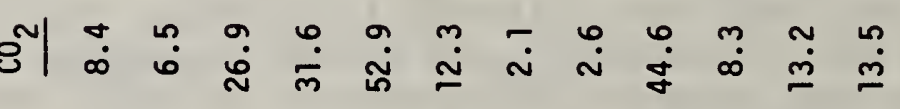

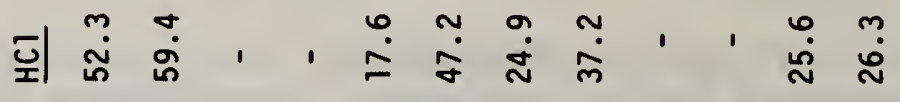

$\frac{\infty}{2}$

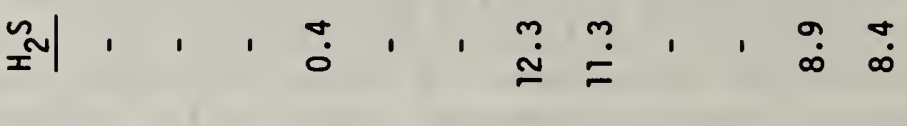

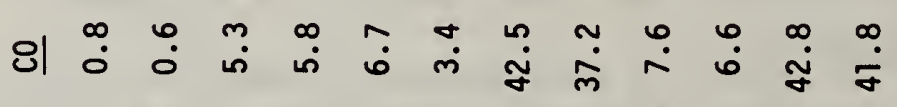

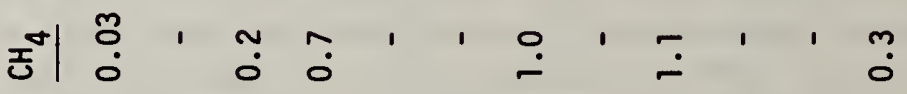

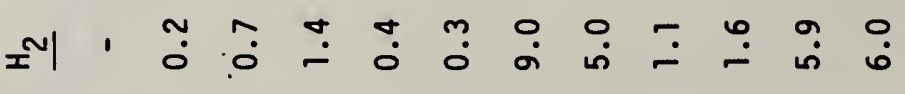

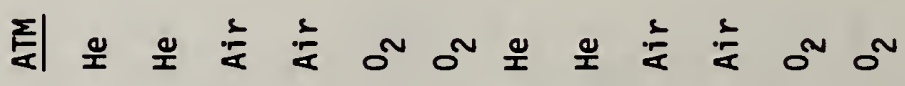

$\frac{\infty}{\circ}$

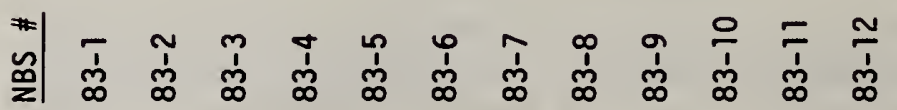


10. Mathematical Chemistry: Design and Evaluation of the Analytical Measurement Process, Detection Limits, and Multidimensional Data (15330)

Lloyd A. Currie, George A. Klouda

The program to apply advanced statistical and mathematical tools to the analytical measurement process (AMP) has continued during the past year with special emphasis on: (a) multiparameter data acquisition and multiple quality control charts for environmental radiocarbon measurements; (b) development of multidimensional (multispecies) AMP design criteria taking into account the effects of measurement imprecision and collinearity; and (c) the assessment of the state-of-the-art and the development of improved techniques for evaluating detection limits, particularly with regard to multidimensional measurements and regulatory issues. The overall objective of these activities is improved chemical measurement, using computational and statistical tools (plus physicochemical knowledge) to improve the design, evaluation and reliability of analytical measurements--especially multidimensional measurements (and "chemical fingerprints") and those having potential impact on public perceptions or policy.

The first activity noted above relates to the continued development of our unique ultrasensitive ${ }^{14} \mathrm{C}$ counting system, in which we are monitoring on-line a very large number of parameters for each counting event. The approach taken has not only led to a highly reliable system for very difficult measurements, but is providing an important case study of multiparameter quality control and new information on the behavior of background events in low-level counting systems. An interesting by-product is the generation of large data sets of (presumably) random events which are of interest to the statistical community for "physical" random number generation [1].

The importance of multidimensional AMP design has grown with the evolution of multielement analysis and "chemical fingerprinting" (or pattern recognition) techniques. Efforts in this area are of increasing significance, however, because of the enormous growth of linked spectroscopic and chromatographic techniques and because of the growing use of multispecies measurements to identify sources of environmental pollutants, "acid rain," and climaticallyactive species (as elemental carbon). From our work in FY82 on the mathematical evaluation of multivariate Receptor Modeling (RM) data [2], it became clear that a unified approach to the design and evaluation of RM experiments was called for, with special emphasis on what to measure, precision and sensitivity requirements, and the effects of collinearity. As a contribution to a meeting at NBS in May, 1983 concerning multispecies measurement, receptor modeling and acid rain, we illustrated the application of such design criteria and measurement limitations to the resolution of sources of Washington, DC aerosol. As shown in figures 47 and 48 , the decision of what and how to measure, together with collinearity profoundly influences the ability to discrimate between particle sources, such as coal and soil [3]. 


\section{Collinearity}

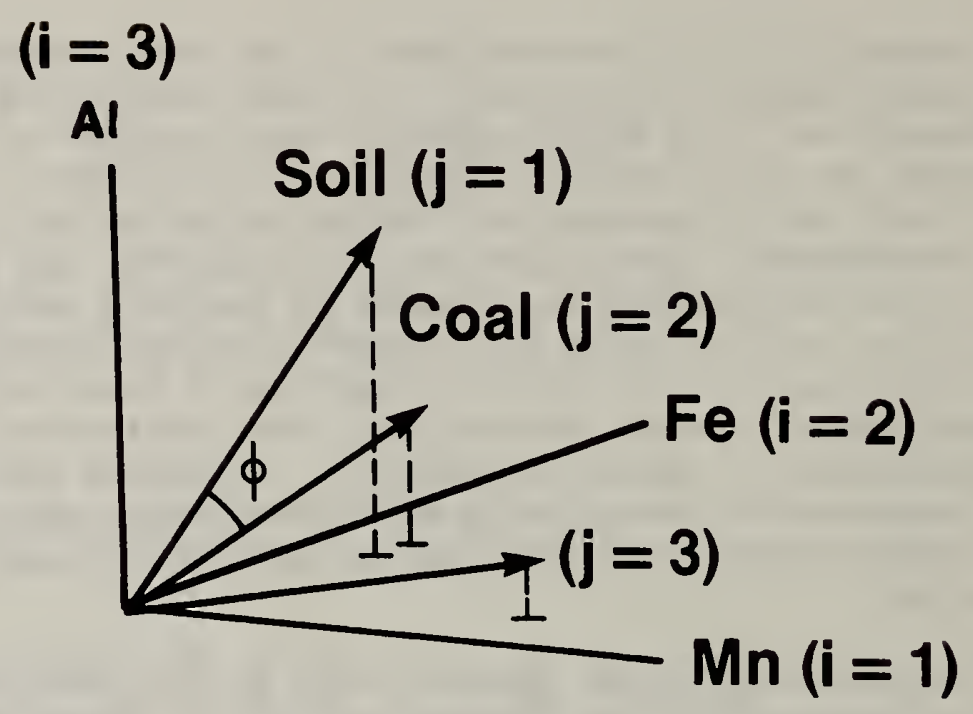

$(S / N)_{i} \approx \sin \phi \sqrt{\sum_{i}(S / N)_{i j}^{2}}$

Figure 47. The effect of collinearity (similar source profiles or "chemical spectra") on the precision of analysis. The signal/noise for a given source estimate (e.g., $j=1$, soil) is by definition the reciprocal of its relative standard deviation. For a 1-component system (or orthogonal multicomponent system) the length of this $(\mathrm{S} / \mathrm{N})_{j}$ vector is simply the vector sum of the individual contributions for each chemical measurement, $(S / N)_{i j}$. (This follows directly from conventional error-propagation formulas). For two non-orthogonal components, the overall $\mathrm{S} / \mathrm{N}$ "length" is decreased by the measure and collinearity, sin $\phi$. [Really, better termed the "measure of orthogonality," since sin $\phi \rightarrow 1$ when orthogonal.] The simple expression [Eq (1) in Fig. (47)] must be viewed as an approximation for a many component system because of the complicating effects of multicollinearity. 


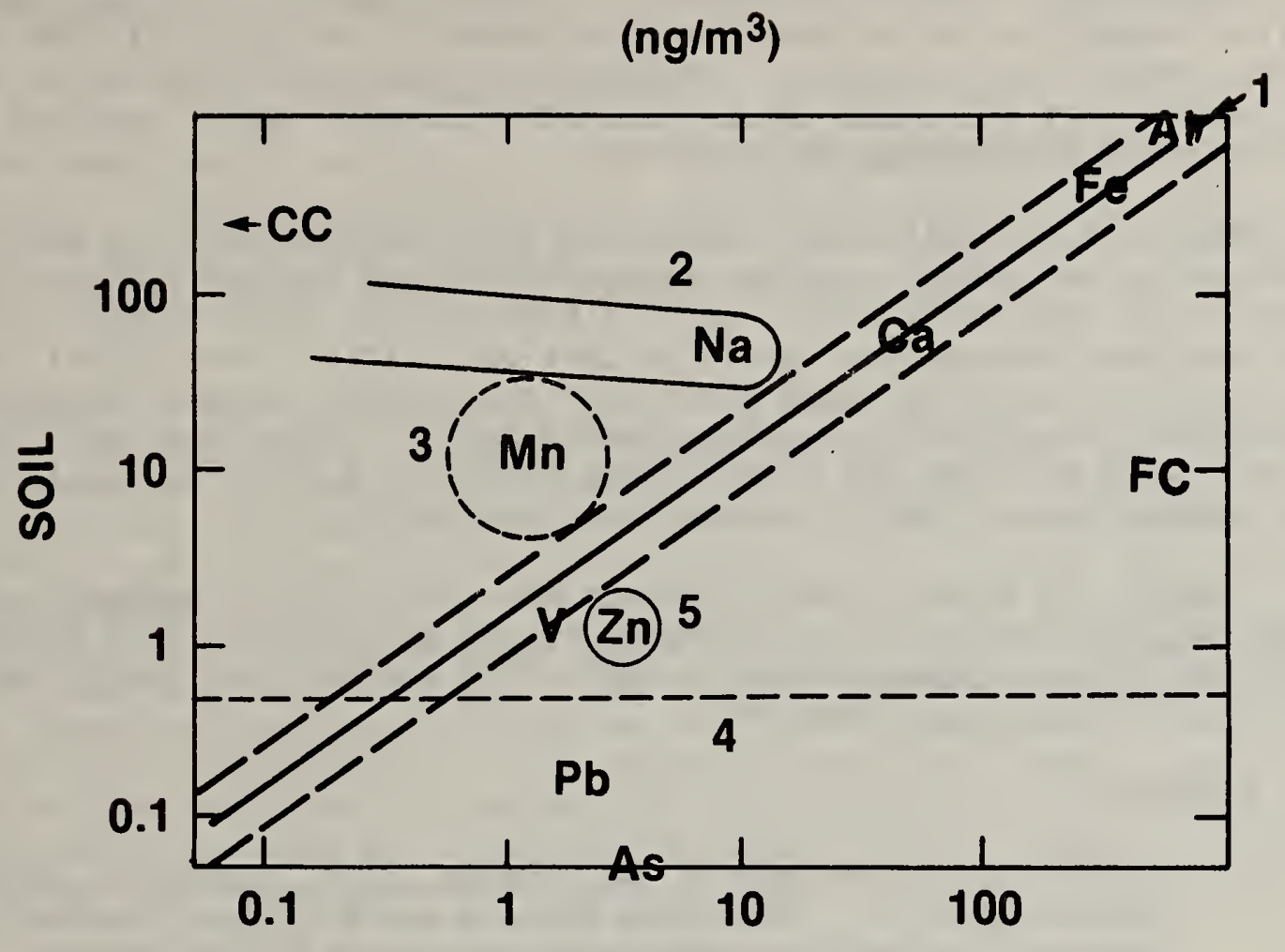

COAL

\section{Limitations}

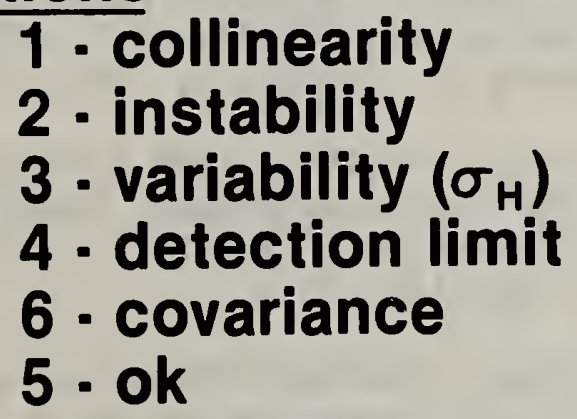

Figure 48. Correlation plot for trace element patterns of soil vs coal aerosol sources. The solid line, which would indicate complete correlation, shows that $\mathrm{Ca}, \mathrm{Fe}$, and $\mathrm{Al}$ lead to collinearity. Species well removed from the line (As, $\mathrm{Pb}$, contemporary [CC], and fossil carbon $[F C], \ldots$ ) are important for source resolution. Imperfect characteristics (numbered conditions) tend to limit resolution or reliability. 
The third area of endeavor relates to the general issue of detection in multidimensional chemical measurements, and the specific issue of the "lower limit of detection" of effluent and environmental radioactivity for the Nuclear Regulatory Commission. These topics have been addressed at two special ACS symposia, an NBS Symposium on Regulatory Issues, and at meetings with NRC officials and contracting laboratories.

Assessment of detection from the perspective of Regulatory Limits, and exposure to the actual practices among utility and (nuclear) contractor laboratories, have shown that widely varying terminology, formulations, reporting practices, and assumptions remain in this basic area of analytical chemistry. Having completed a literature survey for the NRC [5], we are gathering practical information from facility and laboratory visits. During the next fiscal year, we shall work with the NRC to help them extablish a uniform position regarding LLDS, and produce a "NUREG" document on this topic.

Finally, in order to contribute to the furthering of mathematical-chemical education, Dr. Currie will participate on the faculty of a NATO Advanced Study Institute (ASI) on Chemometrics in Sept. 1983 treating the subject of Data Analysis in Analytical Chemistry.

Reference

[1] Currie, L.A., Gerlach, R. W., KTouda, G. A., Ruegg, F. C. and Tompkins, G. B., "Miniature Signals and Miniature Counters: Accuracy Assurance via Microprocessors and Multiparameter Control Techniques," Radiocarbon, $1983 \underline{25} 553$.

[2] Gerlach, R. W., Currie, L. A., and Lewis, C. W., "Review of the Quail Roost II Receptor Model Simulation Exercise," in Proc. APCA Specialty Conference on Receptor Models Applied to Contemporary Air Pollution, 1983 (in press).

[3] $L$.A. Currie, "Design of the Multidimensional Measurement Process for Optional Receptor Modeling Source Resolution and Information Content: The Effect of Collinearity", talk given at NBS in May 1983.

[4] L. A. Currie, "The Many Dimensions of Detection in Chemical Analysis", ACS Symposium Series on Chemometric Techniques in Pesticide Analysis (1983).

[5] L. A. Currie, "Survey of 60 Years of the Detection Limit Literature (1923-1982)," NRC Report, Jan. 1983. 
11. Origins of Carbonaceous Gases and Aerosols: Survey of Recent Measurement Advances and Results (15330)

Lloyd A. Currie and George A. Klouda

The development and application of advanced analytical techniques for the assessment of natural and anthropogenic sources of carbonaceous gases and aerosols has continued during the past year and new data on ${ }^{14} \mathrm{C}$ in atmospheric methane, carbonaceous particles and accelerator techniques have been obtained $[1,2]$.

Accelerator Mass Spectrometry (AMS) continues to be one of the most exciting new techniques in fields ranging from oceanography to astrophysics to environmental chemistry. The tandem accelerator at the University of Arizona, Tucson has become a vital tool for our studies of atmospheric carbon. Experiments demonstrated that we could measure ${ }^{14} \mathrm{C}$ in $100-500 \mu \mathrm{g}-\mathrm{C}$ of atmospheric samples with an imprecision of 22 percent (RSD). Evaluation of the precision and accuracy was accomplished by using three radiocarbon dating standards and two samples of known age. Extensive measurements of blanks and carbon from atmospheric methane and particles also took place. One of the most important consequences of this improved sensitivity (small sample size) is that we now can determine the biogenic carbon contribution to individual particle size fractions and individual chemical forms--as shown in Table 40, which summarizes our recent AMS results.

Table 40. AMS Measurements of Standards and Blanks. ${ }^{2}$

\begin{tabular}{|c|c|c|c|c|}
\hline Sample & ia] Mass $-C(\mu \mathrm{g})$ & $\begin{array}{r}\% M_{0} \\
{[ \pm \quad 1 \sigma-P} \\
\end{array}$ & $\begin{array}{l}\text { Oder } \\
\text { Pois }\end{array}$ & $\begin{array}{l}\mathrm{rn} \\
\text { sson] }\end{array}$ \\
\hline${ }^{14} \mathrm{C}$ Dating Std (SRM 4990B) & 500 & [Defined & as & $1.05]$ \\
\hline New ${ }^{14} \mathrm{C}$ Std (RM49) & 500 & 1.24 & \pm & 0.02 \\
\hline ANU-Sucrose & 500 & 1.49 & \pm & 0.02 \\
\hline USGS-Wood" & 500 & 0.42 & \pm & 0.02 \\
\hline Arizona-1890 wood & $<300$ & 0.96 & \pm & 0.03 \\
\hline $\begin{array}{l}\text { Our blanks with chem. } \\
\text { (coal, two graphites) }\end{array}$ & $300-500$ & 0.098 & \pm & 0.01 \\
\hline $\begin{array}{l}\text { Arizona, } \mathrm{CaCO}_{3} \mathrm{Blk} \\
\text { NS-Graphite (direct to Fe-carbide) }\end{array}$ & $\begin{array}{r}2000 \\
500\end{array}$ & 0.005 & \pm 2 & 20.02 \\
\hline
\end{tabular}


Besides the measurements by AMS, we have continued collaborative efforts on ${ }^{14} \mathrm{C}$ in particles from Norway [3], the Yukon (both cases of significant winter-time wood-burning pollution, confirmed), and Colorado. The lastmentioned work is in collaboration with Kent Voorhees, supported by NSF, and it is directed at ${ }^{14} \mathrm{C}$ and pyrolysis-mass spectrometry of particles from vegetative emmissions (remote forests), auto emissions (Eisenhower tunnel experiment), and forest fires. This combination of techniques is continuing to provide strong confirmation of suspected sources of carbonaceous particles in the Rocky Mountain area [4].

Measurements on two urban particulate SRMs $(1648,1649)$ have taken place during the past year on different chemical fractions, in cooperation with Division 552. Results are given in Table 41.

New developments in our studies of carbon chemistry have focused on identifying and separating important chemical species from particles (e.g., elemental carbon); in miniaturizing of separation and AMS preparation apparatus for improved yields and blanks; and in developing methods to assay $\mathrm{CO}_{3}^{-}$in particles via thermogravimetry and microacidification $\left(\mathrm{H}_{3} \mathrm{PO}_{4}\right)$ followed by PVT measurement.

Research recently started includes improved isotope separator-AMS linkage $\left(\right.$ for ${ }^{14} \mathrm{C}$ ); a new process of AMS sample preparation based on closed-system $\mathrm{CO}_{2}$ reduction followed by $\mathrm{CO}$ "cracking"; and the evaluation of ${ }^{13} \mathrm{C}$ as a supplementary tool to determine the origin of atmospheric carbon.

Finally our high sensitivity gas counting system is undergoing a major evolutionary phase, with the addition of (a) a new data acquisition computer with $5 \mathrm{MB}$ storage to permit recording of the full individual pulse time record, and (b) a transient digitizer, which has already given us considerable insight into the nature (waveform) of the individual pulses.

\section{Reference}

[1] Currie, L. A., Klouda, G. A., Continetti, R. E., Kaplan, I. R., Wang, W. W., Dzubay, J. D., and Stevens, R. K., "Of the Origin of Carbonaceous Particles in American Cities: Results of Radiocarbon 'Dating' and Chemical Characterization." Radiocarbon 25, p. 603 (1983).

[2] L. A. Currie, "Application of Tandem AMS to Measurement of Submilligram Amounts of Carbon in Atmospheric Gases and Particles."

[3] Ramsde11, R., Schjoldager, J., Currie, L. A., Moller, M., Hassendoff, E., Klouda, G. A., and Afheim, I., "Air Pollution from Wood Combustion: Ambient Air Measurements in Elverum, Norway," (Submitted to Environmental Science \& Technology, 1983).

[4] Voorhees, K. J., Schulz, W. D., Currie, L. A., and Klouda, G. A., A., "An Investigation of the Insoluble Carbonaceous Material in Airborne Particulates from Vehicular Emissions," (Submitted to Analytical Chemistry 1983). 
Table 41. Report on the Urban Dust Standards, Carbon Results.

\begin{tabular}{|c|c|c|c|c|}
\hline SRM & $\begin{array}{l}\text { Chemical } \\
\text { Fraction. }\end{array}$ & Percent Carbon $(S E, n)$ & $\begin{array}{l}f_{M}^{a}(S E)^{b} \\
\left(X^{2} / d f, d f\right)\end{array}$ & $f_{C}^{c}(S E)$ \\
\hline & & Extraction Procedure \#1 & & \\
\hline 1648 & Total & $\begin{array}{c}13.1 \\
(0.3,6)\end{array}$ & $\begin{array}{c}60(3) \\
(0.66,2)\end{array}$ & $43(2)$ \\
\hline 1648 & PAH & $\begin{array}{l}80 \\
(2,3)\end{array}$ & $\begin{array}{c}22(4) \\
(3.45,4)\end{array}$ & $16(3)$ \\
\hline 1649 & Total & $\begin{array}{c}17.5 \\
(0.4,6)\end{array}$ & $\begin{array}{c}61(4) \\
(0.89,2)\end{array}$ & $45(3)$ \\
\hline 1649 & PAH & $\begin{array}{c}87 \\
(2,3)\end{array}$ & $\begin{array}{c}17(4) \\
(1.88,2)\end{array}$ & $13(3)$ \\
\hline 1649 & Nonextractable & $\begin{array}{l}14 \\
(1,4)\end{array}$ & $\begin{array}{c}55(4) \\
(0.00,1)\end{array}$ & $41(3)$ \\
\hline 1649 & Benz/Meth & $\begin{array}{c}63 \\
(3,3)\end{array}$ & $\begin{array}{c}46(6) \\
(0.74,1)\end{array}$ & $34(4)$ \\
\hline
\end{tabular}

Extraction Procedure \#2

$\begin{array}{lcccc}1649 & \text { Hexane Sol. } & 78 & 17(3) & 13(2) \\ 1649 & \text { Methanol Sol. } & (0.7,2) & (0.02,1) & \\ & & (11,2) & 58(4) & 43(3) \\ 1649 & \text { Benzene Sol. } & 65 & (2,50,2) & \\ & & (0.7,2) & (0.12,1) & 26(3) \\ 1649 & \text { Water Sol. } & 22 & 54(4) & 40(3) \\ & & (8,2) & (11.23,1) & \end{array}$

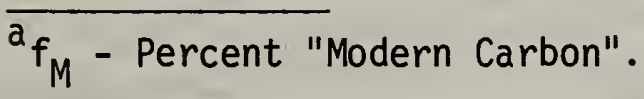

${ }^{b} S E$ - The standard error, SE, is the Poisson standard deviation of the mean.

${ }^{c_{f_{C}}}$ - Percent contemporary carbon for SRM 1648 is $f_{C}=f_{M} / 1.40[1975$ vegetation] and for SRM $1649 \mathrm{f}_{C}=\mathrm{f}_{\mathrm{M}} / 1.36$ [1977 vegetation]. 


\section{Detection of $\mathrm{HCl}$ at the Sub-Parts-Per-Billion Level Using Tunable}

Diode Laser Absorption (15330)

\section{Alan Fried and Robert Sams (550)}

In our 1982 report, we presented results of a laboratory study for detecting the important atmospheric molecule, $\mathrm{HCl}$, using a photoacoustic detection system. The present investigation is a continuation of this study as part of our long term effort to develop and characterize sensitive and selective laser-based techniques for the measurement of such molecules.

Measurement of $\mathrm{HCl}$ concentrations has received a great deal of attention in stratospheric studies because of its importance in the stratospheric $\mathrm{O}_{3}$ destruction cycle. Despite this large data set, the altitude profiles collectively derived from such measurements exhibit discrepancies, and thus further independent measurements in the critical region between 20 and $40 \mathrm{~km}$ are essential. The purpose of the present study is to demonstrate that the sensitive and selective technique of tunable diode laser absorption can be successfully employed for detection of $\mathrm{HCl}$ at the sub-parts-per-billion levels found in the stratosphere. Since the pressure in this critical region ranges between 2 and 41 torr, photoacoustic techniques are not quite as sensitive.

In the present investigation, we report the results of a laboratory study for detection of $\mathrm{HCl}$ in this pressure regime using the NBS tunable diode laser spectrometer coupled with a multipass White cell. The experimental apparatus is illustrated schematically in figure 49 . In contrast to many prototype laboratory studies, this work was carried out near the concentration range of interest. This is important, since highly polar molecules such as $\mathrm{HCl}$ present

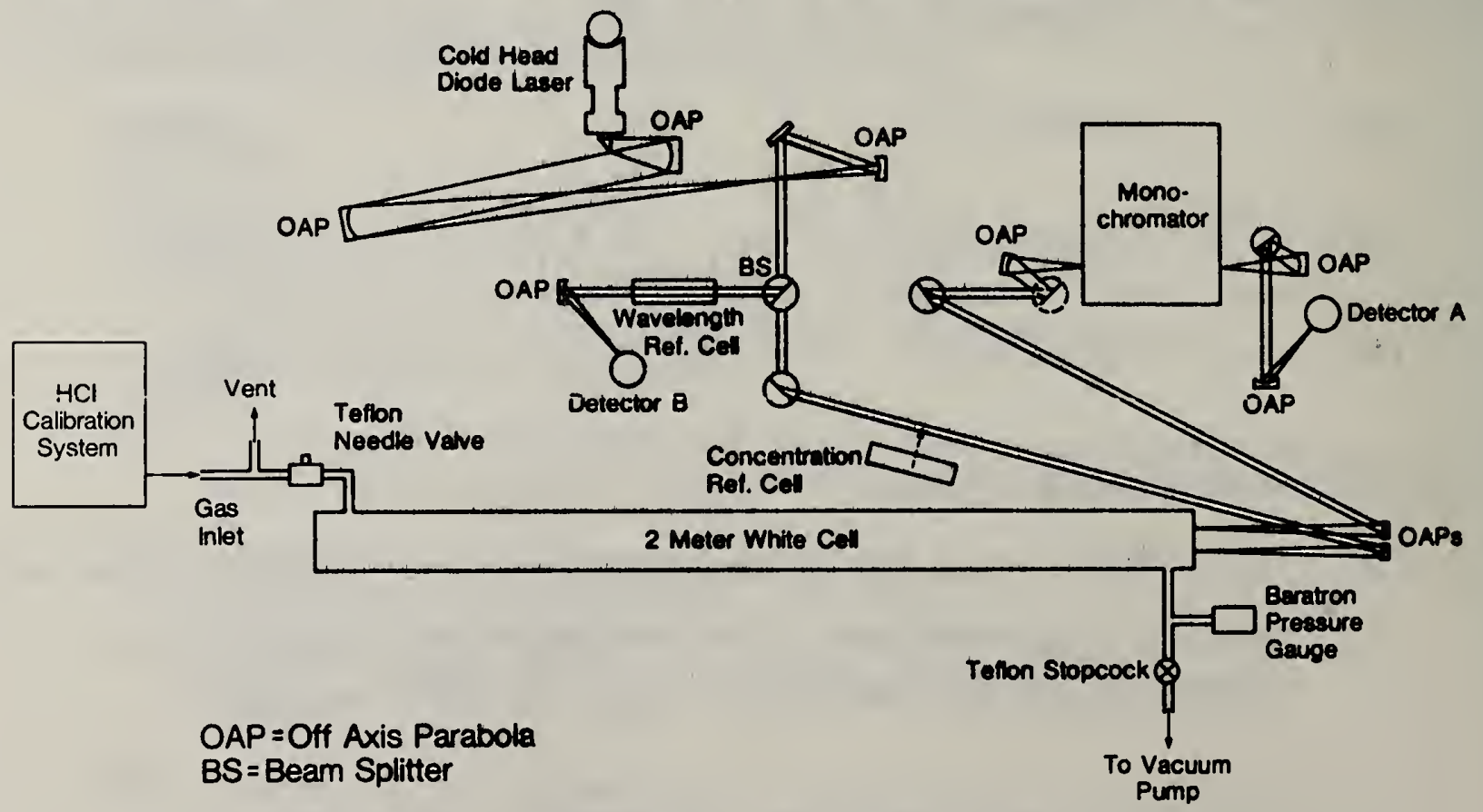

Figure 49. Schematic of optical system. 


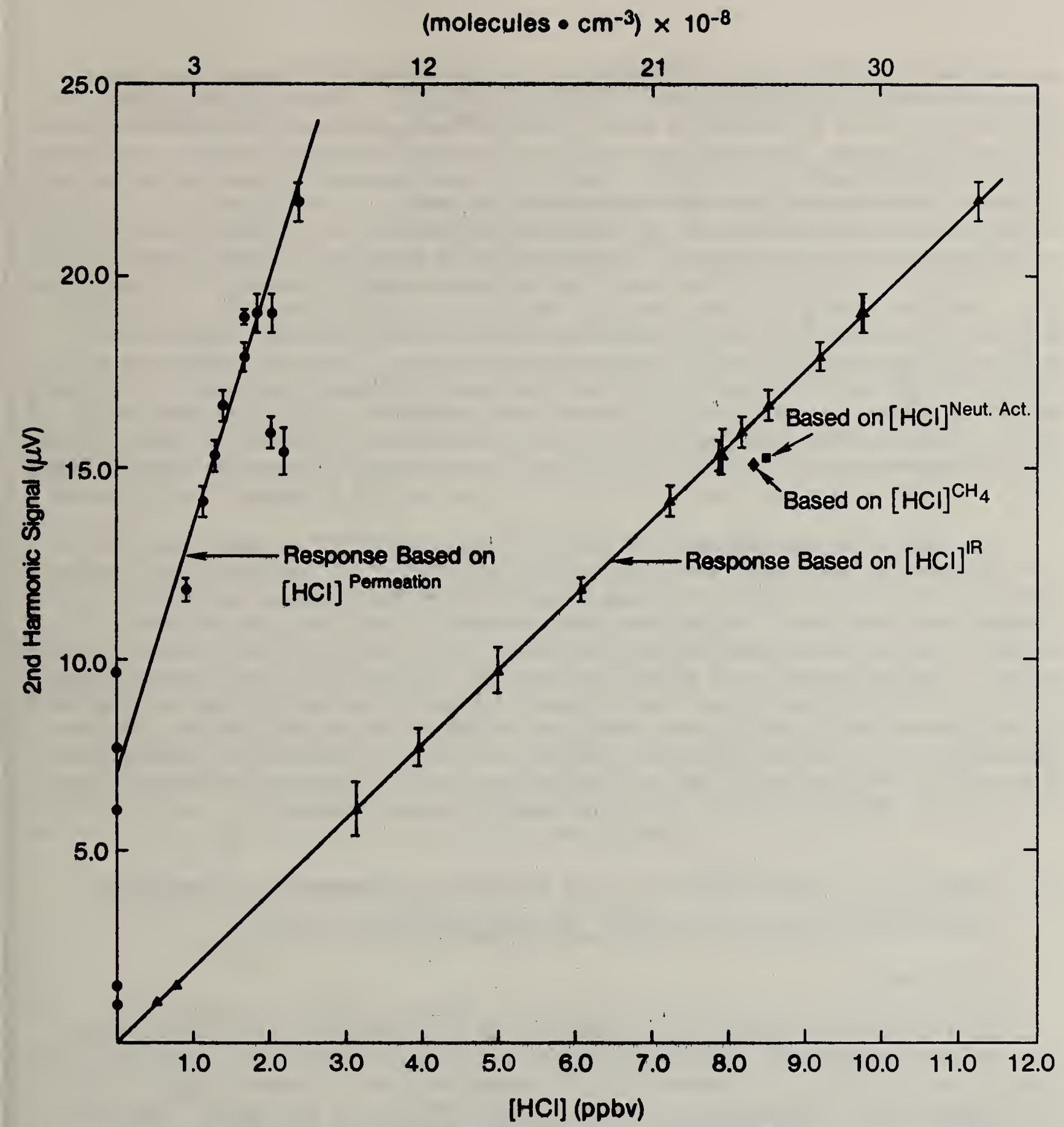

Figure 50. The second harmonic response as a function of the volume mixing ratio for $\mathrm{HCl}$, in normal isotopic abundance, at 8-9 torr based upon four independent determinations. The points $[\mathrm{HCl}]^{\mathrm{IR}}$ and $[\mathrm{HCl}]^{\mathrm{CH}_{4}}$ are based upon ir ratioing techniques using $\mathrm{HCl}$ and $\mathrm{CH}_{4}$ respectively, as the reference gas. The point $[\mathrm{HCl}]^{\text {Neut. Act. }}$ is based upon the quantitative trapping of $\mathrm{HCl}$ on nylon filters followed by neutron activation analysis of $\mathrm{Cl}$. The $[\mathrm{HCl}]^{\text {permeation }}$ response curve is based on a commerical permeation calibration system. The uncertainties shown are the measurement standard deviations. The uncertainties in the concentration determinations are not shown but are discussed in the text. For readers more familiar with other concentration units, $1 \mathrm{ppb}=1627 \mathrm{ng} / \mathrm{m}^{3}$ (STP). 
measurement problems associated with losses and/or adsorption on instrument surfaces when the concentration is in the ppb range. Trace concentrations of $\mathrm{HCl}$ in the 0.8 to $16 \mathrm{ppb}$ range were generated using a commerical permeation calibration system and were continually drawn through the White cell at a flow rate of approximately $0.5 \mathrm{~L} / \mathrm{min}$. The absolute $\mathrm{HCl}$ concentration in the White cell was determined using four independent techniques: the calculated calibration system output based upon the measured permeation and diluent flow rates; ratioing the second derivative response from the White cell to that obtained by alternately placing cells containing reference concentrations of $\mathrm{HCl}$ or of $\mathrm{CH}_{4}$, into the beam path; and finally, from measurements obtained by diverting the calibration output flow through ultrapure nylon filters and subsequently measuring the quantitatively trapped acid-chloride by neutron activation. The results based upon all four system calibration techniques are shown in figure 50. From a detailed analysis of the various measurement uncertainties, the latter three calibration techniques are in statistical agreement. This agreement firmly establishes the $\mathrm{HCl}$ White cell concentrations used throughout this work. Attempts are being made to find the bias in the permeation calibration.

Using a $39.4 \mathrm{~m}$ pathlength and the $\mathrm{R}(1)$ line of $\mathrm{H}^{35} \mathrm{Cl}$ at $2925.897 \mathrm{~cm}^{-1}$, we have achieved a detection limit $(S / N=1)$ in the 0.2 to $0.3 \mathrm{ppb}$ range at a cel1 pressure of about 9 torr. We have also investigated the dependence of pressure and modulation depth upon the detection limit. We have further investigated possible interferences from $\mathrm{CH}_{4}, \mathrm{NO}_{2}$, and $\mathrm{H}_{2} \mathrm{O}$. At their respective ambient concentrations, a 11 three constituents do not present any interference when small modulation depths are employed. Finally, we anticipate that modest system improvements would lower the limit of detection to well below $0.1 \mathrm{ppb}$. Such sensitivity is more than sufficient for detecting $\mathrm{HCl}$ in the stratosphere. The results of this investigation, therefore, are of extreme importance to researchers carrying out in situ stratospheric measurements.

\section{Completion of Measurements of the Temperature Dependence of the Ozone} Absorption Cross-section in the Vacuum Ultraviolet (15330)

A. M. Bass

We are now approaching the completion of this project for the determination of the temperature dependence of the ultraviolet absorption cross-sections. These cross-sections are needed for the interpretation of observational data and for modeling calculations of ozone concentrations in the earth's atmosphere. The only previous comprehensive study of the temperature dependence of the ozone absorption cross-sections was done in 1953 by Vigroux [1]. The older data were recorded under less favorable experimental conditions and over irregularly spaced wavelength intervals. We have been able to take advantage of the technological developments of the past two decades to make measurements of much greater photometric precision on a better characterized sample of ozone. We use a computer to control the data collection and reduction and have been able to make measurements at much smaller wavelength intervals and thus describe the spectrum more completely. 
The objective of this study is to determine the ozone cross-section values over the wavelength range 230 to $350 \mathrm{~nm}$, and over the temperature range 200 to $300 \mathrm{~K}$. We have now completed these measurements for the specified wavelength range $(230-350 \mathrm{~nm})$ using a spectral resolution of about $0.025 \mathrm{~nm}$ in steps of $0.05 \mathrm{~nm}$. The measurements have been completed for four temperatures: $295 \mathrm{~K}, 243 \mathrm{~K}, 228 \mathrm{~K}, 193 \mathrm{~K}$. These results are being analyzed for statistical consistency by Dr. R. Paur of the EPA, and a final data set will be ready for publication by the end of 1983. Preparation of the results of these measurements for publication will complete the work planned.

In the measurement procedure used for these data, all cross-sections are referenced to the value of the cross-section at $253.7 \mathrm{~nm}$. We have now completed detailed measurements of the temperature variation from $193 \mathrm{~K}$ to $295 \mathrm{~K}$ of the cross-section at $253.7 \mathrm{~nm}$. These measurements indicate that, over this temperature interval the cross-section increases by about $11 / 2$ percent. This result contradicts the results reported earlier [1] which indicated a decrease in the cross-section of about 3 percent.

We have proposed to follow this study by a new measurement of the absolute cross-section of ozone at $253.7 \mathrm{~nm}$. The literature value for this cross-section has an uncertainty of about 2 percent, which is the principal source of error in our ozone concentration measurements. We plan to collaborate with Prof. Mauersberger of the University of Minnesota in a joint project to redetermine the cross-section. By combining his techniques of mass spectrometry and accurate pressure measurement with our high precision photometry, we believe we can redetermine the cross-section to an accuracy of approximately 0.5 percent. Since we have made the measurement of the entire ozone spectrum relative to the value at $253.7 \mathrm{~nm}$, we will then be able to normalize the entire data set to the new value and thus achieve the same over all accuracy for the cross-section measurement.

\section{Reference}

$$
\text { [1] Vigroux, E., Ann Physique } \underline{8}, 709 \text { (1953). }
$$

14. Reference Ultraviolet Photometer for Ozone Calibration and Assay (15330)
A. M. Bass

We have discussed in previous reports the rationale for the development of a reference photometer for the calibration of ozone monitors [1]. To summarize briefly, in 1979 the US Environmental Protection Agency promulgated a new calibration procedure for ozone based on ultraviolet abosrption photometry. In response to a request for assistance by the EPA, we undertook to design and construct a new photometer that would serve as a primary standard for the EPA laboratories, and that would also replace the existing NBS standard ultraviolet ozone photometer.

This development, which was done in collaboration with Dr. R. Paur of the $E P A$, has been completed and the instruments that have been constructed have met or exceeded the design criteria in all respects. 
Three photometers have been fully completed. One instrument remains at NBS as our new standard; the second instrument was delivered to the EPA in February, 1983; the third photometer is now being compared with the NBS standard prior to delivery to the EPA. Completion of photometer No. 4 has been delayed while waiting for delivery of components, but we expect to deliver the instrument to the EPA before October 1, 1983.

The new standard is a double-beam photometer with an effective pathlength of about $172 \mathrm{~cm}$. Absorption of the $253.7 \mathrm{~nm}$ line of the mercury spectrum by ozone is determined by means of a very sensitive detection system. From the measured absorbance and known pathlengths, application of the Beer-Lambert law yields the ozone concentration of the sample. The temperature and pressure of the sample are continuously recorded and corrections for these parameters are made automatically. The entire measurement process is controlled by a desk-top computer, and the data are automatically reduced and stored on magnetic tape.

Detailed testing and comparison of the new photometers has confirmed the high precision of the instruments. Over the range of concentrations from 100 to $1000 \mathrm{ppb}$, the precision of the concentration determination is better than \pm 0.3 percent. At concentrations below $100 \mathrm{ppb}$ the precision is $\pm 0.2 \mathrm{ppb}$. The accuracy of the measurement is 1 imited to approximately \pm 2 percent principally because of the uncertainty in the value of the ozone absorption cross-section at $253.7 \mathrm{~nm}[2]$.

In the period May 15 to June 25, the NBS photometer was used at NBS and the launch site as the standard for calibration of 19 instruments that will fly on the NASA Balloon Ozone Intercomparison Campaign (BOIC). In this NASA project, a large number of ozone monitors will be carried aloft on two balloons in order to make simultaneous measurements in the stratosphere. These measurements will provide a basis for intercomparison of data collected over a long period of time by these instruments. This comparison provided valuable guidance to the experimenters as to the flight-readiness of their instruments, and will serve as a basis for the accurate reduction of the data acquired in flight.

References

[1] NBSIR 82-2620 CAC Annual Report (1982).

[2] Vigroux, E., Ann. Physique 8,709 (1953). 


\section{Completion of the Respirable Quartz SRM (15330)}

R. L. McKenzie

There has been a long-standing need for an SRM for the calibration and performance verification of techniques (primarily $x$-ray scattering used for the identification and quantification of $\alpha-$ Quartz in the respirable size range. We have completed the developmental and certification research on a respirable quartz material that will be released for sale through the Office of Standard Reference Materials as an SRM.

The primary properties of concern for the SRM are the size distribution (respirable, i.e. in the size range of 0.5 to $5 \mu \mathrm{m}$ ) and the purity of the crystalline phase ( $\alpha$-quartz). A commercially-available material (min-U-Sil 5) was selected as a candidate material. The size distribution of the powder was determined using sedimentation and aerodynamic size measurement techniques. Sedimentation size distribution analys is indicated that 95 percent of the mass of the material had an equivalent spherical dimater (E.S.D.) less than $5 \mu \mathrm{m}$ and that the mean E.S.D. was $2.15 \mu \mathrm{m}$. Analysis of the number distribution of the aerodynamic diameter of the powder indicated that 98 percent of the particles had an aerodynamic diameter of $3.5 \mu \mathrm{m}$ or less. The powder had an off-white egg-shell color, not white as the powder should appear, so an acid washing procedure was developed to clean the material before further characterization was done. The acid washing (HCl) resulted in a canary-yellow, clear supernatant characteristic of $\mathrm{FeCl}_{2}$, and a white powder. Because the powder may be used for calibration of other techniques than $x$-ray scattering, it is desirable to make the powder as pure as feasible. The washing and drying of the powder resulted in caking and aggregation of the powder such that, though composed of respirable particles, much of the powder was not respirable. A technique was developed for dispersing the powder in an air stream and then collecting that portion of the powder that passed through an impaction stage designed to have penetration characteristics approximating the American Conference of Governmental Industrial Hygienists (ACGIH) respirable criteria. We discovered that even when the powder is dispersed as a respirable powder, if it is subsequently collected a large percentage (up to $40 \%$ ) reaggregates such that it is not in the respirable size range. (This is true of both the original and the acid-washed quartz materials.) Because of the tendency to aggregate and the difficulty in reproducibly dispersing the powder as a respirable dust, the SRM will not be certified to be a respirable dust. He are able however, to disperse the particles in a liquid reproducibly and demonstrated that the powder is composed entirely of respirable size particles, therefore, though the material cannot be certified to be a respirable dust we are able to certify that the powder is comprised of particles that are in the respirable size range.

The size distribution of the powder was established by sedimentation measurements. We verified that the powder was reproducibly and completely dispersed in the sedimentation liquid. Dispersion was accomplished by a sonication and stirring procedure. Size distribution measurements showed that the powder was homogeneous with a mean E.S.D. of $1.62 \pm 0.05 \mu \mathrm{m}$ and 97.5 percent of the mass of the particles with an E.S.D. less that $5 \mu \mathrm{m}$. 
The second important property of the powder is the crystalline phase purity. The material was tested for crystalline homogeneity by several techniques. One test was the measurement of the lattice parameters (at $25{ }^{\circ} \mathrm{C}$ ). The mean values (in angstroms)

$$
\begin{aligned}
& \langle a\rangle=4.9135(1) \\
& \langle c\rangle=5.4057(1),
\end{aligned}
$$

agreed closely with the reported lattice parameters for crystalline a-quartz. Another test of homogeneity consisted of comparing the intensity of the 100 reflection to the intensity of the 101 reflection. No measurable differences were observed for the samples analyzed that were attributal to sample inhomogeneity. The final test was based on the determination of the Reference Intensity Ratio (RIR) of the quartz relative to silicon powder. RIR is defined as the ratio of the intensity of the strongest line of the analyte to the strongest line of silicon for a $1: 1$ mixture by weight. This test also indicated no measurable inhomogeneity exists in the powder. The final measurement was a direct measurement of the crystalline and amorphous quartz percentages of the powder. These measurements demonstrate that the powder is $>95$ percent $\alpha$-quartz. Final determination of the exact value and a statement of precision of the number are in process by NML statisticians.

\section{Use of Monte Carlo Electron Trajectory Simulation for Basic Studies in Analytical Electron Microscopy (26109)}

Dale E. Newbury, Robert L. Myklebust

The NBS Microanalysis Monte Carlo electron trajectory simulation [1] has been applied to the study of two fundamental problems in quantitative analytical electron microscopy: (1) the role of fast secondary electrons in degrading spatial resolution and (2) determination of the correct form of the $x$-ray generation depth distribution function for tilted thin samples.

The question of the limits to spatial resolution in analytical electron microsopy (AEM) has been a subject of great interest in the AEM community in recent years [2]. Previously, all attention has been focused on the role of high angle and multiple elastic scattering in degrading the cylindrical interaction volume defined by the current distribution of the incident.probe and the specimen thickness. Recently, it has been recognized that fast secondary electrons generated by the high energy beam electrons can degrade the spatial resolution [3]. Fast secondary electrons (FSE's) are generated by ejection of atomic electrons during inelastic scattering of the beam electrons. Their kinetic energies can range from zero to half of the incident beam energy. The cross section for production of FSE's follows a $1 / E^{2}$ dependence, so that the majority of FSE energies lie below $0.1 E_{0}$, where $E_{0}$ is the incident beam energy. Most importantly, FSE's in this energy range are scattered at angles from $60^{\circ}$ to $90^{\circ}$ from the incident beam trajectory. For an electron beam set normal to the surface of a foil, the FSE's tend to propagate laterally into the foil, degrading spatial resolution. Moreover, the cross section for inner 
shell ionization, which leads to the production of the characteristic $x$-rays used for analysis, tends to peak at low values of the overvoltage $U\left(U=E / E_{c}\right)$ where $E$ is the energy of the exciting electron and $E_{C}$ is the critical excitation energy for the atomic she11). The influence of FSE's upon the excitation of $x$-rays of low energy $(0.5$ to $5 \mathrm{keV})$ is larger than the number of FSE's relative to the number of beam electrons would suggest.

The Monte Carlo electron trajectory simulation has been modified to calculate FSE generation. The simulation has become, in effect, a "double Monte Carlo". A primary trajectory is followed until FSE generation occurs, and then the primary trajectory is interrupted while the FSE is followed until it either escapes the foil or is absorbed, after which the primary trajectory is resumed. An example of the results of the calculation is shown in figure 51, which compares the interaction in a silicon target from beam electron excitation and from FSE excitation. The two interaction volumes are significantly different in shape. The interaction volume due to beam electrons is cone-shaped with the apex at the beam entry point, while the interaction volume due to FSE's is cylindrical, a result of the fact that FSE's are created with nearly uniform probability along the beam electron trajectory. The significance of these calculations is that there exists a fundamental physical limit to spatial resolution, independent of elastic scattering of the beam electrons. FSE-generated $x$-rays will have to be considered in models for de-convolution of high spatial resolution compositional profiles.
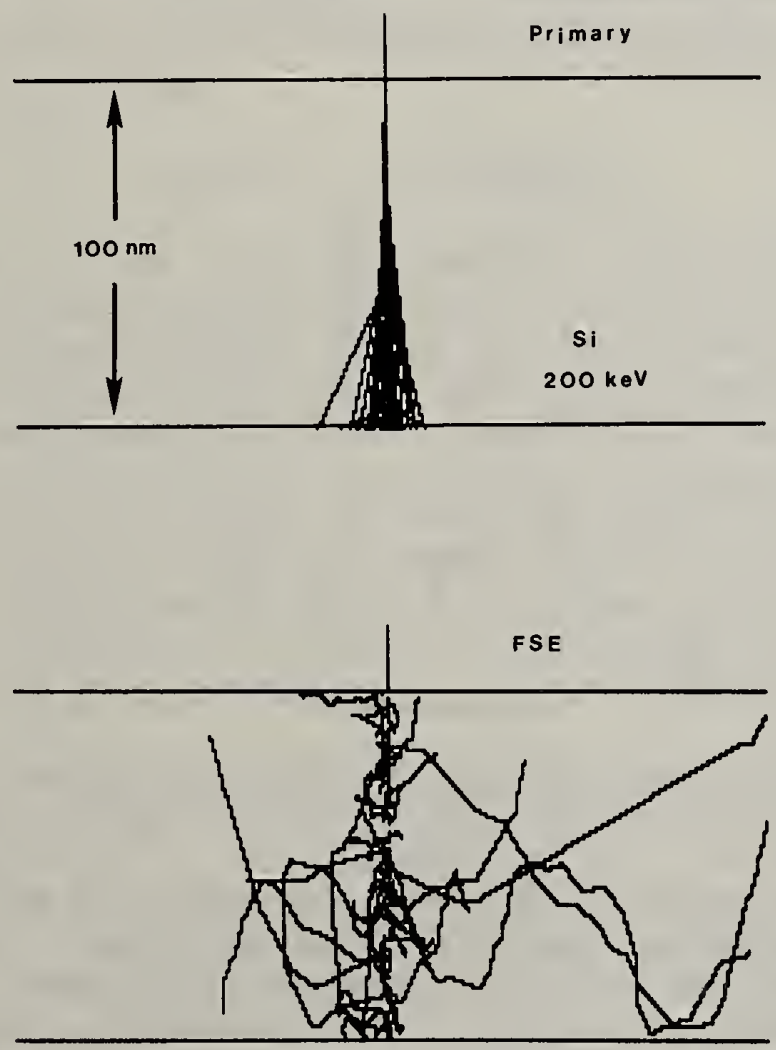

Figure 51. Comparison of electron interaction volumes for primary beam electrons and fast secondary electrons; target silicon, $100 \mathrm{~nm}$ thick; beam energy: $200 \mathrm{keV}$. 
The depth generation function of $x$-rays produced by the beam electrons is an important parameter. in models for the quantitative AEM analysis [4]. At normal beam incidence, the generation function follows a monotonic increase with depth. This behavior has been assumed in current models for correction of $x$-ray absorption effects. Many AEM's require the sample to be tilted to angles as high as $45^{\circ}$. At these angles, scattering parallel to the foil surface will occur which can modify the depth distribution function. To study this phenomenon, the Monte Carlo simulation was employed in conjunction with experimental measurements of $x$-ray production at the exit surface of gold foils [5]. An example of the results is shown in figure 52. The solid curve shows the comparison of the Monte Carlo calculations for the exit surface with experimental measurements made with a tracer layer placed on the exit surface of thin gold foils. Considering the difficulty of the experiment, the agreement is satisfactory. The plotted points give the Monte Carlo results for the $x$-ray depth production function for foils of various thicknesses. The curves are found to deviate significantly from the monotonic increase observed at normal beam incidence. The results of these calculations can be utilized for the development of more accurate models for quantitative AEM analysis.

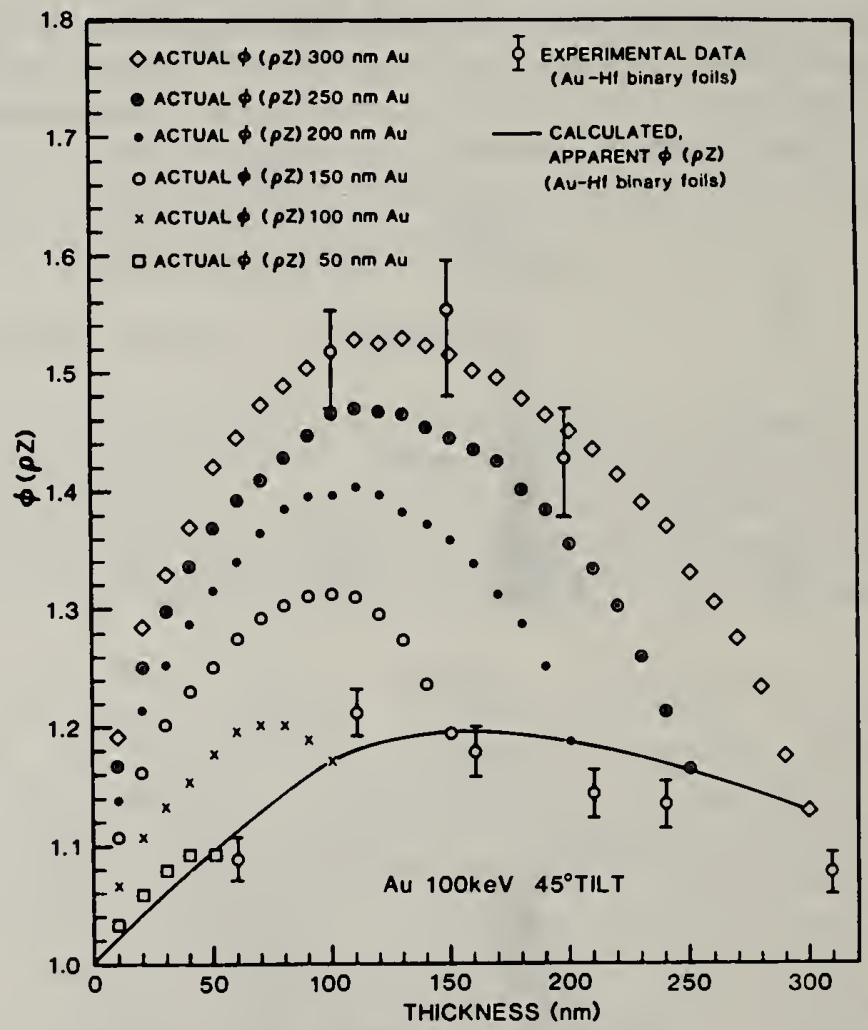

Figure 5. Comparison of $\phi(\rho z)$ curves for thin gold films calculated by Monte Carlo electron trajectory simulation and measured by use of Au-Hf foils; beam energy-100 keV; sample tilt $-45^{\circ}$. 
References

[1] Newbury, D.E. and Myklebust, R.L. (1981) "A Monte Carlo Electron Trajectory Simulation for Analytical Electron Microscopy" in Analytical Electron Microscopy-1981, ed. R. Geiss (San Francisco Press, 1981) 91.

[2] Newbury, D.E. (1982) "Beam Broadening in an Analytical Electron Microscope" in Microbeam Analysis-1982, ed. K.F.J. Heinrich (San Francisco Press) 79.

[3] Joy, D.C., Newbury, D.E., and Myklebust, R.L. (1982) "The Role of Fast Secondary Electrons in Degrading Spatial Resolution in the Analytical Electron Microscope", J. Microscopy, 128, RPI.

[4] Goldstein, J.I. (1979) "Principles of Thin Film Microanalysis" in Introduction to Analytical Electron Microscopy, ed.

J.J. Hren, J.I. Goldstein, and D.C. Joy (Plenum, New York) 83.

[5] Newbury, D.E., Myklebust, R.L., Romig, A.D. and Bieg, K.W. (1983) "Observations on the Determination of $\phi(\rho z)$ Curves for Thin Films in the Analytical Electron Microscope" Microbeam Analysis-1983, ed. R. Gooley (San Francisco Press) 168.

17. Visualization of the Three-Dimensional Nature of the Electron Interaction Volume in Solids (15330)

D. S. Bright, R. L. Myklebust, D. E. Newbury

The development of the Monte Carlo electron trajectory simulation technique for solid specimens has provided a major tool for theoretical calculations in scanning electron microscopy (SEM), electron probe analysis (EPMA), analytical electron microscopy (AEM) and microbeam lithography [1]. Analogous to the development and use of dynamical diffraction calculations in the interpretation of transmission electron microscope images of crystalline objects, Monte Carlo calculations are being employed to aid in the interpretation of SEM images $[2,3]$ and for the prediction of $x$-ray intensities generated in targets, particularly those with unusual shapes $[4,5]$. In many cases, a. graphical plot is particularly valuable in understanding the behavior of electron trajectories at a discontinuity such as a boundary.

Plots of electron trajectories are usually generated by projecting the trajectory segments between scattering points onto a plane which is either perpendicular or parallel to the specimen surface. A continuous record of each trajectory is thus obtained, but the three dimensional nature of the interaction volume is lost. In such a plot, an example of which is shown in figure 53, it is difficult to follow a particular trajectory when several trajectories apparently intersect in the projection plane. Also, as seen by contrasting the flat or planar representation of figure 53 with the 3-D representation in figure 53 , the loss of the third dimension by projecting on to a plane causes serious loss of any notion of true shape. Projection onto a plane makes the overall size of the interaction volume to appear smaller and 
(a)

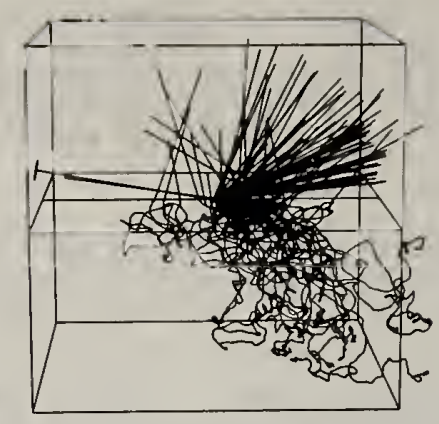

(b)

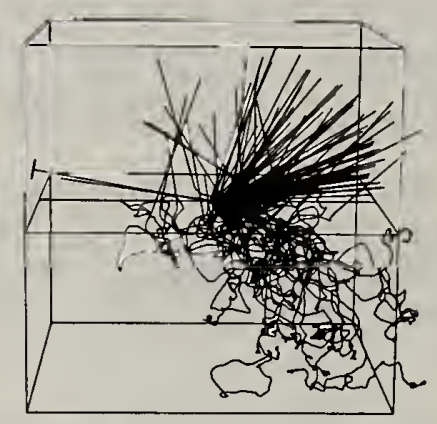

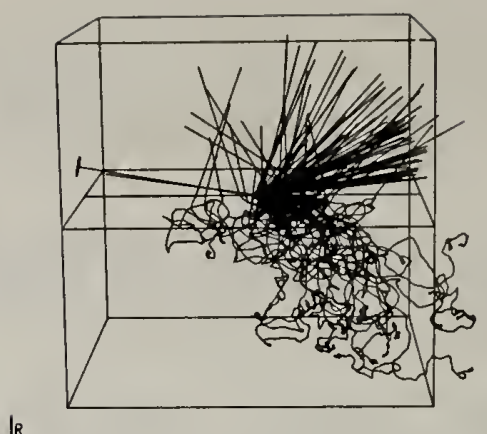

\&.



Figure 53. Side view of a $40 \mathrm{keV}$ beam incident on gold at $10^{\circ}$ from the surface, plotted in stereo as

(a) a two dimensional projection and (b) a three dimensional object. Cube edge $0.3 \mu \mathrm{m}$.

much more dense than the view obtained by stereo projection. Further, most of the trajectories appear to overlap and become confused.

To overcome these limitations, a method of generating matched stereo projection plots of Monte Carlo trajectories has been developed to visualize the true three dimensional structure of the electron interaction volume.

There are advantages and unique capabilities of stereo presentation. The stereo presentation allows a sense of spatial size and often allows one to look behind nearer trajectory segments to follow more distant segments, so that confusion from overlap does not occur. Beyond the improvements over conventional projection plotting noted above, the three-dimensional Monte Carlo plot offers the capability of providing any desired view of the trajectories from different observation points. This facility is illustrated in figure 54, which illustrates three useful views of the interaction volume and backscatter distribution from a highly tilted gold target. 
(a)
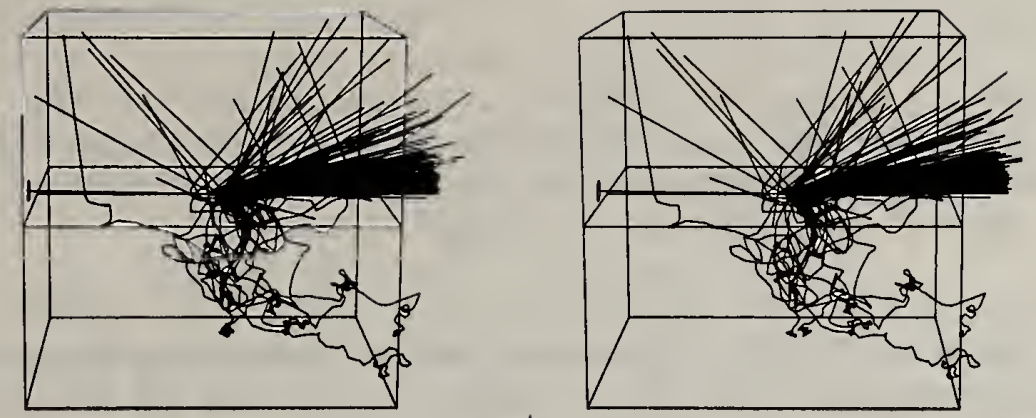

ㄴ.

(b)

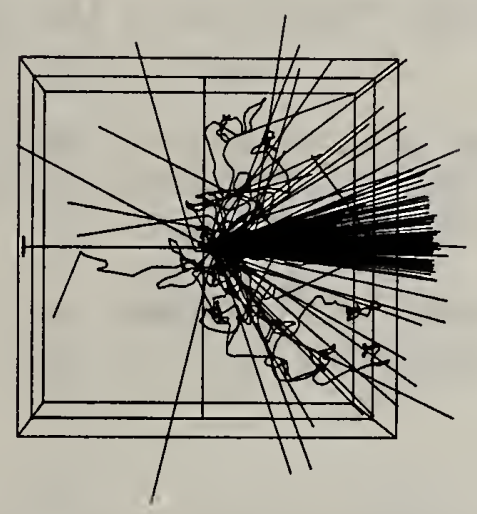

ட.

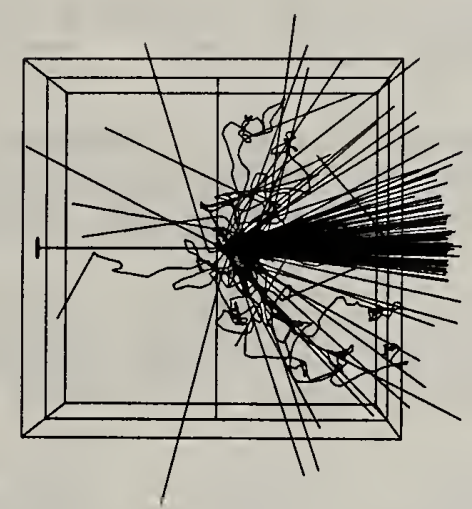

(c)
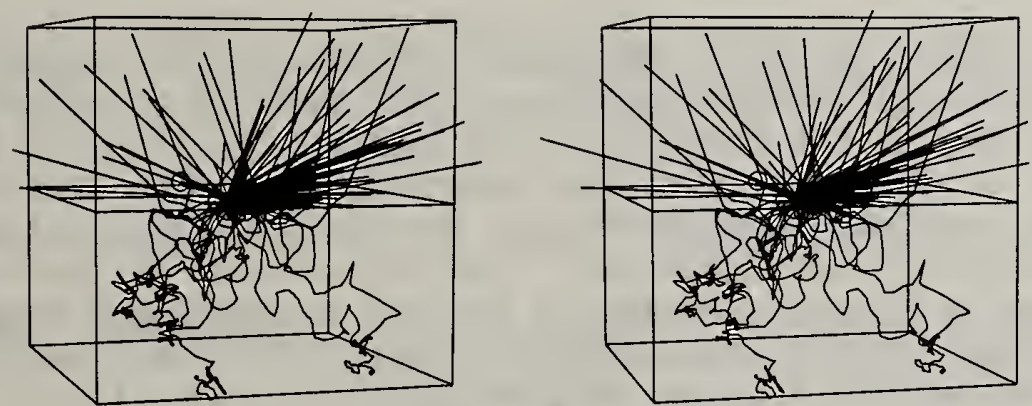

$\underline{R}$

Figure 54. Three views of a $20 \mathrm{keV}$ electron beam incident gold on at a grazing angle (1 deg. from surface). Cube edge $=0.3 \mu \mathrm{m}$.

(a) a few degrees above the tilt axis which is similar to the conventional projection representation; (b) along the surface normal, looking toward the surface; and (c) a few degrees above the surface looking back towards the incident beam. 
Reference

[1] Heinrich, K.F.J., D.E. Newbury, and H. Yakowitz, eds. (1976). "Use of Monte Carlo Calculations in Electron Probe Microanalysis and Scanning Electron Microscopy: National Bureau of Standards (US) Special Publication 460 (Washington, DC).

[2] Fathers, D.J., J.P. Jakubovics, D.C. Joy, D.E. Newbury, and H. Yakowitz (1973), "A new Method of Observing Magnetic Domains by Scanning Electron Microscopy: I. Theory of the Image Contrast", Phys. Stat. Sol. (a) 20:535.

[3] Murata, K. (1973), "Monte Carlo Calculations on Electron Scattering and Secondary Electron Production in the SEM", SEM/1973, 267.

[4] Yakowitz, H., D. E. Newbury, and R. L. Myklebust (1975), "Approaches to Particulate Analysis in the SEM with the Aid of a Monte Carlo Program", SEM/1975, 93.

[5] Newbury, D.E. and R. L. Myklebust (1979), "Monte Carlo Electron Trajectory Simulation of Beam Spreading in Thin Foil Targets", U1tramicroscopy $3: 391$.

18. An Evaluation of X-ray Loss Due to Electron Backscatter (15330)

R. L. Myklebust

One of the most important corrections in the atomic number-absorptionfluorescence matrix correction technique (ZAF) used in quantitative electron probe microanalysis is the so-called "R-factor". The R-factor is an estimate of the fraction of the total possible inner shell ionization which is actually produced in the specimen considering the loss in possible ionization which results from electron backscattering. The formulation of the R-factor currently in use is based on empirical fits of experimental data on electron backscattering and backscattered electron energy distributions [1]. While this formulation has great value in quantitative microanalysis, there exists some uncertainty in its accuracy due to the limited angular integration of the backscattered electron energy distribution in the experimental measurements. Moreover, the expression for $R$ is in the form of a fifth degree polynomial in two separate variables which is inconvenient to calculate.

The loss of $x$-ray intensity due to backscattered electrons has been re-evaluated with the aid of a Monte Carlo (MC) simulation for electron scattering in solids. The advantage of the Monte Carlo simulation is the capability of effectively integrating the energy distribution of backscattered electrons over all possible angles of emission. Additionally, the Monte Carlo simulation can be applied to the calculation of tilted specimens for which virtually no experimental data exists. The Monte Carlo simulation is a single scattering model employing a Rutherford scattering cross-section and a Bethe inner shell ionization cross-section [2]. Initial electron energies in the 
range $4-50 \mathrm{keV}$ were considered in this study. The results are presented as $R$, the ratio of $x$-rays generated within the solid to the total $x$-rays that would have been generated had none of the electrons backscattered. The $R$ values for K X-ray lines agree reasonably well with those of [1]; however, the $R$ values for $L$ and $M X$-ray lines are different. Table 42 lists a few of the values obtained together with values computed with an expression formulated by Duncumb [1]. A third value is listed which was computed with the expression:

$$
R=a Z+b Z^{2}+c Z(\exp (-U))+1
$$

where $Z$ is the atomic number and $U$ is the overvoltage (initial electron energy/ excitation potential). The coefficients $a, b$, and $c$ have different values for each series of $x$-ray lines.

Table 42.

\begin{tabular}{|c|c|c|c|c|c|}
\hline El ement & Line & $\underline{U}$ & $R(M C)$ & $R$ (Duncumb) & $R(c a l c)$ \\
\hline $\begin{array}{l}\mathrm{Na} \\
\mathrm{Fe} \\
\mathrm{Ru}\end{array}$ & $\begin{array}{l}K \\
K \\
K\end{array}$ & $\begin{array}{l}4.0 \\
4.0 \\
2.0\end{array}$ & $\begin{array}{l}0.924 \\
0.846 \\
0.809\end{array}$ & $\begin{array}{l}0.939 \\
0.861 \\
0.837\end{array}$ & $\begin{array}{l}0.930 \\
0.841 \\
0.790\end{array}$ \\
\hline $\begin{array}{l}\mathrm{Zr} \\
\mathrm{Ag} \\
\mathrm{Pb}\end{array}$ & $\begin{array}{l}L \\
L\end{array}$ & $\begin{array}{l}4.0 \\
4.0 \\
2.0\end{array}$ & $\begin{array}{l}0.750 \\
0.726 \\
0.697\end{array}$ & $\begin{array}{l}0.791 \\
0.765 \\
0.747\end{array}$ & $\begin{array}{l}0.758 \\
0.730 \\
0.715\end{array}$ \\
\hline $\begin{array}{l}\text { Ta } \\
\mathrm{Bi} \\
\mathbf{U}\end{array}$ & $\begin{array}{l}M \\
M \\
M\end{array}$ & $\begin{array}{l}6.0 \\
6.0 \\
6.0\end{array}$ & $\begin{array}{l}0.613 \\
0.613 \\
0.596\end{array}$ & $\begin{array}{l}0.674 \\
0.650 \\
0.633\end{array}$ & $\begin{array}{l}0.624 \\
0.600 \\
0.583\end{array}$ \\
\hline
\end{tabular}

The principal differences in quantitative analyses calculated with $R(M C)$ and $R$ (calc) compared to $R$ (Duncumb) are evident for high atomic number elements. Three lead silicate glasses were analyzed using two different lead standards. The results of the analyses are shown in Table 43.

Table 43. Lead Anlyses at $15 \mathrm{keV}$ and $\mathrm{Pb}$ M-alpha X-ray Line.

\begin{tabular}{|c|c|c|c|c|c|}
\hline \multirow[t]{2}{*}{ Glass } & Composition & $\frac{\text { New }}{\text { Sto }}$ & $\frac{\text { factor }}{\text { ards }}$ & \multicolumn{2}{|c|}{$\frac{\text { Duncumb }}{\text { Standards }} \frac{\mathrm{R} \text {-factor }}{}$} \\
\hline & $\mathrm{Pb}$ & PbTe & $K-227$ & PbTe & $K-227$ \\
\hline $\begin{array}{l}K-456 \\
K-493 \\
K-523\end{array}$ & $\begin{array}{l}65.76 \\
63.28 \\
63.45\end{array}$ & $\begin{array}{l}65.70 \\
63.47 \\
63.30\end{array}$ & $\begin{array}{l}65.62 \\
63.41 \\
63.24\end{array}$ & $\begin{array}{l}66.97 \\
64.81 \\
64.64\end{array}$ & $\begin{array}{l}65.99 \\
63.84 \\
63.68\end{array}$ \\
\hline
\end{tabular}

References

[1] Duncumb, P. and Reed, S.J.B., NBS Spec. Pub. 298, K.F.J. Heinrich, ed., (1968) 133.

[2] Mott, N.F. and Massey, H.S.W., The Theory of Atomic Collisions, 2nd Edition, p. 243 (Oxford Univ. Press, London, 1949). 
19. Studies of the Depth Distribution of Boron in Glass by Neutron Depth Profiling and Secondary Ion Mass Spectrometry (15443)

\section{G. Downing, R. Fleming, B. Stallard, D. Simons}

During the past year the first spectra were obtained utilizing the neutron depth profiling (NDP) facility at the 10 MW NBS reactor. This dedicated facility, unique in the United States, allows nondestructive determination of concentration profiles in the near-surface region for selected elements [1]. By coupling the capabilities already available using secondary ion mass spectrometry (SIMS) with the complementary strengths of NDP, a totally new synergism has become available for material analysis.

The NDP technique provides an absolute concentration scale and can be used to nondestructively detect variable ionization or matrix effects which might be present in the SIMS spectra. Initial applications were prompted by the semiconductor industry's interest in the concentration and spatial distribution of boron in processed and unprocessed semiconductor materials. Figure 55 is a comparison of the NDP spectrum, before resolution corrections, and the SIMS depth profile for an annealed boron-10 implant in silicon. The SIMS technique provides sharper resolution and is capable of detecting concentration levels to $10^{14}$ atoms $/ \mathrm{cm}^{3}$. This sample is one of a series analyzed to study the total boron distribution, including the boron component that is not electrically active and thereby not observable by the commonly used spreading resistance probe. The boron, a solid state precipitate, is manifested as the peak that rises above the broader electrically active and thermally mobile boron distribution in the figure.

Figure 55. Depth Profiles obtained by the SIMS and NDP technique for a $70 \mathrm{keV}$ boron-10 implant in silicon at a dost of $10^{16}$ boron- 10 atoms $/ \mathrm{cm}^{2}$ and annealed at $1000^{\circ} \mathrm{C}$ for 30 minutes.

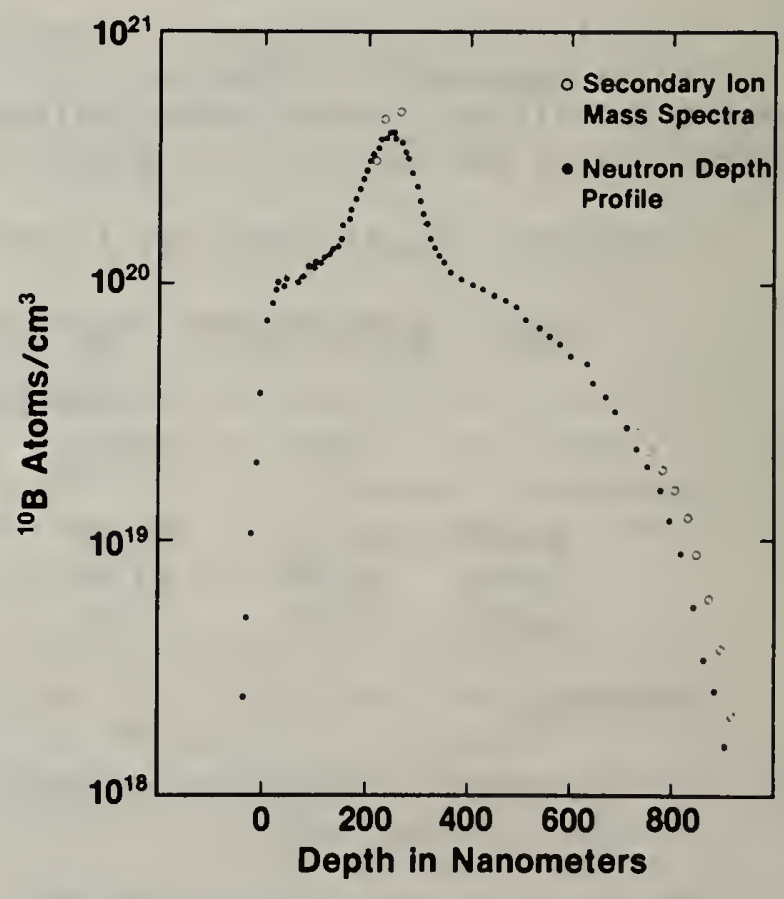


A second example of a joint NDP-SIMS analysis is shown in figure 56. Borophosphosilicate glass (BPSG) is a material often used as the insulating barrier between devices on microcircuits as well as the support for the electrical leads between devices. The integrity of the circuit depends critically on a smooth, continuous glass film over the substrate and therefore, on a uniform boron distribution in the glass. Knowledge of the glass film thickness is also desired. In this case a SIMS and NDP collaborative effort was used to:

(1) study the boron profile in the "as deposited" and "processed" films;

(2) quantify the concentration of boron present in the samples; (3) calibrate

the Fourier transform infrared (FTIR) technique for total boron determinations; and (4) evaluate concentration data from the prompt gamma-ray emission (PGRE) technique. Figure 56 compares spectra obtained from a BPSG film by SIMS and NDP analysis. As before SIMS provides the greater resolution. The NDP measurement, on the other hand, was able to identify and correct measurement artifacts present in the SIMS spectra as a consequence of sample charging during analysis and to establish an absolute boron concentration scale. Measuring the crater depth produced by SIMS enabled a depth scale to be obtained for these spectra. The absolute boron concentration determined from the NDP spectra also allowed comparisons with the values obtained from the FTIR and PGRE techniques and made possible a calibration of the FTIR for future total boron measurements of BPSG films.

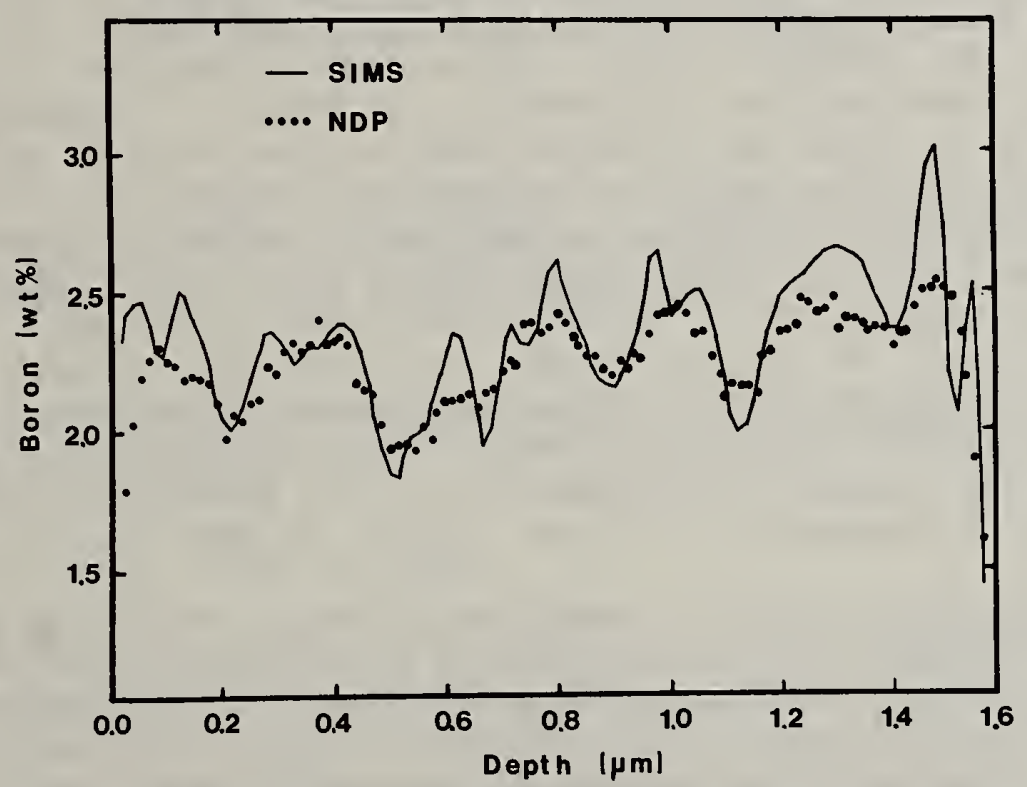

Figure 56. A comparison of depth profiles measured by SIMS and NDP techniques for a thin BPSG film on a silicon substrate.

Other studies now underway include the profiling of sodium in glass, boron and beryllium in gallium arsenide, and analysis of boron diffusion and segregation through $\mathrm{SiO}_{2} / \mathrm{Si}$ boundaries.

Reference

[1] R.G. Downing, R.F. Fleming, J.K. Langland and D.H. Vincent, "Neutron Depth Profiling at the National Bureau of Standards", Sixth International Conference on Ion Beam Analysis, Tempe, $A Z$ May 23-28, 1983. 
20. Detection and Analys is of Organic Monolayers by Laser Microprobe Mass Analysis (13225)

\section{R. A. Fletcher}

The laser microprobe mass analyzer (LAMMA) is an instrument which couples a Nd:YAG laser for ablating and ionizing a sample to a time-of-flight (TOF) mass spectrometer for ion analysis. Usually this instrument is used at high power densities for detection of atomic ions with high sensitivity. By using low laser power densities, we have been able to gently desorb, ionize, and detect an organic monolayer from the surface of a $5 \mathrm{~nm}$ thick silver film. Moreover, this measurement has been carried out with a lateral resolution of 210 micrometers which is a unique capability in organic mass spectrometry.

The thin silver films are made by an evaporative process which results in resonant-active silver microstructures (silver islands) being created which are utilized in surface-enhanced Raman scattering (SERS). The silver film is spin-coated with a $0.001 \mathrm{~mol} / \mathrm{L}$ paranitrobenzoic acid (pNBA) in ethanol solution forming what we believe to be paranitrobenzoate, (DNBA-H) ${ }^{-}$, on the surface. This molecular ion has been observed on the Ag film by SERS. The initial goal was to see whether the silver microstructures which have a surface resonance at the frequency doubled wavelength of the Nd:Yag fundamental, $532 \mathrm{~nm}$, could be used to desorb the (PNBA-H) ions. The resonant effect was not observed, but (pNBA-H) ${ }^{-}$was detected from the monolayer as shown in figure $57(\mathrm{~b})$.

Figure $57(\mathrm{~b})$ shows the negative ion mass spectrum that results when a $5 \mathrm{~nm}$ thick Ag film coated with a monolayer coverage of (pNBA-H) is irradiated by a laser pulse with energy densities (at the sample surface) of $\sim 400 \mathrm{~mJ} / \mathrm{cm}^{2}$. Figure $57(\mathrm{a})$ shows a similar spectrum for the $\mathrm{Ag}$ film without pNBA coverage. The molecular ion peaks of $(\mathrm{M}-\mathrm{H})^{-}$at 166 atomic mass units (amu), and its fragments of $\left(\mathrm{M}-\mathrm{H}-\mathrm{CO}_{2}\right)^{-}$at 122 amu and $\mathrm{NO}_{2}{ }^{-}$at 46 amu are present in figure $57(\mathrm{~b})$ and absent in figure $57(\mathrm{a})$. Contaminant peaks which appear in figure $57(a)$ and (b) most likely result from the film preparation and are not totally unexpected since the samples are exposed to air for several days prior to analysis. The $\mathrm{Ag}^{-}$ion appears at 107 and 109 amu in both spectra.

When the pNBA coated film is irradiated at energy densities of $\sim 100 \mathrm{~mJ} / \mathrm{cm}^{2}$ at either 266 or $532 \mathrm{~nm}$ wavelengths, a gentle desorption process occurs which liberates molecular ions from the surface as shown in figure $57(\mathrm{c})$. The desorption mechanism is thought to be thermal in nature. Evidence for this is the strong power dependence and wavelength independence (for the two wavelengths used). Extinction measurements of the coated Ag films show that the absorption is nearly equal at wavelengths of $266 \mathrm{~nm}$ and $532 \mathrm{~nm}$. The $\mathrm{Ag} f \mathrm{film}$ thus absorbs the radiant energy, heating the surface to liberate the preformed negative ion, paranitrobenzoate. This shows a detection capability for $\sim 10^{6}$ molecules based on irradiated spot size and assuming $10^{14}$ molecules $/ \mathrm{cm}^{2}$ coating density for the monolayer.

This gentle desorption technique should prove to be a useful analytical tool for organic molecular analysis since the molecule is preserved in the desorption process and there appears to be some selectivitity on the sample surface as apparent in figure $57(\mathrm{c})$, where only the molecular ion with its fragments are present in the spectrum. 

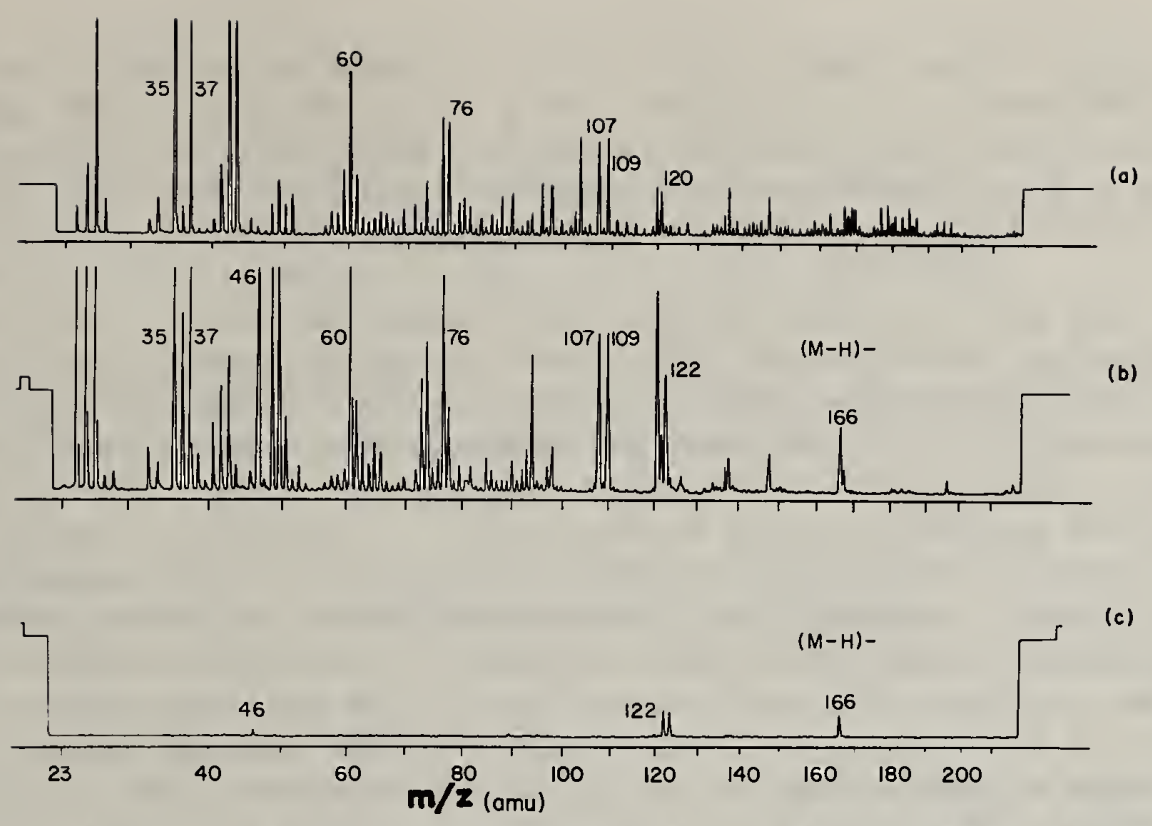

(c)

Figure 57. Negative ion TOF mass spectra of (a) uncoated silver film, (b) paranitrobenzoate monolayer coated film at laser energy densities of $>400 \mathrm{~mJ} / \mathrm{cm}^{2}$ and (c) coated film at lower laser energy density, $\sim 95 \mathrm{~mJ} / \mathrm{cm}^{2}$. The laser wavelength was $532 \mathrm{~nm}$ for (a) and $266 \mathrm{~nm}$ for (b) and (c).

Reference

[1] Fletcher, R.A., I. Chabay, D.A. Weitz, and J.C. Chung "Laser Desorption Mass Spectrometry of Surface-Adsorbed Molecules." Chem. Phys. Letts. (submitted).

21. Standard Reference Material for Analysis of Asbestos by Electron Microscopy (15330)

John A. Small, Eric B. Steel

The determination of asbestos present in the non-workplace environment is currently accomplished by filtering the air sample through a membrane filter and then analyzing the collected particulate by analytical electron microscopy (AEM). A multi-laboratory study was conducted in 1977 by the Environmental Protection Agency (EPA) on ambient air samples with unspecified sample preparation and counting procedures. The results reported from the different laboratories on the analysis of split samples varied by several orders of magnitude for the asbestos fiber loading in the air. Since that time, the EPA has been developing a standard methodology for the analysis of asbestos in non-workplace environments. As part of the EPA's overall asbestos program, the National Bureau of Standards (NBS) and the EPA have been cooperating on the development of a Standard Reference Material (SRM) to aid in the analysis of the asbestos-fiber loading in non-workplace samples. The final goal of our program was to provide an SRM in the form of statistically predictable loadings of chrysotile asbestos fibers on a substrate suitable for sample preparation and analysis by AEM. This set of samples could be used by field laboratories 
to test, refine, and calibrate their sample preparation and analysis procedures. The SRM consists of sections from a polycarbonate filter which contain a medium loading of chrysotile asbestos mixed with an urban particulate matrix. Urban air particles were selected as the matrix material so that the SRM would simulate a sample collected from the environment.

The work on this standard has been completed. It will be released in two batches as SRM 1876 and SRM 1876A. It was decided that the users of the SRM should determine the asbestos fiber loading by AEM on five separate areas of approximately $0.01 \mathrm{~mm}^{2}$ each and average the five values. It was felt that a sample size of five represented the minimum number of samples necessary to make the standard usable in the field. A laboratory cannot be evaluated on the basis of one sample because of the large single-measurement variation in the counts. An additional constraint placed on the users is that the areas counted must come from areas which are distributed evenly over the entire filter section used for preparation of the standard rather than one local area. Table 44 lists the certified values for the two SRM batches, the uncertainties in these values expressed as the 95-95 tolerance intervals for a five measurement mean.

Table 44. Certified Values and Uncertainties.

$\begin{array}{lcc}\text { SRM } & \begin{array}{c}\text { Certified Value } \\ \text { Fibers } / 0.01 \mathrm{~mm}^{2}\end{array} & \begin{array}{c}\text { Tolerance Interval } \\ \text { Fibers } / 0.01 \mathrm{~mm}^{2}\end{array} \\ & 30 & \pm 9 \\ 1876 \mathrm{~A} & 37 & \pm 13\end{array}$

The certified values are means calculated from the analysis of 21 individual $0.01 \mathrm{~mm}^{2}$ areas on SRM 1876 and 23 areas on SRM 1876A. Each individual area was analyzed by 2-5 operators with the verified counting procedure which was developed at NBS [3]. The verified counting procedure uses the relative locations of the fibers recorded by each operator, to construct a map which shows the position of each fiber for each operator. The fiber count on the grid opening is then verified by having an operator reanalyze any fiber for which there were questions. This includes any fibers that were found by only one operator; or any fibers whose identification was disputed because of ambiguous morphology or diffraction data. In this way, a count approaching as closely as possible to the true amount of asbestos is obtained.

Tolerance intervals rather than sample standard deviations were chosen as a robust way of making credible statements about uncertainties in means of counts that the users are likely to obtain in their attempts to characterize pieces of the SRM filter. A 95-95 tolerance interval for a five measurement mean indicates that 95 percent of the population of such 5-count means will fall within the tolerance interval with a 95 percent confidence. The certificate for the SRM will also include the sample standard deviations for the two SRM batches since this value is based directly on experimental measurements. 
References

[1] Leide1, N.A., Bayer S.G., Zumwalde, R.D. and Busch, K.A., USPHS/NIOSH Membraine Filter Method For Evaluating Airborne Asbestos Fibers, Technical Rpt., U.S. Dept. HEW 1979.

[2] Mountgomery County Asbestos Study, EPA Internal rpt. U.S. EPA, Environmental Monitoring Systems Laboratory, Environmental Monitoring Division, Research Triangle Park, N.C. 27711, Oct. 1977.

[3] E.B. Steel, J.A. Small, and P. Sheridan, Analytical Errors in Asbestos Analysis by Analytical Electron Microscopy, NBS SP. 619, Asbestos Standards: Materials and Analytical Methods, 1982 , p 162.

22. Visibility of Asbestos Fibers in the Scanning Electron Microscope

$(15330)$

J. A. Sma11, D. E. Newbury, R. L. Myklebust

The analysis of asbestos mineral fibers in environmental samples requires the careful determination of fiber loading on filter media [1]. We11 established techniques exist for the transfer of asbestos fibers from such filter media to thin carbon films and subsequent counting in the transmission electron microscope (TEM) $[2,3]$. However, due to the cost of TEM instrumentation and the time required for sample preparation, there has been considerable interest in the development of techniques for direct examination of the filter in the scanning electron microscope (SEM). In this work, we have considered the problem of fiber visibility under SEM imaging conditions in a fiber search/ counting procedure.

The asbestos mineral chrysotile is typically found in environmental samples as long fibers composed of one or more fibrils. Individual fibrils have a diameter of approximately $40 \mathrm{~nm}$ and a length which can extend to several micrometers. In order to estimate the visibility of single fibrils and fiber bundles in an SEM image, we have used a three stage procedure: first (1) we calculated the contrast of the asbestos fiber compared to the filter background with the NBS Monte Carlo program; (2) we calculated the threshold beam current necessary to observe that contrast; and (3) we calculated the smallest beam size which could contain the required threshold current. Finally, by comparing the size of the beam calculated in the above steps, with the fiber diameter, the visibility of the fiber was estimated.

Table 45 shows the results of the calculations for several different fiber diameters. These results indicate that asbestos fibers below approximately $200 \mathrm{~nm}$ diameter will not be visible under the imaging conditions in a fiber search/counting procedure. Only for fibers of $240 \mathrm{~nm}$ diameter and above is the threshold visibility condition substantially exceeded, which should ensure adequate image quality. The minimum fiber size for visibility can be reduced by using more efficient detectors and higher brightness electron sources. For practical conditions, such improvements might decrease by a factor of two the minimum fiber diameter which would be visible. However, 
Table 45. Calculations of Asbestos Fiber Contrast and Visibility.

\begin{tabular}{|c|c|c|c|c|}
\hline $\begin{array}{c}\text { Fiber diameter } \\
(\mathrm{nm})\end{array}$ & Contrast & $\begin{array}{l}\text { Threshold Current } \\
\text { (nA) }\end{array}$ & $\begin{array}{c}\text { Beam Diameter } \\
(\mathrm{nm}) \\
\end{array}$ & Visibility \\
\hline 40 & 0.013 & 540 & 1050 & No \\
\hline 80 & 0.025 & 147 & 550 & No \\
\hline 120 & 0.031 & 95 & 440 & No \\
\hline 160 & 0.039 & 60 & 350 & No \\
\hline 200 & 0.049 & 38 & 280 & $?$ \\
\hline 240 & 0.061 & 25 & 223 & Yes \\
\hline
\end{tabular}

since the statistical assumptions used in deriving the threshold equation are based on an average observer, it should be noted that working with objects near the visibility threshold will produce counting results that are erratic and subject to large uncertainties. Since environmental asbestos samples are likely to contain fibers of small diameter, we recommend not using an SEM counting procedure for characterization of such samples.

References

[1] A.V. Samudra, C.F. Harwood, and J.D. Stockham, "Electron Microscope Measurement of Airborne Asbestos Concentrations, A Provisional Methodology Manual", Environmental Protection Agency, EPA-600/2-77-178; revised 1978.

[2] P.M. Cook and D.R. Marklund, "Sample Preparation for Quantitative Electron Microscope Analysis of Asbestos Fiber Concentrations in Air" in Asbestos Standards: Materials and Analytical Methods, National Bureau of Standards (US) Special Publication 619 (Washington, 1982) eds. J. Small and E. Steel, 53.

[3] E.J. Chatfield, "Analytical Procedures and Standardization for Asbestos Fiber Counting in Air, Water, and Solid Samples" NBS SP 619 , op cit., 91.

23. An Analytical Algorithm for Calculation of Spectral Distributions of $X$-ray Tubes for Quantitative $X$-ray Fluorescence Analys is (15330)

L. Feng, P. A. Pella, J. Small

Last year we reported on the initial development of an algorithm for calculating the spectral distribution of $x$-ray tube emission for making fundamental parameter matrix effect corrections in quantitative $x$-ray fluorescence analys is [1]. Fundamental parameter methods require knowledge of the sample excitation function and hence the spectral distribution of the particular $x$-ray tube used for excitation. Several researchers have proposed theoretical approaches to the calculation of spectral distributions but these require rather extensive computer capabilities. In addition, these methods require 
knowledge of parameters such as backscattering and target absorption factors, ionization cross sections, and fluorescence yields which are not known with sufficient accuracy. Spectral distributions have been measured, but only for a few $x$-ray tubes operated at 45-50 kV.

We have completed an initial phase of our work this year in that we have developed an algorithm to calculate $x$-ray tube spectral distributions under various operating conditions by utilizing extensive electron microprobe data obtained with several pure metal targets, covering a wide range of atomic numbers. This proposed analytical algorithm has the advantage that it is simple to program on a computer and combines several fundamental parameters mentioned above into factors determined by the curve-fitting of the corrected electron microprobe experimental data. The algorithm calculates the continuum spectral distribution and the ratio of the target characteristic $x$-ray line intensity to the continuum intensity at the wavelength of the characteristic line. The target characteristic line intensity is then calculated by multiplying the ratio by the associated continuum intensity. For use in quantitative $x$-ray fluorescence analysis, the sample excitation function or primary spectrum is obtained by integrating from the short-wavelength limit to the absorption edge wavelengths for the analyte element(s) of interest in the specimen.

We have performed some limited testing of our proposed algorithm. The continuum spectral distributions calculated from our algorithm were compared to those calculated from another fundamental parameter program called NRLXRF. These calculated primary spectra were also compared to experimental x-ray tube data from the literature [2]. In figure 58, for example, the primary spectra for a tungsten target $x$-ray tube operated at $45 \mathrm{kV}$, a $x$-ray tube take-off angle of 22 degrees, and a beryllium window thickness of $0.25 \mathrm{~mm}$ are compared. These data indicate good relative agreement. To illustrate the validity of our proposed algorithm for analysis purposes, we analyzed a number of wel1characterized $\mathrm{Fe}, \mathrm{Ni}, \mathrm{Cr}$ alloys using just the pure elements as standards for calibration. In Table 46 are summarized the results on selected alloys using the NRLXRF fundamental parameter program. The concentrations were calculated using either the primary spectrum calculated from our proposed algorithm substituted in the NRLXRF or that calculated by NRLXRF. This was done by editing the existing NRLXRF spectral distribution file. In order to be consistent, the intensity data were input over the same wavelength region as the inherent NRLXRF primary spectral data. In this way we could compare results using different sources of primary spectral distributions. The results shown in Table 46 are in reasonably good agreement, with relative errors less than 5 percent. Although these results are encouraging, more extensive work is needed to fully test the proposed algorithm. During the upcoming year we intend to include various sample types such as fused and pressed powder samples, and to analyze them with different $x$-ray tube targets at various operating conditions. 


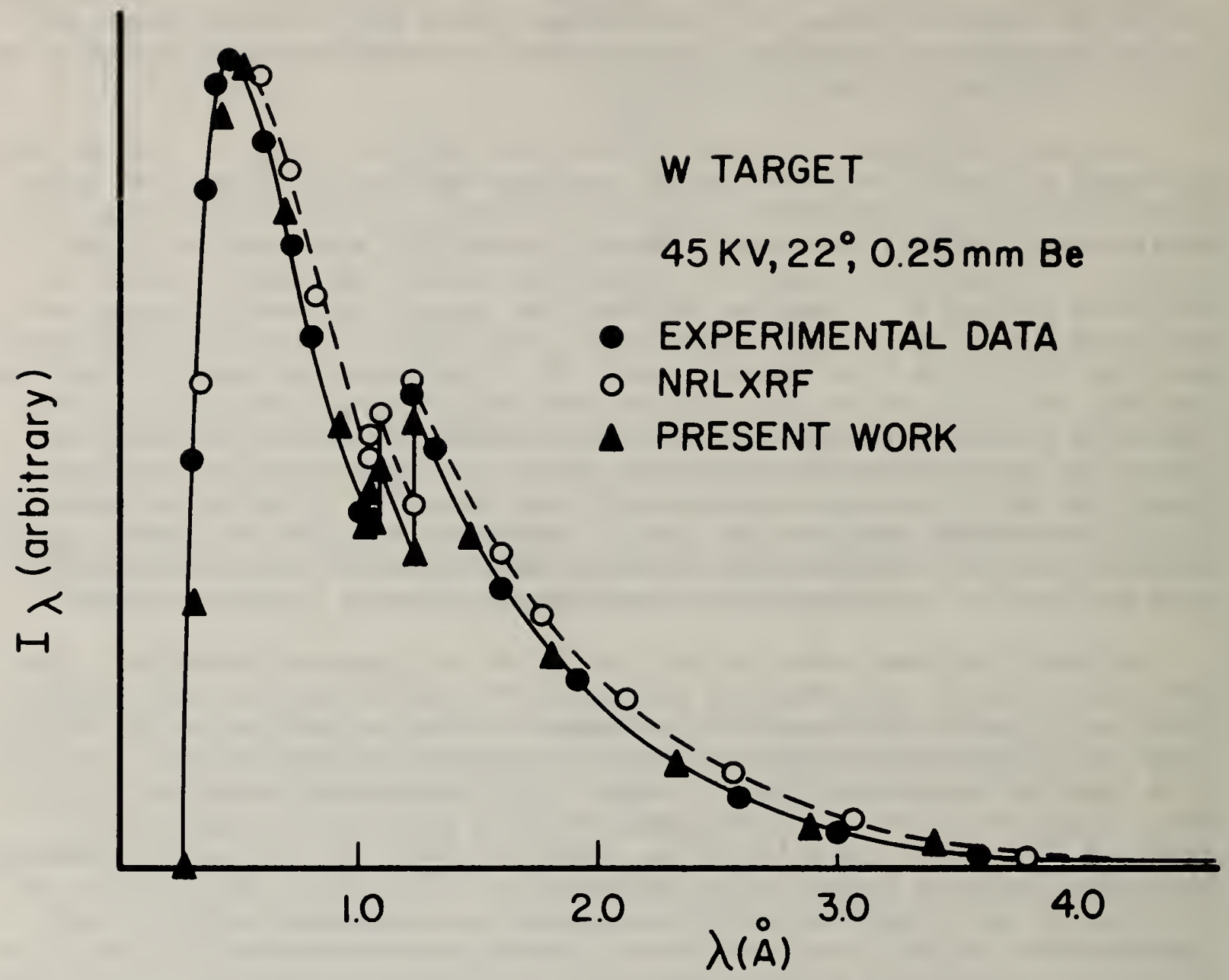

Figure 58. Comparison of experimental and calculated continuum spectral distributions.

References

[1] NBSIR-82-2620, page 208.

[2] Brown, D. B., Gilfrich, J.V., and Peckerar, M. C., J. App1. Phys. 46 , No. 10, 4537 (1975). 




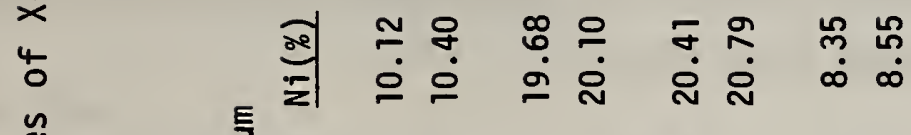

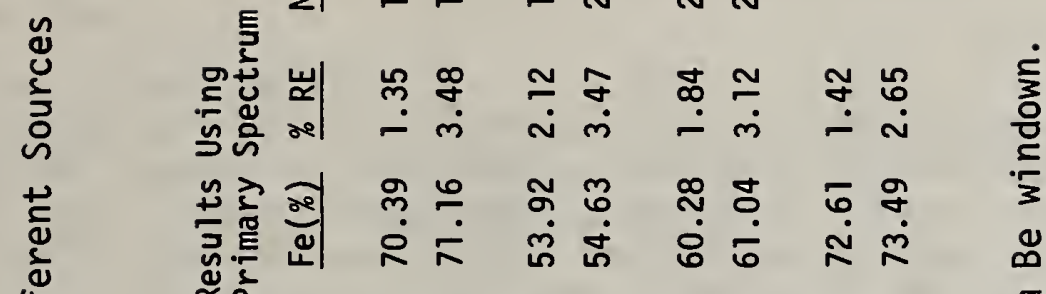

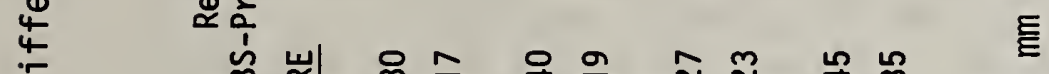

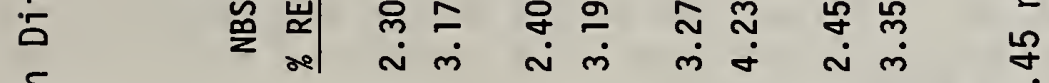

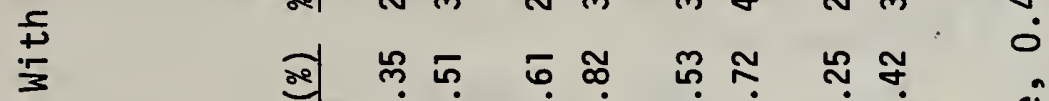

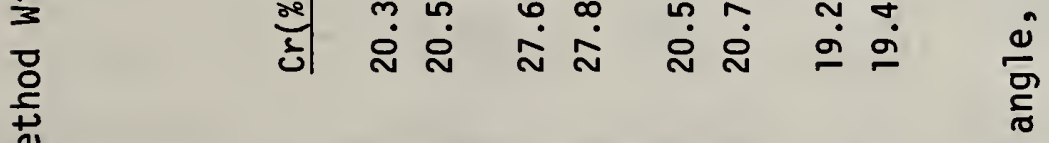

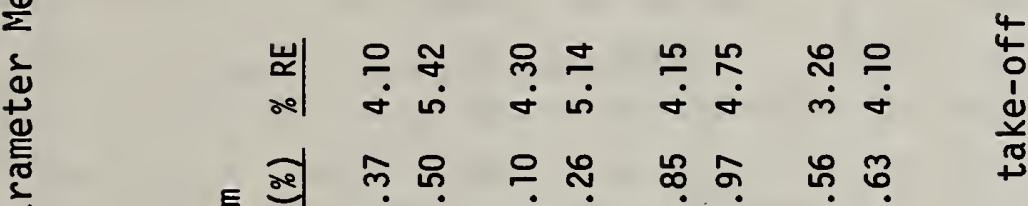

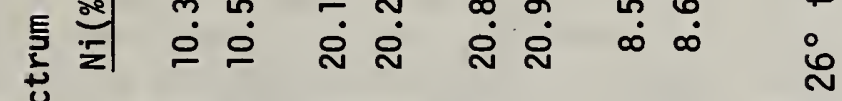

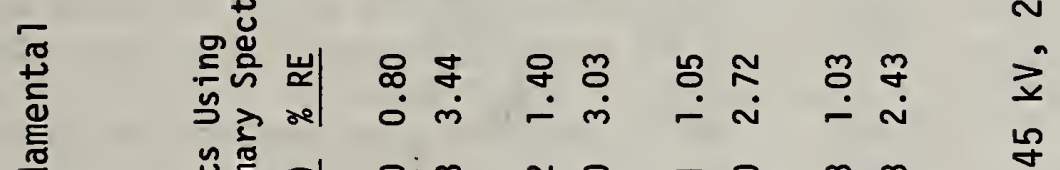

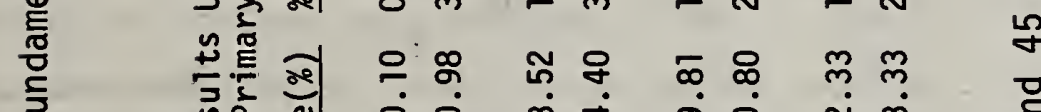

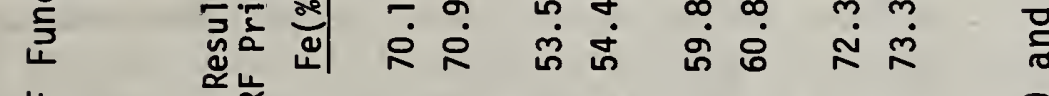

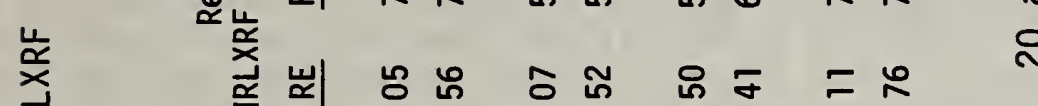

물

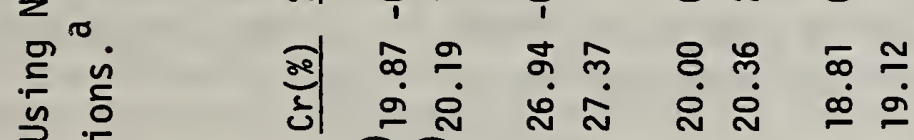

$\stackrel{5}{2}$

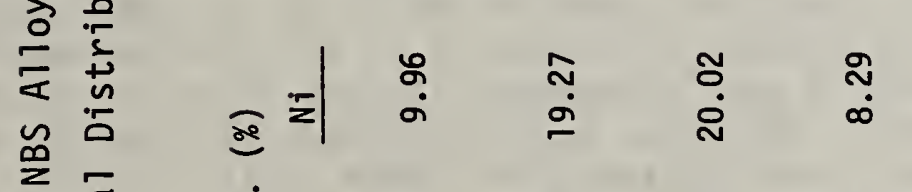

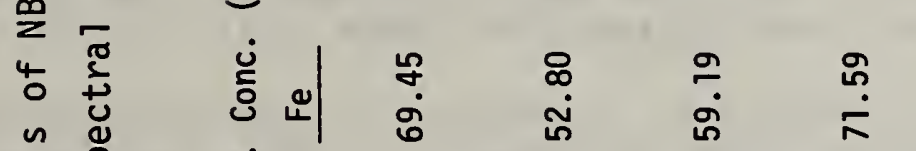

in

ভ্்

疍 $\frac{8}{5}$

s

$\stackrel{\infty}{\infty}$

ஜั

$\stackrel{9}{\infty}$

$\dot{q}$

$\frac{0}{\circ}$

离.

i

స్ల

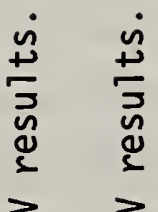

芯 咅 章

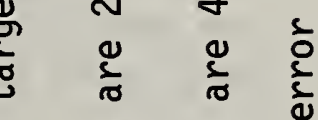

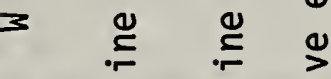

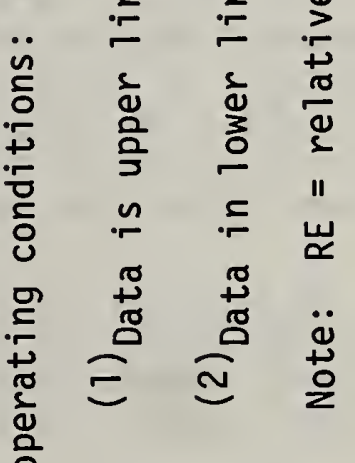


24. Comparison of Theoretical $\alpha$-Coefficient Fundamental Parameter Method with NRLXRF for Quantitative X-ray Analysis (15330)

\section{P.A. Pella, Y. Tao}

Fundamental parameter methods for quantitative $x$-ray fluorescence analysis make use of equations derived from first principles and contain physical constants, absorption coefficients, primary excitation spectra and geometric factors. They can be used to predict the x-ray intensity emitted by an element in any prescribed specimen composition after correction of the matrix effect. The correction term can be visualized as the sum of all coefficients of every element $j$ of the matrix, each one taken in a proportion equal to the concentration $C_{j}$. Expressed mathematically

$$
C_{i}=K_{i} I_{i}\left(1+\Sigma_{j} \alpha_{i j} C_{j}\right)
$$

where each $\alpha_{i j}$ coefficient represents the quantification of all matrix effects due to element $j$ on analyte $i$, and thus has physical meaning. Therefore, the calculation of $C_{i}$ from the measured intensity $I_{i}$ is possible provided that all values of the alpha coefficients are accurately known. Alpha coefficients can be reliably determined experimentally using regression methods, but this depends on the availability of sufficient standards which is often not practicable. A simpler approach is to compute theoretical alpha coefficients from fundamental parameter expressions. Unfortunately, for certain sample types, especially alloys, $\alpha_{i j}$ is not a constant over the entire concentration range of the analyte from 0 to 100 percent. Over the past few years a comprehensive alpha model has been proposed by Lachance [1] which employs three alpha coefficients in addition to so-called "crossed" coefficient term which are calculated to describe the overall matrix correction from the 0 to 100 percent analyte concentration range in accordance with fundamental theory. During the past year we have evaluated this model by analyzing a number of sample types such as alloys, pressed powders and fused minerals and compared the results with those obtained by another fundamental parameter program called NRLXRF. The alpha algorithm was obtained from Lachance in the form of a computer program which we modified somewhat and is on our NBS computer facility. In Table 47 is a summary of the analysis of selected samples of each type. The results are in good agreement with the accepted values and also consistent with NRLXRF. The alpha algorithm presently employs measured primary $x$-ray tube spectral distributions for sample excitation.

We are also currently working to incorporate our proposed NBS algorithm for calculating the primary spectral distribution in this quantitative analys is scheme, so that we can test its relative accuracy with NBS standard reference materials during the next year.

Reference

[1] Lachance, G.R., Claisse, F., Adv. X-ray Anal., 23, 87 (1980). 
Table 47. Comparative Analysis of Selected Samples.

Sample Type

Elements

\begin{tabular}{cllllll} 
Stainiess Steel & Method & Cr & Fe & Co & Ni & Mo \\
\cline { 2 - 7 } 1198 & a & 12.9 & 36.2 & 0.70 & 40.1 & 6.08 \\
& b & 12.80 & 36.19 & 0.72 & 40.21 & 6.12 \\
& C & 13.08 & 36.36 & 0.70 & 40.29 & 6.08
\end{tabular}

Compounds

Fused Minerals

\begin{tabular}{|c|c|c|c|c|c|}
\hline $\mathrm{Al}_{2} \mathrm{O}_{3}$ & $\mathrm{SiO}_{2}$ & $k_{2} 0$ & $\mathrm{CaO}$ & $\mathrm{TiO}_{2}$ & $\mathrm{Fe}_{2} \mathrm{O}_{3}$ \\
\hline 37.67 & 54.68 & 1.37 & 0.27 & 2.21 & 2.38 \\
\hline 37.91 & 55.66 & 1.60 & 0.21 & 2.18 & 2.14 \\
\hline 38.80 & 55.59 & 1.62 & 0.26 & 2.13 & 2.26 \\
\hline
\end{tabular}

Pressed Cement

Powder

$\underline{\mathrm{MgO}} \quad \mathrm{Al}_{2} \mathrm{O}_{3} \quad \mathrm{SiO}_{2} \quad \mathrm{SO}_{3} \quad \mathrm{CaO} \quad \mathrm{Fe}_{2} \mathrm{O}_{3}$

634

$\begin{array}{lllllll}\text { a } & 3.30 & 5.21 & 20.73 & 2.21 & 62.58 & 2.84 \\ \text { b } & 3.12 & 4.97 & 20.77 & 2.14 & 62.56 & 2.71 \\ \text { c } & 3.15 & 4.93 & 20.67 & 2.23 & 62.26 & 2.64\end{array}$

NOTE: under method $a=$ Chemical values in weight percent.

b = COAL (Comprehensive Lachance Algorithm).

$c=$ NRLXRF. 
25. Final Measurements and NBS Certification of Thin-Glass Standard Reference Samples for X-ray Spectrometry and Microanalysis (15601)

P. A. Pella, E. B. Steel

$X$-ray fluorescence spectrometry is a versatile and rapid method for the multi-element analysis of many substances in the form of deposits on a filter, mesh or membrane, or as thin pressed pellets. Collected airborne particulate matter, particulates in waste water, and geochemical samples are just a few examples of this type. The absence of any sizable interelement effects in such "thin samples" makes possible a linear instrument response to element mass-per-unit-area for most elements except low atomic number elements such as aluminum and silicon. For such applications, thin standard reference materials are required for calibration of instrument response where the element mass loading and uniformity across the film diameter are known within acceptable limits. Several types of thin standard materials are available from commercial sources, but the accuracy of these materials is not traceable to any NBS standard as yet. Our efforts at NBS over the past several years have been to provide a reference material that has several advantages over presently available commercial ones. The method of focused-ion beam sputtering was investigated and found to be ideal for producing thin multielement standards for this purpose. Our proposed standards consist of several elements dissolved in a glassy matrix.

In a previous report [1], we described the status of our thin-glass standards program. Essentially we found that in one set of thin glass films the radial inhomogeneity across the film diameter was severe, especially for lead $($ e.g. $\sim 25 \%)$. This year, these problems were overcome through the use of a planetary substrate holder designed and fabricated at NBS. Electron probe microanalys is of films fabricated using the planetary holder where the electron beam was stepped in $1 \mathrm{~mm}$ increments across the film diameter showed that the elements were distributed at random across the film with no observable systematic trends, in marked contrast with previous samples when only sample rotation was used. For example, the relative standard deviation based on 35 measurements across the film diameter was 1.5 percent for lead and 5 percent for zinc. The combination of sample rotation and planetary motion of each sample along its own axis is the key to preparing high quality uniform films independent of elemental composition. Several in-house methods are currently being employed for NBS certification of both sets of thin glass films. These include ICP, $A A$, INAA, and IDMS. In addition, selected samples from both sets have been submitted to five cooperating laboratories for analys is by energy-dispersive $x$-ray spectrometry. A protocol for the analys is has been established at NBS according to a well-defined statistical plan. We expect to receive and compile all analytical data by the end of the fiscal year, and hope to have both sets NBS-certified and available for sale by January 1, 1983.

References

[1] NBSIR-82-2620, page 209. 
C. Outputs and Interactions

(Gas and Particulate Science Division)

1. Publications

Bright, D. S. and Fletcher, R. A., "New Portable Ambient Aerosol Sampler," Journal of Am. Ind. Hygiene Assoc. 44 (7) 523 (in press).

Bright, D. S., Myklebust, R. L. and Newbury, D. E., "Stereo Presentation of Monte Carlo Electron Trajectory Simulations," (in preparation).

Cadoff, B. C. and Hodgeson, J., "Passive Sampler for Ambient Levels of Nitrogen Dioxide, Anal. Chem. (1983).

Currie, L. A., "Contemporary Particulate Carbon," in: Particulate Carbon: Atmospheric Life Cycle, G. T. Wolff and R. L. Klimisch, eds., General Motors Research Laboratories, (1982).

Schell, W. R., Swanson, J. R. and Currie, L. A., "Anthropogenic Changes in Organic Carbon and Trace Metal Input to Lake Washington," Radiocarbon 29, 621 (1983).

Currie, L. A., Klouda, G. A., Continetti, R. E., Kaplan, I. R., Wang, W. W., Dzubay, J. G., and Stevens, R. K., "Of the Orgin of Carbonaceous Particles in American Cities: Results of Radiocarbon "Dating" and Chemical Characterization," Radiocarbon 25, 603 (1983).

Currie, L. A., Gerlach, R. W., Klouda, G. A., Ruegg, F. C. and Tompkins, G. B., "Miniature Signals and Miniature Counters: Accuracy Assurance via Microprocessors and Multiparameter Control Techniques," Radiocarbon (in press).

Voorhees, K. J., Schulz, W. D., Currie, L. A. and Klouda, G. A., "An Investigation of the Insoluble Carbonaceous Material in Airborne Particulates from Vehicular Emissions," (submitted to Anal. Chem).

Randall, R., Schjoldager, J., Currie, L. A., Moller, M., Hassenoff, E., Klouda, G. A. and Afheim, I., "Air Pollution from Wood Combustion: Ambient Air Measurements in Elverum, Norway,: (submitted to Environmental Science \& Technology).

Currie, L. A., Gerlach, R. W., Lewis, C. W., Balfour, W. D., Cooper, J. A., and Dattner, S. L., "Interlaboratory Comparison of Source Apportionment Procedures: REsults for Simulated Data Sets," (submitted to Atmospheric Environment).

Kagann, R. H. and Elkins, J. W., "Absolute Band Strengths of Halocarbons F-11 and F-12 in the 8-10 $\mu \mathrm{m}$ Region," J. of Geophysical Res., 88 (2) 1427-1432 (1983).

Fried, A. and Lin, H-B., "Application of Photothermal Techniques for Trace Detection of $\mathrm{NO}_{2}$ in the Troposphere," NASA Workshop Publication (1983). 
Fried, A. and Berg, W., "Photoacoustic Detection of $\mathrm{HCl}$, Opt. Lett., $\underline{8}$ (3) 160 (1983).

Fried, A., Sams, R. and Berg, W., "Detection of $\mathrm{HCl}$ at the Sub-Parts-Per Billion Level Using Tunable Diode Laser Absorption," Proceedings of the Society of Photo-Optical Instrumentation Engineers: Tunable Diode Laser Development and Spectroscopy Applications, 438 (1983).

Fletcher, R. A. and Bright D. S., "NBS Portable Ambient Particulate Sampler," Final Report for the Environmental Protection Agency, NBSIR-2561 (1983).

Fletcher, R. A., "Chemical Surface Analysis on Particles Using the LAMMA," J. Aerosol Science and Technology, 2, 156 (1983).

Solomon, P. A., Moyers, J. L. and Fletcher, R. A., "A High Volume Dichotomous Virtual Impactor for the Fractionation and Collection of Particles According to Aerodynamic Size," J. Aerosol Science and Technology (in press).

Fletcher, R. A., Chabay, I., Weitz, D., and Chung, J., "Laser Desorption Mass Spectroscopy of Surface-Adsorbed Molecules," Chem. Phys. Letts. (submitted).

Gerlach, R. W., Currie, L. A. and Lewis, C. W., "Review of the Quail Roost II Receptor Model Simulation Exercise," in Proc. APCA Speciality Conference on Receptor Models Applied to Contemporary Air Pollution (1983).

Hughes, E. E., "Nitric Oxide in Nitrogen Appendix D to "A Procedure for Establishing Traceability of Gas Mixtures of Certain National Bureau of Standards SRM's," NBS EPA Joint Report EPA-600/7-81-010, (1981).

Ibid, "Sulfur Dioxide in Nitrogen," Appendix E

Ibid, "Propane in Air or Nitrogen," Appendix F

Hughes, E. and Myers, R., "Measurement of the Concentration of Sulfur Dioxide, Nitrogen Oxides and Ozone in the National Archives Bldg." NBSIR (In review).

KTouda, G. A., Currie, L. A., Gerlach, R. W., Continetti, R. E. and Tompkins, G. B., "Estimating the Impact of Atmospheric Carbonaceous Particulates on Urban and Rural Enviornments by Radiocarbon Measurements," Proc. Residential Wood and Coal Combustion Specialty Conference of Air Pollution Control Association 189-206 (1982).

Marinenko, R. B., Steel, E. B., Smal1, J. A., and Newbury D. E., "Standards for Microbeam Analysis, Bul1. of the Electron Microscopy Soc. of Am., 13 (1) 67 (1983). 
Liu, B. Y. H., Pui, D. Y. H., McKenzie, R. L., Argawal, J. K., Janicke, R., Poh1, F. G., Preining, O., Reisch1, G., Szymanki, W., and Wagner, P. E., "Intercomparison of Different "Absolute" Instruments for Measurement of Aerosol Number Concentration," J. Aerosol Science 13, 429-450 (1982).

McKenzie, R. L., Bright D. S., Fletcher, R. A., and Hodgeson, J. A., "Development of a Personal Exposure Monitor for Two Sizes of Inhalable Particulates," Environ. Internat. 8, 229-233 (1982)

Camp, D. C., Stevens, R. K., Cobourn, W. G., Husar, R. B., Collins, J. F., Huntzicker, J. D., Husar, J. D., Jaklevic, J. M., McKenzie, R. L., Tanner, R. L., and Tesch, J. W., "Intercomparison of Concentration Results from Fine Particle Sulfur Monitors," Atmos. Environ. 16, 911-916 (1982).

Liu, B., Pui, D., McKenzie, R. L., Argawal, J. K., Pohl, F. G., Preining, 0., Reischl, G., Szymanski, W., and Wagner, P. E., "Measurement of KelvinEquivalent Size Distributions of Well-Defined Aerosols with Particle Diameters above $13 \mathrm{~nm}, "$ (submitted to Aerosol Science And Technology)

Myklebust, R. L., Pella, P. A. and Thorne, B., "An Overview of EXFNBS - A Data Reduction Procedure for Energy-Dispersive XRF with Secondary Target Excitation," X-Ray Spectrometry, $\underline{2}$ (4) 170-172 (1982).

Myklebust, R. L., "An Evaluation of X-Ray Loss Due to Electron Backscatter", 10th International Cong. on X-Ray Optics and Microanalysis, Toulouse, France (1983).

Kelley, R. D., Candela, G. A., Madey, T. E., Newbury, D. E. and Schehl, "Surface and Bulk Analys is of a Deactivated Raney Nickel Methanation Catalyst," J. Catalysis $80,235-248$ (1983).

Dunn, P. J. and Newbury, D. E., "Loudonite: A New Zirconium Silicate from Virginia" Canadian Mineralogist 21, 37-40 (1983).

Newbury, D. E., "Recent Developments in Microbeam Analysis," Proceedings of the Electron Microscopy Society of Southern Africa, 12 5-8 (1982).

Joy, D. C., Newbury, D. E., and Myklebust, R. L., "The Role of Fast Secondary Electrons in Degrading Spatial Resolution in the Analytical Electron Microscope," J. Microscopy 128 (1982).

Newbury, D. E., Myklebust, R. L., Romig, A. D., Jr., and Bieg, K. W., "Determinatio of the Depth Distribution of X-ray Generation, $\emptyset(\mathrm{pz})$, for Tilted Specimens of Au in the Analytical Electron Microscope," Microbeam Analysis/1983 (San Francisco Press, 1983)

Butrymowicz, D. B., Piccone, T. J., Manning, J. R., and Newbury, D. E., "Color Metallography of Diffusion -- Induced Grain Boundary Migration in Cu-Zn and Cu-As Alloy, (submitted to Intl. J. Metallography). 
Taylor, H. F. W. and Newbury, D. E., "Calcium Hydroxide Distribution and Calcium Silicate Hydrate Composition in Tircalcium Silicate and betaDicalcium Silicate Pastes," (submitted to Cement and Concrete Research).

Williams, D. B. and Newbury, D. E., "Recent Advances in the Electron Microscopy of Materials," (submitted to Advances in Electronics and Electron Physics).

Small, J. A. and Steel, E. B., "Reference Filter Standards for the Analys is of Chrysotile Asbestos in Non-Workplace Environments (to be published in the Proc. of the 4 th Intl. Conf. on Dust Measuring Technique and Strategy, Edinbourgh, Scotland 1982).

Small, J. A., "Limits of Visibility for Chrysotile Asbestos in the Scanning Electron Microscope, (to be published in the Proc. of the 4th Intl. Conf. on Dust Measuring Technique and Strategy, Edinbourgh, Scotland 1982).

Sma11, J. A., Newbury D. E., and Myklebust, R. L., "The Visibility of Asbestos Fibers in the Scanning Electron Microscope, Microbeam Analys is (1982) San Francisco Press, Ed.

Sma11, J. A., Norris, J. A., and McKenzie, R. L., Fabrication of Metals and Metals Alloys as Particle Standards, Microbeam Analysis (1983) San Francisco Press, Ed.

Small, J. A. and Newbury, D. E., "The Visibility of Serpentine and Amphibole Asbestos in the Scanning Electron Microscope and the Scanning Transmission Electron Microscope, (submitted to the $\underline{\mathrm{J}}$. of Microscopy).

Small, J. A, Newbury, D. E., Leigh, S. D., and Myklebust, R. L., "Development of an Analytical Expression for the Generation of Bremsstrahlung in Pure Element Targets (submitted).

Small, J. A., Steel, E. B. and Leigh, S. D., "The Development of Filter Standards for the Analysis of Chrysotile Asbestos at Non-Workplace Environments (submitted to Anal. Chem.).

Conzemius, R. J., Simons, D. S., Shankai, Z., and Byrd, G. D., "Laser Spectrometry of Solids: A Bibliography 1963 - 1982," Microbeam Anaylsis (1983), R. Gooley, Ed.

Finkelman, R. B., Simons, D. S., and Dulong, F. T., "Semi-Quantitaitve Ion Microprobe Mass Analyses of Mineral-rich Particles in the Upper Freeport Coal," (accepted for publication in Coal Geology(1983).

Simons, D. S., "Isotopic Analys is with the Laser Microprobe Mass Analyzer," (accepted for publication in the Intl. J. of of Mass Spectrometry and Ion Physics (1983).

Stallard, B. R., Champion, P. M., Callis, P. R., and Albrecht, A. C., "Some Advances in Calculating Raman Excitation Profiles by Means of the Transform Theory," J. Chem. Phys. 78712 (1983). 
Lewis, A., Grubb, S. and Stallard, B. R., "Raman Difference Studies of Isotopically Labeled Bacterial Rhodopsin," (submitted to Biochemistry).

Stallard, B. R., Champion, P. M. and Albrecht, A. C., "The Inverse Transform in Resonance Raman Scattering," (submitted to J. Chem. Phys.).

Pella, P. A., Kingston, H. M. Sieber, J. R. and Feng, L., "Effect of Sample Dissolution Procedures on X-ray Spectrometric Analysis of Biological Materials," Anal. Chem. 551193 (1983).

Pella, P. A., Feng, L., and Sma11, J. A., "An Analytical Algorithm for Calculation of Spectral Distributions of X-Ray Tube Emission for Quantitative X-Ray Spectrometry," (submitted for publication)

Zielinski, W. L., "A Summary of Activities Concerning Coal Gasification and Liquidfaction at the National Bureau of Standards $1976-1980 . "$ EPA-600/7-82-002 (1982).

Zielinski, W. L., "Recent Research Trends in Environmental Measurements," Trends in Analytical Chemistry $\underline{2}$ (5) XI-XII (1983).

\section{Talks}

Cadoff, B. C., "Applications of FTIR and Reaction Modeling to Problems of Instability of NO in $\mathrm{N}_{2}$ Gas Mixtures," 25th Rocky Mountain Conference, Denver, C0 $(8 / 15 / 83)$.

Cadoff, B. C., "Design and Construction of Constant Flow Sampling Systems," 1983 Federation of Analytical Chemistry and Spectroscopy Societies (FACCS), Philadelphia, PA (9/29/83).

Currie, L. A., "Identification \& Quantification of the Sources of Ambient Particulate Matter by Receptor Oriented Techniques," APCA Specialty Meeting, Danvers, MA (10/18/82).

Currie, L. A., "The Analytical Measurement Process: Assumptions and Rea 1istic Measures of Uncertainty," Symposium on Improving the Analytical Chemistry/Regulatory Interface, NBS, Gaithersburg, MD (10/20/82).

Currie, L. A., "Radiocarbon Dating of Atmospheric Particles," Oak Ridge National Labs, Oak Ridge, TN (1/10/83).

Currie, L. A., "The Many Dimensions of Detection in Trace Analysis," ACS Symposium The Role of Chemonetrics in Pesticide Analysis, Seattle, WA $(3 / 23 / 83)$.

Currie, L. A., "Detection and Quantitation in Nuclear and Analytical Chemistry," Colorado State University, Fort Collins, CO (6/3/83).

Dorko, W. and Fried, A., "Certification of High Concentration Level Nitrogen Dioxide in Air Gas Standards," 185th American Chemical Society National Meeting, Seattle, WA (3/20-25/83). 
Downing, R. G., "Neutron Depth Profiling at the National Bureau of Standards," Sixth International Conference on Ion Beam Analysis, Arizona State University, Tempe, AZ (5/23/83).

Elkins, J. W., "Gas Analysis by Fourier Transform Infrared Spectroscopy," 2nd NBS-ADABSE Analytical Chemistry Workshop, (10/25-26/82).

Elkins, J. W., Sams, R. L., Kagann, R. H., "Infrared Band Strengths Measurements for Halocarbons $\mathrm{F}-11, \mathrm{~F}-12, \mathrm{CF}_{4}$, and $\mathrm{CH}_{3} \mathrm{Cl}$ in the "Atmospheric Windows", American Geophysical Union, Baltimore, MD $(6 / 2 / 83)$.

Fletcher, R. A., "Chemical Surface Analysis on Particles Using the LAMMA," American Association for Aerosol Research Conference, Washington, D.C. $(4 / 83)$.

Fletcher, R. A., "Laser Desorption of an Organic Monolayer from a 50A Thin Silver Film," Texas Symposium on Particle Induced Desorption Mass Spectrometry, College Station, TX (5/83).

Marinenko, R. B., Blackburn, D. H., "Glasses for Microanalysis: New NBS Standard Reference Materials," 10th International Congress on X-ray Optics and Microanalysis, Toulouse, France $(9 / 83)$.

Mitchell, G. D., "Origin of Entropies in Polymeric Permeation Devices," Southern University, Baton Rouge, LA (3/11/83).

Mitchell, G. D., "The Metrology of Gases: High Accuracy $\mathrm{CO}_{2}$ Analysis," Southern University, Baton Rouge, LA (3/11/83).

Mitchell, G. D., "Cross-Coupled Interaction of Thermal, Electrical, Mechanical, and Chemical Parameters of Polymeric Permeation Devices," 185th American Chemical Society National Meeting, Seattle, WA $(3 / 20-25 / 83)$.

Mitchell, G. D., "Primary and Secondary Contribution to Entropy-Related Parameters in Polymeric Permeation Devices," Norfolk State College, Norfolk, VA $(4 / 29 / 83)$.

Mitchell, G. D., "Cross-Coupled Interaction of Thermal, Electrical, Mechanical, and Chemical Parameters of Polymeric Permeation Devices," National Meeting, National Organization of Black Chemists and Chemical Engineers, Knoxvilie, TN $(5 / 27 / 83)$.

Newbury, D. E., "Ion Microscopy for Compositional Mapping," NIH Biomedical Instrumentation Symposium, Washington, DC $(11 / 17 / 83)$.

Newbury, D. E., "Electron Probe Microanalysis of Failures in Aluminum Wire Electrical Junctions," University of Port Elizabeth, South Africa $(11 / 29 / 82)$.

Newbury, D. E., "Recent Developments in Microbeam Analysis," Electron Microscopy Society of Southern Africa, Rhodes University, Grahamston, South Africa (12/21/82). 
Newbury, D. E., "Electron Beam Microanalysis of Particles and Rough Surfaces," Department of Metallurgy, Univeristy of CapeTown, South Africa (12/6/82).

Newbury, D. E., "Microbeam Mass Spectrometry," Anglo American Research Labs, Johannesburg, South Africa (12/8/82).

Newbury, D. E., "Quantitative Electron Probe Microanalysis With the Energy Dispersive X-ray Spectrometer," Physics Department, University of Witswatersrand, Johannesburg, South Africa (12/8/82).

Newbury, D. E., "Mass Spectrometry at High Spatial Resolution," Commonwealth Science and Industrial Research, Pretoria, South Africa (12/10/82).

Newbury, D. E., "Accuracy of Microprobe Analysis," Workshop on Evaluation of Binary Alloy Phase Diagrams, NBS, Gaithersburg, MD (1/31/83).

Newbury, D. E., "Microprobe Analysis Methods for Quantitative Materials Characterization," Alloy Phase Diagrams Conference, NBS, Gaithersburg, MD $(3 / 83)$.

Newbury, D. E., "Microbeam Analys is for Particle Characterization," International Congress on X-ray Optics and Microanalysis, Toulouse, France $(9 / 5 / 83)$.

Pella, P. A., "X-ray Spectrometric Analys is of NBS-SRM Bovine Lever and Pine Needles After Separation and Preconcentration," ACS, Division of Analytical Chemistry, Seattle, WA (3/23/83).

Pella, P. A., "Approach to Calculation of X-ray Tube Spectral Distributions for X-ray Analys is," 23rd Colloquium Spectroscopicum Internationale, Ams terdam, The Netherlands $(6 / 28 / 83)$.

Pella, P. A., "The Application of Chelex-100 and SA-2 Resins to the Preconcentration of Elements Prior to XRF Analysis," Center for Radiochemistry and Activation Analys is in Pavia, Italy (7/5/83).

Pella, P. A., "The Application of Chelex-100 and SA-2 Resins to the Preconcentration of Elements Prior to XRF Analysis," Institute for Analytical, Micro-, and Radiochemistry, Technical University of Graz, Graz, Austria $(7 / 8 / 83)$.

Pella, P. A., "The Application of Chelex-100 and SA-2 Resins to the Preconcentration of Elements Prior to XRF Analysis," Institute for Radiochemistry, Nuclear Research Center, Karlsruhe, West Germany (7/12/83).

Simons, D. S., "Isotopic Analysis with the Laser Microprobe Mass Analyzer," Microbeam Analysis Society, Washington, DC (8/82).

Simons, D. S., "Microanalysis of Particles by Mass Spectrometry," University of North Carolina, Chapel Hill, NC (11/1/82). 
Simons, D. S., "The Laser Microprobe Mass Analyzer in Biomedical Research," NIH Biomedical Instrumentation Symposium, Washington, DC $(11 / 17 / 82)$. Invited

Simons, D. S., "Quantitative Compositional Mapping," Special Symposium on NBS Research, National Bureau of Standards, Gaithersburg, MD (1/10/83). Invited

Simons, D. S., "Microanalys is Research at NBS," Physics Colloquium, Catholic University of America, Washington, DC (4/21/83). Invited

Simons, D. S., "Isotopic Analys is by LAMMA," Workshop on Laser Microprobe Mass Analysis, Research Institute Borstel, Borstel, W. Germany (9/2/83).

Sma11, J. A., "Analysis of Micrometer and Submicrometer Particles," Metropolitan Chapter, Microbeam Analysis Society, Paramus, NJ $(4 / 28 / 83)$.

Sma11, J. A., "Quantitative Particle Analysis in Electron Beam Instruments," Metropolitan Chapter, Microbeam Analysis Society, Paramus, NJ $(4 / 28 / 83)$.

Small, J. A., "Particle Analysis: A Tutorial," Scanning Electron Microscopy Meeting, Detroit, MI (4/83).

Small, J. A., "Fabrication of Metals and Metal Alloys as Particle Standards," Microbeam Analys is Society Meeting, Pheonix, AZ (8/83).

Small, J. A., "Limits of Visibility for Chrysotile Asbestos in the Scanning ning Electron Microscope," Interantional Conference on Dust Measuring Techniques and Strategy, Edinbourgh, Scotland (9/82). Invited

Small, J. A., "Reference Filter Standards for the Analys is of Chrysotile Asbestos in Non-workplace Environments," International Conference on Dust Measuring Techniques and Strategy, Edinbourgh, Scotland (9/82). Invited

Sma11, J. A., "Microanalytical Techniques for the Analysis of Particles," Microbeam Analys is Society Metropolitan Chapter Meeting, Paramus, NJ (4/83). Invited

Sma11, J. A., "Quantitative Analysis of Individual Particles with Electron Beam Instruments," Microbeam Analys is Society Metropolitan Chapter Meeting, Paramus, NJ 94/83). Invited

Small, J. A., "Visibility of Asbestos Fibers in the Scanning Electron Microscope," Microbeam Analys is Society Meeting, Pheonix, AZ (8/83). Invited

Zielinski, W. L., Jr., "Recent Developments in Environmental Standard Reference Materials to Assist Data Quality in Environmental Measurements," 1972 Eastern Analytical Symposium, New York, NY (11/17-19/82). 
Zielinski, W. L., Jr., Ulma, G. A., Miller, M., and Wasik, S. P., "GLC Separation Behavior of Polychlorinated Biphenyl Isomers Between a Neumatic Liquid Crystal Stationary Phase and a Non-Polar Hydrocarbon Phase (Apolane-87)," 1983 Pittsburgh Conference on Analytical Chemistry and Applied Spectroscopy, Atlantic City, NJ (3/7-12/83).

Zielinski, W. L., Jr. and Martire, D. E., "A Stationary Phase-Independent Predictive Scheme for Retention Data of Solutes in Gas-Liquid Chromatography," 1983 Pittsburgh Conference on Analytical Chemistry and Applied Spectroscopy, Atlantic City, NJ (3/7-12/83).

\section{Committee Assignments}

Harry Rook

Member, ASTM D-22 Sampling and Analys is of Atmospheres

Member, ASTM D-22.05 Calibration

Member, ASTM D-22.07 Precision and Accuracy of Methods

Member, Office of Water Data Coordination, Committee $5 \mathrm{~F}$

Raymond McKenzie

Member, ASTM D-22 Sampling and Analys is of Atmospheres

Member, ASTM D-22.03 Monitoring Ins trumentation

Member, ASTM D-22.05 Calibration

Member, ASTM D-22.07 Precision and Accuracy of Methods

Member, ASTM E-29 Particle Size Measurement

David Bright

Member, ASTM E-29 Particle Size Measurement

Member, ASTM SC. 10 Liquid Particle Measurement

Lloyd Currie

Member, Intersociety Committee - Methods of Air Sampling and Analysis

Member, Model Validation Subcommittee - Receptor Modeling Workshop

Member, CAC Computer Utilization Committee

William Dorko

Member, American Chemical Society, Washington Section, Board of Managers

Edgar Etz

Member, ASTM D-22 Methods of Sampling and Analysis of Atmospheres

Ernest Hughes

Member, ISO/TC 158 Gas Analysis

Member, ISO/TC 158/SC-1 Methods for Preparation and Definition of Gas Mixtures for Calibration

Member, ISO/TC 158/WG-2 Transfer Lines and Sampling

Member, ISO/TC 158/WG-3 Evaluation of the Characteristics of Analysis

Member, ISO/TC 158/WG-4 Analysis of Natural Gas

Member, ISO/TC 158/WG-1 Terminology 
Robert Myklebust

Member, Microbeam Analysis Society (National Council)

Member, ASTM E-2 Emission Spectroscopy

Member, ASTM E-2.04 Standard Reference Materials

Da le Newbury

Member, Microbeam Analysis Society

Member, Technical Program Committee

Member, ASTM E-42 Surface Analysis

Member, Journal of Microscopy, Editorial Review Board

Member, Scanning, Editorial Review Board

Member, J. Trace and Microprobe Techniques, Editorial Review Board

Member, Sigma $X i$

Peter Pella

Member, ASTM E-2 Emission Spectroscopy

Member, ASTM E-2.02 Statistics, Calibration and Standardization

Gerald Sleater

Member, ASTM D-22 Sampling and Analysis of Atmospheres

Member, ASTM D-22.06 Source Sampling

Member, ASTM D-22.07 Precision and Accuracy of Methods

Walter Zielinski

Member, Interagency Committee on $\mathrm{CO}_{2}$ and $\mathrm{Cl}$ imate

Member, Stratospheric Ozone Protection

Member, Indoor Air Quality

Member, ASTM D-22 Sampling and Analysis of Atmospheres

Member, Center for Analytical Chemistry Colloquium Committee

4. Other

a. Seminars

April 19, 1983

- Jorgen Schjoldager, Norwegian Inst.

for Air Research, "The Presence of

Photochemical Oxidants in Scandinavia"

(NBS Sponsor: L. Currie)

March 22, 1983

- Joseph P. Krasnec, Demaray Scientific

Inst., "Quality Assurance for Gaseous

SRM Work" (NBS Sponsor: J. Elkins)

June 28,1983

- Richard Linton, Univ. of North Carolina, "Dynamic Secondary Ion Mass Spectrometry of Organic Matrices -- Applications to the Biological and Polymer Sciences" (NBS Sponsor: D. Simons) 
b. Standard Reference Materials

\section{$X$-ray Fluorescence}

Steel Alloys

C2400 and C2400A

C2401 and C2401A

C2402 and C2402A

C1290 and C1290A

C1291 and C1291A

C1292

Brass A7loys

\section{C2403}

C2404

C2405

C2406

$\mathrm{C} 2408$

C2409

$\mathrm{C} 2410$

other

$0.5 \%$ Austenite in Ferrite

Borosilicate Glass

Glass Sand

Powdered Phosphate Rocks

Powdered Mineral Samples (drilling mud, potential SRM) 
1625 Sulfur Dioxide Permeation Tube,

1626 Sulfur Dioxide Permeation Tube,

1629 Nitrogen Dioxide

1911 Benzene

1658a Methane in Air $\mathrm{CH}_{4}$

1659a Methane in Air $\mathrm{CH}_{4}$

1660a Methane-Propane in Air $\mathrm{CH}_{4}$

$$
\mathrm{C}_{3} \mathrm{H}_{8}
$$

$1 \mathrm{ppm}$

$10 \mathrm{ppm}$

$4 \mathrm{ppm}$

$1 \mathrm{ppm}$

166la Sulfur Dioxide in Nitrogen $\mathrm{SO}_{2}$

1662a Sulfur Dioxide in Nitrogen $\mathrm{SO}_{2}$

1663a Sulfur Dioxide in Nitrogen $\mathrm{SO}_{2}$

1664a Sulfur Dioxide in Nitrogen $\mathrm{SO}_{2}$

1683a Nitric Oxide in Nitrogen NO

1684a Nitric Oxide in Nitrogen NO

1686b Nitric Oxide in Nitrogen NO

1693 Sulfur Dioxide in Nitrogen $\mathrm{SO}_{2}$

1694 Sulfur Dioxide in Nitrogen $\mathrm{SO}_{2}$

$500 \mathrm{ppm}$

$1000 \mathrm{ppm}$

$1500 \mathrm{ppm}$

$2500 \mathrm{ppm}$

1805 Benzene in Nitrogen $\mathrm{C}_{6} \mathrm{H}_{6}$

1806 Benzene in Nitrogen $\mathrm{C}_{6} \mathrm{H}_{6}$

$50 \mathrm{ppm}$

$100 \mathrm{ppm}$

$500 \mathrm{ppm}$

$50 \mathrm{ppm}$

$100 \mathrm{ppm}$

1808 Perchloroethylene in $\mathrm{N}_{2}$

1809 Perchloroethylene in $N_{2}$

$0.25 \mathrm{ppm}$

$10 \mathrm{ppm}$

$0.25 \mathrm{ppm}$

$10 \mathrm{ppm}$

2612a Carbon Monoxide in Air CO

2613a Carbon Monoxide in Air CO

$10 \mathrm{ppm}$

$20 \mathrm{ppm}$

2614a Carbon Monoxide in Air CO

$45 \mathrm{ppm}$

AU.8. GOVEANMENT PAINTINO OFFICE: $1983 \quad 421997 \quad 5254$ 
SHEET (See in structions)

4. TITLE AND SUBTITLE

Technical Activities, 1983, Center for Analytical Chemistry

5. AUTHOR(S)

H. S. Hertz, R. A. Velapoldi, J. K. Taylor

6. PERFORMING ORGANIZATION (If joint or other than NBS, see instructions)

7. Contract/Grant No.

MATIONAL BUREAU OF STANDARDS

DEPARTMENT OF COMMERCE

WASHINGTON, D.C. 20234

9. SPONSORING ORGANIZATION NAME AND COMPLETE ADDRESS (Street, City, StOTe, ZIP)

Same as above.

10. SUPPLEMENTARY NOTES

[Document describes a computer program; SF-185, FIPS Software Summary, is attached.

11. ABSTRACT (A 200-word or less factual summary of most significant information. If document includes a significant bibliography or literature survey. mention it here)

This report summarizes the technical activities of the Center for Analytical Chemistry at the National Bureau of Standards. It emphasizes activities over the Fiscal Year 1983 in the Laser Analytical Chemistry Group, the Inorganic Analytical Research Division, the Organic Analytical Research Division, and the Gas and Particulate Science Division. In addition, it describes certain special activities in the Center including quality assurance and voluntary standardization coordination.

12. KEY WORDS (Six to twelve entries: alphabetical order: capitalize only proper names; and separate key words by semicolons) analytical chemistry; analytical services; gas and particulate science; inorganic analytical chemistry; organic analytical chemistry; quality assurance; voluntary standardization.

13. AVAILABILITY

Unlimited

14. NO. OF PRINTED PAGES

XX] For Official Distribution. Do Not Release to NTIS

$\square$ Order From Superintendent of Documents, U.S. Government Printing Office, Washington, D.C. 20402.

Order From National Technical Information Service (NTIS), Springfield, VA. 22161 
